\title{
GEOCHEMICAL ANALYSES OF SOILS AND SEDIMENTS, COEUR D'ALENE DRAINAGE BASIN, IDAHO: SAMPLING, ANALYTICAL METHODS, AND RESULTS
}

by Stephen E. Box ${ }^{1}$, Arthur A. Bookstrom ${ }^{1}$, Mohammed Ikramuddin ${ }^{2}$, and James Lindsay ${ }^{3}$

Open-File Report 01-139

2001

This report is preliminary and has not been reviewed for conformity with U.S. Geological Survey editorial standards or with the North American Stratigraphic Code. Any use of trade, firm, or product names is for descriptive purposes only and does not imply endorsement by the U.S. Government.

U.S. DEPARTMENT OF THE INTERIOR

U.S. GEOLOGICAL SURVEY

${ }^{1}$ U.S. Geological Survey, 904 West Riverside Avenue, Room 202, Spokane, WA 99201; sbox@usgs.gov; abookstrom@usgs.gov

${ }^{2}$ Geology Department, Eastern Washington University, Mail Stop 70, Cheney, WA 99004; midramuddin@ewu.edu

${ }^{3} 1231$ West Racine St., Bellingham, WA 98226 


\section{TABLE OF CONTENTS}

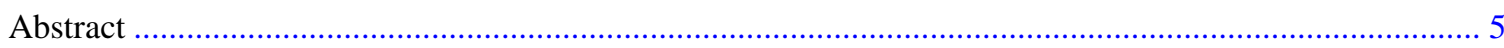

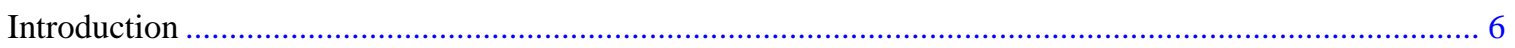

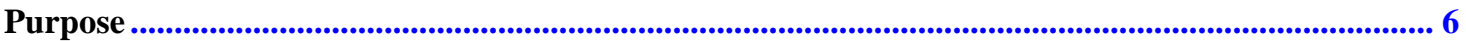

Participants and Cooperators.................................................................................................................... 7

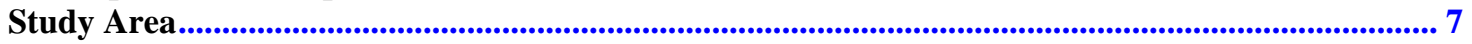

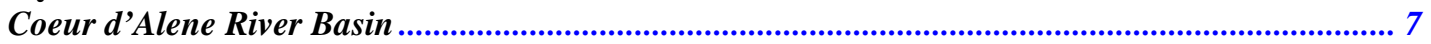

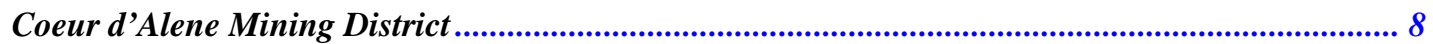

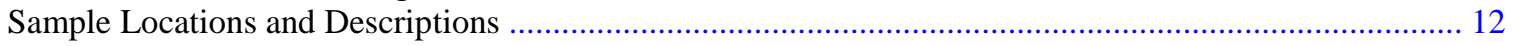

Sample-Site Location Methods ........................................................................................................... 12

Sample Descriptions ................................................................................................................................. 13

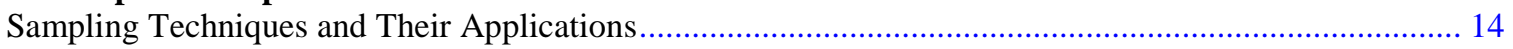

1. Surface Grab ............................................................................................................................ 14

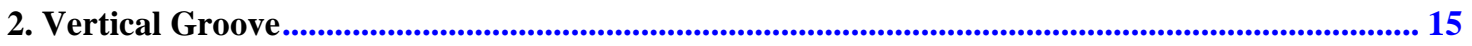

3. Depth-Bracket Sampling .................................................................................................................... 16

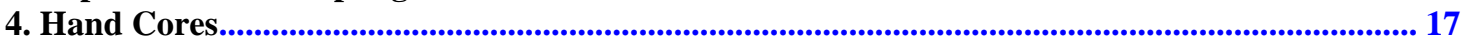

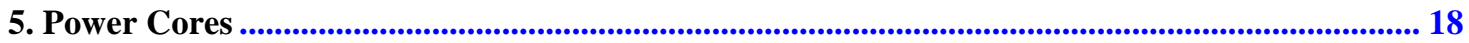

6. Vibro-Cores ....................................................................................................................................................... 19

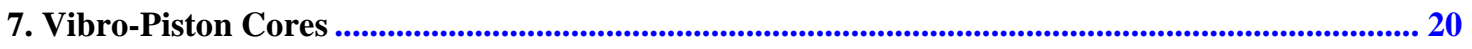

8. Auger In Vibro-Core Casing .......................................................................................................... 21

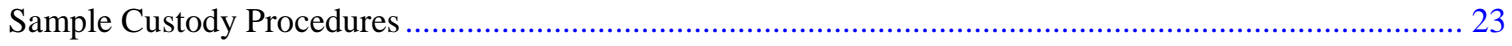

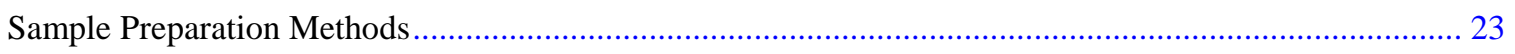

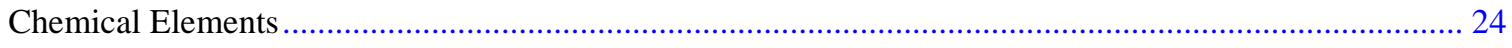

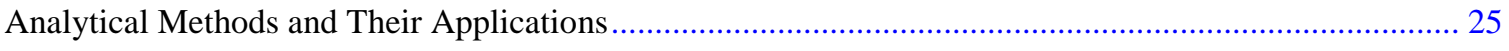

US Geological Survey energy dispersive Xray fluorescence analysis (USGS-EDXRF) .................. 25

Eastern Washington University Geochemistry Laboratory (EWU) Analysis .................................. 25

EWU sample digestion procedures (4-acid) ............................................................................. 25

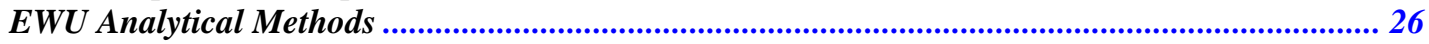

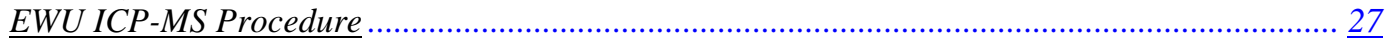

EWU ICP-AES Procedure ................................................................................................. 27

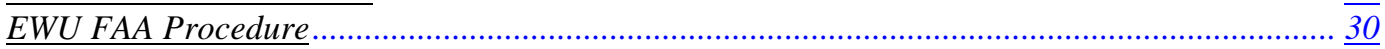

CHEMEX Labs Analysis .............................................................................................................. 30

XRAL Laboratories Analysis ........................................................................................................ 31

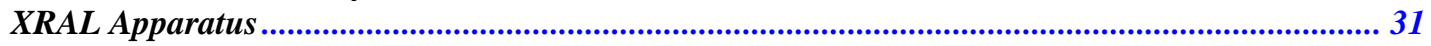

XRAL Reagents ............................................................................................................... 31

XRAL Sample Digestion Procedure (4-acid) ............................................................................... 32

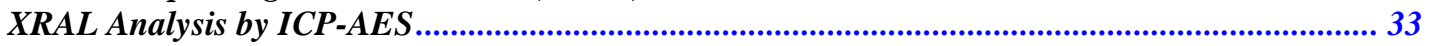

ACZ Laboratories....................................................................................................................................... 33

ACZ Microwave-Assisted Nitric Acid Digestion Procedure ...................................................... 34

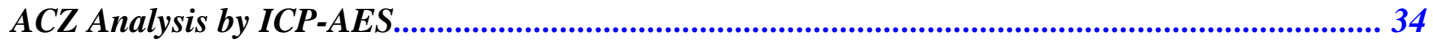

Analytical Quality Control, Assessment, and Assurance ........................................................... 34

Quality Control Procedures........................................................................................................... 35

US Geological Survey EDXRF Quality Control ...................................................................... 38

Eastern Washington University Geochemistry Lab (EWU) Quality Control................................. 38

EWU analyses of known Standard Reference Materials ..................................................... 38

EWU Duplicates and Blanks ........................................................................................ 40

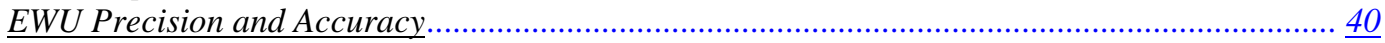

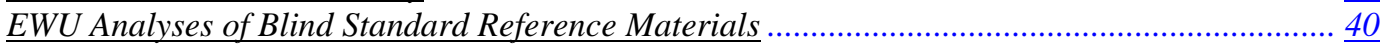

CHEMEX Laboratories Quality Control ..................................................................................... 40

XRAL Laboratories Quality Control .........................................................................................42

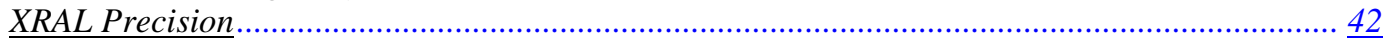




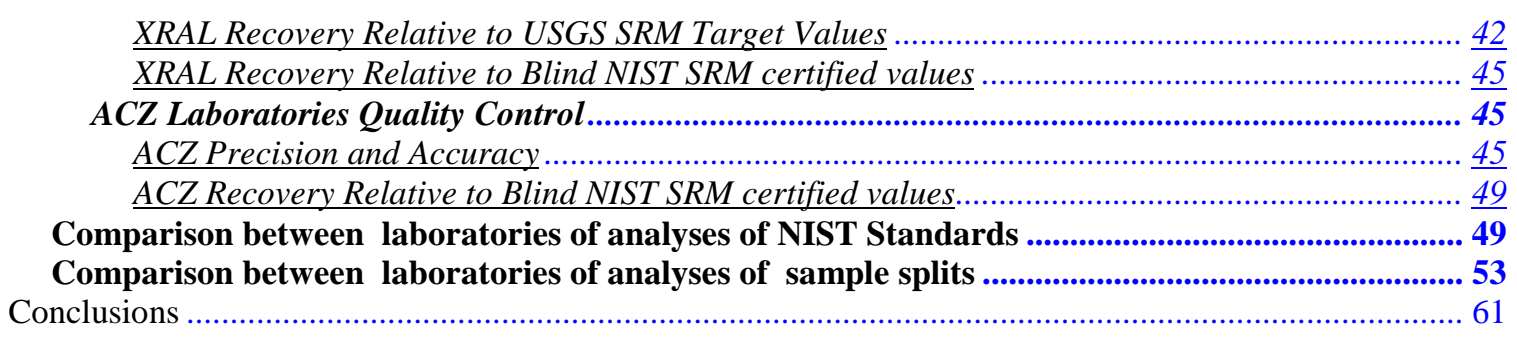

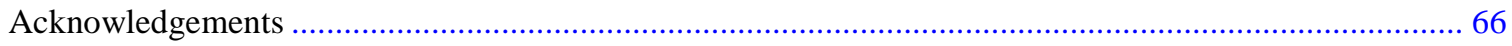

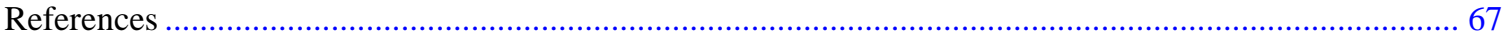

\section{FIGURES}

Figure 1. Location map of study area within Spokane River basin, Idaho and Washington. .................... 10 Figure 2. Index map of CdA River basin study area with outlines of sample site location maps............... 11

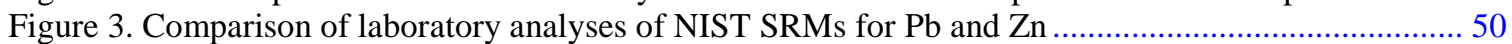

Figure 4. Comparison of laboratory analyses of NIST SRMs for Fe and Mn ................................... 51

Figure 5. Comparison of laboratory analyses of NIST SRMs for As and Cd....................................52

Figure 6. Comparison of $\mathrm{Pb}$ analyses of sample splits by EWU with those of other laboratories.............. 54

Figure 7. Comparison of $\mathrm{Zn}$ analyses of sample splits by EWU with those of other laboratories. ............. 55

Figure 8. Comparison of Fe analyses of sample splits by EWU with those of other laboratories.............. 58

Figure 9. Comparison of Mn analyses of sample splits by EWU with those of other laboratories.............59

Figure 10. Comparison of As analyses of sample splits by EWU with those of other laboratories............. 62

Figure 11. Comparison of Cd analyses of sample splits by EWU with those of other laboratories. ........... 63

\section{TABLES}

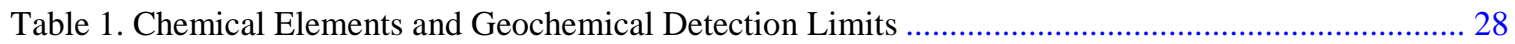

Table 2. USGS-EDXRF Instrumental Run Parameters ........................................................................ 29

Table 3. Quality Control Procedures for Geochemical Analysis ..................................................... 37

Table 4. USGS-EDXRF analysis of four NIST Standard Reference Materials ..................................... 39

Table 5. EWU Precision and Accuracy Relative to NIST-Certified Values............................................ 41

Table 6. XRAL Precision and Accuracy Relative to USGS SRM SAR-L. ..............................................43

Table 7. XRAL Precision and Accuracy Relative to USGS SRM SAR-M........................................... 44

Table 8. Precision and accuracy of ACZ analyses of calibration solutions and SRMs ........................... 46

Table 9. EWU, XRAL and ACZ Blind Analyses Relative to NIST-Certified Values for SRM 2711........ 47

Table 10. EWU, XRAL and ACZ Blind Analyses Relative to NIST-Certified Values for SRM 2710....... 48

Table 11. Comparisons of $\mathrm{Pb}$ and $\mathrm{Zn}$ values by EWU and by NIST with those of other labs. ...................56

Table 12. Comparisons of Fe and Mn values by EWU and by NIST with those of other labs................... 60

Table 13. Comparisons of As and Cd values by EWU and by NIST with those of other labs.................. 64 


\section{APPENDICES}
A. Sample location maps (A-1 to A-10)
B. Sample site location information
C. Lithologic descriptions of samples
D. USGS EDXRF analytical data
E. EWU 4-acid ICP-MS, ICP-AES, and FAA analytical data
F. CHEMEX nitric/aqua regia ICP-AES analytical data
G. XRAL 4-acid ICP-AES analytical data
H. ACZ microwave-assisted nitric-acid ICP-AES analytical data
J. List of digital files associated with this report 


\begin{abstract}
This report presents the locations, descriptions, analytical procedures used, and an inter-lab comparison of over 1100 geochemical analyses of samples of soil and sediment in and downstream of a major lead-zinc-silver mining district in the Coeur d'Alene (CdA) drainage basin of northern Idaho. The samples fall in 3 broad categories: (1) samples from vertical profiles of floodplain soils in the valley of the main stem of the CdA River (767 samples) and of the South Fork of the CdA River (38 samples), (2) size fractionated surficial samples of sediment bedload within the channel of the South Fork of the CdA River (68 samples), and (3) samples from vertical profiles of sediment bedload within the channel of the main stem of the CdA River (260 samples).
\end{abstract}

Five different laboratories contributed geochemical data for this report. Four of the five laboratories employed analytical methods that require sample dissolution prior to analysis; one laboratory (US Geological Survey) used analytical instrumentation (energy dispersive $\mathrm{X}$-ray fluorescence [EDXRF]) that is applied to pulverized samples. Some dissolution procedures use four acids (hydrochloric, nitric, perchloric, and hydrofluoric; Eastern Washington University [EWU] Geochemical Laboratory and XRAL Laboratories, Inc.), others use two acids (nitric acid and aqua regia; CHEMEX Labs, Inc.), and some use only concentrated nitric acid (ACZ Laboratories, Inc.). Most analyses of dissolved samples were done by Inductively Coupled Plasma - Atomic Emission Spectroscopy (ICP-AES) or by ICP - MS (Mass Spectroscopy). Some analyses for Ag and $\mathrm{K}$ were done by Flame Atomic Absorption (FAA).

Inter-laboratory comparisons are made for 6 elements: lead $(\mathrm{Pb})$, zinc $(\mathrm{Zn})$, iron $(\mathrm{Fe})$, manganese $(\mathrm{Mn})$, arsenic (As), and cadmium $(\mathrm{Cd})$. In general inter-laboratory correlations are better for samples within the compositional range of the Standard Reference Materials (SRMs) from the National Institute of Standards and Technology (NIST). Analyses by EWU are the most accurate relative to the NIST standards (mean recoveries within $1 \%$ for $\mathrm{Pb}, \mathrm{Fe}, \mathrm{Mn}$, and $\mathrm{As}, 3 \%$ for $\mathrm{Zn}$ and $5 \%$ for $\mathrm{Cd}$ ) and are the most precise (within $7 \%$ of the mean at the $95 \%$ confidence interval). USGS-EDXRF is similarly accurate for $\mathrm{Pb}$ and $\mathrm{Zn}$. XRAL and $\mathrm{ACZ}$ are relatively accurate for $\mathrm{Pb}$ (within 
5-8\% of certified NIST values), but were considerably less accurate for the other 5 elements of concern (10-25\% of NIST values). However, analyses of sample splits by more than one laboratory reveal that, for some elements, XRAL ( $\mathrm{Pb}, \mathrm{Mn}, \mathrm{Cd})$ and $\mathrm{ACZ}$ $(\mathrm{Pb}, \mathrm{Mn}, \mathrm{Zn}, \mathrm{Fe}$ ) analyses were comparable to EWU analyses of the same samples (when values are within the range of NIST SRMs). These results suggest that, for some elements, XRAL and ACZ dissolutions are more effective on the matrix of the CdA samples than on the matrix of the NIST samples (obtained from soils around Butte, Montana). Splits of CdA samples analyzed by CHEMEX were the least accurate, yielding values $10-25 \%$ less than those of EWU.

\section{Introduction}

This report presents a compilation of about 1,000 geochemical analyses of alluvial sediments, collected from stream- and river- beds, banks, and floodplains downstream from mines and mills of the Coeur d'Alene (CdA) silver-lead-zinc mining district in north Idaho. Methods of sample collection, preparation, and analysis are described. Locations of sample sites are shown on maps and listed in tables showing latitude and longitude (in degrees to four decimal places), sample-depth intervals ( $\mathrm{cm}$ ), sample descriptions, and chemical analytical results for as many as 40 chemical elements.

\section{Purpose}

The purpose of this report is to document the results of about 1,000 chemical analyses of samples of sediments that were deposited in the CdA drainage basin before and during the era of large-scale mining in the CdA mining district, in north Idaho. These samples were collected as part of an effort to determine the character, distribution, thickness, volume, and metals contents of sediments and soils within the CdA drainage basin. Such information is needed for a Natural Resource Damage Assessment (NRDA), and for an environmental Remediation Investigation/Feasibility Study (RI/FS) being conducted by the Environmental Protection Agency. In response to requests for information from litigants on both sides of an ongoing court case, descriptions of sampling and analytical methods and procedures given here are more inclusive than those 
that would accompany most purely scientific publications.

\section{Participants and Cooperators}

Employees and associates of the U.S. Geological Survey - Geologic Division (USGS-GD), Mineral Resources Program (MRP), Spokane Field Office (SFO) collected these sediment samples in the course of a series of geo-environmental studies conducted from 1993 to 1999. The geochemistry laboratory in the Department of Geology at Eastern Washington University provided many of the chemical analyses, and the University of Idaho provided a Livingston core sampler. This work was funded by USGS-GD-MRP. It was done in cooperation with the Coeur d'Alene Tribe, the U.S. Geological SurveyWater Resources Division (USGS-WRD), U.S. Fish and Wildlife Service, U.S. Environmental Protection Agency, U.S. Bureau of Land Management (USBLM), and U.S. Department of Agriculture-Forest Service (USDA-FS).

\section{Study Area}

The study area covered by this report is mostly within the CdA River basin in north Idaho (figure 1). The CdA mining district, which lies mostly within the South Fork drainage basin (figure 2), is one of the largest past-producers of silver in the world, and is also a giant past-producer of lead and zinc (Long, De Young, and Ludington, 1998).

\section{Coeur d'Alene River Basin}

The CdA River drains a large part of the north Idaho panhandle -- from a divide that defines Idaho's eastern border, to CdA Lake, near Idaho's western border (figure 1). The CdA River Basin occupies the western side of the northern Bitterroot Range, between the Clark Fork River Basin to the northeast, and the St. Joe River Basin to the south. The North Fork of the CdA River drains an area of about $900 \mathrm{sq}$ mi, and its

average discharge is about 2,000 $\mathrm{ft}^{3} / \mathrm{s}$. The South Fork of the CdA River drains an area of about $300 \mathrm{sq} \mathrm{mi}$, and its average discharge is about $500 \mathrm{ft}^{3} / \mathrm{s}$.

The North and South Forks of the CdA River join near Enaville, Idaho, to form the main stem of the CdA River, which meanders about $58 \mathrm{~km}$ (36 mi) southwesterly to 
CdA Lake, near Harrison, Idaho (figures 1 and 2). Relatively steep gradients of the North and South Forks flatten downstream, and approach a nearly flat gradient from Cataldo Flats to CdA Lake. The cobble-gravel bottom of the river channel upstream from Cataldo Flats to the mouth of the CdA River into CdA Lake. The cobble-gravel bottom of the river channel upstream from Cataldo Flats gives way to a large central sand bar, which occupies a wide bend in the river channel at Cataldo boat landing. Metal-enriched riverbottom sediments are predominantly sandy from there to the toe of the delta front on CdA Lake. Most of the bottom of CdA Lake beyond the delta is covered by fine-grained metal-enriched sediment (Horowitz, Elrick and Cook, 1993).

The Post Falls Dam, on the Spokane River west of the northwest outlet of CdA Lake, holds summer lake level at 2,125 ft elev, and backs lake water up to about Cataldo Landing (figure 2). Thus, in summer, the CdA River becomes a long, narrow, meandering, eastern extension of the Post Falls - CdA Lake reservoir, with little or no current along the $47 \mathrm{~km}$ (29 mi) river distance between Cataldo Flats and CdA Lake (figure 2).

\section{Coeur d'Alene Mining District}

The CdA mining district is one of the giant silver-lead-zinc mining areas in the world. Its past production ranks first in the United States for silver and third for lead and zinc. Remaining resources of silver rank fourth in the United States (Long, De Young, and Ludington, 2000). The CdA district includes the Bunker Hill mine, mill, tailings impoundment, smelter, and smelter-emissions fallout zone, all of which are in the Bunker Hill Superfund Site (figure 1). The CdA district also includes about 30 other significant mine/mill complexes, and more than 100 relatively small mines and prospects, some of which are in the North Fork drainage basin. To date, the CdA district has produced about 7 million metric tonnes of lead, 3 million tonnes of zinc, and 30 thousand tonnes of silver (Long, 1998a). Significant resources remain (Long, DeYoung, and Ludington, 1998), and, as of mid-2000, production continues at a few major mines.

Mining and milling in the CdA mining region have produced approximately 109 million tonnes of tailings, containing over 1 million tonnes of lead, 1 million tonnes of 
zinc, and 3 thousand tonnes of silver (Long, 1998b). From 1896 to about 1910, the predominant milling technology included hand sorting, crushing with stamp mills, and gravity separation. Jigs sorted particles according to their settling velocities by "jigging" them up and down on under-water screens, or by forcing pulses of water up through the screens and particles. Zinc was not intentionally recovered, and lead recoveries commonly ranged from 50 to 80 percent. Tailings commonly contained up to $5 \mathrm{wt}$. percent each of lead and zinc (Long, 1998b). Addition of other gravity separation devices improved recoveries somewhat, but recovery from slimes (silt and finer grain sizes) remained poor. Most mill-sites were on hillsides near the bottoms of narrow canyons, and their tailings commonly were discarded directly or indirectly into passing streams. Slimes tended to wash away rapidly, but sand- and pebble-sized tailings tended to accumulate near mills, to be washed downstream during high stream-flow episodes.

The flotation process was introduced in the early 1910's to treat tailings from gravity separators. By the early 1930's, most mills had converted to flotation as their principal recovery method. In flotation cells, ore-mineral particles preferentially adhere to surfaces of bubbles formed by agitation and injection of air into a slurry of finely ground mineral particles, water, and oily frothing agents. The bubbles rise through the froth, collecting ore particles, and carrying them to the surface, where they are paddled into collecting troughs. Mineral particles that do not attach to the bubbles sink, forming a slurry of tailings in oily water. Successive sink-float cycles are necessary to concentrate each recoverable ore mineral (generally lead sulfide and zinc sulfide in this case). Adoption and improvement of flotation techniques gradually increased metal recoveries, allowing mines to produce larger amounts of lower-grade ores. This resulted in production of larger quantities of finer-grained tailings (fine sand and finer grain sizes) with lower metal contents. 


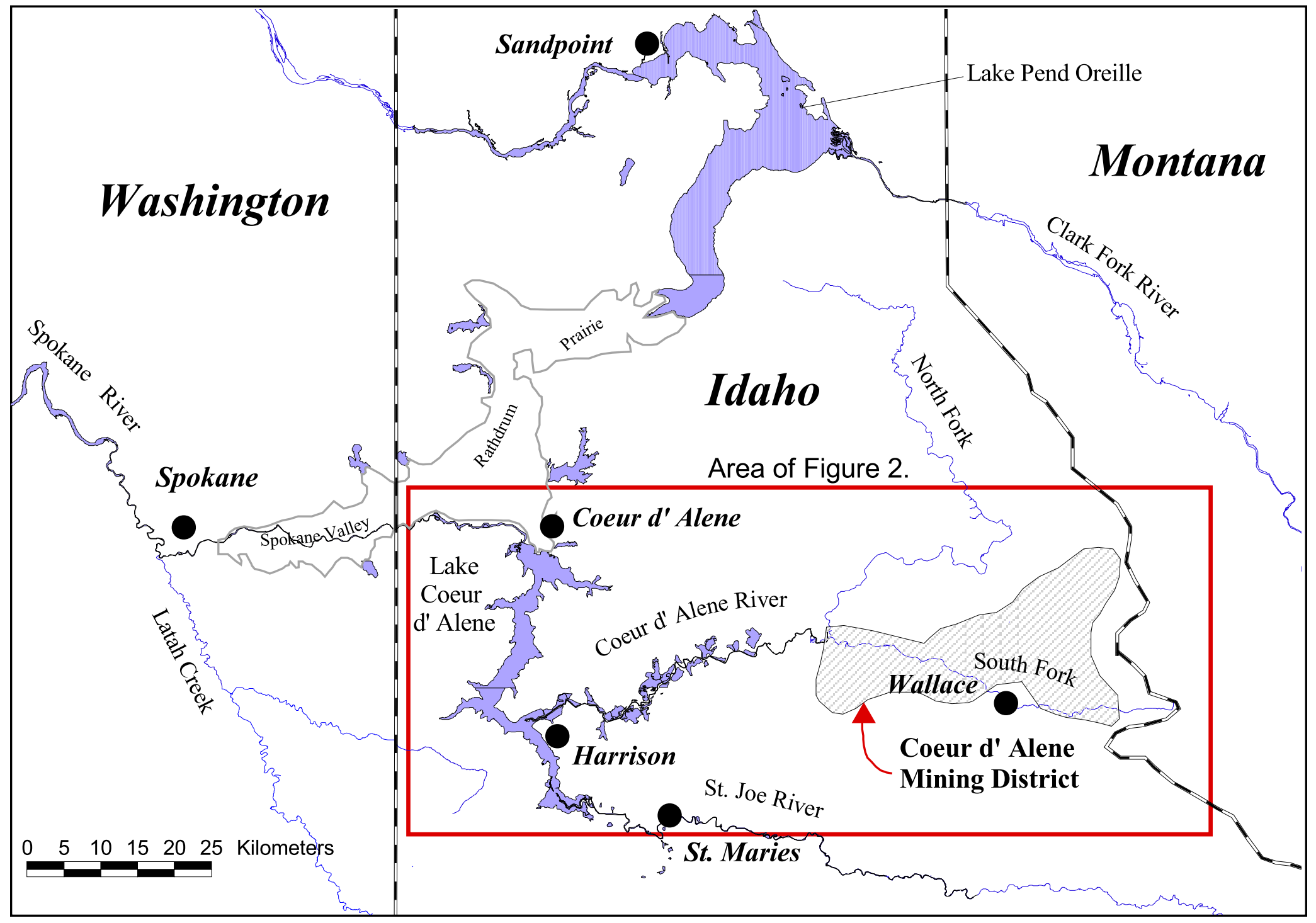

Figure 1. Regional map of the Coeur d'Alene River system and other major tributary streams and rivers of the Spokane River basin in northern Idaho, eastern Washington and western Montana. Area of figure 2 shown by outline. 


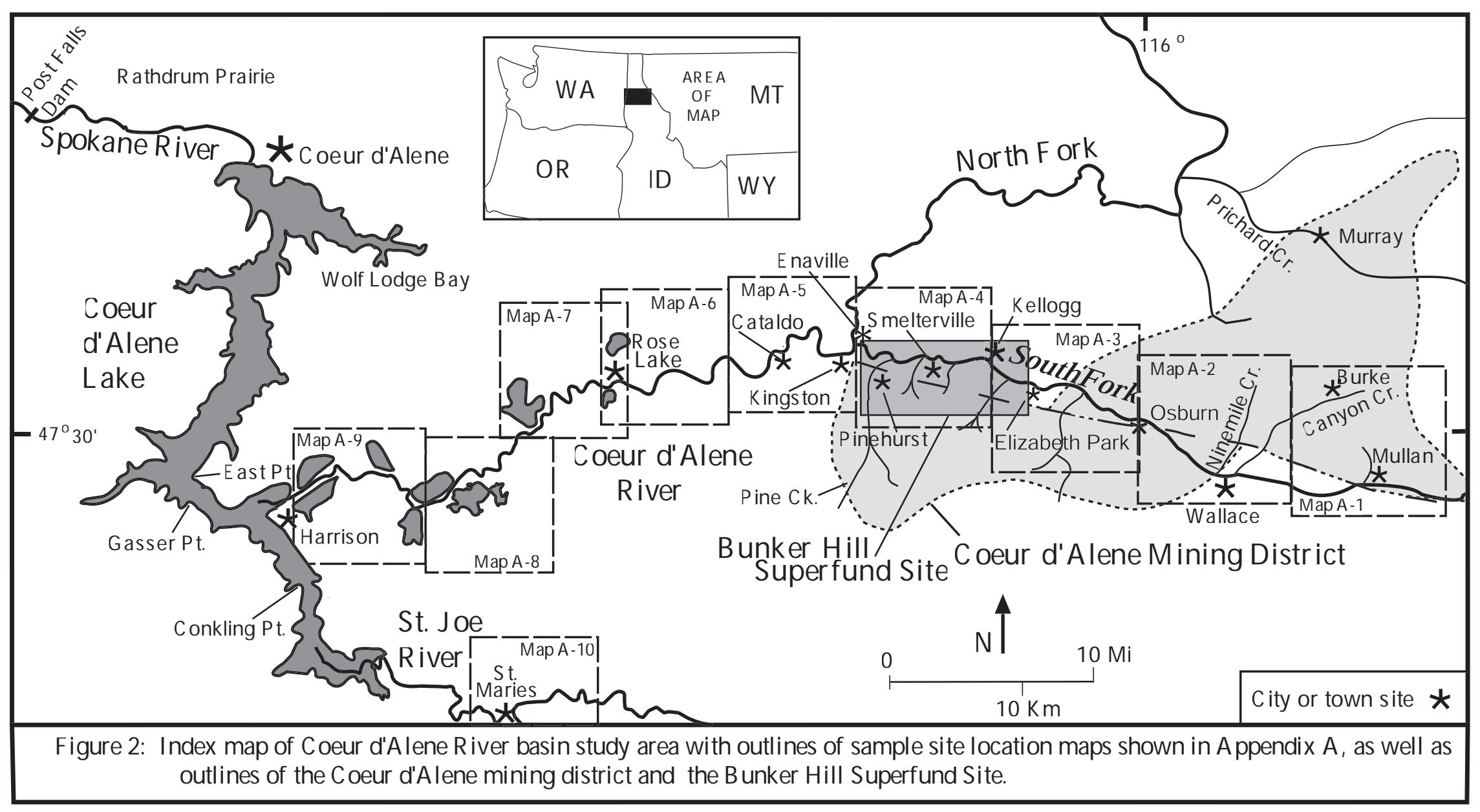


Metal-enriched sediments of the CdA drainage basin are derived mostly from mining and milling wastes, discarded into the South Fork and its tributaries. Mill tailings were washed down-valley and onto floodplains by fluvial processes, including annual spring run-offs, and sudden winter floods, caused by rain-on-snow episodes.

Approximately 51 percent of the tailings generated in the $\mathrm{CdA}$ district were discarded directly into creeks that are tributary to the CdA River (Long, 1998b). The Bunker Hill and Page mills used tailings-settling ponds beginning in 1927, but most other mills discarded tailings into creeks until 1968, when that was prohibited by Federal law. Prior to 1968 , an average of about 2,000 metric tonnes of metal-bearing mine slimes were being discarded into streams each day (Hoffman, 1995), and the South Fork ran "the color of "dirty dough"” with suspended mill tailings (Rabe and Flaherty, 1974). At the confluence of the North and South Forks the flow volume of muddy South Fork water met and mixed with about 4 times its flow volume of relatively clear North-Fork water, to form the larger CdA River, which ran turbid with suspended sediment.

From 1932 to 1967 a suction dredge removed metal-enriched sediment from the river bottom near Cataldo Landing, and placed it on Cataldo Flats, forming extensive dredge-spoil deposits on the floodplain there. Each year the dredge excavated an area of about $10 \mathrm{hm}^{2}$ (25 acres) of river channel to a depth of about $6.7 \mathrm{~m}(22 \mathrm{ft})$, forming a crescent-shaped dredge pond about $180 \mathrm{~m}$ (600 ft) across and 1,200 m (2,800 ft) long (Grant, 1952). Dredging was discontinued in 1967, after which tailings were no longer discarded into streams. Aerial photographs made in 1983 show that by then the dredge pond had filled, and the central sand bar had formed in approximately its present location, size and shape.

\section{Sample Locations and Descriptions}

\section{Sample-Site Location Methods}

Sample locations are tabulated in Appendix B as decimal degrees of latitude and longitude, keyed to the North American datum of 1927 (NAD27) and given to 4 decimal places. Appendix B also lists the sample site location method used to locate each sample 
site. Samples collected prior to 1996 were located on 1:24,000 scale U.S. Geological Survey topographic maps by matching identifiable features on the map to corresponding features on the ground, and (or) by measuring or triangulating from such features. Latitude and longitude of sample-site map locations were determined by measurement from the map grid, and are tabulated in decimal degrees to 4 places (Appendix B). Locations are considered accurate to within about 50 to $150 \mathrm{ft}$, depending on the distinctiveness of the site and (or) its distance from features recognized on both the ground and the map (Appendix B, site-location method "a"). Reference locations on riverbanks at opposite ends of river-channel core transects were staked and flagged. The reference locations were marked on 1:24,000-scale maps, as described above. Drill holes in the channel of the river were located by compass-and-tape measurements from the reference locations. Sample sites along onshore extensions of river transects also were located by compass-and-tape measurements from the reference locations (Appendix B, site-location method "b"). The reference location stakes were re-visited in 1996 and locations were derived with a GPS instrument. After 1996 all sample sites were located with the NAVSTAR Global Positioning System (GPS) Precise Positioning Service (PPS), using a PLGGR receiver for Federal Civilian Users (Appendix B, site-location method "c"). Locations determined in this way are considered accurate to within about $10 \mathrm{~m}(33 \mathrm{ft})$.

All sample locations are plotted on digital raster graphic topographic maps (DRGs) in Appendix A (maps A1 to A10). Sample locations were also plotted on Digital Orthophoto Quarter Quadrangles (DOQQs) with a scale of 1:12000 (not shown). Sample locations on these two electronic base maps were compared to the original field maps in order to check the accuracy of the sample-site latitudes and longitudes. In some cases sample locations were corrected to reflect the known position relative to some feature on the DRG or DOQQ.

\section{Sample Descriptions}

Lithologic descriptions of the samples are given in Appendix C. Samples were described in the field (or laboratory) for grain size, sediment color, organic content, sedimentary and biogenic structures, and degree of cementation. Grain size was estimated using a hand lens by comparison with silt and sand samples of six classified sieve sizes, 
mounted on a card, and named according to the size classification used by most North American geologists and engineers (Compton, 1985). Color was estimated by comparing sediment color to the Rock Color Chart of the Geological Society of America (Goddard and others, 1970).

\section{Sampling Techniques and Their Applications}

Sampling of soil and sediment within the CdA valley fall in 3 broad categories: (1) samples from vertical profiles of floodplain soils in the valley of the main stem of the CdA River (767 samples) and of the South Fork of the CdA River (38 samples), (2) surficial samples of sediment bedload within the channel of the South Fork of the CdA River (68 samples), and (3) samples from vertical profiles of sediment bedload within the channel of the main stem of the CdA River (260 samples). The range of surficial environments and sample target depths necessitated a diverse array of sampling techniques. The sampling technique used at each sample site is given in Appendix B and described in some detail below.

\section{Surface Grab}

Surface grab samples are collected by scooping or scraping sediment from small sample sites, generally at or near the exposed sediment surface, without much digging. Spot-grab samples of sediment can be taken from relatively steep exposures, such as riverbanks, or from relatively flat exposures, such as subaerially exposed stream-beds or floodplains (Appendix B, sampling technique 1).

In the summer of 1994 Julie Eddy, an Eastern Washington University student under the direction of Stephen Box, collected surface grab samples of stream sediments of the South Fork of the Coeur d'Alene River and its tributaries (94JE samples, Appendix B). Samples were collected during summer low flow from wet or dry sediment bars within the high water channel of the South Fork of the Coeur d'Alene River and its tributaries. Each sample is a composite of 3-5 samples collected from the upper $5 \mathrm{~cm}$ of the bar over a 2-5 m distance with a shovel or plastic trowel. Finer grained accumulations behind obstacles in the river channel were generally targeted for 
collection. The samples were screened in the field with a $2 \mathrm{~mm}$ mesh screen, lightly washing the sample through the screen with river water. The oversized material was discarded. The pan with undersized material and water was allowed to settle for 10 minutes and the overlying water was gently poured off. The wet sample was placed in a pre-labeled cambric cloth bag and stored for transport in a plastic box. Samples were kept in the locked vehicle until they were transported to the locked USGS lab at Eastern Washington University (within 1-3 days of collection).

\section{Vertical Groove}

Vertical groove samples were scooped or scraped from a steep exposure, to form a vertical groove, roughly perpendicular to sedimentary layering. Vertical groove samples are representative of the stratigraphic interval from which they are taken. Vertical groove sampling has several advantages over auger- or core drilling. It takes less equipment and time. The material is visible before, during, and after the sampling. Sampling can be done without compressing or disrupting layering or other sedimentary structures.

Contamination of samples from vertically deeper samples by overlying materials, a problem with core sampling, can be avoided.

Vertical groove sampling was the main technique used to sample riverbank exposures (Appendix B, sampling technique 2, Rbw map unit). Sample intervals were selected based on subdivision of the stratigraphic section into lithologically distinct intervals. Groove sampling also was used to sample floodplain sediments exposed in test pits dug into floodplain sediments above the zone of water saturation. However, groove sampling is not well suited to sampling below the water table, where pit walls tend to collapse.

Before sampling, the exposure was cleaned by cutting a fresh exposure, working from top to bottom with a clean, sharp steel shovel. A stainless steel spoon or knife was used to scoop or scrape samples from the exposure, working from the bottom up, to avoid downward contamination by falling sediment. Removed sediment was caught and placed in new, finely woven, tightly sewn, 5.5 by 10.5 -inch cambric cloth bags, labeled with a sample site identifier and the sample interval depth range. Sampling tools and hands were 
well rinsed after each sample was collected to avoid cross-sample contamination.

\section{Depth-Bracket Sampling}

In 1998 EWU students John Wallis and Patrick Blair, under the direction of A.A. Bookstrom, collected 130 samples from 37 sites (labeled "T98-xx" in Appendix B) on the floodplain of the Coeur d'Alene River to fill gaps in the database on thickness and metal content of metal-enriched sediment, particularly in upland and palustrine environments. The sampling was done from late September to early November, as water levels declined, and access to marshy locations improved. Sample locations were determined from the NAVSTAR Global Positioning System (GPS) Precise Positioning Service (PPS), using a PLGGR receiver for Federal Civil Users. Locations are considered accurate to within about 30 to $50 \mathrm{ft}$.

At each sample site, a pit was dug and sampled, and (or) a hole was made and sampled with a core tube or auger (Appendix B, sampling technique 3). Samples were collected from a series of $4 \pm 1 \mathrm{~cm}$ depth intervals, vertically spaced at about $20 \mathrm{~cm}$ centers (at depths of 0 to the 1980 volcanic ash layer, or to $4 \mathrm{~cm}, 18$ to $22 \mathrm{~cm}, 38$ to 42 $\mathrm{cm}$, etc.). Spaced intervals were sampled to limit the size and number of samples, thus maximizing the number of sites that could be sampled and analyzed within budget. In most cases, visible contrast in the character of sediment between the bracketing samples indicates the bottom depth and thickness of the layer of metal-enriched sediment more precisely. At the outset we used a field lead-test kit to indicate whether the entire section of metal-enriched sediment had been sampled. However, by checking with a field XRF analyzer, we found that the field lead test is not dependable in oxidized metal-enriched sediment. After that, we took our samples to the BLM office in Coeur d'Alene, Idaho, where rapid preliminary analyses were done for lead using their portable Outukumpu ® XRF analyzer. This gave a reliable indication of whether we had reached the bottom of the section of metal-enriched sediment. If not, we could return to the site, and try to sample deeper.

A bucket auger was often used in 1998 to sample below the water table, where it was encountered in test pits. Bucket-auger samples are taken by pushing and twisting 
auger teeth into the ground, thus cutting a cylindrical hole, and forcing the cut-sediment into a collection cylinder, or "bucket," above the cutter teeth. Our stainless-steel bucket auger has curved triangular bit teeth that overlap, and extend about $5 \mathrm{~cm}$ ahead of an open-ended sample cylinder, $6.5 \mathrm{~cm}$ in diameter, and $20 \mathrm{~cm}$ long. The diameter of the cutting head is about $7 \mathrm{~cm}$. A "bucket handle" is welded to the top of the sample cylinder, and the drive pipe is screwed into a fitting at the top of the handle. Multiple sections of pipe can be added to lengthen the reach of the bucket auger. A cross bar at the top of the pipe is used to twist and press the auger into the soil, thus cutting and forcing sediment into the bucket. After penetrating $20 \mathrm{~cm}$, the auger is pulled, and the sample extracted. Sediment from the top third of the bucket is discarded to avoid down-hole contamination. Sediment from the bottom part of the bucket is collected for chemical analysis. Sample intervals were determined by the penetration of the auger required to fill the auger bucket.

\section{Hand Cores}

Various hand-driven coring devices were used to sample floodplain sediments below the water table, where test pits tend to collapse (Appendix B, sampling technique 4). One commercially available hand-driven coring device has a $2 \mathrm{~cm}(0.75$-inch)diameter transparent plastic core barrel inside a stainless steel drive tube with a slidehammer attachment. Because of excessive friction along the walls of that small-diameter core tube, it tended to penetrate faster than it accepted sediment into the core barrel. Therefore, core recoveries generally were poor, except in soft, water-saturated sediment. Our best use of this tool was to extend our sampling below the water table in hand-dug test pits. After recovery the filled plastic core tube was transported to the lab and split. Sample intervals were selected based on subdivision of the cored section into lithologically distinct intervals. Samples were collected into labeled cambric cloth bags.

To improve core recovery in marsh environments, we experimented with largerdiameter PVC plastic tubes, which we sharpened on one end, and split lengthwise by sawing. We taped the two halves of the split tube together to make a core tube that could be opened. We cleared away the mat of surface vegetation, placed a block of wood over the top end of the tube, and pushed and pounded it into the sediment as far as possible. 
We measured from the top of the tube to the ground, outside the tube to indicate penetration, and inside the tube to indicate core recovery. We taped shut the top of the tube to create a near vacuum, as we pulled it to extract the core. We experimented with 1, 2-, and 3-inch diameter tubes, and found that with increasing tube diameter, core recovery increased, but core retention during withdrawal decreased. Multiple attempts were often required to successfully extract full core tubes. When we successfully extracted a core, we opened the tube lengthwise, split the core with a stainless steel knife, measured and examined the core, marked sample intervals, and sampled. We collected samples into labeled cambric cloth bags for analysis from one half of the core, and for archive from the other split half.

In 1998 we used a relatively thin-walled aluminum pipe for hand coring. The thinwalled pipe was easier drive into the sediment than the thicker-walled PVC plastic pipe. However, the aluminum pipe, which was 3 inches in diameter, was not split. To remove the core, we tilted the pipe and tapped it to dislodge it, so that gravity would pull it from the pipe. The core was extruded onto a clean plywood board or plastic sheet, where it was split lengthwise with a knife, measured, described, and sampled into labeled cambric cloth bags.

\section{Power Cores}

In the summer of 1994 a Giddings power-impact coring machine was used to drill six core holes (Appendix B, sampling technique 5) along a transect northwest from the riverbank across Strobl Marsh, east of Killarney Lake (Appendix B, sites 94GID2 to 94GID6). The trailer-mounted Giddings coring machine is powered by a small engine, which rotates the drill string, and hydraulically presses the rotating steel drill pipe downward. Core is forced into a 2-inch-diameter transparent plastic core barrel inside the drill pipe. The machine can drill to about $20 \mathrm{ft}$, using 4-ft sections of drill pipe. Penetration and recovery were noted during drilling. The transparent core tubes with recovered sediment were transported back to the lab for sampling. In the lab the transparent core tubes were split lengthwise, and the recovered core was measured and described. Sample intervals were selected based on subdivision of the cored section into lithologically distinct intervals. Samples were collected into labeled cambric cloth bags. 
Because of uncertainty in the consistency of core recovery, sample intervals in Appendix $\mathrm{C}$ are the measured intervals plus the penetration depth of the start of the four foot core section. The gaps in sampled intervals in the GID- cores represent the difference between the core recovery and the 4 foot $(122 \mathrm{~cm})$ penetration interval.

\section{Vibro-Cores}

Vibro-core drilling is particularly well suited to core sampling in sandy unconsolidated sediment that is water-saturated. A vibrating drill pipe agitates the intergranular pore water, which lifts and separates sand grains, so that the drill pipe sinks more-or-less as it would in quicksand. Vibro-core drilling of CdA River bottom sediment was done from a USGS-WRD research boat in 1994, using 9 to $12 \mathrm{~m}$ (30-40 ft) lengths of $7.6 \mathrm{~cm}$ ( 3 inch) diameter aluminum pipe (Appendix B, sampling technique 6). A concrete vibrator, driven by an electric motor, and powered by a portable generator, was clamped to the pipe. The vibrator was tightly clamped perpendicular to the pipe by a specially built steel bracket. Eccentric rotation of a cable-driven mechanism in the head of the vibrator creates very strong vibrations, which are transmitted to the drill pipe. We lowered the vertical pipe, with vibrator attached, over the side of the boat, until it touched bottom, measured the bottom depth, and then turned on the vibrator. At first, the vibrating pipe penetrated rapidly, but as it filled with core, its vibration was dampened, and its penetration rate decreased. In some cases we increased penetration by standing on the vibrator clamp, and bouncing up and down as it vibrated. When penetration stopped, we measured the length of pipe above the water line and plugged the top of the tube with an expanding rubber plug, to create a near vacuum as we extracted the sediment-filled core tube, using the boat's motorized winch, with its cable wrapped around the vibrator clamp. Sandy material at the bottom of a hole tended to fall out of the tube as it was pulled up through the water column, but cohesive inter-beds of clay-rich sediment acted as plugs.

After transporting the horizontal core to the shore, we measured the length of the empty core tube above (and sometimes below, in the case of lost sand) the recovered core. The core was removed from the pipes by tipping them up to about $45^{\circ}$, and tapping their sides with a rubber mallet. Tapping reduced adhesion between the core and the tube, 
and gravity pulled the core downward, extruding it from the lower end of the tube. We extruded the core onto clean plastic sheets or wooden trays. We measured core length, and compared it to the depth of penetration. Except for sand lost from the bottom of the hole during retrieval, core recovery generally was nearly 100 percent. We split the core longitudinally and described litho-stratigraphic units. The core was divided into $33.3 \mathrm{~cm}$ intervals for sampling. We collected one half of the split core for analysis, and the other for reference. The samples were placed in labeled, 30 by $50 \mathrm{~cm}$ plastic bags, marked with the drill-hole number and depth interval (in $\mathrm{cm}$ ), and transported to the lab.

\section{Vibro-Piston Cores}

In the summer of 1995 we rented a Livingston Piston core sampler from the University of Idaho, and William C. Rember joined us in testing it in combination with our vibro-coring equipment, in an effort to penetrate completely through the metalenriched sediment in the river channel (Appendix B, sampling technique 7). None of the 1994 vibro-cores had completely penetrated the historic, metal-enriched sediment into pre-mining sediment with background metal concentrations. The piston-core sampler, as described by Sprenke and others (2000), had been used to core metal-enriched sediment on the bottoms of several lateral lakes in the lower CdA valley. It is operated from two inflatable neoprene rafts, joined by an aluminum frame and derrick. The drill string consists of 1 to $2 \mathrm{~m}$ sections of 5-cm outer pipe, attached at a downward-flared, and vented conical coupling, to which a 7.6-cm PVC plastic core tube is fitted and screwed. An inner 2-cm "stinger" pipe passes through a hole in the top of the conical coupling, into the core barrel, where a rubber piston is attached to its end. The piston fits snuggly into the lower end of the core tube. As the core tube is forced into the sediment, the rubber piston is forced toward the cap at the upper end of the tube. This creates a partial vacuum, which holds the sediment core in place as the core tube is pulled out of the hole. If a hole can be re-entered, it can be deepened in two-meter increments.

This piston core device had to be modified to successfully penetrate into the sandy sediments of the river bottom. Although the piston core device works well in relatively soft, cohesive lake-bottom sediment, sand is difficult to penetrate by pushing down on the core tube, and difficult to hold in the core tube when pulling up on it, 
because sand is strong under compression but weak under tension. To penetrate sand found in the river channel, the concrete vibrator used with our vibro-core device was attached to the drive pipe of the piston-coring rig to allow core tube penetration by vibrating the grains apart. To keep the hole from collapsing when the first core tube is pulled out of the hole, a $20 \mathrm{~cm}$ diameter PVC pipe was first vibrated down to about $2 \mathrm{~m}$. The vibro-piston core was begun within the $20 \mathrm{~cm}$ PVC casing. Penetration and recovery were carefully measured. Except for loss of most of the recovered sediment from the first core segment of 95PCK1 (sand slid out of the core tube while lifting the core out of the hole, accounting for the missing interval $15-168 \mathrm{~cm}$ ), recovery from the other two cores of 95PCK1 and from the one core of 95PCUD2 was 100\%.

The cores were transported to the lab and split longitudinally. The lithology of the split core was described and sample intervals were picked based on lithologic units . We collected one half of the split core for analysis, and the other for archival storage. The samples were placed in labeled, 30 by $50 \mathrm{~cm}$ plastic bags and marked with the drill-hole number and depth interval (in $\mathrm{cm}$ ).

\section{Auger In Vibro-Core Casing}

To increase the depth of vibro-core penetration, we used thin-walled aluminum vibro-core pipe as a casing, and used a bucket auger to remove some of the sediment from within the casing, thus freeing it to vibrate more freely and penetrate deeper (Appendix B, sampling technique 8). Using the dual rafts and aluminum derrick of the piston-core sampler, we vibrated a $9 \mathrm{~m}$ long, $7.6 \mathrm{~cm}$ diameter aluminum pipe into the river-bottom sediment until it stopped. Instead of capping and pulling the pipe, we bailed the water from the pipe, and used a bucket auger (with a $7 \mathrm{~cm}$ diameter and sample barrel $20 \mathrm{~cm}$ long) to remove successive $20-\mathrm{cm}$ increments of sediment. When the auger touched bottom, we marked the auger drive pipe at the top of the casing pipe, measured up $20 \mathrm{~cm}$, and twisted the auger down until the 20 -cm mark was at the top of the casing. We recorded the auger-sample intervals in a field notebook, and on sample bags. Samples were described and the top part of the sample was discarded to minimize possible downhole contamination. The sample for analysis was spooned from the lower end of the auger bucket, and the rest of the interval was bagged for archival storage. After partially 
emptying the aluminum drill pipe, we re-attached the vibrator and vibrated the pipe deeper into the sediment until it stopped again. Then we removed more sediment with the auger, and vibrated down again, until top of the core tube approached water level. By then we were in relatively cohesive sediment, and the hole stayed open enough that we were able to advance the auger into relatively cohesive sediment below the end of the pipe. In this way we were able to recover samples to depths of $486 \mathrm{~cm}$ (about $16 \mathrm{ft}$ ) in drill hole 95VCUD1, and $572 \mathrm{~cm}$ (about $19 \mathrm{ft}$ ) in 95VCD3.

Sediment flowage into hole 95VCUD1 occurred during vibration-penetration of the core tube below $338 \mathrm{~cm}$ sub-bottom. After vibrating the core tip to $338 \mathrm{~cm}$ below the bottom, we augered out the pipe to $306 \mathrm{~cm}$ sub-bottom (leaving $32 \mathrm{~cm}$ in core tube). The core tip was then vibrated to $414 \mathrm{~cm}$ sub-bottom, after which we found that the sediment surface in the core tube had moved up $205 \mathrm{~cm}$ (so our auger hit sediment at $101 \mathrm{~cm}$ subbottom). We augered to the end of the core tube at $414 \mathrm{~cm}$ sub-bottom (recording the new sample depths) and $72 \mathrm{~cm}$ beyond the bottom of the core tube to $486 \mathrm{~cm}$, with the walls of the hole remaining open below the tube. All of the auger samples were analysed and we found that $205 \mathrm{~cm}$ of material with consistent metal concentrations had flowed into the hole at $338 \mathrm{~cm}$, probably when the vibrator was turned on. We interpret that, after vibrating the core tip to $414 \mathrm{~cm}$, the interval augered from 101 to $133 \mathrm{~cm}$ had been floated up above the inflowing sediment from an original depth of 306-338 cm. All of the re-augered samples from 133-338 cm were the consistent metal content material (analyses not included here) that had flowed into the hole. The material below $338 \mathrm{~cm}$ had much higher metal contents and is interpreted to have remained at approximately its original depth during and after the overlying sediment flowage incident. Because of the above caveats, we are highly confident in the stratigraphic section above $306 \mathrm{~cm}$, and less confident in our interpretation of the true sample depths from $306-486 \mathrm{~cm}$.

Drill hole 95VCD3 was drilled after 95VCUD1, and, to prevent any recurrence of sediment flowage into the core tube, we did not remove more than about half of the sediment in the casing before vibrating it deeper, and there were no long time gaps during which sediment could move up into the pipe. The casing was driven to $309 \mathrm{~cm}$, emptied to $84 \mathrm{~cm}$, then driven to $360 \mathrm{~cm}$. From there to the end of the hole at $572 \mathrm{~cm}$, the hole remained open without casing. At 402 to $423 \mathrm{~cm}$ we penetrated a basal layer of lead-rich 
sediment, containing over 26,000 ppm. Below that, lead concentrations decrease downward but are high enough to indicate probable down-hole contamination of premining-era sediment.

\section{Sample Custody Procedures}

The chain of sample custody was not formally documented for any of the samples listed in this report. However, samples were labeled and tracked to avoid sample loss or confusion, and were kept in secure facilities to prevent sample tampering or contamination. Sample collectors collected and transported samples in lockable government vehicles, and retained custody of the samples until they were stored at lockable government-owned or rented offices, labs, or storage facilities in Spokane, and (or) Cheney, WA. Sample collectors made an effort to separate probable metal-rich and metal-poor samples, packaging and transporting them separately to avoid possible crosscontamination between metal-rich and metal-poor samples. Informal sample lists were used to track samples at EWU. Sample-submittal forms were prepared for samples submitted to commercial labs for sample preparation or chemical analysis. Pulps of most pulverized and chemically analyzed samples are stored in the USGS Lab at EWU. However, pulps of samples analyzed by XRAL Laboratories, Inc. (XRAL), are stored at the USGS sample archive in Denver, CO. Bulk reference samples are stored at a warehouse near Cheney, WA, rented by USGS and EWU.

\section{Sample Preparation Methods}

Sample preparation for samples collected before 1995 (except those labeled "94JE-", discussed below) was done in the USGS Lab at Eastern Washington University (EWU), by university students working under the direction of James Lindsay, Mohammed Ikramuddin, Stephen Box, Arthur Bookstrom, and funded through a cooperative agreement between USGS and EWU. Samples collected in 1995 and 1996 were prepared at SVL Analytical, Inc., in Kellogg, ID. Sample preparation for the 1998 samples was done at the USGS sample-control/sample-preparation facility in Denver, 
CO.

Upon arrival in the lab, samples were dried in a warm oven at $60-80^{\circ} \mathrm{C}$ (except samples labeled "T98-", which were freeze-dried at the sample-preparation facility in Denver, CO). Dried samples were passed through a minus 20-mesh sieve (grain diameters less than $0.83 \mathrm{~mm}$ ) to remove sticks, leaves, and roots. The minus 20 -mesh fraction was pulverized to minus 200 mesh (grain diameters less than $0.074 \mathrm{~mm}$ ) in a shatter-box with a chrome-molybdenum steel barrel.

The stream sediment samples labeled "94JE-" were handled differently. Samples were wet sieved in the field to capture only the less than $2 \mathrm{~mm}$ grain size fraction. After drying samples were split and one half sample was archived in labeled plastic sample jar. The other sample split was poured into two nested sieves (250 and 63 micrometer mesh size) and agitated with a Rotap device for 20 minutes. Each sieve fraction (0.250-2.0 $\mathrm{mm}, 0.063-0.250 \mathrm{~mm}$, and $<0.063 \mathrm{~mm}$ fractions) was weighed and placed in labeled plastic sample jars. The sieves and bottom pan were cleaned with a brush and blown out with compressed air between each sample fractionation. 8-10 grams of the 0.250-2.0 mm fraction was hand ground using an agate mortar and pestle to pass through a $0.318 \mathrm{~mm}$ sieve and placed in a separate labeled plastic sample jar. The ground 0.250-2.0 mm fraction from all the sample sites and the other two fractions from a subset of the sample sites were shipped to the Chemex labs in Reno, Nevada for analyses.

\section{Chemical Elements}

Table 1 lists chemical elements mentioned in this paper, gives their chemical symbols, and specifies detection limits for elements analyzed by Inductively-Coupled Plasma Atomic Emission Spectroscopy (ICP-AES). The elements are divided into those of generally major and minor geo chemical abundance. Concentrations of the major elements generally are expressed in wt. percent, whereas concentrations of the minor elements generally are expressed in parts per million parts (ppm). Some elements are listed without concentration units, because they are mentioned as reagents used in sample digestion procedures, but their abundance in the samples was not determined. 


\section{Analytical Methods and Their Applications}

\section{US Geological Survey energy dispersive Xray fluorescence analysis (USGS-EDXRF)}

Most of the samples collected in 1993 were analyzed for $\mathrm{Cu}, \mathrm{Pb}$, and $\mathrm{Zn}$, by USGS geochemists, James Lindsay and Bi Shea King, using Energy Dispersive X-Ray Fluorescence Spectroscopy (EDXRF) at the USGS Analytical Laboratory in Menlo Park, California (Johnson and King, 1987). Appendix D lists the analytical results. EDXRF analyses were performed as follows.

A prepared sample was placed in a plastic sample cup, the bottom of which is covered with 3.5- micrometer mylar film. The film was held tight with plastic ring around the outside. Each sample was analyzed in air, using a Kevex G700/7000 EDXRF Spectrometer, equipped with a $\mathrm{Rh}$ target $\mathrm{X}$-ray tube, $\mathrm{Si}$ (Li) detector, and 6 secondary targets, using 200-second count times, and the run-parameters summarized in table 2. Analyses were done for $\mathrm{Pb}, \mathrm{Zn}$, and $\mathrm{Cu}$. Matrix effects were compensated by ratio to the Compton scatter for the secondary target.

\section{Eastern Washington University Geochemistry Laboratory (EWU) Analysis}

Some of the 1993 samples and all of the 1994, 1995, and 1996 samples were analyzed at the Geochemistry Laboratory of Eastern Washington University by Dr. Mohammed Ikramuddin. All analyses were preceded by 4-acid "total extraction." Analysis of minor and trace elements was by Induced-Coupled Plasma - Mass Spectroscopy (ICP-MS). Major-element analysis was by Induced-Coupled Plasma Atomic Emission Spectroscopy (ICP-AES). Analysis for Ag and K were by Flame Atomic Absorption (FAA). Appendix E lists the analytical results. The sample digestions and analyses were performed as follows.

\section{EWU sample digestion procedures (4-acid)}

Nearly total digestion of most minerals is provided by 4-acid digestion procedures, which use nitric ( $\mathrm{HNO} 3)$, perchloric $(\mathrm{HClO} 4)$, hydrofluoric $(\mathrm{HF})$, and hydrochloric $(\mathrm{HCl})$ acids 
to extract chemical elements from solids for analysis. Nitric and perchloric acids are strong, oxidizing acids, which attack non-silicate minerals and oxidize organic matter. Hydrofluoric acid breaks strong silicate bonds, and $\mathrm{HCl}$ decomposes sulfides and dissolves the salts remaining after evaporation (Hall, 1999). Such digestion extracts most chemical elements from rock-forming silicate minerals, as well as from sulfide ore minerals, and their associated gangue minerals and weathering products. Minerals that are resistant to attack include cassiterite, rutile, monazite, ilmenite, garnet, wolframite spinels, sphene, beryl, zircon, tourmaline and high concentrations of barite, none of which are minerals of environmental importance in the CdA drainage basin.

1. A 250-mg sub-sample of the pulverized sample was weighed and placed in a teflon beaker. Two $\mathrm{ml}$ of $\mathrm{HNO}_{3}$ and one $\mathrm{ml}$ of $\mathrm{HClO}_{4}$ were added to the sample and heated until the volume was reduced to half. Then $2 \mathrm{ml}$ of $\mathrm{HF}$ and $5 \mathrm{ml}$ of aqua regia were added and the sample solution heated to dryness. Another $5 \mathrm{ml}$ of aqua regia is later added to the sample to completely break down any sulfide minerals.

2. The solution was heated to incipient dryness, and $4 \mathrm{ml}$ of $1: 1: 2 \mathrm{HCl}: \mathrm{HNO}_{3}: \mathrm{H}_{2} \mathrm{O}$ were added and the solution warmed. Finally, the solution was diluted to $25 \mathrm{ml}$ with ASTM Type I water (giving a final dilution factor of 100), and the solution was transferred to 60-ml polyethylene bottles. For ICP-MS analysis, the sample solutions were further diluted by a facto of 10 with Type I water.

3. For analysis of Ag a separate digestion was used. For this purpose, a prepared sample $(1.0 \mathrm{~g})$ was digested with aqua regia and diluted to $20 \mathrm{ml}$ with $2 \mathrm{~N} \mathrm{HCl}$.

4. Preparation blanks, duplicate samples, and reference standards were carried through the same procedure. Only Baker instra-analysed acids and Type I water were used during the digestion procedure.

\section{EWU Analytical Methods}

Elemental concentrations of $\mathrm{As}, \mathrm{Ba}, \mathrm{Be}, \mathrm{Cd}, \mathrm{Ce}, \mathrm{Co}, \mathrm{Cr}, \mathrm{Cs}, \mathrm{Cu}, \mathrm{Ga}, \mathrm{La}, \mathrm{Mo}, \mathrm{Ni}$, $\mathrm{P}, \mathrm{Pb}, \mathrm{Rb}, \mathrm{Sb}, \mathrm{Sn}, \mathrm{Sr}$, Th, Tl, U, V, W, Y:, Zn and Zr were determined by ICP-MS, using a Perkins Elmer Sciex model 5000 ICP-MS instrument. Concentrations of Al, Ca, Fe, 
Mg, Mn, and Ti were determined by ICP-AES, using a Perkin Elmer model ICP/6000 ICP-AES instrument. When concentrations of $\mathrm{Pb}$ and $\mathrm{Zn}$ were very high, their concentrations were determined by ICP-AES. Ag and $\mathrm{K}$ were analyzed using a Flame Atomic Absorption (FAA) Spectrophotometer, Perkin Elmer model 5000.

\section{EWU ICP-MS Procedure}

For all ICP-MS quantitative measurements an external calibration was used, and a linear regression (forced through zero) was applied to establish a calibration line. ICPMS calibration was performed using a reagent blank and multi-element standard solution the following concentrations: $\underline{50 \mathrm{ppb}}$ for $\mathrm{Ce}, \mathrm{Co}, \mathrm{Cr}, \mathrm{Cs}, \mathrm{Mo}, \mathrm{Pb}, \mathrm{Rb}, \mathrm{Sb}, \mathrm{Sr}, \mathrm{Th}, \mathrm{Tl}, \mathrm{U}$,

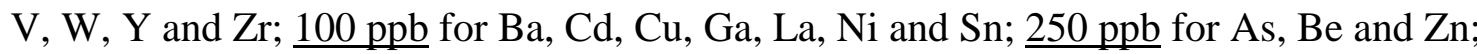
and $1000 \mathrm{ppb}$ for $\mathrm{P}$. These solutions were prepared by serial dilution of $1000 \mathrm{ppm}$ stock solutions of each element, obtained from Alfa Aesar Chemicals and Baker Chemicals. Internal standards ( $\mathrm{Sc}$, In and $\mathrm{Ho}$; or $\mathrm{Sc}, \mathrm{Rh}$ and $\mathrm{Pt}$ ), which provided a range of atomic masses used in the analyses, were added to each blank, standard and sample solution, to correct for instrument drift and physical interferences. The final concentrations of these elements were $50 \mathrm{ppb}$ of In, Rh and Ho, and $100 \mathrm{ppb}$ of Sc and Pt. For ICP-MS analysis the original 4-acid digestion solutions $\left(0.25 \mathrm{~g}\right.$ sample in $25 \mathrm{ml}$ with $4 \% \mathrm{HCl}$ and $\mathrm{HNO}_{3}$ ) were further diluted by a factor of 10 with Type I water, so that the final solutions contained $0.4 \% \mathrm{HCl}$ and $\mathrm{HNO}_{3}$. In some cases it was necessary to dilute the samples further to bring the concentrations of certain elements within the linear range.

\section{$\underline{\text { EWU ICP-AES Procedure }}$}

ICP-AES calibration was performed using a reagent-blank, and standard solutions with concentrations of $50 \mathrm{ppm}$ for $\mathrm{Ti}$, and $100 \mathrm{ppm}$ for $\mathrm{Al}, \mathrm{Ca}, \mathrm{Fe}, \mathrm{Mg}, \mathrm{Mn}, \mathrm{Pb}$ and $\mathrm{Zn}$. $\mathrm{Ca}, \mathrm{Mg}$. $\mathrm{Mn}, \mathrm{Pb}, \mathrm{Ti}$ and $\mathrm{Zn}$ were analyzed directly from 4-acid digestion-sample solutions, whereas for the determination of $\mathrm{Al}$ and $\mathrm{Fe}$, samples were further diluted by a factor of 10 and 20 respectively. Samples containing high concentrations of elements were further diluted to bring them within the linear range. 
Table 1. Chemical elements and geochemical detection limits

\begin{tabular}{|c|c|c|c|c|c|c|c|}
\hline $\begin{array}{l}\text { Major } \\
\text { Element } \\
\text { Symbol }\end{array}$ & $\begin{array}{l}\text { Major } \\
\text { Element } \\
\text { Name }\end{array}$ & $\begin{array}{l}\text { ICP AES } \\
\text { Detection } \\
\text { Limits }^{\mathrm{a}}\end{array}$ & $\begin{array}{l}\text { Chemex } \\
\text { Upper } \\
\text { Limit }^{\mathrm{b}}\end{array}$ & $\begin{array}{c}\text { Minor } \\
\text { Element } \\
\text { Symbol }\end{array}$ & $\begin{array}{l}\text { Minor } \\
\text { Element } \\
\text { Name }\end{array}$ & $\begin{array}{l}\text { ICP AES } \\
\text { Detection } \\
\text { Limits }^{a}\end{array}$ & $\begin{array}{c}\text { Chemex } \\
\text { Upper } \\
\text { Limit }^{\mathrm{b}}\end{array}$ \\
\hline & & wt \% & wt \% & & & ppm & $\overline{\mathrm{ppm}}$ \\
\hline$\overline{\mathrm{Al}}$ & Aluminum & 0.01 & 15 & $\overline{\mathrm{Ag}}$ & Silver & 0.1 & \\
\hline $\mathrm{Ca}$ & Calcium & 0.01 & 15 & As & Arsenic & 2 to 3 & 10,000 \\
\hline $\mathrm{Cl}$ & Chlorine & & & $\mathrm{Au}$ & Gold & & \\
\hline $\mathrm{F}$ & Fluorine & & & $\mathrm{Ba}$ & Barium & 1 to 10 & 10,000 \\
\hline $\mathbf{F e}$ & Iron & 0.01 & 15 & $\mathrm{Be}$ & Beryllium & 0.5 & 100 \\
\hline $\mathrm{H}$ & Hydrogen & & & $\mathrm{Bi}$ & Bismuth & 2 to 3 & 10,000 \\
\hline K & Potassium & 0.01 & 10 & Cd & Cadmium & 0.5 to 1 & 500 \\
\hline $\mathrm{Mg}$ & Magnesium & 0.01 & 15 & $\mathrm{Ce}$ & Cerium & & \\
\hline $\mathrm{N}$ & Nitrogen & & & Co & Cobalt & 1 & 10,000 \\
\hline $\mathrm{Na}$ & Sodium & 0.01 & & $\mathrm{Cr}$ & Chromium & 1 & 10,000 \\
\hline $\mathrm{O}$ & Oxygen & & & $\mathrm{Cu}$ & Copper & 0.5 to 1 & 10,000 \\
\hline$P$ & Phosphorus & 0.001 to 0.01 & 10 & $\mathrm{Eu}$ & Europium & & \\
\hline \multirow[t]{26}{*}{$\mathrm{Ti}$} & Titanium & 0.01 & & $\mathrm{Ga}$ & Gallium & 10 & 10,000 \\
\hline & & & & $\mathrm{Ge}$ & Germanium & & \\
\hline & & & & $\mathrm{Ho}$ & Holmium & & \\
\hline & & & & $\ln$ & Indium & & \\
\hline & & & & La & Lanthanum & 0.5 to 10 & 10,000 \\
\hline & & & & $\mathrm{Li}$ & Lithium & & \\
\hline & & & & Lu & Lutetium & & \\
\hline & & & & Mn & Manganese & 0.01 to 5 & 10,000 \\
\hline & & & & Mo & Molybdenum & 1 & 10,000 \\
\hline & & & & $\mathrm{Nb}$ & Niobium & & \\
\hline & & & & $\mathrm{Nd}$ & Neodymium & & \\
\hline & & & & $\mathrm{Ni}$ & Nickel & 1 & 10,000 \\
\hline & & & & $\mathbf{P b}$ & Lead & 2 & 10,000 \\
\hline & & & & $\mathrm{Sb}$ & Antimony & 2 to 5 & 10,000 \\
\hline & & & & Sc & Scandium & 0.5 & \\
\hline & & & & Sn & Tin & 10 & \\
\hline & & & & $\mathrm{Sr}$ & Strontium & 0.5 & \\
\hline & & & & $\mathrm{Ta}$ & Tantalum & 1 & \\
\hline & & & & Th & Thorium & & \\
\hline & & & & $U$ & Uranium & & \\
\hline & & & & $\mathrm{V}$ & Vanadium & 2 & \\
\hline & & & & W & Tungsten & 10 & \\
\hline & & & & $\mathrm{Y}$ & Yttrium & 0.1 & \\
\hline & & & & $\mathrm{Yb}$ & Ytterbium & & \\
\hline & & & & $\mathbf{Z n}$ & Zinc & 0.5 & \\
\hline & & & & $\mathrm{Zr}$ & Zirconium & 0.5 & \\
\hline
\end{tabular}

${ }^{a}$ Lower detection limits for analysis by ICP-AES, according to XRAL and Chemex catalogues.

${ }^{b}$ Upper limits for Chemex G32 package. Higher concentrations require dilution or chemical assay. 
Table 2. USGS-EDXRF instrumental run parameters

\begin{tabular}{|c|c|c|c|c|}
\hline Element & $\begin{array}{c}\text { Secondary } \\
\text { Target }\end{array}$ & Kv & MA & Line \\
\hline \hline $\mathrm{Pb}$ & $\mathrm{Ag}$ & 35 & 1.7 & L beta \\
\hline & & & & \\
\hline $\mathrm{Zn}$ & $\mathrm{Ge}$ & 20 & 2.0 & K alpha \\
\hline & & & & \\
\hline $\mathrm{Cu}$ & $\mathrm{Ge}$ & 20 & 2.0 & K alpha \\
\hline
\end{tabular}




\section{EWU FAA Procedure}

Three single-element standards of $\mathrm{Ag}$ and $\mathrm{K}(1,3$ and $6 \mathrm{ppm})$ were used for FAA calibration. Analyses for Ag in samples containing low concentrations of Ag were done using a single standard of $0.5 \mathrm{ppm}$ and a high sensitivity nebulizer. For analysis of $\mathrm{K}$, original 4-acid digestion solutions of samples were further diluted by a factor of 50 and blanks, standards and samples contained $1000 \mathrm{ppm}$ of Cs or Na to compensate for ionization interferences.

\section{CHEMEX Labs Analysis}

Splits of the 1993 samples, previously analyzed for $\mathrm{Pb}, \mathrm{Zn}$, and $\mathrm{Cu}$ by EDXRF, as well as samples labeled "94JE-", were analyzed for 32 elements by CHEMEX Labs, Ltd., in Reno, Nevada, using Induced-Coupled Plasma Atomic Emission Spectroscopy (ICP-AES) after extraction by nitric acid and aqua regia. Appendix F lists the analytical results.

Chemex Labs used a nitric acid - aqua regia digestion to extract chemical constituents from pulverized samples for ICP-AES analysis. Aqua regia is a mixture of 3 parts $\mathrm{HCl}$ to 1 part $\mathrm{HNO} 3$. It is an efficient solvent for numerous metal sulfides, many sulfates (except barite), some simple oxide minerals and their hydrates, phosphates, carbonates, and organically bound elements. However, most silicates and some oxides are only slightly attacked (Hall, 1999). Chemex Labs performed the digestions and analyses as follows.

A pulverized sample (1.0g) was digested with concentrated nitric acid for at least one hour. After cooling, hydrochloric acid was added to produce aqua regia, and the mixture was then digested for an additional hour and a half. The resulting solution was diluted to $25 \mathrm{ml}$ with demineralized water, mixed and analyzed by ICP-AES. Elemental concentrations were determined for 32 elements, using a Jarrell Ash 1100 plasma spectrometer after calibration with proper standards. The analytical results were corrected for spectral inter-element interferences. Concentrations higher than the Chemex upper detection limit (table 1) were reported as greater than that limit. 


\section{XRAL Laboratories Analysis}

Since 1997, XRAL Laboratories, Inc. has been the contract laboratory for the Mineral Resources Program of the Geologic Division of the U.S. Geological Survey. XRAL Laboratories analyzed our CdA soil samples collected in 1998 for 40 elements, using 4-acid digestion, followed by ICP-AES analysis. Appendix G lists the results. Apparatus, reagents, digestion procedures, and analytical methods used by XRAL Laboratories to perform these analyses are described below.

\section{XRAL Apparatus}

- Thermo Jarrell Ash, Model 1160 Plasma Atomcomp simultaneous ICP-AES or Perkin Elmer Optima 3000 simultaneous ICP-AES

- Hot plate with 50-position aluminum heating block

- 30-mL Teflon vessels with caps (Savillex)

- Acid dispensers (Labindustries)

- Repeating pipet (Eppendorf)

- Drying oven set at $95^{\circ} \mathrm{C}$

- $13 \times 100 \mathrm{~mm}$ disposable polypropylene test tubes with caps

\section{XRAL Reagents}

- Hydrochloric acid, $\mathrm{HCl}$ reagent grade, 37 percent

- Nitric acid, $\mathrm{HNO}_{3}$ reagent grade, 70 percent

- Hydrofluoric acid, HF reagent grade, 48 percent

- Perchloric acid, $\mathrm{HClO}_{4}$ reagent grade, 70 percent

- De-ionized water (DI)

- One percent nitric acid solution: $10 \mathrm{~mL} 70$ percent concentrated $\mathrm{HNO}_{3}$ diluted in $1000 \mathrm{~mL}$ DI water 
- Aqua regia: three parts concentrated $\mathrm{HCl}$ and one part concentrated $\mathrm{HNO}_{3}$; solution is not stable and must be prepared immediately before use

- Lutetium internal standard (Lu): $500 \mathrm{~g} \mathrm{Lu} / \mathrm{mL}$, as $\mathrm{Lu}_{2} \mathrm{O}_{3}$ in 5 percent (v/v) $\mathrm{HCl}$

\section{XRAL Sample Digestion Procedure (4-acid)}

1. Weigh $0.200 \mathrm{~g}$ sample into Teflon vessel.

2. Add $100 \mu \mathrm{L} \mathrm{Lu} \mathrm{internal} \mathrm{standard} \mathrm{to} \mathrm{each} \mathrm{vessel} \mathrm{with} \mathrm{repeating} \mathrm{pipet.}$

3. Rinse side walls of Teflon vessel with a minimum amount of DI water.

4. In the fume hood, slowly add $3 \mathrm{~mL} \mathrm{HCl}$ and allow any reaction to subside.

5. Add $2 \mathrm{~mL} \mathrm{HNO}_{3}, 1 \mathrm{~mL} \mathrm{HClO}_{4}$, and $2 \mathrm{~mL} \mathrm{HF}$. Place sample solution vessel on hot plate with aluminum heat block at a controlled temperature of $110^{\circ} \mathrm{C}$ in a perchloric acid fume hood.

6. Evaporate sample solution to hard dryness on hot plate (usually overnight).

7. Remove from hot plate, cool to touch and add $1 \mathrm{~mL} \mathrm{HClO}_{4}$ and 2 to $3 \mathrm{~mL}$ DI water.

8. Return to hot plate, and evaporate to hard dryness. The temperature of the hot plate is increased to $160^{\circ} \mathrm{C}$. This step usually takes a few hours.

9. Remove dried sample from hot plate and cool.

10. Add $1.0 \mathrm{~mL}$ aqua regia with repeating pipet and let react for $15 \mathrm{~min}$.

11. Add $9.0 \mathrm{~mL} 1$ percent $\mathrm{HNO}_{3}$ and thread screw cap tightly on vessel. Place vessel in drying oven for 1 hour at a controlled temperature of $95^{\circ} \mathrm{C}$.

12. Remove sample solution and cool. Transfer solution into labeled disposable polypropylene test tube and cap with test tube cap.

13. Analyze sample solution by ICP-AES.

Sample decomposition using this multi-acid digestion technique is suited to dissolve certain rock types, soils, and sediments. The method does not fully dissolve 
refractory or resistant minerals and some secondary minerals. Examples of incomplete digestion are as follows: $\mathrm{Ba}$ in barite, $\mathrm{Cr}$ in chromite, $\mathrm{Ti}$ in rutile, $\mathrm{Sn}$ in cassiterite, $\mathrm{Al}$ in corundum, and rare earth elements in monazite. Samples that contain elements in high concentrations where normally the element is a trace constituent or beyond the linear working range have to be diluted (i.e., $\mathrm{Mg}$ in a dolomite, $\mathrm{Pb}$ in a galena, $\mathrm{Zn}$ in a sphalerite, $\mathrm{Cu}$ in a chalcopyrite). This dilution increases the lower reporting limits.

\section{XRAL Analysis by ICP-AES}

The ICP-AES instrument is calibrated at the start of each day using four-element solutions for the Jarrell Ash 1160, and nine solutions for the Perkin-Elmer Optima. Calibrations are checked against certified total mean values for established geological Standard Reference Materials, including SO3 and STILL4 from the Canadian Certified Reference Materials Project, and USGS Basalt BHVO-1. The major and trace elements are determined by comparing the element intensities obtained from the standards to those obtained from the samples. There are three method preparation blanks digested with each sample set. A blank subtraction is performed to negate the effect of the reagents.

\section{ACZ Laboratories}

Campbell and others (1999), of the U.S. Fish and Wildlife Service, reported the results analyses of about 800 surface-sediment samples from wetlands of the CdA River valley. ACZ Laboratories, Inc. analyzed these samples for $\mathrm{Pb}, \mathrm{Zn}, \mathrm{Fe}, \mathrm{Mn}, \mathrm{As}$, and $\mathrm{Cd}$, using microwave-assisted nitric-acid digestion, and ICP-AES. To test whether we could legitimately compile and use the USFWS and USGS data together, we needed data from analyses of the same samples, using nitric-acid digestion, as compared to EWU and XRAL four-acid digestions. Spits from the set of 27 previously analyzed samples, sent to XRAL Laboratories for analysis of four-acid extractions, were sent to ACZ Laboratories, for nitric-acid digestion and analysis by ICP-AES. Appendix $\mathrm{H}$ lists the results of analyses by ACZ Laboratories.

ACZ Laboratories used concentrated nitric acid digestion to prepare samples for ICP-AES analysis. Nitric acid is a strong oxidizing acid, however it is less effective than $\mathrm{HCl}$ in attacking sulfides, and is relatively ineffective in dissolving iron and manganese 
oxides because of its oxidizing properties. It is relatively ineffective in attacking silicate minerals. Digestion procedures and analytical methods used by ACZ Laboratories are described below.

\section{ACZ Microwave-Assisted Nitric Acid Digestion Procedure}

Splits of pulverized samples were digested using a microwave-assisted nitric-acid procedure specified by USEPA method 3051, in which a representative sample of up to $0.5 \mathrm{~g}$ is digested in $10 \mathrm{~mL}$ of concentrated nitric acid for 10 minutes using a suitable laboratory microwave heating unit. The sample and acid are placed in a fluorocarbon (PFA or TFM) microwave vessel. The vessel is capped and heated in the microwave unit. After cooling, the vessel contents are filtered, centrifuged, or allowed to settle and then diluted to volume and analyzed by the appropriate method.

\section{ACZ Analysis by ICP-AES}

Digested samples produced by the above procedure were analyzed for $\mathrm{Pb}, \mathrm{Zn}, \mathrm{Fe}$, Mn, As, and Cd by ICP-AES, according to EPA method CLP 3/90.

\section{Analytical Quality Control, Assessment, and Assurance}

"Quality Control (QC) includes those activities undertaken to control the quality of services delivered by analytical laboratories" (Kane, 1991)." These services need to be timely and economic as well as consistent and accurate to within acceptable limits. "QC can be maintained only if accuracy can be measured in some manner on an on-going basis; such measurement activities in the laboratory comprise quality assessment" (Kane, 1991). Quality Assurance (QA) "involves those activities undertaken by management to ensure that both the control and the assessment activities are performed routinely and documented adequately so that the quality of services delivered is defined for, and can be demonstrated to, the clients."

Economic constraints required the use of instrumental multi-element geochemical analyses rather than single element assays, even though single-element assays may be 
more accurate for high concentrations of Fe (over 15 wt \%), Pb, Zn, Mn or As (over 1000 ppm), or Cd (over 500 ppm).

\section{Quality Control Procedures}

Quality control procedures consist of a number of routine actions taken by the analytical laboratory to test the precision and accuracy of its analytical techniques. Repeated analysis of materials of known composition is the primary tool to assess the accuracy of the laboratory analyses, and repeated analyses of materials both of known and of unknown composition are used to assess analytical precision. Accuracy is a measure of how closely a result or an average result approaches the true value (Levinson, 1974), as represented by an accepted standard, or target value. Quantification of accuracy (relative to a common standard) provides a basis for comparison of results from different laboratories. Accuracy is expressed as mean percent recovery (mean of concentrations determined by the laboratory / target concentration) x $100 \%$. Precision is a measure of consistency, or the ability to reproduce or repeat the same result (Levinson, 1974). Random errors are assumed to follow a normal Gaussian distribution about their concentration, and analytical precision is then specified as the percent relative variation at the two standard deviation (95\%) confidence level (Fletcher, 1981). Analytical precision is calculated relative to the mean concentration, as $\mathrm{Pm}=(2$ standard deviations $/$ mean $) \mathrm{x}$ $100 \%$.

All of the samples analyzed for this report are in solid form, but these solids must first be dissolved using chemical reagents before analysis using the ICP and AA analytical techniques (EDXRF analysis is performed directly on the pulverized sample). Typically the ICP and AA analytical instruments are calibrated initially and checked at regular intervals using lab-prepared solutions of laboratory reagents in known proportions, while the EDXRF is calibrated and checked with pulverized SRMs. Analyses of blanks ( distilled water and/or chemical reagents used in preparation of sample solutions) are done to assure no elements of interest are being added during sample digestion and preparation of the sample solution. Reanalysis of analyzed samples and analysis of sample splits provide additional checks on the precision (repeatability) of replicate analyses of the same material. "Spikes" are samples to which a known 
concentration of elements of interest are added, and the analyses of spiked and unspiked samples are compared.

Pulverized Standard Reference Materials (SRMs) of known composition that encompass the range of compositions of the unknown samples are periodically analyzed (after dissolution for ICP and AA methods) to check the accuracy of the analytical method from sample preparation to instrumental analysis. The USGS-EDXRF and EWU geochemical laboratories used United States NIST SRMs for these check standards, whereas Chemex and XRAL used Canadian SRMs, and ACZ used standards commercially prepared by Environmental Resources Associates (ERA).

Typically, samples of known composition and replicate samples are also submitted "blind" to the analyst (so the analyst doesn't know they are a standard or replicate sample, and does not treat them with any special care) as an additional test of precision and accuracy. Blind submittals of USGS SRMs were used to assess the precision and accuracy of XRAL data. Accuracy of EWU, XRAL, and ACZ data were also tested by analysis of blind submittals of NIST SRMs, and comparison of laboratory results with NIST-certified values for the SRMs analyzed.

Table 3 summarizes what Quality Control procedures were performed routinely by each of the five laboratories that provided chemical analyses included in this report. The commercial laboratories (ACZ, XRAL, and CHEMEX) have computerized Laboratory Information Management Systems (LIMS), in which internal records of results from quality control procedures are maintained. However, only ACZ Laboratories, Inc. provided us with printed records of those results. USGS also maintains a LIMS for XRAL data on USGS Standard Reference Materials (SRMs), which are routinely submitted for analysis with each batch of samples analyzed by XRAL. The EWU academic research laboratory maintains internal records of results from its quality control procedures, but it is not staffed to make those records public. The USGS EDXRF Laboratory is no longer staffed, but we were able to retrieve some of the Quality Control information. 
Table 3. Quality control procedures, precision, and accuracy for geochemical analysis at the laboratories used for this report.

\begin{tabular}{|c|c|c|c|c|c|}
\hline subject & USGS-EDXRF & EWU & CHEMEX & XRAL & $\mathrm{ACZ}$ \\
\hline Laboratory Type & $\begin{array}{l}\text { government, } \\
\text { research }\end{array}$ & $\begin{array}{l}\text { academic, } \\
\text { research }\end{array}$ & $\begin{array}{l}\text { commercial, } \\
\text { accredited }\end{array}$ & $\begin{array}{l}\text { commercial, } \\
\text { USGS contractor }\end{array}$ & $\begin{array}{l}\text { commercial, } \\
\text { USFWS contractor }\end{array}$ \\
\hline Number of Analyses & 203 & 336 & 236 & 331 & 27 \\
\hline Digestion Method & none & 4-acid & $\begin{array}{l}\text { nitric acid and } \\
\text { aqua regia }\end{array}$ & 4-acid & 4-acid \\
\hline Analytical Method & EDXRF & $\begin{array}{l}\text { ICP-MS, ICP-AES, } \\
\text { FAA }\end{array}$ & ICP-AES & ICP-AES & ACP-AES \\
\hline Analytes & $\mathrm{Cu}, \mathrm{Pb}, \mathrm{Zn}$ & 35 elements & 32 elements & 40 elements & $\begin{array}{l}\mathrm{Pb}, \mathrm{Zn}, \mathrm{Fe}, \mathrm{Mn}, \mathrm{As} \\
\mathrm{Cd}\end{array}$ \\
\hline \multicolumn{6}{|l|}{ Quality Control } \\
\hline Calibration Solutions & & EWU-prepared & CHEMEX-prep. & XRAL-prepared & ACZ-prepared \\
\hline Calibration Checks & yes & yes & yes & yes & yes \\
\hline a. Initial & yes & yes & yes & yes & yes \\
\hline b. Continuing & yes & yes, I in 10 & yes & yes & yes \\
\hline c. Blank & & yes & yes & yes & yes \\
\hline d. Re-analysis & & if $b>5 \%$ off & yes & yes $5 \%$ & \\
\hline e. Duplicate Split & & yes & & yes $5 \%$ & yes $5 \%$ \\
\hline f. spikes & & & & yes (Lu) & yes \\
\hline SRMs used in analyses & $\mathrm{NIST}^{1}$ SRMS & $\mathrm{NIST}^{1}$ SRMS & CANMET $^{3}$ SRMS & CCRMP $^{4}$ SRMS & ERA $^{5}$ SRMS \\
\hline \multirow[t]{6}{*}{ of known standards } & 1645,2704 & 2710,2711 & LKSD-1 to 4 & SS03 & \\
\hline & 2710,2711 & & STSD-1 to 4 & STILL4 & \\
\hline & USGS $^{2}$ SRMS & & RTS-2 and 4 & USGS BHVO-1 & \\
\hline & AGV-1, GXR-1 & & MP-2, SU-1a & & \\
\hline & GXR-2, GXR-4 & & & & \\
\hline & GXR-5, GXR-6 & & & & \\
\hline Percent of checks & $3 \%$ & 2 to $10 \%$ & $3 \%$ & NR & $40 \%$ \\
\hline Precision $(\mathrm{Pb}, \mathrm{Zn})$ & & $\mathrm{Pb} 3.5 \%, \mathrm{Zn} 2.5 \%$ & & & $\mathrm{~Pb} 8 \%, \mathrm{Zn} 12 \%$ \\
\hline Percent Recov. (Pb, Zn) & $101 \%, 98 \%$ & $\mathrm{~Pb} 100 \%, \mathrm{Zn} 98 \%$ & & & $\mathrm{~Pb} 99 \%, \mathrm{Zn} 99 \%$ \\
\hline Prep Blanks & & yes & & & \\
\hline Reagent Blanks & & yes & yes & yes & yes \\
\hline Run Logs & & yes & & yes & yes \\
\hline Lab Info. Mgmt. Syst. & & yes & yes & yes & yes \\
\hline QC Data Available & no longer & not readily & no longer & available & available \\
\hline Blind analyses of SRMs & no & yes & no & yes & yes \\
\hline SRMs used in analyses & & NIST SRMS & & USGS SRMS & NIST SRMS \\
\hline \multirow[t]{3}{*}{ of known standards } & & 2710,2711 & & SAR-L, SAR-M & 2710,2711 \\
\hline & & & & NIST SRMS & \\
\hline & & & & 2710,2711 & \\
\hline$\%$ of blind checks & & $2 \%$ & & $5 \%$ & $9 \%$ \\
\hline Precision $(\mathrm{Pb}, \mathrm{Zn})$ & & & & $11 \%, 15 \%$ & \\
\hline Mean Recov. (vs NIST) & & $\mathrm{Pb} 102 \%, \mathrm{Zn} 99 \%$ & & $\mathrm{~Pb} 97 \%, \mathrm{Zn} 81 \%$ & $\mathrm{~Pb} 88 \%, \mathrm{Zn} 87 \%$ \\
\hline \multicolumn{6}{|c|}{${ }^{1}$ National Institute of Standards \& Technology (NIST) } \\
\hline \multicolumn{6}{|c|}{${ }^{2}$ USGS SRM (Govindaraju, 1994). } \\
\hline \multicolumn{6}{|c|}{${ }^{3}$ Canada Centre for Mineral \& Energy Technology } \\
\hline \multicolumn{6}{|c|}{${ }^{4}$ Canadian Certified Reference Materials Project } \\
\hline
\end{tabular}




\section{US Geological Survey EDXRF Quality Control}

Standard Reference Materials (SRMs) were used to establish an EDXRF calibration curve showing the relation between measured X-ray fluorescence values and accepted values for concentrations of $\mathrm{Pb}, \mathrm{Zn}$, and $\mathrm{Cu}$, in the SRMs. Two types of SRMs were used in preparing the calibration curve. For low concentrations of metals USGS National Rock Standards AVG-1, GXR-1, GXR-2, GXR-4, GXR-5, and GXR-6 were used. Mean compositions of those SRMs are given by Govindaraju, 1994. For moderate to high concentrations of metals, National Institute of Standards and Technology (NIST) Standard Reference Materials 2710 and 2711 were used. Certified values for mean total concentrations of elements in those materials are given by Gills (1993a, 1993b). Results from one set of check analyses of four NIST SRMs indicate calibration accuracy to within or very near the range of precision of the NIST-certified mean total values at the 95\% confidence level (table 4). Of the 203 samples analyzed by USGS-EDXRF, 70 were also analyzed by EWU, 131 by CHEMEX, and 15 by XRAL.

\section{Eastern Washington University Geochemistry Lab (EWU) Quality Control}

EWU quality control procedures are listed in Table 3. Results of repeated analyses of known NIST SRMs were reported by EWU and are given in table 5 and discussed below. Blind analyses of the same NIST SRMs are given in tables 9 and 10 and discussed below. Of the 336 samples analyzed by EWU, 70 were also analyzed by USGS-EDXRF spectroscopy, 30 were analyzed by CHEMEX, and 27 others were analyzed by both XRAL and ACZ laboratories.

\section{EWU analyses of known Standard Reference Materials}

Prepared standard solutions were used to perform instrumental calibrations at 10sample intervals. Acid concentrations in standards and blanks matched those in samples for each instrumental analysis by ICP-MS, ICP-AES, or FAA. NIST SRMs 2710 and 2711 were repeatedly analyzed (table 5). USGS Rock Standards also were analyzed occasionally. The results obtained on NIST and USGS Reference Standards were within 
Table 4. USGS-EDXRF analysis of four NIST Standard Reference Materials for $\mathrm{Pb}, \mathrm{Zn}$ and $\mathrm{Cu}$.

\begin{tabular}{|c|c|c|c|c|c|}
\hline $\begin{array}{c}{ }^{\mathrm{N}} \text { NIST } \\
\text { SRM }\end{array}$ & Element & $\begin{array}{c}\text { NIST certified } \\
\text { value }(\mathrm{ppm})\end{array}$ & $\begin{array}{c}\mathrm{b}(+/-) \text { NIST } \\
\text { precision }\end{array}$ & $\begin{array}{c}{ }^{\mathrm{c}} \text { USGS EDXRF } \\
\text { value }(\mathrm{ppm})\end{array}$ & $\begin{array}{c}{ }^{\mathrm{d}} \text { USGS } \\
\text { Recovery\% }\end{array}$ \\
\hline 2704 & $\mathrm{~Pb}$ & 161 & $\mathrm{~N} \mathrm{R}$ & 184 & $114.3 \%$ \\
\hline 2704 & $\mathrm{Zn}$ & 438 & $\mathrm{~N} \mathrm{R}$ & 454 & $103.7 \%$ \\
\hline 2704 & $\mathrm{Cu}$ & 99 & $\mathrm{~N} \mathrm{R}$ & 116 & $117.2 \%$ \\
\hline \hline 1645 & $\mathrm{~Pb}$ & 714 & $\mathrm{~N} \mathrm{R}$ & 603 & $84.5 \%$ \\
\hline 1645 & $\mathrm{Zn}$ & 1720 & $\mathrm{~N} \mathrm{R}$ & 1514 & $88.0 \%$ \\
\hline 1645 & $\mathrm{Cu}$ & & $\mathrm{N} \mathrm{R}$ & & \\
\hline 2711 & $\mathrm{~Pb}$ & 1162 & 31 & 1200 & $103.3 \%$ \\
\hline 2711 & $\mathrm{Zn}$ & 350 & 5 & 350 & $100.0 \%$ \\
\hline 2711 & $\mathrm{Cu}$ & 114 & 2 & 122 & $107.0 \%$ \\
\hline \hline 2710 & $\mathrm{~Pb}$ & 5532 & 80 & 5600 & $101.2 \%$ \\
\hline 2710 & $\mathrm{Zn}$ & 6952 & 91 & 6940 & $99.8 \%$ \\
\hline 2710 & $\mathrm{Cu}$ & 2950 & 130 & 2970 & $100.7 \%$ \\
\hline
\end{tabular}

${ }^{\text {a }}$ National Institute of Standards and Technology (NIST) Standard Reference Material (SRM).

b at $95 \%$ confidence level (NR is "not reported").

${ }^{c}$ Bi-Shia King, USGS, analyst, 7/8/ 94.

${ }^{\mathrm{d}}$ Recovery $\%=$ (USGS EDXRF value/ NIST value) $* 100 \%$ 
$4 \%$ of certified values for all elements except for refractory $\mathrm{Ba}, \mathrm{Ni}, \mathrm{P}$, Ti. Measured concentrations of $\mathrm{Ba}, \mathrm{Ni}$, and $\mathrm{P}$ were up to $6.5 \%$ lower than NIST-certified values, while Ti was up to $15 \%$ lower.

\section{$\underline{\text { EWU Duplicates and Blanks }}$}

Five percent of the samples and a few reference standards were analyzed in duplicate. The results obtained on these samples were reproducible within the range of \pm $5 \%$. Preparation blanks were processed and analyzed with each batch of sediment samples to check possible contributions from acids, glassware, teflon-ware and the environment. The contributions from blanks were negligible and concentrations generally were below minimum analytical detection limits.

\section{EWU Precision and Accuracy}

EWU precision (relative to the means of EWU analyses of NIST SRMs 2710 and 2711 ) averages \pm 2.5 percent for $\mathrm{Zn}$ and $\mathrm{Mn}, \pm 3.5 \%$ for $\mathrm{Pb}, \pm 5.5 \%$ for $\mathrm{Fe}$ and $\mathrm{As}$, and \pm $6.5 \%$ for $\mathrm{Cd}$ (table 6). EWU calibration accuracy (expressed as percent-recovery relative to NIST-certified mean total values for SRMs 2710 and 2711) averages $96 \%$ for Mn and $\mathrm{Cd}, 98 \%$ for $\mathrm{Zn}, 100 \%$ for $\mathrm{As}, \mathrm{Fe}$ and $\mathrm{Pb}$ (table 5).

\section{EWU Analyses of Blind Standard Reference Materials}

NIST SRMs 2710 and 2711 were submitted to the EWU Geochemistry Laboratory as blind samples in a large analytical job in 1996. The analytical results and percent recovery are given in Tables 9 and 10. For $\mathrm{Pb}, \mathrm{Zn}, \mathrm{Mn}$ and As, the reported values are within $3 \%$ of the certified values. Fe was high by $8 \%$ for 2711 , and $\mathrm{Cd}$ was low by almost $8 \%$ for each SRM.

\section{CHEMEX Laboratories Quality Control}

CHEMEX quality control procedures are summarized in table 3. Results of quality control analyses were not included in reports of analytical results received from Chemex, nor were any blind NIST standards submitted with the CdA samples. Of 
Table 5. Analytical values, precision, and accuracy of EWU analyses of known and blind NIST SRMs 2710 and 2711.

\begin{tabular}{|c|c|c|c|c|c|c|c|c|}
\hline SRM 2710 & units & $\begin{array}{l}\text { NIST } \\
\text { mean }\end{array}$ & $\begin{array}{l}\text { NIST } \\
\mathrm{Pm}^{\mathrm{a}}\end{array}$ & $\begin{array}{c}\text { EWU } \\
\text { mean }^{\mathrm{b}}\end{array}$ & $\begin{array}{c}\text { EWU } \\
\text { std dev }\end{array}$ & $\begin{array}{l}\mathrm{EWU} \\
\mathrm{Pm}^{\mathrm{a}}\end{array}$ & $\begin{array}{c}\text { EWU mean } \\
\text { recov\% }\end{array}$ & $\begin{array}{l}\text { EWU blind } \\
\text { recov\% }{ }^{d}\end{array}$ \\
\hline$\overline{\mathrm{Al}}$ & wt\% & 6.44 & $1.2 \%$ & 6.34 & 0.14 & $4.5 \%$ & $98.4 \%$ & 97.5\% \\
\hline $\mathrm{Ca}$ & wt\% & 1.25 & $2.4 \%$ & 1.26 & 0.06 & $10.2 \%$ & $100.5 \%$ & $87.2 \%$ \\
\hline $\mathbf{F e}$ & $w t \%$ & 3.38 & $3.0 \%$ & 3.36 & 0.10 & $6.2 \%$ & $99.5 \%$ & $100.3 \%$ \\
\hline $\bar{K}$ & $w t \%$ & 2.11 & $5.2 \%$ & 2.09 & 0.06 & $6.1 \%$ & $98.9 \%$ & $96.7 \%$ \\
\hline $\mathrm{Mg}$ & wt\% & 0.85 & $4.9 \%$ & 0.88 & 0.02 & $3.9 \%$ & $102.6 \%$ & $99.6 \%$ \\
\hline $\mathrm{Ag}$ & ppm & 35.30 & $4.2 \%$ & 34.65 & 0.93 & $5.4 \%$ & $98.2 \%$ & $99.7 \%$ \\
\hline As & ppm & 626 & $6.1 \%$ & 623 & 17 & $5.6 \%$ & $99.5 \%$ & $98.6 \%$ \\
\hline$\overline{\mathrm{Ba}}$ & ppm & 707 & $7.2 \%$ & 676 & 16 & $4.6 \%$ & $95.7 \%$ & $91.1 \%$ \\
\hline Cd & ppm & 21.8 & $0.9 \%$ & 20.6 & 0.6 & $6.0 \%$ & $94.7 \%$ & $92.2 \%$ \\
\hline $\mathrm{Cu}$ & $\mathrm{ppm}$ & 2950 & $4.4 \%$ & 2857 & 39 & $2.7 \%$ & $96.8 \%$ & $95.4 \%$ \\
\hline Mn & ppm & 10100 & $4.0 \%$ & 9973 & 130 & $2.6 \%$ & $98.7 \%$ & $97.4 \%$ \\
\hline $\mathrm{Ni}$ & $\mathrm{ppm}$ & 14.3 & $7.0 \%$ & 14.0 & 0.5 & $6.9 \%$ & $97.9 \%$ & $90.9 \%$ \\
\hline$P$ & ppm & 1060 & $14.2 \%$ & 991 & 26 & $5.2 \%$ & $93.5 \%$ & $90.6 \%$ \\
\hline $\mathbf{P b}$ & ppm & 5532 & $1.4 \%$ & 5472 & 116 & $4.3 \%$ & 98.9\% & $100.9 \%$ \\
\hline $\mathrm{Sb}$ & ppm & 38.4 & $7.8 \%$ & 37.6 & 0.6 & $3.1 \%$ & $97.9 \%$ & $85.9 \%$ \\
\hline $\mathrm{Sr}$ & ppm & & & & & & & $118.8 \%$ \\
\hline $\mathrm{Ti}$ & ppm & 2830 & $3.5 \%$ & 2624 & 54 & $4.1 \%$ & $92.7 \%$ & $88.3 \%$ \\
\hline $\mathrm{TI}$ & ppm & & & & & & & \\
\hline $\mathrm{V}$ & ppm & 76.6 & $3.0 \%$ & 75.5 & 1.4 & $3.8 \%$ & $98.5 \%$ & $87.5 \%$ \\
\hline Zn & ppm & 6952 & $1.3 \%$ & 6723 & 78 & $2.3 \%$ & $96.7 \%$ & $98.0 \%$ \\
\hline SRM 2711 & units & $\begin{array}{l}\text { NIST } \\
\text { mean }\end{array}$ & $\begin{array}{l}\text { NIST } \\
\mathrm{Pm}^{\mathrm{a}}\end{array}$ & $\begin{array}{c}\text { EWU } \\
\text { mean }^{b}\end{array}$ & $\begin{array}{c}\text { EWU } \\
\text { std dev }\end{array}$ & $\begin{array}{l}\text { EWU } \\
\mathrm{Pm}^{\mathrm{a}}\end{array}$ & $\begin{array}{l}\text { EWU mean } \\
\text { recov\% }{ }^{c}\end{array}$ & $\begin{array}{l}\text { EWU blind } \\
\text { recov\% }{ }^{d}\end{array}$ \\
\hline$\overline{\overline{\mathrm{Al}}}$ & wt\% & "6.53 & 1.4\% & "6.40 & "0.17 & $\overline{\overline{5.5 \%}}$ & 98.0\% & $\overline{998.3 \%}$ \\
\hline $\mathrm{Ca}$ & wt\% & 2.88 & $2.8 \%$ & 2.85 & 0.11 & $7.5 \%$ & $98.9 \%$ & $95.8 \%$ \\
\hline $\mathbf{F e}$ & wt\% & 2.89 & $2.1 \%$ & 2.91 & 0.08 & $5.3 \%$ & $100.8 \%$ & $108.0 \%$ \\
\hline $\bar{K}$ & $w t \%$ & 2.45 & $3.3 \%$ & 2.48 & 0.07 & $5.5 \%$ & $101.2 \%$ & $105.7 \%$ \\
\hline $\mathrm{Mg}$ & wt\% & 1.05 & $2.9 \%$ & 1.07 & 0.03 & $5.5 \%$ & $101.4 \%$ & $110.5 \%$ \\
\hline $\mathrm{Ag}$ & ppm & 4.63 & $8.4 \%$ & 4.63 & 0.13 & $5.7 \%$ & $100 \%$ & $101.5 \%$ \\
\hline As & ppm & 105 & $7.6 \%$ & 105 & 2.6 & $5.0 \%$ & $100.2 \%$ & $99.0 \%$ \\
\hline $\mathrm{Ba}$ & $\mathrm{ppm}$ & 726 & $5.2 \%$ & 683 & 17.5 & $5.1 \%$ & $94.1 \%$ & $98.5 \%$ \\
\hline Cd & ppm & 41.7 & $0.6 \%$ & 40.3 & 1.4 & $6.9 \%$ & $96.6 \%$ & $92.8 \%$ \\
\hline $\mathrm{Cu}$ & $\mathrm{ppm}$ & 114 & $1.8 \%$ & 110 & 1.3 & $2.4 \%$ & $96.2 \%$ & $96.5 \%$ \\
\hline Mn & ppm & 638 & $4.4 \%$ & 639 & 11.5 & $3.6 \%$ & $99.8 \%$ & $102.8 \%$ \\
\hline $\mathrm{Ni}$ & $\mathrm{ppm}$ & 20.6 & $5.3 \%$ & 19.3 & 0.5 & $5.2 \%$ & $93.9 \%$ & $92.2 \%$ \\
\hline$P$ & ppm & 860 & $8.1 \%$ & 822 & 15.6 & $3.8 \%$ & $95.5 \%$ & $93.0 \%$ \\
\hline Pb & ppm & 1162 & $2.7 \%$ & 1181 & 18.6 & $3.2 \%$ & $101.6 \%$ & $103.7 \%$ \\
\hline $\mathrm{Sb}$ & $\mathrm{ppm}$ & 19.4 & $9.3 \%$ & 18.9 & 0.4 & $4.2 \%$ & $97.6 \%$ & $97.9 \%$ \\
\hline $\mathrm{Sr}$ & ppm & 245 & $0.3 \%$ & 239 & 5.2 & $4.4 \%$ & $97.6 \%$ & $93.4 \%$ \\
\hline $\mathrm{Ti}$ & ppm & 3060 & $7.5 \%$ & 2626 & 55.8 & $4.3 \%$ & $85.8 \%$ & $81.7 \%$ \\
\hline $\mathrm{TI}$ & ppm & 2.47 & $6.1 \%$ & 2.40 & 0.03 & $2.6 \%$ & $97.0 \%$ & \\
\hline $\mathrm{V}$ & ppm & 81.6 & $3.6 \%$ & 80.3 & 1.5 & $3.6 \%$ & $98.4 \%$ & $89.5 \%$ \\
\hline Zn & ppm & 350 & $1.4 \%$ & 347.6 & 6.1 & $3.5 \%$ & $99.2 \%$ & $100.6 \%$ \\
\hline
\end{tabular}

a Precision relative to mean of known standard runs

$(\mathrm{Pm}=(2$ standard deviations $/$ mean $) * 100 \%)$

b EWU number of known standard runs $(n)=8$

c EWU mean recovery \% = (EWU [known] mean/ NIST mean $) * 100 \%$

c Blind recovery $\%=($ EWU blind run value/ NIST mean)* $100 \%$ (see Tables $9 \& 10$ ) 
the 236 samples analyzed by Chemex Laboratories, USGS analyzed 131 by EDXRF spectroscopy, EWU analyzed 30 samples, and XRAL analyzed 15 samples.

\section{XRAL Laboratories Quality Control}

XRAL quality control procedures are listed in Table 3. Results of repeated analyses of known NIST SRMs were not included in reports of analytical results received from XRAL. However results of repeated blind analyses of USGS SRMs (Govindaraju, 1994) and a few blind analyses of NIST SRMs is discussed in the following sections. Of the 314 samples analyzed by XRAL Laboratories, 15 were analyzed both by USGSEDXRF and by CHEMEX, and 27 samples were analyzed both by EWU and by ACZ.

\section{$\underline{X R A L ~ P r e c i s i o n}$}

To monitor the precision of XRAL data, USGS Sample Control routinely submits for analysis USGS Standard Reference Materials SAR-L and SAR-M as blind samples, at a rate of 1 SRM sample in every 10 samples. Based on analyses of those samples, XRAL precision relative to mean results for those samples is listed for each element in tables 6 and 7. In summary, XRAL precision for 16 analyses of SAR-L and 15 analyses of SAR$\mathrm{M}$ averaged $\pm 5 \%$ for $\mathrm{Fe}$ and $\mathrm{Mn}, \pm 11 \%$ for $\mathrm{Pb}, \pm 15 \%$ for $\mathrm{Zn}, \pm 23 \%$ for $\mathrm{Cd}$, and $\pm 26 \%$ for As.

\section{XRAL Recovery Relative to USGS SRM Target Values}

Mean recoveries (relative to USGS target values) for 16 splits of SAR-L and 15 splits of SAR-M are listed for each element in tables 6 and 7. In summary, XRAL accuracy, expressed as percent recovery relative to USGS target values, averaged $95 \%$ 
Table 6. Analytical values, precision and accuracy of XRAL blind analyses of USGS Standard Reference Material (SRM) SAR-L

\begin{tabular}{|c|c|c|c|c|c|c|c|}
\hline & & USGS & USGS & XRAL & XRAL & XRAL & XRAL \\
\hline Element & units & mean $^{\mathrm{a}}$ & std dev & mean $^{\mathrm{b}}$ & std dev & $\mathrm{Pm}^{\mathrm{c}}$ & recov\% ${ }^{\mathrm{d}}$ \\
\hline $\mathrm{Al}$ & wt $\%$ & 5.79 & 0.17 & 5.69 & 0.16 & $5.7 \%$ & $98.3 \%$ \\
\hline $\mathrm{Ca}$ & wt \% & 1.06 & 0.02 & 1.07 & 0.03 & $5.6 \%$ & $101 \%$ \\
\hline $\mathbf{F e}$ & wt \% & 2.67 & 0.06 & 2.58 & 0.07 & $5.1 \%$ & $96.7 \%$ \\
\hline $\mathrm{K}$ & wt \% & 2.98 & 0.10 & 2.92 & 0.07 & $4.8 \%$ & $98.1 \%$ \\
\hline $\mathrm{Mg}$ & wt \% & 0.55 & 0.01 & 0.51 & 0.46 & $181 \%$ & $93.2 \%$ \\
\hline $\mathrm{Na}$ & wt \% & 1.53 & 0.01 & 1.42 & 0.04 & $5.9 \%$ & $93.0 \%$ \\
\hline$P$ & wt $\%$ & 0.09 & 0.01 & 0.08 & 0.004 & $10.5 \%$ & $85.8 \%$ \\
\hline $\mathrm{Ti}$ & wt $\%$ & 0.25 & 0.04 & 0.29 & 0.02 & $13.7 \%$ & $115 \%$ \\
\hline $\mathrm{Ag}$ & ppm & 2.56 & 0.24 & 2.58 & 0.90 & $69.7 \%$ & $100.9 \%$ \\
\hline As & $\mathrm{ppm}$ & 16.5 & 1.11 & 21.6 & 2.87 & $26.7 \%$ & $131 \%$ \\
\hline $\mathrm{Au}$ & $\mathrm{ppm}$ & 0.33 & 0.23 & $<8$ & $\mathrm{~N} / \mathrm{A}$ & $\mathrm{N} / \mathrm{A}$ & $\mathrm{N} / \mathrm{A}$ \\
\hline $\mathrm{Ba}$ & $\mathrm{ppm}$ & 879 & 31.8 & 887 & 31.2 & $7.0 \%$ & $100.9 \%$ \\
\hline $\mathrm{Be}$ & $\mathrm{ppm}$ & 3.24 & 0.21 & 3.75 & 0.58 & $30.8 \%$ & $116 \%$ \\
\hline $\mathrm{Bi}$ & $\mathrm{ppm}$ & 1.1 & 0.23 & $<50$ & $\mathrm{~N} / \mathrm{A}$ & $\mathrm{N} / \mathrm{A}$ & N/A \\
\hline Cd & $\mathrm{ppm}$ & 2.5 & 0.15 & 3 & 0.37 & $24.3 \%$ & $120 \%$ \\
\hline $\mathrm{Ce}$ & $\mathrm{ppm}$ & 150 & 4.27 & 153 & 7.74 & $10.1 \%$ & $102 \%$ \\
\hline Co & ppm & 7.5 & 0.61 & 7.3 & 0.95 & $25.9 \%$ & $97.5 \%$ \\
\hline $\mathrm{Cr}$ & $\mathrm{ppm}$ & 110 & 9.01 & 147 & 10.1 & $13.8 \%$ & $133 \%$ \\
\hline $\mathrm{Cu}$ & $\mathrm{ppm}$ & 370 & 15.5 & 348 & 21.1 & $12.1 \%$ & $94.0 \%$ \\
\hline $\mathrm{Eu}$ & $\mathrm{ppm}$ & 1.5 & 0.02 & $<2$ & $\mathrm{~N} / \mathrm{A}^{\mathrm{c}}$ & N/A & N/A \\
\hline $\mathrm{Ga}$ & $\mathrm{ppm}$ & 17 & 1.27 & 21 & 2.62 & $25.0 \%$ & $123 \%$ \\
\hline $\mathrm{Ho}$ & $\mathrm{ppm}$ & 1.9 & 0.60 & $<4$ & $\mathrm{~N} / \mathrm{A}$ & N/A & N/A \\
\hline $\mathrm{La}$ & $\mathrm{ppm}$ & 75 & 3.18 & 76 & 3.57 & $9.4 \%$ & $101.0 \%$ \\
\hline $\mathrm{Li}$ & $\mathrm{ppm}$ & 28 & 0.87 & 26 & 2.87 & $21.8 \%$ & $94.2 \%$ \\
\hline Mn & $\mathrm{ppm}$ & 2094 & 65.0 & 2045 & 42.3 & $4.1 \%$ & $\mathbf{9 7 . 7} \%$ \\
\hline Mo & $\mathrm{ppm}$ & 13 & 0.85 & 16 & 1.09 & $13.3 \%$ & $126.4 \%$ \\
\hline $\mathrm{Nb}$ & ppm & 35 & 2.73 & 27 & 4.15 & $31.2 \%$ & $75.9 \%$ \\
\hline $\mathrm{Nd}$ & ppm & 66 & 2.11 & 66 & 3.58 & $10.8 \%$ & $100.6 \%$ \\
\hline $\mathrm{Ni}$ & $\mathrm{ppm}$ & 52 & 3.49 & 54 & 2.92 & $10.9 \%$ & $103.1 \%$ \\
\hline $\mathbf{P b}$ & $\mathrm{ppm}$ & 578 & 22.2 & 618 & 30.0 & $9.7 \%$ & $107.0 \%$ \\
\hline Sc & $\mathrm{ppm}$ & 7.8 & 0.22 & 7.7 & 0.48 & $12.5 \%$ & $98.6 \%$ \\
\hline $\mathrm{Sn}$ & $\mathrm{ppm}$ & 6 & N/A & $<50$ & $\mathrm{~N} / \mathrm{A}$ & $\mathrm{N} / \mathrm{A}$ & $\mathrm{N} / \mathrm{A}$ \\
\hline $\mathrm{Sr}$ & $\mathrm{ppm}$ & 158 & 11.8 & 148 & 4.37 & $5.9 \%$ & $93.6 \%$ \\
\hline $\mathrm{Ta}$ & $\mathrm{ppm}$ & 2.8 & 0.09 & $<40$ & $\mathrm{~N} / \mathrm{A}$ & N/A & $\mathrm{N} / \mathrm{A}$ \\
\hline Th & $\mathrm{ppm}$ & 19 & 1.21 & 21 & 1.36 & $13.0 \%$ & $109.9 \%$ \\
\hline U & $\mathrm{ppm}$ & 5.2 & 0.06 & $<100$ & $\mathrm{~N} / \mathrm{A}$ & $\mathrm{N} / \mathrm{A}$ & $\mathrm{N} / \mathrm{A}$ \\
\hline V & $\mathrm{ppm}$ & 140 & 3.36 & 129 & 4.16 & $6.4 \%$ & $92.4 \%$ \\
\hline$Y$ & $\mathrm{ppm}$ & 44 & 1.58 & 40 & 2.24 & $11.2 \%$ & $91.1 \%$ \\
\hline $\mathrm{Yb}$ & $\mathrm{ppm}$ & 5.7 & 1.13 & 4.3 & 0.48 & $22.2 \%$ & $75.7 \%$ \\
\hline $\mathrm{Zn}$ & $\mathrm{ppm}$ & 420 & 53.1 & 395 & 29.6 & $15.0 \%$ & $94.0 \%$ \\
\hline \multicolumn{8}{|c|}{ USGS $n=5$ to 24} \\
\hline \multicolumn{8}{|c|}{ XRAL $n=16$} \\
\hline \multicolumn{8}{|c|}{ c Precision relative to mean $(\mathrm{Pm})=(2 \mathrm{std}$. dev. $/$ mean $) * 100 \%$} \\
\hline \multicolumn{8}{|c|}{${ }^{d}$ Recovery $\%=($ XRAL mean/ USGS mean $) * 100 \%$} \\
\hline$N / A=$ & lis & heraus & & & & & \\
\hline
\end{tabular}


Table 7. Analytical values, precision and accuracy of XRAL blind analyses of USGS Standard Reference Material (SRM) SAR-M

\begin{tabular}{|c|c|c|c|c|c|c|c|}
\hline & & USGS & USGS & XRAL & XRAL & XRAL & XRAL \\
\hline Element & units & mean $^{a}$ & std dev & mean $^{b}$ & std dev & $\mathrm{Pm}^{\mathrm{c}}$ & recov\% ${ }^{\mathrm{d}}$ \\
\hline Al & wt \% & 6.06 & 0.20 & 6.01 & 0.15 & $5.0 \%$ & $99.2 \%$ \\
\hline $\mathrm{Ca}$ & wt \% & 0.57 & 0.03 & 0.57 & 0.01 & $3.7 \%$ & $100 \%$ \\
\hline $\mathrm{Fe}$ & wt \% & 3.21 & 0.13 & 3.05 & 0.06 & $3.6 \%$ & $94.8 \%$ \\
\hline$K$ & wt \% & 2.89 & 0.11 & 2.88 & 0.14 & $9.7 \%$ & $99.6 \%$ \\
\hline $\mathrm{Mg}$ & wt \% & 0.50 & 0.02 & 0.46 & 0.01 & $5 \%$ & $93.4 \%$ \\
\hline $\mathrm{Na}$ & wt \% & 1.18 & 0.05 & 1.12 & 0.02 & $4.3 \%$ & $94.6 \%$ \\
\hline $\mathrm{P}$ & wt \% & 0.07977 & 0.01 & 0.07 & 0.00 & $12.1 \%$ & $88.0 \%$ \\
\hline $\mathrm{Ti}$ & wt \% & 0.35 & 0.04 & 0.34 & 0.02 & $11.1 \%$ & $98 \%$ \\
\hline$\overline{\mathrm{Ag}}$ & ppm & 3.18 & 0.40 & 4.20 & 1.01 & $48.3 \%$ & $132.1 \%$ \\
\hline As & $\mathrm{ppm}$ & 37.2 & 4.38 & 39.2 & 4.71 & $24.0 \%$ & $105 \%$ \\
\hline $\mathrm{Au}$ & ppm & 0.03 & 0.03 & $<8$ & $\mathrm{~N} / \mathrm{A}$ & $\mathrm{N} / \mathrm{A}$ & $\mathrm{N} / \mathrm{A}$ \\
\hline $\mathrm{Ba}$ & $\mathrm{ppm}$ & 776 & 44.6 & 788 & 29.5 & $7.5 \%$ & $101.5 \%$ \\
\hline $\mathrm{Be}$ & $\mathrm{ppm}$ & 2.40 & 0.03 & 2.07 & 0.26 & $25.0 \%$ & $86 \%$ \\
\hline $\mathrm{Bi}$ & ppm & 0.98 & 0.84 & $\mathrm{~N} / \mathrm{A}$ & N/A & $\mathrm{N} / \mathrm{A}$ & $\mathrm{N} / \mathrm{A}$ \\
\hline Cd & ppm & 4.64 & 0.35 & 6 & 0.65 & $21.8 \%$ & $129 \%$ \\
\hline $\mathrm{Ce}$ & $\mathrm{ppm}$ & 119 & 6.08 & 117 & 8.72 & $14.9 \%$ & $99 \%$ \\
\hline Co & ppm & 10.3975 & 1.26 & 10.9 & 1.53 & $28.1 \%$ & $105.2 \%$ \\
\hline $\mathrm{Cr}$ & $\mathrm{ppm}$ & 97.0 & 8.39 & 78 & 25.0 & $63.9 \%$ & $81 \%$ \\
\hline $\mathrm{Cu}$ & $\mathrm{ppm}$ & 310 & 24.5 & 303 & 16.5 & $10.9 \%$ & $97.9 \%$ \\
\hline $\mathrm{Eu}$ & $\mathrm{ppm}$ & 0.67 & 0.70 & $\mathrm{~N} / \mathrm{A}$ & $\mathrm{N} / \mathrm{A}$ & $\mathrm{N} / \mathrm{A}$ & N/A \\
\hline $\mathrm{Ga}$ & ppm & 19.7 & 0.44 & 19 & 3.01 & $31.6 \%$ & $97 \%$ \\
\hline $\mathrm{Ho}$ & $\mathrm{ppm}$ & 1.72 & 0.16 & $\mathrm{~N} / \mathrm{A}$ & $\mathrm{N} / \mathrm{A}$ & $\mathrm{N} / \mathrm{A}$ & $\mathrm{N} / \mathrm{A}$ \\
\hline La & ppm & 57.605 & 4.59 & 59 & 3.24 & $10.9 \%$ & $103.0 \%$ \\
\hline $\mathrm{Li}$ & ppm & 29.5 & 0.81 & 27 & 2.09 & $15.5 \%$ & $91.2 \%$ \\
\hline$M n$ & $\mathrm{ppm}$ & 5262.01 & 332 & 5099 & 122 & $4.8 \%$ & $96.9 \%$ \\
\hline Mo & ppm & 13 & 3.50 & 16 & 1.75 & $22.3 \%$ & $121.0 \%$ \\
\hline $\mathrm{Nb}$ & $\mathrm{ppm}$ & 28.0 & 3.10 & 27 & 3.71 & $27.8 \%$ & $95.5 \%$ \\
\hline $\mathrm{Nd}$ & $\mathrm{ppm}$ & 51.4 & 3.36 & 48 & 3.89 & $16.2 \%$ & $93.7 \%$ \\
\hline $\mathrm{Ni}$ & ppm & 42 & 5.00 & 42 & 3.08 & $14.7 \%$ & $100.2 \%$ \\
\hline $\mathbf{P b}$ & ppm & 906 & 76.4 & 1030 & 63.9 & $12.4 \%$ & $113.7 \%$ \\
\hline Sc & $\mathrm{ppm}$ & 8.34 & 0.37 & 7.9 & 0.83 & $21.2 \%$ & $94.4 \%$ \\
\hline Sn & $\mathrm{ppm}$ & 9.4 & 1.70 & $\mathrm{~N} / \mathrm{A}$ & $\mathrm{N} / \mathrm{A}$ & $\mathrm{N} / \mathrm{A}$ & $\mathrm{N} / \mathrm{A}$ \\
\hline $\mathrm{Sr}$ & ppm & 154 & 7.60 & 148 & 2.66 & $3.6 \%$ & $96.1 \%$ \\
\hline $\mathrm{Ta}$ & $\mathrm{ppm}$ & 2.63 & 0.19 & N/A & $\mathrm{N} / \mathrm{A}$ & $\mathrm{N} / \mathrm{A}$ & N/A \\
\hline Th & $\mathrm{ppm}$ & 18.1 & 0.96 & 18 & 2.39 & $26.3 \%$ & $100.2 \%$ \\
\hline $\mathrm{U}$ & $\mathrm{ppm}$ & 5.19 & 0.14 & $\mathrm{~N} / \mathrm{A}$ & $\mathrm{N} / \mathrm{A}$ & $\mathrm{N} / \mathrm{A}$ & $\mathrm{N} / \mathrm{A}$ \\
\hline V & $\mathrm{ppm}$ & 65.5 & 2.85 & 66 & 1.77 & $5.4 \%$ & $100.0 \%$ \\
\hline$Y$ & $\mathrm{ppm}$ & 31.6 & 3.80 & 27 & 1.13 & $8.4 \%$ & $85.4 \%$ \\
\hline $\mathrm{Yb}$ & ppm & 4.17 & 0.84 & 3.0 & 0.00 & $0.0 \%$ & $71.9 \%$ \\
\hline Zn & $\mathrm{ppm}$ & 883 & 102 & 842 & 59.5 & $14.1 \%$ & $95.4 \%$ \\
\hline \multicolumn{8}{|c|}{ USGS $n=4$ to 24} \\
\hline \multicolumn{8}{|c|}{ D XRAL $n=15$} \\
\hline \multicolumn{8}{|c|}{${ }^{c}$ Precision relative to mean $(\mathrm{Pm})=(2 \mathrm{std}$. dev. $/$ mean $) * 100 \%$} \\
\hline \multicolumn{8}{|c|}{${ }^{d}$ Recovery $\%=($ XRAL mean/ USGS mean $) * 100 \%$} \\
\hline$/ A=$ & onlic & because & aulifi & & & & \\
\hline
\end{tabular}


for $\mathrm{Zn}, 97 \%$ for $\mathrm{Fe}$ and $\mathrm{Mn}, 110 \%$ for $\mathrm{Pb}, 118 \%$ for As, and $125 \%$ for $\mathrm{Cd}$. Data are deemed acceptable by USGS when the percent recovery of an element is between the range of 80 and $120 \%$ of the target value for that element, provided the target value of that element is $>5$ times the lower detection limit for that element. (See detection limits, table 1, and XRAL percent recovery, tables 6 and 7). By those criteria, XRAL data for $\mathrm{Pb}, \mathrm{Zn}, \mathrm{Fe}$, and $\mathrm{Mn}$, are of acceptable accuracy, but XRAL data for As and $\mathrm{Cd}$ are of marginal acceptability.

\section{$\underline{\text { XRAL Recovery Relative to Blind NIST SRM certified values }}$}

NIST SRMs 2710 and 2711 were submitted to the XRAL Laboratory as blind samples and the analytical results and percent recovery (relative to NIST certified values) are listed in tables 9 and 10. Mean recoveries for the two NIST SRMs range are more than $90 \%$ for $\mathrm{Pb}$ and $\mathrm{Fe}$ ( $96.9 \%$ and $92.6 \%$, respectively), between $85 \%$ and $90 \%$ for $\mathrm{Mn}$ and $\mathrm{As}(89.3 \%$ and $88.7 \%$, respectively), and between $80 \%$ and $85 \%$ for $\mathrm{Zn}$ and $\mathrm{Cd}(81 \%$ and $82.5 \%$, respectively). Recoveries were considerably less than those from the USGS SRMs.

\section{ACZ Laboratories Quality Control}

ACZ Laboratories listed quality control data in their report of analyses, which included the precision and recovery data for prepared calibration solutions and recovery data for known pulverized SRMs supplied by Environmental Resources Associates (ERA) (table 8). Two blind NIST SRMs were submitted with our analytical jobs and their analytical data and percent recovery are given in tables 9 and 10. All 27 samples analyzed by ACZ Laboratories were also analyzed by both EWU and XRAL laboratories.

\section{$\underline{\text { ACZ Precision and Accuracy }}$}

Based on repeated analyses of prepared calibration solutions (table 8), ACZ precision is \pm $7 \%$ for $\mathrm{Mn}, \pm 8 \%$ for $\mathrm{Pb}$ and $\mathrm{Cd}, \pm 10 \%$ for $\mathrm{Fe}$ and $\mathrm{As}$, and $\pm 12 \%$ for $\mathrm{Zn}$. ACZ recovery relative to ERA standards is $116 \%$ for $\mathrm{Mn}, 113 \%$ for $\mathrm{Zn}, 107 \%$ for $\mathrm{Fe}, 99 \%$ for $\mathrm{Pb}$ and Cd, and $87 \%$ for As. 
Table 8. Analytical values, precision, and accuracy of ACZ analyses of lab-prepared calibration solutions and of nitricacid dissolutions of known, pulverized SRMs.

\begin{tabular}{|c|c|c|c|c|c|c|c|c|c|c|}
\hline \multicolumn{7}{|c|}{ Analyses of lab-prepared calibration solutions } & \multicolumn{4}{|c|}{ Analyses of pulverized SRMs } \\
\hline Element & TRUE $^{1}$ & Tests & $\begin{array}{c}\text { Mean } \\
\text { FOUND }\end{array}$ & $\begin{array}{l}\text { Std. } \\
\text { Dev. }\end{array}$ & $\begin{array}{c}\text { Precis. } \\
\text { Pm }\end{array}$ & $\begin{array}{l}\text { Mean } \\
\text { Recov. }\end{array}$ & $\mathrm{TRUE}^{2}$ & Tests & $\begin{array}{c}\text { Mean } \\
\text { FOUND }\end{array}$ & $\begin{array}{l}\text { Mean } \\
\text { Recov. }\end{array}$ \\
\hline & ppm & $\mathrm{n}$ & ppm & $\mathrm{ppm}$ & $\%$ & $\%$ & $\mathrm{ppm}$ & $\mathrm{n}$ & $\mathrm{ppm}$ & $\%$ \\
\hline & & & & & & & 136 & 2 & 125 & \\
\hline & & & & & & & & & & \\
\hline $\mathrm{Zn}$ & 1250 & 11 & 1240 & 72 & 12 & 99.2 & 70 & 3 & 79 & 112.7 \\
\hline & & & & 123 & 10 & 1006 & 7850 & 1 & 8410 & 1071 \\
\hline & & & & & & & & & & \\
\hline $\mathrm{Mn}$ & 1250 & 8 & 1210 & 42 & 7 & 96.8 & 147 & 2 & 171 & 116.3 \\
\hline As & 5000 & 12 & 4994 & 256 & 10 & 99.9 & 94.2 & 3 & 82 & 86.7 \\
\hline & & & & & & & & & & \\
\hline $\mathrm{Cd}$ & 2500 & 8 & 2470 & 98 & 8 & 98.8 & 98.3 & 3 & 97 & 98.7 \\
\hline
\end{tabular}

1 Concentration of calibration standard solution.

2 Target values supplied Environmental Resources Associates (ERA). 
Table 9. Analyses of blind submittals of NIST SRM 2711 (with moderately elevated metals) by

XRAL, EWU, and ACZ laboratories.

\begin{tabular}{|c|c|c|c|c|c|c|c|c|c|}
\hline Element & $\begin{array}{c}\text { Reporting } \\
\text { units }\end{array}$ & $\begin{array}{l}{ }^{\mathrm{a}} \mathrm{NIST} \\
\text { mean }\end{array}$ & $\begin{array}{l}{ }^{\mathrm{b}} \mathrm{NIST} \text { plus } \\
\text { or minus }\end{array}$ & $\begin{array}{l}\text { EWU } \\
\text { value }\end{array}$ & $\begin{array}{l}{ }^{C} X R A L \\
\text { value }\end{array}$ & $\begin{array}{c}\text { ACZ } \\
\text { value }\end{array}$ & $\begin{array}{c}\text { EWU } \\
{ }^{\mathrm{d}} \text { Recov. \% }\end{array}$ & $\begin{array}{c}\text { XRAL } \\
{ }^{\mathrm{d}} \text { Recov. \% }\end{array}$ & $\begin{array}{c}\text { ACZ } \\
{ }^{\mathrm{d}} \text { Recov. \% }\end{array}$ \\
\hline$\overline{\mathrm{Al}}$ & $\%$ & 6.53 & 0.09 & 6.42 & 6.28 & & $98.3 \%$ & $96.1 \%$ & \\
\hline $\mathrm{Ca}$ & $\%$ & 2.88 & 0.08 & 2.76 & 2.80 & & $95.8 \%$ & $97.3 \%$ & \\
\hline $\mathbf{F e}$ & $\%$ & 2.89 & 0.06 & 3.12 & 2.75 & 1.95 & $108.0 \%$ & $95.0 \%$ & $67.5 \%$ \\
\hline $\bar{K}$ & $\%$ & 2.45 & 0.08 & 2.59 & 2.36 & & $105.7 \%$ & $96.1 \%$ & \\
\hline $\mathrm{Mg}$ & $\%$ & 1.05 & 0.03 & 1.16 & 0.99 & & $110.5 \%$ & $94.3 \%$ & \\
\hline $\mathrm{Na}$ & $\%$ & 1.14 & 0.03 & & 1.07 & & & $94.1 \%$ & \\
\hline$P$ & $\%$ & 0.09 & 0.01 & 0.08 & 0.08 & & $93.0 \%$ & $95.9 \%$ & \\
\hline $\mathrm{Ti}$ & $\%$ & 0.31 & 0.02 & 0.25 & 0.30 & & $81.7 \%$ & $98.9 \%$ & \\
\hline $\mathrm{Ag}$ & ppm & 4.6 & 0.4 & 4.7 & 4.5 & & $101.5 \%$ & $97.2 \%$ & \\
\hline As & $\mathrm{ppm}$ & 105 & 8 & 104 & 98 & 44 & $99.0 \%$ & 93.3\% & $41.6 \%$ \\
\hline $\mathrm{Au}$ & $\mathrm{ppm}$ & 0.03 & & & & & & & \\
\hline $\mathrm{Ba}$ & ppm & 726 & 38 & 715 & 711 & & $98.5 \%$ & $97.9 \%$ & \\
\hline $\mathrm{Be}$ & ppm & & & 2 & 2 & & & & \\
\hline $\mathrm{Bi}$ & ppm & & & & & & & & \\
\hline Cd & ppm & 41.7 & 0.3 & 38.7 & 39.5 & 36.9 & $92.8 \%$ & $94.7 \%$ & $88.5 \%$ \\
\hline $\mathrm{Ce}$ & $\mathrm{ppm}$ & 69 & & 62 & 75 & & $89.9 \%$ & $108.0 \%$ & \\
\hline $\mathrm{Co}$ & ppm & 10 & & 9 & 10 & & $90.0 \%$ & $95.0 \%$ & \\
\hline $\mathrm{Cr}$ & $\mathrm{ppm}$ & 47 & & & 65 & & & $138.3 \%$ & \\
\hline $\mathrm{Cu}$ & $\mathrm{ppm}$ & 114 & 2 & 110 & 102 & & $96.5 \%$ & $89.5 \%$ & \\
\hline $\mathrm{Eu}$ & ppm & 1.1 & & & & & & & \\
\hline $\mathrm{Ga}$ & ppm & 15 & & 14 & 27 & & $93.3 \%$ & $176.7 \%$ & \\
\hline La & ppm & 40 & & 30 & 40 & & $75.0 \%$ & $100.0 \%$ & \\
\hline $\mathrm{Li}$ & $\mathrm{ppm}$ & & & & 23 & & & & \\
\hline$M n$ & $\mathrm{ppm}$ & 638 & 28 & 656 & 585 & 455 & $102.8 \%$ & $91.6 \%$ & $71.3 \%$ \\
\hline Mo & $\mathrm{ppm}$ & 1.6 & & 2.0 & 5.5 & & $125.0 \%$ & $344 \%$ & \\
\hline $\mathrm{Nb}$ & ppm & & & & 9 & & & & \\
\hline $\mathrm{Nd}$ & ppm & 31 & & & 34 & & & $108.1 \%$ & \\
\hline $\mathrm{Ni}$ & ppm & 20.6 & 1.1 & 19 & 19 & & $92.2 \%$ & $92.2 \%$ & \\
\hline $\mathbf{P b}$ & $\mathrm{ppm}$ & 1162 & 31 & 1205 & 1150 & 973 & $103.7 \%$ & $99.0 \%$ & $83.7 \%$ \\
\hline $\mathrm{Sb}$ & ppm & 19.4 & 1.8 & 19 & & & $97.9 \%$ & & \\
\hline Sc & ppm & 9 & & & 10 & & & $111.1 \%$ & \\
\hline Sn & $\mathrm{ppm}$ & & & 3 & & & & & \\
\hline $\mathrm{Sr}$ & ppm & 245.3 & 0.7 & 229 & 230 & & $93.4 \%$ & $93.8 \%$ & \\
\hline $\mathrm{Ta}$ & ppm & & & & & & & & \\
\hline Th & ppm & 14 & & 15 & 12 & & $107.1 \%$ & $85.7 \%$ & \\
\hline$U$ & $\mathrm{ppm}$ & 2.6 & & 2.5 & & & & & \\
\hline V & ppm & 81.6 & 2.9 & 73 & 81 & & $89.5 \%$ & $98.7 \%$ & \\
\hline $\mathrm{Y}$ & ppm & 25 & & 23 & 25 & & $92.0 \%$ & $100.0 \%$ & \\
\hline $\mathrm{Yb}$ & ppm & 2.7 & & & 3 & & & $111.1 \%$ & \\
\hline Zn & $\mathrm{ppm}$ & 350 & 4.8 & 352 & 302 & 307 & $100.6 \%$ & $86.3 \%$ & $87.7 \%$ \\
\hline
\end{tabular}

\footnotetext{
a - Certified values (non-certified in italics).

b - at $95 \%$ confidence level.

c - mean of two analyses

$\mathrm{d}$ - \% recovery relative to NIST mean values.
} 
Table 10. Analyses of blind submittals of NIST SRM 2710 (with highly elevated metals) by XRAL, EWU, and ACZ laboratories.

\begin{tabular}{|c|c|c|c|c|c|c|c|c|c|}
\hline Element & $\begin{array}{c}\text { Reporting } \\
\text { units }\end{array}$ & 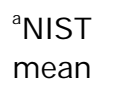 & $\begin{array}{l}{ }^{b} \text { NIST plus } \\
\text { or minus }\end{array}$ & $\begin{array}{l}\text { EWU } \\
\text { value }\end{array}$ & $\begin{array}{l}\text { 'XRAL } \\
\text { value }\end{array}$ & $\begin{array}{c}A C Z \\
\text { value }\end{array}$ & $\begin{array}{c}\text { EWU } \\
\text { dRecov. \% }\end{array}$ & $\begin{array}{c}\text { XRAL } \\
\text { decov. \% }\end{array}$ & $\begin{array}{c}\text { ACZ } \\
{ }^{\mathrm{d}} \text { Recov. \% }\end{array}$ \\
\hline$\overline{\overline{\mathrm{Al}}}$ & $\overline{\%}$ & $\overline{\overline{6.44}}$ & | 0.08 & $\overline{\overline{6.28}}$ & $\mid \overline{5.93}$ & & 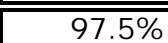 & $\begin{array}{l}92.0 \% \\
\end{array}$ & \\
\hline $\mathrm{Ca}$ & $\%$ & 1.250 & 0.030 & 1.090 & 1.213 & & $87.2 \%$ & $97.1 \%$ & \\
\hline $\mathrm{Fe}$ & $\%$ & 3.38 & 0.10 & 3.39 & 3.05 & 2.44 & $100.3 \%$ & $90.2 \%$ & $72.2 \%$ \\
\hline $\bar{K}$ & $\%$ & 2.11 & 0.11 & 2.04 & 1.92 & & $96.7 \%$ & $91.2 \%$ & \\
\hline $\mathrm{Mg}$ & $\%$ & 0.853 & 0.042 & 0.850 & 0.790 & & $99.6 \%$ & $92.6 \%$ & \\
\hline $\mathrm{Na}$ & $\%$ & 1.14 & 0.06 & & 0.99 & & & $86.4 \%$ & \\
\hline$P$ & $\%$ & 0.106 & 0.015 & 0.096 & 0.100 & & $90.6 \%$ & $94.3 \%$ & \\
\hline $\mathrm{Ti}$ & $\%$ & 0.283 & 0.010 & 0.250 & 0.286 & & $88.3 \%$ & $101.1 \%$ & \\
\hline $\mathrm{Ag}$ & ppm & 35.3 & 1.5 & 35.2 & 40.0 & & $99.7 \%$ & $113.3 \%$ & \\
\hline As & ppm & 626 & 38 & 617 & 526 & 570 & $98.6 \%$ & $84.0 \%$ & $91.1 \%$ \\
\hline $\mathrm{Au}$ & $\mathrm{ppm}$ & 0.6 & & & & & & & \\
\hline $\mathrm{Ba}$ & $\mathrm{ppm}$ & 707 & 51 & 644 & 660 & & $91.1 \%$ & $93.4 \%$ & \\
\hline $\mathrm{Be}$ & ppm & & & 2.4 & 2.0 & & & & \\
\hline $\mathrm{Bi}$ & $\mathrm{ppm}$ & & & & & & & & \\
\hline Cd & ppm & 21.8 & 0.2 & 20.1 & 15.3 & 18.9 & $92.2 \%$ & $70.3 \%$ & $86.7 \%$ \\
\hline $\mathrm{Ce}$ & $\mathrm{ppm}$ & 57 & & 48 & 61 & & $84.2 \%$ & $107.6 \%$ & \\
\hline Co & $\mathrm{ppm}$ & 10 & & 8 & 9 & & $80.0 \%$ & $90.0 \%$ & \\
\hline $\mathrm{Cr}$ & ppm & 39 & & & 50 & & & $128.2 \%$ & \\
\hline $\mathrm{Cu}$ & $\mathrm{ppm}$ & 2950 & 130 & 2815 & 2453 & & $95.4 \%$ & $83.2 \%$ & \\
\hline $\mathrm{Eu}$ & $\mathrm{ppm}$ & 1 & & & & & & & \\
\hline $\mathrm{Ga}$ & ppm & 34 & & 30 & 40 & & $88.2 \%$ & $117.6 \%$ & \\
\hline La & $\mathrm{ppm}$ & 34 & & 24 & 31 & & $70.6 \%$ & $91.2 \%$ & \\
\hline $\mathrm{Li}$ & ppm & & & & 35 & & & & \\
\hline Mn & $\mathrm{ppm}$ & 10100 & 400 & 9841 & 8790 & 7830 & $97.4 \%$ & $87.0 \%$ & $77.5 \%$ \\
\hline Mo & $\mathrm{ppm}$ & 19 & & 18 & 21 & & $94.7 \%$ & $108.8 \%$ & \\
\hline $\mathrm{Nb}$ & $\mathrm{ppm}$ & & & & 7 & & & & \\
\hline $\mathrm{Nd}$ & $\mathrm{ppm}$ & 23 & & & 24 & & & $102.9 \%$ & \\
\hline $\mathrm{Ni}$ & $\mathrm{ppm}$ & 14.3 & 1 & 13 & 14 & & $90.9 \%$ & $97.9 \%$ & \\
\hline $\mathbf{P b}$ & ppm & 5532 & 80 & 5580 & 5243 & 5110 & $100.9 \%$ & $94.8 \%$ & 92.4\% \\
\hline $\mathrm{Sb}$ & $\mathrm{ppm}$ & 38.4 & 3 & 33 & & & 85.9 & & \\
\hline Sc & ppm & 8.7 & & & 9 & & & $103.4 \%$ & \\
\hline $\mathrm{Sn}$ & ppm & & & & & & & & \\
\hline $\mathrm{Sr}$ & ppm & 240 & & 285 & 300 & & $118.8 \%$ & $124.9 \%$ & \\
\hline $\mathrm{Ta}$ & $\mathrm{ppm}$ & & & & & & & & \\
\hline Th & $\mathrm{ppm}$ & 13 & & 14 & 11 & & $107.7 \%$ & $84.6 \%$ & \\
\hline $\mathrm{U}$ & ppm & 25 & & 24.6 & & & & & \\
\hline V & ppm & 76.6 & 2.3 & 67 & 70 & & $87.5 \%$ & $91.8 \%$ & \\
\hline$Y$ & ppm & 23 & & 18 & 20 & & $78.3 \%$ & $87.0 \%$ & \\
\hline $\mathrm{Yb}$ & ppm & 1.3 & & & 2 & & & $179.5 \%$ & \\
\hline$Z n$ & $\mathrm{ppm}$ & 6952 & 91 & 6815 & 5253 & 6000 & $98.0 \%$ & $75.6 \%$ & $86.3 \%$ \\
\hline
\end{tabular}

a - Certified values (non-certified in italics).

b - at $95 \%$ confidence level.

c - mean of three analyses

d - \% recovery relative to NIST mean values. 


\section{$\underline{\text { ACZ Recovery Relative to Blind NIST SRM certified values }}$}

NIST SRMs 2710 and 2711 were submitted to the ACZ Laboratory as blind samples and the analytical results and percent recovery (relative to NIST certified values) are listed in tables 9 and 10. Mean recoveries for the two NIST SRMs are between $85 \%$ and $90 \%$ for $\mathrm{Pb}, \mathrm{Zn}$ and $\mathrm{Cd}(88.1 \%, 87 \%$, and $87.6 \%$, respectively), and are between $65 \%$ and $75 \%$ for $\mathrm{Mn}, \mathrm{Fe}$ and $\mathrm{As}$ (74\%, $70 \%$ and $66.4 \%$, respectively). Recoveries were considerably less than those reported by ACZ from the ERA SRMs.

\section{Comparison between laboratories of analyses of NIST Standards}

Four of the five laboratories (USGS-EDXRF, EWU, XRAL, and ACZ laboratories) analyzed NIST SRMs 2710 and 2711 as either known standards, blind submittals, or as both (discussed earlier); analyses of NIST SRMs are lacking only for CHEMEX laboratories. How well do analyses of NIST SRMs by each lab compare to the NIST-certified values for the main elements of concern here ( $\mathrm{Pb}, \mathrm{Zn}, \mathrm{Fe}, \mathrm{Mn}, \mathrm{As}$, Cd)? The accuracy (or percent recovery) can be compared by element for each analyses or for the means of several analyses (tables 4, 5, 9 and 10). Visual comparison of the accuracy between labs is given by $x-y$ plots (NIST values on the $x$-axis, lab values on the $y$-axis) for each of these elements in figures 3,4 and 5. For reference, the charts include a thin line with a slope of $y=1.0 \mathrm{x}$, indicating a perfect correlation with NIST-certified values. Linear regressions calculated by the least squares method from the data of each lab for each these elements (projected through origin) are given as the slope of the line in tables 11 through 13 (column labeled "slope (y/NIST)". The number of available analyses of NIST SRMs from each lab is given in the same tables. A third type of comparison is given by the mean percent recovery for each element, based on all the analyses of NIST SRMs by each lab. These values are also given in tables 11 through 13.

For all of the elements of concern ( $\mathrm{Pb}, \mathrm{Zn}, \mathrm{Fe}, \mathrm{Mn}, \mathrm{As}$ and $\mathrm{Cd})$, the EWU Geochemical Laboratories gives the closest values to the certified values NIST SRMs (within $1 \%$ for $\mathrm{Pb}, \mathrm{Fe}, \mathrm{Mn}$, and $\mathrm{As}, 3 \%$ for $\mathrm{Zn}$ and 5\% for $\mathrm{Cd}$ ). Analyses by USGSEDXRF ( $\mathrm{Pb}$ and $\mathrm{Zn}$ only) are nearly as accurate as those of EWU. XRAL and ACZ 


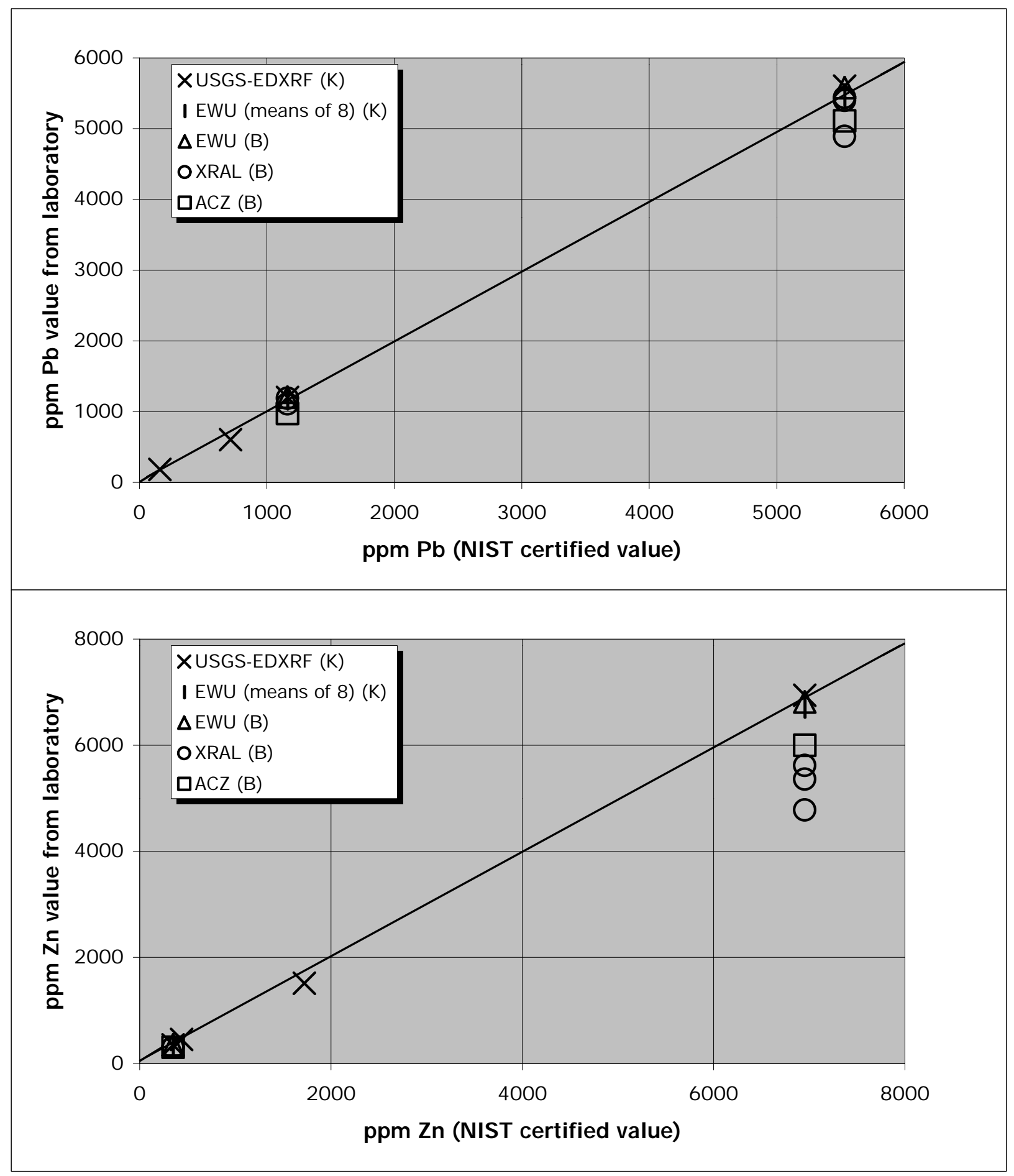

Figure 3. Comparison of laboratory analytical values of known ("K") and blind ("B") NIST SRMs with NIST certified values. Line represents 1:1 correlation for reference. (A) Pb, (B) Zn. 


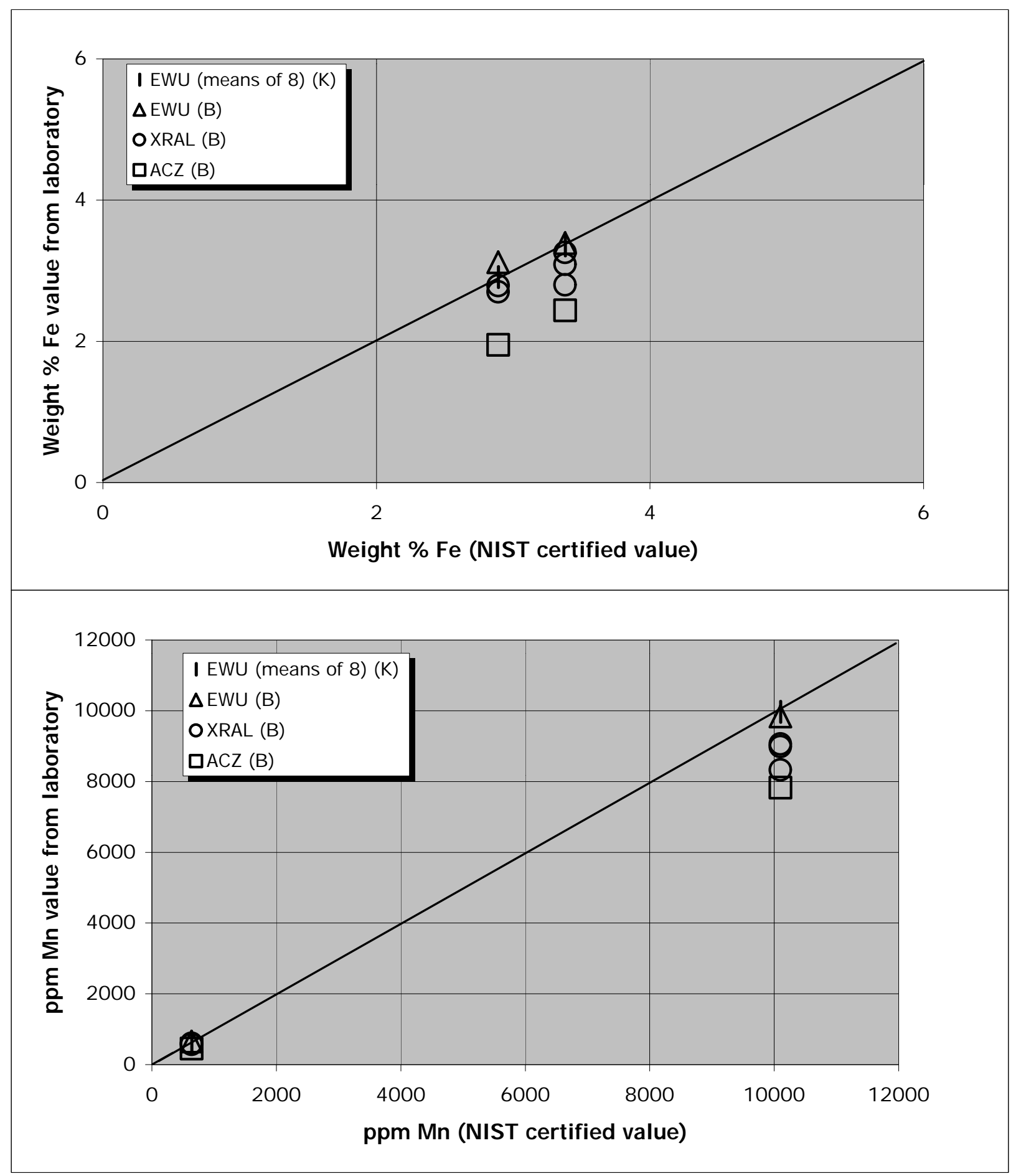

Figure 4. Comparison of laboratory analytical values of known ("K") and blind ("B") NIST SRMs with NIST certified values. Line represents 1:1 correlation for reference. (A) Fe, (B) Mn. 


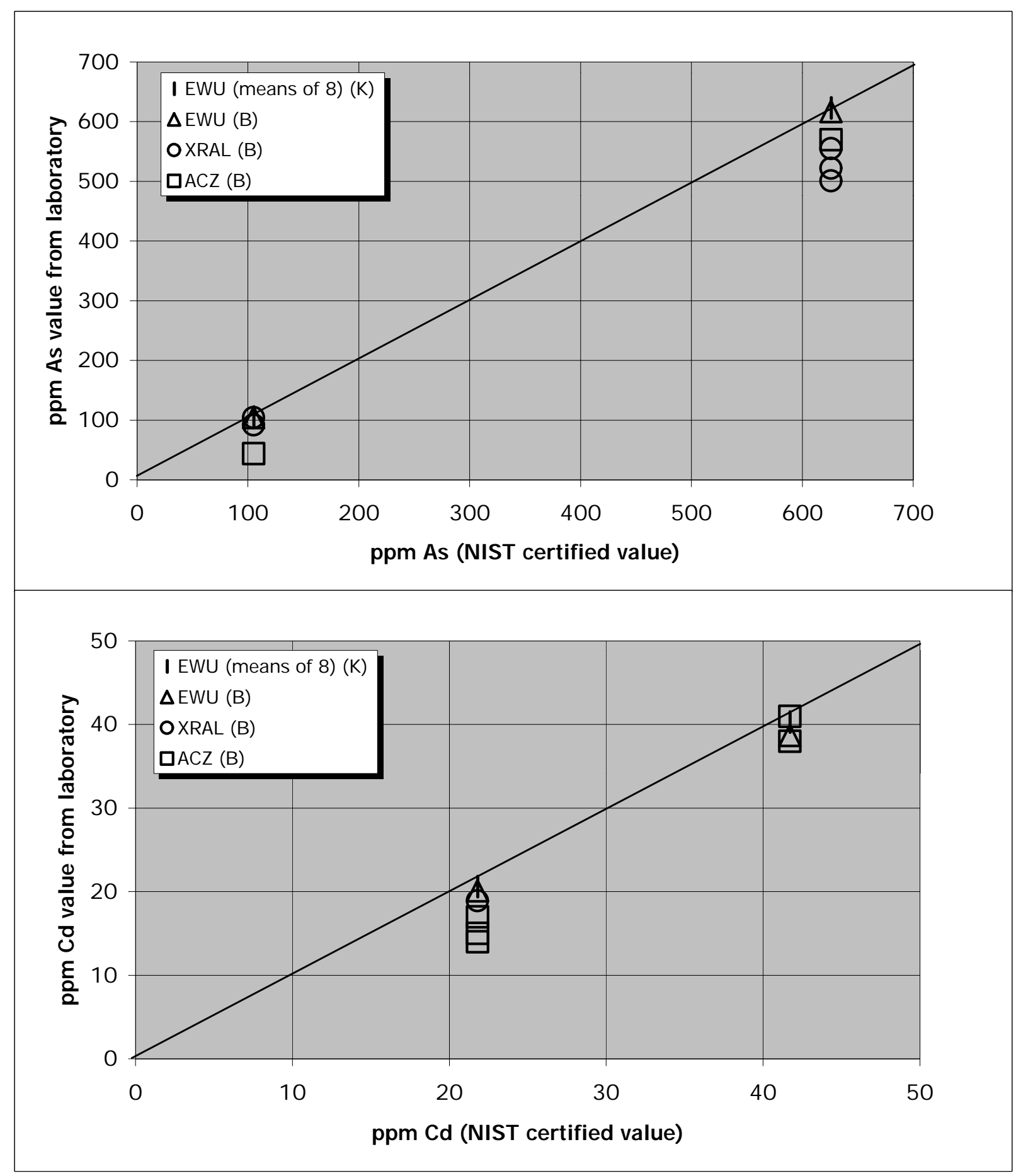

Figure 5. Comparison of laboratory analytical values of known ("K") and blind ("B") NIST SRMs with NIST certified values. Line represents 1:1 correlation for reference. (A) As, (B) Cd. 
values are distinctly less than NIST-certified values for each of the 6 elements. $\mathrm{Pb}$ is 5 to $8 \%$ low by XRAL and ACZ, but the other 5 elements are from $10 \%$ to over $30 \%$ low by these labs.

\section{Comparison between laboratories of analyses of sample splits}

As discussed earlier, a large number of the soil and sediment samples were analyzed by more than one laboratory, each using a different sample preparation or analytical method. Since the EWU Geochemistry Laboratory produced analyses that most closely matched certified values for NIST SRMs, comparisons are made between the values of other labs and of EWU for $\mathrm{Pb}, \mathrm{Zn}, \mathrm{Fe}, \mathrm{Mn}, \mathrm{As}$, and $\mathrm{Cd}$ in figures 6 through 11. For most elements (except Fe) the correlation between labs is better and there is less scatter when elemental values in the analyzed samples are less than that of the NIST SRM with the highest metal values (2710). In tables 11 through 13, the number of commonly analyzed samples between labs, the slope of a least-squares linear regression line (projected through the origin), and the coefficient of determination $\left(\mathrm{R}^{2}\right.$, a measure of the scatter around the regression line) are given for each element at a given range of analytical values between each laboratory and the EWU Geochemistry Laboratory.

Comparison of the inter-laboratory analyses of $\mathrm{Pb}$ (figure 6) indicates that values of USGS-EDXRF are less than 3\% higher and those of XRAL and ACZ are less than 2\% lower than EWU values for $\mathrm{Pb}$ concentrations less than $8,000 \mathrm{ppm}$. The scatter around the linear regression is relatively narrow, with $\mathrm{R}^{2}$ values of 0.96 or better (table 11 ). In other words, for Coeur d'Alene valley samples below 8,000 ppm $\mathrm{Pb}$, the four laboratories (USGS-EDXRF, EWU, XRAL, and ACZ) give comparable analytical values for $\mathrm{Pb}$ (within 5\%). Above 8,000 ppm Pb, the values of USGS-EDXRF, XRAL, and ACZ are 6-11\% lower than those EWU, and the scatter is higher. On the other hand, $\mathrm{Pb}$ analyses by CHEMEX for values less than $8,000 \mathrm{ppm} \mathrm{Pb}$ are considerably lower (23\%) than those of EWU, and the scatter is even higher $\left(\mathrm{R}^{2}=0.85\right)$. Although we are lacking data from CHEMEX on the accuracy of its analyses relative to NIST SRMs, we can infer, from the comparison with EWU analyses of the same samples, that the accuracy or percent recovery of CHEMEX $\mathrm{Pb}$ analyses is roughly $77 \%$. 

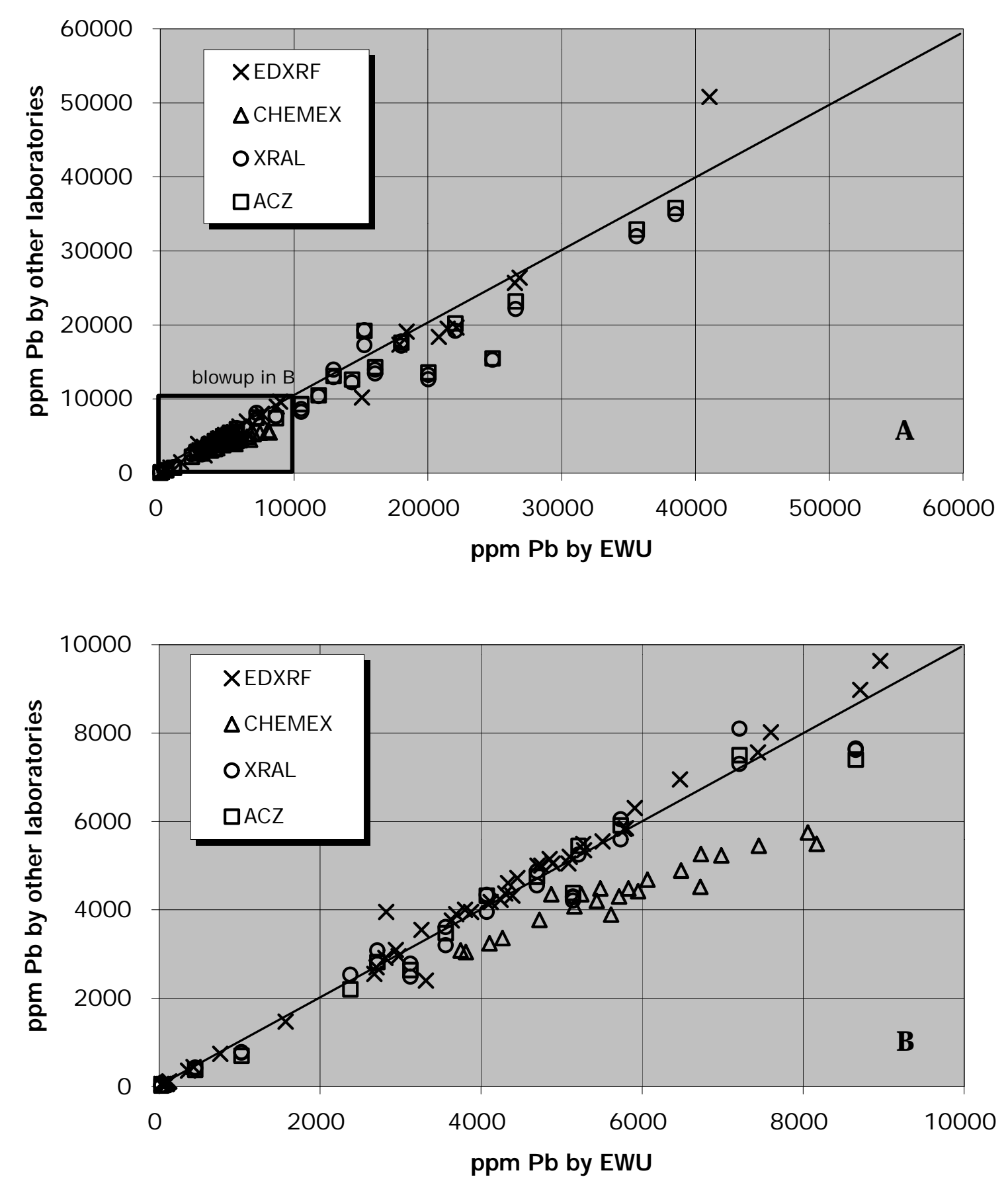

Figure 6. $\mathrm{Pb}$ analyses of sample splits analysed by EWU and by one of the other 4 laboratories. (A) Entire dataset, (B) blowup of the lower left corner of A. Line shows 1:1 correlation of analytical values. 

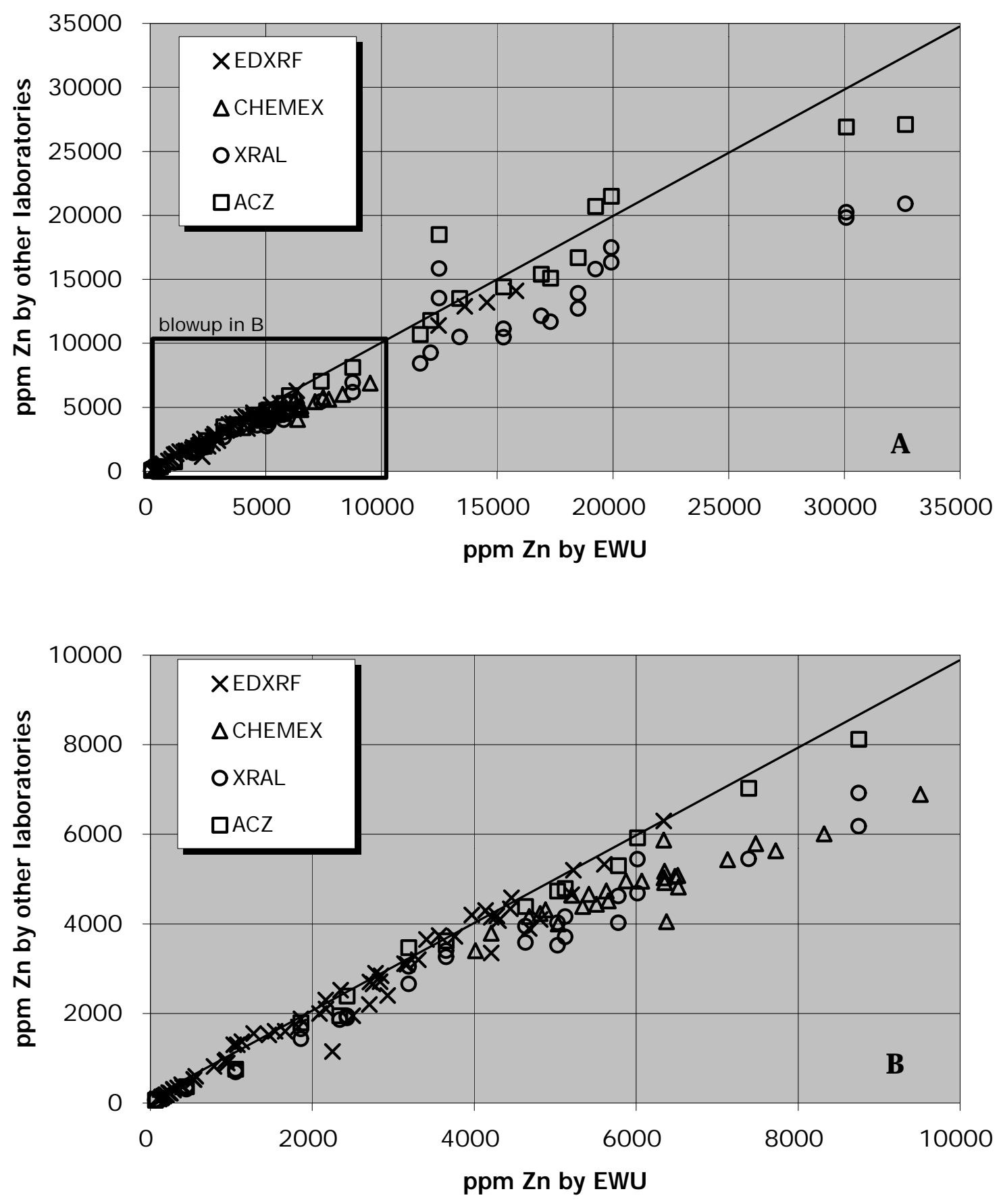

Figure 7. Zn analyses of sample splits analysed by EWU and by one of the other 4 laboratories. (A) Entire dataset, (B) blowup of the lower left corner of A. Line shows 1:1 correlation of analytical values. 
Table 11. Comparisons of $\mathrm{Pb}$ and $\mathrm{Zn}$ values by EWU with those of other labs, and of NIST SRMs by all labs against certified values.

\begin{tabular}{|c|c|c|c|c|c|c|c|c|}
\hline $\begin{array}{l}\text { Analyzed } \\
\text { element }\end{array}$ & $\begin{array}{l}{ }^{1} \text { Laboratory data } \\
\text { compared }(\mathrm{y} / \mathrm{x})\end{array}$ & $\begin{array}{l}{ }^{2} \text { Compositional range } \\
\text { compared (ppm) }\end{array}$ & $\begin{array}{l}\text { Number of } \\
\text { sample } \\
\text { pairs }\end{array}$ & $\begin{array}{c}{ }^{3} \text { coefficient } \\
\text { of determ. } \\
\left(R^{2}\right)\end{array}$ & $\begin{array}{l}{ }^{4} \text { slope } \\
(y / x)\end{array}$ & $\begin{array}{l}\text { Number of } \\
\text { NIST SRM } \\
\text { analyses }\end{array}$ & $\begin{array}{c}\text { Mean Pb recov. } \\
\text { of NIST SRM } \\
\text { values }\end{array}$ & $\begin{array}{c}{ }^{4} \text { slope } \\
\text { (y/ NIST) }\end{array}$ \\
\hline$\overline{\mathrm{Pb}}$ & $\overline{\mathrm{EWU} / \mathrm{NIST}}$ & 1,162 to 5,532 & $\overline{18}$ & 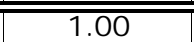 & 0.99 & $\overline{18}$ & $100.0 \%$ & 0.99 \\
\hline $\mathrm{Pb}$ & USGS-EDXRF/EWU & 20 to 8,000 & 59 & 0.99 & 1.03 & 4 & $100.8 \%$ & 1.01 \\
\hline $\mathrm{Pb}$ & USGS-EDXRF/ EWU & 8,000 to 35,000 & 10 & 0.92 & 0.94 & & & \\
\hline $\mathrm{Pb}$ & CHEMEX/EWU & 20 to 8,000 & 20 & 0.85 & 0.77 & & & \\
\hline $\mathrm{Pb}$ & XRAL/ EWU & 20 to 8,000 & 25 & 0.96 & 0.98 & 5 & $96.5 \%$ & 0.95 \\
\hline $\mathrm{Pb}$ & XRAL/ EWU & 8,000 to 35,000 & 18 & 0.94 & 0.92 & & & \\
\hline $\mathrm{Pb}$ & ACZ/EWU & 20 to 8,000 & 13 & 0.98 & 1.00 & 2 & $88.1 \%$ & 0.92 \\
\hline $\mathrm{Pb}$ & ACZ/EWU & 8,000 to 35,000 & 14 & 0.89 & 0.89 & & & \\
\hline $\begin{array}{l}\text { Analyzed } \\
\text { element }\end{array}$ & $\begin{array}{l}\text { Laboratory data } \\
\text { compared }(y / x)\end{array}$ & $\begin{array}{l}\text { Compositional range } \\
\text { compared (ppm) }\end{array}$ & $\begin{array}{l}\text { Number of } \\
\text { sample } \\
\text { pairs }\end{array}$ & $\begin{array}{c}\text { coefficient of } \\
\text { determ. } \\
\left.\mathrm{R}^{2}\right)\end{array}$ & $\begin{array}{l}\text { slope } \\
(y / x)\end{array}$ & $\begin{array}{l}\text { Number of } \\
\text { NIST SRM } \\
\text { analyses }\end{array}$ & $\begin{array}{c}\text { Mean Zn recov. } \\
\text { of NIST SRM } \\
\text { values }\end{array}$ & $\begin{array}{c}\text { slope } \\
\text { (y/ NIST) }\end{array}$ \\
\hline$\overline{\overline{Z n}}$ & 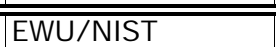 & 350 to 6,952 & $\overline{18}$ & 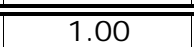 & 0.97 & $\overline{18}$ & $\overline{98.1 \%}$ & $\overline{0.97}$ \\
\hline $\mathrm{Zn}$ & USGS-EDXRF/EWU & 80 to 7,000 & 65 & 0.98 & 0.96 & 4 & $97.9 \%$ & 0.99 \\
\hline $\mathrm{Zn}$ & USGS-EDXRF/EWU & 7,000 to 16,000 & 4 & 0.91 & 0.91 & & & \\
\hline $\mathrm{Zn}$ & CHEMEX/EWU & 4,000 to 7,000 & 22 & 0.52 & 0.81 & & & \\
\hline $\mathrm{Zn}$ & CHEMEX/EWU & 7,000 to 10,000 & 5 & 0.89 & 0.74 & & & \\
\hline $\mathrm{Zn}$ & XRAL/ EWU & 60 to 7,000 & 26 & 0.97 & 0.80 & 5 & $79.8 \%$ & 0.76 \\
\hline $\mathrm{Zn}$ & XRAL/ EWU & 7,000 to 33,000 & 20 & 0.68 & 0.79 & & & \\
\hline $\mathrm{Zn}$ & ACZ/EWU & 60 to 7,000 & 13 & 0.99 & 0.95 & 2 & $87.0 \%$ & 0.86 \\
\hline $\mathrm{Zn}$ & ACZ/EWU & 7,000 to 33,000 & 14 & 0.84 & 0.94 & & & \\
\hline
\end{tabular}

${ }^{1}$ for samples analyzed by both methods ( $y=$ first listed lab, $x=$ second listed lab)

${ }^{2}$ values given from analyses by lab listed second

${ }^{3}$ measure of goodness-of-fit of least squares linear regression through the paired analytical values, projected through origin

${ }^{4}$ slope of least-squares linear regression through paired analytical values and the origin. 
Comparison of the inter-laboratory analyses of Zn (figure 7) indicates that USGSEDXRF and ACZ give values less than 5\% lower than those of EWU for concentrations less than 7,000 ppm, while both CHEMEX and XRAL give values that are about $20 \%$ low (table 11). The relative recoveries by each laboratory relative to EWU are lower for $\mathrm{Zn}$ concentrations above 7,000 ppm, and the scatter is higher.

Comparison of the inter-laboratory analyses of Fe (figure 8) indicates that, for concentrations below $16 \%$ Fe, analyses by ACZ and by XRAL are less than $8 \%$ low relative to EWU values (with considerable scatter: $\mathrm{R}^{2}=0.90-0.92$ ), while CHEMEX analyses are about $16 \%$ low (table 12). Only a few samples (analyzed by XRAL and ACZ) have Fe concentrations greater than $16 \%$, and these few samples appear to have even lower Fe recovery relative to EWU values.

Comparison of the inter-laboratory analyses of Mn (figure 9) indicates that, for concentrations below 10,000 ppm, only XRAL analyses closely compare with those of EWU (within 1\%); ACZ analyses are about 6\% greater than, and CHEMEX are about $18 \%$ less than those of EWU (table 12). For Mn concentrations above 10,000 ppm, the scatter of the inter-laboratory analyses is even greater, comparisons are even worse for ACZ and CHEMEX, but recoveries are similar for XRAL.

Comparison of the inter-laboratory analyses of As (figure 10) indicates that, for low concentrations (less than $70 \mathrm{ppm}$ ) XRAL and ACZ give very similar values to those of EWU, while CHEMEX gives considerably lower values (table 13). At higher concentrations (but still below the As concentration of NIST SRM 2710), all 3 laboratories (XRAL, ACZ and CHEMEX) consistently yield values that are 11-14\% less than those of EWU with relatively narrow scatter $\left(\mathrm{R}^{2}=0.97-0.98\right)$.

Comparison of the inter-laboratory analyses of Cd (figure 11) indicates that, for Cd concentrations less than $50 \mathrm{ppm}$, XRAL analyses are very close to those of EWU (within 2\%) with little scatter, while those of CHEMEX and ACZ are 8-13\% lower (table 13). At concentrations from 50 to $150 \mathrm{ppm} \mathrm{Cd}$, relative recoveries of $\mathrm{Cd}$ by XRAL and ACZ are higher and even closer to EWU values, although scatter is higher. 

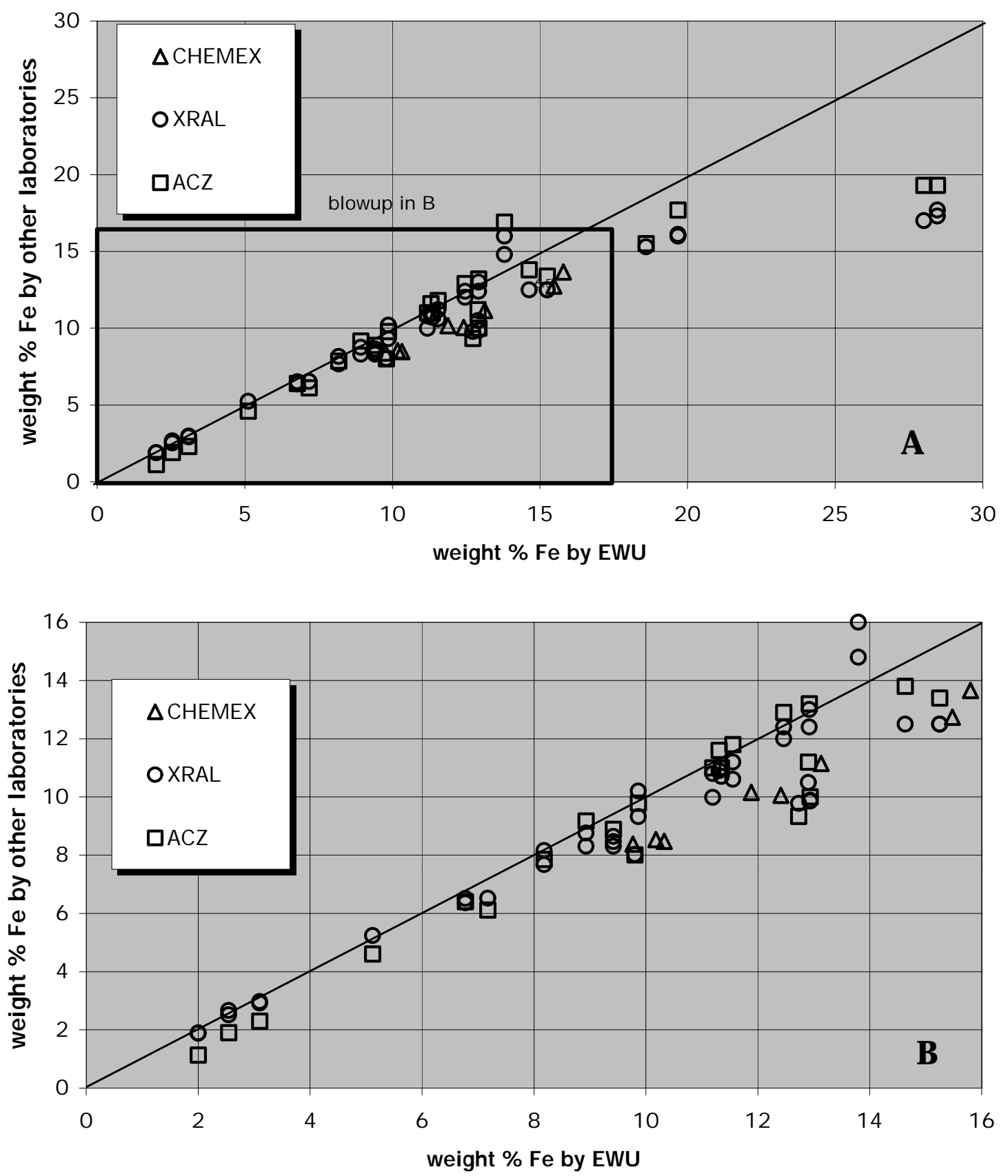

Figure 8. Fe analyses of sample splits analyzed by EWU and by one of the other 4 laboratories. (A) Entire dataset, (B) blowup of the lower left corner of A. Line shows 1:1 correlation of analytical values. 

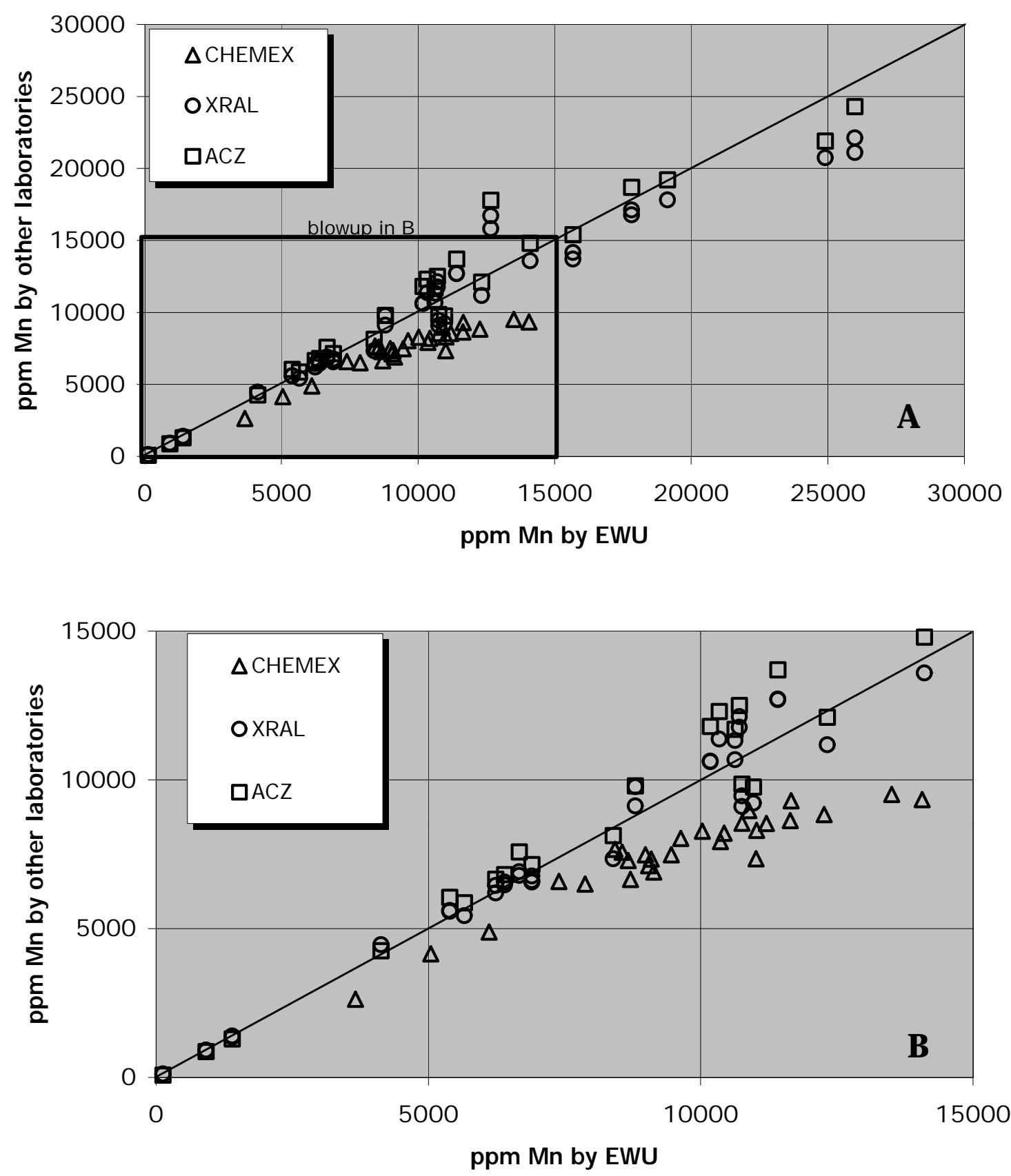

Figure 9. Mn analyses of sample splits analysed by EWU and by one of the other 4 laboratories. (A) Entire dataset, (B) blowup of the lower left corner of A. Line shows 1:1 correlation of analytical values. 
Table 12. Comparisons of Fe and Mn values by EWU with those of other labs, and of NIST SRMs by all labs against certified values.

\begin{tabular}{|c|c|c|c|c|c|c|c|c|}
\hline $\begin{array}{l}\text { Analyzed } \\
\text { element }\end{array}$ & $\begin{array}{l}{ }^{1} \text { Laboratory data } \\
\text { compared }(y / x)\end{array}$ & $\begin{array}{c}{ }^{2} \text { Compositional range } \\
\text { compared (wt } \%)\end{array}$ & $\begin{array}{l}\text { Number of } \\
\text { sample } \\
\text { pairs }\end{array}$ & $\begin{array}{c}{ }^{3} \text { coefficient of } \\
\text { determ. } \\
\left.\mathrm{R}^{2}\right)\end{array}$ & $\begin{array}{l}{ }^{4} \text { slope } \\
(y / x)\end{array}$ & $\begin{array}{c}\text { Number of } \\
\text { NIST SRM } \\
\text { analyses }\end{array}$ & $\begin{array}{c}\text { Mean Fe recov. } \\
\text { of NIST SRM } \\
\text { values }\end{array}$ & $\begin{array}{l}{ }^{4} \text { slope } \\
\text { (y/ NIST) }\end{array}$ \\
\hline$\overline{\mathrm{Fe}}$ & EWU/NIST & 2.7 to 3.3 & $\overline{\overline{18}}$ & 0.82 & $\overline{1.00}$ & $\overline{\overline{18}}$ & $100.6 \%$ & $\overline{1.00}$ \\
\hline $\mathrm{Fe}$ & CHEMEX/EWU & 9 to 16 & 8 & 0.98 & 0.84 & & & \\
\hline $\mathrm{Fe}$ & XRAL/ EWU & 2 to 16 & 40 & 0.92 & 0.93 & 5 & $92.1 \%$ & 0.92 \\
\hline $\mathrm{Fe}$ & ACZ/EWU & 2 to 16 & 23 & 0.90 & 0.95 & 2 & $69.8 \%$ & 0.84 \\
\hline $\begin{array}{l}\text { Analyzed } \\
\text { element }\end{array}$ & $\begin{array}{l}\text { Laboratory data } \\
\text { compared }(\mathrm{y} / \mathrm{x})\end{array}$ & $\begin{array}{l}\text { Compositional range } \\
\text { compared (ppm) }\end{array}$ & $\begin{array}{l}\text { Number of } \\
\text { sample } \\
\text { pairs }\end{array}$ & $\begin{array}{c}\text { coefficient of } \\
\text { determ. } \\
\left.\mathrm{R}^{2}\right)\end{array}$ & $\begin{array}{l}\text { slope } \\
(y / x)\end{array}$ & $\begin{array}{c}\text { Number of } \\
\text { NIST SRM } \\
\text { analyses }\end{array}$ & $\begin{array}{c}\text { Mean Mn } \\
\text { recov. of NIST } \\
\text { SRM values }\end{array}$ & $\begin{array}{c}\text { slope } \\
\text { (y/ NIST) }\end{array}$ \\
\hline$\overline{\mathrm{Mn}}$ & EWU/NIST & 638 to 10,100 & $\overline{18}$ & $\overline{1.00}$ & 0.99 & $\overline{18}$ & 99.5\% & 0.99 \\
\hline $\mathrm{Mn}$ & CHEMEX/EWU & 3500 to 10,000 & 15 & 0.93 & 0.82 & & & \\
\hline $\mathrm{Mn}$ & CHEMEX/EWU & 10,000 to 14,000 & 13 & 0.07 & 0.75 & & & \\
\hline $\mathrm{Mn}$ & XRAL/ EWU & 100 to 10,000 & 22 & 0.98 & 1.01 & 5 & $88.9 \%$ & 0.87 \\
\hline $\mathrm{Mn}$ & XRAL/ EWU & 10,000 to 20,000 & 21 & 0.64 & 1.00 & & & \\
\hline $\mathrm{Mn}$ & ACZ/EWU & 100 to 10,000 & 12 & 0.99 & 1.06 & 2 & $74.4 \%$ & 0.78 \\
\hline $\mathrm{Mn}$ & ACZ/EWU & 10,000 to 20,000 & 13 & 0.69 & 1.08 & & & \\
\hline
\end{tabular}

${ }^{1}$ for samples analyzed by both methods ( $y=$ first listed lab, $x=$ second listed lab)

${ }^{2}$ values given from analyses by lab listed second

${ }^{3}$ measure of goodness-of-fit of least squares linear regression through the paired analytical values, projected through origin.

${ }^{4}$ slope of least-squares linear regression through paired analytical values and the origin. 
Comparison of recoveries relative to EWU for CdA samples and to NIST for NIST SRMs for each laboratory indicates general similarities with a few interesting differences (tables 11 to 13). Recoveries of $\mathrm{Pb}$ and $\mathrm{Zn}$ by USGS-EDXRF are within 4\% both of EWU values of CdA samples and of NIST certified values of NIST SRM. XRAL percent recoveries of certified values of NIST SRMs are similar to percent recoveries of EWU values for CdA samples for most elements except for Mn and Cd. XRAL recovery of $\mathrm{Mn}$ and $\mathrm{Cd}$ from CdA samples relative to EWU values is $98-100 \%$, while $\mathrm{Mn}$ and $\mathrm{Cd}$ recovery by XRAL from NIST SRMs is much lower (87-89\%). In other words for a given CdA sample, XRAL and EWU yielded similar Mn and Cd analytical values, even though XRAL had much poorer recovery of Mn and Cd from NIST SRMs than did EWU. In contrast $\mathrm{ACZ}$ analytical values for $\mathrm{Pb}, \mathrm{Zn}, \mathrm{Fe}$, and $\mathrm{Mn}$ for split $\mathrm{CdA}$ samples are similar to EWU values (within 6\%), even though ACZ analytical values of NIST SRMs were considerably lower (12-30\%) than the certified value. ACZ values for As and $\mathrm{Cd}$ for split CdA samples are much lower than corresponding EWU values (87-89\%), and are similar to ACZ percent recoveries of certified values for NIST SRMs. These differences in recovery for CdA samples versus for NIST SRMs probably reflects differences in the way these elements are held in the matrix of the two sample types and the contrasting efficiency of the several sample dissolution methods used.

\section{Conclusions}

This report documents the results of over 1,100 chemical analyses of samples of sediments and soils that were deposited in the CdA drainage basin before and during the era of large-scale mining in the CdA mining district, in north Idaho. These samples were collected as part of an effort to determine the character, distribution, thickness, volume, and metals contents of sediments and soils within the CdA drainage basin. Five different laboratories using different sample preparation and analytical techniques contributed geochemical data for this report. Analytical accuracy is given by comparison of data from multiple analyses of pulverized NIST SRMs by four of the laboratories. Analytical precision is calculated for data from two laboratories (EWU and XRAL) from repeated analyses of SRMs. Analyses of splits of over $21 \%$ of the soil and sediment samples by 

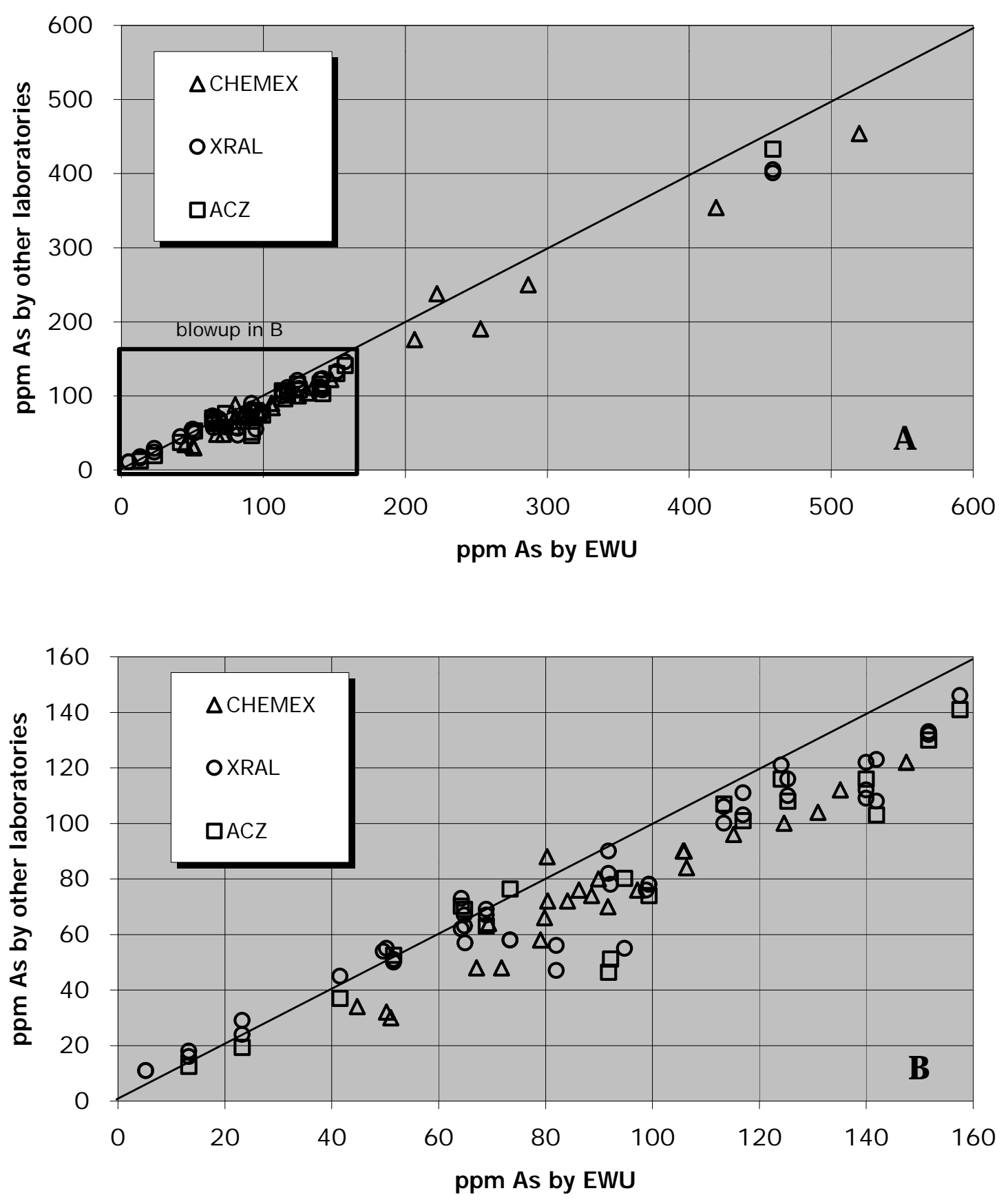

Figure 10. As analyses of sample splits analysed by EWU and by one of the other 4 laboratories. (A) Entire dataset, (B) blowup of the lower left corner of A. Line shows 1:1 correlation of analytical values. 

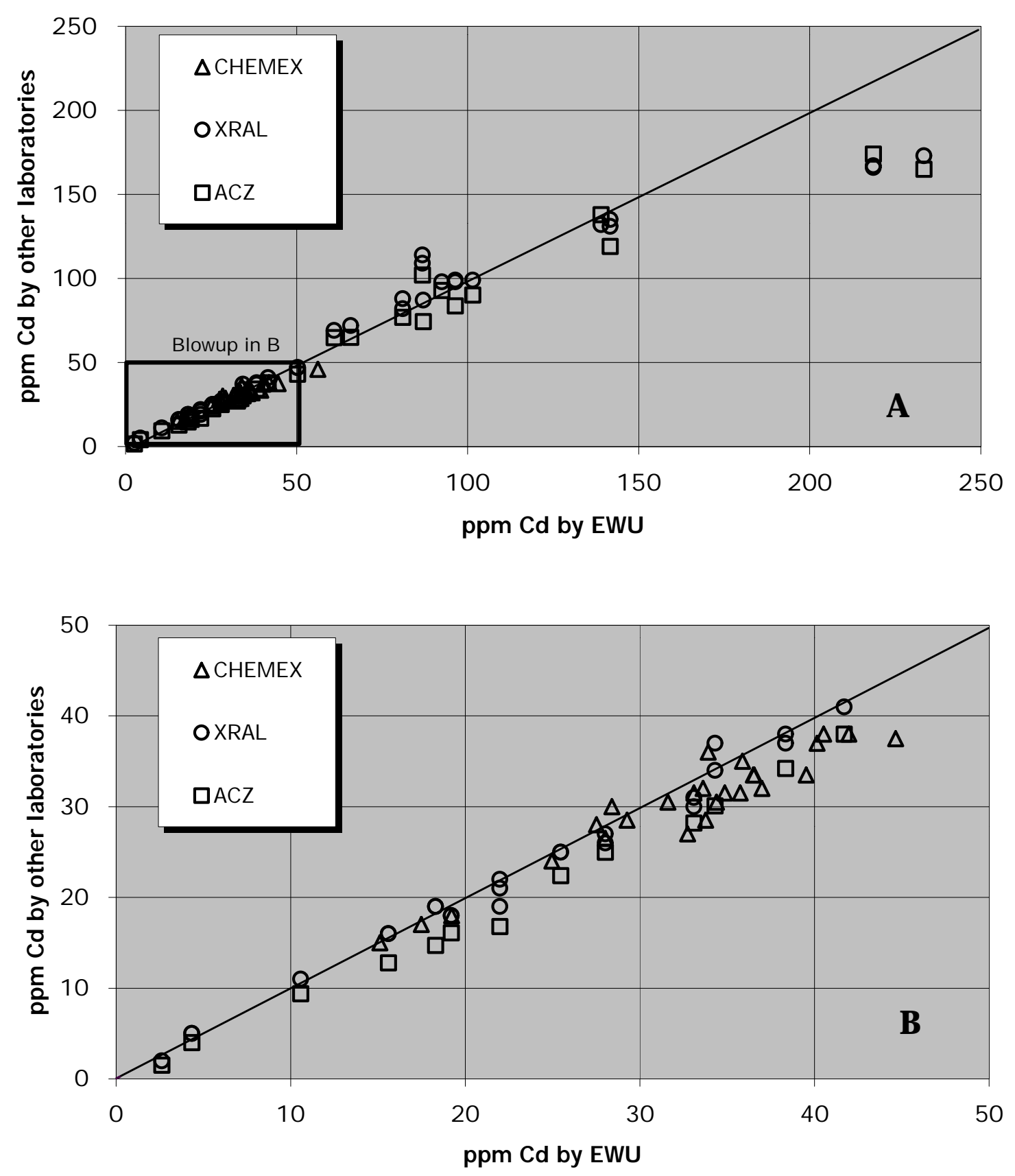

Figure 11. Cd analyses of sample splits analysed by EWU and by one of the other 4 laboratories. (A) Entire dataset, (B) blowup of the lower left corner of A. Line shows 1:1 correlation of analytical values. 
Table 13. Comparisons of As and Cd values by EWU with those of other labs, and of NIST SRMs by all labs against certified values.

\begin{tabular}{|c|c|c|c|c|c|c|c|c|}
\hline $\begin{array}{l}\text { Analyzed } \\
\text { element }\end{array}$ & $\begin{array}{l}{ }^{1} \text { Laboratory data } \\
\text { compared }(y / x)\end{array}$ & $\begin{array}{l}{ }^{2} \text { Compositional range } \\
\text { compared }(\mathrm{ppm})\end{array}$ & $\begin{array}{l}\text { Number of } \\
\text { sample } \\
\text { pairs }\end{array}$ & $\begin{array}{c}{ }^{3} \text { coefficient } \\
\text { of determ. } \\
\left(R^{2}\right)\end{array}$ & $\begin{array}{l}{ }^{4} \text { slope } \\
(y / x)\end{array}$ & $\begin{array}{l}\text { Number of } \\
\text { NIST SRM } \\
\text { analyses }\end{array}$ & $\begin{array}{l}\text { Mean As } \\
\text { recov. of NIST } \\
\text { SRM values }\end{array}$ & $\begin{array}{c}{ }^{4} \text { slope } \\
\text { (y/ NIST) }\end{array}$ \\
\hline$\overline{\overline{A s}}$ & EWU/NIST & 105 to 626 & $\overline{\overline{18}}$ & 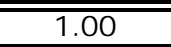 & $\overline{0.99}$ & $\overline{18}$ & 99.8\% & 0.99 \\
\hline As & CHEMEX/EWU & 45 to 520 & 30 & 0.98 & 0.86 & & & \\
\hline As & XRAL/ EWU & 5 to 70 & 18 & 0.96 & 1.01 & & & \\
\hline As & XRAL/ EWU & 70 to 460 & 28 & 0.98 & 0.86 & 5 & $87.8 \%$ & 0.84 \\
\hline As & ACZ/ EWU & 5 to 70 & 7 & 0.96 & 1.00 & & & \\
\hline As & ACZ/ EWU & 70 to 460 & 28 & 0.97 & 0.89 & 2 & $66.3 \%$ & 0.90 \\
\hline $\begin{array}{l}\text { Analyzed } \\
\text { element }\end{array}$ & $\begin{array}{l}\text { Laboratory data } \\
\text { compared }(y / x)\end{array}$ & $\begin{array}{l}\text { Compositional range } \\
\text { compared }(\mathrm{ppm})\end{array}$ & $\begin{array}{l}\text { Number of } \\
\text { sample } \\
\text { pairs }\end{array}$ & $\begin{array}{l}\text { coeffecient } \\
\text { of determ. } \\
\qquad\left(R^{2}\right)\end{array}$ & $\begin{array}{l}\text { slope } \\
(y / x)\end{array}$ & $\begin{array}{c}\text { Number of } \\
\text { NIST SRM } \\
\text { analyses }\end{array}$ & $\begin{array}{c}\text { Mean Cd } \\
\text { recov. of NIST } \\
\text { SRM values }\end{array}$ & $\begin{array}{c}\text { slope } \\
\text { (y/ NIST) }\end{array}$ \\
\hline$\overline{\mathrm{Cd}}$ & EWU/NIST & 21.8 to 41.7 & $\overline{18}$ & 0.99 & $\overline{0.96}$ & $\overline{18}$ & 95.3\% & $\overline{0.96}$ \\
\hline $\mathrm{Cd}$ & CHEMEX/EWU & 15 to 45 & 27 & 0.89 & 0.92 & & & \\
\hline $\mathrm{Cd}$ & XRAL/ EWU & 2 to 50 & 24 & 0.99 & 0.98 & 5 & $80.1 \%$ & 0.88 \\
\hline $\mathrm{Cd}$ & XRAL/ EWU & 50 to 150 & 19 & 0.85 & 1.02 & & & \\
\hline $\mathrm{Cd}$ & ACZ/ EWU & 2 to 50 & 13 & 0.99 & 0.87 & 2 & $87.6 \%$ & 0.88 \\
\hline $\mathrm{Cd}$ & ACZ/ EWU & 50 to 150 & 11 & 0.87 & 0.94 & & & \\
\hline
\end{tabular}

${ }^{1}$ for samples analyzed by both methods ( $y=$ first listed lab, $x=$ second listed lab)

${ }^{2}$ values given from analyses by lab listed second

${ }^{3}$ measure of goodness-of-fit of least squares linear regression through the paired analytical values, projected through origin.

${ }^{4}$ slope of least-squares linear regression through paired analytical values and the origin. 
more than one laboratory allow direct comparison of the laboratories in analysis of $\mathrm{CdA}$ basin materials.

Inter-laboratory comparisons are made for 6 elements: lead $(\mathrm{Pb})$, zinc $(\mathrm{Zn})$, iron $(\mathrm{Fe})$, manganese $(\mathrm{Mn})$, arsenic (As), and cadmium $(\mathrm{Cd})$. In general inter-laboratory correlations are better for samples within the compositional ranges of the NIST SRMs. Analyses by EWU are the most accurate relative to the NIST standards (within 1\% for $\mathrm{Pb}, \mathrm{Fe}, \mathrm{Mn}$, and $\mathrm{As}, 3 \%$ for $\mathrm{Zn}$ and $5 \%$ for $\mathrm{Cd}$ ) and are the most precise (within 7\%). USGS-EDXRF is similarly accurate for $\mathrm{Pb}$ and $\mathrm{Zn}$. CHEMEX were the least accurate, yielding values $10-25 \%$ less than those of EWU. XRAL and ACZ are relatively accurate for $\mathrm{Pb}$ (within 5-8\% of NIST values and of EWU analyses), but were considerably less accurate for the other 5 elements of concern (10-25\% of NIST values). For some elements, however, XRAL and ACZ analyses of CdA samples were comparable to EWU analyses of the same samples, suggesting that, for some elements, XRAL and ACZ dissolutions are more effective on the matrix of the CdA samples than on the matrix of the NIST samples (obtained from soils around Butte, Montana). 


\section{Acknowledgements}

Former USGS-GD employee Cole Smith was instrumental in organizing the Coeur d'Alene geo-environmental project in the Spokane Field Office in 1992-93. BiShiea King helped James Lindsay do XRF analyses in the Menlo Park laboratories. Richard O'Leary and Stephen Wilson provided QA-QC and descriptions of USGS QAQC procedures for samples analyzed at XRAL Laboratories.

USGS-WRD employees Michael Beckwith, Paul Woods, Rick Backson, and Robert Fousek helped with 1994 drilling projects. University of Idaho researcher William Rember helped with drilling in 1995, as did chemistry professor William van der Sluys and his students from Gonzaga University.

Eastern Washington University students, working part-time for USGS, participated as follows: Julie Eddy collected stream sediment samples from the South Fork, Reanette Boese and Nasir Aziz did sample preparation, Andrew Knowles and Nasir Aziz helped with drilling, John Wallis and Patrick Blair did most of the 1998 floodplain sampling.

Julie Campbell, of USFWS, provided information about analytical procedures contracted to ACZ Laboratories by USFWS.

John Wallis completed the digital conversion of all the data and wrote the metadata file. The manuscript was greatly improved by the review of Laurie Balistrieri. 


\section{References}

Abraham, Joju, 1994, Impact of mining on the trace element geochemistry and lead isotopic composition of sediments in Coeur d'Alene River, Idaho: unpublished. M.S. thesis, Eastern Washington University, Cheney, Washington, 149 p.

Balistrieri, L.S., Box, S.E., Ikramuddin, Mohammed, Horowitz, A.J., and Elrick, K.A., 2000, A study of porewater in water saturated sediments of levee banks and marshes in the lower Coeur d'Alene River valley, Idaho: Sampling, analytical methods, and results: U. S. Geological Survey Open-File Report 00-126.

Campbell, J.K., Audet, D.J., Kern, J.W., Reyes, Marie, and McDonald, L.L., 1999, Metal contamination of palustrine and lacustrine habitats in the CdA Basin, Idaho: Draft report, USFWS and Western Ecosystems Technology, Inc., 26 p.

Condoyannis, Nick, 1995, The uptake of heavy metals and associated elements by selected plants on tailings-contaminated soils: An example from the Coeur d'Alene river Basin, Idaho: unpublished. M.S. thesis, Eastern Washington University, Cheney, Washington, 109 p.

Compton, R.R., 1985, Geology in the field: John Wiley and Sons, Inc., New York, 398 p.

Fletcher, 1981, Handbook of exploration geochemistry: Volume1. Analytical Methods in Geochemical Prospecting: Elsevier Scientific Publishing Co., Amsterdam, Oxford, New York.

Fousek, R.S., 1996, Trace-element distributions in the sediments of the flood plain and river banks of the South Fork and Coeur d'Alene Rivers, Shoshone and Kootenai Counties, Idaho: unpublished. M.S. thesis, Auburn University, Auburn, Alabama, 333 p.

Gills, 1993a, Certificate of analysis, Standard Reference Material 2710, Montana soil, highly elevated trace element concentrations: U.S. Dept. of Commerce, National Institute of Standards and Technology (NIST), 5 p., addendum 6 p. 
Gills, 1993b, Certificate of analysis, Standard Reference Material 2711, Montana soil, moderately elevated trace element concentrations: U.S. Dept. of Commerce, National Institute of Standards and Technology (NIST), 5 p., addendum 7 p.

Goddard, E.N., Trask, P.D., De Ford, R.K, Rove, O.N., Singewald, J.T., Jr., and Overbeck, R.M., 1970, Rock-color chart: Geological Society of America, Boulder, CO

Govindaraju, K., 1994, Geostandards newsletter: Vol. XVIII Special Issue: International Working Group "Analytical standards of Minerals, Ores and Rocks, with the assistance of Centre de Recherches Petrographiques et Geochimiques (CNRS), Vandoeuver-les-Nancy, France, July 1994, ISSN 0150 5505, p. 115-117 and Appendix I, p. 01 to 03.

Grant, L.A., 1952, A history of the Cataldo dredge: Fourth Annual Pacific Northwest Industrial Waste Conference Proceedings, Washington State College, Pullman, Washington, p. 101-110.

Hall, Gwendy E.M., 1999, "Near total" acid digestions: Explore, Newsletter for the Association of Exploration Geochemists, no. 104, p. 15-19.

Horowitz, Arthur J., Elrick, Kent A., and Cook, Robert B., 1993, Effect of mining and related activities on the sediment trace element geochemistry of Lake Coeur d'Alene, Idaho, USA Part I. Surface sediments: Hydrological Processes, v. 7, p. 403-423.

Hoffmann, M.L., 1995, Characterization of heavy metal contamination in two lateral lakes of the lower Coeur d'Alene River valley, northern Idaho: unpublished M.S. thesis, University of Idaho, Moscow, Idaho,76 p.

Johnson, R.G., and King, B.-S.L., 1987, Energy dispersive X-ray fluorescence spectrometry, in Beadecker, Philip A., editor, Methods of Geochemical Analyis: U.S. Geological Survey Bulletin 1770, p. F1-F5.

Kane, J.S., 1991, Quality control and reference sample data bases: Geostandards Newsletter, v. 15, no 1, p. 33 to 47. 
Levinson, A.A., 1974, Introduction to exploration geochemistry: Applied Publishing Co., Calgary, $608 \mathrm{p}$.

Long, K.R., 1998a, Grade and tonnage models for Coeur d'Alene-type polymetallic veins: U.S. Geological Survey Open-File Report OF 98-583, 28 p.

Long, K.R., 1998b, Production and disposal of mill tailings in the Coeur d'Alene mining region, Shoshone County, Idaho; Preliminary estimates: U.S. Geological Survey Open-File Report OF 98-595, 14 p.

Long, K.R., DeYoung, J.H.,Jr., and Ludington, Steve, 2000, Significant deposits of gold, silver, copper, lead, and zinc in the United States: Economic Geology, v. 95, no. 3, p. 629-641.

Rabe, F.W., and Flaherty, D.C., 1974, The river of green and gold: A pristine wilderness dramatically affected by man's discovery of gold: Idaho Research Foundation, Inc., Natural Resource Series, no. 4, Moscow, Idaho, 97 p.

Sprenke, K.F., Rember, W.C., Bender, S.F., Hoffmann, M.L., Rabbi, F., Chamberrlain, V.E., 2000, Toxic metal contamination in the lateral lakes of the Coeur d'Alene River valley, Idaho: Environmental Geology, Springer-Verlag, v. 39, p. 575-586.

US Dept. of Health and Human Services (USDHHS), 1994, Toxicological profile for zinc: Public Health Service, Agency for Toxic Substances and Disease Registry Report TP-93/15, 230 p., 2 appendices.

US Dept. of Health and Human Services (USDHHS), 1997, Toxicological profile for manganese (update): Public Health Service, Agency for Toxic Substances and Disease Registry, 201 p., 3 appendices.

US Dept. of Health and Human Services (USDHHS), 1998, Toxicological profile for arsenic (update): Public Health Service, Agency for Toxic Substances and Disease Registry, 349 p., 3 appendices.

US Dept. of Health and Human Services (USDHHS), 1999a, Toxicological profile for lead (update): Public Health Service, Agency for Toxic Substances and Disease Registry, 587 p., 4 appendices. 
US Dept. of Health and Human Services (USDHHS), 1999b, Toxicological profile for cadmium: Public Health Service, Agency for Toxic Substances and Disease Registry, 397 p., 3 appendices.

US Environmental Protection Agency (USEPA), 1998, Sediment contamination in the Lower Coeur d'Alene River Basin (LCDARB): Geophysical and sediment coring investigations in the river channel, lateral lakes, and floodplains: Bunker Hill Facility Basin-Wide RI/FS Data Report, vol. 1 and 2, prepared for USEPA by URS Greiner, Inc. and CH2M Hill. 


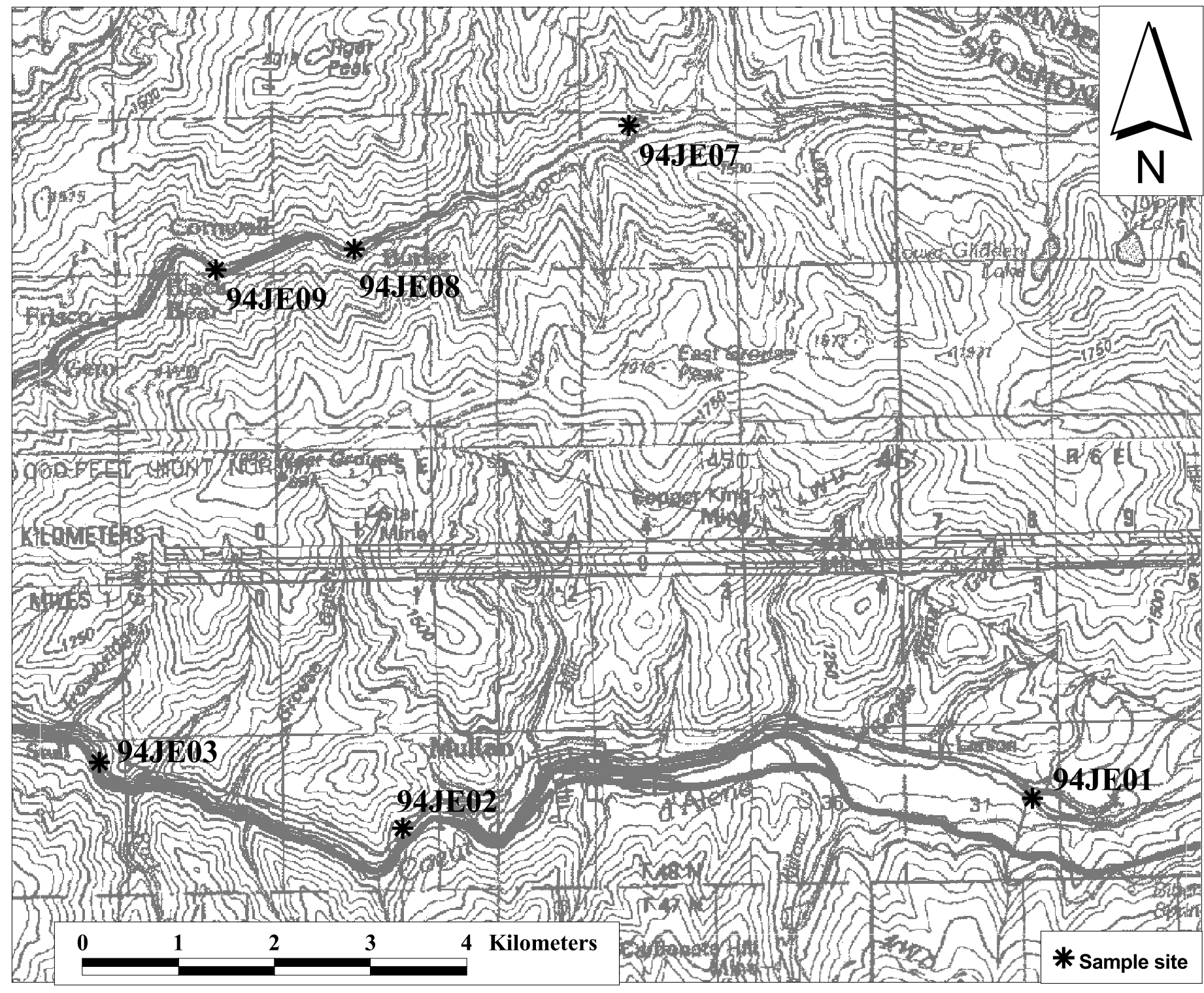

Map A-1: Sample site location map for the South Fork of the Coeur d'Alene River and tributaries near Mullan and Burke, Idaho. Base map from USGS Thomson Falls and Wallace, Idaho 1:100,000 quadrangles. 


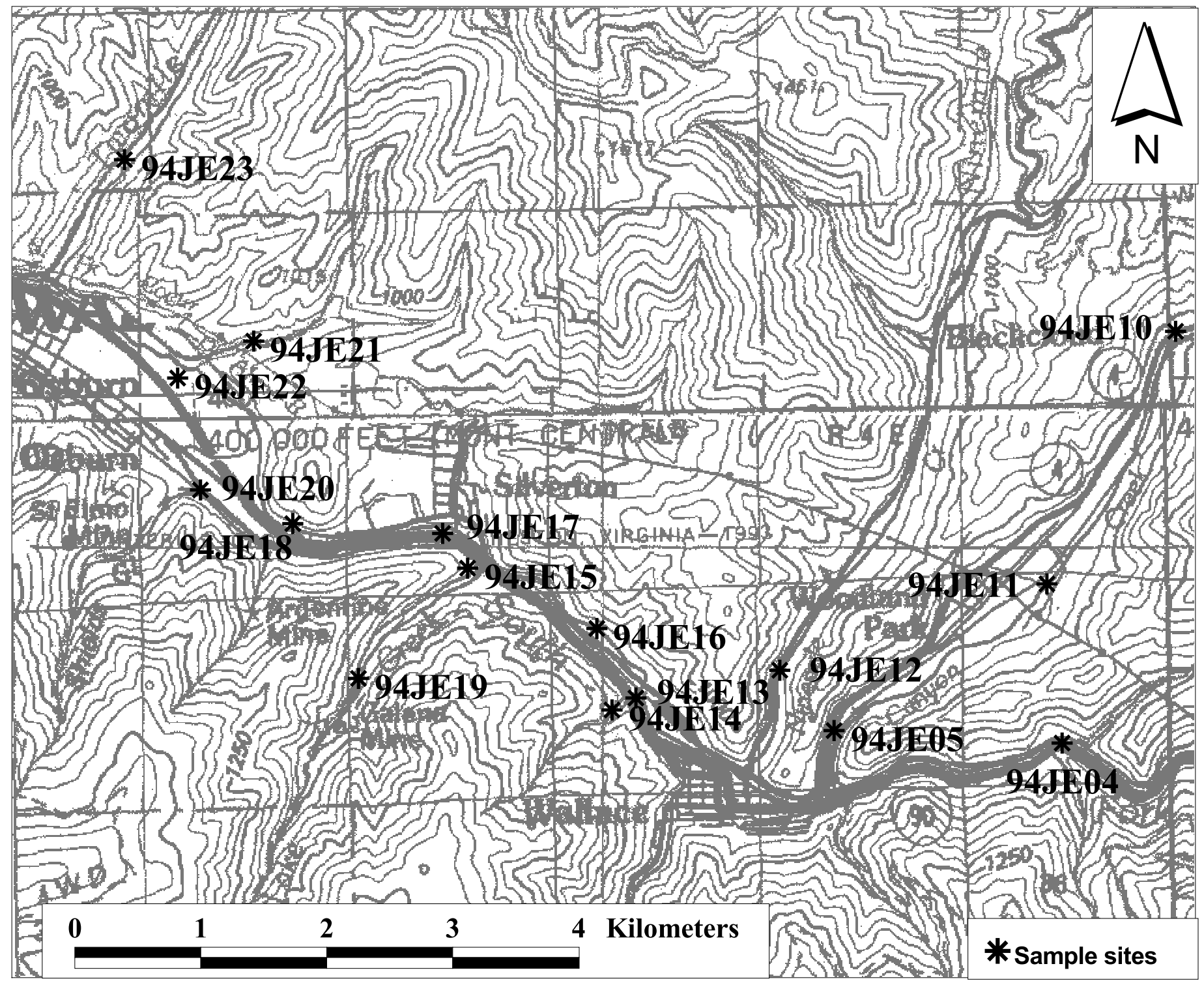

Map A-2: Sample site locations along the South Fork of the Coeur d'Alene River and tributaries near Wallace, Idaho. Base map from USGS Thomson Falls and Wallace, Idaho 1:100,000 quadrangles. 


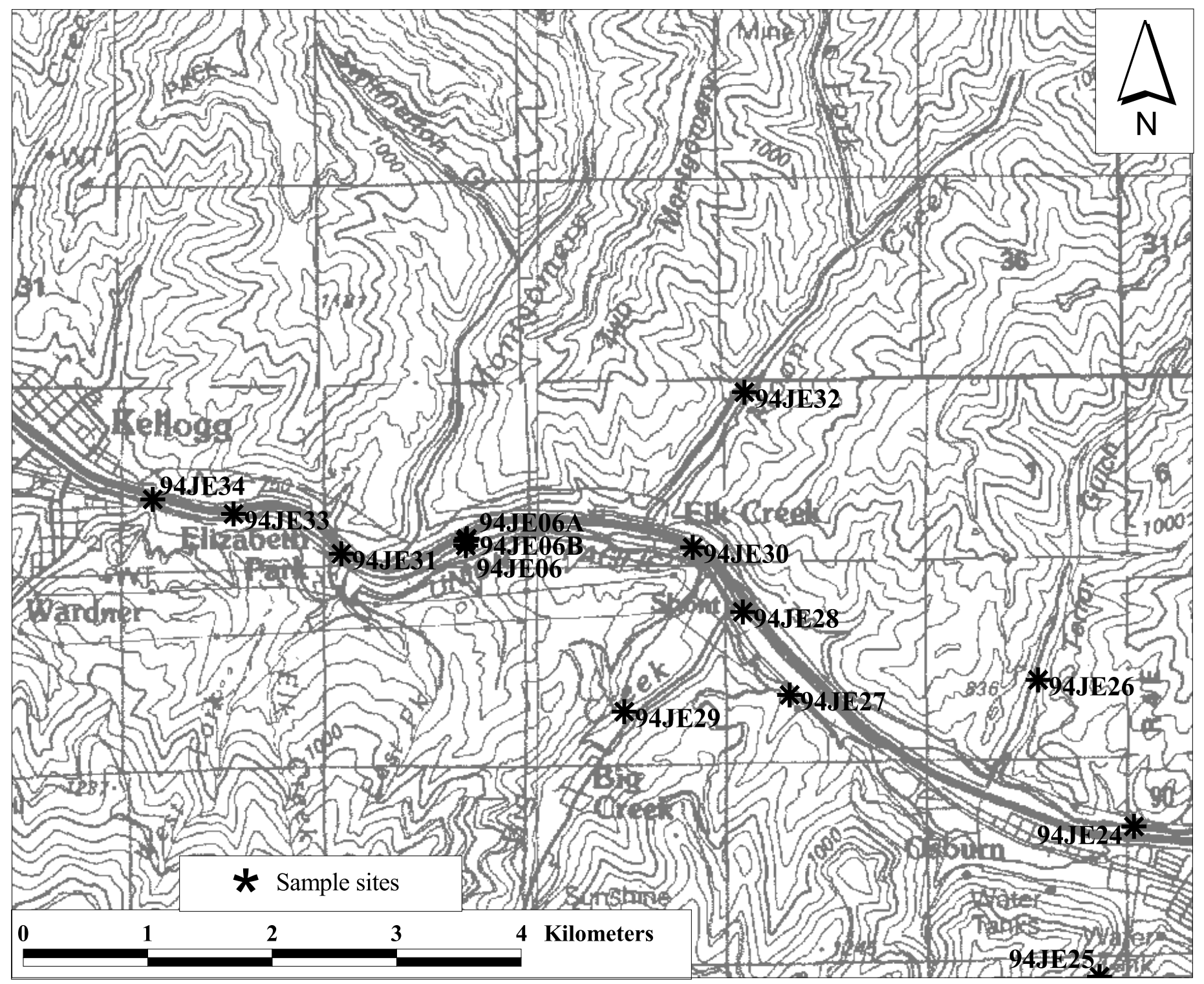

Map A-3: Sample site location map for the South Fork of the Coeur d'Alene River and tributaries between Osburn and Kellogg, Idaho. Base map from USGS Coeur d'Alene, Idaho 1:100,000 quadrangle. 


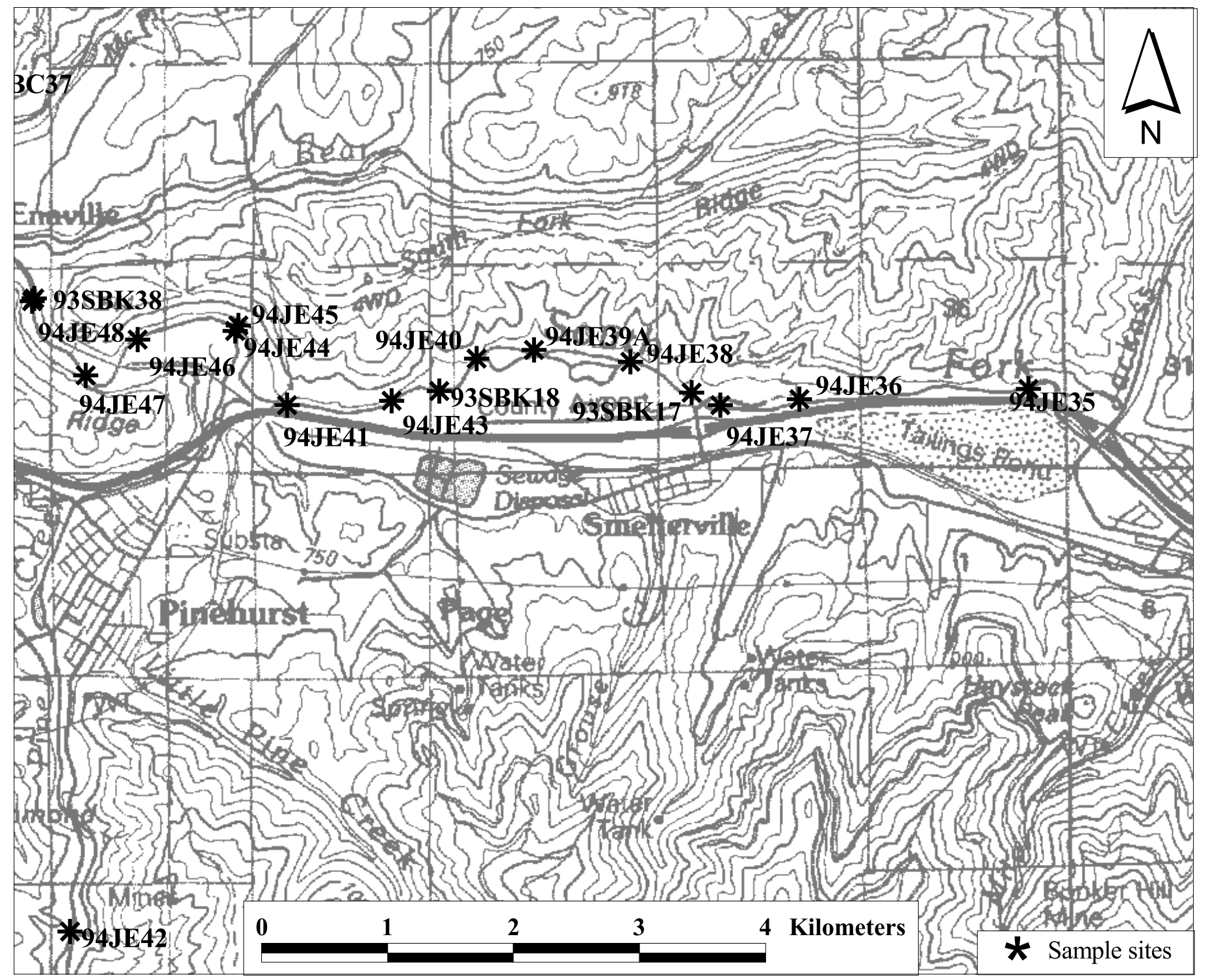

Map A-4: Sample site location map for the South Fork of the Coeur d'Alene River and tributaries between Kellogg and Pinehurst, Idaho. Base map from USGS Coeur d'Alene, Idaho 1:100,000 quadrangle. 


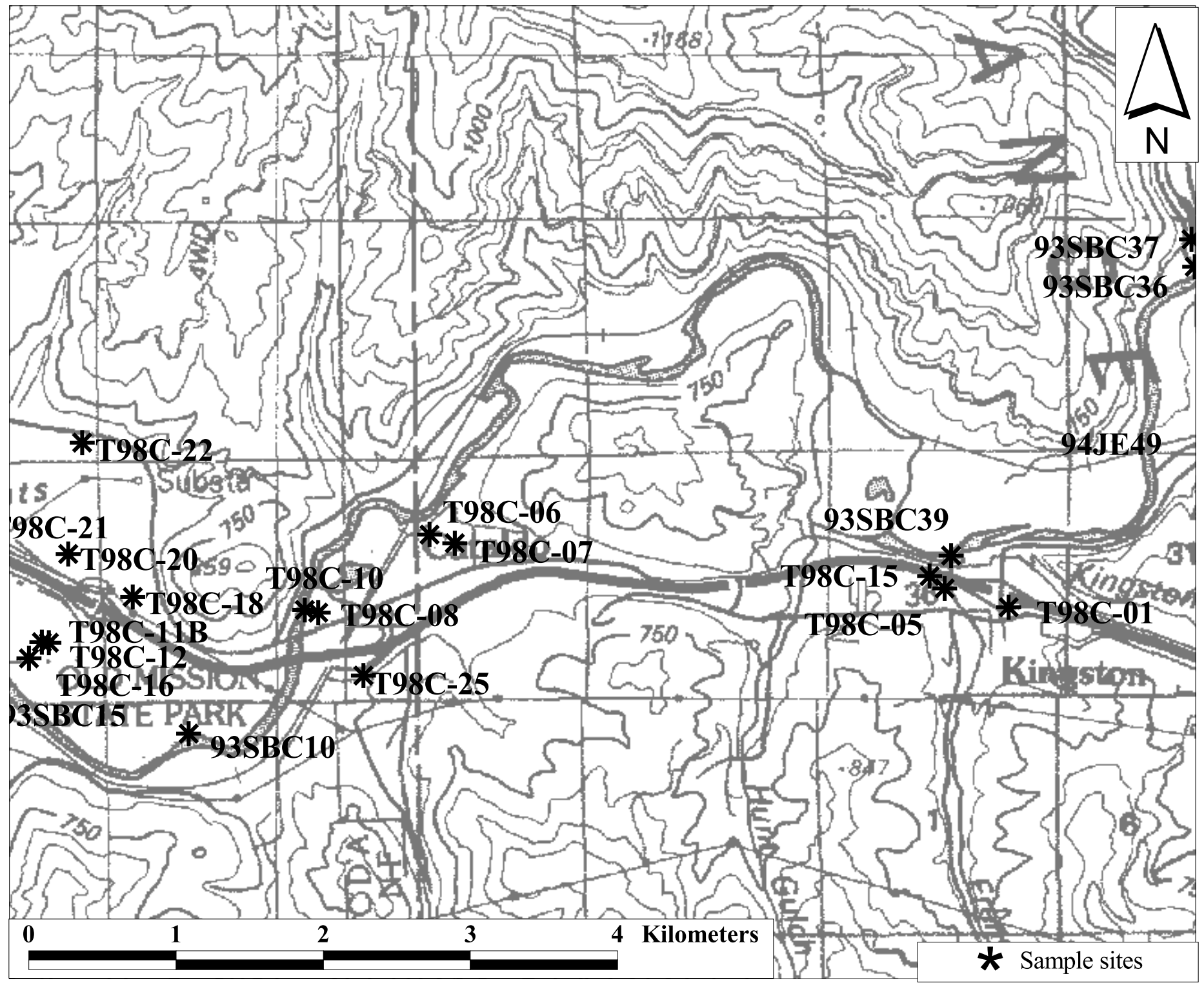

Map A-5: Sample site location map for the North Fork and mainstem of the Coeur d'Alene River between Enaville and the Cataldo Mission, Idaho. Base map from USGS Coeur d'Alene, Idaho 1:100,000 quadrangle. 


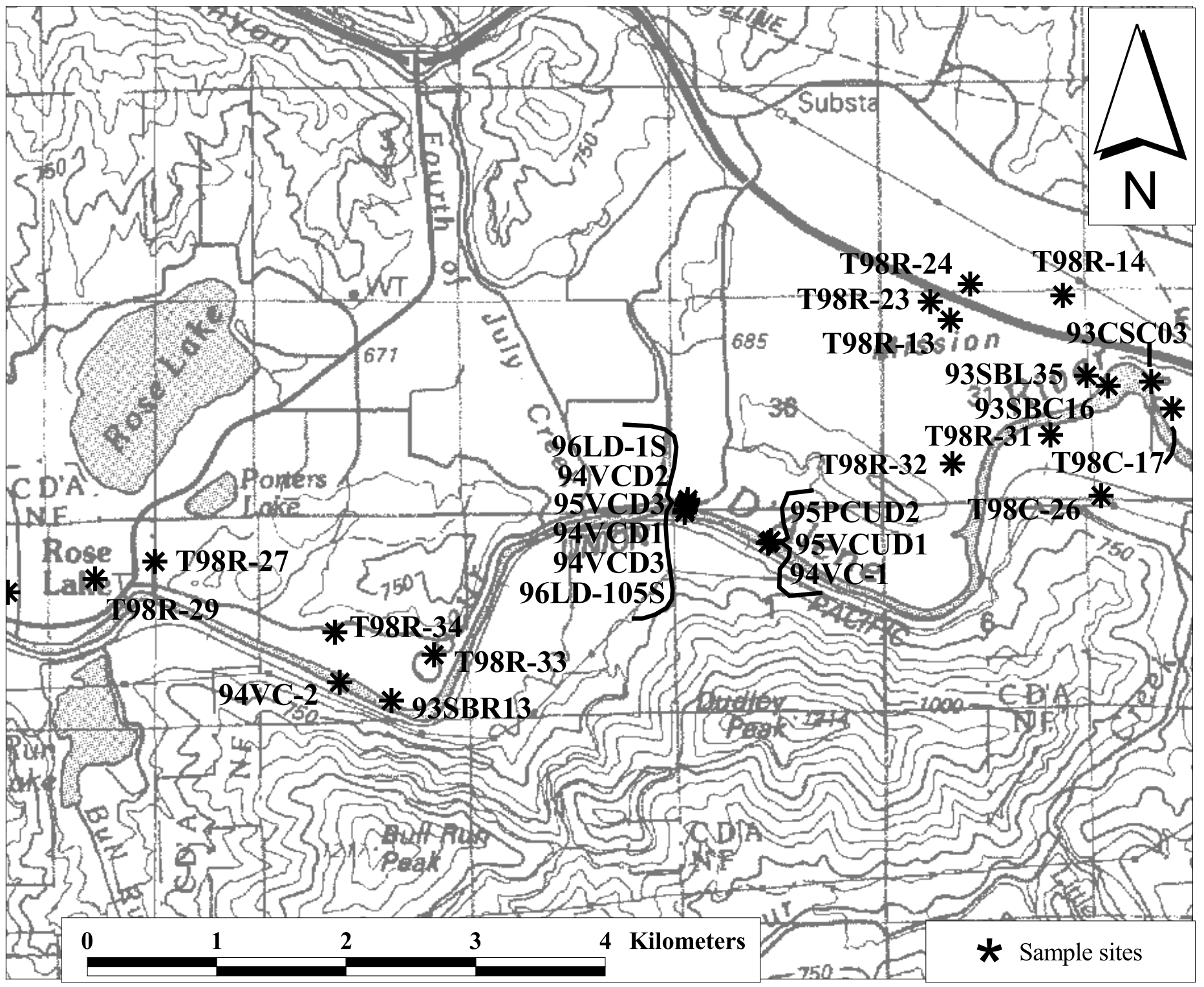

Map A-6: Sample site locations for the mainstem Coeur d'Alene River between Cataldo Mission and Rose Lake, Idaho. Base map from USGS Coeur d'Alene, Idaho 1:100,000 quadrangle. 


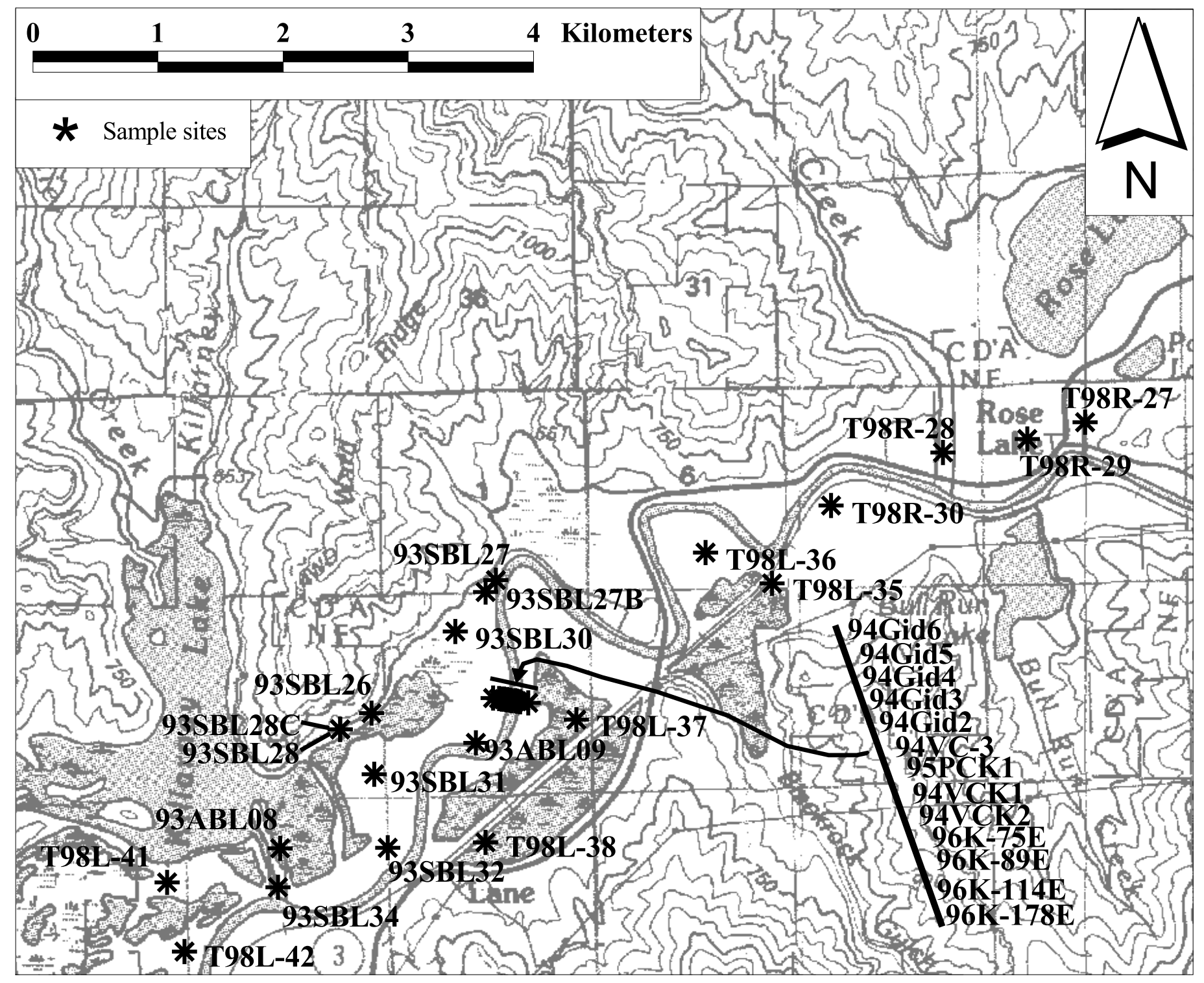

Map A-7: Sample site locations for the main stem of the Coeur d'Alene River between Rose and Killarney Lakes, Idaho. Base map from USGS Coeur d'Alene, Idaho 1:100,000 quadrangle. 


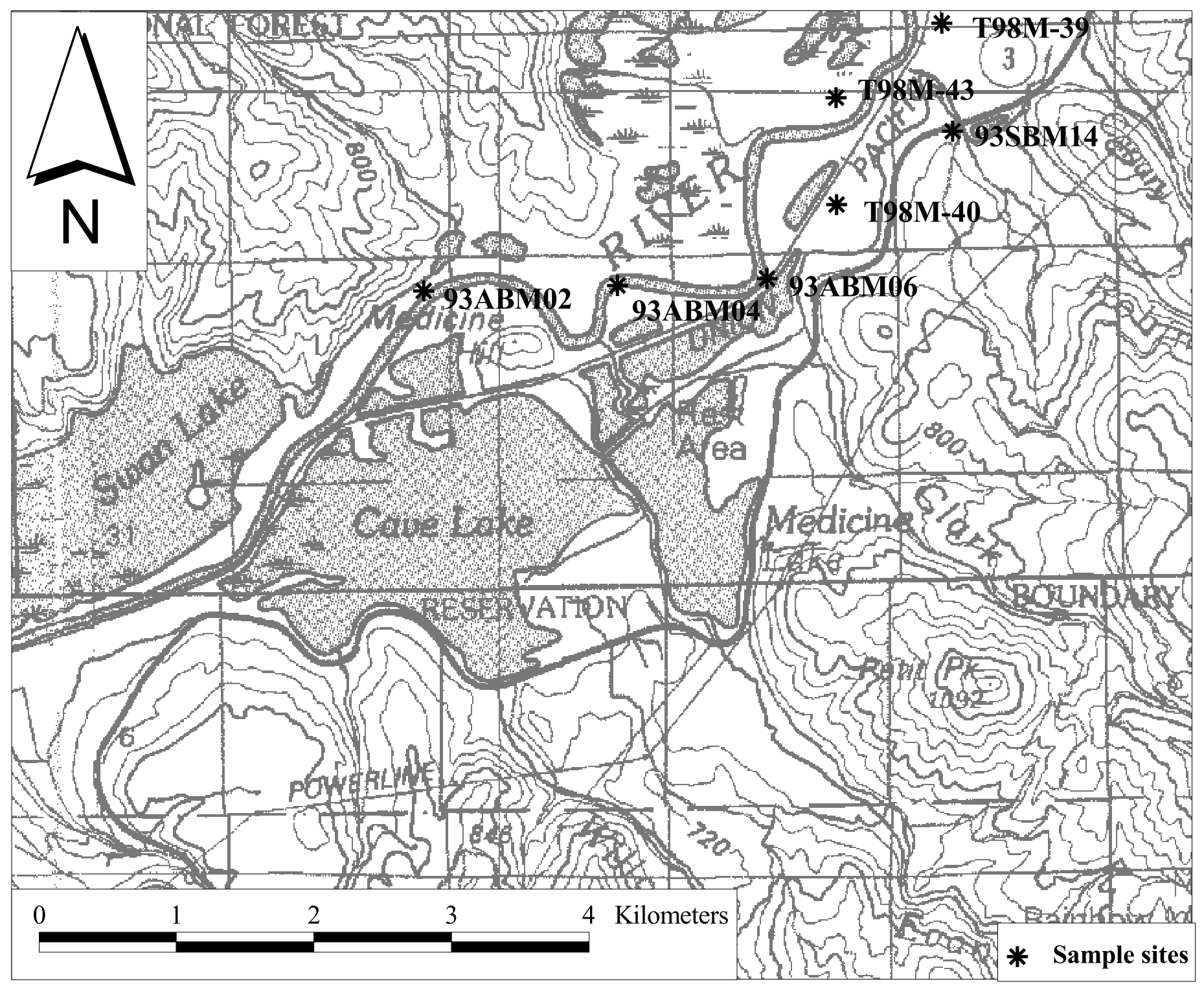

Map A-8: Sample site location map for the main stem of the Coeur d'Alene River near Cave and Medicine Lakes, Idaho. Base map from USGS St. Maries, Idaho 1:100,000 quadrangle. 


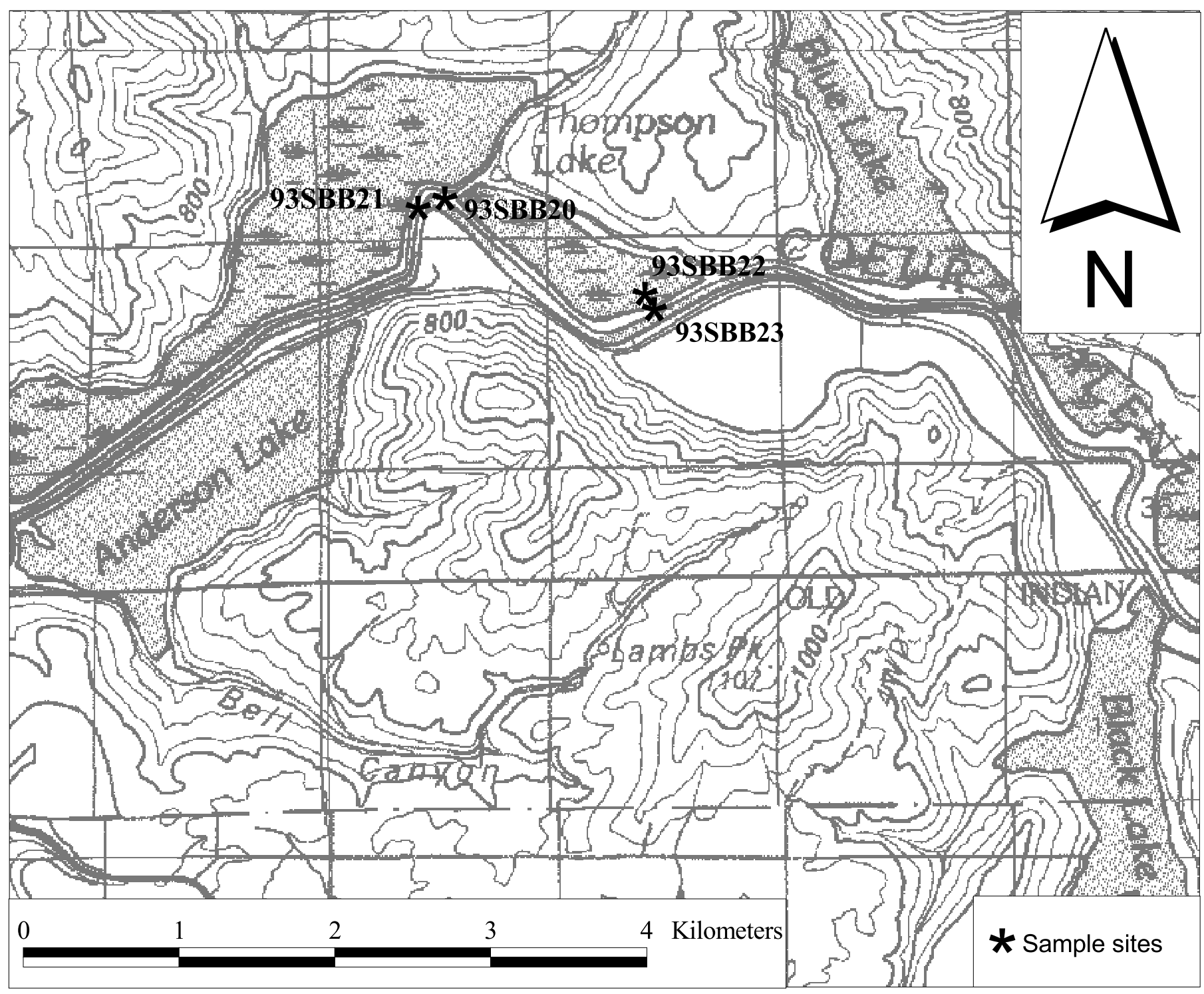

Map A-9: Sample site location map for the mainstem of the Coeur d'Alene River near Thompson Lake, Idaho. Base map from USGS St. Maries, Idaho 1:100,000 quadrangle. 


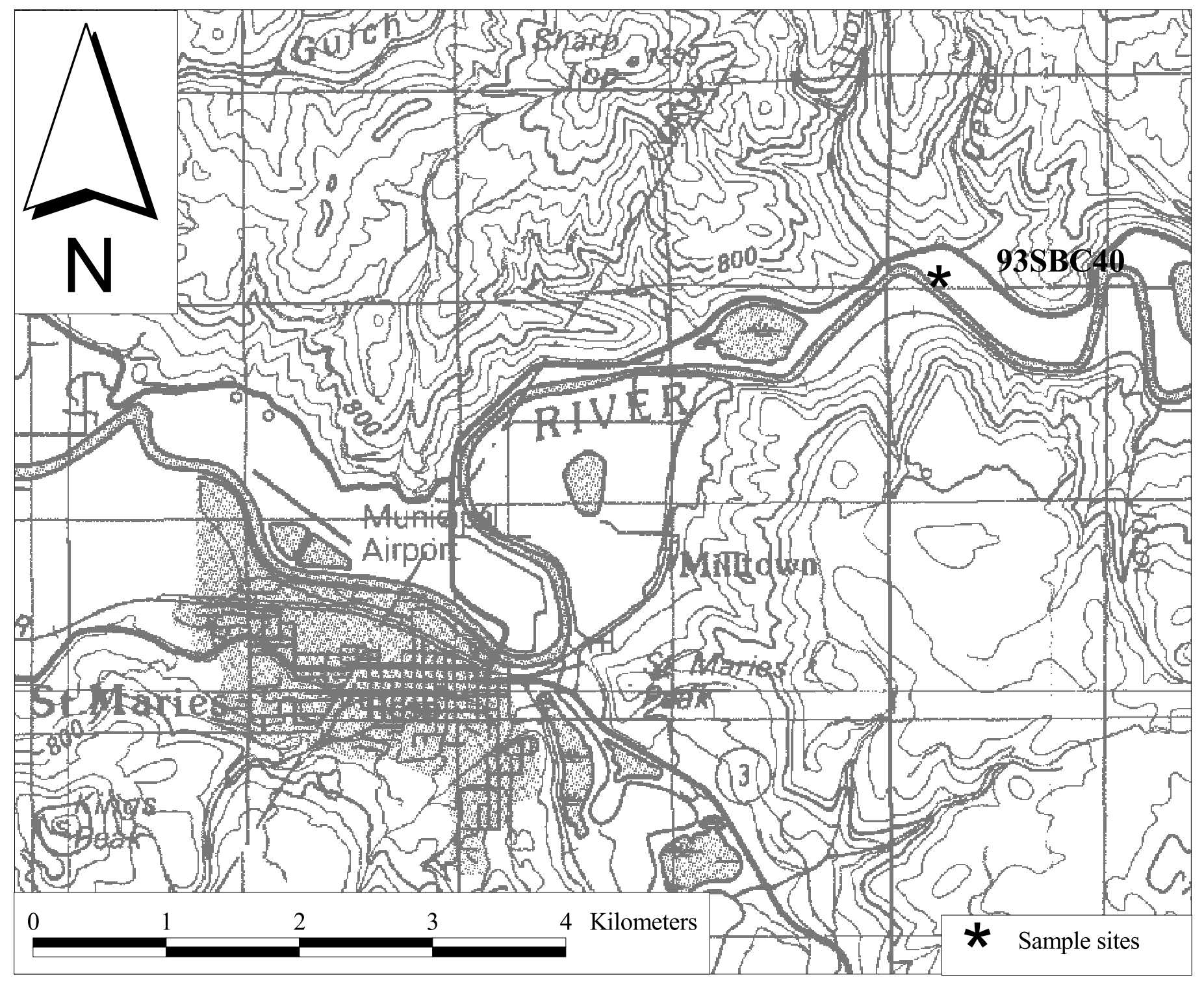

Map A-10: Sample site location map for the St. Joe River near St. Maries, Idaho. Base map from USGS St. Maries, Idaho 1:100,000 quadrangle. 
Appendix B. Sample site locations, depositional environments, depositional map units, sampling techniques and laboratory and analytical methods used on samples from that site (all=samples from site; some=some samples from site).

\begin{tabular}{|c|c|c|c|c|c|c|c|c|}
\hline Site ID & Latitude & Longitude & $\begin{array}{c}\text { Water depth } \\
(\mathrm{m})\end{array}$ & $\begin{array}{c}\text { Site Location } \\
\text { Map in } \\
\text { Appendix A }\end{array}$ & $\begin{array}{c}\text { Site- } \\
\text { Location } \\
\text { Method* }^{*}\end{array}$ & Environment & USGS map unit description & $\begin{array}{c}\text { USGS map } \\
\text { unit }\end{array}$ \\
\hline SITE_ID & LATITUDE & LONGITUDE & WTR_DEPTH & APDX_MAPNO & LOC_METHOD & ENVIRONMNT & UNIT_DESC & UNIT_LABEL \\
\hline 93ABM02 & 47.4817 & -116.6092 & & MAP A-8 & $\mathrm{a}$ & Subaerial floodplain & Riverbank wedge & Rbw \\
\hline 93CSC03 & 47.5520 & -116.3661 & & MAP A-6 & $\mathrm{a}$ & Subaerial floodplain & Riverbank wedge & Rbw \\
\hline 93ABM04 & 47.4820 & -116.5905 & & MAP A-8 & $\mathrm{a}$ & Subaerial floodplain & Upland, levee backslope & Ulb \\
\hline 93ABM06 & 47.4824 & -116.5760 & & MAP A-8 & $\mathrm{a}$ & Subaerial floodplain & Riverbank wedge & Rbw \\
\hline 93ABL08 & 47.5097 & -116.5542 & & MAP A-7 & $a$ & Marsh & Palustrine, perennial & PEph \\
\hline 93ABL09 & 47.5172 & -116.5333 & & MAP A-7 & $a$ & Exposed channel bar & Riverbank wedge & Rbw \\
\hline $93 \mathrm{SBC} 10$ & 47.5418 & -116.3435 & & MAP A-5 & $\mathrm{a}$ & Subaerial floodplain & Upland, alluvial terrace & Uat3 \\
\hline 93SBR13 & 47.5302 & -116.4443 & & MAP A-6 & $\mathrm{a}$ & Subaerial floodplain & Riverbank wedge & Rbw \\
\hline 93SBM14 & 47.4919 & -116.5581 & & MAP A-8 & $\mathrm{a}$ & Miocene lake seds & Highland, Miocene seds. & HMs \\
\hline 93SBC15 & 47.5447 & -116.3608 & & MAP A-5 & $\mathrm{a}$ & Subaerial floodplain & Upland levee sand & Uls \\
\hline 93SBC16 & 47.5517 & -116.3706 & 0.1 & MAP A-6 & $\mathrm{a}$ & Submerged channel & River sand bar & Rscb \\
\hline 93SBK17 & 47.5489 & -116.1750 & & MAP A-4 & $\mathrm{a}$ & Subaerial floodplain & Jig-era overbank sediments & Jos \\
\hline 93SBK18 & 47.5492 & -116.2016 & & MAP A-4 & $\mathrm{a}$ & Subaerial floodplain & Jig-era overbank sediments & Jos \\
\hline 93SBB20 & 47.4858 & -116.7239 & & MAP A-9 & $a$ & Subaerial floodplain & Riverbank wedge & Rbw \\
\hline $93 \mathrm{SBB} 21$ & 47.4856 & -116.7258 & & MAP A-9 & $\mathrm{a}$ & Subaerial floodplain & Riverbank wedge & Rbw \\
\hline 93SBB22 & 47.4794 & -116.7039 & & MAP A-9 & $\mathrm{a}$ & Marsh & Seasonal marsh with emergent vegetation & PEs \\
\hline $93 \mathrm{SBB} 23$ & 47.4786 & -116.7036 & & MAP A-9 & $\mathrm{a}$ & Subaerial floodplain & Riverbank wedge & Rbw \\
\hline 93SBL26 & 47.5194 & -116.5444 & & MAP A-7 & $\mathrm{a}$ & Marsh & Palustrine, perennial & PEph \\
\hline 93SBL27 & 47.5289 & -116.5311 & & MAP A-7 & $\mathrm{a}$ & Subaerial floodplain & Riverbank wedge & Rbw \\
\hline 93SBL27B & 47.5281 & -116.5322 & & MAP A-7 & $\mathrm{a}$ & Subaerial floodplain & Upland levee sand & Uls \\
\hline 93SBL28 & 47.5182 & -116.5478 & & MAP A-7 & $\mathrm{a}$ & Marsh & Palustrine, perennial & PEph \\
\hline 93SBL28C & 47.5182 & -116.5478 & & MAP A-7 & $\mathrm{a}$ & Marsh & Palustrine, perennial & PEph \\
\hline 93SBL30 & 47.5252 & -116.5355 & & MAP A-7 & $\mathrm{a}$ & Subaerial floodplain & Upland levee sand & Ulso \\
\hline 93SBL31 & 47.5150 & -116.5442 & & MAP A-7 & $\mathrm{a}$ & Subaerial floodplain & Upland sand splay & Ussc \\
\hline 93SBL32 & 47.5097 & -116.5428 & & MAP A-7 & $\mathrm{a}$ & Subaerial floodplain & Upland meander scroll set & Umsf \\
\hline 93SBL34 & 47.5069 & -116.5544 & & MAP A-7 & $\mathrm{a}$ & Subaerial floodplain & Riverbank wedge & Rbw \\
\hline 93SBC35 & 47.5524 & -116.3728 & & MAP A-6 & $\mathrm{a}$ & Subaerial dredge spoils & Dredge spoils, subaerial & Ads1 \\
\hline
\end{tabular}

${ }^{*} \mathrm{a}=\mathrm{map}, \mathrm{b}=$ compass and tape, $\mathrm{c}=\mathrm{GPS} .{ }^{* *} 1=$ Bookstom and others (1999), $2=$ Box and others(in prep. $)^{\star \star \star} 1=$ grab, $2=$ grove,

$3=$ depth-bracket,4=hand core,5=power core,6=vibro core,7=vibro-piston core,8=auger in casing. 
Appendix B. Sample site locations, depositional environments, depositional map units, sampling techniques and laboratory and analytical methods used on samples from that site (all=samples from site; some=some samples from site).

\begin{tabular}{|l|c|c|c|c|c|c|c|}
\hline Site ID & $\begin{array}{c}\text { USGS } \\
\text { map ** }\end{array}$ & $\begin{array}{c}\text { Sampling } \\
\text { Technique** }\end{array}$ & $\begin{array}{c}\text { USGS } \\
\text { EDXRF }\end{array}$ & $\begin{array}{c}\text { EWU } \\
\text { 4-acid }\end{array}$ & $\begin{array}{c}\text { CHEMEX } \\
\text { nitric-aqua } \\
\text { regia }\end{array}$ & $\begin{array}{c}\text { XRAL } \\
\text { 4-acid }\end{array}$ & $\begin{array}{c}\text { ACZ } \\
\text { nitric }\end{array}$ \\
\hline SITE_ID & REF_MAP & SAMP_TECH & EDXRF & EWU-4-ACII & NITRIC-AQR & XRL-4-ACID & ACZ-NITRIC \\
\hline 93ABM02 & 1 & 2 & all & & all & some & \\
\hline 93CSC03 & 1 & 2 & & all & & & \\
\hline 93ABM04 & 1 & 2 & all & & all & some & \\
\hline 93ABM06 & 1 & 1 & all & & all & & \\
\hline 93ABL08 & 1 & 1 & all & & all & & \\
\hline 93ABL09 & 1 & 1 & all & & all & & \\
\hline 93SBC10 & 1 & 2 & all & & all & some & \\
\hline 93SBR13 & 1 & 2 & all & & all & & \\
\hline 93SBM14 & 1 & 2 & all & & all & & \\
\hline 93SBC15 & 1 & 2 & all & all & & & \\
\hline 93SBC16 & 1 & 2 & all & & all & & \\
\hline 93SBK17 & 2 & 2 & all & & all & some & \\
\hline 93SBK18 & 2 & 2 & all & & all & some & \\
\hline 93SBB20 & 1 & 1 & all & & all & some & \\
\hline 93SBB21 & 1 & 2,4 & all & all & & & \\
\hline 93SBB22 & 1 & 2 & all & & all & some & \\
\hline 93SBB23 & 1 & 2 & all & & all & some & \\
\hline 93SBL26 & 1 & 4 & all & & all & some & \\
\hline 93SBL27 & 1 & 2 & all & all & & some & \\
\hline 93SBL27B & 1 & 4,2 & all & & all & & \\
\hline 93SBL28 & 1 & 4 & all & & all & & \\
\hline 93SBL28C & 1 & 4 & all & & all & & \\
\hline 93SBL30 & 1 & 2,4 & all & all & & & \\
\hline 93SBL31 & 1 & 2,4 & all & & all & some & \\
\hline 93SBL32 & 1 & 2 & all & & all & some & \\
\hline 93SBL34 & 1 & 2 & all & all & all & & \\
\hline 93SBC35 & 1 & 1 & all & & all & & \\
\hline
\end{tabular}

${ }^{*} \mathrm{a}=\mathrm{map}, \mathrm{b}=\mathrm{compass}$ and tape, $\mathrm{c}=\mathrm{GPS} .{ }^{* *} 1=$ Bookstom and others (1999), $2=$ Box and others(in prep.) ${ }^{\star * *} 1=$ grab, $2=$ grove,

3=depth-bracket,4=hand core,5=power core,6=vibro core,7=vibro-piston core,8=auger in casing. 
Appendix B. Sample site locations, depositional environments, depositional map units, sampling techniques and laboratory and analytical methods used on samples from that site (all=samples from site; some=some samples from site).

\begin{tabular}{|c|c|c|c|c|c|c|c|c|}
\hline Site ID & Latitude & Longitude & $\begin{array}{c}\text { Water depth } \\
(\mathrm{m})\end{array}$ & $\begin{array}{l}\text { Site Location } \\
\text { Map in } \\
\text { Appendix A }\end{array}$ & $\begin{array}{c}\text { Site- } \\
\text { Location } \\
\text { Method* }^{*}\end{array}$ & Environment & USGS map unit description & $\begin{array}{c}\text { USGS map } \\
\text { unit }\end{array}$ \\
\hline 93SBC36 & 47.5697 & -116.2522 & & MAP A-5 & $\mathrm{a}$ & Exposed channel bar & River, gravel bottomed & $\mathrm{Rg}$ \\
\hline 93SBC37 & 47.5714 & -116.2525 & & MAP A-5 & $\mathrm{a}$ & Exposed channel bar & River, gravel bottomed & $\mathrm{Rg}$ \\
\hline 93SBK38 & 47.5561 & -116.2442 & & MAP A-4 & $\mathrm{a}$ & Exposed channel bar & Sandy gravels of the present channel & $\mathrm{Pcg}$ \\
\hline 93SBC39 & 47.5522 & -116.2744 & & MAP A-5 & $\mathrm{a}$ & Subaerial floodplain & Upland alluvial terrace & Uat1 \\
\hline $93 \mathrm{SBC} 40$ & 47.3420 & -116.5165 & & MAP A-10 & $\mathrm{a}$ & Subaerial floodplain & Riverbank wedge & \\
\hline 94Gid2 & 47.5203 & -116.5301 & & MAP A-7 & $\mathrm{b}$ & Subaerial floodplain & Riverbank wedge & Rbw \\
\hline 94Gid3 & 47.5203 & -116.5304 & & MAP A-7 & $\mathrm{b}$ & Subaerial floodplain & Upland levee sand & Ulso \\
\hline 94Gid4 & 47.5204 & -116.5306 & & MAP A-7 & $\mathrm{b}$ & Subaerial floodplain & Upland levee sand & Ulso \\
\hline 94Gid5 & 47.5204 & -116.5309 & & MAP A-7 & $\mathrm{b}$ & Subaerial floodplain & Upland meander scroll set, farmed & Umsf \\
\hline 94Gid6 & 47.5204 & -116.5315 & & MAP A-7 & $\mathrm{b}$ & Subaerial floodplain & Upland meander scroll set, farmed & Umsf \\
\hline 94JE01 & 47.4662 & -115.7321 & & MAP A-1 & $\mathrm{a}$ & Exposed channel bar & Sandy gravels of the present channel & Pcg \\
\hline 94JE02 & 47.4643 & -115.8187 & & MAP A-1 & $\mathrm{a}$ & Exposed channel bar & Sandy gravels of the present channel & Pcg \\
\hline 94JE03 & 47.4709 & -115.8603 & & MAP A-1 & $\mathrm{a}$ & Exposed channel bar & Sandy gravels of the present channel & Pcg \\
\hline 94JE04 & 47.4758 & -115.8896 & & MAP A-2 & $\mathrm{a}$ & Exposed channel bar & Sandy gravels of the present channel & Pcg \\
\hline 94JE05 & 47.4770 & -115.9136 & & MAP A-2 & $\mathrm{a}$ & Exposed channel bar & Sandy gravels of the present channel & Pcg \\
\hline 94JE06 & 47.5318 & -116.0775 & & MAP A-3 & $\mathrm{a}$ & Subaerial floodplain & Jig-era overbank sediments & Jos \\
\hline 94JE06A & 47.5319 & -116.0774 & & MAP A-3 & $\mathrm{a}$ & Exposed channel bar & Sandy gravels of the present channel & Pcg \\
\hline 94JE06B & 47.5314 & -116.0775 & & MAP A-3 & $\mathrm{a}$ & Subaerial floodplain & Jig-era overbank sediments & Jos \\
\hline 94JE07 & 47.5294 & -115.7861 & & MAP A-1 & $\mathrm{a}$ & Exposed channel bar & Sandy gravels of the present channel & Pcg \\
\hline 94JE08 & 47.5183 & -115.8242 & & MAP A-1 & $\mathrm{a}$ & Exposed channel bar & Sandy gravels of the present channel & Pcg \\
\hline 94JE09 & 47.5166 & -115.8432 & & MAP A-1 & $\mathrm{a}$ & Exposed channel bar & Sandy gravels of the present channel & Pcg \\
\hline 94JE10 & 47.5051 & -115.8771 & & MAP A-2 & $\mathrm{a}$ & Exposed channel bar & Sandy gravels of the present channel & Pcg \\
\hline 94JE11 & 47.4872 & -115.8910 & & MAP A-2 & $\mathrm{a}$ & Exposed channel bar & Sandy gravels of the present channel & Pcg \\
\hline 94JE12 & 47.4813 & -115.9192 & & MAP A-2 & $\mathrm{a}$ & Exposed channel bar & Sandy gravels of the present channel & Pcg \\
\hline 94JE13 & 47.4795 & -115.9344 & & MAP A-2 & $\mathrm{a}$ & Exposed channel bar & Sandy gravels of the present channel & Pcg \\
\hline 94JE14 & 47.4786 & -115.9369 & & MAP A-2 & $\mathrm{a}$ & Exposed channel bar & Sandy gravels of the present channel & Pcg \\
\hline 94JE15 & 47.4889 & -115.9519 & & MAP A-2 & $\mathrm{a}$ & Exposed channel bar & Sandy gravels of the present channel & Pcg \\
\hline 94JE16 & 47.4844 & -115.9385 & & MAP A-2 & $\mathrm{a}$ & Exposed channel bar & Sandy gravels of the present channel & Pcg \\
\hline
\end{tabular}

${ }^{*} \mathrm{a}=\mathrm{map}, \mathrm{b}=\mathrm{compass}$ and tape, $\mathrm{c}=\mathrm{GPS} .{ }^{* \star} 1=$ Bookstom and others (1999), $2=$ Box and others(in prep.) ${ }^{\star \star *} 1=$ grab, $2=$ grove,

$3=$ depth-bracket, $4=$ hand core,5=power core,6=vibro core,7=vibro-piston core, $8=$ auger in casing. 
Appendix B. Sample site locations, depositional environments, depositional map units, sampling techniques and laboratory and analytical methods used on samples from that site (all=samples from site; some=some samples from site).

\begin{tabular}{|l|c|c|c|c|c|c|c|}
\hline Site ID & $\begin{array}{c}\text { USGS } \\
\text { map ** }\end{array}$ & $\begin{array}{c}\text { Sampling } \\
\text { Technique** }\end{array}$ & $\begin{array}{c}\text { USGS } \\
\text { EDXRF }\end{array}$ & $\begin{array}{c}\text { EWU } \\
\text { 4-acid }\end{array}$ & $\begin{array}{c}\text { CHEMEX } \\
\text { nitric-aqua } \\
\text { regia }\end{array}$ & $\begin{array}{c}\text { XRAL } \\
\text { 4-acid }\end{array}$ & $\begin{array}{c}\text { ACZ } \\
\text { nitric }\end{array}$ \\
\hline 93SBC36 & 1 & 1 & all & all & & & \\
\hline 93SBC37 & 1 & 2 & all & all & & & \\
\hline 93SBK38 & 2 & 2 & all & all & & & \\
\hline 93SBC39 & 1 & 2 & all & all & & & \\
\hline 93SBC40 & --- & 2 & & all & & & \\
\hline 94Gid2 & 1 & 5 & & all & & some & some \\
\hline 94Gid3 & 1 & 5 & & all & & some & some \\
\hline 94Gid4 & 1 & 5 & & all & & some & some \\
\hline 94Gid5 & 1 & 5 & & all & & & \\
\hline 94Gid6 & 1 & 5 & & all & & some & some \\
\hline 94JE01 & 2 & 1 & & & all & & \\
\hline 94JE02 & 2 & 1 & & & all & & \\
\hline 94JE03 & 2 & 1 & & & all & & \\
\hline 94JE04 & 2 & 1 & & & all & & \\
\hline 94JE05 & 2 & 1 & & & all & & \\
\hline 94JE06 & 2 & 1 & & all & & & \\
\hline 94JE06A & 2 & 1 & & & all & & \\
\hline 94JE06B & 2 & 1 & & all & & & \\
\hline 94JE07 & 2 & 1 & & & all & & \\
\hline 94JE08 & 2 & 1 & & & all & & \\
\hline 94JE09 & 2 & 1 & & & all & & \\
\hline 94JE10 & 2 & 1 & & & all & & \\
\hline 94JE11 & 2 & 1 & & & all & & \\
\hline 94JE12 & 2 & 1 & & & all & & \\
\hline 94JE13 & 2 & 1 & & & all & & \\
\hline 94JE14 & 2 & 1 & & & all & & \\
\hline 94JE15 & 2 & 1 & & & all & & \\
\hline 94JE16 & 2 & 1 & & & all & & \\
\hline
\end{tabular}

${ }^{*} \mathrm{a}=\mathrm{map}, \mathrm{b}=\mathrm{compass}$ and tape, $\mathrm{c}=$ GPS. ${ }^{* *} 1=$ Bookstom and others (1999), $2=$ Box and others(in prep. ${ }^{\star \star *} 1=$ grab, $2=$ grove,

3=depth-bracket,4=hand core,5=power core,6=vibro core,7=vibro-piston core,8=auger in casing. 
Appendix B. Sample site locations, depositional environments, depositional map units, sampling techniques and laboratory and analytical methods used on samples from that site (all=samples from site; some=some samples from site).

\begin{tabular}{|c|c|c|c|c|c|c|c|c|}
\hline Site ID & Latitude & Longitude & $\begin{array}{c}\text { Water depth } \\
(\mathrm{m})\end{array}$ & $\begin{array}{l}\text { Site Location } \\
\text { Map in } \\
\text { Appendix A }\end{array}$ & $\begin{array}{c}\text { Site- } \\
\text { Location } \\
\text { Method* }^{*}\end{array}$ & Environment & USGS map unit description & $\begin{array}{c}\text { USGS map } \\
\text { unit }\end{array}$ \\
\hline 94JE17 & 47.4914 & -115.9545 & & MAP A-2 & $\mathrm{a}$ & Exposed channel bar & Sandy gravels of the present channel & Pcg \\
\hline 94JE18 & 47.4922 & -115.9703 & & MAP A-2 & $\mathrm{a}$ & Exposed channel bar & Sandy gravels of the present channel & Pcg \\
\hline 94JE19 & 47.4811 & -115.9636 & & MAP A-2 & $\mathrm{a}$ & Exposed channel bar & Sandy gravels of the present channel & Pcg \\
\hline 94JE20 & 47.4947 & -115.9800 & & MAP A-2 & $\mathrm{a}$ & Exposed channel bar & Sandy gravels of the present channel & Pcg \\
\hline 94JE21 & 47.5053 & -115.9742 & & MAP A-2 & $\mathrm{a}$ & Exposed channel bar & Sandy gravels of the present channel & Pcg \\
\hline 94JE22 & 47.5027 & -115.9822 & & MAP A-2 & $\mathrm{a}$ & Exposed channel bar & Sandy gravels of the present channel & Pcg \\
\hline 94JE23 & 47.5183 & -115.9875 & & MAP A-2 & $\mathrm{a}$ & Exposed channel bar & Sandy gravels of the present channel & Pcg \\
\hline 94JE24 & 47.5106 & -116.0067 & & MAP A-3 & $\mathrm{a}$ & Exposed channel bar & Sandy gravels of the present channel & Pcg \\
\hline 94JE25 & 47.4997 & -116.0105 & & MAP A-3 & $\mathrm{a}$ & Exposed channel bar & Sandy gravels of the present channel & Pcg \\
\hline 94JE26 & 47.5212 & -116.0167 & & MAP A-3 & $\mathrm{a}$ & Exposed channel bar & Sandy gravels of the present channel & Pcg \\
\hline 94JE27 & 47.5203 & -116.0432 & & MAP A-3 & $\mathrm{a}$ & Exposed channel bar & Sandy gravels of the present channel & Pcg \\
\hline 94JE28 & 47.5264 & -116.0481 & & MAP A-3 & $\mathrm{a}$ & Exposed channel bar & Sandy gravels of the present channel & Pcg \\
\hline 94JE29 & 47.5193 & -116.0608 & & MAP A-3 & $\mathrm{a}$ & Exposed channel bar & Sandy gravels of the present channel & Pcg \\
\hline 94JE30 & 47.5311 & -116.0533 & & MAP A-3 & $\mathrm{a}$ & Exposed channel bar & Sandy gravels of the present channel & Pcg \\
\hline 94JE31 & 47.5309 & -116.0908 & & MAP A-3 & $\mathrm{a}$ & Exposed channel bar & Sandy gravels of the present channel & Pcg \\
\hline 94JE32 & 47.5423 & -116.0476 & & MAP A-3 & $\mathrm{a}$ & Exposed channel bar & Sandy gravels of the present channel & Pcg \\
\hline 94JE33 & 47.5339 & -116.1022 & & MAP A-3 & $\mathrm{a}$ & Exposed channel bar & Sandy gravels of the present channel & Pcg \\
\hline 94JE34 & 47.5350 & -116.1108 & & MAP A-3 & $\mathrm{a}$ & Exposed channel bar & Sandy gravels of the present channel & Pcg \\
\hline 94JE35 & 47.5489 & -116.1394 & & MAP A-4 & $\mathrm{a}$ & Exposed channel bar & Sandy gravels of the present channel & Pcg \\
\hline 94JE36 & 47.5483 & -116.1636 & & MAP A-4 & $\mathrm{a}$ & Exposed channel bar & Sandy gravels of the present channel & Pcg \\
\hline 94JE37 & 47.5480 & -116.1720 & & MAP A-4 & $\mathrm{a}$ & Exposed channel bar & Sandy gravels of the present channel & $\mathrm{Pcg}$ \\
\hline 94JE38 & 47.5511 & -116.1814 & & MAP A-4 & $\mathrm{a}$ & Exposed channel bar & Sandy gravels of the present channel & Pcg \\
\hline 94JE39A & 47.5521 & -116.1915 & & MAP A-4 & $\mathrm{a}$ & Exposed channel bar & Sandy gravels of the present channel & Pcg \\
\hline 94JE40 & 47.5515 & -116.1976 & & MAP A-4 & $\mathrm{a}$ & Exposed channel bar & Sandy gravels of the present channel & Pcg \\
\hline 94JE41 & 47.5483 & -116.2177 & & MAP A-4 & $\mathrm{a}$ & Exposed channel bar & Sandy gravels of the present channel & Pcg \\
\hline 94JE42 & 47.5108 & -116.2411 & & MAP A-4 & $\mathrm{a}$ & Exposed channel bar & Sandy gravels of the present channel & Pcg \\
\hline 94JE43 & 47.5485 & -116.2066 & & MAP A-4 & $\mathrm{a}$ & Exposed channel bar & Sandy gravels of the present channel & Pcg \\
\hline 94JE44 & 47.5536 & -116.2231 & & MAP A-4 & $\mathrm{a}$ & Exposed channel bar & Sandy gravels of the present channel & Pcg \\
\hline
\end{tabular}

${ }^{*} \mathrm{a}=\mathrm{map}, \mathrm{b}=$ compass and tape, $\mathrm{c}=\mathrm{GPS} .{ }^{* *} 1=$ Bookstom and others (1999), $2=$ Box and others(in prep. ${ }^{\star \star *} 1=$ grab, $2=$ grove,

$3=$ depth-bracket, $4=$ hand core,5=power core, $6=$ vibro core, $7=$ vibro-piston core, $8=$ auger in casing. 
Appendix B. Sample site locations, depositional environments, depositional map units, sampling techniques and laboratory and analytical methods used on samples from that site (all=samples from site; some=some samples from site)

\begin{tabular}{|l|c|c|c|c|c|c|c|}
\hline Site ID & $\begin{array}{c}\text { USGS } \\
\text { map ** }\end{array}$ & $\begin{array}{c}\text { Sampling } \\
\text { Technique*** }\end{array}$ & $\begin{array}{c}\text { USGS } \\
\text { EDXRF }\end{array}$ & $\begin{array}{c}\text { EWU } \\
\text { 4-acid }\end{array}$ & $\begin{array}{c}\text { CHEMEX } \\
\text { nitric-aqua } \\
\text { regia }\end{array}$ & $\begin{array}{c}\text { XRAL } \\
\text { 4-acid }\end{array}$ & $\begin{array}{c}\text { ACZ } \\
\text { nitric }\end{array}$ \\
\hline 94JE17 & 2 & 1 & & & all & & \\
\hline 94JE18 & 2 & 1 & & & all & & \\
\hline 94JE19 & 2 & 1 & & & all & & \\
\hline 94JE20 & 2 & 1 & & & all & & \\
\hline 94JE21 & 2 & 1 & & & all & & \\
\hline 94JE22 & 2 & 1 & & & all & & \\
\hline 94JE23 & 2 & 1 & & & all & & \\
\hline 94JE24 & 2 & 1 & & & all & & \\
\hline 94JE25 & 2 & 1 & & & all & & \\
\hline 94JE26 & 2 & 1 & & & all & & \\
\hline 94JE27 & 2 & 1 & & & all & & \\
\hline 94JE28 & 2 & 1 & & & all & & \\
\hline 94JE29 & 2 & 1 & & & all & & \\
\hline 94JE30 & 2 & 1 & & & all & & \\
\hline 94JE31 & 2 & 1 & & & all & & \\
\hline 94JE32 & 2 & 1 & & & all & & \\
\hline 94JE33 & 2 & 1 & & & all & & \\
\hline 94JE34 & 2 & 1 & & & all & & \\
\hline 94JE35 & 2 & 1 & & & all & & \\
\hline 94JE36 & 2 & 1 & & & all & & \\
\hline 94JE37 & 2 & 1 & & & all & & \\
\hline 94JE38 & 2 & 1 & & & all & & \\
\hline 94JE39A & 2 & 1 & & & all & & \\
\hline 94JE40 & 2 & 1 & & & all & & \\
\hline 94JE41 & 2 & 1 & & & all & & \\
\hline 94JE42 & 2 & 1 & & & all & & \\
\hline 94JE43 & 2 & 1 & & & all & & \\
\hline 94JE44 & 2 & 1 & & & all & & \\
\hline
\end{tabular}

${ }^{*} \mathrm{a}=\mathrm{map}, \mathrm{b}=\mathrm{compass}$ and tape, $\mathrm{c}=\mathrm{GPS} .{ }^{* *} 1=$ Bookstom and others (1999), $2=$ Box and others(in prep.) ${ }^{\star * *} 1=$ grab, $2=$ grove,

3=depth-bracket,4=hand core,5=power core,6=vibro core,7=vibro-piston core,8=auger in casing. 
Appendix B. Sample site locations, depositional environments, depositional map units, sampling techniques and laboratory and analytical methods used on samples from that site (all=samples from site; some=some samples from site).

\begin{tabular}{|c|c|c|c|c|c|c|c|c|}
\hline Site ID & Latitude & Longitude & $\begin{array}{c}\text { Water depth } \\
(\mathrm{m})\end{array}$ & $\begin{array}{l}\text { Site Location } \\
\text { Map in } \\
\text { Appendix A }\end{array}$ & $\begin{array}{c}\text { Site- } \\
\text { Location } \\
\text { Method* }^{*}\end{array}$ & Environment & USGS map unit description & $\underset{\text { unit }}{\text { USGS map }}$ \\
\hline 94JE45 & 47.5540 & -116.2227 & & MAP A-4 & $a$ & Exposed channel bar & Sandy gravels of the present channel & Pcg \\
\hline 94JE46 & 47.5531 & -116.2333 & & MAP A-4 & $a$ & Exposed channel bar & Sandy gravels of the present channel & Pcg \\
\hline 94JE47 & 47.5506 & -116.2389 & & MAP A-4 & $a$ & Exposed channel bar & Sandy gravels of the present channel & Pcg \\
\hline 94JE48 & 47.5559 & -116.2445 & & MAP A-4 & $a$ & Exposed channel bar & Sandy gravels of the present channel & Pcg \\
\hline 94JE49 & 47.5595 & -116.2520 & & MAP A-5 & $\bar{a}$ & Exposed channel bar & Sandy gravels of the present channel & $\mathrm{Pcg}$ \\
\hline 94VC-1 & 47.5409 & -116.4055 & 3.9 & MAP A-6 & $\mathrm{b}$ & Submerged channel & Sand-bottomed river channel & Rs \\
\hline 94VC-2 & 47.5315 & -116.4497 & 4.2 & MAP A-6 & $\mathrm{b}$ & Submerged channel & Sand-bottomed river channel & Rs \\
\hline 94VC-3 & 47.5203 & -116.5300 & 2.7 & MAP A-7 & $\mathrm{b}$ & Submerged channel & Sand-bottomed river channel & Rs \\
\hline 94VCD1 & 47.5436 & -116.4139 & 5.1 & MAP A-6 & $\mathrm{b}$ & Submerged channel & Sand-bottomed river channel & Rs \\
\hline 94VCD2 & 47.5438 & -116.4138 & 2.0 & MAP A-6 & $\mathrm{b}$ & Submerged channel & Sand-bottomed river channel & Rs \\
\hline 94VCD3 & 47.5433 & -116.4141 & 3.4 & MAP A-6 & $\mathrm{b}$ & Submerged channel & Pre-mining era sediments in channel & Rpm \\
\hline 94VCK1 & 47.5203 & -116.5297 & 4.4 & MAP A-7 & $\mathrm{b}$ & Submerged channel & Sand-bottomed river channel & Rs \\
\hline 94VCK2 & 47.5203 & -116.5296 & 5.9 & MAP A-7 & $\mathrm{b}$ & Submerged channel & Sand-bottomed river channel & Rs \\
\hline 95PCK1 & 47.5203 & -116.5300 & 2.0 & MAP A-7 & $\mathrm{b}$ & Submerged channel & Sand-bottomed river channel & Rs \\
\hline 95PCUD2 & 47.5410 & -116.4055 & 3.9 & MAP A-6 & $\mathrm{b}$ & Submerged channel & Sand-bottomed river channel & Rs \\
\hline 95VCD3 & 47.5437 & -116.4139 & 4.4 & MAP A-6 & $\mathrm{b}$ & Submerged channel & Sand-bottomed river channel & Rs \\
\hline 95VCUD1 & 47.5411 & -116.4053 & 4.0 & MAP A-6 & $\mathrm{b}$ & Submerged channel & Sand-bottomed river channel & Rs \\
\hline $96 \mathrm{~K}-75 \mathrm{E}$ & 47.5202 & -116.5291 & 3.0 & MAP A-7 & $\mathrm{b}$ & Submerged channel & Pre-mining era sediments in channel & Rpm \\
\hline $96 \mathrm{~K}-89 \mathrm{E}$ & 47.5202 & -116.5290 & & MAP A-7 & $\mathrm{b}$ & Subaerial floodplain & Upland sand splay & Uss \\
\hline $96 \mathrm{~K}-114 \mathrm{E}$ & 47.5202 & -116.5286 & & MAP A-7 & $\mathrm{b}$ & Subaerial floodplain & Palustrine, perennial & PEp \\
\hline $96 \mathrm{~K}-178 \mathrm{E}$ & 47.5201 & -116.5278 & & MAP A-7 & $\mathrm{b}$ & Subaerial floodplain & Palustrine, perennial & PEp \\
\hline 96LD-1S & 47.5440 & -116.4138 & & MAP A-6 & $\mathrm{b}$ & Subaerial floodplain & Riverbank wedge & Rbw \\
\hline 96LD-105S & 47.5431 & -116.4141 & & MAP A-6 & $\mathrm{b}$ & Subaerial floodplain & Riverbank wedge & Rbw \\
\hline T98C-01 & 47.5490 & -116.2693 & & MAP A-5 & c & Marsh & Palustrine, seasonal, emergent plants & PEs \\
\hline T98C-05 & 47.5502 & -116.2751 & & MAP A-5 & C & Marsh & Palustrine, seasonal, emergent plants & PEs \\
\hline T98C-06 & 47.5538 & -116.3215 & & MAP A-5 & c & Subaerial floodplain & Upland, levee sand & Ulso \\
\hline T98C-07 & 47.5533 & -116.3193 & & MAP A-5 & C & Subaerial floodplain & Upland, upper alluvial terrace & Uat3 \\
\hline T98C-08 & 47.5491 & -116.3316 & & MAP A-5 & C & Subaerial floodplain & Upland, middle alluvial terrace & Uat2 \\
\hline
\end{tabular}

${ }^{*} \mathrm{a}=\mathrm{map}, \mathrm{b}=\mathrm{compass}$ and tape, $\mathrm{c}=\mathrm{GPS} .{ }^{* \star} 1=$ Bookstom and others (1999), $2=$ Box and others(in prep.) ${ }^{\star \star *} 1=$ grab, $2=$ grove,

$3=$ depth-bracket, $4=$ hand core,5=power core, $6=$ vibro core, $7=$ vibro-piston core, $8=$ auger in casing. 
Appendix B. Sample site locations, depositional environments, depositional map units, sampling techniques and laboratory and analytical methods used on samples from that site (all=samples from site; some=some samples from site).

\begin{tabular}{|c|c|c|c|c|c|c|c|}
\hline Site ID & $\begin{array}{l}\text { USGS } \\
\text { map ** }\end{array}$ & $\begin{array}{c}\text { Sampling } \\
\text { Technique }^{\star * \star}\end{array}$ & $\begin{array}{l}\text { USGS } \\
\text { EDXRF }\end{array}$ & $\begin{array}{l}\text { EWU } \\
\text { 4-acid }\end{array}$ & $\begin{array}{c}\text { CHEMEX } \\
\text { nitric-aqua } \\
\text { regia }\end{array}$ & $\begin{array}{l}\text { XRAL } \\
\text { 4-acid }\end{array}$ & $\begin{array}{l}\mathrm{ACZ} \\
\text { nitric }\end{array}$ \\
\hline 94JE45 & 2 & 1 & & & all & & \\
\hline 94JE46 & 2 & 1 & & & all & & \\
\hline 94JE47 & 2 & 1 & & & all & & \\
\hline 94JE48 & 2 & 1 & & & all & & \\
\hline 94JE49 & 2 & 1 & & & all & & \\
\hline 94VC-1 & 1 & 6 & & all & & some & some \\
\hline 94VC-2 & 1 & 6 & & & all & & \\
\hline 94VC-3 & 1 & 6 & & all & all & & \\
\hline 94VCD1 & 1 & 6 & & all & & & \\
\hline 94VCD2 & 1 & 6 & & all & & & \\
\hline 94VCD3 & 1 & 6 & & all & & some & some \\
\hline 94VCK1 & 1 & 6 & & all & & some & some \\
\hline 94VCK2 & 1 & 6 & & all & & some & some \\
\hline 95PCK1 & 1 & 7 & & all & & some & some \\
\hline 95PCUD2 & 1 & 7 & & all & & some & some \\
\hline 95VCD3 & 1 & 8 & & all & & some & some \\
\hline 95VCUD1 & 1 & 8 & & all & & some & some \\
\hline $96 \mathrm{~K}-75 \mathrm{E}$ & 1 & 2 & & all & & & \\
\hline $96 \mathrm{~K}-89 \mathrm{E}$ & 1 & 2 & & all & & & \\
\hline 96K-114E & 1 & 2 & & all & & some & some \\
\hline $96 \mathrm{~K}-178 \mathrm{E}$ & 1 & 2 & & all & & & \\
\hline 96LD-1S & 1 & 2 & & all & & & \\
\hline 96LD-105S & 1 & 2 & & all & & & \\
\hline T98C-01 & 1 & 3 & & & & all & \\
\hline T98C-05 & 1 & 3 & & & & all & \\
\hline T98C-06 & 1 & 3 & & & & all & \\
\hline T98C-07 & 1 & 3 & & & & all & \\
\hline T98C-08 & 1 & 3 & & & & all & \\
\hline
\end{tabular}

${ }^{*} \mathrm{a}=\mathrm{map}, \mathrm{b}=\mathrm{compass}$ and tape, $\mathrm{c}=\mathrm{GPS} .{ }^{* \star} 1=$ Bookstom and others (1999), $2=$ Box and others(in prep.) ${ }^{\star \star *} 1=$ grab, $2=$ grove,

3=depth-bracket,4=hand core,5=power core,6=vibro core,7=vibro-piston core,8=auger in casing. 
Appendix B. Sample site locations, depositional environments, depositional map units, sampling techniques and laboratory and analytical methods used on samples from that site (all=samples from site; some=some samples from site).

\begin{tabular}{|c|c|c|c|c|c|c|c|c|}
\hline Site ID & Latitude & Longitude & $\begin{array}{c}\text { Water depth } \\
(\mathrm{m})\end{array}$ & $\begin{array}{l}\text { Site Location } \\
\text { Map in } \\
\text { Appendix A }\end{array}$ & $\begin{array}{c}\text { Site- } \\
\text { Location } \\
\text { Method* }^{*}\end{array}$ & Environment & USGS map unit description & $\begin{array}{c}\text { USGS map } \\
\text { unit }\end{array}$ \\
\hline T98C-10 & 47.5493 & -116.3329 & & MAP A-5 & $\mathrm{c}$ & Exposed channel bar & Upland, lower terrace base at river shore & $\mathrm{Rg}$ \\
\hline T98C-11B & 47.5474 & -116.3560 & & MAP A-5 & $\mathrm{c}$ & Marsh & Riverine, high-water channel on terrace & Rhc \\
\hline T98C-12 & 47.5474 & -116.3566 & & MAP A-5 & C & Subaerial floodplain & Upland, levee of slough on terrace & Uat2 \\
\hline T98R-13 & 47.5563 & -116.3867 & & MAP A-6 & $\mathrm{c}$ & Marsh & Dredge spoils, palustrine, common reed & AdsPEcr \\
\hline T98R-14 & 47.5580 & -116.3751 & & MAP A-6 & $\mathrm{c}$ & Subaerial dredge spoils & Dredge spoils, subaerial & Ads1 \\
\hline T98C-15 & 47.5510 & -116.2764 & & MAP A-5 & $\mathrm{c}$ & Marsh & Palustrine, seasonal, emergent plants & PEs \\
\hline T98C-16 & 47.5465 & -116.3578 & & MAP A-5 & $\mathrm{c}$ & Subaerial floodplain & Upland, middle alluvial terrace & Uat2 \\
\hline T98C-17 & 47.5501 & -116.3640 & & MAP A-6 & $\mathrm{c}$ & Subaerial floodplain & Upland, levee sand & Ulso \\
\hline T98C-18 & 47.5502 & -116.3484 & & MAP A-5 & $\mathrm{c}$ & Subaerial floodplain & Upland, middle alluvial terrace & Uat2 \\
\hline T98C-20 & 47.5528 & -116.3542 & & MAP A-5 & $\mathrm{c}$ & Subaerial floodplain & Upland, leveelpalustrine, aquatic shore & UlsolPA \\
\hline T98C-21 & 47.5523 & -116.3607 & & MAP A-5 & $\mathrm{c}$ & Marsh & Palustrine, perennial, emergent plants & PEp \\
\hline T98C-22 & 47.5596 & -116.3528 & & MAP A-5 & c & Marsh & Palustrine, emergent common reed & PEcr \\
\hline T98R-23 & 47.5576 & -116.3887 & & MAP A-6 & $\mathrm{c}$ & Marsh & Dredge spoils, palustrine, common reed & AdsPEcr \\
\hline T98R-24 & 47.5588 & -116.3846 & & MAP A-6 & C & Marsh & Dredge spoils, palustrine, common reed & AdsPEcr \\
\hline T98C-25 & 47.5452 & -116.3276 & & MAP A-5 & $\mathrm{c}$ & Subaerial floodplain & Upland, middle alluvial terrace & Uat2 \\
\hline T98C-26 & 47.5441 & -116.3713 & & MAP A-6 & c & Marsh & Palustrine, seasonal, emergent plants & PEs \\
\hline T98R-27 & 47.5400 & -116.4685 & & MAP A-6 & c & Marsh & Palustrine, distributary, blocked & PdisbE \\
\hline T98R-28 & 47.5379 & -116.4836 & & MAP A-7 & $\mathrm{c}$ & Marsh & Palustrine, seasonal, bushes and trees & PEsT \\
\hline T98R-29 & 47.5388 & -116.4746 & & MAP A-6 & $\mathrm{c}$ & Subaerial floodplain & Upland, levee of blocked distributary & Udisb \\
\hline T98R-30 & 47.5341 & -116.4955 & & MAP A-7 & $\mathrm{c}$ & Subaerial floodplain & Upland, levee of channel scar & Ucsl \\
\hline T98R-31 & 47.5483 & -116.3765 & & MAP A-6 & $\mathrm{c}$ & Marsh & Upland, channel scar (filled with sediment) & Ucs \\
\hline T98R-32 & 47.5464 & -116.3866 & & MAP A-6 & c & Subaerial floodplain & Upland, distributary levee & Udis \\
\hline T98R-33 & 47.5334 & -116.4400 & & MAP A-6 & $\mathrm{c}$ & Subaerial floodplain & Upland, levee sand & Ulso \\
\hline T98R-34 & 47.5350 & -116.4501 & & MAP A-6 & $\mathrm{c}$ & Shallow lake & Lacustrine, littoral & LltE \\
\hline T98L-35 & 47.5286 & -116.5018 & & MAP A-7 & c & Marsh & Palustrine, perennial, emergent plants & PEp \\
\hline T98L-36 & 47.5308 & -116.5089 & & MAP A-7 & $\mathrm{c}$ & Subaerial floodplain & Upland, sand splay & Uss \\
\hline T98L-37 & 47.5189 & -116.5227 & & MAP A-7 & c & Marsh & Palustrine, aquatic plants & $\mathrm{PA}$ \\
\hline T98L-38 & 47.5101 & -116.5324 & & MAP A-7 & $\mathrm{c}$ & Marsh & Palustrine, perennial, emergent plants & PEp \\
\hline
\end{tabular}

${ }^{*} \mathrm{a}=\mathrm{map}, \mathrm{b}=\mathrm{compass}$ and tape, $\mathrm{c}=\mathrm{GPS} .{ }^{* \star} 1=$ Bookstom and others (1999), $2=$ Box and others(in prep.) ${ }^{\star \star *} 1=$ grab, $2=$ grove,

$3=$ depth-bracket, $4=$ hand core,5=power core, $6=$ vibro core, $7=$ vibro-piston core, $8=$ auger in casing. 
Appendix B. Sample site locations, depositional environments, depositional map units, sampling techniques and laboratory and analytical methods used on samples from that site (all=samples from site; some=some samples from site).

\begin{tabular}{|l|c|c|c|c|c|c|c|}
\hline Site ID & $\begin{array}{c}\text { USGS } \\
\text { map ** }\end{array}$ & $\begin{array}{c}\text { Sampling } \\
\text { Technique*** }\end{array}$ & $\begin{array}{c}\text { USGS } \\
\text { EDXRF }\end{array}$ & $\begin{array}{c}\text { EWU } \\
\text { 4-acid }\end{array}$ & $\begin{array}{c}\text { CHEMEX } \\
\text { nitric-aqua } \\
\text { regia }\end{array}$ & $\begin{array}{c}\text { XRAL } \\
\text { 4-acid }\end{array}$ & $\begin{array}{c}\text { ACZ } \\
\text { nitric }\end{array}$ \\
\hline T98C-10 & 1 & 3 & & & & all & \\
\hline T98C-11B & 1 & 3 & & & & all & \\
\hline T98C-12 & 1 & 3 & & & & all & \\
\hline T98R-13 & 1 & 3 & & & & all & \\
\hline T98R-14 & 1 & 3 & & & & all & \\
\hline T98C-15 & 1 & 3 & & & & all & \\
\hline T98C-16 & 1 & 3 & & & & all & \\
\hline T98C-17 & 1 & 3 & & & & all & \\
\hline T98C-18 & 1 & 3 & & & & all & \\
\hline T98C-20 & 1 & 3 & & & & all & \\
\hline T98C-21 & 1 & 3 & & & & all & \\
\hline T98C-22 & 1 & 3 & & & & all & \\
\hline T98R-23 & 1 & 3 & & & & all & \\
\hline T98R-24 & 1 & 3 & & & & all & \\
\hline T98C-25 & 1 & 3 & & & & all & \\
\hline T98C-26 & 1 & 3 & & & & all & \\
\hline T98R-27 & 1 & 3 & & & & all & \\
\hline T98R-28 & 1 & 3 & & & & all & \\
\hline T98R-29 & 1 & 3 & & & & all & \\
\hline T98R-30 & 1 & 3 & & & & all & \\
\hline T98R-31 & 1 & 3 & & & & all & \\
\hline T98R-32 & 1 & 3 & & & & all & \\
\hline T98R-33 & 1 & 3 & & & & all & \\
\hline T98R-34 & 1 & 3 & & & & all & \\
\hline T98L-35 & 1 & 3 & & & & all & \\
\hline T98L-36 & 1 & 3 & & & & all & \\
\hline T98L-37 & 1 & 3 & & & & all & \\
\hline T98L-38 & 1 & 3 & & & & all & \\
\hline
\end{tabular}

${ }^{*} \mathrm{a}=\mathrm{map}, \mathrm{b}=\mathrm{compass}$ and tape, $\mathrm{c}=\mathrm{GPS} .{ }^{* *} 1=$ Bookstom and others (1999), $2=$ Box and others(in prep.) ${ }^{* * *} 1=$ grab,2=grove,

3=depth-bracket,4=hand core,5=power core,6=vibro core,7=vibro-piston core,8=auger in casing. 
Appendix B. Sample site locations, depositional environments, depositional map units, sampling techniques and laboratory and analytical methods used on samples from that site (all=samples from site; some=some samples from site)

\begin{tabular}{|c|c|c|c|c|c|c|c|c|}
\hline Site ID & Latitude & Longitude & $\begin{array}{c}\text { Water depth } \\
\text { (m) }\end{array}$ & $\begin{array}{l}\text { Site Location } \\
\text { Map in } \\
\text { Appendix A }\end{array}$ & $\begin{array}{c}\text { Site- } \\
\text { Location } \\
\text { Method }^{*}\end{array}$ & Environment & USGS map unit description & $\begin{array}{c}\text { USGS map } \\
\text { unit }\end{array}$ \\
\hline T98M-39 & 47.4990 & -116.5590 & & MAP A-8 & $\mathrm{C}$ & Drained marsh & Palustrine, seasonal, drained, farmed & PESdf \\
\hline T98M-40 & 47.4872 & -116.5693 & & MAP A-8 & $\mathrm{C}$ & Drained marsh & Palustrine, seasonal to perennial, drained & Pespdf \\
\hline T98L-41 & 47.5073 & -116.5662 & & MAP A-7 & $c$ & Marsh & Palustrine, perennial, emergent rice & PEpr \\
\hline T98L-42 & 47.5024 & -116.5644 & & MAP A-7 & $\mathrm{C}$ & Marsh & Palustrine, perennial, emergent plants & PEp \\
\hline T98M-43 & 47.4942 & -116.5693 & & MAP A-8 & $\mathrm{C}$ & Subaerial floodplain & Upland, levee backslope & Ulb \\
\hline
\end{tabular}

${ }^{*} \mathrm{a}=\mathrm{map}, \mathrm{b}=$ compass and tape, $\mathrm{c}=\mathrm{GPS} .{ }^{* *} 1=$ Bookstom and others (1999), $2=$ Box and others(in prep. ${ }^{\star \star *} 1=$ grab, $2=$ grove, 
Appendix B. Sample site locations, depositional environments, depositional map units, sampling techniques and laboratory and analytical methods used on samples from that site (all=samples from site; some=some samples from site)

\begin{tabular}{|l|c|c|c|c|c|c|c|}
\hline Site ID & $\begin{array}{c}\text { USGS } \\
\text { map ** }\end{array}$ & $\begin{array}{c}\text { Sampling } \\
\text { Technique }\end{array}$ & $\begin{array}{c}\text { USGS } \\
\text { EDXRF }\end{array}$ & $\begin{array}{c}\text { EWU } \\
\text { 4-acid }\end{array}$ & $\begin{array}{c}\text { CHEMEX } \\
\text { nitric-aqua } \\
\text { regia }\end{array}$ & $\begin{array}{c}\text { XRAL } \\
\text { 4-acid }\end{array}$ & $\begin{array}{c}\text { ACZ } \\
\text { nitric }\end{array}$ \\
\hline T98M-39 & 1 & 3 & & & & all & \\
\hline T98M-40 & 1 & 3 & & & & all & \\
\hline T98L-41 & 1 & 3 & & & & all & \\
\hline T98L-42 & 1 & 3 & & & & all & \\
\hline T98M-43 & 1 & 3 & & & & all & \\
\hline
\end{tabular}

${ }^{*} \mathrm{a}=\mathrm{map}, \mathrm{b}=\mathrm{compass}$ and tape, $\mathrm{c}=\mathrm{GPS} .{ }^{* *} 1=$ Bookstom and others (1999), $2=$ Box and others(in prep.) ${ }^{* * *} 1=$ grab, $2=$ grove, 
Appendix C. Lithologic descriptions of soil and sediment samples for which chemical analyses are given in this report (Appendix_C.xls, Appendix_C.dbf)

\begin{tabular}{|c|c|c|c|c|}
\hline Sample No. & Site ID & $\begin{array}{c}\text { Depth } \\
\text { interval, } \\
\text { top }(\mathrm{cm})\end{array}$ & $\begin{array}{c}\text { Depth } \\
\text { interval, } \\
\text { bottom } \\
(\mathrm{cm})\end{array}$ & Lithologic description \\
\hline SAMPLE_NO. & SITE_ID & INT_TOP_CM & INT_BTM_CM & LITH_DESC \\
\hline 93ABM02(0-10) & 93ABM02 & 0 & 10 & light brown fine sand \\
\hline 93ABM02(10-11) & 93ABM02 & 10 & 11 & gray-white ash (May, 1980 Mt. St. Helens) \\
\hline 93ABM02(11-22) & 93ABM02 & 11 & 22 & medium brown medium-fine sand \\
\hline 93ABM02(22-36) & 93ABM02 & 22 & 36 & orange laminated silt with $3 \mathrm{~cm}$ dark brown fine sand at $25-28$ \\
\hline 93ABM02(36-44) & 93ABM02 & 36 & 44 & dark brown, ripple-laminated fine sand with wavy bedding top \\
\hline 93ABM02(44-66) & 93ABM02 & 44 & 66 & 8 couplets of dark brown, very fine sand and orange silt \\
\hline 93ABM02(66-85) & 93ABM02 & 66 & 85 & orange-brown fine sand with climbing ripple laminations and 3 grass-mat \\
\hline 93ABM02(85-97) & 93ABM02 & 85 & 97 & gray silty mud with irregular lens of orange cemented silty mud \\
\hline 93ABM02(97-110) & 93ABM02 & 97 & 110 & massive gray silty mud \\
\hline 93ABM02(110-126) & 93ABM02 & 110 & 126 & massive gray silty mud with sparse orange $0.5 \mathrm{~cm}$ spots \\
\hline 93ABM02(0-97) & 93ABM02 & 0 & 97 & composite 0.5 liter channel sample of orange, sandy section \\
\hline 93ABM02(97-126) & 93ABM02 & 97 & 126 & composite 0.5 liter sample of gray muddy section \\
\hline 93ABM02(0-97) & 93ABM02 & 0 & 97 & composite 6 liter channel sample of orange, sandy section \\
\hline 93ABM02(97-129) & 93ABM02 & 97 & 129 & composite 6 liter sample of gray muddy section \\
\hline $93 \mathrm{CSC} 03(0-6)$ & 93CSC03 & 0 & 6 & brown fine-very fine sand \\
\hline 93CSC03(6-6.5) & 93CSC03 & 6 & 6.5 & gray-white ash (May, 1980 Mt. St. Helens) \\
\hline $93 \operatorname{CSC} 03(6.5-10)$ & $93 \mathrm{CSC} 03$ & 6.5 & 10 & pale brown medium and fine sand \\
\hline $93 \mathrm{CSC} 03(10-43)$ & $93 \mathrm{CSC03}$ & 10 & 43 & $\tan$ silt-very fine sand \\
\hline 93CSC03(43-46) & 93CSC03 & 43 & 46 & red brown silt \\
\hline 93CSC03(46-55) & 93CSC03 & 46 & 55 & red brown very fine sand \\
\hline $93 \operatorname{CSC} 03(55-61)$ & $93 \mathrm{CSC} 03$ & 55 & 61 & red-brown fine and medium sand \\
\hline $93 \operatorname{CSC} 03(61-66)$ & $93 \mathrm{CSC} 03$ & 61 & 66 & tan very fine sand and silt \\
\hline $93 \operatorname{CSC} 03(66-71)$ & $93 \mathrm{CSC} 03$ & 66 & 71 & interbedded coarse and medium sand \\
\hline 93CSC03(71-77) & 93CSC03 & 71 & 77 & pale brown very fine sand and silt \\
\hline 93CSC03(77-95) & 93CSC03 & 77 & 95 & pale brown silt \\
\hline 93CSC03(95-112) & 93CSC03 & 95 & 112 & chocolate brown silt \\
\hline $93 \mathrm{CSC} 03(112-121.5)$ & 93CSC03 & 112 & 121.5 & muddy red-brown silt \\
\hline $93 \mathrm{CSC} 03(121.5-133)$ & 93CSC03 & 121.5 & 133 & chocolate brown fine sand \\
\hline 93CSC03(133-145) & 93CSC03 & 133 & 145 & pale brown silt-very fine sand \\
\hline $93 \operatorname{CSC} 03(145-155)$ & 93CSC03 & 145 & 155 & pale brown silt-very fine sand \\
\hline 93CSC03(155-168) & 93CSC03 & 155 & 168 & gray silt and fine sand \\
\hline 93CSC03(168-192) & 93CSC03 & 168 & 192 & gray very fine sand \\
\hline $93 \mathrm{CSC} 03(192-232)$ & 93CSC03 & 192 & 232 & medium sand \\
\hline $93 \operatorname{CsC03(232-256)}$ & 93CSC03 & 232 & 256 & gray silt and fine sand \\
\hline 93ABM04(0-12) & 93ABM04 & 0 & 12 & $\tan$ unconsolidated fine-grained sand \\
\hline
\end{tabular}


Appendix C. Lithologic descriptions of soil and sediment samples for which chemical analyses are given in this report (Appendix_C.xls, Appendix_C.dbf)

\begin{tabular}{|c|c|c|c|c|}
\hline Sample No. & Site ID & $\begin{array}{c}\text { Depth } \\
\text { interval, } \\
\text { top }(\mathrm{cm})\end{array}$ & $\begin{array}{c}\text { Depth } \\
\text { interval, } \\
\text { bottom } \\
(\mathrm{cm})\end{array}$ & Lithologic description \\
\hline SAMPLE_NO. & SITE_ID & INT_TOP_CM & INT_BTM_CM & LITH_DESC \\
\hline 93ABM04(12-13) & 93ABM04 & 12 & 13 & gray-white ash (May,1980 Mt. St. Helens) \\
\hline 93ABM04(13-18) & 93ABM04 & 13 & 18 & rusty red-brown fine sand, weakly cemented by iron hydroxide \\
\hline 93ABM04(22-27) & 93ABM04 & 22 & 27 & rusty red-brown fine sand, weakly cemented by iron hydroxide \\
\hline 93ABM04(32-37) & $93 \mathrm{ABM} 04$ & 32 & 37 & rusty red-brown fine sand, weakly cemented by iron hydroxide \\
\hline 93ABM04(42-48) & 93ABM04 & 42 & 48 & rusty red-brown fine sand, weakly cemented by iron hydroxide \\
\hline 93ABM04(48-76) & 93ABM04 & 48 & 76 & orange-brown laminated silt, weakly cemented by iron hydroxide \\
\hline 93ABM04(76-142) & 93ABM04 & 76 & 142 & gray massive silt, obvious root bioturbation in upper $25 \mathrm{~cm}$; lake level \\
\hline 93ABM06 & 93ABM06 & & & $\begin{array}{l}\text { crs. grained, } 2 \mathrm{~cm} \text { thick red-brown quartz sand lens under angular } 0.5 \mathrm{~m} \\
\text { auartzite block of } \mathrm{RR} \text { embankment fill }\end{array}$ \\
\hline 93ABM06 & 93ABM06 & & & gray silt $45 \mathrm{~cm}$ below $6 \mathrm{~A}$ : lake level $\left(2128^{\prime}\right) 50 \mathrm{~cm}$ below \\
\hline 93ABL08(0-5) & 93ABL08 & 0 & 5 & red-brown silty mud \\
\hline 93ABL09(0-83) & 93ABL09 & 0 & 83 & tan unconsolidated fine sand \\
\hline 93SBC10(0-3) & 93SBC10 & 0 & 3 & tan unconsolidated fine-grained sand \\
\hline 93SBC10(3-3.5) & 93SBC10 & 3 & 3.5 & gray-white ash (May, 1980 Mt. St. Helens) \\
\hline 93SBC10(3.5-30) & 93SBC10 & 3.5 & 30 & pale brown fine sand, weakly cemented \\
\hline 93SBC10(30-70) & 93SBC10 & 30 & 70 & red-brown fine-medium sand, ripple-laminated with black cross-laminae, \\
\hline 93SBC10(70-90) & 93SBC10 & 70 & 90 & brown fine sand, weakly cemented, with irregular leaf-rich seams \\
\hline 93SBC10(90-100) & 93SBC10 & 90 & 100 & brown fine sand, weakly cemented, with irregular leaf-rich seams \\
\hline 93SBC10(100-130) & 93SBC10 & 100 & 130 & $\begin{array}{l}\text { mottled dark to light tan silty sand with } 5 \mathrm{~cm} \text { wood-rich seam at top and } 1 \\
\mathrm{~cm} \text { charcoal seam at base }\end{array}$ \\
\hline 93SBC10(130-160) & $93 \mathrm{SBC} 10$ & 130 & 160 & mottled dark to light tan silty sand \\
\hline 93SBC10(160-360) & $93 \mathrm{SBC} 10$ & 160 & 360 & pebbly to cobbly gravel, clast-supported, clasts to $10 \mathrm{~cm}$; river level at 360 \\
\hline 93SBR13(0-15) & 93SBR13 & 0 & 15 & tan unconsolidated fine-grained sand \\
\hline 93SBR13(15-16) & 93SBR13 & 15 & 16 & gray-white ash (May, 1980 Mt. St. Helens) \\
\hline 93SBR13(16-52) & 93SBR13 & 16 & 52 & tan unconsolidated medium sand with climbing-ripple laminations \\
\hline 93SBR13(57-65) & 93SBR13 & 57 & 65 & $\begin{array}{l}\text { orange-brown laminated silt, weakly cemented by iron hydroxide; } 2 \mathrm{~cm} \\
\text { verv fine sand in center }\end{array}$ \\
\hline 93SBR13(221-237) & 93SBR13 & 221 & 237 & dark red-brown medium-fine sand, weakly cemented by iron hydroxide \\
\hline 93SBR13(237-251) & 93SBR13 & 237 & 251 & dark red-brown fine sand, weakly cemented by iron hydroxide \\
\hline 93SBR13(251-291) & 93SBR13 & 251 & 291 & orange-brown laminated silt, weakly cemented by iron hydroxide \\
\hline 93SBR13(291-351) & 93SBR13 & 291 & 351 & gray clayey silt with irregular orange-cemented fractures; lake level (2128') \\
\hline 93SBM14 & 93SBM14 & & & moderately cemented tan mudstone with Miocene leaf impressions \\
\hline 93SBC15(0-15) & 93SBC15 & 0 & 15 & tan unconsolidated fine-grained sand \\
\hline 93SBC15(15-16) & 93SBC15 & 15 & 16 & gray-white ash (May, 1980 Mt. St. Helens) \\
\hline 93SBC15(16-35) & 93SBC15 & 16 & 35 & tan very fine sand with $4 \mathrm{~cm}$ medium fine sand in center \\
\hline
\end{tabular}


Appendix C. Lithologic descriptions of soil and sediment samples for which chemical analyses are given in this report (Appendix_C.xls, Appendix_C.dbf)

\begin{tabular}{|c|c|c|c|c|}
\hline Sample No. & Site ID & $\begin{array}{c}\text { Depth } \\
\text { interval, } \\
\text { top }(\mathrm{cm})\end{array}$ & $\begin{array}{l}\text { Depth } \\
\text { interval, } \\
\text { bottom } \\
(\mathrm{cm})\end{array}$ & Lithologic description \\
\hline SAMPLE_NO. & SITE_ID & INT_TOP_CM & INT_BTM_CM & LITH_DESC \\
\hline 93SBC15(35-90) & 93SBC15 & 35 & 90 & $\begin{array}{l}\text { alternating } 1-3 \mathrm{~cm} \text { thick layers of laminated orange silt and weakly } \\
\text { cemented pale brown fine sand }\end{array}$ \\
\hline 93SBC15(90-165) & 93SBC15 & 90 & 165 & orange laminated silt with several $1 \mathrm{~cm}$ dark fine sand layers \\
\hline 93SBC15(165-214) & 93SBC15 & 165 & 214 & orange massive silt with several $0.5-1.0 \mathrm{~cm}$ very fine sand layers \\
\hline 93SBC15(214-242) & 93SBC15 & 214 & 242 & mottled gray, black, orange and brown clayey silt \\
\hline 93SBC15(242-261) & 93SBC15 & 242 & 261 & gray clayey silt with irregular orange streaks: lake level (2127.8') at 261 \\
\hline 93SBC16(0-5) & 93SBC16 & 0 & 5 & $\begin{array}{l}\text { poorly sorted gravelly sand with considerable mud; } 5 \mathrm{~cm} \text { below lake } \\
\text { surface }\left(2128^{\prime}\right) \text { on mid-river sand bar }\end{array}$ \\
\hline 93SBK17(0-8) & 93SBK17 & 0 & 8 & artificially placed anqular white quartzite gravel \\
\hline 93SBK17(8-25) & 93SBK17 & 8 & 25 & black, magnetite-rich coarse-grained sand with pea gravel lenses \\
\hline 93SBK17(25-68) & 93SBK17 & 25 & 68 & $\begin{array}{l}\text { maroon and tan ripple cross-laminated medium to coarse-grained sand } \\
\text { with lens of imbricated aravel with rounded. } 1 \mathrm{~cm} \text { disc-shaned clasts }\end{array}$ \\
\hline 93SBK17(68-106) & 93SBK17 & 68 & 106 & $\begin{array}{l}\text { dark red-orange clayey silt, well laminated, weathered surface partially } \\
\text { coated with white sulfate }\end{array}$ \\
\hline 93SBK17(106-152) & 93SBK17 & 106 & 152 & $\begin{array}{l}\text { bioturbated tan silty sand with some clay; upper } 15 \mathrm{~cm} \text { is organic-rich } \\
\text { clavev silt: floating } 3-5 \mathrm{~cm} \text { pebbles in lower } 25 \mathrm{~cm}\end{array}$ \\
\hline 93SBK17(152-230) & 93SBK17 & 152 & 230 & $\begin{array}{l}\text { unconsolidated cobble conglomerate, matrix-supported in upper } 20 \mathrm{~cm} \text {, } \\
\text { clast-sunoorted below: average clast size: } 5 \mathrm{~cm} \text { max clast size: } 15 \mathrm{~cm}\end{array}$ \\
\hline 93SBK17(230-300) & 93SBK17 & 230 & 300 & similar to above conglomerate; river at 300 \\
\hline 93SBK18(0-7) & 93SBK18 & 0 & 7 & artificially placed wood chips \\
\hline 93SBK18(7-10) & 93SBK18 & 7 & 10 & orange-brown silt, weakly cemented \\
\hline 93SBK18(10-11) & 93SBK18 & 10 & 11 & gray-white ash (May,1980 Mt. St. Helens) \\
\hline 93SBK18(11-26) & 93SBK18 & 11 & 26 & orange-brown silt, weakly cemented \\
\hline 93SBK18(26-82) & 93SBK18 & 26 & 82 & $\begin{array}{l}\text { ripple-laminated, very fine sand in } 1 \mathrm{~cm} \text { beds (weathered-orange, } \\
\text { unweathered-tan) with numerous } 0.3 \mathrm{~cm} \text { orange silt seams; several finely }\end{array}$ \\
\hline 93SBK18(82-92) & 93SBK18 & 82 & 92 & gray clay with black sooty organics within and at base \\
\hline 93SBK18(92-109) & 93SBK18 & 92 & 109 & gray massive clay with $5-10 \%$ floating pebbles to $3 \mathrm{~cm}$ in diameter \\
\hline 93SBK18(109-160) & 93SBK18 & 109 & 160 & $\begin{array}{l}\text { clast-supported round-cobble gravel coarsening downward from } 2-3 \mathrm{~cm} \\
\text { clasts to areater than } 10 \mathrm{~cm} \text { clasts }\end{array}$ \\
\hline 93SBB20(0-5) & 93SBB20 & 0 & 5 & $\begin{array}{l}\text { red-orange silt at edge of gravelled parking lot; overlies earlier white gravel } \\
\text { and underlies black aravel of most recent surfacina }\end{array}$ \\
\hline 93SBB21(0-9) & 93SBB21 & 0 & 9 & unconsolidated fine sand, grading from red to black to tan upwards \\
\hline 3B21(9-10) & 93SBB21 & 9 & 10 & gray-white ash (May,1980 Mt. St. Helens) \\
\hline 93SBB21(10-41) & 93SBB21 & 10 & 41 & $y$ bedded dark-brown fine sand with a few $1 \mathrm{~cm}$ white mud layers \\
\hline
\end{tabular}


Appendix C. Lithologic descriptions of soil and sediment samples for which chemical analyses are given in this report (Appendix_C.xls, Appendix_C.dbf)

\begin{tabular}{|c|c|c|c|c|}
\hline Sample No. & Site ID & $\begin{array}{c}\text { Depth } \\
\text { interval, } \\
\text { top }(\mathrm{cm})\end{array}$ & $\begin{array}{l}\text { Depth } \\
\text { interval, } \\
\text { bottom } \\
(\mathrm{cm})\end{array}$ & Lithologic description \\
\hline SAMPLE_NO. & SITE_ID & INT_TOP_CM & INT_BTM_CM & LITH_DESC \\
\hline 93SBB21(41-72) & 93SBB21 & 41 & 72 & $\begin{array}{l}\text { alternated } 1 \mathrm{~cm} \text { layers of light orange silt and dark brown fine sand; } 3 \mathrm{~cm} \\
\text { maanetite-rich riople-laminated fine sand at base }\end{array}$ \\
\hline 93SBB21(72-84) & 93SBB21 & 72 & 84 & orange silt with $3.5 \mathrm{~cm}$ ripple-laminated fine sand in center \\
\hline 93SBB21(84-99) & 93SBB21 & 84 & 99 & $\begin{array}{l}\text { light tan-orange muddy silt in lower half; alternated dark red-brown fine } \\
\text { sand-orange silt in unper half }\end{array}$ \\
\hline 93SBB21(99-111) & 93SBB21 & 99 & 111 & pale gray silt (irregularly mottled orange) with disseminated black woody \\
\hline 93SBB21(111-120) & 93SBB21 & 111 & 120 & gray clay with black organic flecks and rare red blotches \\
\hline 93SBB21(120-130) & 93SBB21 & 120 & 130 & light brown medium sand with irregular orange streaks \\
\hline 93SBB21(130-148) & 93SBB21 & 130 & 148 & dark gray-brown silty mud with rare orange spots \\
\hline 93SBB21(148-160) & 93SBB21 & 148 & 160 & light brown medium sand \\
\hline 93SBB21(160-180) & 93SBB21 & 160 & 180 & gray-brown thinly bedded fine sand, silt, and mud; lake level (2125.87') at \\
\hline 93SBB21(173-180) & 93SBB21 & 173 & 180 & gray-brown mud \\
\hline 93SBB21(180-192) & 93SBB21 & 180 & 192 & $\tan$ fine sand \\
\hline 93SBB21(192-203) & 93SBB21 & 192 & 203 & gray silty clay \\
\hline 93SBB22(0-4) & 93SBB22 & 0 & 4 & matted vegetation \\
\hline 93SBB22(4-4.5) & 93SBB22 & 4 & 4.5 & gray-white ash (May,1980 Mt. St. Helens) \\
\hline 93SBB22(4.5-7.5) & 93SBB22 & 4.5 & 7.5 & vegetation mat with minor silt component \\
\hline 93SBB22(7.5-12) & 93SBB22 & 7.5 & 12 & orange-brown silty clay \\
\hline 93SBB22(12-15) & 93SBB22 & 12 & 15 & orange-brown silty clay with abundant roots \\
\hline 93SBB22(15-19) & 93SBB22 & 15 & 19 & orange-brown clay with silty seams \\
\hline 93SBB22(19-26) & 93SBB22 & 19 & 26 & orange-brown silty clay, mottled gray at base \\
\hline 93SBB22(26-29) & 93SBB22 & 26 & 29 & gray and orange mottled clay, gray near base \\
\hline 93SBB22(29-36) & 93SBB22 & 29 & 36 & gray-black organic-rich mud \\
\hline 93SBB22(36-48) & 93SBB22 & 36 & 48 & dark-gray peat \\
\hline 93SBB22(48-60) & 93SBB22 & 48 & 60 & dark-gray peat with preserved vegetative mats \\
\hline 93SBB22(60-82) & 93SBB22 & 60 & 82 & gray-brown weakly layered peat \\
\hline 93SBB23(0-5) & 93SBB23 & 0 & 5 & $\tan$ unconsolidated fine-grained sand \\
\hline 93SBB23(5-6) & 93SBB23 & 5 & 6 & gray-white ash (May,1980 Mt. St. Helens) \\
\hline 93SBB23(6-12) & 93SBB23 & 6 & 12 & lower $3 \mathrm{~cm}$-dark brown fine sand; upper $3 \mathrm{~cm}$-light tan silt \\
\hline 93SBB23(12-17) & 93SBB23 & 12 & 17 & brown silt to very fine sand; grass mat layer at base \\
\hline 93SBB23(17-22) & 93SBB23 & 17 & 22 & brown silt to very fine sand; grass mat layer at base \\
\hline 93SBB23(22-30) & 93SBB23 & 22 & 30 & dark brown fine sand; grass mat layer at base \\
\hline 93SBB23(30-46) & 93SBB23 & 30 & 46 & dark to light brown fine sand, ripple cross-laminated, irregular channelized \\
\hline 93SBB23(46-53) & 93SBB23 & 46 & 53 & range laminated silt, with $1 \mathrm{~cm}$ organic rich layer at base \\
\hline
\end{tabular}


Appendix C. Lithologic descriptions of soil and sediment samples for which chemical analyses are given in this report (Appendix_C.xls, Appendix_C.dbf)

\begin{tabular}{|c|c|c|c|c|}
\hline Sample No. & Site ID & $\begin{array}{c}\text { Depth } \\
\text { interval, } \\
\text { top }(\mathrm{cm})\end{array}$ & $\begin{array}{c}\text { Depth } \\
\text { interval, } \\
\text { bottom } \\
(\mathrm{cm})\end{array}$ & Lithologic description \\
\hline SAMPLE_NO. & SITE_ID & INT_TOP_CM & INT_BTM_CM & LITH_DESC \\
\hline 93SBB23(53-86) & 93SBB23 & 53 & 86 & $\tan$ to light tan mottled and massive silty clay, grossly layered in upper 10 \\
\hline 93SBB23(86-117) & 93SBB23 & 86 & 117 & tan to light tan mottled and massive silty clay \\
\hline 93SBL26(0-6) & 93SBL26 & 0 & 6 & $60 \%$ horsetail stalks, $40 \%$ gray mud; mud is brown with red tinge at top \\
\hline 93SBL26(6-12) & 93SBL26 & 6 & 12 & gray brown mud with reddish tinge; $40 \%$ horsetail stalks and dark organic \\
\hline 93SBL26(12-20) & 93SBL26 & 12 & 20 & gray brown mud with $30 \%$ horsetail stalks and dark organic matter \\
\hline 93SBL26(20-28) & 93SBL26 & 20 & 28 & gray brown mud with $50 \%$ vegetative matter (including horsetail stalks) \\
\hline 93SBL26(28-34) & 93SBL26 & 28 & 34 & upper $3 \mathrm{~cm}$ - brown-black muddy peat; lower $3 \mathrm{~cm}$ - vegetative matter \\
\hline 93SBL26(34-47) & 93SBL26 & 34 & 47 & brown-black peat with $1.5 \mathrm{~cm}$ vegetative mat of horsetail stalks and grass \\
\hline 93SBL26(47-58) & 93SBL26 & 47 & 58 & brown-black peat with $1.5 \mathrm{~cm}$ vegetative mat of horsetail stalks and grass \\
\hline 93SBL27(0-7) & 93SBL27 & 0 & 7 & $\tan$ unconsolidated fine-grained sand \\
\hline 93SBL27(7-7.5) & 93SBL27 & 7 & 7.5 & gray-white ash (May, 1980 Mt. St. Helens) \\
\hline 93SBL27(7.5-19) & 93SBL27 & 7.5 & 19 & medium-grained sand with black-streaked ripple-laminations \\
\hline 93SBL27(19-23) & 93SBL27 & 19 & 23 & orange laminated silt \\
\hline 93SBL27(23-39) & 93SBL27 & 23 & 39 & dark brown fine-grained sand with two $1 \mathrm{~cm}$ orange silt layers \\
\hline 93SBL27(39-46) & 93SBL27 & 39 & 46 & alternating dark brown fine-grained sand and orange silt \\
\hline 93SBL27(46-50) & 93SBL27 & 46 & 50 & $1 \mathrm{~cm}$ orange silt beds with fine sand partings \\
\hline 93SBL27(50-60) & 93SBL27 & 50 & 60 & alternating dark brown fine-grained sand and orange silt \\
\hline 93SBL27(60-76) & 93SBL27 & 60 & 76 & gray brown bioturbated muddy very fine sand \\
\hline 93SBL27(63-65) & 93SBL27 & 63 & 65 & orange-brown silt filling burrow(?) \\
\hline 93SBL27(76-90) & 93SBL27 & 76 & 90 & gray brown bioturbated muddy very fine sand \\
\hline 93SBL27(90-99) & 93SBL27 & 90 & 99 & gray brown bioturbated muddy very fine sand \\
\hline 93SBL27B(0-4) & 93SBL27B & 0 & 4 & $\tan$ unconsolidated fine-grained sand \\
\hline 93SBL27B(4-5) & 93SBL27B & 4 & 5 & gray-white ash (May, 1980 Mt. St. Helens) \\
\hline 93SBL27B(5-16) & 93SBL27B & 5 & 16 & dark brown fine to medium-grained sand \\
\hline 93SBL27B(16-23) & 93SBL27B & 16 & 23 & alternating $\mathrm{cm}$-thick orange silt and very fine sand beds \\
\hline 93SBL27B(23-34) & 93SBL27B & 23 & 34 & dark brown-orange silty fine sand, weakly cemented by iron hydroxide \\
\hline 93SBL27B(34-43) & 93SBL27B & 34 & 43 & laminated pale tan to orange silt and very fine sand \\
\hline 93SBL27B(43-53) & 93SBL27B & 43 & 53 & laminated pale tan to orange silt and very fine sand \\
\hline 93SBL27B(53-69) & 93SBL27B & 53 & 69 & laminated pale tan to orange silt and very fine sand \\
\hline 93SBL27B(69-85) & 93SBL27B & 69 & 85 & gray bioturbated silty fine sand \\
\hline 93SBL28(0-11) & 93SBL28 & 0 & 11 & gray mud with $70 \%$ horsetail stalks \\
\hline 93SBL28(11-16) & 93SBL28 & 11 & 16 & gray mud with $50 \%$ organic material (decomposed horsetail stalks) \\
\hline 93SBL28(16-25) & 93SBL28 & 16 & 25 & gray mud with $<25 \%$ organic material \\
\hline 93SBL28(25-34) & 93SBL28 & 25 & 34 & gray mud with $<25 \%$ organic material \\
\hline
\end{tabular}


Appendix C. Lithologic descriptions of soil and sediment samples for which chemical analyses are given in this report (Appendix_C.xls, Appendix_C.dbf)

\begin{tabular}{|c|c|c|c|c|}
\hline Sample No. & Site ID & $\begin{array}{c}\text { Depth } \\
\text { interval, } \\
\text { top }(\mathrm{cm})\end{array}$ & $\begin{array}{l}\text { Depth } \\
\text { interval, } \\
\text { bottom } \\
(\mathrm{cm})\end{array}$ & Lithologic description \\
\hline SAMPLE_NO. & SITE_ID & INT_TOP_CM & INT_BTM_CM & LITH_DESC \\
\hline 93SBL28(34-37) & 93SBL28 & 34 & 37 & gray mud with $<25 \%$ organic material (horsetail mat parting at base) \\
\hline 93SBL28(37-41) & 93SBL28 & 37 & 41 & gray mud with $<25 \%$ organic material (horsetail mat parting at base) \\
\hline 93SBL28(41-46) & 93SBL28 & 41 & 46 & gray mud with $<25 \%$ organic material \\
\hline 93SBL28C $(0-25)$ & 93SBL28C & 0 & 25 & vegetative mat (horsetails) with minor reddish mud \\
\hline 93SBL28C(25-28) & 93SBL28C & 25 & 28 & brown mud with $75 \%$ horsetail stalks and roots \\
\hline 93SBL28C(28-37) & 93SBL28C & 28 & 37 & brownish gray mud with $30-40 \%$ wiry black organic material \\
\hline 93SBL28C(37-48) & 93SBL28C & 37 & 48 & dark gray mud with $15 \%$ black organic material \\
\hline 93SBL28C(48-54) & 93SBL28C & 48 & 54 & dark gray clay with $30 \%$ wiry black organic material \\
\hline 93SBL28C(54-64) & 93SBL28C & 54 & 64 & dark gray muddy compost with $60 \%$ black organic material \\
\hline 93SBL28C(64-72) & 93SBL28C & 64 & 72 & brown compost (95-100\% organic) with gray coating \\
\hline 93SBL28C(72-83) & 93SBL28C & 72 & 83 & brown compost with dark smooth coating \\
\hline 93SBL28C(83-93) & 93SBL28C & 83 & 93 & brown-black composted horsetail stalks/roots \\
\hline 93SBL28C(93-97) & 93SBL28C & 93 & 97 & brown-black composted horsetail stalks/roots \\
\hline 93SBL30(0-4) & 93SBL30 & 0 & 4 & orange-brown clayey silt to very fine sand \\
\hline 93SBL30(4-4.5) & 93SBL30 & 4 & 4.5 & gray-white ash (May,1980 Mt. St. Helens) \\
\hline 93SBL30(4.5-9) & 93SBL30 & 4.5 & 9 & orange-brown clayey silt to very fine sand, grass root network \\
\hline 93SBL30(9-18) & 93SBL30 & 9 & 18 & orange-brown clayey silt to very fine sand; grass mat parting at base \\
\hline 93SBL30(18-23) & 93SBL30 & 18 & 23 & vellow-brown clayey silt to very fine sand; grass mat parting at base \\
\hline 93SBL30(23-28.5) & 93SBL30 & 23 & 28.5 & vellow-orange clayey silt to very fine sand; grass mat parting at base \\
\hline 93SBL30(28.5-31.5) & 93SBL30 & 28.5 & 31.5 & vellow-orange clayey silt to very fine sand; grass mat parting at base \\
\hline 93SBL30(31.5-37) & 93SBL30 & 31.5 & 37 & bright red-orange, moderately cemented fine sand \\
\hline 93SBL30(37-48) & 93SBL30 & 37 & 48 & black clayey silt with $20 \%$ irregular gray lenses \\
\hline 93SBL30(48-62) & 93SBL30 & 48 & 62 & black clayey silt \\
\hline 93SBL30(62-77) & 93SBL30 & 62 & 77 & gray-black clayey silt with $1 \%$ orange spots \\
\hline 93SBL30(77-100) & 93SBL30 & 77 & 100 & homogenous gray clayey silt with minor orange specks \\
\hline 93SBL30(100-122) & 93SBL30 & 100 & 122 & homogenous gray clayey silt \\
\hline 93SBL30(127-137.5) & 93SBL30 & 127 & 137.5 & pale gray clayey silt with horizontal cracks every $0.5 \mathrm{~cm}$ \\
\hline 93SBL30 $(137.5-144.5$ & 93SBL30 & 137.5 & 144.5 & gray-brown unlayered clayey silt \\
\hline 93SBL30(144.5-158.5 & $93 S B L 30$ & 144.5 & 158.5 & gray-brown clayey silt with horizontal cracks every $1.5 \mathrm{~cm}$ \\
\hline 93SBL30(158.5-172) & 93SBL30 & 158.5 & 172 & gray clayey silt with horizontal cracks every $2 \mathrm{~cm}$ \\
\hline 93SBL30(172-180) & 93SBL30 & 172 & 180 & gray clayey silt with horizontal cracks every $1 \mathrm{~cm}$ \\
\hline 93SBL30(180-187) & 93SBL30 & 180 & 187 & gray clayey silt with horizontal cracks every $1 \mathrm{~cm}$ \\
\hline 93SBL31(0-6) & 93SBL31 & 0 & 6 & medium brown, very fine unconsolidated sand \\
\hline 93SBL31(6-6.5) & 93SBL31 & 6 & 6.5 & gray-white ash (May,1980 Mt. St. Helens) \\
\hline
\end{tabular}


Appendix C. Lithologic descriptions of soil and sediment samples for which chemical analyses are given in this report (Appendix_C.xls, Appendix_C.dbf)

\begin{tabular}{|c|c|c|c|c|}
\hline Sample No. & Site ID & $\begin{array}{c}\text { Depth } \\
\text { interval, } \\
\text { top }(\mathrm{cm})\end{array}$ & $\begin{array}{l}\text { Depth } \\
\text { interval, } \\
\text { bottom } \\
(\mathrm{cm})\end{array}$ & Lithologic description \\
\hline SAMPLE_NO. & $\overline{S I T E \_I D}$ & INT_TOP_CM & INT_BTM_CM & LITH_DESC \\
\hline 93SBL31(6.5-18) & 93SBL31 & 6.5 & 18 & $\begin{array}{l}\text { alternating cm-thick dusky brown and medium brown silt couplets; lower } 3 \\
\mathrm{~cm} \text {-dark brown fine sand }\end{array}$ \\
\hline 93SBL31(18-23) & 93SBL31 & 18 & 23 & $3 \mathrm{~cm}$ ripple-laminated salt \& pepper fine sand underlain by $2 \mathrm{cmm}$ pale \\
\hline 93SBL31(23-30) & 93SBL31 & 23 & 30 & $\begin{array}{l}\text { dark brown fine sand with ripple laminations defined by alternating black } \\
\text { and pale orange streaks }\end{array}$ \\
\hline 93SBL31(30-35) & 93SBL31 & 30 & 35 & dark brown fine sand grades up to $0.5 \mathrm{~cm}$ silt at top \\
\hline 93SBL31(35-42) & 93SBL31 & 35 & 42 & tan very fine sand with black ripple-laminations, grade \\
\hline 93SBL31(42-48) & 93SBL31 & 42 & 48 & light brown very fine sand with $1 \mathrm{~cm}$ silt at top \\
\hline 93SBL31(48-61) & 93SBL31 & 48 & 61 & orange-brown silt with fine laminae; bright orange-brown very fine sand \\
\hline 93SBL31(61-67) & 93SBL31 & 61 & 67 & $1 \mathrm{~cm}$ orange silt at base, capped by two $3 \mathrm{~cm}$ sands with orange silt \\
\hline 93SBL31(67-73) & 93SBL31 & 67 & 73 & orange silt with black spots; black organic parting at base \\
\hline 93SBL31(73-87) & 93SBL31 & 73 & 87 & $\begin{array}{l}\text { dark orange, tan and black fine- to medium-grained sand with ripple } \\
\text { laminations: orange silt parting at base }\end{array}$ \\
\hline 93SBL31(87-108) & 93SBL31 & 87 & 108 & $\begin{array}{l}\text { coarsely banded orange and tan fine sand with ripple-laminations } \\
\text { decreasing in heiaht from } 2 \mathrm{~cm} \text { at bottom to } 0.5 \mathrm{~cm} \text { at ton: aradational with }\end{array}$ \\
\hline 93SBL31(108-130) & 93SBL31 & 108 & 130 & homogenous tan fine sand with irregular black and orange streaks \\
\hline 93SBL31(130-137) & 93SBL31 & 130 & 137 & pale yellow brown very fine sand with orange laminae \\
\hline 93SBL31(137-146) & 93SBL31 & 137 & 146 & alternating dark gray muddy silt and very fine sand in $0.5 \mathrm{~cm}$ thick layers \\
\hline 93SBL31(146-160) & 93SBL31 & 146 & 160 & medium gray to light olive gray silty very fine sand \\
\hline 93SBL31(185-200) & 93SBL31 & 185 & 200 & alternating gray silt and tan very fine sand in $0.5 \mathrm{~cm}$ layers \\
\hline 93SBL31(200-211) & 93SBL31 & 200 & 211 & three $3 \mathrm{~cm}$ tan fine sand lavers separated by $1 \mathrm{~cm}$ dark gray silt layers \\
\hline 93SBL32(0-3) & 93SBL32 & 0 & 3 & medium brown very fine sand with roots \\
\hline 93SBL32(3-3.5) & 93SBL32 & 3 & 3.5 & gray-white ash (May,1980 Mt. St. Helens) \\
\hline 93SBL32(3.5-10.5) & 93SBL32 & 3.5 & 10.5 & light red-orange brown very fine sand \\
\hline 93SBL32(10.5-16) & 93SBL32 & 10.5 & 16 & dusky brown silt \\
\hline 93SBL32(16-24) & 93SBL32 & 16 & 24 & irregular lens of dark red-brown silt in homogenous moderate brown silt \\
\hline 93SBL32(24-31) & 93SBL32 & 24 & 31 & dusky brown silt with mottles of light brown \\
\hline 93SBL32(31-48) & 93SBL32 & 31 & 48 & moderate brown silty mud with no mottling \\
\hline 93SBL34a & 93SBL34 & & & gray black mud with $10 \%$ red spots at water's edge at $2121.4^{\prime}$ above sea \\
\hline 93SBL34b & 93SBL34 & & & $\begin{array}{l}\text { red very fine sand and silt with gray streaks vertical meter above } \\
93 \mathrm{SB} \perp 34 \mathrm{a} \text { and } 10 \mathrm{~cm} \text { below } 93 \mathrm{SB} \perp 34 \mathrm{c}\end{array}$ \\
\hline 93SBL34C & 93SBL34 & & & gray black mud $10 \mathrm{~cm}$ above 93SBL34b and adjacent to 93SBL34d \\
\hline 93SBL34d & 93SBL34 & & & $\begin{array}{l}\text { irregular blob of red-orange, moderately cemented mud penetrating } \\
\text { downward into laver of } 93 \mathrm{SBL} 34 \mathrm{c}\end{array}$ \\
\hline
\end{tabular}


Appendix C. Lithologic descriptions of soil and sediment samples for which chemical analyses are given in this report (Appendix_C.xls, Appendix_C.dbf)

\begin{tabular}{|c|c|c|c|c|}
\hline Sample No. & Site ID & $\begin{array}{c}\text { Depth } \\
\text { interval, } \\
\text { top }(\mathrm{cm})\end{array}$ & $\begin{array}{c}\text { Depth } \\
\text { interval, } \\
\text { bottom } \\
(\mathrm{cm})\end{array}$ & Lithologic description \\
\hline SAMPLE_NO. & SITE_ID & INT_TOP_CM & INT_BTM_CM & LITH_DESC \\
\hline 93SBL34e & 93SBL34 & & & $\begin{array}{l}\text { gray black mud with minor red streaks } 2 \mathrm{~m} \text { east of } 93 \mathrm{SBL} 34 \mathrm{~d} \text { and about } 1.5 \\
\text { m below around surface }\end{array}$ \\
\hline 93SBL34f & 93SBL34 & & & $\begin{array}{l}\text { red cemented silty mud along vertical fracture }(3 \mathrm{~cm} \text { wide) cutting gray- } \\
\text { black mud laver of } 93 \mathrm{SBL} 34 \mathrm{e}\end{array}$ \\
\hline 93SBL34g & 93SBL34 & & & $25 \mathrm{~cm}$ thick red-orange layered silt $50 \mathrm{~cm}$ above $93 \mathrm{SBL} 34 \mathrm{e}$ \\
\hline 93SBL34h & 93SBL34 & & & $2 \mathrm{~cm}$ gray lens in red-orange layered silt between layers of 93SBL34g and \\
\hline 93SBL34i & 93SBL34 & & & $\begin{array}{l}25 \mathrm{~cm} \text { red-orange layered silt-very fine sand above 93SBL34h with layer } \\
\text { ton } 65 \mathrm{~cm} \text { below around surface }\end{array}$ \\
\hline 93SBC35A $(0-5)$ & 93SBC35A & 0 & 5 & gravelly coarse unconsolidated sand from dredge pile west of Cataldo boat \\
\hline 93SBC35B(0-5) & 93SBC35B & 0 & 5 & gravelly coarse unconsolidated sand from dredge pile west of Cataldo boat \\
\hline 93SBC36(0-5) & 93SBC36 & 0 & 5 & coarse to fine sand from modern sandbar \\
\hline 93SBC37(0-30) & 93SBC37 & 0 & 30 & tan medium-coarse unconsolidated sand with scattered $1-2 \mathrm{~cm}$ clasts in \\
\hline 93SBK38(0-2) & 93SBK38 & 0 & 2 & tan unconsolidated fine sand in small patches on modern gravel point bar \\
\hline 93SBK38(0-11) & 93SBK38 & 0 & 11 & brown muddy fine sand (no Mt. St. Helens ash) \\
\hline 93SBK38(11-20) & 93SBK38 & 11 & 20 & red-brown medium sand \\
\hline 93SBK38(20-55) & 93SBK38 & 20 & 55 & gravelly coarse sand with $25 \% 2-\mathrm{cm}$ rounded quartzite clasts \\
\hline 93SBK38(55-100) & 93SBK38 & 55 & 100 & red-brown sandy gravel $(70 \% 2-5 \mathrm{~cm}$ clasts; $30 \%$ coarse sand) \\
\hline 93SBC39(0-2) & 93SBC39 & 0 & 2 & medium-fine sand from eddy bar in modern channel \\
\hline 93SBC39(0-5) & 93SBC39 & 0 & 5 & light tan fine unconsolidated sand \\
\hline 93SBC39(5-5.5) & 93SBC39 & 5 & 5.5 & gray-white ash (May,1980 Mt. St. Helens) \\
\hline 93SBC39(5.5-10) & 93SBC39 & 5.5 & 10 & orange-brown fine sand \\
\hline 93SBC39(10-20) & 93SBC39 & 10 & 20 & marble-cake (brown, orange, black) fine sand \\
\hline 93SBC39(20-27) & 93SBC39 & 20 & 27 & dark brown and black fine sand \\
\hline 93SBC39(27-32) & 93SBC39 & 27 & 32 & red-brown fine sand \\
\hline 93SBC39(32-40) & 93SBC39 & 32 & 40 & dark brown clayey very fine sand \\
\hline 93SBC39(40-58) & 93SBC39 & 40 & 58 & light brown clayey very fine sand \\
\hline 93SBC39(58-72) & 93SBC39 & 58 & 72 & dark brown clayey fine sand \\
\hline 93SBC39(72-225) & 93SBC39 & 72 & 225 & unconsolidated round cobble conglomerate; river at 225 \\
\hline 94SB40(0-2) & 94SB40 & 0 & 2 & unconsolidated medium sand from modern point bar deposit \\
\hline 94SB40(0-15) & 94SB40 & 0 & 15 & grassroot-filled fine sand with visible mica flakes \\
\hline 94SB40(15-16) & 94SB40 & 15 & 16 & gray-white ash (May, 1980 Mt. St. Helens) \\
\hline 94SB40(16-50) & $94 S B 40$ & 16 & 50 & brown silt to very fine sand, unlayered, with visible mica flakes \\
\hline $94 S B 40(50-75)$ & 94SB40 & 50 & 75 & mottled gray-orange-brown fine sand with clayey matrix; no layering \\
\hline
\end{tabular}


Appendix C. Lithologic descriptions of soil and sediment samples for which chemical analyses are given in this report (Appendix_C.xls, Appendix_C.dbf)

\begin{tabular}{|c|c|c|c|c|}
\hline Sample No. & Site ID & $\begin{array}{c}\text { Depth } \\
\text { interval, } \\
\text { top }(\mathrm{cm})\end{array}$ & $\begin{array}{l}\text { Depth } \\
\text { interval, } \\
\text { bottom } \\
\text { (cm) }\end{array}$ & Lithologic description \\
\hline SAMPLE_NO. & SITE_ID & INT_TOP_CM & INT_BTM_CM & LITH_DESC \\
\hline $94 S B 40(75-100)$ & $94 S B 40$ & 75 & 100 & $\begin{array}{l}\text { mottled gray-orange-brown fine sand with clayey matrix with two distinct 2- } \\
\mathrm{cm} \text { dark brown silts with dark organics }\end{array}$ \\
\hline $94 S B 40(100-125)$ & 94SB40 & 100 & 125 & $\begin{array}{l}\text { mottled gray-orange-brown fine sand with clayey matrix with } 1 \mathrm{~cm} \text { dark } \\
\text { brown silt laver with dark organics: base is } 2 \mathrm{~m} \text { above lake surface }\end{array}$ \\
\hline 94Gid2(0-3) & 94Gid2 & 0 & 3 & grassy duff with disseminated fine sand \\
\hline 94Gid2(3-4) & 94Gid2 & 3 & 4 & fine sand \\
\hline 94Gid2(4-9) & 94Gid2 & 4 & 9 & mottled red-brown to gray very fine sand and silt layers, $1-2 \mathrm{~cm}$ thick \\
\hline 94Gid2(9-10) & 94Gid2 & 9 & 10 & fine sand \\
\hline 94Gid2(10-27) & 94Gid2 & 10 & 27 & mottled red-brown to gray very fine sand and silt layers, $1-2 \mathrm{~cm}$ thick \\
\hline 94Gid2(27-29) & 94Gid2 & 27 & 29 & red-brown fine sand with organics \\
\hline 94Gid2(29-52) & 94Gid2 & 29 & 52 & mottled gray silt with organics \\
\hline 94Gid2(52-56) & 94Gid2 & 52 & 56 & fine sand \\
\hline 94Gid2(56-86) & 94Gid2 & 56 & 86 & dark gray silt with two $0.5 \mathrm{~cm}$ very fine sand layers \\
\hline 94Gid2(86-90) & 94Gid2 & 86 & 90 & fine-very fine sand and silt layers $(0.5 \mathrm{~cm}$ each) \\
\hline 94Gid2(90-142) & 94Gid2 & 90 & 142 & homogenous gray fine sand \\
\hline 94Gid2(142-181) & 94Gid2 & 142 & 181 & $\mathrm{~cm}$-thick layered gray muddy silt with rootlets \\
\hline 94Gid2(181-203) & 94Gid2 & 181 & 203 & gray very fine sand \\
\hline 94Gid2(203-215) & 94Gid2 & 203 & 215 & gray fine sand \\
\hline 94Gid2(215-245) & 94Gid2 & 215 & 245 & gray very fine sand \\
\hline 94Gid2(245-250) & 94Gid2 & 245 & 250 & gray silt \\
\hline 94Gid2(250-280) & 94Gid2 & 250 & 280 & gray silt and muddy silt with rootlets; layered in $0.2-1.0 \mathrm{~cm}$ layers of gray \\
\hline 94Gid3(0-3) & 94Gid3 & 0 & 3 & $\tan$ very fine sand \\
\hline 94Gid3(3-3.5) & 94Gid3 & 3 & 3.5 & gray white ash, 1980 Mt. St. Helens \\
\hline 94Gid3(3.5-12) & 94Gid3 & 3.5 & 12 & dark gray fine sand \\
\hline 94Gid3(12-67.5) & 94Gid3 & 12 & 67.5 & laminated dark brown and yellow-orange very fine sand and silt layers, 0.1 - \\
\hline 94Gid3(122-130) & 94Gid3 & 122 & 130 & laminated dark brown and yellow-orange very fine sand and silt layers, 0.1 - \\
\hline 94Gid3(130-136) & 94Gid3 & 130 & 136 & fine salt \& pepper dark gray sand \\
\hline 94Gid3(136-151) & 94Gid3 & 136 & 151 & orange laminated silt with $5 \%$ irregular gray splotches \\
\hline 94Gid3(151-190) & 94Gid3 & 151 & 190 & dark gray muddy silt with fine sand 155-157; plant debris rich layers at 153 \\
\hline 94Gid3(190-223) & 94Gid3 & 190 & 223 & tan-gray muddy silt with indistinct layers \\
\hline 94Gid3(244-252) & 94Gid3 & 244 & 252 & tan gray muddy silt \& very fine sand \\
\hline 94Gid3(252-284) & 94Gid3 & 252 & 284 & $\tan$, massive medium-fine sand \\
\hline 94Gid3(284-297) & 94Gid3 & 284 & 297 & $\tan$ muddy silt; black plant debris rich seam at $286 \mathrm{~cm}$ \\
\hline 94Gid3(297-300) & 94Gid3 & 297 & 300 & tan fine sand \\
\hline 94Gid3(300-321) & 94Gid3 & 300 & 321 & $\tan$ muddy silt \\
\hline
\end{tabular}


Appendix C. Lithologic descriptions of soil and sediment samples for which chemical analyses are given in this report (Appendix_C.xls, Appendix_C.dbf)

\begin{tabular}{|c|c|c|c|c|}
\hline Sample No. & Site ID & $\begin{array}{c}\text { Depth } \\
\text { interval, } \\
\text { top }(\mathrm{cm})\end{array}$ & $\begin{array}{c}\text { Depth } \\
\text { interval, } \\
\text { bottom } \\
(\mathrm{cm})\end{array}$ & Lithologic description \\
\hline SAMPLE_NO. & SITE_ID & INT_TOP_CM & INT_BTM_CM & LITH_DESC \\
\hline 94Gid3(321-326) & 94Gid3 & 321 & 326 & tan fine sand \\
\hline 94Gid3(326-337) & 94Gid3 & 326 & 337 & $\tan$ muddy silt \\
\hline 94Gid4(0-2) & 94Gid4 & 0 & 2 & grassy duff layer \\
\hline 94Gid4(2-10) & 94Gid4 & 2 & 10 & $\tan$ medium-fine sand with grass rootlets \\
\hline 94Gid4(10-16) & 94Gid4 & 10 & 16 & very fine sand and silt layers, dark brown \\
\hline 94Gid4(16-40) & 94Gid4 & 16 & 40 & orange brown laminated silt \\
\hline 94Gid4(40-46) & 94Gid4 & 40 & 46 & dark brown-black fine sand \\
\hline 94Gid4(46-52) & 94Gid4 & 46 & 52 & orange-brown silt \\
\hline 94Gid4(52-57) & 94Gid4 & 52 & 57 & brown fine sand \\
\hline 94Gid4(57-63.5) & 94Gid4 & 57 & 63.5 & laminated orange silt, gray on bottom \\
\hline 94Gid4(63.5-122) & 94Gid4 & 63.5 & 122 & No recovery \\
\hline 94Gid4(122-128) & 94Gid4 & 122 & 128 & black-brown silty clay, blotchy layers with red, eye-shaped lenses in upper \\
\hline 94Gid4(128-132) & 94Gid4 & 128 & 132 & medium-fine brown sand \\
\hline 94Gid4(132-194) & 94Gid4 & 132 & 194 & tan silty clay with dark gray laminae visible below 160 \\
\hline 94Gid4(194-198) & 94Gid4 & 194 & 198 & $\tan$ silty medium-fine sand \\
\hline 94Gid4(198-215) & 94Gid4 & 198 & 215 & $\tan$ silty clay with irregular black laminae \\
\hline 94Gid4(215-244) & 94Gid4 & 215 & 244 & No recovery \\
\hline 94Gid4(244-251) & 94Gid4 & 244 & 251 & tan silty clay with thin fine sand laminae \\
\hline 94Gid4(251-255) & 94Gid4 & 251 & 255 & very fine sand \\
\hline 94Gid4(255-256) & 94Gid4 & 255 & 256 & tan silty clay with thin fine sand laminae \\
\hline 94Gid4(256-267) & 94Gid4 & 256 & 267 & fine sand \\
\hline 94Gid4(267-273) & 94Gid4 & 267 & 273 & tan silty clay with thin fine sand laminae \\
\hline 94Gid4(273-279) & 94Gid4 & 273 & 279 & very fine sand \\
\hline 94Gid4(279-351) & 94Gid4 & 279 & 351 & tan silty clay with irregular rusty blotches; sporadic thin fine sand layers \\
\hline 94Gid5(0-5) & 94Gid5 & 0 & 5 & grassy duff layer $0-1.5 \mathrm{~cm}$; tan fine sand with grass roots $1.5-5.0 \mathrm{~cm}$ \\
\hline 94Gid5(5-5.5) & 94Gid5 & 5 & 5.5 & gray white ash, 1980 Mt. St. Helens \\
\hline 94Gid5(5.5-10) & 94Gid5 & 5.5 & 10 & $\tan$ fine sand \\
\hline 94Gid5(10-18) & 94Gid5 & 10 & 18 & fine sand, gradually changing from orange to dark brown downward \\
\hline 94Gid5(18-43) & 94Gid5 & 18 & 43 & $\begin{array}{l}\text { dark brown silty very fine sand with irregular lenses of orange silt in upper } \\
5 \mathrm{~cm} \text {. orange fine sand } 35-36\end{array}$ \\
\hline 94Gid5(43-90) & 94Gid5 & 43 & 90 & $\begin{array}{l}\text { homogenous dark brown-black silt becoming somewhat lighter in lower } \\
\text { half: sharn basal contact }\end{array}$ \\
\hline 94Gid5(90-96) & 94Gid5 & 90 & 96 & $0.2-0.4 \mathrm{~cm}$ layers of tan very fine sand and dark brown silt \\
\hline 94Gid5(96-122) & 94Gid5 & 96 & 122 & No recovery \\
\hline 94Gid5(122-128) & 94Gid5 & 122 & 128 & dark gray brown silt, fine light and dark layers \\
\hline
\end{tabular}


Appendix C. Lithologic descriptions of soil and sediment samples for which chemical analyses are given in this report (Appendix_C.xls, Appendix_C.dbf)

\begin{tabular}{|c|c|c|c|c|}
\hline Sample No. & Site ID & $\begin{array}{c}\text { Depth } \\
\text { interval, } \\
\text { top }(\mathrm{cm})\end{array}$ & $\begin{array}{l}\text { Depth } \\
\text { interval, } \\
\text { bottom } \\
(\mathrm{cm})\end{array}$ & Lithologic description \\
\hline SAMPLE_NO. & SITE_ID & INT_TOP_CM & INT_BTM_CM & LITH_DESC \\
\hline 94Gid5(128-131) & 94Gid5 & 128 & 131 & chocolate brown fine sand with twigs \\
\hline 94Gid5(131-225) & 94Gid5 & 131 & 225 & $\begin{array}{l}\text { homogenous, unlayered silty clay and very fine sand, dark at top becoming } \\
\text { tan bv } 180 \text { to bottom. irreaular orange blotches throughout }\end{array}$ \\
\hline 94Gid6(0-3) & 94Gid6 & 0 & 3 & grassy duff with fine sand content increasing downward \\
\hline 94Gid6(3-4) & 94Gid6 & 3 & 4 & gray white ash, 1980 Mt. St. Helens \\
\hline 94Gid6(4-8) & 94Gid6 & 4 & 8 & tan-orange very fine sand \\
\hline 94Gid6(8-11) & 94Gid6 & 8 & 11 & orange silt \\
\hline 94Gid6(11-16) & 94Gid6 & 11 & 16 & orange very fine sand \\
\hline 94Gid6(16-20) & 94Gid6 & 16 & 20 & mottled orange and gray very fine sand \\
\hline 94Gid6(20-70) & 94Gid6 & 20 & 70 & $\begin{array}{l}\text { dark brown very fine sand-silt grading downward to light brown, very fine } \\
\text { sand-silt_no lavering }\end{array}$ \\
\hline 94Gid6(70-79) & 94Gid6 & 70 & 79 & laminated gray-tan silt with pale rust splotches \\
\hline 94Gid6(79-122) & 94Gid6 & 79 & 122 & No recovery \\
\hline 94Gid6(122-127) & 94Gid6 & 122 & 127 & laminated gray-tan silt \\
\hline 94Gid6(127-130) & 94Gid6 & 127 & 130 & red-brown fine sand with grass stalks \\
\hline 94Gid6(130-226) & 94Gid6 & 130 & 226 & $\begin{array}{l}\text { homogenous gray-tan silty clay and very fine sand as gradational } 1-3 \mathrm{~cm} \\
\text { lavers becomina liahter downward }\end{array}$ \\
\hline 94VC-1(0-91) & 94VC-1 & 0 & 91 & light olive brown medium-grained sand \\
\hline 94VC-1(91-97) & 94VC-1 & 91 & 97 & olive gray silt \\
\hline 94VC-1(97-110) & 94VC-1 & 97 & 110 & olive gray muddy silt \\
\hline 94VC-1(110-130) & 94VC-1 & 110 & 130 & light olive gray fine-grained sand \\
\hline 94VC-1(130-135) & 94VC-1 & 130 & 135 & olive gray muddy silt \\
\hline 94VC-1(135-148) & 94VC-1 & 135 & 148 & light olive gray very fine-grained sand \\
\hline 94VC-1(148-159) & 94VC-1 & 148 & 159 & olive gray muddy silt \\
\hline 94VC-1(159-172) & 94VC-1 & 159 & 172 & light olive gray fine-grained sand (with woody debris) \\
\hline 94VC-1(172-176) & 94VC-1 & 172 & 176 & olive gray muddy silt \\
\hline 94VC-1(176-180) & 94VC-1 & 176 & 180 & light olive gray fine-grained sand (with woody debris) \\
\hline 94VC-1(180-191) & 94VC-1 & 180 & 191 & olive gray muddy silt \\
\hline 94VC-1(191-207) & 94VC-1 & 191 & 207 & light olive gray medium-fine-grained sand \\
\hline 94VC-2(0-15) & 94VC-2 & 0 & 15 & brown, medium-grained sand with black wood fragments \\
\hline 94VC-2(15-30) & 94VC-2 & 15 & 30 & inclined contact between brown medium-grained sand above and olive \\
\hline 94VC-2(30-70) & 94VC-2 & 30 & 70 & dark olive gray silty mud \\
\hline 94VC-2(70-80) & 94VC-2 & 70 & 80 & dark olive gray silty mud with $30 \%$ very fine sand \\
\hline 94VC-2(80-147) & 94VC-2 & 80 & 147 & dark olive gray silty mud with $30-60 \%$ fine sand \\
\hline 94VC-2(147-164) & 94VC-2 & 147 & 164 & dark olive gray medium-grained sand with black wood chips, sticks \\
\hline
\end{tabular}


Appendix C. Lithologic descriptions of soil and sediment samples for which chemical analyses are given in this report (Appendix_C.xls, Appendix_C.dbf)

\begin{tabular}{|c|c|c|c|c|}
\hline Sample No. & Site ID & $\begin{array}{c}\text { Depth } \\
\text { interval, } \\
\text { top }(\mathrm{cm})\end{array}$ & $\begin{array}{l}\text { Depth } \\
\text { interval, } \\
\text { bottom } \\
(\mathrm{cm})\end{array}$ & Lithologic description \\
\hline SAMPLE_NO. & SITE_ID & INT_TOP_CM & INT_BTM_CM & LITH_DESC \\
\hline 94VC-3(0-55) & 94VC-3 & 0 & 55 & light olive brown medium-fine-grained sand \\
\hline 94VC-3(55-120) & 94VC-3 & 55 & 120 & light olive gray fine-grained sand \\
\hline 94VC-3(120-130) & 94VC-3 & 120 & 130 & dark gray silt \\
\hline 94VC-3(130-140) & 94VC-3 & 130 & 140 & light olive gray medium-fine-grained sand \\
\hline 94VC-3(140-170) & 94VC-3 & 140 & 170 & light olive gray fine-grained sand \\
\hline 94VC-3(170-173) & 94VC-3 & 170 & 173 & dark gray silt \\
\hline 94VC-3(173-190) & 94VC-3 & 173 & 190 & olive gray very fine-grained sand \\
\hline 94VC-3(190-195) & 94VC-3 & 190 & 195 & light olive gray fine-grained sand \\
\hline 94VC-3(195-209) & 94VC-3 & 195 & 209 & olive gray very fine-grained sand \\
\hline 94VC-3(209-211) & 94VC-3 & 209 & 211 & olive gray very fine-grained sand with black woody debris \\
\hline 94VC-3(211-214) & 94VC-3 & 211 & 214 & olive gray very fine-grained sand \\
\hline 94VC-3(214-220) & 94VC-3 & 214 & 220 & olive gray very fine-grained sand with black woody debris \\
\hline 94VC-3(220-230) & 94VC-3 & 220 & 230 & olive gray fine- to very fine-grained sand \\
\hline 94VCD1(0-103) & 94VCD1 & 0 & 103 & light olive gray coarse-grained sand (wood fragments in upper $10 \mathrm{~cm}$ ) \\
\hline 94VCD1(103-155) & 94VCD1 & 103 & 155 & olive gray medium-grained sand \\
\hline 94VCD1(155-171) & 94VCD1 & 155 & 171 & dark gray muddy silt \\
\hline 94VCD1(171-229) & 94VCD1 & 171 & 229 & olive gray medium-grained sand (abun wood chips $=190-200,210-216,225$ \\
\hline 94VCD1(229-235) & 94VCD1 & 229 & 235 & dark gray very fine-grained sand \\
\hline 94VCD1(235-260) & 94VCD1 & 235 & 260 & dark olive gray silt (black leaf debris @ $257 \mathrm{~cm}$ ) \\
\hline 94VCD1(260-282) & 94VCD1 & 260 & 282 & olive gray medium-fine-grained sand \\
\hline 94VCD2(0-40) & 94VCD2 & 0 & 40 & olive gray medium-fine-grained sand \\
\hline 94VCD2(40-100) & 94VCD2 & 40 & 100 & light gray muddy silt \\
\hline 94VCD2(100-188) & 94VCD2 & 100 & 188 & light gray medium-grained sand \\
\hline 94VCD2(188-192) & 94VCD2 & 188 & 192 & light gray muddy silt \\
\hline 94VCD2(192-260) & 94VCD2 & 192 & 260 & light gray medium-grained sand \\
\hline 94VCD2(260-295) & 94VCD2 & 260 & 295 & light gray medium-fine-grained sand \\
\hline 94VCD2(295-303) & 94VCD2 & 295 & 303 & very light gray muddy silt \\
\hline 94VCD2(303-325) & 94VCD2 & 303 & 325 & olive gray fine-grained sand \\
\hline 94VCD3(0-24) & 94VCD3 & 0 & 24 & olive gray fine-grained sand (black leaf layers @ 15 \& $22 \mathrm{~cm}$ ) \\
\hline 94VCD3(24-38) & 94VCD3 & 24 & 38 & alternating $2 \mathrm{~cm}$ lavers of fine-grained sand and silt \\
\hline 94VCD3(38-42) & 94VCD3 & 38 & 42 & gray silt \\
\hline 94VCD3(42-56) & 94VCD3 & 42 & 56 & olive gray fine-grained sand \\
\hline 94VCD3(56-73) & 94VCD3 & 56 & 73 & olive gray medium-grained sand (woody layer @ $73 \mathrm{~cm}$ ) \\
\hline 94VCD3(73-81) & 94VCD3 & 73 & 81 & olive gray fine-grained sand \\
\hline 94VCD3(81-91) & 94VCD3 & 81 & 91 & alternating $2 \mathrm{~cm}$ layers of fine-grained sand and silt \\
\hline
\end{tabular}


Appendix C. Lithologic descriptions of soil and sediment samples for which chemical analyses are given in this report (Appendix_C.xls, Appendix_C.dbf)

\begin{tabular}{|c|c|c|c|c|}
\hline Sample No. & Site ID & $\begin{array}{c}\text { Depth } \\
\text { interval, } \\
\text { top }(\mathrm{cm})\end{array}$ & $\begin{array}{l}\text { Depth } \\
\text { interval, } \\
\text { bottom } \\
(\mathrm{cm})\end{array}$ & Lithologic description \\
\hline SAMPLE_NO. & SITE_ID & INT_TOP_CM & INT_BTM_CM & LITH_DESC \\
\hline 94VCD3(91-100) & 94VCD3 & 91 & 100 & medium-fine-grained sand \\
\hline 94VCD3(100-125) & 94VCD3 & 100 & 125 & alternating $2 \mathrm{~cm}$ layers of fine-grained sand and silt \\
\hline 94VCD3(125-140) & 94VCD3 & 125 & 140 & medium-grained sand \\
\hline 94VCD3(140-175) & 94VCD3 & 140 & 175 & alternating $2 \mathrm{~cm}$ layers of fine-grained sand and silt (black leaf horizon @ \\
\hline 94VCK1(0-133) & 94VCK1 & 0 & 133 & light olive medium-grained sand \\
\hline 94VCK1(133-180) & 94VCK1 & 133 & 180 & light olive medium-fine-grained sand \\
\hline 94VCK1(180-325) & 94VCK1 & 180 & 325 & gravish-olive fine-grained sand \\
\hline 94VCK1(325-348) & 94VCK1 & 325 & 348 & olive gray clayey silt \\
\hline 94VCK1(348-350) & 94VCK1 & 348 & 350 & plant debris rich layer \\
\hline 94VCK1(350-360) & 94VCK1 & 350 & 360 & olive gray clayey silt \\
\hline 94VCK1(360-367) & 94VCK1 & 360 & 367 & gravish-olive fine-grained sand \\
\hline 94VCK1(367-380) & 94VCK1 & 367 & 380 & olive gray clayey silt \\
\hline 94VCK2(0-90) & 94VCK2 & 0 & 90 & light olive gray medium-grained sand \\
\hline 94VCK2(90-240) & 94VCK2 & 90 & 240 & Medium olive gray medium-grained sand \\
\hline 94VCK2(240-267) & 94VCK2 & 240 & 267 & medium olive gray medium-fine-grained sand \\
\hline 94VCK2(267-300) & 94VCK2 & 267 & 300 & medium olive gray fine-grained sand with $\sim 10 \%$ mud \\
\hline 94VCK2(300-325) & 94VCK2 & 300 & 325 & olive gray fine- and very fine-grained sand with $\sim 10 \%$ mud \\
\hline 94VCK2(325-333) & 94VCK2 & 325 & 333 & gray silt with black wood chips \\
\hline 94VCK2(333-340) & 94VCK2 & 333 & 340 & olive gray fine- and very fine-grained sand with $\sim 10 \%$ mud \\
\hline 95PCK1(0-15) & 95PCK1 & 0 & 15 & tan medium-grained sand \\
\hline 95PCK1(168-193) & 95PCK1 & 168 & 193 & olive gray, fine-grained sand \\
\hline 95PCK1(193-204) & 95PCK1 & 193 & 204 & dark gray, very fine-grained sand \\
\hline 95PCK1(204-227) & 95PCK1 & 204 & 227 & light gray very fine-grained sand, laminated \\
\hline 95PCK1(227-229) & 95PCK1 & 227 & 229 & dark gray organic silt \\
\hline 95PCK1(229-241) & 95PCK1 & 229 & 241 & finely laminated, light gray very fine-grained sand \\
\hline 95PCK1(241-249) & 95PCK1 & 241 & 249 & alternating fine- and very fine-grained sand \\
\hline 95PCK1(249-250) & 95PCK1 & 249 & 250 & silty mud \\
\hline 95PCK1(250-260) & 95PCK1 & 250 & 260 & alternating fine- and very fine-grained sand \\
\hline 95PCK1(260-262) & 95PCK1 & 260 & 262 & silty mud \\
\hline 95PCK1(262-297) & 95PCK1 & 262 & 297 & fine-grained sand with occasional silty mud rip-up clasts \\
\hline 95PCK1(297-326) & 95PCK1 & 297 & 326 & interlayered silt/silty mud \\
\hline 95PCK1(326-343) & 95PCK1 & 326 & 343 & fine-medium-grained sand with interlayered $1 \mathrm{~cm}$ silt beds \\
\hline 95PCK1(343-351) & 95PCK1 & 343 & 351 & medium-fine-grained sand \\
\hline 95PCK1(351-360) & 95PCK1 & 351 & 360 & peaty silty mud with sticks, roots; $1 \mathrm{~cm}$ fine-grained sand layers at 353 , \\
\hline 95PCK1(360-363) & 95PCK1 & 360 & 363 & medium-grained sand \\
\hline
\end{tabular}


Appendix C. Lithologic descriptions of soil and sediment samples for which chemical analyses are given in this report (Appendix_C.xls, Appendix_C.dbf)

\begin{tabular}{|c|c|c|c|c|}
\hline Sample No. & Site ID & $\begin{array}{c}\text { Depth } \\
\text { interval, } \\
\text { top }(\mathrm{cm})\end{array}$ & $\begin{array}{l}\text { Depth } \\
\text { interval, } \\
\text { bottom } \\
(\mathrm{cm})\end{array}$ & Lithologic description \\
\hline SAMPLE_NO. & SITE_ID & INT_TOP_CM & INT_BTM_CM & LITH_DESC \\
\hline 95PCK1(363-366) & 95PCK1 & 363 & 366 & peaty silty mud \\
\hline 95PCK1(366-369) & 95PCK1 & 366 & 369 & medium-grained sand \\
\hline 95PCK1(369-375) & 95PCK1 & 369 & 375 & fine-grained sand \\
\hline 95PCK1(375-381) & 95PCK1 & 375 & 381 & medium-grained sand \\
\hline 95PCK1(381-393) & 95PCK1 & 381 & 393 & alternating organic-debris-rich muddy silt and fine-grained sand \\
\hline 95PCK1(393-400) & 95PCK1 & 393 & 400 & medium-fine sand \\
\hline 95PCK1(400-407) & 95PCK1 & 400 & 407 & medium sand \\
\hline 95PCK1(407-411) & 95PCK1 & 407 & 411 & peaty silty mud \\
\hline 95PCK1(411-415) & 95PCK1 & 411 & 415 & med sand \\
\hline 95PCK1(415-418) & 95PCK1 & 415 & 418 & peaty silty mud \\
\hline 95PCUD2(0-25) & 95PCUD2 & 0 & 25 & muddy silt \\
\hline 95PCUD2(25-50) & 95PCUD2 & 25 & 50 & muddy silt \\
\hline 95PCUD2(50-75) & 95PCUD2 & 50 & 75 & muddy silt \\
\hline 95PCUD2(75-100) & 95PCUD2 & 75 & 100 & muddy silt \\
\hline 95PCUD2(100-125) & 95PCUD2 & 100 & 125 & muddy silt \\
\hline 95PCUD2(125-150) & 95PCUD2 & 125 & 150 & muddy silt \\
\hline 95PCUD2(150-165) & 95PCUD2 & 150 & 165 & sandy silt \\
\hline 95VCD3(0-28) & 95VCD3 & 0 & 28 & fine-grained sand \\
\hline 95VCD3(28-56) & 95VCD3 & 28 & 56 & olive gray medium-grained sand \\
\hline 95VCD3(56-84) & 95VCD3 & 56 & 84 & olive gray medium-grained sand \\
\hline 95VCD3(84-177) & 95VCD3 & 84 & 177 & olive fine-grained sand \\
\hline 95VCD3(177-195.5) & 95VCD3 & 177 & 195.5 & olive gray silt \\
\hline 95VCD3(195.5-214) & 95VCD3 & 195.5 & 214 & olive gray fine-grained sand \\
\hline 95VCD3(214-230) & 95VCD3 & 214 & 230 & olive black fine-grained sand \\
\hline 95VCD3(230-262) & 95VCD3 & 230 & 262 & olive black medium-grained sand \\
\hline 95VCD3(262-277) & 95VCD3 & 262 & 277 & olive gray medium-coarse-grained sand \\
\hline 95VCD3(277-298) & 95VCD3 & 277 & 298 & olive black fine-grained sand \\
\hline 95VCD3(298-340) & 95VCD3 & 298 & 340 & olive gray very fine-grained sand \\
\hline 95VCD3(340-402) & 95VCD3 & 340 & 402 & olive black very fine-grained sand \\
\hline 95VCD3(402-423) & 95VCD3 & 402 & 423 & olive-brown silt \\
\hline 95VCD3(423-547) & 95VCD3 & 423 & 547 & olive gray muddy silt with fibrous wood fragments \\
\hline 95VCD3(547-572) & 95VCD3 & 547 & 572 & olive gray silt \\
\hline 95VCUD1(0-25) & 95VCUD1 & 0 & 25 & coarse-grained sand \\
\hline 95VCUD1(25-50) & 95VCUD1 & 25 & 50 & coarse-grained sand with wood fragments \\
\hline 95VCUD1(50-75) & 95VCUD1 & 50 & 75 & coarse-grained sand with wood fragments \\
\hline
\end{tabular}


Appendix C. Lithologic descriptions of soil and sediment samples for which chemical analyses are given in this report (Appendix_C.xls, Appendix_C.dbf)

\begin{tabular}{|c|c|c|c|c|}
\hline Sample No. & Site ID & $\begin{array}{c}\text { Depth } \\
\text { interval, } \\
\text { top }(\mathrm{cm})\end{array}$ & $\begin{array}{l}\text { Depth } \\
\text { interval, } \\
\text { bottom } \\
(\mathrm{cm})\end{array}$ & Lithologic description \\
\hline SAMPLE_NO. & SITE_ID & INT_TOP_CM & INT_BTM_CM & LITH_DESC \\
\hline 95VCUD1(75-100) & 95VCUD1 & 75 & 100 & medium-coarse-grained sand grading down to fine-grained sand \\
\hline 95VCUD1(100-125) & 95VCUD1 & 100 & 125 & [Data missing] \\
\hline 95VCUD1(125-150) & 95VCUD1 & 125 & 150 & [Data missing] \\
\hline 95VCUD1(150-175) & 95VCUD1 & 150 & 175 & [Data missing] \\
\hline 95VCUD1(175-200) & 95VCUD1 & 175 & 200 & [Data missing] \\
\hline 95VCUD1(200-224) & 95VCUD1 & 200 & 224 & [Data missing] \\
\hline 95VCUD1(224-245) & 95VCUD1 & 224 & 245 & [Data missing] \\
\hline 95VCUD1(245-266) & 95VCUD1 & 245 & 266 & [Data missing] \\
\hline 95VCUD1(266-286) & 95VCUD1 & 266 & 286 & [Data missing] \\
\hline 95VCUD1(286-306) & 95VCUD1 & 286 & 306 & [Data missing] \\
\hline 95VCUD1(311-328) & 95VCUD1 & 311 & 328 & [Data missing] \\
\hline 95VCUD1(328-344) & 95VCUD1 & 328 & 344 & [Data missing] \\
\hline 95VCUD1(344-360) & 95VCUD1 & 344 & 360 & [Data missing] \\
\hline 95VCUD1(360-377) & 95VCUD1 & 360 & 377 & [Data missing] \\
\hline 95VCUD1(377-393) & 95VCUD1 & 377 & 393 & [Data missing] \\
\hline 95VCUD1(393-409) & 95VCUD1 & 393 & 409 & [Data missing] \\
\hline 95VCUD1(409-435) & 95VCUD1 & 409 & 435 & fine sand and silt \\
\hline 95VCUD1(435-461) & 95VCUD1 & 435 & 461 & fine sand and silt \\
\hline 95VCUD1(461-486) & 95VCUD1 & 461 & 486 & fine sand and silt \\
\hline 95VCUD1(486-512) & 95VCUD1 & 486 & 512 & [Data missing] \\
\hline 95VCUD1(512-538) & 95VCUD1 & 512 & 538 & [Data missing] \\
\hline 95VCUD1(538-564) & 95VCUD1 & 538 & 564 & [Data missing] \\
\hline 95VCUD1(564-589) & 95VCUD1 & 564 & 589 & medium-fine sand \\
\hline $96 \mathrm{~K}-75 \mathrm{E}(0-10)$ & $96 \mathrm{~K}-75 \mathrm{E}$ & 0 & 10 & gray clayey silt \\
\hline $96 \mathrm{~K}-89 \mathrm{E}(0-23)$ & $96 \mathrm{~K}-89 \mathrm{E}$ & 0 & 23 & tan medium-fine grained sand, 4 layers, oranger colored near base; $1 \mathrm{~cm}$ \\
\hline $96 \mathrm{~K}-89 \mathrm{E}(23-49)$ & 96K-89E & 23 & 49 & $\begin{array}{l}\text { alternating } 2-4 \mathrm{~cm} \text { layers of very fine sand, fine sand, and red-brown silt } \\
(\text { duff laver } @ 42 \mathrm{~cm})\end{array}$ \\
\hline $96 \mathrm{~K}-89 \mathrm{E}(49-85)$ & 96K-89E & 49 & 85 & $\begin{array}{l}\text { mostly red-brown silt to very fine sand with }(3) 3 \mathrm{~cm} \text { fine-grained, rippled } \\
\text { sand beds (duff @ } 52 \mathrm{~cm} \text { ): irreaular arav lenses in middle part }\end{array}$ \\
\hline 96K-89E(85-93) & 96K-89E & 85 & 93 & medium-grained sand with ripple laminations \\
\hline $96 \mathrm{~K}-89 \mathrm{E}(93-110)$ & 96K-89E & 93 & 110 & alternating layers of red-brown silt and very fine sand, heavily cemented by \\
\hline $96 \mathrm{~K}-89 \mathrm{E}(110-132)$ & $96 \mathrm{~K}-89 \mathrm{E}$ & 110 & 132 & dark gray muddy silt with (2) $1 \mathrm{~cm}$ red-brown horizons @ 116 and $124 \mathrm{~cm}$ \\
\hline $96 \mathrm{~K}-89 \mathrm{E}(132-162)$ & $96 \mathrm{~K}-89 \mathrm{E}$ & 132 & 162 & light gray muddy silt with disseminated $\mathrm{cm}$-sized orange spots \\
\hline $96 \mathrm{~K}-114 \mathrm{E}(0-15)$ & 96K-114E & 0 & 15 & $\tan$ medium-fine grained sand \\
\hline $96 \mathrm{~K}-114 \mathrm{E}(15-33)$ & 96K-114E & 15 & 33 & medium brown fine sand $(1 \mathrm{~cm}$ MSH ash @ $33 \mathrm{~cm})$ \\
\hline
\end{tabular}


Appendix C. Lithologic descriptions of soil and sediment samples for which chemical analyses are given in this report (Appendix_C.xls, Appendix_C.dbf)

\begin{tabular}{|c|c|c|c|c|}
\hline Sample No. & Site ID & $\begin{array}{c}\text { Depth } \\
\text { interval, } \\
\text { top }(\mathrm{cm})\end{array}$ & $\begin{array}{c}\text { Depth } \\
\text { interval, } \\
\text { bottom } \\
(\mathrm{cm})\end{array}$ & Lithologic description \\
\hline SAMPLE_NO. & SITE_ID & INT_TOP_CM & INT_BTM_CM & LITH_DESC \\
\hline 96K-114E(33-43) & 96K-114E & 33 & 43 & dark brown-black medium fine sand \\
\hline $96 \mathrm{~K}-114 \mathrm{E}(43-61)$ & $96 \mathrm{~K}-114 \mathrm{E}$ & 43 & 61 & interlayered brown fine sand and orange silt layers \\
\hline $96 \mathrm{~K}-114 \mathrm{E}(61-80)$ & $96 \mathrm{~K}-114 \mathrm{E}$ & 61 & 80 & mostly silt with thin fine sand layers \\
\hline $96 \mathrm{~K}-114 \mathrm{E}(80-104)$ & $96 \mathrm{~K}-114 \mathrm{E}$ & 80 & 104 & dark brown-black medium fine sand with black streaks \\
\hline 96K-114E(104-130) & $96 \mathrm{~K}-114 \mathrm{E}$ & 104 & 130 & medium brown fine sand \\
\hline 96K-114E(130-162) & $96 \mathrm{~K}-114 \mathrm{E}$ & 130 & 162 & light gray fine sand \\
\hline 96K-114E(162-175) & $96 \mathrm{~K}-114 \mathrm{E}$ & 162 & 175 & dark gray muddy silt \\
\hline $96 \mathrm{~K}-178 \mathrm{E}(0-9)$ & $96 \mathrm{~K}-178 \mathrm{E}$ & 0 & 9 & tan fine sand with minimal roots \\
\hline $96 \mathrm{~K}-178 \mathrm{E}(9-13)$ & $96 \mathrm{~K}-178 \mathrm{E}$ & 9 & 13 & tan fine sand with abundant roots and black plant matter seam on top \\
\hline $96 \mathrm{~K}-178 \mathrm{E}(13-18)$ & $96 \mathrm{~K}-178 \mathrm{E}$ & 13 & 18 & orange-brown very fine sand, laminated \\
\hline $96 \mathrm{~K}-178 \mathrm{E}(18-19)$ & $96 \mathrm{~K}-178 \mathrm{E}$ & 18 & 19 & gray-white ash, $1980 \mathrm{Mt}$ St. Helens \\
\hline $96 \mathrm{~K}-178 \mathrm{E}(19-35)$ & $96 \mathrm{~K}-178 \mathrm{E}$ & 19 & 35 & orange-brown mottled silt \& very fine sand, laminated \\
\hline 96K-178E(35-70) & $96 \mathrm{~K}-178 \mathrm{E}$ & 35 & 70 & orange-brown silt and very fine sand, bioturbated \\
\hline $96 \mathrm{~K}-178 \mathrm{E}(70-84)$ & $96 \mathrm{~K}-178 \mathrm{E}$ & 70 & 84 & orange-brown silt and very fine sand, finely layered \\
\hline 96K-178E(84-102) & $96 \mathrm{~K}-178 \mathrm{E}$ & 84 & 102 & dark gray very fine sand with rare red streaks \\
\hline $96 \mathrm{~K}-178 \mathrm{E}(102-140)$ & $96 \mathrm{~K}-178 \mathrm{E}$ & 102 & 140 & dark gray silt to very fine sand, layered \\
\hline 96LD-1S(0-16) & 96LD-1S & 0 & 16 & 8 medium sand-silt couplets, light brown above $10 \mathrm{~cm}$, medium brown \\
\hline 96LD-1S(16-43) & 96LD-1S & 16 & 43 & alternating silt and very-fine sand, with several thin medium sand layers \\
\hline 96LD-1S(43-70) & 96LD-1S & 43 & 70 & orange-brown fine and very fine sand lavers, with moderate iron-oxide \\
\hline 96LD-1S(70-93) & 96LD-1S & 70 & 93 & $\begin{array}{l}\text { Orange brown silt with occasional very fine sand layers, stronger iron-oxide } \\
\text { cementation than above }\end{array}$ \\
\hline 96LD-1S(105-120) & 96LD-1S & 105 & 120 & very fine sand, silt, clay and woody debris; med gray with $25 \%$ orange- \\
\hline 96LD-105S(0-5) & 96LD-105S & 0 & 5 & $3 \mathrm{~cm}$ forest duff overlain by $2 \mathrm{~cm}$ of tan, medium-grained sand \\
\hline 96LD-105S(5-18) & 96LD-105S & 5 & 18 & red-brown medium-fine grained sand capped by $0.5 \mathrm{~cm} \mathrm{Mt} \mathrm{St} \mathrm{Helens} \mathrm{ash}$ \\
\hline 96LD-105S(18-34) & 96LD-105S & 18 & 34 & dark red-brown-black medium-fine sand with $1 \mathrm{~cm}$ orange silt at base \\
\hline 96LD-105S(34-49) & 96LD-105S & 34 & 49 & $\begin{array}{l}\text { gray homogenous fine sand with } 5 \mathrm{~cm} \text { charcoal layer at top, scattered } \\
\text { charcoal fraaments below }\end{array}$ \\
\hline T98C-1(0-3) & T98C-1 & 0 & 3 & silt, dark brown, with organic debris \\
\hline T98C-1(3-4) & T98C-1 & 3 & 4 & 1980 Mt. St. Helens ash, very fine-grained, very pale gray \\
\hline T98C-1(4-15) & T98C-1 & 4 & 15 & silt, dark brown, with sparse fragments of gray clay (plowed?) \\
\hline T98C-1(15-30) & T98C-1 & 15 & 30 & silt, rusty colored, with abundant fragments of gray clay (plowed?) \\
\hline T98C-1(30-50) & T98C-1 & 30 & 50 & silt, rusty colored \\
\hline T98C-1(50-65) & T98C-1 & 50 & 65 & clay, gray \\
\hline T98C-5(0-5) & T98C-5 & 0 & 5 & soil, blackish brown, with organic debris \\
\hline T98C-5(5-20) & T98C-5 & 5 & 20 & silt, red-brown (hematitic, goethitic) \\
\hline
\end{tabular}


Appendix C. Lithologic descriptions of soil and sediment samples for which chemical analyses are given in this report (Appendix_C.xls, Appendix_C.dbf)

\begin{tabular}{|c|c|c|c|c|}
\hline Sample No. & Site ID & $\begin{array}{c}\text { Depth } \\
\text { interval, } \\
\text { top }(\mathrm{cm})\end{array}$ & $\begin{array}{c}\text { Depth } \\
\text { interval, } \\
\text { bottom } \\
(\mathrm{cm})\end{array}$ & Lithologic description \\
\hline SAMPLE_NO. & SITE_ID & INT_TOP_CM & INT_BTM_CM & LITH_DESC \\
\hline T98C-5(20-24) & T98C-5 & 20 & 24 & sand, coarse-grained, red-brown \\
\hline T98C-5(24-34) & T98C-5 & 24 & 34 & clay, red-brown \\
\hline T98C-5(34-36) & T98C-5 & 34 & 36 & sand, coarse-grained, red-brown \\
\hline T98C-5(36-44) & T98C-5 & 36 & 44 & clay, gray \\
\hline T98C-5(44-60) & T98C-5 & 44 & 60 & clay, gray, with fragments of red-brown clay (plowed?) \\
\hline T98C-5(60-110) & T98C-5 & 60 & 110 & clay, gray (water saturated), with fragments of red-brown clay \\
\hline T98C-5(110-200) & T98C-5 & 110 & 200 & clay, dark gray to black, organic-rich, water saturated \\
\hline T98C-6(0-3) & T98C-6 & 0 & 3 & soil, black-brown \\
\hline T98C-6(3-3.2) & T98C-6 & 3 & 3.2 & 1980 Mt. St. Helens ash, very fine-grained, very pale gray \\
\hline T98C-6(3.2-11) & T98C-6 & 3.2 & 11 & sand and silt, very fine-grained, red-brown \\
\hline T98C-6(11-25) & T98C-6 & 11 & 25 & sandy silt, red-brown \\
\hline T98C-6(25-80) & T98C-6 & 25 & 80 & silt, red-brown \\
\hline T98C-6(80-120) & T98C-6 & 80 & 120 & sand and silt, very fine-grained, red-brown \\
\hline T98C-6(120-200) & T98C-6 & 120 & 200 & sand, very fine-grained, red-brown \\
\hline T98C-7(0-5) & T98C-7 & 0 & 5 & silt, dark brown, organic-bearing \\
\hline T98C-7(5-5.2) & T98C-7 & 5 & 5.2 & 1980 Mt. St. Helens ash, very fine-grained, very pale gray \\
\hline T98C-7(5.2-25) & T98C-7 & 5.2 & 25 & silt, orange-brown \\
\hline T98C-7(25-35) & T98C-7 & 25 & 35 & sand, brown-gray \\
\hline T98C-8(0-3) & T98C-8 & 0 & 3 & silt, dark brown, organic-bearing \\
\hline T98C-8(3-3.2) & T98C-8 & 3 & 3.2 & 1980 Mt. St. Helens ash, very fine-grained, very pale gray \\
\hline T98C-8(3.2-23) & T98C-8 & 3.2 & 23 & silt, red brown \\
\hline T98C-8(23-38) & T98C-8 & 23 & 38 & sand, medium-grained, gray \\
\hline T98C-10(0-23) & T98C-10 & 0 & 23 & silt, dark brown (at edge of river bed) \\
\hline T98C-10(23-23.6) & T98C-10 & 23 & 23.6 & 1980 Mt. St. Helens ash, very fine-grained, very pale gray; thick, inclined \\
\hline T98C-10(23.6-40) & T98C-10 & 23.6 & 40 & silt, very fine-grained, red-brown, grading downward to clay \\
\hline T98C-10(40-45) & T98C-10 & 40 & 45 & gravel \\
\hline T98C-11B(0-25) & T98C-11B & 0 & 25 & silt, gray, with fragments of black peat \\
\hline T98C-11B(25-68) & T98C-11B & 25 & 68 & silt, gray, with fragments of black peat \\
\hline T98C-11B(68-111) & T98C-11B & 68 & 111 & sand, very fine-grained, dark gray \\
\hline T98C-12(0-5) & T98C-12 & 0 & 5 & soil, light brown to gray, organic-bearing \\
\hline T98C-12(5-5.2) & T98C-12 & 5 & 5.2 & 1980 Mt. St. Helens ash, very fine-grained, very pale gray \\
\hline T98C-12(5.2-30) & T98C-12 & 5.2 & 30 & silt, red-brown \\
\hline T98C-12(30-79) & T98C-12 & 30 & 79 & silt, red-brown \\
\hline T98R-13(0-4) & T98R-13 & 0 & 4 & soil, dark gray to black, organic-rich \\
\hline T98R-13(4-8) & T98R-13 & 4 & 8 & soil containing dispersed 1980 volcanic ash \\
\hline
\end{tabular}


Appendix C. Lithologic descriptions of soil and sediment samples for which chemical analyses are given in this report (Appendix_C.xls, Appendix_C.dbf)

\begin{tabular}{|c|c|c|c|c|}
\hline Sample No. & Site ID & $\begin{array}{c}\text { Depth } \\
\text { interval, } \\
\text { top }(\mathrm{cm})\end{array}$ & $\begin{array}{c}\text { Depth } \\
\text { interval, } \\
\text { bottom } \\
(\mathrm{cm})\end{array}$ & Lithologic description \\
\hline SAMPLE_NO. & SITE_ID & INT_TOP_CM & INT_BTM_CM & LITH_DESC \\
\hline T98R-13(8-35) & T98R-13 & 8 & 35 & silt, red-brown (hematitic), with thin clay interbeds \\
\hline T98R-13(35-60) & T98R-13 & 35 & 60 & clay, gray, with sandy to silty interbeds throughout \\
\hline T98R-14(0-3) & T98R-14 & 0 & 3 & silt, dark brown, organic-bearing \\
\hline T98R-14(3-3.5) & T98R-14 & 3 & 3.5 & 1980 Mt. St. Helens ash, very fine-grained, very pale grav \\
\hline T98R-14(3.5-35) & T98R-14 & 3.5 & 35 & silt, red-brown, rusty, with fragments of gray silt \\
\hline T98R-14(35-50) & T98R-14 & 35 & 50 & sand, yellowish brown \\
\hline T98R-14(50-60) & T98R-14 & 50 & 60 & sand, gray \\
\hline T98R-14(60-90) & T98R-14 & 60 & 90 & sand, gray \\
\hline T98R-14(90-120) & T98R-14 & 90 & 120 & sand, gray, water saturated \\
\hline T98C-15(0-3) & T98C-15 & 0 & 3 & silt, dark brown, organic-bearing \\
\hline T98C-15(3-3.2) & T98C-15 & 3 & 3.2 & 1980 Mt. St. Helens ash, very fine-grained, very pale gray \\
\hline$\overline{T 98 C-15(3.2-30)}$ & T98C-15 & 3.2 & 30 & silt, dark brown and black, grading down to red-brown \\
\hline T98C-15(30-46) & T98C-15 & 30 & 46 & silt, layers of red, brown, gray, and red-brown \\
\hline T98C-15(46-80) & T98C-15 & 46 & 80 & clay, gray, with red-brown fragments (plowed?) \\
\hline T98C-15(80-120) & T98C-15 & 80 & 120 & clay, gray \\
\hline T98C-16(0-9.9) & T98C-16 & 0 & 9.9 & silty mud, dark brown, organic-rich \\
\hline T98C-16(9.9-10.1) & T98C-16 & 9.9 & 10.1 & 1980 Mt. St. Helens ash, very fine-grained, very pale gray \\
\hline T98C-16(10.1-34) & T98C-16 & 10.1 & 34 & silt, fine-grained, red-brown with gray interbeds \\
\hline T98C-16(34-50) & T98C-16 & 34 & 50 & silt, fine-grained, dark red-brown, with interbeds of coarse, black silt \\
\hline T98C-16(50-62) & T98C-16 & 50 & 62 & sand, fine-grained, red-brown, with black interbeds \\
\hline T98C-16(62-133) & T98C-16 & 62 & 133 & silt, light brown and red-brown \\
\hline T98C-16(133-160) & T98C-16 & 133 & 160 & sand, very fine grained, dark gray \\
\hline T98C-16(160-182) & T98C-16 & 160 & 182 & sand, fine-grained, brownish gray \\
\hline T98C-16(182-210) & T98C-16 & 182 & 210 & sand, medium-grained, light brown \\
\hline T98C-17(0-8) & T98C-17 & 0 & 8 & silt to clay, red-brown, with roots and black organic fragments \\
\hline T98C-17(8-9) & T98C-17 & 8 & 9 & 1980 Mt. St. Helens ash, very fine-grained, very pale gray; thick layer (1 \\
\hline T98C-17(9-20) & T98C-17 & 9 & 20 & silt grades downward to medium-grained sand, red brown \\
\hline T98C-17(20-92) & T98C-17 & 20 & 92 & silt, red-brown \\
\hline T98C-17(92-155) & T98C-17 & 92 & 155 & silt, brown-red, grading downward to \\
\hline T98C-17(155-173) & T98C-17 & 155 & 173 & silt, red-brown \\
\hline T98C-17(173-194) & T98C-17 & 173 & 194 & silt, grading downward to clay, reddish gray \\
\hline T98C-17(194-202) & T98C-17 & 194 & 202 & clay, light brownish gray \\
\hline T98C-18(0-3) & T98C-18 & 0 & 3 & soil, brown, with mossy organic material \\
\hline T98C-18(3-3.5) & T98C-18 & 3 & 3.5 & 1980 Mt. St. Helens ash, very fine-grained, very pale gray \\
\hline T98C-18(3.5-8) & T98C-18 & 3.5 & 8 & silt, light brown \\
\hline
\end{tabular}


Appendix C. Lithologic descriptions of soil and sediment samples for which chemical analyses are given in this report (Appendix_C.xls, Appendix_C.dbf)

\begin{tabular}{|c|c|c|c|c|}
\hline Sample No. & Site ID & $\begin{array}{c}\text { Depth } \\
\text { interval, } \\
\text { top }(\mathrm{cm})\end{array}$ & $\begin{array}{l}\text { Depth } \\
\text { interval, } \\
\text { bottom } \\
(\mathrm{cm})\end{array}$ & Lithologic description \\
\hline SAMPLE_NO. & SITE_ID & INT_TOP_CM & INT_BTM_CM & LITH_DESC \\
\hline T98C-18(8-10) & T98C-18 & 8 & 10 & silt, red brown \\
\hline T98C-18(10-17) & T98C-18 & 10 & 17 & sand, fine-grained, dark brown \\
\hline T98C-18(17-31) & T98C-18 & 17 & 31 & silt, red brown \\
\hline T98C-18(31-55) & T98C-18 & 31 & 55 & silt, gray, with fragments of red-brown silt (plowed?) \\
\hline T98C-18(55-130) & T98C-18 & 55 & 130 & silt, dark brown \\
\hline T98C-18(130-175) & T98C-18 & 130 & 175 & silt, dark brown, with about 25 percent of clay \\
\hline T98C-18(175-192) & T98C-18 & 175 & 192 & silt and clay, red-brown \\
\hline T98C-18(192-200) & T98C-18 & 192 & 200 & clay, reddish brown \\
\hline T98C-20(0-2.5) & T98C-20 & 0 & 2.5 & silty mud, dark brown, organic-bearing \\
\hline T98C-20(2.5-32) & T98C-20 & 2.5 & 32 & silt, red-brown \\
\hline T98C-20(32-41) & T98C-20 & 32 & 41 & silt, organic-rich, black \\
\hline$\overline{T 98 C-20(41-46)}$ & T98C-20 & 41 & 46 & clay, gray \\
\hline T98C-21(0-14) & T98C-21 & 0 & 14 & silt, light brown, organic-bearing \\
\hline T98C-21(14-15) & T98C-21 & 14 & 15 & 1980 Mt. St. Helens ash, very fine-grained, very pale gray \\
\hline T98C-21(15-30) & T98C-21 & 15 & 30 & silt, light brown, with fragments of gray clay and plant roots (plowed?) \\
\hline T98C-21(30-45) & T98C-21 & 30 & 45 & silt, red-brown, laminated \\
\hline T98C-21(45-60) & T98C-21 & 45 & 60 & sand, dark gray \\
\hline T98C-21B(60-125) & T98C-21B & 60 & 125 & sand, medium-grained, gray \\
\hline T98C-21B(125-200) & T98C-21B & 125 & 200 & sand, gray \\
\hline T98C-22(0-30) & T98C-22 & 0 & 30 & clay, dark brown, organic-rich, with fragments of red-brown clay (plowed?) \\
\hline T98C-22(30-47) & T98C-22 & 30 & 47 & clay, dark brown, with fragments of red-brown clay (plowed?) \\
\hline T98C-22(47-80) & T98C-22 & 47 & 80 & clay, gray \\
\hline T98C-23(0-13) & T98C-23 & 0 & 13 & silt, brown-black, organic-rich (smells like a sewer) \\
\hline T98C-23(13-24) & T98C-23 & 13 & 24 & clay, broken fragments of red and gray clay (plowed?) \\
\hline T98C-23(24-40) & T98C-23 & 24 & 40 & silt, dark gray \\
\hline T98C-23(40-50) & T98C-23 & 40 & 50 & clay \\
\hline T98R-24(0-5) & T98R-24 & 0 & 5 & mud, black, organic-rich \\
\hline T98R-24(5-50) & T98R-24 & 5 & 50 & silt, red-brown, with lenses of red-gray sand, and gray clay (plowed?) \\
\hline T98R-24(50-60) & T98R-24 & 50 & 60 & clay, gray \\
\hline T98R-24(60-75) & T98R-24 & 60 & 75 & sand, coarse, gray to red-brown \\
\hline T98R-24(75-95) & T98R-24 & 75 & 95 & clay, gray \\
\hline T98C-25(0-5) & T98C-25 & 0 & 5 & silt, light brown, organic-bearing \\
\hline T98C-25(5-6) & T98C-25 & 5 & 6 & 1980 Mt. St. Helens ash, very fine-grained, very pale gray \\
\hline T98C-25(5-15) & T98C-25 & 5 & 15 & silt, red-brown \\
\hline T98C-25(15-21) & T98C-25 & 15 & 21 & silt, organic-bearing, brown-black \\
\hline
\end{tabular}


Appendix C. Lithologic descriptions of soil and sediment samples for which chemical analyses are given in this report (Appendix_C.xls, Appendix_C.dbf)

\begin{tabular}{|c|c|c|c|c|}
\hline Sample No. & Site ID & $\begin{array}{c}\text { Depth } \\
\text { interval, } \\
\text { top }(\mathrm{cm})\end{array}$ & $\begin{array}{l}\text { Depth } \\
\text { interval, } \\
\text { bottom } \\
(\mathrm{cm})\end{array}$ & Lithologic description \\
\hline SAMPLE_NO. & SITE_ID & INT_TOP_CM & INT_BTM_CM & LITH_DESC \\
\hline T98C-25(21-26) & T98C-25 & 21 & 26 & silt, with dark brown and red-brown horizontal lenses \\
\hline T98C-25(26-140) & T98C-25 & 26 & 140 & (a) \\
\hline T98C-25(140-150) & T98C-25 & 140 & 150 & silt and clay, red-brown \\
\hline T98C-25(150-165) & T98C-25 & 150 & 165 & silty clay, gray \\
\hline T98C-25(165-175) & T98C-25 & 165 & 175 & clay, light gray \\
\hline T98C-26(0-3) & T98C-26 & 0 & 3 & soil, dark brown, organic-rich \\
\hline T98C-26(3-25) & T98C-26 & 3 & 25 & clay, red-brown, with horizontal lenses of gray clay \\
\hline T98C-26(25-40) & T98C-26 & 25 & 40 & clay, gray \\
\hline T98R-27(0-15) & T98R-27 & 0 & 15 & silt, red-brown, organic-rich \\
\hline T98R-27(15-60) & T98R-27 & 15 & 60 & silt, light gray with small fragments of red-brown silt (plowed?) \\
\hline T98R-27(60-100) & T98R-27 & 60 & 100 & silt, light gray with small fragments of red-brown silt (plowed?) \\
\hline T98R-27(100-134) & T98R-27 & 100 & 134 & silt, light gray with small fragments of red-brown silt (plowed?) \\
\hline T98R-27(134-161) & T98R-27 & 134 & 161 & sand, very fine-grained, with small fragments of red silt (plowed?) \\
\hline T98R-27(161-180) & T98R-27 & 161 & 180 & silt and sand, red-brown, with fragments of red silt (plowed?) \\
\hline T98R-27(180-190) & T98R-27 & 180 & 190 & silt, red brown, with small fragments of gray silt (plowed?) \\
\hline T98R-27(190-200) & T98R-27 & 190 & 200 & fine-grained silt, blue gray \\
\hline T98R-28(0-2.5) & T98R-28 & 0 & 2.5 & silt, black-gray-brown, organic-bearing \\
\hline T98R-28(2.5-3) & T98R-28 & 2.5 & 3 & 1980 Mt. St. Helens ash, very fine-grained, very pale grav \\
\hline T98R-28(3-11) & T98R-28 & 3 & 11 & silt, black-gray-brown, organic-bearing \\
\hline T98R-28(11-17) & T98R-28 & 11 & 17 & silt, light brown \\
\hline T98R-28(17-30) & T98R-28 & 17 & 30 & clay, red \\
\hline T98R-28(30-45) & T98R-28 & 30 & 45 & clay, black to gray \\
\hline T98R-28(45-60) & T98R-28 & 45 & 60 & clay, black \\
\hline T98R-28(60-100) & T98R-28 & 60 & 100 & clay, gray \\
\hline T98R-28(100-135) & T98R-28 & 100 & 135 & clay, gray, with small fragments of red clay \\
\hline T98R-29(0-10) & T98R-29 & 0 & 10 & clay, red-brown, organic-bearing \\
\hline T98R-29(10-30) & T98R-29 & 10 & 30 & clay, red-brown, with fragments of black organics and gray clay \\
\hline T98R-29(30-60) & T98R-29 & 30 & 60 & clay, gray, with small fragments of very red clay throughout (plowed?) \\
\hline T98R-29(60-75) & T98R-29 & 60 & 75 & clay, gray with fragments of very red clay, decreasing downward \\
\hline T98R-29(75-100) & T98R-29 & 75 & 100 & clay, gray \\
\hline T98R-29(100-120) & T98R-29 & 100 & 120 & silt, gray, grading downward to medium-grained sand, mostly gray \\
\hline T98R-30(0-4) & T98R-30 & 0 & 4 & silty soil, red-brown, organic-bearing \\
\hline T98R-30(4-4.9) & T98R-30 & 4 & 4.9 & 1980 Mt. St. Helens ash, very fine-grained, very pale gray \\
\hline T98R-30(4.9-14) & T98R-30 & 4.9 & 14 & silt, red-brown, with rootlets \\
\hline T98R-30(14-80) & T98R-30 & 14 & 80 & silt, black-gray \\
\hline
\end{tabular}


Appendix C. Lithologic descriptions of soil and sediment samples for which chemical analyses are given in this report (Appendix_C.xls, Appendix_C.dbf)

\begin{tabular}{|c|c|c|c|c|}
\hline Sample No. & Site ID & $\begin{array}{c}\text { Depth } \\
\text { interval, } \\
\text { top }(\mathrm{cm})\end{array}$ & $\begin{array}{l}\text { Depth } \\
\text { interval, } \\
\text { bottom } \\
(\mathrm{cm})\end{array}$ & Lithologic description \\
\hline SAMPLE_NO. & SITE_ID & INT_TOP_CM & INT_BTM_CM & LITH_DESC \\
\hline T98R-31(0-5) & T98R-31 & 0 & 5 & silt, red-brown, with organic debris \\
\hline T98R-31(5-6) & T98R-31 & 5 & 6 & 1980 Mt. St. Helens ash, very fine-grained, very pale gray \\
\hline T98R-31(6-35) & T98R-31 & 6 & 35 & silt, with rootlets \\
\hline T98R-31(35-52) & T98R-31 & 35 & 52 & silt, with horizontal lenses of gray sand, 4 to $5 \mathrm{~cm}$ thick \\
\hline T98R-31(52-63) & T98R-31 & 52 & 63 & silt \\
\hline T98R-31(63-135) & T98R-31 & 63 & 135 & sand, very fine-grained, dark red-brown \\
\hline T98R-31(135-155) & T98R-31 & 135 & 155 & silt, coarse, orange-red \\
\hline T98R-31(155-165) & T98R-31 & 155 & 165 & silt, orange red, with fragments of gray clay \\
\hline T98R-31(165-185) & T98R-31 & 165 & 185 & silt and very fine sand, orange-red \\
\hline T98R-31(185-190) & T98R-31 & 185 & 190 & silt, red-brown, with fragments of gray clay \\
\hline T98R-32(0-2) & T98R-32 & 0 & 2 & silt, dark brown, organic-rich \\
\hline T98R-32(2-55) & T98R-32 & 2 & 55 & silt, red-brown, with organic debris, and sand layers, dark red to black \\
\hline T98R-32(55-85) & T98R-32 & 55 & 85 & silt, red-brown \\
\hline T98R-32(85-110) & T98R-32 & 85 & 110 & silt, red-brown, with horizontal layers of red-orange clay \\
\hline T98R-32(110-180) & T98R-32 & 110 & 180 & silt, red-orange, with fragments of gray silt and clay \\
\hline T98R-33(0-5) & T98R-33 & 0 & 5 & silt, dark brown, organic-rich \\
\hline T98R-33(5-10) & T98R-33 & 5 & 10 & silt, red-brown \\
\hline T98R-33(10-21) & T98R-33 & 10 & 21 & silt, brown \\
\hline T98R-33(21-40) & T98R-33 & 21 & 40 & silt, red, with lenses of fine sand, dark brown \\
\hline T98R-33(40-45) & T98R-33 & 40 & 45 & silt, dark gray with fragments of red silt \\
\hline T98R-33(45-60) & T98R-33 & 45 & 60 & silt, light gray, with fragments of red silt \\
\hline T98R-33(60-145) & T98R-33 & 60 & 145 & silt, yellow to reddish yellow \\
\hline T98R-33(145-185) & T98R-33 & 145 & 185 & clay \\
\hline T98R-34(0-15) & T98R-34 & 0 & 15 & silt, black-gray, organic-bearing \\
\hline T98R-34(15-22) & T98R-34 & 15 & 22 & silt \\
\hline T98R-34(22-40) & T98R-34 & 22 & 40 & clay, brown-black \\
\hline T98R-34(40-60) & T98R-34 & 40 & 60 & silty clay, dark brown \\
\hline T98R-34(60-90) & T98R-34 & 60 & 90 & clay, dark brown \\
\hline T98L-35(0-18) & T98L-35 & 0 & 18 & clay, brown and gray, organic-bearing \\
\hline T98L-35(18-52) & T98L-35 & 18 & 52 & clay, gray, water-saturated \\
\hline T98L-35(52-72) & T98L-35 & 52 & 72 & silt, gray \\
\hline T98L-35(72-92) & T98L-35 & 72 & 92 & sand to silt, gray \\
\hline T98L-35(92-115) & T98L-35 & 92 & 115 & mixed silt, sand and clay, in order of decreasing abundance \\
\hline T98L-35(115-139) & T98L-35 & 115 & 139 & sand, fine-grained, gray \\
\hline T98L-35(139-161) & T98L-35 & 139 & 161 & silt, gray \\
\hline
\end{tabular}


Appendix C. Lithologic descriptions of soil and sediment samples for which chemical analyses are given in this report (Appendix_C.xls, Appendix_C.dbf)

\begin{tabular}{|c|c|c|c|c|}
\hline Sample No. & Site ID & $\begin{array}{c}\text { Depth } \\
\text { interval, } \\
\text { top }(\mathrm{cm})\end{array}$ & $\begin{array}{l}\text { Depth } \\
\text { interval, } \\
\text { bottom } \\
(\mathrm{cm})\end{array}$ & Lithologic description \\
\hline SAMPLE_NO. & SITE_ID & INT_TOP_CM & INT_BTM_CM & LITH_DESC \\
\hline T98L-35(161-195) & T98L-35 & 161 & 195 & clay, gray \\
\hline T98L-36(0-10) & T98L-36 & 0 & 10 & sand, medium-grained, light brown \\
\hline T98L-36(10-16) & T98L-36 & 10 & 16 & sand, fine-grained, gray, cross-bedded \\
\hline T98L-36(16-16.5) & T98L-36 & 16 & 16.5 & 1980 Mt. St. Helens ash, very fine grained, very pale gray \\
\hline T98L-36(16.5-25) & T98L-36 & 16.5 & 25 & sand, medium-grained \\
\hline T98L-36(25-30) & T98L-36 & 25 & 30 & silt, red-brown \\
\hline T98L-36(30-65) & T98L-36 & 30 & 65 & sand, fine-grained, gray, cross-bedded \\
\hline T98L-36(65-90) & T98L-36 & 65 & 90 & sand, fine-grained, red \\
\hline T98L-36(90-105) & T98L-36 & 90 & 105 & sand, yellow \\
\hline T98L-36(105-120) & T98L-36 & 105 & 120 & sand, yellow, with clasts of gray clay \\
\hline T98L-36(120-165) & T98L-36 & 120 & 165 & sand, yellow-gray \\
\hline T98L-36(165-180) & T98L-36 & 165 & 180 & silt, gray \\
\hline T98L-36(180-195) & T98L-36 & 180 & 195 & sand, fine-grained, gray \\
\hline T98L-37(0-35) & T98L-37 & 0 & 35 & clay, gray, with organic debris and fragments of red clay (plowed?) \\
\hline T98L-37(35-45) & T98L-37 & 35 & 45 & clay, gray, water-saturated, with fragments of red clay (plowed?) \\
\hline T98L-37(45-65) & T98L-37 & 45 & 65 & clay, dark gray, with fragments of light gray clay (plowed?) \\
\hline T98L-37(65-200) & T98L-37 & 65 & 200 & clay, gray, dark gray, and light gray \\
\hline T98L-38(0-5) & T98L-38 & 0 & 5 & clay, dark brown, with limonite-cemented tubes of red clay around roots \\
\hline T98L-38(5-45) & T98L-38 & 5 & 45 & clay, dark brown, organic-brearing \\
\hline T98L-38(45-55) & T98L-38 & 45 & 55 & clay, gray, organic-bearing \\
\hline T98L-38(55-75) & T98L-38 & 55 & 75 & clay, medium gray, organic-bearing \\
\hline T98L-38(75-105) & T98L-38 & 75 & 105 & clay, gray, organic-bearing \\
\hline T98L-38(105-125) & T98L-38 & 105 & 125 & clay, dark gray, organic-bearing \\
\hline T98L-38(125-185) & T98L-38 & 125 & 185 & clay, brown-red, organic-bearing \\
\hline T98L-38(185-195) & T98L-38 & 185 & 195 & clay, organic-bearing, with fragments of light gray and red-brown clay \\
\hline T98L-38(195-205) & T98L-38 & 195 & 205 & clay \\
\hline T98M-39(0-5) & T98M-39 & 0 & 5 & silt, dark brown, organic-bearing, with fragments of $1980 \mathrm{Mt}$. St. Helens \\
\hline T98M-39(5-30) & T98M-39 & 5 & 30 & silt, fine-grained, dark brown, organic-bearing \\
\hline T98M-39(30-58) & T98M-39 & 30 & 58 & clay, gray-brown, organic-bearing \\
\hline T98M-39(58-85) & T98M-39 & 58 & 85 & clay, gray to dark brown \\
\hline T98M-39(85-135) & T98M-39 & 85 & 135 & clay, gray, with fragments of light yellow to red-brown clay \\
\hline T98M-39(135-147) & T98M-39 & 135 & 147 & silt, yellow-gray \\
\hline T98M-39(147-175) & T98M-39 & 147 & 175 & clay, gray, with fragments of red-brown clay \\
\hline T98M-39(175-205) & T98M-39 & 175 & 205 & clay, gray \\
\hline T98M-40(0-15) & T98M-40 & 0 & 15 & silt, dark brown, organic-bearing, with small red fragments \\
\hline
\end{tabular}


Appendix C. Lithologic descriptions of soil and sediment samples for which chemical analyses are given in this report (Appendix_C.xls, Appendix_C.dbf)

\begin{tabular}{|c|c|c|c|c|}
\hline Sample No. & Site ID & $\begin{array}{c}\text { Depth } \\
\text { interval, } \\
\text { top (cm) }\end{array}$ & $\begin{array}{l}\text { Depth } \\
\text { interval, } \\
\text { bottom } \\
(\mathrm{cm})\end{array}$ & Lithologic description \\
\hline SAMPLE_NO. & SITE_ID & INT_TOP_CM & INT_BTM_CM & LITH_DESC \\
\hline T98M-40(15-48) & T98M-40 & 15 & 48 & silt, gray, organic-bearing, with red fragments, and red stain around \\
\hline T98M-40(48-130) & T98M-40 & 48 & 130 & silty clay, dark brown \\
\hline T98M-40(130-155) & T98M-40 & 130 & 155 & clay, light aray, with small fragments of red-brown clay \\
\hline T98M-40(155-168) & T98M-40 & 155 & 168 & clay \\
\hline T98M-40(168-180) & T98M-40 & 168 & 180 & clay, light gray with small fragments of red clay \\
\hline T98M-40(180-195) & T98M-40 & 180 & 195 & silt, light gray \\
\hline T98L-41(0-10) & $\overline{T 98 L-41}$ & 0 & 10 & clay, gray, organic-bearing \\
\hline T98L-41(10-40) & T98L-41 & 10 & 40 & silt, red-brown \\
\hline T98L-41(40-52) & T98L-41 & 40 & 52 & clay, gray, with fragments of red-black clay \\
\hline T98L-41(52-70) & T98L-41 & 52 & 70 & peat, black \\
\hline T98L-41(70-105) & T98L-41 & 70 & 105 & clay, gray \\
\hline T98L-42(0-11) & T98L-42 & 0 & 11 & clay, red-brown, organic-bearing \\
\hline T98L-42(11-25) & T98L-42 & 11 & 25 & clay, gray, organic-bearing, with fragments of red clay \\
\hline T98L-42(25-35) & T98L-42 & 25 & 35 & clay, organic-rich, dark gray \\
\hline T98L-42(35-45) & T98L-42 & 35 & 45 & peat, dark gray \\
\hline T98L-42(45-110) & T98L-42 & 45 & 110 & clay, gray \\
\hline T98L-42(110-120) & T98L-42 & 110 & 120 & silt \\
\hline T98M-43(0-2) & T98M-43 & 0 & 2 & silt, organic-bearing \\
\hline T98M-43(2-2.5) & T98M-43 & 2 & 2.5 & 1980 Mt. St. Helens ash, very fine-grained, very pale gray \\
\hline T98M-43(2.5-20) & T98M-43 & 2.5 & 20 & silt, organic-bearing \\
\hline T98M-43(20-30) & T98M-43 & 20 & 30 & silt, dark brown \\
\hline T98M-43(30-135) & T98M-43 & 30 & 135 & silt, light brown to brown \\
\hline T98M-43(135-155) & T98M-43 & 135 & 155 & fine silt, brown \\
\hline T98M-43(155-190) & T98M-43 & 155 & 190 & silt, yellowish brown \\
\hline
\end{tabular}


Appendix D. Chemical composition of samples analyzed by energy dispersive X-ray fluorescence (EDXRF) at the USGS labs, Menlo Park, CA (Appendix_D.xls, Appendix_D.dbf)

\begin{tabular}{|c|c|c|c|c|c|c|c|}
\hline Sample No. & Site ID & $\begin{array}{c}\text { Depth } \\
\text { interval, } \\
\text { top }(\mathrm{cm})\end{array}$ & $\begin{array}{c}\text { Depth } \\
\text { interval, } \\
\text { bottom }(\mathrm{cm})\end{array}$ & $\begin{array}{c}{ }^{*} \text { Other } \\
\text { analyses } \\
\text { (Appendix \#) }\end{array}$ & $\begin{array}{c}\mathrm{Cu} \\
(\mathrm{ppm})\end{array}$ & $\begin{array}{c}\mathrm{Pb} \\
(\mathrm{ppm})\end{array}$ & $\begin{array}{c}\mathrm{Zn} \\
(\mathrm{ppm})\end{array}$ \\
\hline SAMPLE_NO. & $\begin{array}{l}\text { SITE_ID } \\
\end{array}$ & INT_TOP_CM & INT_BTM_CM & OTHR_ANLYS & CU_PPM & PB_PPM & $\overline{Z N \_P P M}$ \\
\hline 93ABM02(0-10) & 93ABM02 & 0 & 10 & $\mathrm{~F}$ & 128 & 4200 & 3450 \\
\hline 93ABM02(11-22) & 93ABM02 & 11 & 22 & $F, G$ & 130 & 4000 & 3150 \\
\hline 93ABM02(22-36) & 93ABM02 & 22 & 36 & $\mathrm{~F}$ & 138 & 4150 & 5450 \\
\hline 93ABM02(36-44) & 93ABM02 & 36 & 44 & $\bar{F}$ & 138 & 4150 & 2250 \\
\hline 93ABM02(44-66) & 93ABM02 & 44 & 66 & $\mathrm{~F}$ & 120 & 5050 & 2700 \\
\hline 93ABM02(66-85) & 93ABM02 & 66 & 85 & $\mathrm{~F}$ & 108 & 5900 & 1650 \\
\hline 93ABM02(85-97) & 93ABM02 & 85 & 97 & $\bar{F}$ & 106 & 4600 & 3200 \\
\hline 93ABM02(97-110) & 93ABM02 & 97 & 110 & $\mathrm{~F}$ & 56 & 70 & 620 \\
\hline 93ABM02(110-126) & 93ABM02 & 110 & 126 & $\bar{F}$ & 60 & 70 & 525 \\
\hline 93ABM02(0-97) & 93ABM02 & 0 & 97 & $\bar{F}$ & 120 & 4700 & 3000 \\
\hline 93ABM02(97-126) & 93ABM02 & 97 & 126 & $\mathrm{~F}$ & 48 & 60 & 555 \\
\hline 93ABM02(0-97)L & 93ABM02 & 0 & 97 & $\mathrm{~F}$ & 110 & 4900 & 3350 \\
\hline 93ABM02(97-129)L & 93ABM02 & 97 & 126 & $\mathrm{~F}$ & 42 & 57 & 570 \\
\hline $93 \mathrm{ABM} 04(0-12)$ & 93ABM04 & 0 & 12 & $\mathrm{~F}$ & 118 & 3700 & 4000 \\
\hline 93ABM04 $(13-18)$ & 93ABM04 & 13 & 18 & $\mathrm{~F}$ & 38 & 3900 & 2750 \\
\hline 93ABM04(22-27) & 93ABM04 & 22 & 27 & $\mathrm{~F}$ & 122 & 3850 & 6000 \\
\hline 93ABM04(32-37) & 93ABM04 & 32 & 37 & $\mathrm{~F}$ & $\overline{98}$ & 3350 & 3450 \\
\hline 93ABM04(42-48) & 93ABM04 & 42 & 48 & $\bar{F}$ & 97 & 3700 & 2700 \\
\hline 93ABM04(48-76) & 93ABM04 & 48 & 76 & $\bar{F}$ & 160 & 10400 & 4200 \\
\hline 93ABM04(76-142) & 93ABM04 & 76 & 142 & $\mathrm{~F}, \mathrm{G}$ & 48 & 340 & 340 \\
\hline 93ABM6A & 93ABM06 & & & $\mathrm{F}$ & 64 & 2800 & 1050 \\
\hline 93ABM6B & 93ABM06 & & & $\bar{F}$ & 40 & 83 & 129 \\
\hline $93 A B L 08(0-5)$ & 93ABL08 & 0 & 5 & $\mathrm{~F}$ & 145 & 4750 & 3450 \\
\hline 93ABL09(0-83) & 93ABL09 & 0 & 83 & $\mathrm{~F}$ & 134 & 4050 & 5700 \\
\hline $93 \mathrm{SBC} 10(0-3)$ & 93SBC10 & 0 & 3 & $\mathrm{~F}$ & 140 & 3310 & 1840 \\
\hline 93SBC10(3-30) & 93SBC10 & 3 & 30 & $\mathrm{~F}$ & 130 & 3820 & 2110 \\
\hline $93 \mathrm{SBC} 10(30-70)$ & 93SBC10 & 30 & 70 & $\bar{F}$ & 230 & 10700 & 3080 \\
\hline $93 \mathrm{SBC} 10(70-90)$ & 93SBC10 & 70 & 90 & $F, G$ & 270 & 11300 & 2460 \\
\hline 93SBC10(90-100) & 93SBC10 & 90 & 100 & $\mathrm{~F}$ & 200 & 12900 & 1950 \\
\hline 93SBC10(100-130) & 93SBC10 & 100 & 130 & $\bar{F}$ & 100 & 640 & 1100 \\
\hline 93SBC10(130-160) & 93SBC10 & 130 & 160 & $\bar{F}$ & 55 & 55 & 1500 \\
\hline 93SBR13(0-15) & 93SBR13 & 0 & 15 & $\mathrm{~F}$ & 184 & 5350 & 4550 \\
\hline 93SBR13(16-52) & 93SBR13 & 16 & 52 & $\mathrm{~F}$ & 149 & 5650 & 4050 \\
\hline 93SBR13(57-65) & 93SBR13 & 57 & 65 & $\bar{F}$ & 136 & 6850 & 4200 \\
\hline 93SBR13(221-237) & 93SBR13 & 221 & 237 & $\mathrm{~F}$ & 365 & 21150 & 5850 \\
\hline 93SBR13(237-251) & 93SBR13 & 237 & 251 & $\mathrm{~F}$ & 220 & 13300 & 5450 \\
\hline 93SBR13(251-291) & 93SBR13 & 251 & 291 & $\mathrm{~F}$ & 190 & 11850 & 5300 \\
\hline 93SBM14 & 93SBM14 & & & $\mathrm{F}$ & 49 & 46 & 102 \\
\hline 93SBC15(0-15) & 93SBC15 & 0 & 15 & $\bar{E}$ & 160 & 740 & 520 \\
\hline 93SBC15(15-35) & 93SBC15 & 15 & 35 & $\bar{E}$ & 130 & 1470 & 820 \\
\hline 93SBC15(35-90) & 93SBC15 & 35 & 90 & $\bar{E}$ & 150 & 2910 & 1520 \\
\hline 93SBC15(90-165) & $93 \mathrm{SBC} 15$ & 90 & 165 & $\overline{\mathrm{E}}$ & 220 & 7560 & 2710 \\
\hline 93SBC15(165-214) & 93SBC15 & 165 & 214 & $\bar{E}$ & 190 & 8980 & 2660 \\
\hline 93SBC15(214-242) & $93 \mathrm{SBC} 15$ & 214 & 242 & $\overline{\mathrm{E}}$ & 160 & 9630 & 3720 \\
\hline 93SBC15(242-261) & 93SBC15 & 242 & 261 & $\bar{E}$ & 170 & 8020 & 3740 \\
\hline 93SBC16(0-5) & 93SBC16 & 0 & 5 & $\bar{F}$ & 65 & 1880 & 1240 \\
\hline 93SBK17(8-25) & 93SBK17 & 8 & 25 & $\overline{---}$ & 370 & 50800 & 12900 \\
\hline 93SBK17(25-68) & $93 \mathrm{SBK} 17$ & 25 & 68 & $F, G$ & 390 & $\begin{array}{l}18300 \\
18300\end{array}$ & 10200 \\
\hline 93SBK17(68-106)L & 93SBK17 & 68 & 106 & $\mathrm{~F}$ & 355 & 60600 & 5900 \\
\hline
\end{tabular}


Appendix D. Chemical composition of samples analyzed by energy dispersive X-ray fluorescence (EDXRF) at the USGS labs, Menlo Park, CA (Appendix_D.xls, Appendix_D.dbf)

\begin{tabular}{|c|c|c|c|c|c|c|c|}
\hline Sample No. & Site ID & $\begin{array}{c}\text { Depth } \\
\text { interval, } \\
\text { top }(\mathrm{cm})\end{array}$ & $\begin{array}{c}\text { Depth } \\
\text { interval, } \\
\text { bottom }(\mathrm{cm})\end{array}$ & $\begin{array}{c}{ }^{*} \text { Other } \\
\text { analyses } \\
\text { (Appendix \#) }\end{array}$ & $\begin{array}{c}\mathrm{Cu} \\
(\mathrm{ppm})\end{array}$ & $\begin{array}{c}\mathrm{Pb} \\
(\mathrm{ppm})\end{array}$ & $\begin{array}{c}\mathrm{Zn} \\
(\mathrm{ppm})\end{array}$ \\
\hline SAMPLE_NO. & $\begin{array}{l}\text { SITE_ID } \\
\end{array}$ & INT_TOP_CM & INT_BTM_CM & OTHR_ANLYS & CU_PPM & PB_PPM & ZN_PPM \\
\hline 93SBK17(106-152) & 93SBK17 & 106 & 152 & $\mathrm{~F}, \mathrm{G}$ & 70 & 3320 & 1880 \\
\hline 93SBK17(152-230) & 93SBK17 & 152 & 230 & $\mathrm{~F}$ & 58 & 1080 & 1310 \\
\hline 93SBK18(7-10) & 93SBK18 & 7 & 10 & $\mathrm{~F}, \mathrm{G}$ & 370 & 24500 & 5560 \\
\hline 93SBK18(10-26) & $93 \mathrm{SBK} 18$ & 10 & 26 & $\mathrm{~F}$ & 450 & 33800 & 5820 \\
\hline 93SBK18(26-82)L & 93SBK18 & 26 & 82 & $\mathrm{~F}, \mathrm{G}$ & 670 & 37450 & 5300 \\
\hline 93SBK18(82-92) & 93SBK18 & 82 & 92 & $\bar{F}$ & 100 & 3230 & 2730 \\
\hline 93SBK18(92-109) & 93SBK18 & 92 & 109 & $\mathrm{~F}$ & 56 & 260 & 5920 \\
\hline 93SBK18(109-160) & 93SBK18 & 109 & 160 & $\mathrm{~F}$ & 39 & 220 & 1700 \\
\hline 93SBB20(0-5) & 93SBB20 & 0 & 5 & $\mathrm{~F}, \mathrm{G}$ & 110 & 3220 & 2930 \\
\hline 93SBB21(0-9) & 93SBB21 & $\overline{0}$ & $\overline{9}$ & $\mathrm{E}$ & 110 & 3090 & 4580 \\
\hline 93SBB21(41-72) & 93SBB21 & 41 & 72 & $\bar{E}$ & 120 & 3760 & 3200 \\
\hline 93SBB21(72-84) & 93SBB21 & 72 & 84 & E & 110 & 4720 & 2520 \\
\hline 93SBB21 (84-99) & 93SBB21 & 84 & 99 & $\mathrm{E}$ & 110 & 5350 & 1880 \\
\hline 93SBB21(99-111) & 93SBB21 & 99 & 111 & $\mathrm{E}$ & 110 & 5830 & 2840 \\
\hline 93SBB21(111-120) & 93SBB21 & 111 & 120 & $\overline{\mathrm{E}}$ & 43 & 360 & 1370 \\
\hline 93SBB21(120-130) & 93SBB21 & 120 & 130 & $\overline{\mathrm{E}}$ & 31 & 50 & 230 \\
\hline 93SBB21(130-148) & 93SBB21 & 130 & 148 & $\overline{\mathrm{E}}$ & 32 & 46 & 180 \\
\hline 93SBB21(148-160) & 93SBB21 & 148 & 160 & $\bar{E}$ & 35 & 40 & 97 \\
\hline 93SBB21(160-180) & 93SBB21 & 160 & 180 & $\overline{\mathrm{E}}$ & 30 & 47 & 89 \\
\hline 93SBB21(173-180) & 93SBB21 & 173 & 180 & $\overline{\mathrm{E}}$ & 340 & 120 & 340 \\
\hline 93SBB21(180-192) & 93SBB21 & 180 & 192 & $\bar{E}$ & 180 & 98 & 220 \\
\hline 93SBB21(192-203) & 93SBB21 & 192 & 203 & $\overline{\mathrm{E}}$ & 160 & 40 & 160 \\
\hline 93SBB22(0-4) & 93SBB22 & 0 & 4 & $\bar{F}$ & 87 & 1180 & 480 \\
\hline 93SBB22(4.5-7.5) & 93SBB22 & 4.5 & 7.5 & $\mathrm{~F}$ & 200 & 2620 & 970 \\
\hline 93SBB22(7.5-12) & 93SBB22 & 7.5 & 12 & $\mathrm{~F}$ & 120 & 4170 & 1860 \\
\hline 93SBB22(12-15) & 93SBB22 & 12 & 15 & $\mathrm{~F}$ & 160 & 4200 & 4060 \\
\hline 93SBB22(15-19) & 93 SBB22 & 15 & 19 & $\bar{F}$ & 120 & 5140 & 3240 \\
\hline 93SBB22(19-26) & $93 \mathrm{SBB} 22$ & 19 & 26 & $\mathrm{~F}, \mathrm{G}$ & 150 & 9740 & 3370 \\
\hline 93SBB22(26-29) & 93 SBB22 & 26 & 29 & $\mathrm{~F}$ & 210 & 16800 & 1680 \\
\hline 93SBB22(29-36) & $93 \mathrm{SBB} 22$ & 29 & 36 & $\bar{F}$ & 85 & 580 & 1090 \\
\hline 93SBB22(36-48) & 93SBB22 & 36 & 48 & $\bar{F}$ & 190 & 74 & 450 \\
\hline 93SBB22(48-60) & 93SBB22 & 48 & 60 & $\bar{F}$ & 160 & 40 & 270 \\
\hline 93SBB22(60-82) & $93 \mathrm{SBB} 22$ & 60 & 82 & $\mathrm{~F}$ & 54 & 50 & 120 \\
\hline $93 S B B 23(0-5)$ & 93SBB23 & 0 & 5 & $\mathrm{~F}$ & 120 & 3950 & 2400 \\
\hline 93SBB23(5-12) & 93SBB23 & 5 & 12 & $\mathrm{~F}$ & 98 & 2530 & 3310 \\
\hline 93SBB23(12-17) & 93SBB23 & 12 & 17 & $\mathrm{~F}$ & 140 & 3810 & 4430 \\
\hline 93SBB23(17-22) & 93SBB23 & 17 & 22 & $\mathrm{~F}$ & 130 & 4550 & 3590 \\
\hline 93SBB23(22-30) & $93 \mathrm{SBB} 23$ & 22 & 30 & $\mathrm{~F}$ & 120 & 4760 & 2540 \\
\hline 93SBB23(30-46) & 93SBB23 & 30 & 46 & $\bar{F}$ & 150 & 6280 & 2430 \\
\hline 93SBB23(46-53) & 93SBB23 & 46 & 53 & $\mathrm{~F}, \mathrm{G}$ & 170 & 7030 & 5800 \\
\hline 93SBB23(53-86) & 93SBB23 & 53 & 86 & $\mathrm{~F}$ & 36 & 63 & 620 \\
\hline 93SBB23(86-117) & 93SBB23 & 86 & 117 & $\bar{F}$ & 40 & 63 & 290 \\
\hline 93SBL26(0-6) & 93SBL26 & 0 & 6 & $F, G$ & 230 & 2850 & 1490 \\
\hline $93 \mathrm{SBL} 26(6-12)$ & 93SBL26 & 6 & 12 & $\mathrm{~F}$ & 390 & 4830 & 6800 \\
\hline 93SBL26(12-20) & 93SBL26 & 12 & 20 & $\bar{F}$ & 280 & 5120 & 3860 \\
\hline 93SBL26(20-28) & 93SBL26 & 20 & 28 & $\mathrm{~F}$ & 340 & 7470 & 2100 \\
\hline 93SBL26(28-34) & 93SBL26 & 28 & 34 & $\mathrm{~F}$ & 130 & 1430 & 2170 \\
\hline 93SBL26(34-47) & 93SBL26 & 34 & 47 & $\bar{F}$ & 97 & 270 & 240 \\
\hline 93SBL26(47-58) & 93SBL26 & 47 & 58 & $\bar{F}$ & 220 & 280 & 340 \\
\hline
\end{tabular}

*Other analyses (Appendix \#): $\quad \mathrm{E}=\mathrm{EWU}, \mathrm{F}=\mathrm{CHEMEX}, \mathrm{G}=\mathrm{XRAL},------=$ none. 
Appendix D. Chemical composition of samples analyzed by energy dispersive X-ray fluorescence (EDXRF) at the USGS labs, Menlo Park, CA (Appendix_D.xls, Appendix_D.dbf)

\begin{tabular}{|c|c|c|c|c|c|c|c|}
\hline Sample No. & Site ID & $\begin{array}{c}\text { Depth } \\
\text { interval, } \\
\text { top }(\mathrm{cm})\end{array}$ & $\begin{array}{c}\text { Depth } \\
\text { interval, } \\
\text { bottom }(\mathrm{cm})\end{array}$ & $\begin{array}{c}{ }^{*} \text { Other } \\
\text { analyses } \\
\text { (Appendix \#) }\end{array}$ & $\begin{array}{c}\mathrm{Cu} \\
(\mathrm{ppm})\end{array}$ & $\begin{array}{c}\mathrm{Pb} \\
(\mathrm{ppm})\end{array}$ & $\begin{array}{c}\mathrm{Zn} \\
\text { (ppm) }\end{array}$ \\
\hline SAMPLE_NO. & SITE_ID & INT_TOP_CM & INT_BTM_CM & OTHR_ANLYS & CU_PPM & PB_PPM & ZN_PPM \\
\hline 93SBL27(0-7) & 93SBL27 & 0 & 7 & $\bar{E}$ & 150 & 4610 & 4200 \\
\hline 93SBL27(7-19) & 93SBL27 & 7 & 19 & $\bar{E}$ & 140 & 4230 & 4340 \\
\hline 93SBL27(19-23) & 93SBL27 & 19 & 23 & $\bar{E}$ & 160 & 4370 & 3100 \\
\hline 93SBL27(23-39) & 93SBL27 & 23 & 39 & $\overline{\mathrm{E}}$ & 120 & 4320 & 4070 \\
\hline 93SBL27(39-46) & 93SBL27 & 39 & 46 & $\bar{E}$ & 130 & 4180 & 4180 \\
\hline 93SBL27(46-50) & 93SBL27 & 46 & 50 & $\bar{E}$ & 160 & 5490 & 4150 \\
\hline $93 \mathrm{SBL} 27(50-60)$ & 93SBL27 & 50 & 60 & $\overline{\mathrm{E}}$ & 150 & 5050 & 5330 \\
\hline 93SBL27 $60-76)$ & 93SBL27 & 60 & 76 & $\overline{\mathrm{E}}$ & 54 & 63 & 960 \\
\hline 93SBL27(63-65) & 93SBL27 & 63 & 65 & $\overline{----}$ & 120 & 4440 & 3530 \\
\hline 93SBL27(76-90) & 93SBL27 & 76 & 90 & $\bar{E}$ & 54 & 57 & 530 \\
\hline 93SBL27(90-99) & 93SBL27 & 90 & 99 & $\bar{E}$ & 72 & 60 & 310 \\
\hline 93SBL27B(0-4) & 93SBL27B & 0 & 4 & $\mathrm{~F}$ & 210 & 4460 & 3460 \\
\hline 93SBL27B(4-16) & 93SBL27B & 4 & 16 & $\bar{F}$ & 160 & 4910 & 4330 \\
\hline 93SBL27B(16-23) & 93SBL27B & 16 & 23 & $\mathrm{~F}$ & 160 & 4570 & 4890 \\
\hline 93SBL27B(23-34) & 93SBL27B & 23 & 34 & $\mathrm{~F}$ & 160 & 5030 & 3380 \\
\hline 93SBL27B(34-43) & 93SBL27B & $\overline{34}$ & 43 & $\mathrm{~F}$ & 130 & 5390 & 2000 \\
\hline 93SBL27B(43-53) & 93SBL27B & 43 & 53 & $\mathrm{~F}$ & 150 & 6090 & 2100 \\
\hline 93SBL27B(53-69) & 93SBL27B & 53 & 69 & $\bar{F}$ & 120 & 5330 & 1740 \\
\hline 93SBL27B $(69-85)$ & 93SBL27B & 69 & 85 & $\bar{F}$ & 110 & $<40$ & 1580 \\
\hline 93SBL28(0-11) & 93SBL28 & 0 & 11 & $\bar{F}$ & 180 & 3280 & 2820 \\
\hline 93SBL28(11-16) & 93SBL28 & 11 & 16 & $\bar{F}$ & 190 & 2970 & 980 \\
\hline 93SBL28(16-25) & 93SBL28 & 16 & 25 & $\mathrm{~F}$ & 200 & 3980 & 4000 \\
\hline 93SBL28(25-34) & 93SBL28 & 25 & 34 & $\mathrm{~F}$ & 130 & 4250 & 2980 \\
\hline 93SBL28(34-37) & 93SBL28 & 34 & 37 & $\mathrm{~F}$ & 150 & 5030 & 3230 \\
\hline 93SBL28(37-41) & 93SBL28 & 37 & 41 & $\mathrm{~F}$ & 210 & 7150 & 3480 \\
\hline 93SBL28(41-46) & 93SBL28 & 41 & 46 & $\mathrm{~F}$ & 170 & 5880 & 1580 \\
\hline $93 \mathrm{SBL} 28 \mathrm{C}(0-25)$ & 93SBL28C & $\overline{0}$ & 25 & $\bar{F}$ & 210 & 1960 & 1530 \\
\hline 93SBL28C(25-28) & 93SBL28C & 25 & 28 & $\bar{F}$ & 302 & 3900 & 1550 \\
\hline 93SBL28C(28-37) & 93SBL28C & 28 & 37 & $\bar{F}$ & 203 & 3650 & 3900 \\
\hline 93SBL28C(37-48) & 93SBL28C & 37 & 48 & $\bar{F}$ & 160 & 4330 & 2910 \\
\hline 93SBL28C(48-54) & 93SBL28C & 48 & 54 & $\bar{F}$ & 220 & 5840 & 3380 \\
\hline 93SBL28C(54-64) & 93SBL28C & 54 & 64 & $\mathrm{~F}$ & 170 & 6820 & 1840 \\
\hline 93SBL28C (64-72) & 93SBL28C & 64 & 72 & $\mathrm{~F}$ & 160 & 350 & 2280 \\
\hline 93SBL28C (72-83) & 93SBL28C & 72 & 83 & $\bar{F}$ & 160 & 79 & 910 \\
\hline 93SBL28C(83-93) & 93SBL28C & 83 & 93 & $\mathrm{~F}$ & 180 & 68 & 540 \\
\hline 93SBL28C(93-97) & 93SBL28C & 93 & 97 & $\mathrm{~F}$ & 430 & 300 & 490 \\
\hline 93SBL30 $(0-4)$ & 93SBL30 & 0 & 4 & $\mathrm{E}$ & 140 & 5200 & 4300 \\
\hline 93SBL30(4-9) & 93SBL30 & 4 & 9 & $\bar{E}$ & 198 & 5150 & 5200 \\
\hline 93SBL30(9-18) & 93SBL30 & 9 & 18 & $\bar{E}$ & 182 & 5000 & 3650 \\
\hline 93SBL30(18-23) & 93SBL30 & 18 & 23 & $\bar{E}$ & 1650 & 6300 & 9800 \\
\hline 93SBL30(23-28.5) & 93SBL30 & 23 & 28.5 & $\bar{E}$ & 154 & 6950 & 2900 \\
\hline 93SBL30(28.5-31.5) & 93SBL30 & 28.5 & 31.5 & $\bar{E}$ & 150 & 5850 & 2700 \\
\hline 93SBL30(31.5-37) & 93SBL30 & 31.5 & 37 & $\bar{E}$ & 120 & 5550 & 3110 \\
\hline $93 \mathrm{SBL} 30(37-48)$ & 93SBL30 & 37 & 48 & $\overline{\mathrm{E}}$ & 60 & 440 & 930 \\
\hline 93SBL30(48-62) & 93SBL30 & 48 & 62 & $\bar{E}$ & 55 & 79 & 600 \\
\hline 93SBL30(62-77) & 93SBL30 & 62 & 77 & $\bar{E}$ & 61 & 40 & 410 \\
\hline 93SBL30(77-100) & 93SBL30 & 77 & 100 & $E$ & 50 & 48 & 290 \\
\hline 93SBL30(100-122) & 93SBL30 & 100 & 122 & $\overline{---}$ & 42 & 48 & 240 \\
\hline 93SBL30(127-137.5) & 93SBL30 & 127 & 137.5 & $\bar{E}$ & 120 & 46 & 130 \\
\hline
\end{tabular}

*Other analyses (Appendix \#): $\quad \mathrm{E}=\mathrm{EWU}, \mathrm{F}=\mathrm{CHEMEX}, \mathrm{G}=\mathrm{XRAL},------=$ none. 
Appendix D. Chemical composition of samples analyzed by energy dispersive X-ray fluorescence (EDXRF) at the USGS labs, Menlo Park, CA (Appendix_D.xls, Appendix_D.dbf)

\begin{tabular}{|c|c|c|c|c|c|c|c|}
\hline Sample No. & Site ID & $\begin{array}{c}\text { Depth } \\
\text { interval, } \\
\text { top }(\mathrm{cm})\end{array}$ & $\begin{array}{c}\text { Depth } \\
\text { interval, } \\
\text { bottom }(\mathrm{cm})\end{array}$ & $\begin{array}{c}{ }^{*} \text { Other } \\
\text { analyses } \\
\text { (Appendix \#) }\end{array}$ & $\begin{array}{c}\mathrm{Cu} \\
\text { (ppm) }\end{array}$ & $\begin{array}{c}\mathrm{Pb} \\
(\mathrm{ppm})\end{array}$ & $\begin{array}{c}\mathrm{Zn} \\
(\mathrm{ppm})\end{array}$ \\
\hline SAMPLE_NO. & $\begin{array}{l}\text { SITE_ID } \\
\end{array}$ & INT_TOP_CM & INT_BTM_CM & OTHR_ANLYS & CU_PPM & PB_PPM & ZN_PPM \\
\hline 93SBL30(137.5-144.5) & 93SBL30 & 137.5 & 144.5 & $\bar{E}$ & 160 & 40 & 360 \\
\hline 93SBL30(144.5-158.5) & 93SBL30 & 144.5 & 158.5 & $\bar{E}$ & 91 & 47 & 180 \\
\hline 93SBL30(158.5-172) & 93SBL30 & 158.5 & 172 & $\bar{E}$ & 110 & 45 & 110 \\
\hline 93SBL30(172-180) & 93SBL30 & 172 & 180 & $\bar{E}$ & 180 & 47 & 140 \\
\hline 93SBL30(180-187) & 93SBL30 & 180 & 187 & $\bar{E}$ & 260 & 71 & 220 \\
\hline 93SBL31(0-6) & 93SBL31 & $\overline{0}$ & 6 & $\bar{F}$ & 140 & 4720 & 1900 \\
\hline 93SBL31(6.5-18) & 93SBL31 & 6.5 & 18 & $\bar{F}$ & 136 & 4300 & 3800 \\
\hline 93SBL31(18-23) & 93SBL31 & 18 & 23 & $\bar{F}$ & 132 & 3650 & 3300 \\
\hline 93SBL31(23-30) & 93SBL31 & 23 & 30 & $\bar{F}$ & 117 & 4000 & 2300 \\
\hline 93SBL31(30-35) & 93SBL31 & 30 & 35 & $\bar{F}$ & 116 & 3950 & 1850 \\
\hline 93SBL31(35-42) & 93SBL31 & 35 & 42 & $\bar{F}$ & 108 & 3450 & 1450 \\
\hline 93SBL31(42-48) & 93SBL31 & 42 & 48 & $\mathrm{~F}$ & 107 & 3700 & 1600 \\
\hline 93SBL31(48-61) & 93SBL31 & 48 & 61 & $\overline{----}$ & 108 & 4050 & 2200 \\
\hline 93SBL31(61-67) & 93SBL31 & 61 & 67 & $\overline{----}$ & 94 & 3950 & 1950 \\
\hline 93SBL31(67-73) & 93SBL31 & 67 & 73 & $-\cdots-$ & 96 & 3950 & 2400 \\
\hline 93SBL31(73-87) & 93SBL31 & 73 & 87 & $\mathrm{~F}$ & 120 & 4550 & 2150 \\
\hline 93SBL31(87-108) & 93SBL31 & 87 & 108 & ---- & $\overline{91}$ & 4300 & 2350 \\
\hline 93SBL31(108-130) & 93SBL31 & 108 & 130 & $\overline{----}$ & 97 & 4650 & 2850 \\
\hline 93SBL31(130-137) & 93SBL31 & 130 & 137 & $\bar{F}$ & 140 & 5200 & 5380 \\
\hline 93SBL31(137-146) & 93SBL31 & 137 & 146 & $\mathrm{~F}, \mathrm{G}$ & 150 & 5150 & 4850 \\
\hline 93SBL31(146-160) & 93SBL31 & 146 & 160 & $\mathrm{~F}$ & 160 & 5280 & 7500 \\
\hline 93SBL31(185-200) & 93SBL31 & 185 & 200 & $\mathrm{~F}, \mathrm{G}$ & 240 & 6240 & 4780 \\
\hline 93SBL31(200-211) & 93SBL31 & 200 & 211 & $\mathrm{~F}$ & 190 & 5320 & 4780 \\
\hline 93SBL32(0-3) & 93SBL32 & 0 & 3 & $\bar{F}$ & 167 & 2650 & 1700 \\
\hline 93SBL32(3-10.5) & 93SBL32 & 3 & 10.5 & $\mathrm{~F}$ & 129 & 4800 & 2200 \\
\hline 93SBL32(10.5-16) & 93SBL32 & 10.5 & 16 & $\mathrm{~F}, \mathrm{G}$ & 82 & 2050 & 577 \\
\hline 93SBL32(16-24) & $93 \mathrm{SBL} 32$ & 16 & $\overline{24}$ & $\mathrm{~F}$ & 284 & 23000 & 950 \\
\hline 93SBL32(24-31) & 93SBL32 & 24 & 31 & $\mathrm{~F}$ & 58 & 830 & 328 \\
\hline 93SBL32(31-48) & 93SBL32 & 31 & 48 & $\bar{F}$ & 305 & 72 & 38 \\
\hline 93SBL34a & 93SBL34a & & & $\mathrm{F}$ & 140 & 6170 & 6440 \\
\hline 93SBL34b & 93SBL34b & & & $\mathrm{E}, \mathrm{F}$ & 184 & 10200 & 3350 \\
\hline 93SBL34c & 93SBL34c & & & $\mathrm{E}, \mathrm{F}$ & 380 & 25700 & 13200 \\
\hline 93SBL34d & 93SBL34d & & & $\mathrm{E}, \mathrm{F}$ & 248 & 19650 & 3900 \\
\hline 93SBL34e & 93SBL34e & & & $\mathrm{E}, \mathrm{F}$ & 380 & 26400 & 14100 \\
\hline 93SBL34f & 93SBL34f & & & $\bar{E}, F$ & 215 & 19500 & 4100 \\
\hline 93SBL34g & 93SBL34g & & & $E, F$ & 232 & 18400 & 4650 \\
\hline 93SBL34h & 93SBL34h & & & $\bar{E}, F$ & 300 & 19100 & 11400 \\
\hline 93SBL34i & 93SBL34i & & & $\mathrm{E}, \mathrm{F}$ & 254 & 17350 & 6300 \\
\hline $93 \mathrm{SBC} 35 \mathrm{~A}(0-5)$ & 93SBC35A & 0 & 5 & $\overline{----}$ & 98 & 2950 & 2600 \\
\hline $93 \mathrm{SBC} 35 \mathrm{~B}(0-5)$ & 93SBC35B & 0 & 5 & $\overline{----}$ & 79 & 2350 & 1800 \\
\hline $93 \mathrm{SBC} 36(0-5)$ & 93SBC36 & 0 & 5 & $\overline{----}$ & 61 & 29 & 117 \\
\hline 93SBC37(0-5) & 93SBC37 & $\overline{0}$ & 5 & $\overline{----}$ & 34 & 72 & 104 \\
\hline 93SBK38(SAND) & 93SBK38 & 0 & 2 & $\mathrm{E}$ & 118 & 2950 & 2000 \\
\hline $93 \mathrm{SBK} 38(0-11)$ & 93SBK38 & 0 & $\overline{11}$ & $\overline{\mathrm{E}}$ & 108 & 2700 & 1600 \\
\hline 93SBK38(11-20) & 93SBK38 & 11 & 20 & $\mathrm{E}$ & 144 & 5050 & 1600 \\
\hline 93SBK38(20-55) & 93SBK38 & 20 & 55 & $\bar{E}$ & 70 & 2550 & 890 \\
\hline 93SBK38(55-100) & 93SBK38 & 55 & 100 & $E$ & 96 & 5000 & 1700 \\
\hline 93SBC39(SAND) & 93SBC39 & 0 & 2 & $\bar{E}$ & 101 & 3550 & 2300 \\
\hline 93SBC39(0-5) & 93SBC39 & 0 & 5 & $\bar{E}$ & 105 & 2400 & 1150 \\
\hline
\end{tabular}

*Other analyses (Appendix \#): $\quad \mathrm{E}=\mathrm{EWU}, \mathrm{F}=\mathrm{CHEMEX}, \mathrm{G}=\mathrm{XRAL},------=$ none. 
Appendix D. Chemical composition of samples analyzed by energy dispersive X-ray fluorescence (EDXRF) at the USGS labs, Menlo Park, CA (Appendix_D.xls, Appendix_D.dbf)

\begin{tabular}{|c|c|c|c|c|c|c|c|}
\hline Sample No. & Site ID & $\begin{array}{c}\text { Depth } \\
\text { interval, } \\
\text { top }(\mathrm{cm})\end{array}$ & $\begin{array}{c}\text { Depth } \\
\text { interval, } \\
\text { bottom }(\mathrm{cm})\end{array}$ & $\begin{array}{c}{ }^{*} \text { Other } \\
\text { analyses } \\
\text { (Appendix \#) }\end{array}$ & $\begin{array}{c}\mathrm{Cu} \\
(\mathrm{ppm})\end{array}$ & $\begin{array}{c}\mathrm{Pb} \\
(\mathrm{ppm})\end{array}$ & $\begin{array}{c}\text { Zn } \\
(\mathrm{ppm})\end{array}$ \\
\hline SAMPLE_NO. & SITE_ID & INT_TOP_CM & INT_BTM_CM & OTHR_ANLYS & CU_PPM & PB_PPM & ZN_PPM \\
\hline 93SBC39(5-10) & 93SBC39 & 5 & 10 & $\mathrm{E}$ & 104 & 3950 & 2200 \\
\hline 93SBC39(10-20) & 93SBC39 & 10 & 20 & $\mathrm{E}$ & 117 & 4000 & 1950 \\
\hline 93SBC39(20-27) & 93SBC39 & 20 & 27 & $\mathrm{E}$ & 126 & 3950 & 2400 \\
\hline 93SBC39(27-32) & 93SBC39 & 27 & 32 & $\mathrm{E}$ & 104 & 3900 & 2100 \\
\hline 93SBC39(32-40) & 93SBC39 & 32 & 40 & $\mathrm{E}$ & 42 & 70 & 1550 \\
\hline 93SBC39(40-58) & 93SBC39 & 40 & 58 & $\mathrm{E}$ & 40 & 76 & 1300 \\
\hline 93SBC39(58-72) & 93SBC39 & 58 & 72 & $\mathrm{E}$ & 47 & 56 & 1300 \\
\hline
\end{tabular}


Appendix E. Chemical composition of samples analyzed, using 4 acid dissolution, by ICP-AES and ICP-MS at Eastern Washington University (EWU), Cheney, WA (Appendix_E.xls, Appendix_E.dbf).

\begin{tabular}{|c|c|c|c|c|c|c|c|c|c|c|c|c|c|c|c|c|}
\hline Sample No. & Site ID & $\begin{array}{c}\text { Depth } \\
\text { Interval, } \\
\text { top (cm) }\end{array}$ & $\begin{array}{c}\text { Depth } \\
\text { Interval, } \\
\text { bottom }(\mathrm{cm})\end{array}$ & $\begin{array}{c}{ }^{*} \text { Other } \\
\text { analyses } \\
\text { (Appendix \#) }\end{array}$ & $\begin{array}{c}\mathrm{Ag} \\
(\mathrm{ppm})\end{array}$ & $\begin{array}{c}\mathrm{Al} \\
(\%)\end{array}$ & $\begin{array}{c}\text { As } \\
(\mathrm{ppm})\end{array}$ & $\begin{array}{c}\mathrm{Ba} \\
\text { (ppm) }\end{array}$ & $\begin{array}{c}\mathrm{Be} \\
(\mathrm{ppm})\end{array}$ & $\begin{array}{c}\mathrm{Ca} \\
(\%)\end{array}$ & $\mid \begin{array}{c}\text { Cd } \\
(p p m)\end{array}$ & $\begin{array}{c}\mathrm{Ce} \\
(\mathrm{ppm})\end{array}$ & $\begin{array}{c}\text { Co } \\
\text { (ppm) }\end{array}$ & $\begin{array}{c}\mathrm{Cr} \\
(\mathrm{ppm})\end{array}$ & $\begin{array}{c}\text { Cs } \\
(\mathrm{ppm})\end{array}$ & $\begin{array}{c}\mathrm{Cu} \\
(\mathrm{ppm})\end{array}$ \\
\hline SAMPLE_NO. & $\overline{\text { SITE_ID }}$ & INT_TOP_CM & INT_BTM_CM & OTHR_ANLYS & $\overline{A G_{-} P P M}$ & AL_\% & AS_PPM & BA_PPM & BE_PPM & CA_\% & CD_PPM & CE_PPM & CO_PPM & \begin{tabular}{|l|} 
CR_PPM \\
\end{tabular} & CS_PPM & CU_PPM \\
\hline 94VC1(0-10) & 94VC1 & 0 & 10 & ---- & 8.3 & 4.0 & 53 & 466 & 1.2 & 0.19 & 17.9 & 38 & 6 & 113 & 7 & 85 \\
\hline $94 \mathrm{VC} 1(10-20)$ & $94 \mathrm{VC1}$ & 10 & 20 & $\mathrm{G}, \mathrm{H}$ & 8.3 & 4.1 & 52 & 489 & 1.1 & 0.20 & 18.3 & 35 & 6 & 106 & 7 & $\overline{84}$ \\
\hline 94VC1(20-30) & 94VC1 & 20 & 30 & ---- & 9.7 & 3.6 & 56 & 444 & 1.2 & 0.23 & 23.3 & 32 & 6 & 102 & 6 & 93 \\
\hline 94VC1 $(30-40)$ & 94VC1 & 30 & 40 & $\begin{array}{ll}--- \\
\end{array}$ & 8.7 & 3.8 & 50 & 469 & 1.3 & 0.19 & 16.7 & 21 & 6 & 104 & 7 & 82 \\
\hline 94VC1(40-50) & 94VC1 & 40 & 50 & $\begin{array}{ll}--- \\
-\end{array}$ & 10.3 & 3.6 & 55 & 449 & 1.0 & 0.23 & 27.2 & 23 & 6 & 95 & 6 & 103 \\
\hline 94VC1(50-60) & 94VC1 & 50 & 60 & ---- & 12.3 & 3.7 & 49 & 460 & 1.2 & 0.26 & 31.3 & 33 & 7 & 97 & 7 & 117 \\
\hline 94VC1(60-70) & $94 \mathrm{VC1}$ & 60 & 70 & ---- & 12.8 & 3.7 & 53 & 491 & 1.3 & 0.29 & 34.2 & 45 & 8 & 98 & 7 & 119 \\
\hline 94VC1 $1(70-80)$ & 94VC1 & 70 & 80 & $-\overline{---}$ & 12.9 & 3.2 & 47 & 430 & 1.2 & 0.28 & 37.8 & 27 & 8 & 92 & 6 & 126 \\
\hline 94VC1 $(80-90)$ & 94VC1 & 80 & 90 & ---- & 16.6 & 3.5 & 57 & 539 & 1.3 & 0.38 & 42.4 & 25 & 10 & 93 & 7 & 149 \\
\hline 94VC1(90-97) & 94VC1 & 90 & 97 & --- & 18.1 & 3.1 & 64 & 575 & 1.2 & 0.44 & 45.9 & 26 & 10 & 90 & 6 & 156 \\
\hline 94VC1(97-110) & 94VC1 & 97 & 110 & ---- & 31.4 & 2.9 & 54 & 1103 & 1.5 & 0.83 & 98.7 & 32 & 12 & 56 & 9 & 254 \\
\hline 94VC1(110-120) & 94VC1 & 110 & 120 & ---- & 33.3 & 2.3 & 58 & 812 & 1.0 & 0.74 & 145.7 & 20 & 12 & 68 & 6 & 343 \\
\hline 94VC1(120-130) & 94VC1 & 120 & 130 & ---- & 35.7 & 2.5 & 60 & 850 & 0.9 & 0.84 & 147.1 & 23 & 12 & 71 & 6 & 336 \\
\hline 94VC1(130-140) & $94 \mathrm{VC1}$ & 130 & 140 & ---- & 31.1 & 3.2 & 58 & 841 & 1.2 & 0.84 & 135.0 & 21 & 13 & 78 & 8 & 318 \\
\hline 94VC1(140-150) & $94 \mathrm{VC1}$ & 140 & 150 & $\mathrm{G}, \mathrm{H}$ & 31.3 & 2.3 & 73 & 838 & 1.0 & 0.89 & 141.7 & 17 & 13 & 57 & 6 & 280 \\
\hline 94VC1(150-160) & $94 \mathrm{VC1}$ & 150 & 160 & $\mathrm{G}, \mathrm{H}$ & 39.8 & 3.1 & 99 & 1038 & 1.3 & 0.93 & 139.0 & 25 & 15 & 58 & 10 & 269 \\
\hline 94VC1(160-170) & $94 \mathrm{VC1}$ & 160 & 170 & ---- & 31.5 & 2.5 & 57 & 624 & 0.8 & 0.57 & 119.1 & 24 & 10 & 67 & 5 & 312 \\
\hline 94VC1(170-180) & 94VC1 & 170 & 180 & ---- & 36.3 & 3.0 & 65 & 966 & 1.2 & 0.71 & 131.1 & 33 & 12 & 72 & 7 & 334 \\
\hline 94VC1(180-190) & 94VC1 & 180 & 190 & $\mathrm{G}, \mathrm{H}$ & 29.5 & 2.2 & 65 & 824 & 0.8 & 0.64 & 86.8 & 25 & 11 & 40 & 6 & 203 \\
\hline 94VC1(190-200) & 94VC1 & 190 & 200 & $\mathrm{G}, \mathrm{H}$ & 60.0 & 2.8 & 95 & 1138 & 1.0 & 0.92 & 233.4 & 35 & 16 & 71 & 7 & 601 \\
\hline 94VC1(200-207) & 94VC1 & 200 & 207 & $\mathrm{G}, \mathrm{H}$ & 52.3 & 2.5 & 82 & 916 & 1.1 & 0.97 & 218.6 & 35 & 15 & 96 & 6 & 553 \\
\hline $94 \mathrm{VC} 3(0-11)$ & 94VC3 & 0 & 11 & $\mathrm{~F}$ & 15.1 & 5.7 & 106 & 557 & 1.2 & 0.39 & 35.9 & 35 & 9 & $\mathrm{NA}$ & 5 & 106 \\
\hline $94 \mathrm{VC3}(11-22)$ & 94VC3 & 11 & 22 & $\mathrm{~F}$ & 15.6 & 5.9 & 89 & 553 & 1.3 & 0.38 & 31.6 & 42 & 8 & $\overline{N A}$ & 5 & 100 \\
\hline 94VC3(22-33) & 94VC3 & 22 & 33 & $\mathrm{~F}$ & 14.2 & 6.1 & 131 & 570 & 1.4 & 0.46 & 36.5 & 37 & 10 & $\mathrm{NA}$ & 6 & 103 \\
\hline 94VC3(33-44) & 94VC3 & 33 & 44 & $\bar{F}$ & 12.1 & 5.8 & 90 & 523 & 1.3 & 0.38 & 29.3 & 36 & 8 & NA & 5 & 89 \\
\hline 94VC3(44-55) & 94VC3 & 44 & 55 & $\mathrm{~F}$ & 14.4 & 5.5 & 80 & 565 & 1.3 & 0.51 & 33.1 & 36 & 9 & NA & 6 & 102 \\
\hline 94VC3(55-66) & 94VC3 & 55 & 66 & $\mathrm{~F}$ & 16.1 & 5.0 & 86 & 556 & 1.4 & 0.54 & 35.7 & 35 & 10 & NA & 5 & 946 \\
\hline 94VC3(66-77) & 94VC3 & 66 & 77 & $\mathrm{~F}$ & 15.6 & 4.9 & 84 & 554 & 1.3 & 0.54 & 33.6 & 38 & 10 & NA & 5 & 100 \\
\hline 94VC3(77-88) & 94VC3 & 77 & 88 & $\mathrm{~F}$ & 15.5 & 5.2 & 97 & 588 & 1.4 & 0.53 & 34.4 & 35 & 10 & $\mathrm{NA}$ & 5 & 104 \\
\hline 94VC3(88-99) & 94VC3 & 88 & 99 & $F$ & 16.2 & 5.6 & 92 & 613 & 1.2 & 0.55 & 33.8 & 43 & 10 & $\overline{\mathrm{NA}}$ & 6 & 107 \\
\hline 94VC3(99-110) & 94VC3 & 99 & 110 & $F$ & 16.6 & 5.2 & 115 & 644 & 1.1 & 0.58 & 39.5 & 39 & 11 & $\mathrm{NA}$ & 5 & 117 \\
\hline 94VC3(110-120) & 94VC3 & 110 & 120 & $\mathrm{~F}$ & 17.2 & 4.7 & 147 & 611 & 1.1 & 0.56 & 36.5 & 33 & 10 & $\mathrm{NA}$ & 5 & 108 \\
\hline 94VC3(120-130) & 94VC3 & 120 & 130 & $\mathrm{~F}$ & 18.4 & 4.7 & 206 & 635 & 1.3 & 0.58 & 34.9 & 36 & 12 & $\mathrm{NA}$ & 5 & 104 \\
\hline 94VC3(130-140) & 94VC3 & 130 & 140 & $\mathrm{~F}$ & 19.3 & 5.4 & 287 & 731 & 1.4 & 0.63 & 37.0 & 29 & 15 & $\mathrm{NA}$ & 6 & 123 \\
\hline 94VC3(140-150) & 94VC3 & 140 & 150 & $\mathrm{~F}$ & 18.1 & 5.3 & 253 & 667 & 1.2 & 0.56 & 32.7 & 32 & 14 & $\mathrm{NA}$ & 6 & 107 \\
\hline 94VC3(150-160) & 94VC3 & 150 & 160 & $\mathrm{~F}$ & 17.6 & 5.6 & 222 & 639 & 1.5 & 0.53 & 28.4 & 40 & 12 & $\mathrm{NA}$ & 6 & 98 \\
\hline
\end{tabular}


Appendix E. Chemical composition of samples analyzed, using 4 acid dissolution, by ICP-AES and ICP-MS at Eastern Washington University (EWU), Cheney, WA (Appendix_E.xls, Appendix_E.dbf).

\begin{tabular}{|c|c|c|c|c|c|c|c|c|c|c|c|c|c|c|c|c|c|c|}
\hline Sample No. & $\begin{array}{l}\mathrm{Fe} \\
(\%)\end{array}$ & $\begin{array}{c}\mathrm{Ga} \\
(\mathrm{ppm})\end{array}$ & $\begin{array}{c}K \\
(\%)\end{array}$ & $\begin{array}{c}\text { La } \\
(\mathrm{ppm})\end{array}$ & $\begin{array}{l}\mathrm{Mg} \\
(\%)\end{array}$ & $\begin{array}{c}\mathrm{Mn} \\
(\mathrm{ppm})\end{array}$ & $\begin{array}{c}\text { Mo } \\
(\mathrm{ppm})\end{array}$ & $\begin{array}{c}\mathrm{Ni} \\
(\mathrm{ppm})\end{array}$ & $\begin{array}{c}P \\
(p p m)\end{array}$ & $\begin{array}{c}\mathrm{Pb} \\
(\mathrm{ppm})\end{array}$ & $\begin{array}{c}\mathrm{Rb} \\
(\mathrm{ppm})\end{array}$ & $\begin{array}{c}\mathrm{Sb} \\
(\mathrm{ppm})\end{array}$ & $\begin{array}{c}\mathrm{Sn} \\
(\mathrm{ppm})\end{array}$ & $\begin{array}{c}\mathrm{Sr} \\
(\mathrm{ppm})\end{array}$ & $\begin{array}{c}\text { Th } \\
\text { (ppm) }\end{array}$ & $\begin{array}{c}\mathrm{Ti} \\
(\%)\end{array}$ & $\begin{array}{c}\mathrm{TI} \\
(\mathrm{ppm})\end{array}$ & $\begin{array}{c}\mathrm{U} \\
\text { (ppm }\end{array}$ \\
\hline SAMPLE_NO. & FE_\% & GA_PPM & K_\% & LA_PPM & MG_\% & MN_PPM & MO_PPM & NI_PPM & P_PPM & PB_PPM & RB_PPM & SB_PPM & SN_PPM & SR_PPM & TH_PPM & TI_\% & TL_PPM & U_PPM \\
\hline 94VC1(0-10) & 6.83 & 9 & 1.95 & 16 & 0.57 & 5379 & 4 & 13 & 193 & 2981 & 97 & 32 & $\overline{2}$ & 32 & 8 & 0.0679 & 0.6 & 3.2 \\
\hline 94VC1(10-20) & 6.78 & 9 & 1.91 & 12 & 0.59 & 5391 & 2 & 11 & 189 & 3121 & 101 & 34 & 2 & 34 & 8 & 0.0721 & 0.6 & 1.9 \\
\hline 94VC1(20-30) & 8.53 & 8 & 1.70 & 13 & 0.60 & 7179 & 4 & 17 & 170 & 3199 & 91 & 35 & 2 & 31 & 7 & 0.0685 & 0.6 & 1.3 \\
\hline 94VC1(30-40) & 7.12 & 9 & 1.82 & 10 & 0.56 & 5625 & 2 & 11 & 169 & 3285 & 96 & 34 & 2 & 32 & 7 & 0.0750 & 0.6 & 1.6 \\
\hline 94VC1(40-50) & 9.47 & 8 & 1.70 & 9 & 0.64 & 7994 & 3 & 12 & 169 & 3633 & 90 & 40 & 2 & 30 & 7 & 0.0659 & 0.5 & 1.3 \\
\hline 94VC1(60-70) & 10.08 & 8 & 1.76 & 18 & 0.65 & 8566 & 4 & 13 & 175 & 5195 & 94 & 40 & $\overline{2}$ & 33 & 9 & 0.0683 & 0.5 & 1.5 \\
\hline 94VC1 $(70-80)$ & 9.90 & 8 & 1.52 & 11 & 0.60 & 8873 & 2 & 10 & 146 & 5421 & 79 & 48 & $\overline{2}$ & 29 & 7 & 0.0597 & 0.5 & 1.2 \\
\hline 94VC1 $(80-90)$ & 12.11 & 9 & 1.70 & 10 & 0.69 & 10824 & 4 & 14 & 172 & 6639 & 91 & 57 & 3 & 29 & 7 & 0.0609 & 0.6 & 1.4 \\
\hline 94VC1 $(90-97)$ & 11.93 & 8 & 1.53 & 11 & 0.59 & 10918 & 2 & 13 & 169 & 7911 & 84 & 55 & 2 & 30 & 7 & 0.0572 & 0.5 & 1.3 \\
\hline 94VC1(97-110) & 18.82 & 8 & 1.35 & 12 & 0.77 & 16932 & 1 & 17 & 194 & 17922 & 77 & 56 & 3 & 40 & 7 & 0.0563 & 0.5 & 1.8 \\
\hline 94VC1(110-120) & 18.22 & 7 & 1.14 & 8 & 0.73 & 16761 & 2 & 14 & 130 & 14774 & 64 & 92 & 2 & 39 & 5 & 0.0649 & 0.4 & 1.3 \\
\hline 94VC1(120-130) & 19.47 & 7 & 1.18 & 9 & 0.78 & 18139 & 3 & 16 & 146 & 15368 & 69 & 92 & 2 & 42 & 6 & 0.0582 & 0.4 & 1.3 \\
\hline 94VC1(130-140) & 18.48 & 8 & 1.48 & 9 & 0.86 & 17422 & 2 & 17 & 189 & 15673 & 81 & 95 & 3 & 46 & 6 & 0.0738 & 0.5 & 1.6 \\
\hline 94VC1(140-150) & 19.67 & 7 & 1.05 & 7 & 0.81 & 17824 & 3 & 17 & 137 & 16072 & 60 & 87 & 2 & 42 & 5 & 0.0594 & 0.4 & 1.3 \\
\hline 94VC1(170-180) & 17.02 & 8 & 1.37 & 14 & 0.80 & 16573 & 2 & 15 & 161 & 16935 & 80 & 90 & 3 & 41 & 7 & 0.0752 & 0.5 & 1.7 \\
\hline 94VC1(180-190) & 13.80 & 5 & 0.91 & 10 & 0.60 & 12668 & 2 & 14 & 152 & 15269 & 55 & 38 & 2 & 32 & 6 & 0.0522 & 0.4 & 1.5 \\
\hline 94VC1(190-200) & 28.01 & 8 & 1.33 & 14 & 1.08 & 24913 & 2 & 18 & 175 & 24840 & 77 & 121 & 3 & 44 & 8 & 0.0965 & 0.6 & 1.9 \\
\hline 94VC1(200-207) & 28.46 & 7 & 1.19 & 15 & 1.13 & 25996 & 4 & 18 & 154 & 20035 & 69 & 124 & 2 & 42 & 7 & 0.0965 & 0.5 & 1.6 \\
\hline $94 \mathrm{VC} 3(0-11)$ & 15.08 & 7 & 2.51 & 15 & 0.96 & 8559 & 1 & 20 & 279 & 3745 & 71 & 36 & 2 & 28 & 7 & 0.0863 & 0.55 & 1.8 \\
\hline 94VC3(11-22) & 14.75 & 8 & 2.75 & 18 & 0.98 & 8669 & 1 & 12 & 257 & 3810 & 75 & 38 & $<1$ & 27 & 7 & 0.0928 & 0.57 & 1.8 \\
\hline 94VC3(22-33) & 13.83 & 8 & 2.66 & 16 & 0.96 & 7878 & 1 & 13 & 428 & 4265 & 76 & 44 & $<1$ & 34 & 7 & 0.0846 & 0.70 & 1.8 \\
\hline 94VC3(33-44) & 12.60 & 7 & 2.65 & 15 & 0.90 & 7400 & 1 & 12 & 241 & 4105 & $\overline{74}$ & 43 & $\overline{1}$ & 26 & 6 & 0.0699 & 0.56 & $\overline{1.6}$ \\
\hline 94VC3(44-55) & 14.75 & 8 & 2.56 & 15 & 0.91 & 8984 & 1 & 12 & 184 & 4725 & 75 & 44 & 1 & 26 & 6 & 0.0672 & 0.51 & 1.7 \\
\hline 94VC3(55-66) & 16.55 & 7 & 2.35 & 15 & 0.90 & 10365 & 1 & 26 & 181 & 5435 & 67 & 45 & 23 & 25 & 6 & 0.0712 & 0.42 & 1.9 \\
\hline 94VC3(66-77) & 15.37 & 6 & 2.24 & 16 & 0.86 & 9633 & 1 & 15 & 175 & 5480 & 66 & 46 & 1 & 25 & 6 & 0.0694 & 0.41 & 1.7 \\
\hline 94VC3(77-88) & 15.20 & 7 & 2.39 & 15 & 0.92 & 9459 & 1 & 17 & 201 & 5245 & 69 & 43 & $<1$ & 27 & 6 & 0.0813 & 0.46 & $\overline{1.7}$ \\
\hline 94VC3(88-99) & 14.61 & 8 & 2.55 & 18 & 0.94 & 9138 & 1 & 18 & 221 & 5160 & 73 & 41 & $\overline{2}$ & 28 & 7 & 0.0731 & 0.49 & 1.5 \\
\hline 94VC3(99-110) & 17.21 & 7 & 2.43 & 16 & 1.00 & 11026 & 1 & 28 & 197 & 5715 & 70 & 44 & 1 & 27 & 6 & 0.0630 & 0.50 & 1.4 \\
\hline 94VC3(110-120) & 16.68 & 6 & 2.17 & 14 & 0.94 & 10760 & 1 & 23 & 187 & 5830 & 62 & 43 & 1 & 25 & 6 & 0.0559 & 0.42 & 1.3 \\
\hline 94VC3(120-130) & 18.36 & 6 & 2.15 & 15 & 0.97 & 11643 & 1 & 13 & 207 & 6065 & 60 & 42 & 1 & 25 & 6 & 0.0491 & 0.45 & 1.4 \\
\hline 94VC3(130-140) & 19.71 & 7 & 2.42 & 12 & 1.05 & 12266 & 2 & 21 & 261 & 6730 & 68 & 58 & 1 & 29 & 6 & 0.0600 & 0.49 & 1.8 \\
\hline 94VC3(140-150) & 17.14 & 7 & 2.40 & 13 & 0.96 & 10432 & 2 & 22 & 396 & 5615 & 69 & 50 & 2 & 28 & 6 & 0.0763 & 0.44 & 1.7 \\
\hline 94VC3(150-160) & 16.20 & 7 & 2.55 & 17 & 0.97 & 10028 & 3 & 23 & 260 & 4870 & 70 & 66 & 4 & 28 & 7 & 0.0696 & 0.49 & 1.7 \\
\hline
\end{tabular}


Appendix E. Chemical composition of samples analyzed, using 4 acid dissolution, by ICP-AES and ICP-MS at Eastern Washington University (EWU), Cheney, WA (Appendix_E.xls, Appendix_E.dbf).

\begin{tabular}{|l|r|r|r|r|r|}
\hline \multicolumn{1}{|c|}{ Sample No. } & $\begin{array}{c}\text { V } \\
\text { (ppm) }\end{array}$ & $\begin{array}{c}\text { W } \\
(\mathrm{ppm})\end{array}$ & $\begin{array}{c}\mathrm{Y} \\
(\mathrm{ppm})\end{array}$ & $\begin{array}{c}\text { Zn } \\
\text { (ppm) }\end{array}$ & $\begin{array}{c}\text { Zr } \\
(\mathrm{ppm})\end{array}$ \\
\hline SAMPLE_NO. & V_PPM & w_PPM & Y_PPM & ZN_PPM & ZR_PPM \\
\hline 94VC1(0-10) & 31 & 1 & 6 & 3193 & 27 \\
\hline 94VC1(10-20) & 31 & 1 & 6 & 3190 & 28 \\
\hline 94VC1(20-30) & 28 & 1 & 7 & 3995 & 27 \\
\hline 94VC1(30-40) & 29 & 1 & 6 & 3117 & 33 \\
\hline 94VC1(40-50) & 28 & 1 & 7 & 4724 & 28 \\
\hline 94VC1(50-60) & 27 & 1 & 8 & 5275 & 30 \\
\hline 94VC1(60-70) & 28 & 1 & 8 & 5648 & 27 \\
\hline 94VC1(70-80) & 24 & 1 & 7 & 6199 & 25 \\
\hline 94VC1(80-90) & 27 & 1 & 7 & 6777 & 22 \\
\hline 94VC1(90-97) & 24 & 1 & 7 & 7234 & 25 \\
\hline 94VC1(97-110) & 23 & 1 & 10 & 14019 & 29 \\
\hline 94VC1(110-120) & 24 & 2 & 9 & 19947 & 24 \\
\hline 94VC1(120-130) & 24 & 1 & 9 & 20264 & 20 \\
\hline 94VC1(130-140) & 29 & 2 & 10 & 18773 & 25 \\
\hline 94VC1(140-150) & 24 & 1 & 9 & 19937 & 20 \\
\hline 94VC1(150-160) & 28 & 1 & 11 & 19252 & 25 \\
\hline 94VC1(160-170) & 23 & 1 & 8 & 17200 & 28 \\
\hline 94VC1(170-180) & 27 & 1 & 10 & 18410 & 27 \\
\hline 94VC1(180-190) & 21 & 1 & 8 & 12494 & 23 \\
\hline 94VC1(190-200) & 28 & 2 & 13 & 32635 & 31 \\
\hline 94VC1(200-207) & 24 & 1 & 13 & 30090 & 25 \\
\hline 94VC3(0-11) & 26 & 3 & 8 & 6070 & 30 \\
\hline 94VC3(11-22) & 27 & 2 & 8 & 6350 & 31 \\
\hline 94VC3(22-33) & 29 & 1 & 8 & 5655 & 32 \\
\hline 94VC3(33-44) & 25 & 1 & 7 & 5340 & 25 \\
\hline 94VC3(44-55) & 25 & 1 & 7 & 5415 & 24 \\
\hline 94VC3(55-66) & 23 & 2 & 7 & 6520 & 25 \\
\hline 94VC3(66-77) & 22 & 1 & 7 & 6350 & 25 \\
\hline 94VC3(77-88) & 23 & 1 & 7 & 5875 & 31 \\
\hline 94VC3(88-99) & 24 & 1 & 7 & 5630 & 30 \\
\hline 94VC3(99-110) & 23 & 3 & 7 & 6515 & 23 \\
\hline 94VC3(110-120) & 21 & 1 & 6 & 6480 & 22 \\
\hline 94VC3(120-130) & 19 & 2 & 6 & 6345 & 19 \\
\hline 94VC3(130-140) & 24 & 2 & 7 & 5510 & 26 \\
\hline 94VC3(140-150) & 23 & 1 & 7 & 6375 & 26 \\
\hline 94VC3(150-160) & 24 & 1 & 7 & 4880 & 33 \\
\hline
\end{tabular}


Appendix E. Chemical composition of samples analyzed, using 4 acid dissolution, by ICP-AES and ICP-MS at Eastern Washington University (EWU), Cheney, WA (Appendix_E.xls, Appendix_E.dbf).

\begin{tabular}{|c|c|c|c|c|c|c|c|c|c|c|c|c|c|c|c|c|}
\hline Sample No. & Site ID & $\begin{array}{c}\text { Depth } \\
\text { Interval, } \\
\text { top }(\mathrm{cm})\end{array}$ & $\begin{array}{c}\text { Depth } \\
\text { Interval, } \\
\text { bottom }(\mathrm{cm})\end{array}$ & $\begin{array}{c}{ }^{*} \text { Other } \\
\text { analyses } \\
\text { (Appendix \#) }\end{array}$ & $\begin{array}{c}\mathrm{Ag} \\
(\mathrm{ppm})\end{array}$ & $\begin{array}{c}\mathrm{Al} \\
(\%)\end{array}$ & $\begin{array}{c}\text { As } \\
(\mathrm{ppm})\end{array}$ & $\begin{array}{c}\mathrm{Ba} \\
\text { (ppm) }\end{array}$ & $\begin{array}{c}\mathrm{Be} \\
(\mathrm{ppm})\end{array}$ & $\begin{array}{c}\mathrm{Ca} \\
(\%)\end{array}$ & $\mid \begin{array}{c}\text { Cd } \\
(p p m)\end{array}$ & $\begin{array}{c}\mathrm{Ce} \\
(\mathrm{ppm})\end{array}$ & $\begin{array}{c}\text { Co } \\
\text { (ppm) }\end{array}$ & $\begin{array}{c}\mathrm{Cr} \\
\text { (ppm) }\end{array}$ & $\begin{array}{c}\text { Cs } \\
(p p m)\end{array}$ & $\begin{array}{c}\mathrm{Cu} \\
(\mathrm{ppm})\end{array}$ \\
\hline SAMPLE_NO. & $\overline{\text { SITE_ID }}$ & INT_TOP_CM & INT_BTM_CM & OTHR_ANLYS & $\overline{A G_{-} P P M}$ & AL_\% & AS_PPM & BA_PPM & BE_PPM & CA_\% & CD_PPM & CE_PPM & CO_PPM & \begin{tabular}{|l|} 
CR_PPM \\
\end{tabular} & CS_PPM & CU_PPM \\
\hline 94VC3(160-170) & 94VC3 & 160 & 170 & $\bar{F}$ & 23.1 & 5.7 & 520 & 855 & 1.3 & 0.67 & 25.0 & 34 & 22 & $\mathrm{NA}$ & 6 & $\overline{111}$ \\
\hline 94VC3(170-180) & 94VC3 & 170 & 180 & $\mathrm{~F}$ & 25.3 & 6.4 & 419 & 974 & 1.7 & 0.63 & 28.0 & 48 & 22 & $\overline{\mathrm{NA}}$ & 8 & 128 \\
\hline 94VC3(180-190) & 94VC3 & 180 & 190 & $\mathrm{~F}$ & 22.7 & 5.0 & 135 & 739 & 1.3 & 0.63 & 42.0 & 40 & 12 & $\mathrm{NA}$ & 6 & 124 \\
\hline 94VC3(190-200) & 94VC3 & 190 & 200 & $\mathrm{~F}$ & 20.8 & 4.8 & 106 & 641 & 1.4 & 0.59 & 40.5 & 38 & 10 & $\mathrm{NA}$ & 5 & 114 \\
\hline 94VC3(200-210) & 94VC3 & 200 & 210 & $\mathrm{~F}$ & 23.7 & 5.1 & 125 & 738 & 1.3 & 0.73 & 56.2 & 34 & 12 & NA & 5 & 148 \\
\hline 94VC3(210-220) & 94VC3 & 210 & 220 & $\mathrm{~F}$ & 18.2 & 5.8 & 106 & 694 & 1.7 & 0.63 & 44.6 & 40 & 11 & $\mathrm{NA}$ & 6 & 125 \\
\hline 94VC3(220-230) & 94VC3 & 220 & 230 & $\mathrm{~F}$ & 16.6 & 4.4 & 80 & 536 & 1.1 & 0.50 & 33.9 & 31 & 9 & $\mathrm{NA}$ & 5 & 107 \\
\hline 94VCD1 (0-33) & 94VCD1 & 0 & 33 & $-\overline{---}$ & 8.7 & 4.0 & 54 & 428 & 1.5 & 0.17 & 12.0 & 29 & 5 & $\mathrm{NA}$ & 6 & 69 \\
\hline 94VCD1(33-67) & 94VCD1 & 33 & 67 & ---- & 1.2 & 4.0 & 61 & 441 & 1.3 & 0.20 & 15.7 & 39 & 6 & $\mathrm{NA}$ & 6 & 81 \\
\hline 94VCD1(67-100) & 94VCD1 & 67 & 100 & ---- & 0.8 & 3.8 & 55 & 416 & 1.4 & 0.19 & 15.9 & 29 & 5 & $\mathrm{NA}$ & 5 & 76 \\
\hline 94VCD1(133-167) & 94VCD1 & 133 & 167 & ---- & 34.1 & 3.8 & 59 & 939 & 1.7 & 0.57 & 90.1 & 41 & 10 & NA & 7 & 271 \\
\hline 94VCD1(167-200) & 94VCD1 & 167 & 200 & ---- & 29.2 & 3.8 & 40 & 756 & 1.5 & 0.44 & 87.0 & 35 & 9 & $\mathrm{NA}$ & 6 & 256 \\
\hline 94VCD1(200-233) & 94VCD1 & 200 & 233 & ---- & 30.1 & 3.2 & 39 & 711 & 1.2 & 0.49 & 88.0 & 30 & 9 & $\overline{\mathrm{NA}}$ & 6 & 252 \\
\hline 94VCD1(233-267) & 94VCD1 & 233 & 267 & ---- & 45.6 & 4.1 & 56 & 1053 & 1.4 & 0.56 & 89.9 & 39 & 11 & $\mathrm{NA}$ & 8 & 304 \\
\hline 94VCD1(267-282) & 94VCD1 & 267 & 282 & ---- & 28.5 & 4.4 & 29 & 698 & 1.5 & 0.26 & 57.1 & 27 & 6 & $\mathrm{NA}$ & 7 & 194 \\
\hline 94VCD2(0-33) & 94VCD2 & 0 & 33 & ---- & 21.4 & 3.5 & 180 & 486 & 1.2 & 0.30 & 55.0 & 18 & 11 & $\mathrm{NA}$ & 5 & 161 \\
\hline 94VCD2(33-67) & 94VCD2 & 33 & 67 & ---- & 24.6 & 3.1 & 544 & 561 & 1.3 & 0.31 & 61.0 & 29 & 18 & $\mathrm{NA}$ & 6 & 191 \\
\hline 94VCD2(67-100) & 94VCD2 & 67 & 100 & ---- & 20.1 & 4.2 & 319 & 655 & 1.5 & 0.42 & 31.6 & 46 & 17 & $\mathrm{NA}$ & 8 & 159 \\
\hline 94VCD2(100-133) & 94VCD2 & 100 & 133 & $-\cdots$ & 18.5 & 3.3 & 131 & 547 & 1.2 & 0.31 & 38.6 & 20 & 11 & $\mathrm{NA}$ & 5 & 135 \\
\hline 94VCD2(133-167) & 94VCD2 & 133 & 167 & ---- & 23.2 & 3.9 & 78 & 717 & 1.7 & 0.45 & 73.2 & 30 & 11 & $\overline{N A}$ & 6 & 215 \\
\hline 94VCD2(167-200) & 94VCD2 & 167 & 200 & $\begin{array}{ll}--- \\
--1\end{array}$ & 27.1 & 2.6 & 50 & 689 & 1.3 & 0.38 & 73.2 & 27 & 8 & $\mathrm{NA}$ & 4 & 188 \\
\hline 94VCD2(200-233) & 94VCD2 & 200 & 233 & ---- & 26.3 & 2.7 & 62 & 694 & 1.1 & 0.45 & 77.9 & 19 & 9 & $\mathrm{NA}$ & 4 & 194 \\
\hline 94VCD2(233-267) & 94VCD2 & 233 & 267 & ---- & 25.2 & 2.9 & 98 & 538 & 1.2 & 0.36 & 71.8 & 24 & 9 & $\mathrm{NA}$ & 5 & 199 \\
\hline 94VCD2(267-300) & 94VCD2 & 267 & 300 & ---- & 23.5 & 2.8 & 74 & 977 & 1.1 & 0.51 & 61.7 & 28 & 10 & $\mathrm{NA}$ & 5 & 164 \\
\hline 94VCD2(300-325) & 94VCD2 & 300 & 325 & ---- & 27.8 & 2.9 & 77 & 898 & 1.2 & 0.52 & 71.4 & 22 & 10 & $\mathrm{NA}$ & 5 & 200 \\
\hline $94 \operatorname{VCD} 3(0-33)$ & 94VCD3 & 0 & 33 & $\begin{array}{ll}--- \\
\end{array}$ & 1.2 & 5.5 & 10 & 511 & 1.8 & 0.21 & 3.2 & $\mathrm{NA}$ & NA & 32 & NA & 21 \\
\hline 94VCD3(33-67) & 94VCD3 & 33 & 67 & $\begin{array}{ll}--- \\
-\cdots\end{array}$ & 0.21 & 5.5 & 4.9 & 489 & 1.7 & 0.19 & 0.33 & NA & NA & 27 & NA & 14 \\
\hline 94VCD3(67-100) & 94VCD3 & 67 & 100 & $\mathrm{G}, \mathrm{H}$ & 0.19 & 5.6 & 5.2 & 518 & 1.6 & 0.21 & 0.2 & NA & NA & 28 & NA & 15 \\
\hline 94VCD3(100-133) & 94VCD3 & 100 & 133 & ---- & 0.13 & 5.7 & 5.5 & 508 & 1.7 & 0.21 & 0.18 & $\mathrm{NA}$ & $\mathrm{NA}$ & 27 & $\mathrm{NA}$ & 15 \\
\hline 94VCD3(133-167) & 94VCD3 & 133 & 167 & --- & 0.15 & 5.2 & 4.7 & 474 & 1.8 & 0.18 & 0.16 & $\mathrm{NA}$ & $\mathrm{NA}$ & 28 & $\mathrm{NA}$ & $\overline{14}$ \\
\hline 94VCD3(167-175) & 94VCD3 & 167 & 175 & ---- & 0.18 & 5.8 & 6.3 & 516 & 1.7 & 0.19 & 0.2 & $\mathrm{NA}$ & $\mathrm{NA}$ & 29 & NA & 16 \\
\hline 94VCK1 $(0-33)$ & 94VCK1 & 0 & 33 & $\mathrm{G}, \mathrm{H}$ & 10.4 & 3.4 & 92 & 483 & 0.9 & 0.24 & 34.3 & 34 & 8 & $\mathrm{NA}$ & 5 & 104 \\
\hline 94VCK1(33-67) & 94VCK1 & 33 & 67 & ---- & 10.5 & 3.7 & 94 & 534 & 1.0 & 0.25 & 36.8 & 40 & 8 & $\mathrm{NA}$ & 6 & 108 \\
\hline 94VCK1(67-100) & 94VCK1 & 67 & 100 & ---- & 6.2 & 3.7 & 111 & 490 & 0.8 & 0.24 & 26.3 & 39 & 9 & $\mathrm{NA}$ & 6 & 90 \\
\hline 94VCK1(100-133) & 94VCK1 & 100 & 133 & ---- & 9.7 & 3.3 & 101 & 537 & 0.8 & 0.34 & 40.0 & 33 & 11 & $\mathrm{NA}$ & 6 & 117 \\
\hline 94VCK1(133-167) & 94VCK1 & 133 & 167 & ---- & 13.8 & 3.1 & 129 & 303 & 0.8 & 0.39 & 43.3 & 39 & 12 & $\mathrm{NA}$ & 5 & 121 \\
\hline
\end{tabular}


Appendix E. Chemical composition of samples analyzed, using 4 acid dissolution, by ICP-AES and ICP-MS at Eastern Washington University (EWU), Cheney, WA (Appendix_E.xls, Appendix_E.dbf).

\begin{tabular}{|c|c|c|c|c|c|c|c|c|c|c|c|c|c|c|c|c|c|c|}
\hline Sample No. & $\begin{array}{l}\mathrm{Fe} \\
(\%)\end{array}$ & $\begin{array}{c}\mathrm{Ga} \\
(\mathrm{ppm})\end{array}$ & $\begin{array}{c}K \\
(\%)\end{array}$ & $\begin{array}{c}\text { La } \\
(\mathrm{ppm})\end{array}$ & $\begin{array}{l}\mathrm{Mg} \\
(\%)\end{array}$ & $\begin{array}{c}\mathrm{Mn} \\
(\mathrm{ppm})\end{array}$ & $\begin{array}{c}\text { Mo } \\
(\mathrm{ppm})\end{array}$ & $\begin{array}{c}\mathrm{Ni} \\
(\mathrm{ppm})\end{array}$ & $\begin{array}{c}\mathrm{P} \\
(\mathrm{ppm})\end{array}$ & $\begin{array}{c}\mathrm{Pb} \\
\text { (ppm) }\end{array}$ & $\begin{array}{c}\mathrm{Rb} \\
(\mathrm{ppm})\end{array}$ & $\begin{array}{c}\mathrm{Sb} \\
\text { (ppm) }\end{array}$ & $\begin{array}{c}\text { Sn } \\
\text { (ppm) }\end{array}$ & $\begin{array}{c}\mathrm{Sr} \\
\text { (ppm) }\end{array}$ & $\begin{array}{c}\text { Th } \\
\text { (ppm) }\end{array}$ & $\begin{array}{c}\mathrm{Ti} \\
(\%)\end{array}$ & $\begin{array}{c}\mathrm{Tl} \\
\text { (ppm) }\end{array}$ & $\begin{array}{c}U \\
\text { (ppm }\end{array}$ \\
\hline SAMPLE_NO. & FE_\% & GA_PPM & K_\% & LA_PPM & MG_\% & $\begin{array}{l}\text { MN_PPM } \\
\end{array}$ & MO_PPM & NI_PPM & P_PPM & PB_PPM & RB_PPM & SB_PPM & SN_PPM & SR_PPM & TH_PPM & TI_\% & TL_PPM & U_PPM \\
\hline 94VC3(160-170) & 23.20 & 8 & 2.60 & 14 & 1.13 & 14171 & 2 & 33 & 317 & 5950 & 72 & 63 & 3 & 29 & 7 & 0.0523 & 0.54 & 1.8 \\
\hline 94VC3(170-180) & 22.06 & 9 & 2.90 & 20 & 1.08 & 13508 & 3 & 21 & 321 & 6725 & 83 & 72 & 2 & 28 & 8 & 0.0568 & 0.57 & 2.1 \\
\hline 94VC3(180-190) & 18.56 & 7 & 2.26 & 17 & 0.99 & 11659 & 1 & 17 & 230 & 8170 & 64 & 55 & 1 & 29 & 6 & 0.0622 & 0.53 & 1.6 \\
\hline 94VC3(190-200) & 17.23 & 7 & 2.23 & 16 & 0.97 & 11207 & 1 & 12 & 211 & 6985 & 62 & 39 & 1 & 26 & 6 & 0.0595 & 0.47 & $\overline{1.4}$ \\
\hline 94VC3(200-210) & 21.87 & 7 & 2.33 & 14 & 1.17 & 14069 & 1 & 15 & 216 & 8060 & 66 & 56 & 1 & 29 & 6 & 0.0586 & 0.56 & $\overline{1.7}$ \\
\hline 94VC3(220-230) & 13.43 & 6 & 1.97 & 13 & 0.82 & 8423 & 1 & 16 & 177 & 6485 & 56 & 39 & 1 & 23 & 5 & 0.0556 & 0.42 & $\overline{1.4}$ \\
\hline 94VCD1(0-33) & 8.89 & 8 & 1.87 & 12 & 0.64 & 7693 & $\overline{2}$ & 10 & 236 & 2760 & 86 & 34 & $\overline{2}$ & 31 & 6 & 0.0808 & 0.59 & 1.5 \\
\hline 94VCD1(33-67) & 11.25 & 8 & 1.86 & 17 & 0.73 & 10283 & 2 & 9 & 240 & 3125 & 86 & 39 & 2 & 30 & 7 & 0.0765 & 0.57 & 1.5 \\
\hline 94VCD1(67-100) & 10.24 & 8 & 1.81 & 12 & 0.67 & 9444 & 2 & 9 & 245 & 2980 & 80 & 38 & 2 & 28 & 6 & 0.0686 & 0.59 & 1.4 \\
\hline 94VCD1(133-167) & 15.35 & 8 & 1.67 & 17 & 0.77 & 14818 & 1 & 13 & 233 & 14910 & 77 & 63 & 2 & 37 & 7 & 0.0824 & 0.58 & 2.0 \\
\hline 94VCD1(167-200) & 11.74 & 8 & 1.73 & 15 & 0.70 & 11146 & 2 & 12 & 202 & 11460 & 77 & 76 & 2 & 35 & 7 & 0.0994 & 0.53 & 1.9 \\
\hline 94VCD1(200-233) & 10.74 & 7 & 1.34 & 12 & 0.62 & 10345 & 1 & 11 & 168 & 12520 & 68 & 82 & 1 & 36 & 6 & 0.0919 & 0.41 & 1.7 \\
\hline 94VCD1(233-267) & 12.00 & 9 & 1.65 & 16 & 0.69 & 11197 & 2 & 15 & 296 & 20370 & 82 & 114 & 1 & 44 & 8 & 0.0942 & 0.57 & 2.5 \\
\hline 94VCD1(267-282) & 6.91 & 9 & 1.88 & 11 & 0.63 & 5892 & 1 & 11 & 236 & 15210 & 87 & 85 & 2 & 35 & 7 & 0.0897 & 0.57 & 2.1 \\
\hline 94VCD2(33-67) & 14.31 & 7 & 1.32 & 13 & 0.63 & 12376 & 2 & 16 & 515 & 4610 & 65 & 109 & 2 & 23 & 6 & 0.0695 & 0.63 & 2.4 \\
\hline 94VCD2(67-100) & 12.79 & 9 & 1.84 & 19 & 0.63 & 11176 & 3 & 19 & 266 & 6625 & 86 & 72 & 2 & 27 & 8 & 0.0691 & 0.54 & 2.3 \\
\hline 94VCD2(100-133) & 12.15 & 7 & 1.51 & 8 & 0.62 & 11555 & 1 & 12 & 185 & 6170 & 65 & 56 & $\overline{2}$ & 20 & 5 & 0.0484 & 0.47 & 2.6 \\
\hline 94VCD2(133-167) & 17.16 & 8 & 1.79 & 13 & 0.89 & 16945 & 2 & 14 & 219 & 8265 & 79 & 64 & 2 & 29 & 6 & 0.0683 & 0.59 & 2.3 \\
\hline 94VCD2(167-200) & 13.19 & 6 & 1.19 & 11 & 0.66 & 13431 & 2 & 10 & 142 & 9880 & 53 & 57 & 1 & 22 & 4 & 0.0416 & 0.41 & 1.1 \\
\hline 94VCD2(200-233) & 15.26 & 6 & 1.23 & 8 & 0.73 & 15694 & 1 & 11 & 168 & 9975 & 54 & 64 & 2 & 24 & 5 & 0.0520 & 0.47 & 1.3 \\
\hline 94VCD2(233-267) & 15.21 & 6 & 1.37 & 10 & 0.76 & 14996 & 2 & 11 & 167 & 7740 & 60 & 65 & 2 & 22 & 5 & 0.0659 & 0.49 & 1.3 \\
\hline 94VCD2(267-300) & 14.98 & 6 & 1.18 & 12 & 0.69 & 14895 & 2 & 13 & 162 & 9650 & 60 & 49 & 2 & 28 & 5 & 0.0531 & 0.57 & 1.5 \\
\hline 94VCD2(300-325) & 15.47 & 6 & 1.23 & 9 & 0.73 & 15595 & 2 & 13 & 175 & 11290 & 59 & 75 & $<1$ & 30 & 5 & 0.0545 & 0.44 & 1.6 \\
\hline 94VCD3(0-33) & 2.5 & $\mathrm{NA}$ & $\mathrm{NA}$ & $\mathrm{NA}$ & 0.64 & 775 & 0.53 & 13 & & 230 & & 4.2 & & 50 & 11 & 0.1940 & 0.55 & 3.2 \\
\hline 94VCD3(33-67) & 2.1 & $\mathrm{NA}$ & $\mathrm{NA}$ & $\mathrm{NA}$ & 0.63 & 175 & 0.4 & 11 & & 37 & & 1.5 & & 49 & 10 & 0.1920 & 0.52 & 3.1 \\
\hline 94VCD3(67-100) & 2 & $\mathrm{NA}$ & $\mathrm{NA}$ & $\mathrm{NA}$ & 0.64 & 128 & 0.45 & 12 & & 26 & & 1.4 & & 52 & 11 & 0.1825 & 0.53 & 3.4 \\
\hline 94VCD3(100-133) & 2.1 & $\mathrm{NA}$ & $\mathrm{NA}$ & $\mathrm{NA}$ & 0.64 & 123 & 0.43 & 12 & & 25 & & 1.4 & & 54 & 12 & 0.1955 & 0.51 & 3.3 \\
\hline 94VCD3(133-167) & 1.9 & $\mathrm{NA}$ & $\mathrm{NA}$ & $\mathrm{NA}$ & 0.6 & 116 & 0.41 & 11 & & 21 & & 1.3 & & 53 & 11 & 0.1865 & 0.53 & 3.5 \\
\hline 94VCD3(167-175) & 2.2 & $\mathrm{NA}$ & $\mathrm{NA}$ & $\mathrm{NA}$ & 0.64 & 163 & 0.49 & 12 & & 28 & & 1.6 & & 53 & 12 & 0.1960 & 0.54 & 3.6 \\
\hline 94VCK1(0-33) & 9.87 & 9 & 1.51 & 15 & 0.60 & 8801 & 7 & 11 & 187 & 2712 & 74 & 43 & 2 & 26 & 6 & 0.0814 & 0.51 & 1.3 \\
\hline 94VCK1(33-67) & 9.79 & 10 & 1.64 & 18 & 0.63 & 8613 & 11 & 12 & 215 & 3010 & 83 & 46 & 2 & 29 & 7 & 0.0819 & 0.52 & 1.4 \\
\hline 94VCK1(67-100) & 7.70 & 10 & 1.63 & 17 & 0.54 & 6732 & 6 & 11 & 191 & 3123 & 81 & 45 & 4 & 27 & 6 & 0.0811 & 0.50 & 1.4 \\
\hline 94VCK1(100-133) & 10.06 & 9 & 1.41 & 15 & 0.56 & 9150 & 12 & 14 & 166 & 4391 & 73 & 50 & 2 & 25 & 6 & 0.0682 & 0.47 & 1.4 \\
\hline
\end{tabular}


Appendix E. Chemical composition of samples analyzed, using 4 acid dissolution, by ICP-AES and ICP-MS at Eastern Washington University (EWU), Cheney, WA (Appendix_E.xls, Appendix_E.dbf).

\begin{tabular}{|c|c|c|c|c|c|}
\hline Sample No. & $\begin{array}{c}\mathrm{V} \\
(\mathrm{ppm})\end{array}$ & $\begin{array}{c}\text { W } \\
(\mathrm{ppm})\end{array}$ & $\begin{array}{c}\mathrm{Y} \\
(\mathrm{ppm})\end{array}$ & $\begin{array}{c}\mathrm{Zn} \\
(\mathrm{ppm})\end{array}$ & $\begin{array}{c}\mathrm{Zr} \\
(\mathrm{ppm})\end{array}$ \\
\hline SAMPLE_NO. & $\begin{array}{ll}\text { V_PPM } \\
\end{array}$ & W_PPM & Y_PPM & ZN_PPM & ZR_PPM \\
\hline 94VC3(160-170) & 23 & 1 & 7 & 4015 & 25 \\
\hline 94VC3(170-180) & 28 & 1 & 8 & 5035 & 28 \\
\hline 94VC3(180-190) & 23 & 2 & 7 & 8320 & 25 \\
\hline 94VC3(190-200) & 20 & 1 & 7 & 7475 & 24 \\
\hline 94VC3(200-210) & 23 & 1 & 8 & 9510 & 21 \\
\hline 94VC3(210-220) & 24 & 7 & 8 & 7725 & 27 \\
\hline 94VC3(220-230) & 19 & 1 & 6 & 7130 & 23 \\
\hline $94 \mathrm{VCD} 1(0-33)$ & 28 & 1 & 7 & 2605 & 30 \\
\hline 94VCD1(33-67) & 27 & 1 & 8 & 3360 & 29 \\
\hline 94VCD1(67-100) & 24 & 2 & 7 & 3510 & 26 \\
\hline 94VCD1(133-167) & 28 & 1 & 9 & 14630 & 32 \\
\hline 94VCD1(167-200) & 27 & 2 & 8 & 13610 & 31 \\
\hline 94VCD1(200-233) & 24 & 1 & 8 & 16020 & 29 \\
\hline 94VCD1(233-267) & 31 & 2 & 10 & 16850 & 32 \\
\hline 94VCD1(267-282) & 29 & 2 & 7 & 10020 & 30 \\
\hline 94VCD2(0-33) & 26 & 1 & 8 & 9035 & 23 \\
\hline 94VCD2(33-67) & 25 & 2 & 9 & 7605 & 24 \\
\hline 94VCD2(67-100) & 29 & 2 & 8 & 5540 & 24 \\
\hline 94VCD2(100-133) & 21 & 1 & 6 & 7690 & 19 \\
\hline 94VCD2(133-167) & 26 & 1 & 8 & 11720 & 24 \\
\hline 94VCD2(167-200) & 17 & 1 & 6 & 15080 & 14 \\
\hline 94VCD2(200-233) & 18 & 1 & 7 & 14185 & 19 \\
\hline 94VCD2(233-267) & 22 & 3 & 7 & 13290 & 22 \\
\hline 94VCD2(267-300) & 21 & 1 & 8 & 11410 & 22 \\
\hline 94VCD2(300-325) & 21 & 1 & 7 & 12280 & 21 \\
\hline 94VCD3(0-33) & 44 & NA & NA & 510 & NA \\
\hline 94VCD3(33-67) & 42 & NA & NA & 83 & NA \\
\hline 94VCD3(67-100) & 45 & NA & NA & 62 & NA \\
\hline 94VCD3(100-133) & 44 & NA & NA & 55 & NA \\
\hline 94VCD3(133-167) & 42 & NA & NA & 54 & $\mathrm{NA}$ \\
\hline 94VCD3(167-175) & 45 & NA & NA & 65 & $\overline{N A}$ \\
\hline 94VCK1 $10-33)$ & 27 & 1 & 7 & 6015 & 33 \\
\hline 94VCK1(33-67) & 29 & 1 & 7 & 6450 & 36 \\
\hline 94VCK1(67-100) & 29 & 2 & 7 & 4740 & 34 \\
\hline 94VCK1(100-133) & 25 & 1 & 6 & 6650 & 27 \\
\hline 94VCK1(133-167) & 24 & 1 & 7 & 7235 & 40 \\
\hline
\end{tabular}


Appendix E. Chemical composition of samples analyzed, using 4 acid dissolution, by ICP-AES and ICP-MS at Eastern Washington University (EWU), Cheney, WA (Appendix_E.xls, Appendix_E.dbf).

\begin{tabular}{|c|c|c|c|c|c|c|c|c|c|c|c|c|c|c|c|c|}
\hline Sample No. & Site ID & $\begin{array}{c}\text { Depth } \\
\text { Interval, } \\
\text { top (cm) }\end{array}$ & $\begin{array}{c}\text { Depth } \\
\text { Interval, } \\
\text { bottom }(\mathrm{cm})\end{array}$ & $\begin{array}{c}{ }^{*} \text { Other } \\
\text { analyses } \\
\text { (Appendix \#) }\end{array}$ & $\begin{array}{c}\mathrm{Ag} \\
(\mathrm{ppm})\end{array}$ & $\begin{array}{c}\mathrm{Al} \\
(\%)\end{array}$ & $\begin{array}{c}\text { As } \\
(\mathrm{ppm})\end{array}$ & $\begin{array}{c}\mathrm{Ba} \\
\text { (ppm) }\end{array}$ & $\begin{array}{c}\mathrm{Be} \\
(\mathrm{ppm})\end{array}$ & $\begin{array}{l}\mathrm{Ca} \\
(\%)\end{array}$ & $\mid \begin{array}{c}\text { Cd } \\
(p p m)\end{array}$ & $\begin{array}{c}\mathrm{Ce} \\
(\mathrm{ppm})\end{array}$ & $\begin{array}{c}\text { Co } \\
\text { (ppm) }\end{array}$ & $\begin{array}{c}\mathrm{Cr} \\
(\mathrm{ppm})\end{array}$ & $\begin{array}{c}\text { Cs } \\
(\mathrm{ppm})\end{array}$ & $\begin{array}{c}\mathrm{Cu} \\
(\mathrm{ppm})\end{array}$ \\
\hline SAMPLE_NO. & SITE_ID & INT_TOP_CM & INT_BTM_CM & OTHR_ANLYS & AG_PPM & AL_\% & AS_PPM & BA_PPM & BE_PPM & CA_\% & CD_PPM & CE_PPM & CO_PPM & \begin{tabular}{|l|} 
CR_PPM \\
\end{tabular} & CS_PPM & \begin{tabular}{|l|} 
CU_PPM \\
\end{tabular} \\
\hline 94VCK1(167-200) & 94VCK1 & 167 & 200 & ---- & 16.6 & 2.9 & 268 & 370 & 0.7 & 0.43 & 43.6 & 41 & 14 & NA & 5 & 122 \\
\hline 94VCK1(200-233) & 94VCK1 & 200 & 233 & ---- & 13.7 & 3.0 & 288 & 633 & 0.8 & 0.40 & 39.8 & 40 & 15 & NA & 5 & 115 \\
\hline 94VCK1(233-267) & 94VCK1 & 233 & 267 & $\mathrm{G}, \mathrm{H}$ & 14.5 & 3.1 & 99 & 302 & 0.9 & 0.40 & 50.3 & 35 & 10 & NA & 5 & 128 \\
\hline 94VCK1(267-300) & 94VCK1 & 267 & 300 & --- & 15.0 & 3.1 & 94 & 429 & 0.8 & 0.40 & 52.3 & 39 & 10 & NA & 5 & 127 \\
\hline 94VCK1(300-333) & 94VCK1 & 300 & 333 & ---- & 14.7 & 2.9 & 109 & 195 & 0.7 & 0.39 & 45.7 & 37 & 10 & NA & 5 & 121 \\
\hline 94VCK1(333-367) & 94VCK1 & 333 & 367 & ---- & 18.9 & 3.3 & 82 & 497 & 0.7 & 0.50 & 67.5 & 48 & 10 & $\mathrm{NA}$ & 6 & 173 \\
\hline 94VCK1(367-380) & 94VCK1 & 367 & 380 & ---- & 19.7 & 4.5 & 48 & 546 & 0.9 & 0.33 & 52.2 & 49 & 8 & NA & 7 & 172 \\
\hline 94VCK2(0-33) & 94VCK2 & 0 & 33 & $-\overline{---}$ & 9.7 & 3.3 & 107 & 500 & 0.8 & 0.28 & 41.5 & 39 & 9 & NA & 5 & 113 \\
\hline 94VCK2(33-67) & 94VCK2 & 33 & 67 & ---- & 8.0 & 3.9 & 84 & 494 & 0.8 & 0.23 & 30.9 & 45 & 7 & NA & 6 & 88 \\
\hline 94VCK2(67-100) & 94VCK2 & 67 & 100 & --- & 10.7 & 3.1 & 128 & 513 & 0.6 & 0.33 & 45.1 & 38 & 10 & $\mathrm{NA}$ & 5 & 117 \\
\hline 94VCK2(100-133) & 94VCK2 & 100 & 133 & ---- & 11.5 & 3.3 & 111 & 558 & 0.6 & 0.34 & 41.8 & 39 & 10 & $\mathrm{NA}$ & 6 & 109 \\
\hline 94VCK2(133-167) & 94VCK2 & 133 & 167 & ---- & 11.1 & 3.1 & 113 & 536 & 0.7 & 0.33 & 42.3 & 38 & 10 & $\mathrm{NA}$ & 5 & 113 \\
\hline 94VCK2(167-200) & 94VCK2 & 167 & 200 & ---- & 11.4 & 3.0 & 117 & 489 & 1.0 & 0.34 & 41.3 & 37 & 10 & NA & 5 & 114 \\
\hline 94VCK2(200-233) & 94VCK2 & 200 & 233 & ---- & 13.5 & 3.0 & 110 & 507 & 1.0 & 0.35 & 46.5 & 38 & 10 & NA & 5 & 124 \\
\hline 94VCK2(233-267) & 94VCK2 & 233 & 267 & ---- & 13.3 & 3.1 & 105 & 539 & 1.0 & 0.38 & 44.2 & 35 & 11 & NA & 5 & 120 \\
\hline 94VCK2(267-300) & 94VCK2 & 267 & 300 & ---- & 9.9 & 3.2 & 96 & 541 & 1.1 & 0.37 & 39.8 & 32 & 10 & NA & 6 & 111 \\
\hline 94VCK2(300-333) & 94VCK2 & 300 & 333 & $\mathrm{G}, \mathrm{H}$ & 13.1 & 3.0 & 124 & 214 & 1.0 & 0.39 & 41.7 & 28 & 11 & NA & 5 & 119 \\
\hline 94VCK2(333-340) & 94VCK2 & 333 & 340 & --- & 12.6 & 3.4 & 152 & 585 & 1.1 & 0.38 & 35.4 & 37 & 14 & $\mathrm{NA}$ & 6 & 111 \\
\hline 95PCK1(0-15) & 95PCK1 & 0 & 15 & $-\cdots$ & 15.6 & 3.6 & 202 & 570 & 1.3 & 0.32 & 46.1 & 27 & 12 & NA & 5 & 128 \\
\hline 95PCK1(168-193) & 95PCK1 & 168 & 193 & $-\cdots$ & 16.7 & 3.3 & 141 & 655 & 1.3 & 0.44 & 32.8 & 27 & 12 & NA & 5 & 102 \\
\hline 95PCK1(193-204) & 95PCK1 & 193 & 204 & ---- & 16.8 & 3.6 & 205 & 623 & 1.4 & 0.42 & 33.9 & 29 & 14 & NA & 6 & 125 \\
\hline 95PCK1(204-227) & 95PCK1 & 204 & 227 & ---- & 18.3 & 3.5 & 206 & 772 & 1.3 & 0.52 & 32.3 & 33 & 15 & $\mathrm{NA}$ & 6 & 109 \\
\hline 95PCK1(227-229) & 95PCK1 & 227 & 229 & ---- & 24.7 & 4.0 & 353 & 881 & 1.5 & 0.54 & 36.4 & 33 & 21 & NA & 7 & 139 \\
\hline 95PCK1(229-241) & 95PCK1 & 229 & 241 & ---- & 18.5 & 3.2 & 299 & 823 & 1.1 & 0.61 & 23.8 & 25 & 19 & NA & 6 & 96 \\
\hline 95PCK1(241-249) & 95PCK1 & 241 & 249 & ---- & 16.7 & 3.3 & 193 & 744 & 1.2 & 0.50 & 31.6 & 25 & 15 & NA & 6 & 100 \\
\hline 95PCK1(249-250) & 95PCK1 & 249 & 250 & $\begin{array}{ll}--- \\
\end{array}$ & 20.5 & 5.3 & 212 & 958 & 1.5 & 0.50 & 26.7 & 36 & 17 & NA & 10 & 114 \\
\hline 95PCK1(250-260) & 95PCK1 & 250 & 260 & $\begin{array}{ll}--- \\
-\cdots\end{array}$ & 18.1 & 3.3 & 160 & 627 & 1.1 & 0.46 & 34.6 & 20 & 12 & NA & 6 & 119 \\
\hline 95PCK1(260-262) & 95PCK1 & 260 & 262 & $\mathrm{G}, \mathrm{H}$ & 30.2 & 4.4 & 158 & 863 & 1.7 & 0.50 & 65.8 & 29 & 13 & NA & 9 & 181 \\
\hline 95PCK1(262-297) & 95PCK1 & 262 & 297 & --- & 40.8 & 3.3 & $\overline{72}$ & 1057 & 1.4 & 0.61 & 108.7 & 36 & 12 & $\mathrm{NA}$ & 6 & 300 \\
\hline 95PCK1(297-326) & 95PCK1 & 297 & 326 & $\mathrm{G}, \mathrm{H}$ & 76.5 & 5.4 & 65 & 1108 & 1.8 & 0.45 & 92.4 & 51 & 10 & $\mathrm{NA}$ & 10 & 335 \\
\hline 95PCK1(326-343) & 95PCK1 & 326 & 343 & ---- & 0.1 & 6.0 & 8 & 554 & 1.9 & 0.25 & 1.4 & 70 & 5 & NA & 7 & 35 \\
\hline 95PCK1(343-360) & 95PCK1 & 343 & 360 & ---- & 0.1 & 6.1 & 4 & 536 & 2.0 & 0.23 & 0.3 & 60 & 6 & NA & 8 & 17 \\
\hline 95PCK1(360-381) & 95PCK1 & 360 & 381 & ---- & 0.1 & 6.3 & 2 & 507 & 1.7 & 0.18 & 0.2 & 52 & 5 & $\mathrm{NA}$ & 7 & 12 \\
\hline 95PCK1(381-393) & 95PCK1 & 381 & 393 & ---- & 0.1 & 6.0 & 2 & 528 & 1.6 & 0.20 & 0.3 & 60 & 6 & NA & 8 & 13 \\
\hline 95PCK1(393-407) & 95PCK1 & 393 & 407 & ---- & 0.1 & 5.6 & 3 & 523 & 1.7 & 0.17 & 0.1 & 62 & 5 & $\mathrm{NA}$ & 7 & 11 \\
\hline 95PCK1(407-411) & 95PCK1 & 407 & 411 & ---- & 0.1 & 6.1 & 3 & 530 & 1.8 & 0.19 & 0.1 & 75 & 5 & $\mathrm{NA}$ & 8 & 15 \\
\hline
\end{tabular}


Appendix E. Chemical composition of samples analyzed, using 4 acid dissolution, by ICP-AES and ICP-MS at Eastern Washington University (EWU), Cheney, WA (Appendix_E.xls, Appendix_E.dbf).

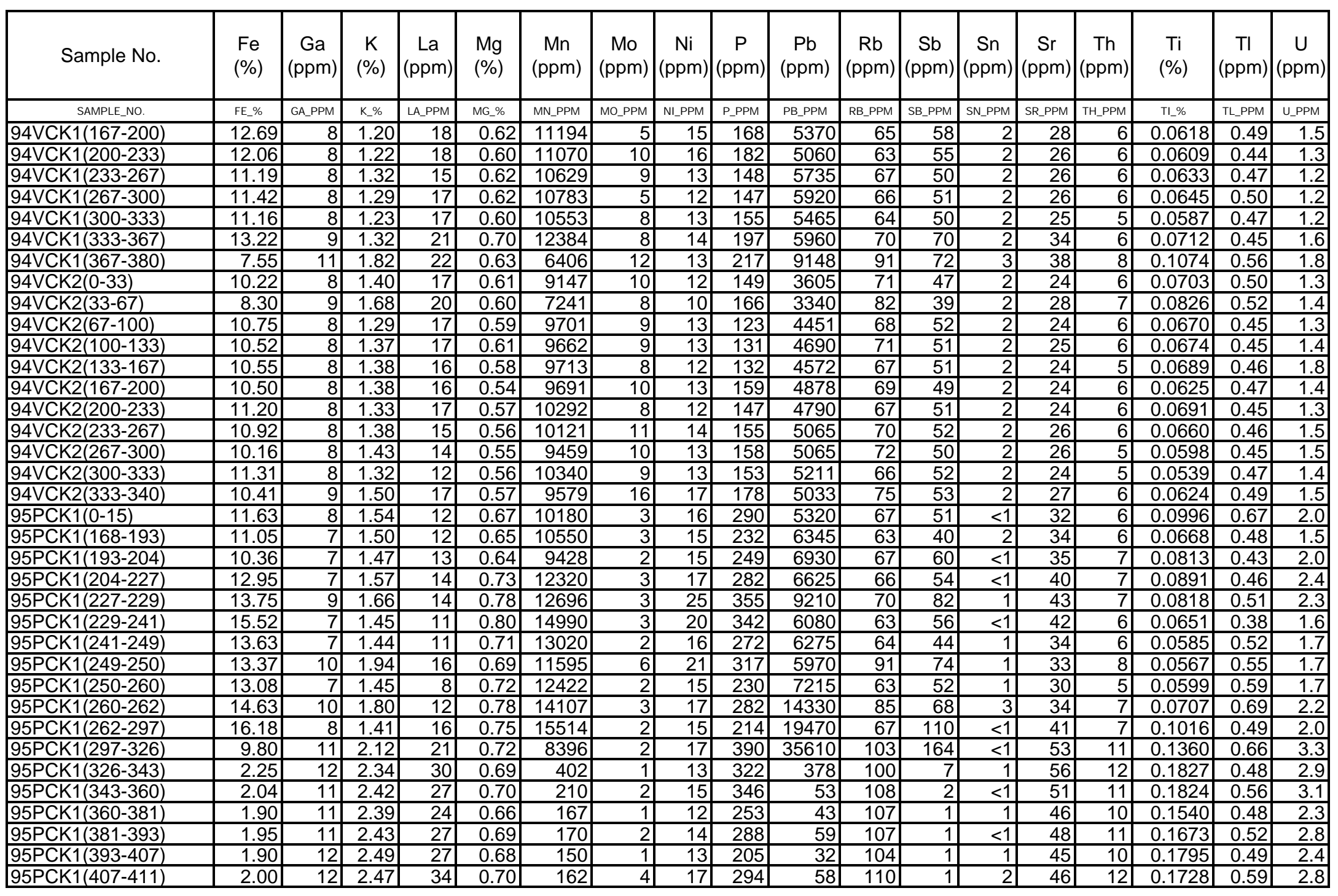


Appendix E. Chemical composition of samples analyzed, using 4 acid dissolution, by ICP-AES and ICP-MS at Eastern Washington University (EWU), Cheney, WA (Appendix_E.xls, Appendix_E.dbf).

\begin{tabular}{|l|r|r|r|r|r|}
\hline Sample No. & $\begin{array}{c}\text { V } \\
(\mathrm{ppm})\end{array}$ & $\begin{array}{c}\text { W } \\
(\mathrm{ppm})\end{array}$ & $\begin{array}{c}\mathrm{Y} \\
(\mathrm{ppm})\end{array}$ & $\begin{array}{c}\mathrm{Zn} \\
(\mathrm{ppm})\end{array}$ & $\begin{array}{c}\mathrm{Zr} \\
(\mathrm{ppm})\end{array}$ \\
\hline SAMPLE_NO. & V_PPM & w_PPM & Y_PPM & ZN_PPM & ZR_PPM \\
\hline 94VCK1(167-200) & 24 & 1 & 7 & 7050 & 33 \\
\hline 94VCK1(200-233) & 21 & 1 & 7 & 6100 & 29 \\
\hline 94VCK1(233-267) & 23 & 1 & 7 & 8750 & 28 \\
\hline 94VCK1(267-300) & 24 & 1 & 7 & 8560 & 29 \\
\hline 94VCK1(300-333) & 23 & 1 & 7 & 7615 & 26 \\
\hline 94VCK1(333-367) & 25 & 1 & 9 & 10620 & 34 \\
\hline 94VCK1(367-380) & 32 & 1 & 9 & 8550 & 44 \\
\hline 94VCK2(0-33) & 24 & 1 & 7 & 5765 & 27 \\
\hline 94VCK2(33-67) & 28 & 1 & 7 & 7190 & 34 \\
\hline 94VCK2(67-100) & 24 & 3 & 6 & 8160 & 26 \\
\hline 94VCK2(100-133) & 24 & 1 & 7 & 7105 & 30 \\
\hline 94VCK2(133-167) & 24 & 1 & 6 & 7525 & 29 \\
\hline 94VCK2(167-200) & 22 & 1 & 6 & 7350 & 28 \\
\hline 94VCK2(200-233) & 22 & 1 & 7 & 8435 & 28 \\
\hline 94VCK2(233-267) & 23 & 2 & 7 & 7570 & 32 \\
\hline 94VCK2(267-300) & 23 & 1 & 6 & 7345 & 28 \\
\hline 94VCK2(300-333) & 22 & 2 & 6 & 7390 & 25 \\
\hline 94VCK2(333-340) & 22 & 3 & 7 & 6035 & 32 \\
\hline 95PCK1(0-15) & 29 & 1 & 8 & 8115 & 32 \\
\hline 95PCK1(168-193) & 22 & 1 & 7 & 6005 & 25 \\
\hline 95PCK1(193-204) & 23 & 1 & 8 & 5610 & 34 \\
\hline 95PCK1(204-227) & 25 & 1 & 7 & 5495 & 37 \\
\hline 95PCK1(227-229) & 29 & 1 & 9 & 6010 & 35 \\
\hline 95PCK1(229-241) & 24 & 1 & 7 & 4165 & 31 \\
\hline 95PCK1(241-249) & 23 & 1 & 7 & 5610 & 28 \\
\hline 95PCK1(249-250) & 27 & 1 & 8 & 5405 & 27 \\
\hline 95PCK1(250-260) & 23 & 1 & 7 & 6615 & 24 \\
\hline 95PCK1(260-262) & 29 & 2 & 9 & 12120 & 30 \\
\hline 95PCK1(262-297) & 27 & 2 & 10 & 18850 & 38 \\
\hline 95PCK1(297-326) & 41 & 4 & 13 & 17310 & 55 \\
\hline 95PCK1(326-343) & 43 & 2 & 12 & 361 & 57 \\
\hline 95PCK1(343-360) & 43 & 2 & 13 & 112 & 57 \\
\hline 95PCK1(360-381) & 41 & 1 & 11 & 83 & 48 \\
\hline 95PCK1(381-393) & 43 & 1 & 11 & 92 & 52 \\
\hline 95PCK1(393-407) & 43 & 2 & 9 & 82 & 49 \\
\hline 95PCK1(407-411) & 43 & 1 & 12 & 148 & 52 \\
\hline
\end{tabular}


Appendix E. Chemical composition of samples analyzed, using 4 acid dissolution, by ICP-AES and ICP-MS at Eastern Washington University (EWU), Cheney, WA (Appendix_E.xls, Appendix_E.dbf).

\begin{tabular}{|c|c|c|c|c|c|c|c|c|c|c|c|c|c|c|c|c|}
\hline Sample No. & Site ID & $\begin{array}{c}\text { Depth } \\
\text { Interval, } \\
\text { top }(\mathrm{cm})\end{array}$ & $\begin{array}{c}\text { Depth } \\
\text { Interval, } \\
\text { bottom }(\mathrm{cm})\end{array}$ & $\begin{array}{c}{ }^{*} \text { Other } \\
\text { analyses } \\
\text { (Appendix \#) }\end{array}$ & $\begin{array}{c}\mathrm{Ag} \\
(\mathrm{ppm})\end{array}$ & $\begin{array}{c}\text { Al } \\
(\%)\end{array}$ & $\begin{array}{c}\text { As } \\
\text { (ppm) }\end{array}$ & $\begin{array}{c}\mathrm{Ba} \\
(\mathrm{ppm})\end{array}$ & $\begin{array}{c}\mathrm{Be} \\
\text { (ppm) }\end{array}$ & $\begin{array}{l}\mathrm{Ca} \\
(\%)\end{array}$ & $\begin{array}{c}\text { Cd } \\
(p p m)\end{array}$ & $\begin{array}{c}\mathrm{Ce} \\
(\mathrm{ppm})\end{array}$ & $\begin{array}{c}\text { Co } \\
\text { (ppm) }\end{array}$ & $\begin{array}{c}\mathrm{Cr} \\
\text { (ppm) }\end{array}$ & $\begin{array}{c}\text { Cs } \\
\text { (ppm) }\end{array}$ & $\begin{array}{c}\mathrm{Cu} \\
\text { (ppm) }\end{array}$ \\
\hline SAMPLE_NO. & SITE_ID & INT_TOP_CM & INT_BTM_CM & OTHR_ANLYS & AG_PPM & AL_\% & AS_PPM & BA_PPM & BE_PPM & CA_\% & CD_PPM & CE_PPM & CO_PPM & CR_PPM & CS_PPM & CU_PPM \\
\hline 95PCK1(411-415) & 95PCK1 & 411 & 415 & ---- & 0.1 & 7.7 & 3 & 590 & 1.9 & 0.20 & 0.1 & 86 & 6 & $\overline{\mathrm{NA}}$ & 9 & 14 \\
\hline 95PCK1(415-418) & 95PCK1 & 415 & 418 & $\mathrm{G}, \mathrm{H}$ & 1.4 & 6.1 & 23 & 541 & 1.7 & 0.22 & 4.3 & 66 & 6 & $\overline{N A}$ & 7 & 25 \\
\hline 95PCUD2(0-25) & 95PCUD2 & 0 & 25 & $\mathrm{G}, \mathrm{H}$ & 44.6 & 6.0 & 92 & 892 & 2.3 & 0.47 & 101.5 & 36 & $\overline{13}$ & $\mathrm{NA}$ & $\overline{11}$ & 366 \\
\hline 95PCUD2(25-50) & 95PCUD2 & 25 & 50 & ---- & 34.5 & 5.4 & 73 & 592 & 2.0 & 0.25 & 79.8 & 37 & $\overline{11}$ & $\mathrm{NA}$ & 9 & 288 \\
\hline 95PCUD2(50-75) & 95PCUD2 & 50 & 75 & ---- & 13.1 & 6.2 & 15 & 537 & 1.7 & 0.20 & 11.6 & 57 & 6 & $\mathrm{NA}$ & 8 & 50 \\
\hline 95PCUD2(75-100) & 95PCUD2 & 75 & 100 & ---- & 8.2 & 5.7 & 15 & 529 & 2.0 & 0.19 & 8.4 & 49 & 5 & $\mathrm{NA}$ & $\overline{7}$ & 39 \\
\hline 95PCUD2(100-125) & 95PCUD2 & 100 & 125 & ---- & 2.5 & 5.7 & 4 & 516 & 1.8 & 0.19 & 2.3 & 64 & 4 & $\mathrm{NA}$ & 7 & 21 \\
\hline 95PCUD2(125-150) & 95PCUD2 & 125 & 150 & $-\overline{---}$ & 0.5 & 5.7 & 3 & 508 & 1.6 & 0.19 & 1.1 & 72 & 4 & $\mathrm{NA}$ & 8 & 17 \\
\hline 95PCUD2(150-165) & 95PCUD2 & 150 & 165 & ---- & 0.1 & 6.0 & 4 & 525 & 1.7 & 0.22 & 0.4 & 72 & 5 & $\mathrm{NA}$ & 8 & 18 \\
\hline 95VCD3 $(0-28)$ & 95VCD3 & 0 & 28 & ---- & 12.7 & 4.1 & 71 & 481 & 1.5 & 0.22 & 24.9 & 32 & 7 & $\mathrm{NA}$ & 6 & 102 \\
\hline 95VCD3(28-56) & 95VCD3 & 28 & 56 & ---- & 17.4 & 3.7 & 218 & 523 & 1.2 & 0.27 & 40.5 & 32 & 10 & $\mathrm{NA}$ & 6 & 131 \\
\hline 95VCD3(56-84) & 95VCD3 & 56 & 84 & ---- & 20.3 & 3.2 & 147 & 646 & 1.4 & 0.38 & 49.6 & 25 & 10 & $\mathrm{NA}$ & 5 & 154 \\
\hline 95VCD3(84-112) & 95VCD3 & 84 & 112 & ---- & 16.7 & 3.8 & 159 & 659 & 1.5 & 0.35 & 43.4 & 29 & 10 & $\mathrm{NA}$ & 6 & 155 \\
\hline 95VCD3(112-140) & 95VCD3 & 112 & 140 & ---- & 27.3 & 4.0 & 100 & 1115 & 1.8 & 0.63 & 103.1 & 41 & 13 & $\mathrm{NA}$ & 7 & 282 \\
\hline 95VCD3(140-158.5) & 95VCD3 & 140 & 158.5 & ---- & 23.6 & 3.3 & 83 & 914 & 1.2 & 0.49 & 72.2 & 33 & 10 & $\mathrm{NA}$ & 6 & 202 \\
\hline 95VCD3(158.5-177) & 95VCD3 & 158.5 & 177 & ---- & 23.8 & 3.0 & 71 & 1000 & 1.3 & 0.53 & 64.3 & 31 & 10 & NA & 5 & 180 \\
\hline 95VCD3(177-195.5) & 95VCD3 & 177 & 195.5 & ---- & 26.4 & 3.7 & 92 & 1140 & 1.5 & 0.62 & 46.3 & 29 & 13 & $\mathrm{NA}$ & 7 & 178 \\
\hline 95VCD3(195.5-214) & 95VCD3 & 195.5 & 214 & $\mathrm{G}, \mathrm{H}$ & 28.1 & 3.2 & 64 & 791 & 1.3 & 0.49 & 81.0 & 30 & 10 & NA & 6 & 232 \\
\hline 95VCD3(214-230) & 95VCD3 & 214 & 230 & ---- & 26.5 & 3.3 & 62 & 755 & 1.4 & 0.49 & 82.2 & 38 & 9 & NA & 5 & 223 \\
\hline 95VCD3(230-246) & 95VCD3 & 230 & 246 & ---- & 22.1 & 3.7 & 54 & 672 & 1.2 & 0.45 & 69.6 & 35 & 9 & $\mathrm{NA}$ & 6 & 201 \\
\hline 95VCD3(246-262) & 95VCD3 & 246 & 262 & ---- & 28.5 & 3.2 & 47 & 630 & 1.4 & 0.48 & 92.7 & 26 & 9 & NA & 5 & 261 \\
\hline 95VCD3(262-277) & 95VCD3 & 262 & 277 & ---- & 26.3 & 3.1 & 58 & 776 & 1.3 & 0.51 & 87.2 & 28 & 10 & NA & 5 & 248 \\
\hline 95VCD3(277-298) & 95VCD3 & 277 & 298 & ---- & 27.2 & 3.4 & 57 & 832 & 1.1 & 0.56 & 92.6 & 29 & 11 & NA & 6 & 264 \\
\hline 95VCD3(298-319) & 95VCD3 & 298 & 319 & ---- & 24.8 & 3.3 & 61 & 805 & 1.5 & 0.50 & 82.1 & 29 & 9 & $\mathrm{NA}$ & 6 & 229 \\
\hline 95VCD3(319-340) & 95VCD3 & 319 & 340 & $\begin{array}{ll}--- \\
\end{array}$ & 27.6 & 3.5 & 54 & 735 & 1.5 & 0.55 & 92.2 & 33 & 10 & $\overline{N A}$ & 6 & 257 \\
\hline 95VCD3(340-360) & 95VCD3 & 340 & 360 & $\begin{array}{ll}--- \\
\end{array}$ & 28.3 & 3.6 & 59 & 899 & 1.6 & 0.56 & 96.6 & 31 & 11 & $\mathrm{NA}$ & 6 & 265 \\
\hline 95VCD3(360-381) & 95VCD3 & 360 & 381 & $\begin{array}{ll}--- \\
\end{array}$ & 33.8 & 4.4 & 70 & 1126 & 1.7 & 0.67 & 128.0 & 40 & 13 & $\mathrm{NA}$ & 8 & 371 \\
\hline 95VCD3(381-402) & 95VCD3 & 381 & 402 & --- & 22.1 & 4.7 & 68 & 902 & 1.7 & 0.46 & 114.5 & 37 & 12 & $\mathrm{NA}$ & 8 & 397 \\
\hline 95VCD3(402-423) & 95VCD3 & 402 & 423 & $\mathrm{G}, \mathrm{H}$ & 18.2 & 5.1 & 50 & 709 & 1.9 & 0.32 & 61.0 & 42 & 8 & $\mathrm{NA}$ & 8 & 235 \\
\hline 95VCD3(423-547) & 95VCD3 & 423 & 547 & --- & 6.7 & 6.2 & 15 & 562 & 2.1 & 0.22 & 9.9 & 45 & 5 & $\mathrm{NA}$ & 8 & 54 \\
\hline 95VCD3(547-572) & 95VCD3 & 547 & 572 & ---- & 1.8 & 5.6 & 8 & 537 & 1.9 & 0.21 & 2.9 & 57 & 5 & $\mathrm{NA}$ & 8 & 32 \\
\hline 95VCUD1(0-25) & 95VCUD1 & 0 & 25 & ---- & 7.8 & 4.2 & 47 & 457 & 1.5 & 0.16 & 10.4 & 34 & 5 & $\mathrm{NA}$ & 6 & 63 \\
\hline 95VCUD1(25-50) & 95VCUD1 & 25 & 50 & $\mathrm{G}, \mathrm{H}$ & 7.3 & 3.5 & 42 & 413 & 1.0 & 0.13 & 10.6 & 32 & 5 & $\mathrm{NA}$ & 5 & 56 \\
\hline 95VCUD1(50-75) & 95VCUD1 & 50 & 75 & --- & 6.8 & 3.7 & 53 & 432 & 1.2 & 0.15 & 15.6 & 41 & 5 & $\mathrm{NA}$ & 6 & 61 \\
\hline 95VCUD1(75-100) & 95VCUD1 & 75 & 100 & ---- & 9.7 & 3.2 & 42 & 380 & 1.1 & 0.16 & 17.9 & 29 & 5 & $\mathrm{NA}$ & 5 & 66 \\
\hline 95VCUD1(100-125) & 95VCUD1 & 100 & 125 & ---- & 10.1 & 3.5 & 43 & 408 & 1.2 & 0.20 & 22.7 & 25 & 6 & $\mathrm{NA}$ & 5 & 87 \\
\hline
\end{tabular}


Appendix E. Chemical composition of samples analyzed, using 4 acid dissolution, by ICP-AES and ICP-MS at Eastern Washington University (EWU), Cheney, WA (Appendix_E.xls, Appendix_E.dbf).

\begin{tabular}{|c|c|c|c|c|c|c|c|c|c|c|c|c|c|c|c|c|c|c|}
\hline Sample No. & $\begin{array}{c}\mathrm{Fe} \\
(\%)\end{array}$ & $\begin{array}{c}\mathrm{Ga} \\
(\mathrm{ppm})\end{array}$ & $\begin{array}{c}\mathrm{K} \\
(\%)\end{array}$ & $\begin{array}{c}\text { La } \\
(\mathrm{ppm})\end{array}$ & $\begin{array}{l}\mathrm{Mg} \\
(\%)\end{array}$ & $\begin{array}{c}\mathrm{Mn} \\
(\mathrm{ppm})\end{array}$ & $\begin{array}{c}\text { Mo } \\
(\mathrm{ppm})\end{array}$ & $\begin{array}{c}\mathrm{Ni} \\
(\mathrm{ppm})\end{array}$ & $\begin{array}{c}\mathrm{P} \\
(\mathrm{ppm})\end{array}$ & $\begin{array}{c}\mathrm{Pb} \\
(\mathrm{ppm})\end{array}$ & $\begin{array}{c}\mathrm{Rb} \\
(\mathrm{ppm})\end{array}$ & $\begin{array}{c}\mathrm{Sb} \\
(\mathrm{ppm})\end{array}$ & $\begin{array}{c}\mathrm{Sn} \\
(\mathrm{ppm})\end{array}$ & $\begin{array}{c}\mathrm{Sr} \\
(\mathrm{ppm})\end{array}$ & $\begin{array}{c}\text { Th } \\
\text { (ppm) }\end{array}$ & $\begin{array}{c}\mathrm{Ti} \\
(\%)\end{array}$ & $\begin{array}{c}\mathrm{TI} \\
(\mathrm{ppm})\end{array}$ & $\begin{array}{c}\mathrm{U} \\
(\mathrm{ppm})\end{array}$ \\
\hline SAMPLE_NO. & FE_\% & GA_PPM & K_\% & LA_PPM & MG_\% & MN_PPM & MO_PPM & NI_PPM & P_PPM & PB_PPM & RB_PPM & SB_PPM & SN_PPM & SR_PPM & TH_PPM & TI_\% & TL_PPM & U_PPM \\
\hline 95PCK1(411-415) & 2.24 & 13 & 2.78 & 38 & 0.80 & 164 & 5 & 18 & 271 & 34 & 126 & 2 & 2 & 52 & $\overline{14}$ & 0.2216 & 0.65 & 2.9 \\
\hline 95PCK1(415-418) & 3.10 & 10 & 2.13 & 29 & 0.67 & 1399 & 2 & 14 & 298 & 1025 & 98 & 7 & 1 & 45 & 11 & 0.1544 & 0.41 & 2.6 \\
\hline 95PCUD2(0-25) & 12.73 & 12 & 2.38 & 15 & 0.83 & 10970 & 3 & 20 & 414 & 38520 & 106 & 259 & $<1$ & 47 & 10 & 0.1323 & 0.75 & 3.9 \\
\hline 95PCUD2(25-50) & 7.54 & 11 & 2.11 & 15 & 0.60 & 5573 & 1 & 16 & 393 & 36460 & 96 & 199 & $<1$ & 38 & 9 & 0.1300 & 0.65 & 3.6 \\
\hline 95PCUD2(50-75) & 2.80 & 11 & 2.39 & 24 & 0.69 & 866 & 2 & 13 & 397 & 5210 & 101 & 29 & 1 & 47 & 11 & 0.1620 & 0.53 & 3.6 \\
\hline 95PCUD2(100-125) & 2.02 & 11 & 2.27 & 27 & 0.65 & 334 & 1 & 13 & 225 & 922 & 101 & 6 & 1 & 48 & 11 & 0.1759 & 0.47 & 3.4 \\
\hline 95PCUD2(125-150) & 1.92 & 11 & 2.28 & 30 & 0.65 & 216 & 1 & 12 & 211 & 498 & 96 & 4 & 1 & 49 & 11 & 0.1723 & 0.43 & 3.4 \\
\hline 95PCUD2(150-165) & 1.92 & 11 & 2.33 & 31 & 0.69 & 149 & 1 & 14 & 265 & 170 & 103 & 2 & 2 & 51 & 12 & 0.1822 & 0.54 & 3.8 \\
\hline 95VCD3(0-28) & 10.49 & 9 & 2.07 & 14 & 0.70 & 9668 & 4 & 13 & 224 & 3460 & 90 & 40 & 2 & 29 & 6 & 0.0780 & 0.55 & 1.7 \\
\hline 95VCD3(28-56) & 12.12 & 7 & 1.77 & 14 & 0.68 & 11285 & 2 & 13 & 362 & 4850 & 75 & 55 & 2 & 26 & 7 & 0.0724 & 0.58 & 2.3 \\
\hline 95VCD3(56-84) & 13.06 & 7 & 1.52 & 11 & 0.67 & 12985 & 2 & 14 & 246 & 6470 & 67 & 53 & 1 & 26 & 5 & 0.0605 & 0.50 & 1.7 \\
\hline 95VCD3(84-112) & 13.47 & 8 & 1.86 & 13 & 0.74 & 13137 & 2 & 15 & 290 & 5615 & 80 & 52 & 2 & 27 & 6 & 0.0623 & 0.60 & 1.8 \\
\hline 95VCD3(112-140) & 20.57 & 9 & 1.94 & 17 & 1.01 & 20911 & 3 & 18 & 226 & 11630 & 85 & 81 & 1 & 38 & 7 & 0.0836 & 0.55 & 2.1 \\
\hline 95VCD3(140-158.5) & 14.60 & 6 & 1.48 & 14 & 0.76 & 15197 & 2 & 16 & 206 & 10960 & 69 & 65 & $<1$ & 30 & 6 & 0.0703 & 0.45 & 1.8 \\
\hline 95VCD3(177-195.5) & 17.52 & 8 & 1.68 & 12 & 0.81 & 18183 & 2 & 20 & 259 & 13620 & 79 & 87 & 1 & 32 & 7 & 0.0591 & 0.54 & 2.5 \\
\hline 95VCD3(195.5-214) & 15.25 & $\overline{6}$ & 1.47 & 13 & 0.78 & 15670 & $\overline{3}$ & 15 & 168 & 10540 & 66 & 70 & $<1$ & 28 & 6 & 0.0740 & 0.42 & 1.7 \\
\hline 95VCD3(214-230) & 14.88 & 7 & 1.55 & 16 & 0.80 & 15443 & 1 & 14 & 178 & 10770 & 68 & 60 & $<1$ & 29 & 6 & 0.0670 & 0.38 & 1.6 \\
\hline 95VCD3(230-246) & 12.55 & 7 & 1.76 & 15 & 0.75 & 12880 & 2 & 14 & 184 & 9240 & 77 & 64 & $<1$ & 32 & 7 & 0.1028 & 0.48 & 1.9 \\
\hline 95VCD3(246-262) & 14.49 & 7 & 1.48 & 11 & 0.79 & 14578 & 2 & 13 & 154 & 10410 & 65 & 70 & $<1$ & 30 & 5 & 0.0682 & 0.44 & 1.5 \\
\hline 95VCD3(262-277) & 14.30 & 7 & 1.44 & 12 & 0.75 & 14297 & 1 & 16 & 172 & 10820 & 63 & 67 & $<1$ & 31 & 6 & 0.0748 & 0.43 & 1.7 \\
\hline 95VCD3(277-298) & 15.29 & 7 & 1.53 & 12 & 0.81 & 15279 & 2 & 17 & 173 & 11080 & 70 & 70 & $<1$ & 34 & 6 & 0.0778 & 0.44 & 1.6 \\
\hline 95VCD3(298-319) & 14.59 & 7 & 1.58 & 12 & 0.78 & 14702 & 2 & 14 & 186 & 10410 & 68 & 61 & $<1$ & 31 & 6 & 0.0637 & 0.48 & 1.5 \\
\hline 95VCD3(319-340) & 15.24 & 7 & 1.58 & 14 & 0.81 & 14712 & $\overline{2}$ & 15 & 232 & 13210 & 67 & 52 & $\overline{2}$ & 35 & 7 & 0.0890 & 0.50 & 2.4 \\
\hline 95VCD3(340-360) & 15.53 & 8 & 1.63 & 13 & 0.85 & 15314 & 3 & 16 & 212 & 13660 & 70 & 78 & 1 & 36 & 7 & 0.0842 & 0.48 & 2.0 \\
\hline 95VCD3(360-381) & 15.58 & 10 & 1.91 & 17 & 0.89 & 15524 & 2 & 17 & 251 & 17510 & 94 & 127 & 2 & 46 & 8 & 0.1062 & 0.63 & 2.5 \\
\hline 95VCD3(381-402) & 12.39 & 10 & 2.08 & 16 & 0.81 & 11663 & 3 & 16 & 276 & 23540 & 92 & 157 & 2 & 40 & 8 & 0.1072 & 0.59 & 2.4 \\
\hline 95VCD3(402-423) & 7.18 & 10 & 2.14 & 18 & 0.69 & 5659 & 1 & 15 & 300 & 26570 & 89 & 127 & 2 & 42 & 9 & 0.1305 & 0.59 & 2.9 \\
\hline 95VCD3(423-547) & 2.89 & 11 & 2.67 & 20 & 0.73 & 998 & $\overline{3}$ & 17 & 339 & 3680 & 108 & 24 & 2 & 45 & 10 & 0.1590 & 0.55 & 2.9 \\
\hline 95VCD3(547-572) & 2.36 & 11 & 2.47 & 24 & 0.65 & 447 & 1 & 17 & 382 & 965 & 91 & 7 & 2 & 47 & 10 & 0.1729 & 0.54 & 3.4 \\
\hline 95VCUD1 $(0-25)$ & 6.43 & $\overline{9}$ & 2.03 & 15 & 0.56 & 5302 & 3 & 12 & 208 & 2780 & 89 & 34 & $<1$ & 35 & 7 & 0.0810 & 0.51 & 1.4 \\
\hline 95VCUD1(25-50) & 5.12 & 8 & 1.68 & 14 & 0.46 & 4130 & 2 & 10 & 166 & 2375 & 78 & 65 & $<1$ & 31 & 5 & 0.0652 & 0.34 & 1.3 \\
\hline 95VCUD1(50-75) & 5.51 & 8 & 1.80 & 17 & 0.51 & 4401 & 4 & 13 & 216 & 3505 & 79 & 38 & $<1$ & 34 & 6 & 0.1062 & 0.49 & 2.0 \\
\hline 95VCUD1(75-100) & 6.40 & 7 & 1.52 & 12 & 0.49 & 5669 & 2 & 9 & 167 & 3690 & 67 & 39 & $<1$ & 27 & 5 & 0.0727 & 0.39 & 1.3 \\
\hline
\end{tabular}


Appendix E. Chemical composition of samples analyzed, using 4 acid dissolution, by ICP-AES and ICP-MS at Eastern Washington University (EWU), Cheney, WA (Appendix_E.xls, Appendix_E.dbf).

\begin{tabular}{|l|r|r|r|r|r|}
\hline Sample No. & $\begin{array}{c}\text { V } \\
(\mathrm{ppm})\end{array}$ & $\begin{array}{c}\text { W } \\
(\mathrm{ppm})\end{array}$ & $\begin{array}{c}\mathrm{Y} \\
(\mathrm{ppm})\end{array}$ & $\begin{array}{c}\text { Zn } \\
(\mathrm{ppm})\end{array}$ & $\begin{array}{c}\mathrm{Zr} \\
(\mathrm{ppm})\end{array}$ \\
\hline \multicolumn{1}{|c|}{ SAMPLE_NO. } & V_PPM & w_PPM & Y_PPM & ZN_PPM & ZR_PPM \\
\hline 95PCK1(411-415) & 47 & 2 & 14 & 83 & 66 \\
\hline 95PCK1(415-418) & 38 & 1 & 13 & 1052 & 52 \\
\hline 95PCUD2(0-25) & 44 & 12 & 12 & 16910 & 48 \\
\hline 95PCUD2(25-50) & 41 & 12 & 11 & 15840 & 50 \\
\hline 95PCUD2(50-75) & 42 & 4 & 12 & 2285 & 52 \\
\hline 95PCUD2(75-100) & 40 & 2 & 11 & 1465 & 52 \\
\hline 95PCUD2(100-125) & 41 & 2 & 12 & 521 & 54 \\
\hline 95PCUD2(125-150) & 40 & 2 & 11 & 315 & 51 \\
\hline 95PCUD2(150-165) & 42 & 1 & 14 & 171 & 61 \\
\hline 95VCD3(0-28) & 29 & 1 & 7 & 5015 & 28 \\
\hline 95VCD3(28-56) & 26 & 1 & 8 & 6470 & 27 \\
\hline 95VCD3(56-84) & 24 & 1 & 7 & 8615 & 22 \\
\hline 95VCD3(84-112) & 27 & 1 & 8 & 7030 & 21 \\
\hline 95VCD3(112-140) & 29 & 1 & 10 & 16560 & 31 \\
\hline 95VCD3(140-158.5) & 22 & 1 & 9 & 13970 & 28 \\
\hline 95VCD3(158.5-177) & 22 & 1 & 8 & 12020 & 25 \\
\hline 95VCD3(177-195.5) & 26 & 1 & 9 & 9325 & 30 \\
\hline 95VCD3(195.5-214) & 23 & 1 & 8 & 15280 & 28 \\
\hline 95VCD3(214-230) & 23 & 1 & 8 & 15750 & 25 \\
\hline 95VCD3(230-246) & 26 & 2 & 9 & 12810 & 39 \\
\hline 95VCD3(246-262) & 24 & 1 & 8 & 16980 & 23 \\
\hline 95VCD3(262-277) & 23 & 1 & 8 & 15730 & 25 \\
\hline 95VCD3(277-298) & 27 & 1 & 9 & 16420 & 25 \\
\hline 95VCD3(298-319) & 25 & 1 & 8 & 15050 & 22 \\
\hline 95VCD3(319-340) & 26 & 1 & 10 & 15980 & 29 \\
\hline 95VCD3(340-360) & 27 & 2 & 9 & 16360 & 29 \\
\hline 95VCD3(360-381) & 34 & 2 & 11 & 20050 & 36 \\
\hline 95VCD3(381-402) & 33 & 2 & 10 & 19410 & 38 \\
\hline 95VCD3(402-423) & 36 & 3 & 10 & 11680 & 48 \\
\hline 95VCD3(423-547) & 44 & 2 & 10 & 1525 & 51 \\
\hline 95VCD3(547-572) & 44 & 2 & 10 & 596 & 54 \\
\hline 95VCUD1(0-25) & 29 & 1 & 7 & 2185 & 31 \\
\hline 95VCUD1(25-50) & 24 & 1 & 6 & 2340 & 26 \\
\hline 95VCUD1(50-75) & 25 & 2 & 8 & 2715 & 39 \\
\hline 95VCUD1(75-100) & 21 & 1 & 6 & 3620 & 28 \\
\hline 95VCUD1(100-125) & 22 & 1 & 6 & 4845 & 25 \\
\hline
\end{tabular}


Appendix E. Chemical composition of samples analyzed, using 4 acid dissolution, by ICP-AES and ICP-MS at Eastern Washington University (EWU), Cheney, WA (Appendix_E.xls, Appendix_E.dbf).

\begin{tabular}{|c|c|c|c|c|c|c|c|c|c|c|c|c|c|c|c|c|}
\hline Sample No. & Site ID & $\begin{array}{c}\text { Depth } \\
\text { Interval, } \\
\text { top }(\mathrm{cm})\end{array}$ & $\begin{array}{c}\text { Depth } \\
\text { Interval, } \\
\text { bottom }(\mathrm{cm})\end{array}$ & $\begin{array}{c}{ }^{*} \text { Other } \\
\text { analyses } \\
\text { (Appendix \#) }\end{array}$ & $\begin{array}{c}\mathrm{Ag} \\
(\mathrm{ppm})\end{array}$ & $\begin{array}{c}\text { Al } \\
(\%)\end{array}$ & $\begin{array}{c}\text { As } \\
\text { (ppm) }\end{array}$ & $\begin{array}{c}\mathrm{Ba} \\
(\mathrm{ppm})\end{array}$ & $\begin{array}{c}\mathrm{Be} \\
(\mathrm{ppm})\end{array}$ & $\begin{array}{l}\mathrm{Ca} \\
(\%)\end{array}$ & $\begin{array}{c}\text { Cd } \\
\text { (ppm) }\end{array}$ & $\begin{array}{c}\mathrm{Ce} \\
(\mathrm{ppm})\end{array}$ & $\begin{array}{c}\text { Co } \\
\text { (ppm) }\end{array}$ & $\begin{array}{c}\mathrm{Cr} \\
\text { (ppm) }\end{array}$ & $\begin{array}{c}\text { Cs } \\
\text { (ppm) }\end{array}$ & $\begin{array}{c}\mathrm{Cu} \\
\text { (ppm) }\end{array}$ \\
\hline SAMPLE_NO. & SITE_ID & INT_TOP_CM & INT_BTM_CM & OTHR_ANLYS & AG_PPM & AL_\% & AS_PPM & BA_PPM & BE_PPM & CA_\% & CD_PPM & CE_PPM & CO_PPM & CR_PPM & CS_PPM & CU_PPM \\
\hline 95VCUD1(125-150) & 95VCUD1 & 125 & 150 & ---- & 12.7 & 3.4 & 45 & 417 & 1.3 & 0.21 & 25.3 & 31 & 7 & $\overline{\mathrm{NA}}$ & 5 & 90 \\
\hline 95VCUD1(150-175) & 95VCUD1 & 150 & 175 & ---- & 14.3 & 3.6 & 53 & 445 & 1.3 & 0.24 & 31.9 & 34 & 6 & $\mathrm{NA}$ & 5 & 104 \\
\hline 95VCUD1(175-200) & 95VCUD1 & 175 & 200 & ---- & 14.8 & 3.3 & 48 & 437 & 1.3 & 0.25 & 34.8 & 28 & 7 & $\mathrm{NA}$ & 5 & 112 \\
\hline 95VCUD1(200-224) & 95VCUD1 & 200 & 224 & ---- & 17.2 & 3.7 & 49 & 592 & 1.4 & 0.36 & 54.6 & 29 & $\overline{9}$ & $\mathrm{NA}$ & $\overline{6}$ & 159 \\
\hline 95VCUD1(224-245) & 95VCUD1 & 224 & 245 & ---- & 18.6 & 3.0 & 41 & 485 & 1.1 & 0.32 & 55.1 & 28 & 7 & $\mathrm{NA}$ & 5 & 147 \\
\hline 95VCUD1(245-266) & 95VCUD1 & 245 & 266 & ---- & 35.7 & 3.7 & 74 & 970 & 1.4 & 0.76 & 118.3 & 30 & $\overline{13}$ & $\mathrm{NA}$ & $\overline{8}$ & 302 \\
\hline 95VCUD1(266-286) & 95VCUD1 & 266 & 286 & $\mathrm{G}, \mathrm{H}$ & 27.6 & 3.9 & 50 & 808 & 1.3 & 0.55 & 87.1 & 30 & 10 & $\mathrm{NA}$ & 8 & 248 \\
\hline 95VCUD1(286-306) & 95VCUD1 & 286 & 306 & --- & 21.8 & 3.7 & 40 & 683 & 1.1 & 0.50 & 72.8 & 27 & $\overline{9}$ & $\mathrm{NA}$ & 6 & 233 \\
\hline 95VCUD1(311-328) & 95VCUD1 & 311 & 328 & --- & 26.1 & 3.3 & 36 & 692 & 1.1 & 0.45 & 78.0 & 33 & 8 & $\mathrm{NA}$ & 5 & 244 \\
\hline 95VCUD1(328-344) & 95VCUD1 & 328 & 344 & ---- & 25.8 & 3.1 & 39 & 647 & 1.0 & 0.41 & 72.9 & 28 & 8 & $\mathrm{NA}$ & 5 & 229 \\
\hline 95VCUD1(344-360) & 95VCUD1 & 344 & 360 & ---- & 28.3 & 3.1 & 37 & 694 & 1.1 & 0.46 & 80.1 & 35 & 8 & $\mathrm{NA}$ & 5 & 246 \\
\hline 95VCUD1(360-377) & 95VCUD1 & 360 & 377 & ---- & 24.7 & 3.7 & 37 & 757 & 1.5 & 0.46 & 74.5 & 39 & 8 & $\mathrm{NA}$ & 6 & 231 \\
\hline 95VCUD1(377-393) & 95VCUD1 & 377 & 393 & ---- & 33.2 & 3.2 & 42 & 821 & 1.2 & 0.45 & 73.4 & 27 & 8 & $\mathrm{NA}$ & 6 & 220 \\
\hline 95VCUD1(393-409) & 95VCUD1 & 393 & 409 & ---- & 32.4 & 3.6 & 54 & 827 & 1.2 & 0.49 & 86.8 & 30 & 9 & $\mathrm{NA}$ & 6 & 278 \\
\hline 95VCUD1(409-435) & 95VCUD1 & 409 & 435 & ---- & 33.1 & 3.2 & 46 & 821 & 1.2 & 0.47 & 104.1 & 33 & 9 & $\mathrm{NA}$ & 5 & 291 \\
\hline 95VCUD1(435-461) & 95VCUD1 & 435 & 461 & ---- & 41.2 & 3.3 & 55 & 1169 & 1.1 & 0.50 & 120.9 & 37 & 10 & NA & 6 & 333 \\
\hline 95VCUD1(461-486) & 95VCUD1 & 461 & 486 & ---- & 42.7 & 3.6 & 58 & 928 & 1.1 & 0.49 & 126.7 & 31 & 11 & $\mathrm{NA}$ & 6 & 400 \\
\hline 95VCUD1(486-512) & 95VCUD1 & 486 & 512 & ---- & 27.1 & 3.0 & 38 & 632 & 1.2 & 0.40 & 79.8 & 24 & 8 & NA & 5 & 233 \\
\hline 95VCUD1(512-538) & 95VCUD1 & 512 & 538 & ---- & 28.3 & 3.5 & 41 & 762 & 1.3 & 0.44 & 86.8 & 29 & 9 & $\mathrm{NA}$ & 6 & 252 \\
\hline 95VCUD1(538-564) & 95VCUD1 & 538 & 564 & ---- & 24.5 & 3.1 & 30 & 625 & 1.1 & 0.35 & 68.4 & 25 & 7 & $\mathrm{NA}$ & 5 & 195 \\
\hline 95VCUD1(564-589) & 95VCUD1 & 564 & 589 & ---- & 7.8 & 4.8 & 41 & 804 & 1.8 & 0.49 & 91.5 & 27 & 10 & NA & 7 & 264 \\
\hline $96 \mathrm{~K} 75 \mathrm{E}(0-10)$ & 96K75E & 0 & 10 & $\begin{array}{ll}--- \\
\end{array}$ & 0.3 & 8.2 & 9 & 773 & 2.6 & 0.28 & $<0.5$ & 90 & 13 & 78 & 13 & 28 \\
\hline 94Gid2(0-30) & 94Gid2 & 0 & 30 & $\mathrm{G}, \mathrm{H}$ & 11.9 & 4.3 & 140 & 623 & 1.4 & 0.33 & 22.0 & 57 & 7 & NA & 6 & 110 \\
\hline 94Gid2(30-60) & 94Gid2 & 30 & 60 & $\mathrm{G}, \mathrm{H}$ & 10.4 & 4.0 & 152 & 599 & 1.2 & 0.25 & 38.3 & 42 & 7 & $\mathrm{NA}$ & 6 & 105 \\
\hline 94Gid2(60-90) & 94Gid2 & 60 & 90 & $\mathrm{G}, \mathrm{H}$ & 10.7 & 2.7 & 459 & 478 & 0.8 & 0.29 & 33.1 & 39 & 17 & $\overline{\mathrm{NA}}$ & 5 & 112 \\
\hline 94Gid2(90-142) & 94Gid2 & 90 & 142 & ---- & 13.0 & 2.6 & 402 & 263 & 0.8 & 0.35 & 29.8 & 38 & 15 & $\mathrm{NA}$ & 4 & 114 \\
\hline 94Gid2(142-181) & 94Gid2 & 142 & 181 & $\begin{array}{ll}--- \\
\end{array}$ & 17.8 & 3.3 & 122 & 348 & 1.2 & 0.51 & 46.2 & 37 & 13 & $\mathrm{NA}$ & 6 & 140 \\
\hline 94Gid2(181-215) & 94Gid2 & 181 & 215 & ---- & 12.8 & 2.7 & 370 & 550 & 0.9 & 0.34 & 31.6 & 38 & 14 & $\mathrm{NA}$ & 5 & 116 \\
\hline 94Gid2(215-250) & 94Gid2 & 215 & 250 & --- & 17.2 & 2.5 & 212 & 389 & 0.8 & 0.46 & 54.2 & 45 & 12 & $\mathrm{NA}$ & 4 & 143 \\
\hline 94Gid2(250-280) & 94Gid2 & 250 & 280 & $\mathrm{G}, \mathrm{H}$ & 40.2 & 3.7 & 69 & 175 & 1.2 & 0.57 & 96.4 & 37 & 12 & $\mathrm{NA}$ & 8 & 221 \\
\hline 94Gid3(0-3) & 94Gid3 & 0 & 3 & --- & 12.3 & 3.8 & 129 & 581 & 1.2 & 0.24 & 18.4 & 48 & 9 & $\mathrm{NA}$ & 6 & 104 \\
\hline 94Gid3(3-12) & 94Gid3 & 3 & 12 & $\mathrm{G}, \mathrm{H}$ & 11.6 & 3.8 & 125 & 642 & 1.0 & 0.16 & 25.5 & 54 & 9 & $\mathrm{NA}$ & 6 & 121 \\
\hline 94Gid3(12-50) & 94Gid3 & 12 & 50 & ---- & 10.7 & 3.8 & 245 & 584 & 1.0 & 0.10 & 23.9 & 55 & 14 & $\mathrm{NA}$ & 6 & 110 \\
\hline 94Gid3(50-67.5) & 94Gid3 & 50 & 67.5 & ---- & 15.8 & 3.7 & 139 & 700 & 1.2 & 0.16 & 15.6 & 48 & 10 & $\mathrm{NA}$ & 6 & 123 \\
\hline 94Gid3(122-151) & 94Gid3 & 122 & 151 & ---- & 29.8 & 4.2 & 100 & 1106 & 1.3 & 0.20 & 17.3 & 49 & 6 & $\mathrm{NA}$ & 8 & 255 \\
\hline 94Gid3(151-190) & 94Gid3 & 151 & 190 & --- & 0.2 & 6.6 & 6 & 520 & 1.5 & 0.20 & 6.0 & 75 & 17 & $\mathrm{NA}$ & 9 & 19 \\
\hline
\end{tabular}


Appendix E. Chemical composition of samples analyzed, using 4 acid dissolution, by ICP-AES and ICP-MS at Eastern Washington University (EWU), Cheney, WA (Appendix_E.xls, Appendix_E.dbf).

\begin{tabular}{|c|c|c|c|c|c|c|c|c|c|c|c|c|c|c|c|c|c|c|}
\hline Sample No. & $\begin{array}{c}\mathrm{Fe} \\
(\%)\end{array}$ & $\begin{array}{c}\mathrm{Ga} \\
(\mathrm{ppm})\end{array}$ & $\begin{array}{c}\mathrm{K} \\
(\%)\end{array}$ & $\begin{array}{c}\text { La } \\
(\mathrm{ppm})\end{array}$ & $\begin{array}{l}\mathrm{Mg} \\
(\%)\end{array}$ & $\begin{array}{c}\mathrm{Mn} \\
(\mathrm{ppm})\end{array}$ & $\begin{array}{c}\text { Mo } \\
(\mathrm{ppm})\end{array}$ & $\begin{array}{c}\mathrm{Ni} \\
(\mathrm{ppm})\end{array}$ & $\begin{array}{c}\mathrm{P} \\
(\mathrm{ppm})\end{array}$ & $\begin{array}{c}\mathrm{Pb} \\
(\mathrm{ppm})\end{array}$ & $\begin{array}{c}\mathrm{Rb} \\
(\mathrm{ppm})\end{array}$ & $\begin{array}{c}\mathrm{Sb} \\
(\mathrm{ppm})\end{array}$ & $\begin{array}{c}\mathrm{Sn} \\
(\mathrm{ppm})\end{array}$ & $\begin{array}{c}\mathrm{Sr} \\
(\mathrm{ppm})\end{array}$ & $\begin{array}{c}\text { Th } \\
\text { (ppm) }\end{array}$ & $\begin{array}{c}\mathrm{Ti} \\
(\%)\end{array}$ & $\begin{array}{c}\text { TI } \\
\text { (ppm) }\end{array}$ & $\underset{(\mathrm{ppm}}{\mathrm{U}}$ \\
\hline SAMPLE_NO. & FE_\% & GA_PPM & K_\% & LA_PPM & MG_\% & MN_PPM & MO_PPM & NI_PPM & P_PPM & PB_PPM & RB_PPM & SB_PPM & SN_PPM & SR_PPM & TH_PPM & TI_\% & TL_PPM & U_PPM \\
\hline 95VCUD1(125-150) & 8.43 & 7 & 1.61 & 13 & 0.58 & 7882 & 2 & 10 & 153 & 4680 & 72 & 40 & $<1$ & 27 & 6 & 0.0660 & 0.43 & $\overline{1.4}$ \\
\hline 95VCUD1(150-175) & 10.35 & 7 & 1.70 & 15 & 0.68 & 9968 & 3 & 11 & 170 & 5210 & 74 & 44 & $<1$ & 27 & 6 & 0.0635 & 0.44 & 1.5 \\
\hline 95VCUD1(175-200) & 10.20 & 7 & 1.57 & 12 & 0.65 & 9843 & 2 & 10 & 154 & 5525 & 71 & 47 & $<1$ & 27 & 5 & 0.0588 & 0.40 & 1.3 \\
\hline 95VCUD1(200-224) & 11.23 & 8 & 1.69 & 12 & 0.71 & 11062 & 3 & 13 & 168 & 7350 & 78 & 53 & $<1$ & 32 & 6 & 0.0726 & 0.47 & $\overline{1.6}$ \\
\hline 95VCUD1(224-245) & 11.02 & 6 & 1.38 & 12 & 0.66 & 11110 & 2 & 10 & 135 & 7410 & 61 & 47 & $<1$ & 25 & 5 & 0.0688 & 0.39 & 1.3 \\
\hline 95VCUD1(266-286) & 12.90 & $\overline{9}$ & 1.78 & 13 & 0.77 & 12326 & 2 & 14 & 179 & 11850 & 90 & 82 & $<1$ & 38 & 6 & 0.0806 & 0.49 & $\overline{1.8}$ \\
\hline 95VCUD1(286-306) & 12.16 & 8 & 1.70 & 12 & 0.72 & 11856 & $\overline{2}$ & 13 & 151 & 8725 & 79 & 68 & $<1$ & 35 & 6 & 0.0903 & 0.48 & 1.5 \\
\hline 95VCUD1(311-328) & 12.36 & 7 & 1.50 & 14 & 0.69 & 11776 & 1 & 11 & 143 & 9480 & 66 & 63 & 1 & 31 & 6 & 0.0686 & 0.44 & 1.3 \\
\hline 95VCUD1(328-344) & 11.39 & 7 & 1.44 & 12 & 0.65 & 10983 & 2 & 11 & 136 & 9105 & 64 & 64 & 1 & 30 & 5 & 0.0692 & 0.39 & 1.6 \\
\hline 95VCUD1(344-360) & 12.11 & 7 & 1.42 & 15 & 0.67 & 11714 & 1 & 12 & 139 & 10630 & 65 & 68 & 1 & 32 & 6 & 0.0851 & 0.40 & 1.5 \\
\hline 95VCUD1(360-377) & 11.26 & 8 & 1.65 & 17 & 0.68 & 10991 & 2 & 13 & 179 & 10380 & 74 & 65 & 1 & 36 & 7 & 0.0914 & 0.44 & 1.8 \\
\hline 95VCUD1(377-393) & 11.50 & 7 & 1.37 & 11 & 0.63 & 11174 & 1 & 12 & 168 & 15160 & 65 & 77 & 1 & 31 & 6 & 0.0686 & 0.43 & 1.6 \\
\hline 95VCUD1(393-409) & 12.07 & 8 & 1.61 & 13 & 0.69 & 11851 & 2 & 14 & 186 & 13210 & 75 & 78 & 2 & 34 & 6 & 0.0727 & 0.49 & 1.6 \\
\hline 95VCUD1(409-435) & 11.94 & 7 & 1.42 & 14 & 0.67 & 11995 & 2 & 12 & 155 & 12760 & 65 & 78 & 1 & 32 & 6 & 0.0697 & 0.41 & 1.5 \\
\hline 95VCUD1(461-486) & 14.38 & 8 & 1.64 & 13 & 0.77 & 14031 & 2 & 14 & 190 & 15660 & 74 & 86 & 2 & 34 & 6 & 0.0752 & 0.57 & $\overline{1.8}$ \\
\hline 95VCUD1(486-512) & 10.94 & $\overline{6}$ & 1.35 & 10 & 0.63 & 10951 & 2 & 12 & 138 & 9810 & 60 & 61 & $\overline{2}$ & 29 & 5 & 0.0628 & 0.43 & $\overline{1.3}$ \\
\hline 95VCUD1(512-538) & 11.51 & 7 & 1.59 & 12 & 0.68 & 11274 & 2 & 13 & 165 & 9970 & 73 & 64 & $\overline{2}$ & 34 & 6 & 0.0781 & 0.49 & 1.5 \\
\hline 95VCUD1( & 9.51 & 6 & 1.42 & 11 & 0.59 & 9198 & 3 & 12 & 137 & 8625 & 64 & 43 & $\overline{1}$ & 30 & 5 & 0.0623 & 0.46 & 1.2 \\
\hline 95VCUD1 & 13.05 & 10 & 2.28 & 11 & 0.88 & 12482 & 2 & 16 & 225 & 9850 & 99 & 72 & 2 & 46 & 7 & 0.0942 & 0.72 & 1.7 \\
\hline $96 \mathrm{~K} 75 \mathrm{E}(0-$ & 2.26 & 20 & 3.21 & 37 & 1.02 & 139 & 2 & 26 & 174 & 59 & 177 & 3 & 4 & 77 & 20 & 0.2782 & 1.0 & 4.9 \\
\hline 94Gid2(0-30) & 9.42 & 11 & 1.59 & 25 & 0.58 & 6901 & 16 & 13 & 536 & 5142 & 79 & 47 & 3 & 52 & 9 & 0.1617 & 0.79 & 2.6 \\
\hline 94Gid2(30-60) & 8.93 & 9 & 1.53 & 19 & 0.56 & 6671 & 10 & 13 & 428 & 4695 & 78 & 54 & 3 & 38 & 7 & 0.0937 & 0.63 & 2.2 \\
\hline 94Gid2(60-90) & 12.92 & 7 & 1.08 & 18 & 0.57 & 10713 & 8 & 16 & 282 & 3560 & 55 & 75 & $\overline{2}$ & 24 & 6 & 0.0627 & 0.52 & $\overline{1.8}$ \\
\hline 94Gid2(90-142) & 13.84 & 6 & 1.08 & 16 & 0.60 & 11923 & 7 & 15 & 161 & 4943 & 55 & 67 & $\overline{2}$ & 22 & 5 & 0.0588 & 0.39 & 1.3 \\
\hline 94Gid2(142-181) & 13.04 & 8 & 1.31 & 16 & 0.68 & 12301 & 8 & 18 & 236 & 9704 & 68 & 71 & $\overline{2}$ & 33 & 7 & 0.0643 & 0.59 & 1.9 \\
\hline 94Gid2(181-215) & 12.59 & 7 & 1.11 & 17 & 0.58 & 10790 & 8 & 15 & 195 & 5119 & 56 & 69 & 2 & 24 & 6 & 0.0590 & 0.45 & 1.5 \\
\hline 94Gid2(215-250) & 14.89 & 6 & 1.01 & 19 & 0.68 & 13421 & 5 & 13 & 151 & 8038 & 52 & 65 & 2 & 27 & 6 & 0.0584 & 0.40 & 1.3 \\
\hline 94Gid2(250-280) & 12.46 & 10 & 1.41 & 16 & 0.71 & $\overline{11416}$ & 5 & 15 & 230 & 18020 & 77 & 106 & $\overline{3}$ & 42 & 7 & 0.0814 & 0.51 & 2.1 \\
\hline 94Gid3(0-3) & 9.23 & 9 & 1.41 & 22 & 0.54 & 7621 & 33 & 16 & 354 & 4935 & 70 & 48 & $\overline{2}$ & 39 & 7 & 0.0984 & 0.59 & 2.0 \\
\hline 94Gid3(3-12) & 11.55 & $\overline{9}$ & 1.56 & 24 & 0.46 & 10179 & 13 & 12 & 193 & 4070 & 75 & 54 & $\overline{2}$ & 28 & 7 & 0.0830 & 0.53 & 1.7 \\
\hline 94Gid3(12-50) & 10.99 & 9 & 1.44 & 24 & 0.31 & 9112 & 9 & 15 & 227 & 5970 & 71 & 60 & 3 & 24 & 8 & 0.0826 & 0.49 & 2.1 \\
\hline 94Gid3(50-67.5) & 12.02 & 9 & 1.46 & 21 & 0.45 & 10248 & 6 & 13 & 235 & 7310 & 72 & 67 & 3 & 27 & 7 & 0.0701 & 0.51 & 2.1 \\
\hline 94Gid3(122-151) & 11.52 & 10 & 1.55 & 21 & 0.57 & 7902 & 8 & 12 & 248 & 17750 & 80 & 140 & 3 & 33 & 8 & 0.0913 & 0.61 & 2.2 \\
\hline
\end{tabular}


Appendix E. Chemical composition of samples analyzed, using 4 acid dissolution, by ICP-AES and ICP-MS at Eastern Washington University (EWU), Cheney, WA (Appendix_E.xls, Appendix_E.dbf).

\begin{tabular}{|l|r|r|r|r|r|}
\hline \multicolumn{1}{|c|}{ Sample No. } & $\begin{array}{c}\text { V } \\
\text { (ppm) }\end{array}$ & $\begin{array}{c}\text { W } \\
(\mathrm{ppm})\end{array}$ & $\begin{array}{c}\mathrm{Y} \\
(\mathrm{ppm})\end{array}$ & $\begin{array}{c}\text { Zn } \\
\text { (ppm) }\end{array}$ & $\begin{array}{c}\text { Zr } \\
(\mathrm{ppm})\end{array}$ \\
\hline \multicolumn{1}{|c|}{ SAMPLE_NO. } & V_PPM & w_PPM & Y_PPM & ZN_PPM & ZR_PPM \\
\hline 95VCUD1(125-150) & 21 & 1 & 7 & 5405 & 32 \\
\hline 95VCUD1(150-175) & 21 & 1 & 7 & 6710 & 29 \\
\hline 95VCUD1(175-200) & 22 & 1 & 7 & 6825 & 25 \\
\hline 95VCUD1(200-224) & 25 & 2 & 8 & 9270 & 29 \\
\hline 95VCUD1(224-245) & 20 & 1 & 7 & 11250 & 27 \\
\hline 95VCUD1(245-266) & 30 & 5 & 11 & 19540 & 29 \\
\hline 95VCUD1(266-286) & 30 & 1 & 9 & 13380 & 30 \\
\hline 95VCUD1(286-306) & 26 & 2 & 8 & 11840 & 27 \\
\hline 95VCUD1(311-328) & 23 & 1 & 7 & 13470 & 19 \\
\hline 95VCUD1(328-344) & 22 & 1 & 7 & 13230 & 21 \\
\hline 95VCUD1(344-360) & 23 & 1 & 8 & 15240 & 27 \\
\hline 95VCUD1(360-377) & 25 & 1 & 9 & 13280 & 31 \\
\hline 95VCUD1(377-393) & 22 & 1 & 7 & 15120 & 24 \\
\hline 95VCUD1(393-409) & 25 & 3 & 7 & 14860 & 25 \\
\hline 95VCUD1(409-435) & 21 & 1 & 7 & 18020 & 23 \\
\hline 95VCUD1(435-461) & 22 & 1 & 8 & 21240 & 28 \\
\hline 95VCUD1(461-486) & 24 & 1 & 8 & 19570 & 26 \\
\hline 95VCUD1(486-512) & 19 & 2 & 6 & 14420 & 20 \\
\hline 95VCUD1(512-538) & 23 & 1 & 7 & 14250 & 26 \\
\hline 95VCUD1(538-564) & 19 & 1 & 6 & 13120 & 21 \\
\hline 95VCUD1(564-589) & 32 & 2 & 8 & 13860 & 29 \\
\hline 96K75E(0-10) & 85 & 3 & 18 & 105 & 98 \\
\hline 94Gid2(0-30) & 31 & 1 & 13 & 2430 & 73 \\
\hline 94Gid2(30-60) & 29 & 1 & 9 & 5125 & 43 \\
\hline 94Gid2(60-90) & 20 & 1 & 8 & 5780 & 32 \\
\hline 94Gid2(90-142) & 19 & 1 & 7 & 5670 & 25 \\
\hline 94Gid2(142-181) & 25 & 1 & 9 & 10450 & 39 \\
\hline 94Gid2(181-215) & 20 & 1 & 7 & 6035 & 28 \\
\hline 94Gid2(215-250) & 19 & 1 & 8 & 9670 & 29 \\
\hline 94Gid2(250-280) & 31 & 1 & 10 & 18500 & 42 \\
\hline 94Gid3(0-3) & 19 & 1 & 10 & 3025 & 45 \\
\hline 94Gid3(3-12) & 25 & 1 & 9 & 5030 & 37 \\
\hline 94Gid3(12-50) & 27 & 1 & 9 & 4015 & 42 \\
\hline 94Gid3(50-67.5) & 27 & 1 & 8 & 2320 & 35 \\
\hline 94Gid3(122-151) & 31 & 2 & 10 & 3740 & 43 \\
\hline 94Gid3(151-190) & 45 & 118 & 11 & 796 & 58 \\
\hline
\end{tabular}


Appendix E. Chemical composition of samples analyzed, using 4 acid dissolution, by ICP-AES and ICP-MS at Eastern Washington University (EWU), Cheney, WA (Appendix_E.xls, Appendix_E.dbf).

\begin{tabular}{|c|c|c|c|c|c|c|c|c|c|c|c|c|c|c|c|c|}
\hline Sample No. & Site ID & $\begin{array}{c}\text { Depth } \\
\text { Interval, } \\
\text { top }(\mathrm{cm})\end{array}$ & $\begin{array}{c}\text { Depth } \\
\text { Interval, } \\
\text { bottom }(\mathrm{cm})\end{array}$ & $\begin{array}{c}{ }^{*} \text { Other } \\
\text { analyses } \\
\text { (Appendix \#) }\end{array}$ & $\begin{array}{c}\mathrm{Ag} \\
(\mathrm{ppm})\end{array}$ & $\begin{array}{c}\mathrm{Al} \\
(\%)\end{array}$ & $\begin{array}{c}\text { As } \\
(p p m)\end{array}$ & $\begin{array}{c}\mathrm{Ba} \\
(\mathrm{ppm})\end{array}$ & $\begin{array}{c}\mathrm{Be} \\
(\mathrm{ppm})\end{array}$ & $\begin{array}{l}\mathrm{Ca} \\
(\%)\end{array}$ & $\begin{array}{c}\mathrm{Cd} \\
(\mathrm{ppm})\end{array}$ & $\begin{array}{c}\mathrm{Ce} \\
(\mathrm{ppm})\end{array}$ & $\begin{array}{c}\text { Co } \\
\text { (ppm) }\end{array}$ & $\begin{array}{c}\mathrm{Cr} \\
\text { (ppm) }\end{array}$ & $\begin{array}{c}\text { Cs } \\
(\mathrm{ppm})\end{array}$ & $\begin{array}{c}\mathrm{Cu} \\
\text { (ppm) }\end{array}$ \\
\hline SAMPLE_NO. & $\overline{\text { SITE_ID }}$ & INT_TOP_CM & INT_BTM_CM & OTHR_ANLYS & AG_PPM & AL_\% & AS_PPM & BA_PPM & BE_PPM & CA_\% & CD_PPM & CE_PPM & CO_PPM & \begin{tabular}{|l|} 
CR_PPM \\
\end{tabular} & CS_PPM & CU_PPM \\
\hline 94Gid3(190-223) & 94Gid3 & 190 & 223 & --- & 0.1 & 7.3 & 6 & 567 & 1.6 & 0.19 & 2.1 & 75 & 23 & $\overline{\mathrm{NA}}$ & 10 & $\overline{16}$ \\
\hline 94Gid3(244-284) & 94Gid3 & 244 & 284 & $\mathrm{G}, \mathrm{H}$ & 0.4 & 5.4 & 13 & 488 & 1.4 & 0.14 & 2.6 & 60 & 12 & NA & 7 & 18 \\
\hline 94Gid3(284-310) & 94Gid3 & 284 & 310 & --- & 0.1 & 6.8 & 5 & 557 & 1.7 & 0.19 & 1.3 & 82 & 16 & NA & 9 & 15 \\
\hline 94Gid3(310-337) & 94Gid3 & 310 & 337 & $\begin{array}{ll}--- \\
--\end{array}$ & 0.1 & 6.3 & 5 & 542 & 1.7 & 0.18 & 0.9 & 81 & 24 & NA & 9 & 14 \\
\hline 94Gid4(0-31) & 94Gid4 & 0 & 31 & ---- & 10.3 & 4.1 & 166 & 622 & 1.2 & 0.16 & 22.4 & 50 & 9 & $\mathrm{NA}$ & 7 & $\overline{113}$ \\
\hline 94Gid4(31-63) & 94Gid4 & 31 & 63 & $\mathrm{G}, \mathrm{H}$ & 25.2 & 4.3 & 113 & 958 & 1.2 & 0.14 & 19.2 & 61 & 7 & NA & 8 & 213 \\
\hline 94Gid4(63-157) & 94Gid4 & 63 & 157 & --- & 1.7 & 6.5 & 23 & 593 & 1.5 & 0.21 & 3.7 & 82 & 15 & NA & 9 & 39 \\
\hline 94Gid4(157-186) & 94Gid4 & 157 & 186 & $\overline{----}$ & 0.1 & 2.6 & 6 & 379 & 1.8 & 0.14 & 1.6 & 47 & 11 & NA & 8 & 21 \\
\hline 94Gid4(186-215) & 94Gid4 & 186 & 215 & $\begin{array}{ll}--- \\
--\end{array}$ & 0.1 & 6.6 & 6 & 551 & 2.1 & 0.23 & 1.6 & 79 & 19 & NA & 9 & 19 \\
\hline 94Gid4(244-279) & 94Gid4 & 244 & 279 & ---- & 0.2 & 5.7 & 8 & 484 & 1.6 & 0.19 & 1.2 & 77 & 17 & NA & 8 & 16 \\
\hline 94Gid4(279-315) & 94Gid4 & 279 & 315 & ---- & 0.1 & 3.5 & 9 & 448 & 1.6 & 0.19 & 1.2 & 61 & 14 & $\mathrm{NA}$ & 8 & 17 \\
\hline 94Gid4(315-351) & 94Gid4 & 315 & 351 & ---- & 0.1 & 6.7 & 10 & 547 & 1.8 & 0.24 & 1.1 & 85 & 21 & $\mathrm{NA}$ & 9 & 23 \\
\hline 94Gid5(0-18) & 94Gid5 & 0 & 18 & ---- & 17.8 & 4.0 & 165 & 718 & 1.3 & 0.25 & 19.1 & 58 & 11 & $\overline{\mathrm{NA}}$ & 6 & 128 \\
\hline 94Gid5(18-43) & 94Gid5 & 18 & 43 & ---- & 8.3 & 5.0 & 22 & 670 & 1.6 & 0.30 & 21.6 & 59 & 7 & $\mathrm{NA}$ & 7 & 77 \\
\hline 94Gid5(43-70) & 94Gid5 & 43 & 70 & ---- & 0.1 & 5.2 & 6 & 478 & 1.5 & 0.24 & 6.2 & 63 & 19 & NA & 7 & 14 \\
\hline 94Gid5(70-96) & 94Gid5 & 70 & 96 & $\begin{array}{ll}--- \\
--\end{array}$ & 0.1 & 5.4 & 5 & 470 & 1.6 & 0.22 & 0.4 & 71 & 17 & NA & 7 & 16 \\
\hline 94Gid5(96-155) & 94Gid5 & 96 & 155 & $\begin{array}{ll}--- \\
-\end{array}$ & 0.4 & 5.5 & 10 & 477 & 1.6 & 0.22 & 0.6 & 77 & 13 & NA & 7 & 19 \\
\hline 94Gid5(155-190) & 94Gid5 & 155 & 190 & ---- & 0.1 & 3.3 & 6 & 401 & 1.4 & 0.15 & 0.1 & 58 & 13 & $\mathrm{NA}$ & 7 & 16 \\
\hline 94Gid5(190-225) & 94Gid5 & 190 & 225 & $-\cdots$ & 0.1 & 5.7 & 6 & 481 & 1.6 & 0.24 & 0.1 & 71 & 13 & $\mathrm{NA}$ & 7 & 16 \\
\hline 94Gid6(0-20) & 94Gid6 & 0 & 20 & $\bar{G}, \mathrm{H}$ & 18.2 & 4.8 & 117 & 730 & 1.4 & 0.27 & 15.6 & 63 & 10 & NA & 7 & 134 \\
\hline 94Gid6(20-50) & 94Gid6 & 20 & 50 & --- & 0.4 & 5.9 & 7 & 606 & 1.9 & 0.29 & 12.4 & 73 & 20 & NA & 8 & 20 \\
\hline 94Gid6(50-79) & 94Gid6 & 50 & 79 & ---- & 0.1 & 6.7 & 7 & 566 & 1.7 & 0.24 & 0.8 & 83 & 17 & $\mathrm{NA}$ & 9 & 22 \\
\hline 94Gid6(122-155) & 94Gid6 & 122 & 155 & $\overline{----}$ & 0.3 & 6.4 & 11 & 564 & 1.7 & 0.27 & 0.6 & 85 & 28 & $\overline{N A}$ & 8 & 25 \\
\hline 94Gid6(155-190) & 94Gid6 & 155 & 190 & ---- & 0.1 & 7.1 & 9 & 583 & 1.9 & 0.29 & 0.1 & 82 & 15 & NA & 8 & 22 \\
\hline 94Gid6(190-226) & 94Gid6 & 190 & 226 & ---- & 0.1 & 6.5 & 8 & 537 & 1.8 & 0.34 & 0.1 & 70 & 14 & NA & 7 & 20 \\
\hline $96 \mathrm{~K}-89 \mathrm{E}(0-23)$ & $96 \mathrm{~K}-89 \mathrm{E}$ & 0 & 23 & ---- & 13.2 & 3.2 & 140 & 574 & 1.0 & 0.25 & 23.5 & 32 & 9 & NA & 6 & 122 \\
\hline $96 \mathrm{~K}-89 \mathrm{E}(23-49)$ & $96 \mathrm{~K}-89 \mathrm{E}$ & 23 & 49 & ---- & 14.0 & 3.1 & 322 & 523 & 1.4 & 0.12 & 33.5 & 29 & 13 & NA & 6 & 125 \\
\hline 96K-89E(49-85) & $96 \mathrm{~K}-89 \mathrm{E}$ & 49 & 85 & ---- & 15.1 & 3.7 & 186 & 667 & 1.2 & 0.18 & 11.6 & 34 & 9 & NA & 8 & 132 \\
\hline $96 \mathrm{~K}-89 \mathrm{E}(85-110)$ & $96 \mathrm{~K}-89 \mathrm{E}$ & 85 & 110 & $-\overline{---}$ & 13.0 & 3.0 & 71 & 539 & 1.3 & 0.15 & 12.8 & 31 & 8 & $\mathrm{NA}$ & 6 & 82 \\
\hline $96 \mathrm{~K}-89 \mathrm{E}(110-132)$ & 96K-89E & 110 & 132 & $-\overline{---}$ & 9.7 & 6.8 & 21 & 719 & 1.7 & 0.27 & 0.9 & 85 & 12 & $\overline{\mathrm{NA}}$ & 11 & $\overline{70}$ \\
\hline $96 \mathrm{~K}-89 \mathrm{E}(132-162)$ & 96K-89E & 132 & 162 & ---- & 0.2 & 6.7 & 11 & 604 & 1.6 & 0.21 & $<0.5$ & 77 & 5 & $\mathrm{NA}$ & 10 & 15 \\
\hline $96 \mathrm{~K}-114 \mathrm{E}(0-15)$ & $96 \mathrm{~K}-114 \mathrm{E}$ & 0 & 15 & ---- & 14.7 & 3.2 & 129 & 599 & 0.9 & 0.37 & 45.0 & 33 & 10 & $\mathrm{NA}$ & 6 & 138 \\
\hline $96 \mathrm{~K}-114 \mathrm{E}(15-33)$ & $96 \mathrm{~K}-114 \mathrm{E}$ & 15 & 33 & ---- & 14.7 & 3.2 & 126 & 632 & 1.0 & 0.22 & 27.3 & 38 & 11 & $\mathrm{NA}$ & 6 & 131 \\
\hline $96 \mathrm{~K}-114 \mathrm{E}(33-61)$ & $96 \mathrm{~K}-114 \mathrm{E}$ & 33 & 61 & ---- & 17.9 & 2.9 & 426 & 650 & 1.1 & 0.14 & 34.2 & 39 & 19 & $\mathrm{NA}$ & 6 & 158 \\
\hline $96 \mathrm{~K}-114 \mathrm{E}(61-80)$ & $96 \mathrm{~K}-114 \mathrm{E}$ & 61 & 80 & ---- & 16.1 & 2.8 & 566 & 550 & 1.3 & 0.09 & 35.4 & 22 & 18 & $\mathrm{NA}$ & 6 & 134 \\
\hline 96K-114E(80-104) & $96 \mathrm{~K}-114 \mathrm{E}$ & 80 & 104 & ---- & 11.9 & 3.0 & 129 & 556 & 1.0 & 0.10 & 8.9 & 17 & 12 & $\mathrm{NA}$ & 6 & 108 \\
\hline
\end{tabular}


Appendix E. Chemical composition of samples analyzed, using 4 acid dissolution, by ICP-AES and ICP-MS at Eastern Washington University (EWU), Cheney, WA (Appendix_E.xls, Appendix_E.dbf).

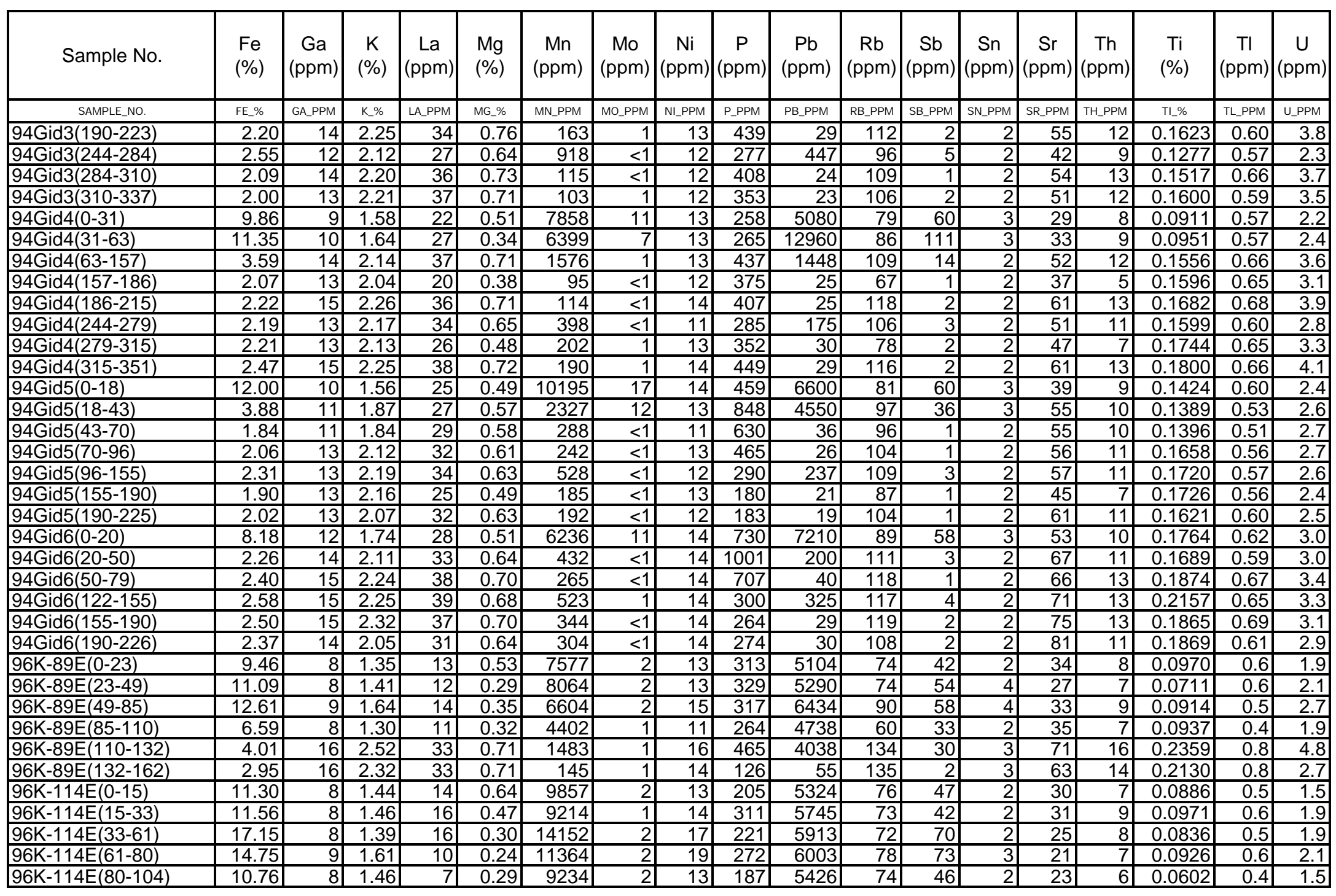


Appendix E. Chemical composition of samples analyzed, using 4 acid dissolution, by ICP-AES and ICP-MS at Eastern Washington University (EWU), Cheney, WA (Appendix_E.xls, Appendix_E.dbf).

\begin{tabular}{|l|r|r|r|r|r|}
\hline \multicolumn{1}{|c|}{ Sample No. } & $\begin{array}{c}\text { V } \\
\text { (ppm) }\end{array}$ & $\begin{array}{c}\text { W } \\
\text { (ppm) }\end{array}$ & $\begin{array}{c}\text { Y } \\
(\mathrm{ppm})\end{array}$ & $\begin{array}{c}\text { Zn } \\
\text { (ppm) }\end{array}$ & $\begin{array}{c}\text { Zr } \\
(\mathrm{ppm})\end{array}$ \\
\hline & V_PPM & w_PPM & Y_pPM & ZN_PPM & ZR_PPM \\
\hline 94Gid3(190-223) & 49 & 168 & 14 & 252 & 78 \\
\hline 94Gid3(244-284) & 41 & 92 & 9 & 445 & 57 \\
\hline 94Gid3(284-310) & 49 & 116 & 13 & 161 & 72 \\
\hline 94Gid3(310-337) & 47 & 214 & 13 & 130 & 71 \\
\hline 94Gid4(0-31) & 28 & 2 & 9 & 4050 & 42 \\
\hline 94Gid4(31-63) & 32 & 2 & 11 & 4635 & 48 \\
\hline 94Gid4(63-157) & 48 & 99 & 14 & 652 & 75 \\
\hline 94Gid4(157-186) & 50 & 57 & 7 & 169 & 67 \\
\hline 94Gid4(186-215) & 53 & 123 & 18 & 183 & 79 \\
\hline 94Gid4(244-279) & 45 & 118 & 13 & 188 & 69 \\
\hline 94Gid4(279-315) & 53 & 77 & 9 & 207 & 74 \\
\hline 94Gid4(315-351) & 56 & 137 & 17 & 222 & 87 \\
\hline 94Gid5(0-18) & 30 & 3 & 12 & 2825 & 67 \\
\hline 94Gid5(18-43) & 39 & 1 & 11 & 1760 & 61 \\
\hline 94Gid5(43-70) & 43 & 115 & 12 & 922 & 63 \\
\hline 94Gid5(70-96) & 48 & 94 & 12 & 517 & 73 \\
\hline 94Gid5(96-155) & 49 & 69 & 13 & 352 & 74 \\
\hline 94Gid5(155-190) & 48 & 59 & 8 & 166 & 70 \\
\hline 94Gid5(190-225) & 48 & 76 & 12 & 87 & 70 \\
\hline 94Gid6(0-20) & 40 & 2 & 14 & 1860 & 83 \\
\hline 94Gid6(20-50) & 50 & 106 & 14 & 832 & 78 \\
\hline 94Gid6(50-79) & 57 & 93 & 15 & 393 & 88 \\
\hline 94Gid6(122-155) & 56 & 184 & 16 & 342 & 100 \\
\hline 94Gid6(155-190) & 57 & 58 & 16 & 296 & 88 \\
\hline 94Gid6(190-226) & 52 & 54 & 14 & 285 & 91 \\
\hline 96K-89E(0-23) & 30 & 1 & 9 & 3357 & 37 \\
\hline 96K-89E(23-49) & 27 & 1 & 8 & 4427 & 27 \\
\hline 96K-89E(49-85) & 33 & 2 & 9 & 2922 & 40 \\
\hline 96K-89E(85-110) & 30 & 1 & 6 & 1966 & 37 \\
\hline 96K-89E(110-132) & 66 & 2 & 14 & 404 & 85 \\
\hline 96K-89E(132-162) & 74 & 2 & 12 & 81 & 71 \\
\hline 96K-114E(0-15) & 28 & 1 & 8 & 6752 & 32 \\
\hline 96K-114E(15-33) & 27 & $<1$ & 9 & 4043 & 38 \\
\hline 96K-114E(33-61) & 25 & 1 & 9 & 4913 & 31 \\
\hline 96K-114E(61-80) & 29 & 3 & 8 & 5294 & 39 \\
\hline 96K-114E(80-104) & 25 & 1 & 6 & 1337 & 20 \\
\hline
\end{tabular}


Appendix E. Chemical composition of samples analyzed, using 4 acid dissolution, by ICP-AES and ICP-MS at Eastern Washington University (EWU), Cheney, WA (Appendix_E.xls, Appendix_E.dbf).

\begin{tabular}{|c|c|c|c|c|c|c|c|c|c|c|c|c|c|c|c|c|}
\hline Sample No. & Site ID & $\begin{array}{c}\text { Depth } \\
\text { Interval, } \\
\text { top }(\mathrm{cm})\end{array}$ & $\begin{array}{c}\text { Depth } \\
\text { Interval, } \\
\text { bottom }(\mathrm{cm})\end{array}$ & $\begin{array}{c}{ }^{*} \text { Other } \\
\text { analyses } \\
\text { (Appendix \#) }\end{array}$ & $\begin{array}{c}\mathrm{Ag} \\
(\mathrm{ppm})\end{array}$ & $\begin{array}{c}\mathrm{Al} \\
(\%)\end{array}$ & $\begin{array}{c}\text { As } \\
(\mathrm{ppm})\end{array}$ & $\begin{array}{c}\mathrm{Ba} \\
\text { (ppm) }\end{array}$ & $\begin{array}{c}\mathrm{Be} \\
(\mathrm{ppm})\end{array}$ & $\begin{array}{c}\mathrm{Ca} \\
(\%)\end{array}$ & $\mid \begin{array}{c}\text { Cd } \\
(p p m)\end{array}$ & $\begin{array}{c}\mathrm{Ce} \\
(\mathrm{ppm})\end{array}$ & $\begin{array}{c}\text { Co } \\
\text { (ppm) }\end{array}$ & $\begin{array}{c}\mathrm{Cr} \\
(\mathrm{ppm})\end{array}$ & $\begin{array}{c}\text { Cs } \\
(\mathrm{ppm})\end{array}$ & $\begin{array}{c}\mathrm{Cu} \\
(\mathrm{ppm})\end{array}$ \\
\hline SAMPLE_NO. & $\overline{\text { SITE_ID }}$ & INT_TOP_CM & INT_BTM_CM & OTHR_ANLYS & $\overline{A G_{-} P P M}$ & AL_\% & AS_PPM & BA_PPM & BE_PPM & CA_\% & CD_PPM & CE_PPM & CO_PPM & \begin{tabular}{|l|} 
CR_PPM \\
\end{tabular} & CS_PPM & CU_PPM \\
\hline $96 \mathrm{~K}-114 \mathrm{E}(104-130)$ & $96 \mathrm{~K}-114 \mathrm{E}$ & 104 & 130 & ---- & 12.9 & 2.8 & 111 & 545 & 1.0 & 0.17 & 12.3 & 21 & 6 & $\mathrm{NA}$ & 5 & 102 \\
\hline $96 \mathrm{~K}-114 \mathrm{E}(140-160)$ & $96 \mathrm{~K}-114 \mathrm{E}$ & 130 & 160 & $\mathrm{G}, \mathrm{H}$ & 20.1 & 3.5 & 142 & 767 & 1.5 & 0.29 & 28.0 & 33 & 9 & $\overline{\mathrm{NA}}$ & 7 & 145 \\
\hline $96 \mathrm{~K}-114 \mathrm{E}(160-175)$ & $96 \mathrm{~K}-114 \mathrm{E}$ & 160 & 175 & $-\cdots$ & 6.8 & 5.0 & 15 & 604 & 1.9 & 0.29 & 17.0 & 42 & 7 & NA & 9 & 56 \\
\hline $96 \mathrm{~K}-178 \mathrm{E}(0-18)$ & $96 \mathrm{~K}-178 \mathrm{E}$ & 0 & 18 & $\begin{array}{ll}--- \\
\end{array}$ & 13.1 & 4.2 & 122 & 585 & 1.2 & 0.42 & 28.4 & 25 & 10 & NA & 8 & 115 \\
\hline $96 \mathrm{~K}-178 \mathrm{E}(18-35)$ & $96 \mathrm{~K}-178 \mathrm{E}$ & 18 & 35 & ---- & 18.6 & 5.0 & 373 & 785 & 1.9 & 0.33 & 26.8 & 56 & 10 & NA & 10 & 162 \\
\hline $96 \mathrm{~K}-178 \mathrm{E}(35-70)$ & $96 \mathrm{~K}-178 \mathrm{E}$ & 35 & 70 & ---- & 17.2 & 4.2 & 561 & 677 & 1.4 & 0.24 & 17.6 & 50 & 11 & $\mathrm{NA}$ & 8 & 163 \\
\hline $96 \mathrm{~K}-178 \mathrm{E}(70-84)$ & $96 \mathrm{~K}-178 \mathrm{E}$ & 70 & 84 & ---- & 14.5 & 3.8 & 192 & 656 & 1.6 & 0.20 & 6.8 & 22 & 8 & $\mathrm{NA}$ & 8 & 130 \\
\hline $96 \mathrm{~K}-178 \mathrm{E}(84-102)$ & 96K-178E & 84 & 102 & $-\overline{---}$ & 11.9 & 2.9 & 113 & 497 & 1.2 & 0.42 & 37.7 & 23 & 13 & $\mathrm{NA}$ & 6 & 113 \\
\hline $96 \mathrm{~K}-178 \mathrm{E}(102-120)$ & $96 \mathrm{~K}-178 \mathrm{E}$ & 102 & 120 & ---- & 16.6 & 3.6 & 144 & 598 & 1.3 & 0.48 & 31.1 & 35 & 18 & $\mathrm{NA}$ & 7 & 140 \\
\hline $96 \mathrm{~K}-178 \mathrm{E}(120-140)$ & $96 \mathrm{~K}-178 \mathrm{E}$ & 120 & 140 & ---- & 14.1 & 4.2 & 100 & 714 & 1.6 & 0.38 & 16.9 & 38 & 13 & NA & 9 & 113 \\
\hline 96LD-1S $(0-16)$ & 96LD-1S & 0 & 16 & ---- & 14.9 & 3.8 & 145 & 598 & 1.5 & 0.31 & 38.4 & 28 & 10 & 43 & 7 & 138 \\
\hline 96LD-1S(16-43) & 96LD-1S & 16 & 43 & ---- & 15.6 & 3.8 & 402 & 546 & 1.1 & 0.20 & 30.1 & 32 & 11 & 41 & 7 & 132 \\
\hline 96LD-1S(43-70) & 96LD-1S & 43 & 70 & ---- & 15.7 & 3.1 & 541 & 506 & 1.2 & 0.15 & 25.2 & 36 & 15 & 44 & 6 & 131 \\
\hline 96LD-1S(70-93) & 96LD-1S & 70 & 93 & ---- & 14.3 & 2.7 & 608 & 456 & 1.0 & 0.12 & 16.2 & 26 & 10 & 48 & 5 & 129 \\
\hline 96LD-1S $(105-120)$ & 96LD-1S & 105 & 120 & ---- & 11.0 & 2.1 & 699 & 342 & 1.0 & 0.18 & 44.6 & 18 & 17 & 37 & 4 & 110 \\
\hline 96LD-105S(0-5) & 96LD-105S & 0 & 5 & ---- & 12.1 & 3.9 & 78 & 543 & 1.3 & 0.27 & 35.1 & 27 & 7 & 46 & 7 & 117 \\
\hline 96LD-105S(5-18) & 96LD-105S & 5 & 18 & ---- & 11.7 & 4.2 & 155 & 600 & 1.4 & 0.15 & 16.4 & 46 & 10 & 52 & 7 & 116 \\
\hline 96LD-105S(18-34) & 96LD-105S & 18 & 34 & ---- & 23.1 & 3.5 & 106 & 780 & 1.3 & 0.08 & 9.8 & 40 & 9 & 43 & 7 & 190 \\
\hline 96LD-105S(34-49) & 96LD-105S & 34 & 49 & $-\cdots$ & 0.4 & 3.4 & 5 & 512 & 1.8 & 0.16 & 26.0 & 47 & 5 & 54 & 7 & 12 \\
\hline $93 \mathrm{CSC} 03(0-6)$ & 93CSC03 & 0 & 6 & ---- & 7.9 & 6.3 & 90 & 679 & 2.2 & 0.60 & 26.9 & 59 & 15 & 70 & 10 & 106 \\
\hline $93 \mathrm{CSC} 03(6-10)$ & 93CSC03 & 6 & 10 & $---\cdot$ & 18.5 & 5.7 & 224 & 768 & 1.8 & 0.29 & 35.0 & 80 & 14 & 83 & 10 & 177 \\
\hline $93 \mathrm{CSC} 03(10-43)$ & 93CSC03 & 10 & 43 & --- & 17.9 & 4.0 & 604 & 607 & 1.6 & 0.23 & 74.3 & 58 & 13 & 63 & 8 & 173 \\
\hline $93 \mathrm{CSC} 03(43-46)$ & $93 \mathrm{CSC} 03$ & 43 & 46 & ---- & 18.1 & 4.9 & 384 & 769 & 1.8 & 0.20 & 31.8 & 47 & 10 & 63 & 11 & 172 \\
\hline $93 \mathrm{CSC} 03(46-55)$ & $93 \mathrm{CSC} 03$ & 46 & 55 & ---- & 16.8 & 4.2 & 229 & 721 & 1.6 & 0.16 & 11.8 & 36 & 14 & 79 & 8 & 149 \\
\hline $93 \mathrm{CSC} 03(55-61)$ & $93 \mathrm{CSC} 03$ & 55 & 61 & ---- & 16.3 & 4.1 & 167 & 706 & 1.3 & 0.17 & 12.0 & 54 & 11 & 63 & 8 & 138 \\
\hline $93 \mathrm{CSC} 03(61-66)$ & $93 \mathrm{CSC} 03$ & 61 & 66 & $\begin{array}{ll}--- \\
\end{array}$ & 23.5 & 4.8 & 188 & 849 & 1.4 & 0.21 & 13.4 & 52 & 12 & 73 & 10 & 189 \\
\hline $93 \mathrm{CSC} 03(66-71)$ & $93 \mathrm{CSC} 03$ & 66 & 71 & ---- & 27.9 & 3.7 & 165 & 838 & 1.4 & 0.15 & 18.6 & 37 & 15 & 69 & 8 & 212 \\
\hline $93 \mathrm{CSC} 03(71-77)$ & $93 \mathrm{CSC} 03$ & 71 & 77 & $-\cdots-$ & 21.1 & 4.9 & 130 & 812 & 1.7 & 0.21 & 23.0 & 66 & 10 & 58 & 11 & 160 \\
\hline 93CSC03(77-95) & $93 \mathrm{CSC} 03$ & 77 & 95 & $-\overline{---}$ & 27.3 & 6.8 & 139 & 1072 & 2.3 & 0.34 & 26.0 & 90 & 21 & 61 & 14 & 191 \\
\hline $93 \mathrm{CSC} 03(95-112)$ & 93CSC03 & 95 & 112 & ---- & 22.8 & 4.1 & 504 & 759 & 1.2 & 0.19 & 20.5 & 48 & 9 & 49 & 8 & 170 \\
\hline 93CSC03(112-122) & 93CSC03 & 112 & 122 & ---- & 18.6 & 4.0 & 180 & 683 & 1.4 & 0.22 & 18.4 & 43 & 6 & 46 & 8 & 117 \\
\hline 93CSC03(122-133) & 93CSC03 & 122 & 133 & ---- & 23.7 & 3.6 & 107 & 1196 & 1.3 & 0.24 & 29.6 & 32 & 6 & 53 & 8 & 157 \\
\hline 93CSC03(133-145) & 93CSC03 & 133 & 145 & ---- & 41.2 & 4.5 & 82 & 1147 & 1.5 & 0.27 & 25.1 & 45 & 6 & 46 & 11 & 236 \\
\hline 93CSC03(145-155) & 93CSC03 & 145 & 155 & ---- & 42.6 & 3.8 & 81 & 1199 & 1.3 & 0.37 & 98.2 & 49 & 8 & 59 & 9 & 302 \\
\hline $93 \mathrm{CSC} 03(155-168)$ & 93CSC03 & 155 & 168 & ---- & 45.9 & 4.5 & 65 & 852 & 1.3 & 0.59 & 139.8 & 22 & 14 & 74 & 10 & 364 \\
\hline 93CSC03(168-192) & $93 \mathrm{CSC} 03$ & 168 & 192 & ---- & 45.4 & 4.8 & 56 & 915 & 1.5 & 0.50 & 103.8 & 38 & 15 & 74 & 10 & 336 \\
\hline
\end{tabular}


Appendix E. Chemical composition of samples analyzed, using 4 acid dissolution, by ICP-AES and ICP-MS at Eastern Washington University (EWU), Cheney, WA (Appendix_E.xls, Appendix_E.dbf).

\begin{tabular}{|c|c|c|c|c|c|c|c|c|c|c|c|c|c|c|c|c|c|c|}
\hline Sample No. & $\begin{array}{l}\mathrm{Fe} \\
(\%)\end{array}$ & $\begin{array}{c}\mathrm{Ga} \\
(\mathrm{ppm})\end{array}$ & $\begin{array}{c}\mathrm{K} \\
(\%)\end{array}$ & $\begin{array}{c}\mathrm{La} \\
(\mathrm{ppm})\end{array}$ & $\begin{array}{l}\mathrm{Mg} \\
(\%)\end{array}$ & $\begin{array}{c}\mathrm{Mn} \\
(\mathrm{ppm})\end{array}$ & $\begin{array}{c}\text { Mo } \\
\text { (ppm) }\end{array}$ & $\begin{array}{c}\mathrm{Ni} \\
(\mathrm{ppm})\end{array}$ & $\begin{array}{c}\mathrm{P} \\
(\mathrm{ppm})\end{array}$ & $\begin{array}{c}\mathrm{Pb} \\
(\mathrm{ppm})\end{array}$ & $\begin{array}{c}\mathrm{Rb} \\
(\mathrm{ppm})\end{array}$ & $\begin{array}{c}\mathrm{Sb} \\
(\mathrm{ppm})\end{array}$ & $\begin{array}{c}\text { Sn } \\
(p p m)\end{array}$ & $\begin{array}{c}\mathrm{Sr} \\
(\mathrm{ppm})\end{array}$ & $\left|\begin{array}{c}\text { Th } \\
(\mathrm{ppm})\end{array}\right|$ & $\begin{array}{c}\mathrm{Ti} \\
(\%)\end{array}$ & $\begin{array}{c}\mathrm{Tl} \\
\text { (ppm) }\end{array}$ & $\underset{(\mathrm{ppm})}{\mathrm{U}}$ \\
\hline SAMPLE_NO. & FE_\% & GA_PPM & K_\% & LA_PPM & MG_\% & MN_PPM & MO_PPM & NI_PPM & P_PPM & PB_PPM & RB_PPM & SB_PPM & SN_PPM & SR_PPM & A_PPM & TI_\% & TL_PPM & U_PPM \\
\hline $96 \mathrm{~K}-114 \mathrm{E}(104-130)$ & 10.90 & 7 & 1.32 & 8 & 0.45 & 8898 & 1 & 10 & 177 & 5742 & 68 & 42 & 3 & 21 & 6 & 0.0598 & 0.4 & 1.4 \\
\hline $96 \mathrm{~K}-114 \mathrm{E}(140-160)$ & 12.94 & 9 & 1.58 & 14 & 0.60 & 10758 & 2 & 18 & 321 & 8656 & 85 & 62 & 4 & 38 & 8 & 0.0772 & 0.6 & 2.2 \\
\hline $96 \mathrm{~K}-114 \mathrm{E}(160-175)$ & 2.28 & 14 & 2.18 & 16 & 0.62 & 350 & 2 & 30 & 674 & 3315 & 117 & 21 & 6 & 63 & 10 & 0.1703 & 0.7 & 3.9 \\
\hline $96 \mathrm{~K}-178 \mathrm{E}(0-18)$ & 7.97 & 10 & 1.71 & 10 & 0.58 & 5619 & 2 & 16 & 629 & 5665 & 97 & 44 & 3 & 55 & 9 & 0.1146 & 0.8 & 2.8 \\
\hline $96 \mathrm{~K}-178 \mathrm{E}(18-35)$ & 11.60 & 12 & 2.03 & 23 & 0.56 & 6470 & 2 & 19 & 751 & 6735 & 118 & 73 & 4 & 55 & 13 & 0.1324 & 0.8 & 4.0 \\
\hline $96 \mathrm{~K}-178 \mathrm{E}(70-84)$ & 11.78 & 10 & 1.66 & 9 & 0.37 & 6543 & 2 & 16 & 343 & 6164 & 89 & 56 & 4 & 30 & 8 & 0.0794 & 0.5 & 2.1 \\
\hline $96 \mathrm{~K}-178 \mathrm{E}(84-102)$ & 10.16 & 8 & 1.36 & 9 & 0.50 & 8290 & 2 & 17 & 222 & 5660 & 75 & 44 & 4 & 33 & 7 & 0.0823 & 0.4 & 1.8 \\
\hline $96 \mathrm{~K}-178 \mathrm{E}(102-120)$ & 12.71 & 10 & 1.64 & 14 & 0.60 & 11135 & 3 & 24 & 304 & 7724 & 88 & 57 & 5 & 44 & 10 & 0.1099 & 0.5 & 2.8 \\
\hline $96 \mathrm{~K}-178 \mathrm{E}(120-140)$ & 7.41 & 11 & 1.89 & 14 & 0.61 & 5825 & 4 & 31 & 568 & 5636 & 89 & 43 & 4 & 53 & 10 & 0.1490 & 0.6 & 3.8 \\
\hline 96LD-1S(0-16) & 10.00 & 9 & 1.68 & 11 & 0.59 & 7978 & 2 & 14 & 351 & 5235 & 91 & 54 & 2 & 40 & 8 & 0.1051 & 0.6 & 2.1 \\
\hline 96LD-1S(16-43) & 9.80 & 9 & 1.56 & 14 & 0.38 & 6738 & 2 & 14 & 599 & 4634 & 86 & 71 & 3 & 39 & 9 & 0.0929 & 0.7 & 2.9 \\
\hline 96LD-1S(43-70) & 13.63 & 8 & 1.36 & 15 & 0.30 & 9447 & 3 & 17 & 421 & 3792 & $\overline{74}$ & 76 & 3 & 31 & 9 & 0.0753 & 0.6 & 2.7 \\
\hline 96LD-1S(70-93) & 15.44 & 7 & \begin{tabular}{ll|}
1.21 \\
\end{tabular} & 10 & 0.33 & 7863 & 3 & 17 & 269 & 2937 & 64 & 84 & 3 & 24 & 7 & 0.0672 & 0.5 & 2.1 \\
\hline 96LD-1S(105-120) & 15.83 & 5 & 0.95 & 7 & 0.54 & 12292 & 2 & 14 & 167 & 2981 & 48 & 59 & 2 & 15 & 5 & 0.0446 & 0.3 & 1.9 \\
\hline 96LD-105 & 12.54 & 9 & 1.63 & 16 & 0.34 & 10103 & 2 & 11 & 254 & 9965 & 91 & 55 & 3 & 27 & 8 & 0.0734 & 0.6 & 2.2 \\
\hline 96LD-105s & 1.58 & 11 & 2.11 & 19 & 0.58 & 389 & 1 & 13 & 293 & 155 & 108 & 2 & 2 & 43 & 9 & 0.1396 & 0.6 & 1.8 \\
\hline $93 \operatorname{CsC} 03(0-6)$ & 4.94 & 14 & 2.14 & 24 & 0.68 & 2565 & 2 & 19 & 1143 & 3211 & 110 & 35 & 4 & 99 & 12 & 0.2036 & 0.9 & 4.3 \\
\hline $93 \mathrm{CSC} 03(6-10)$ & 9.97 & 13 & 2.20 & 34 & 0.63 & 7352 & 3 & 20 & 859 & 6804 & 127 & 71 & 4 & 59 & 15 & 0.1535 & 1.0 & 4.2 \\
\hline $93 \operatorname{CSC} 03(10-43)$ & 15.65 & 10 & 1.70 & 25 & 0.45 & 9868 & 3 & 20 & 482 & 5150 & 93 & 127 & 4 & 37 & 11 & 0.0917 & 0.7 & 3.2 \\
\hline $93 \mathrm{CSC} 03(43-46)$ & 11.41 & 12 & 2.00 & 19 & 0.44 & 7473 & 3 & 22 & 474 & 7198 & 108 & 79 & 6 & 41 & 12 & 0.1157 & 0.6 & 3.3 \\
\hline $93 \mathrm{CSC} 03(46-55)$ & 13.29 & 10 & 1.81 & 15 & 0.36 & 10230 & 2 & 19 & 344 & 7696 & 101 & 68 & 4 & 35 & 9 & 0.1035 & 0.6 & 2.0 \\
\hline 93CSC03(55-61) & 11.04 & 10 & 1.74 & 22 & 0.37 & 8832 & 3 & 18 & 327 & 7076 & 93 & 54 & 4 & 34 & 11 & 0.1067 & 0.5 & 3.7 \\
\hline 93CSC03(61-66) & 12.63 & 11 & 2.06 & 22 & 0.46 & 8765 & 2 & 19 & 405 & 10225 & 111 & 68 & 5 & 40 & 12 & 0.1020 & 0.7 & 2.7 \\
\hline 93CSC03(66-71) & 13.29 & 8 & 1.64 & 15 & 0.39 & 13210 & 3 & 17 & 272 & 12621 & 90 & 68 & 3 & 30 & 8 & 0.0783 & 0.8 & 2.2 \\
\hline 93CSC03(71-77) & 13.02 & 12 & 2.06 & 28 & 0.45 & 8218 & 2 & 20 & 399 & 12028 & 119 & 66 & 5 & 37 & 13 & 0.1113 & 0.7 & 3.1 \\
\hline 93CSC03(77-95) & 8.52 & 16 & 2.46 & 37 & 0.71 & 6873 & $\overline{2}$ & 25 & 677 & 11150 & 145 & 81 & 5 & 67 & 17 & 0.1870 & 0.9 & 5.1 \\
\hline $93 \mathrm{CSC} 03(95-112)$ & 15.89 & $\overline{9}$ & 1.66 & 20 & 0.43 & 5802 & $\overline{2}$ & 20 & 376 & 10919 & 88 & 52 & 4 & 34 & 10 & 0.0763 & 1.0 & 2.8 \\
\hline 93CSC03(112-122) & 12.60 & 8 & 1.50 & 18 & 0.53 & 9029 & 2 & 16 & 356 & 9005 & 82 & 57 & 3 & 31 & 9 & 0.0759 & 0.8 & 2.4 \\
\hline 93CSC03(122-133) & 14.04 & 8 & 1.47 & 13 & 0.63 & 11882 & 2 & 13 & 269 & 12565 & 82 & 71 & 3 & 27 & 8 & 0.0742 & 0.6 & 2.2 \\
\hline 93CSC03(133-145) & 13.01 & 11 & 1.74 & 18 & 0.69 & 10038 & 2 & 16 & 381 & 20908 & 100 & 86 & 4 & 47 & 11 & 0.1105 & 0.6 & 3.0 \\
\hline $93 \mathrm{CSC} 03(145-155)$ & 13.01 & $\overline{9}$ & 1.56 & 20 & 0.68 & 11695 & 1 & 16 & 334 & 21409 & 87 & 116 & 3 & 41 & 10 & 0.0941 & 0.6 & 2.6 \\
\hline $93 \mathrm{CSC} 03(155-168)$ & 11.73 & 10 & 1.78 & 9 & 0.72 & 11097 & 3 & 22 & 305 & 22072 & 103 & 144 & 4 & 50 & 8 & 0.0970 & 0.7 & 2.7 \\
\hline 93CSC03(168-192) & 9.11 & 11 & 1.86 & 15 & 0.74 & 8073 & 2 & 26 & 401 & 20950 & 102 & 92 & 3 & 55 & 11 & 0.1302 & 0.7 & 3.8 \\
\hline
\end{tabular}


Appendix E. Chemical composition of samples analyzed, using 4 acid dissolution, by ICP-AES and ICP-MS at Eastern Washington University (EWU), Cheney, WA (Appendix_E.xls, Appendix_E.dbf).

\begin{tabular}{|c|c|c|c|c|c|}
\hline Sample No. & $\begin{array}{c}\mathrm{V} \\
(\mathrm{ppm})\end{array}$ & $\begin{array}{c}\text { W } \\
(\mathrm{ppm})\end{array}$ & $\begin{array}{c}\mathrm{Y} \\
(\mathrm{ppm})\end{array}$ & $\begin{array}{c}\mathrm{Zn} \\
(\mathrm{ppm})\end{array}$ & $\begin{array}{c}\mathrm{Zr} \\
(\mathrm{ppm})\end{array}$ \\
\hline SAMPLE_NO. & V_PPM & W_PPM & Y_PPM & ZN_PPM & ZR_PPM \\
\hline 96K-114E(104-130) & 24 & 1 & 6 & 1218 & 22 \\
\hline $96 \mathrm{~K}-114 \mathrm{E}(140-160)$ & 32 & 1 & 9 & 3652 & 35 \\
\hline 96K-114E(160-175) & 52 & 4 & 12 & 5835 & 68 \\
\hline $96 \mathrm{~K}-178 \mathrm{E}(0-18)$ & 41 & 2 & 12 & 3404 & 45 \\
\hline $96 \mathrm{~K}-178 \mathrm{E}(18-35)$ & 48 & 2 & 15 & 2881 & 50 \\
\hline $96 \mathrm{~K}-178 \mathrm{E}(35-70)$ & 38 & 2 & 12 & 2884 & 34 \\
\hline 96K-178E(70-84) & 33 & 2 & 9 & 2276 & 28 \\
\hline $96 \mathrm{~K}-178 \mathrm{E}(84-102)$ & 28 & 1 & 7 & 3754 & 29 \\
\hline $96 \mathrm{~K}-178 \mathrm{E}(102-120)$ & 34 & 2 & 11 & 4547 & 52 \\
\hline $96 \mathrm{~K}-178 \mathrm{E}(120-140)$ & 44 & 2 & 11 & 3773 & 63 \\
\hline 96LD-1S(0-16) & 35 & 1 & 10 & 5422 & 38 \\
\hline 96LD-1S(16-43) & 35 & 1 & 11 & 2798 & 35 \\
\hline 96LD-1S(43-70) & 28 & 2 & 10 & 2453 & 31 \\
\hline 96LD-1S(70-93) & 23 & 2 & 8 & 3772 & 32 \\
\hline 96LD-1S(105-120) & 16 & 1 & 7 & 2927 & 20 \\
\hline 96LD-105S(0-5) & 33 & 1 & 8 & 5155 & 38 \\
\hline 96LD-105S(5-18) & 35 & 1 & 9 & 1818 & 36 \\
\hline 96LD-105S(18-34) & 31 & 1 & 7 & 3699 & 26 \\
\hline 96LD-105S(34-49) & 41 & 1 & 6 & 1434 & 41 \\
\hline $93 \operatorname{CSC} 03(0-6)$ & 59 & 3 & 16 & 1942 & $\overline{76}$ \\
\hline $93 \operatorname{CSC} 03(6-10)$ & 51 & 2 & 18 & 2928 & 59 \\
\hline $93 \mathrm{CSC} 03(10-43)$ & 35 & 2 & 12 & 6127 & 41 \\
\hline $93 \csc 03(43-46)$ & 42 & 2 & 13 & 4663 & 49 \\
\hline 93CSC03(46-55) & 36 & 2 & 10 & 2350 & 39 \\
\hline 93CSC03(55-61) & 35 & 2 & 11 & 1938 & 50 \\
\hline $93 \mathrm{CSC} 03(61-66)$ & 41 & 2 & 11 & 2397 & 37 \\
\hline 93CSC03(66-71) & 30 & 1 & 10 & 2038 & 33 \\
\hline 93CSC03(71-77) & 43 & 2 & 13 & 3715 & 51 \\
\hline 93CSC03(77-95) & 62 & 3 & 14 & 3270 & 52 \\
\hline 93CSC03(95-112) & 32 & 1 & 12 & 5648 & 60 \\
\hline 93CSC03(112-122) & 30 & 1 & 11 & 2454 & 38 \\
\hline 93CSC03(122-133) & 29 & 1 & 10 & 2486 & 37 \\
\hline 93CSC03(133-145) & 41 & 2 & 12 & 3638 & 46 \\
\hline 93CSC03(145-155) & 32 & 1 & 12 & 9116 & 36 \\
\hline 93CSC03(155-168) & 38 & 2 & 12 & 19404 & 34 \\
\hline 93CSC03(168-192) & 43 & 4 & 13 & 16249 & 32 \\
\hline
\end{tabular}


Appendix E. Chemical composition of samples analyzed, using 4 acid dissolution, by ICP-AES and ICP-MS at Eastern Washington University (EWU), Cheney, WA (Appendix_E.xls, Appendix_E.dbf).

\begin{tabular}{|c|c|c|c|c|c|c|c|c|c|c|c|c|c|c|c|c|}
\hline Sample No. & Site ID & $\begin{array}{c}\text { Depth } \\
\text { Interval, } \\
\text { top }(\mathrm{cm})\end{array}$ & $\begin{array}{c}\text { Depth } \\
\text { Interval, } \\
\text { bottom }(\mathrm{cm})\end{array}$ & $\begin{array}{c}{ }^{*} \text { Other } \\
\text { analyses } \\
\text { (Appendix \#) }\end{array}$ & $\begin{array}{c}\mathrm{Ag} \\
(\mathrm{ppm})\end{array}$ & $\begin{array}{c}\mathrm{Al} \\
(\%)\end{array}$ & $\begin{array}{c}\text { As } \\
(\mathrm{ppm})\end{array}$ & $\begin{array}{c}\mathrm{Ba} \\
\text { (ppm) }\end{array}$ & $\begin{array}{c}\mathrm{Be} \\
(\mathrm{ppm})\end{array}$ & $\begin{array}{l}\mathrm{Ca} \\
(\%)\end{array}$ & \begin{tabular}{|c} 
Cd \\
$(\mathrm{ppm})$
\end{tabular} & $\begin{array}{c}\mathrm{Ce} \\
(\mathrm{ppm})\end{array}$ & $\begin{array}{c}\text { Co } \\
\text { (ppm) }\end{array}$ & $\begin{array}{c}\mathrm{Cr} \\
(\mathrm{ppm})\end{array}$ & $\begin{array}{c}\text { Cs } \\
(\mathrm{ppm})\end{array}$ & $\begin{array}{c}\mathrm{Cu} \\
(\mathrm{ppm})\end{array}$ \\
\hline SAMPLE_NO. & $\overline{\text { SITE_ID }}$ & INT_TOP_CM & INT_BTM_CM & OTHR_ANLYS & $\overline{A G_{-} P P M}$ & AL_\% & AS_PPM & BA_PPM & BE_PPM & CA_\% & CD_PPM & CE_PPM & CO_PPM & $\begin{array}{ll}\text { CR_PPM } \\
\end{array}$ & CS_PPM & CU_PPM \\
\hline 93CSC03(192-233) & $93 \mathrm{CSC} 03$ & 192 & 233 & ---- & 44.1 & 2.7 & 63 & 870 & 1.1 & 0.59 & 156.8 & 24 & 11 & 81 & 6 & 383 \\
\hline 93CSC03(233-256) & 93CSC03 & 233 & 256 & ---- & 61.3 & 3.0 & 78 & 1610 & 1.1 & 0.92 & 178.3 & 24 & 17 & 126 & 9 & 398 \\
\hline 94JE06(0-80) & 94JE06 & 0 & 80 & $\begin{array}{ll}--- \\
-\cdots\end{array}$ & 67.2 & 2.6 & 77 & 2023 & 1.2 & 0.19 & 56.1 & 29 & 12 & 56 & 7 & 519 \\
\hline 94JE06(80-120) & 94JE06 & 80 & 120 & $\begin{array}{ll}--- \\
--\end{array}$ & 111.0 & 2.1 & 62 & 1301 & 1.0 & 0.21 & 133.1 & 16 & 12 & 51 & 6 & 688 \\
\hline 94JE06(120-150) & 94JE06 & 120 & 150 & $\begin{array}{ll}--- \\
--\end{array}$ & 170.2 & 3.8 & 76 & 1348 & 1.6 & 0.17 & 49.5 & 58 & 6 & 43 & 11 & 636 \\
\hline 94JE06(150-175) & 94JE06 & 150 & 175 & ---- & 1.3 & 5.2 & 18 & 778 & 2.4 & 0.17 & 48.7 & 76 & 31 & 62 & 14 & 37 \\
\hline 94JE06(175-200) & 94JE06 & 175 & 200 & --- & 1.4 & 6.1 & 18 & 808 & 2.2 & 0.18 & 35.0 & 84 & 21 & 68 & 15 & 43 \\
\hline 94JE06B(0-5) & 94JE06B & 0 & 5 & $-\overline{---}$ & 30.1 & 4.6 & 126 & 921 & 1.5 & 0.69 & 26.2 & 26 & 13 & 92 & 8 & 253 \\
\hline 93SBL34b & 93SBL34 & & & $\mathrm{D}, \mathrm{F}$ & 9.3 & 5.1 & 45 & 823 & 1.7 & 0.24 & 17.5 & 53 & 5 & $\mathrm{NA}$ & 7 & 205 \\
\hline 93SBL34c & 93SBL34 & & & $\mathrm{D}, \mathrm{F}$ & 52.1 & 5.4 & 51 & 937 & 1.8 & 0.44 & 487.7 & 60 & 11 & $\mathrm{NA}$ & 10 & 376 \\
\hline 93SBL34d & 93SBL34 & & & $\mathrm{D}, \mathrm{F}$ & 39.2 & 6.4 & 79 & 989 & 2.7 & 0.27 & 15.1 & 50 & 8 & NA & 11 & 281 \\
\hline 93SBL34e & 93SBL34 & & & $\mathrm{D}, \mathrm{F}$ & 54.2 & 5.6 & 50 & 588 & 1.7 & 0.46 & 375.4 & 52 & 11 & $\mathrm{NA}$ & 11 & 388 \\
\hline 93SBL34f & 93SBL34 & & & $\mathrm{D}, \mathrm{F}$ & 33.1 & 5.9 & 80 & 1104 & 2.5 & 0.25 & 19.2 & 64 & 8 & $\overline{\mathrm{NA}}$ & 10 & 242 \\
\hline 93SBL34g & 93SBL34 & & & $\mathrm{D}, \mathrm{F}$ & 34.8 & 5.2 & 69 & 1168 & 1.8 & 0.27 & 40.1 & 60 & 7 & $\mathrm{NA}$ & 9 & 271 \\
\hline 93SBL34h & 93SBL34 & & & $\mathrm{D}, \mathrm{F}$ & 27.6 & 4.1 & 67 & 1161 & 1.6 & 0.51 & 137.6 & 52 & 10 & $\overline{\mathrm{NA}}$ & 8 & 297 \\
\hline 93SBL34i & 93SBL34 & & & $\mathrm{D}, \mathrm{F}$ & 29.7 & 3.9 & 72 & 811 & 1.5 & 0.40 & 27.5 & 26 & 6 & NA & 8 & 272 \\
\hline 93SBC15(0-15) & 93SBC15 & 0 & 15 & D & 2.4 & 5.0 & 24 & 455 & 1.5 & 0.46 & 4.9 & $\mathrm{NA}$ & 7.8 & NA & $\mathrm{NA}$ & 146 \\
\hline 93SBC15(15-35) & 93SBC15 & 15 & 35 & $\mathrm{D}$ & 5.2 & 5.1 & 68 & 500 & 1.5 & 0.36 & 11.0 & $\mathrm{NA}$ & 8.7 & $\mathrm{NA}$ & $\mathrm{NA}$ & 108 \\
\hline 93SBC15(35-90) & 93SBC15 & 35 & 90 & $\mathrm{D}$ & 9.5 & 4.2 & 181 & 511 & 1.3 & 0.20 & 9.6 & $\mathrm{NA}$ & 11 & $\mathrm{NA}$ & $\mathrm{NA}$ & 125 \\
\hline 93SBC15(90-165) & 93SBC15 & 90 & 165 & $\mathrm{D}$ & 20.0 & 4.3 & 97 & 680 & 1.5 & 0.18 & 22 & $\mathrm{NA}$ & 12 & $\mathrm{NA}$ & NA & 205 \\
\hline 93SBC15(165-214) & 93SBC15 & 165 & 214 & $\mathrm{D}$ & 24.0 & 4.4 & 97 & 718 & 1.5 & 0.22 & 20 & $\mathrm{NA}$ & 11 & $\mathrm{NA}$ & NA & 170 \\
\hline 93SBC15(214-242) & 93SBC15 & 214 & 242 & $\mathrm{D}$ & 23.0 & 4.7 & 97 & 755 & 1.5 & 0.26 & 47 & NA & 8.3 & NA & NA & 152 \\
\hline 93SBC15(242-261) & $93 \mathrm{SBC} 15$ & 242 & 261 & $\bar{D}$ & 20.0 & 4.4 & 30 & 665 & 1.6 & 0.28 & 55 & $\mathrm{NA}$ & 8.7 & $\overline{N A}$ & NA & 140 \\
\hline 93SBK17(8-25) & 93SBK17 & 8 & 25 & $\mathrm{D}$ & 115.0 & 5.5 & 38 & 429 & 1.9 & 0.25 & 330 & $\mathrm{NA}$ & 7 & $\mathrm{NA}$ & NA & 360 \\
\hline 93SBB21(0-9) & 93SBB21 & 0 & 9 & $\bar{D}$ & 12.0 & 2.9 & 144 & 493 & 0.96 & 0.61 & 28 & $\mathrm{NA}$ & 9.9 & $\mathrm{NA}$ & NA & 100 \\
\hline 93SBB21(41-72) & 93SBB21 & 41 & 72 & $\mathrm{D}$ & 12.0 & 2.9 & 331 & 462 & 0.99 & 0.13 & 23 & NA & 11 & NA & NA & 142 \\
\hline 93SBB21(72-84) & 93SBB21 & 72 & 84 & $\mathrm{D}$ & 12.0 & 3.0 & 150 & 518 & 1.0 & 0.12 & 10 & NA & 7.6 & NA & NA & 109 \\
\hline 93SBB21(84-99) & 93SBB21 & 84 & 99 & $\bar{D}$ & 12.0 & 3.2 & 102 & 528 & 1.2 & 0.15 & 13 & $\mathrm{NA}$ & 6.5 & $\mathrm{NA}$ & $\mathrm{NA}$ & 115 \\
\hline 93SBB21(99-111) & 93SBB21 & 99 & 111 & $\bar{D}$ & 13.0 & 3.3 & 91 & 568 & 1.1 & 0.27 & 62 & $\mathrm{NA}$ & 8.3 & $\mathrm{NA}$ & $\mathrm{NA}$ & 103 \\
\hline 93SBB21(111-120) & 93SBB21 & 111 & 120 & $\bar{D}$ & 1.1 & 3.5 & 17 & 399 & 1.4 & 0.14 & 3.0 & $\mathrm{NA}$ & 5.3 & $\overline{\mathrm{NA}}$ & $\mathrm{NA}$ & 23 \\
\hline 93SBB21(120-130) & 93SBB21 & 120 & 130 & $\mathrm{D}$ & 0.3 & 4.8 & 4 & 422 & 1.5 & 0.17 & 2.0 & $\mathrm{NA}$ & 3.5 & $\mathrm{NA}$ & NA & 12 \\
\hline 93SBB21(130-148) & 93SBB21 & 130 & 148 & $\mathrm{D}$ & 0.1 & 4.9 & 4 & 450 & 1.5 & 0.21 & 2.0 & $\mathrm{NA}$ & 4.1 & $\mathrm{NA}$ & NA & 17 \\
\hline 93SBB21(148-160) & 93SBB21 & 148 & 160 & $\mathrm{D}$ & 0.2 & 5.1 & 3 & 344 & 1.3 & 0.14 & 0.5 & $\mathrm{NA}$ & 3.9 & $\mathrm{NA}$ & NA & 11 \\
\hline 93SBB21(160-180) & 93SBB21 & 160 & 180 & $\mathrm{D}$ & 0.2 & 5.3 & 4 & 463 & 1.5 & 0.19 & 0.7 & NA & 4.1 & NA & NA & 13 \\
\hline 93SBB21(173-180) & 93SBB21 & 173 & 180 & $\mathrm{D}$ & 0.4 & 5.7 & 7 & 488 & 1.6 & 0.12 & 1.0 & $\mathrm{NA}$ & 6 & $\mathrm{NA}$ & $\mathrm{NA}$ & 325 \\
\hline 93SBB21(180-192) & 93SBB21 & 180 & 192 & $\mathrm{D}$ & 0.5 & 5.8 & 7 & 477 & 1.5 & 0.11 & 0.6 & $\mathrm{NA}$ & 5.2 & $\mathrm{NA}$ & $\mathrm{NA}$ & 165 \\
\hline
\end{tabular}


Appendix E. Chemical composition of samples analyzed, using 4 acid dissolution, by ICP-AES and ICP-MS at Eastern Washington University (EWU), Cheney, WA (Appendix_E.xls, Appendix_E.dbf).

\begin{tabular}{|c|c|c|c|c|c|c|c|c|c|c|c|c|c|c|c|c|c|c|}
\hline Sample No. & $\begin{array}{c}\mathrm{Fe} \\
(\%)\end{array}$ & $\begin{array}{c}\mathrm{Ga} \\
(\mathrm{ppm})\end{array}$ & $\begin{array}{c}\mathrm{K} \\
(\%)\end{array}$ & $\begin{array}{c}\text { La } \\
(\mathrm{ppm})\end{array}$ & $\begin{array}{l}\mathrm{Mg} \\
(\%)\end{array}$ & $\begin{array}{c}\mathrm{Mn} \\
(\mathrm{ppm})\end{array}$ & $\begin{array}{c}\text { Mo } \\
(\mathrm{ppm})\end{array}$ & $\begin{array}{c}\mathrm{Ni} \\
(\mathrm{ppm})\end{array}$ & $\begin{array}{c}\mathrm{P} \\
(\mathrm{ppm})\end{array}$ & $\begin{array}{c}\mathrm{Pb} \\
(\mathrm{ppm})\end{array}$ & $\begin{array}{c}\mathrm{Rb} \\
(\mathrm{ppm})\end{array}$ & $\begin{array}{c}\mathrm{Sb} \\
(\mathrm{ppm})\end{array}$ & $\begin{array}{c}\mathrm{Sn} \\
(\mathrm{ppm})\end{array}$ & $\begin{array}{c}\mathrm{Sr} \\
(\mathrm{ppm})\end{array}$ & $\begin{array}{c}\text { Th } \\
(p p m)\end{array}$ & $\begin{array}{c}\mathrm{Ti} \\
(\%)\end{array}$ & $\begin{array}{c}\text { TI } \\
\text { (ppm) }\end{array}$ & $\begin{array}{c}\mathrm{U} \\
(\mathrm{ppm})\end{array}$ \\
\hline SAMPLE_NO. & FE_\% & GA_PPM & K_\% & LA_PPM & MG_\% & MN_PPM & MO_PPM & NI_PPM & P_PPM & PB_PPM & RB_PPM & SB_PPM & SN_PPM & SR_PPM & TH_PPM & TI_\% & TL_PPM & U_PPM \\
\hline 93CSC03(192-233) & 15.46 & 6 & 1.20 & 10 & 0.76 & 15009 & 3 & 14 & 126 & 16297 & 63 & 84 & $\overline{2}$ & 30 & 6 & 0.0519 & $\overline{0.4}$ & 1.3 \\
\hline $93 \mathrm{CSC} 03(233-256)$ & 16.35 & 8 & 1.32 & 11 & 0.77 & 15350 & 2 & 21 & 205 & 23650 & 76 & 88 & $\overline{3}$ & 44 & 7 & 0.0668 & 0.5 & 2.2 \\
\hline 94JE06(0-80) & 19.47 & 7 & 1.24 & 12 & 0.36 & 18547 & 3 & 19 & 249 & 25476 & 69 & 86 & 3 & 32 & 6 & 0.0663 & 0.5 & 1.5 \\
\hline 94JE06(80-120) & 19.73 & 6 & 1.00 & 7 & 0.50 & 16531 & 2 & 14 & 178 & 40160 & 56 & 218 & 4 & 25 & 5 & 0.0476 & 0.4 & 1.1 \\
\hline 94JE06(120-150) & 12.33 & 11 & 1.64 & 24 & 0.32 & 5447 & 2 & 12 & 461 & 69540 & 93 & 84 & 4 & 75 & 11 & 0.0927 & 0.6 & 3.3 \\
\hline 94JE06(175-200) & 2.82 & 13 & 2.48 & 34 & 0.66 & 3101 & 2 & 15 & 667 & 750 & 138 & 7 & 3 & 57 & 13 & 0.1633 & 0.8 & 6.6 \\
\hline 94JE06B(0-5) & 11.22 & 11 & 1.65 & 12 & 0.45 & 8160 & 2 & 13 & 407 & 12860 & 87 & 115 & 4 & 103 & 8 & 0.1326 & 0.7 & 3.1 \\
\hline 93SBL34b & 9.77 & 10 & 1.93 & 24 & 0.70 & 6113 & 1 & 14 & 307 & 15070 & 87 & 63 & 2 & 45 & 10 & 0.1315 & 0.55 & 2.8 \\
\hline 93SBL34c & 10.33 & 12 & 1.95 & 26 & 0.85 & 9045 & 12 & 35 & 386 & 26510 & 98 & 126 & 2 & 53 & 11 & 0.1350 & 0.68 & 3.9 \\
\hline 93SBL34d & 12.41 & 13 & 2.38 & 21 & 0.67 & 3664 & 1 & 16 & 498 & 22150 & 115 & 161 & 1 & 55 & 10 & 0.1305 & 0.67 & 4.4 \\
\hline 93SBL34e & 10.18 & 12 & 2.02 & 22 & 0.85 & 9090 & 8 & 23 & 397 & 26860 & 104 & 131 & 2 & 54 & 11 & 0.1568 & 0.69 & 4.3 \\
\hline 93SBL34f & 11.89 & 12 & 2.08 & 28 & 0.65 & 5045 & 1 & 15 & 434 & 21480 & 104 & 129 & 2 & 50 & 11 & 0.1260 & 0.61 & 4.2 \\
\hline 93SBL34g & 13.13 & 11 & 1.99 & 26 & 0.70 & 8709 & 1 & 18 & 343 & 20830 & 97 & 105 & $\overline{2}$ & 42 & 11 & 0.1163 & 0.61 & 3.4 \\
\hline 93SBL34h & 15.47 & 9 & 1.57 & 22 & 0.91 & 14615 & 9 & 17 & 276 & 18420 & 75 & 64 & 3 & 41 & 9 & 0.0868 & 0.58 & 2.7 \\
\hline 93SBC15(0-15) & 2.90 & NA & $\mathrm{NA}$ & NA & 0.45 & 1240 & 28 & 17 & NA & 760 & NA & 9 & $\mathrm{NA}$ & 82 & 11 & 0.2150 & 0.52 & 2.9 \\
\hline 93SBC15(15-35) & 4.10 & NA & $\mathrm{NA}$ & NA & 0.46 & 2560 & 12 & 15 & NA & 1570 & NA & 22 & $\mathrm{NA}$ & 69 & 10 & 0.2160 & 0.55 & 3.0 \\
\hline 93SBC15(35-90) & 7.00 & NA & $\mathrm{NA}$ & NA & 0.37 & 5450 & 18 & 15 & NA & 2810 & NA & 40 & $\mathrm{NA}$ & 49 & 8.8 & 0.1550 & 0.46 & 2.5 \\
\hline 93SBC15(90-165) & 9.20 & $\mathrm{NA}$ & $\mathrm{NA}$ & $\mathrm{NA}$ & 0.36 & 8390 & 14 & 17 & $\mathrm{NA}$ & 7440 & $\overline{N A}$ & 65 & $\mathrm{NA}$ & 45 & 8.1 & 0.1590 & 0.53 & 5.9 \\
\hline 93SBC15(1 & 8.30 & $\mathrm{NA}$ & $\mathrm{NA}$ & NA & 0.44 & 6850 & 20 & 16 & NA & 8710 & $\mathrm{NA}$ & 73 & $\mathrm{NA}$ & 49 & 7.5 & 820 & 0.62 & 3.1 \\
\hline 93SBC15(214-242) & 8.60 & $\mathrm{NA}$ & $\mathrm{NA}$ & NA & 0.45 & 5890 & 7.3 & 14 & NA & 8960 & $\mathrm{NA}$ & 71 & $\mathrm{NA}$ & 52 & 8.9 & 0.1610 & 0.64 & 3.0 \\
\hline 93SBC15(242-261) & 5.20 & $\mathrm{NA}$ & $\mathrm{NA}$ & NA & 0.43 & 3610 & 12 & 17 & NA & 7600 & $\mathrm{NA}$ & 57 & $\mathrm{NA}$ & 59 & 7.3 & 0.1970 & 0.56 & 3.1 \\
\hline 93SBK17(8-25) & 7.80 & NA & NA & NA & 0.54 & 7630 & 4.4 & 13 & NA & 41050 & NA & 228 & $\mathrm{NA}$ & 38 & 8.2 & 0.1300 & 0.78 & 3.2 \\
\hline 93SBB21 (0-9) & 11.00 & NA & $\mathrm{NA}$ & NA & 0.42 & 10770 & 9.4 & 12 & NA & 2940 & NA & 40 & $\mathrm{NA}$ & 24 & 5.2 & 0.0980 & 0.47 & 1.5 \\
\hline 93SBB21(41-72) & 12.00 & NA & NA & NA & 0.32 & 11220 & 6.4 & 13 & NA & 3635 & NA & 53 & $\mathrm{NA}$ & 20 & 6 & 0.0780 & 0.41 & 1.7 \\
\hline 93SBB21(72-84) & 11.00 & NA & $\mathrm{NA}$ & NA & 0.32 & 9240 & 8 & 12 & NA & 4450 & NA & 41 & $\mathrm{NA}$ & 21 & 6 & 0.0850 & 0.38 & 1.6 \\
\hline 93SBB21(84-99) & 11.00 & NA & $\mathrm{NA}$ & NA & 0.38 & 9250 & 7.3 & 11 & NA & 5280 & $\mathrm{NA}$ & 45 & $\mathrm{NA}$ & 24 & 6.7 & 0.0980 & 0.42 & $\overline{1.8}$ \\
\hline 93SBB21(99-111) & 11.00 & NA & NA & NA & 0.56 & 11230 & 6.7 & 13 & NA & 5780 & $\mathrm{NA}$ & 50 & $\mathrm{NA}$ & 26 & 6.7 & 0.0910 & 0.44 & 2.1 \\
\hline 93SBB21(111-120) & 2.40 & NA & NA & NA & 0.45 & 354 & 6.9 & 13 & NA & 359 & $\mathrm{NA}$ & 4 & $\mathrm{NA}$ & 37 & 6 & 0.1500 & 0.50 & 2.3 \\
\hline 93SBB21(120-130) & 1.90 & NA & NA & NA & 0.58 & 139 & 9.9 & 10 & NA & 41 & NA & 1 & $\mathrm{NA}$ & 45 & 8.6 & 0.1490 & 0.51 & 1.8 \\
\hline 93SBB21(130-148) & 2.00 & NA & NA & NA & 0.59 & 127 & 6.9 & 12 & NA & 45 & $\mathrm{NA}$ & 1 & $\mathrm{NA}$ & 51 & 8.6 & 0.1710 & 0.58 & 2.5 \\
\hline 93SBB21(148-160) & 1.70 & NA & NA & NA & 0.34 & 96 & 8.8 & 11 & NA & 34 & $\mathrm{NA}$ & 1 & $\mathrm{NA}$ & 43 & 4.4 & 0.1550 & 0.44 & 1.6 \\
\hline 93SBB21(160-180) & 2.20 & NA & NA & NA & 0.61 & 99 & 5.8 & 11 & NA & 30 & NA & 1 & $\mathrm{NA}$ & 50 & 9.1 & 0.1640 & 0.62 & 2.3 \\
\hline 93SBB21(173-180) & 2.80 & NA & NA & NA & 0.64 & 298 & 72 & 24 & NA & 126 & NA & 2 & $\mathrm{NA}$ & 52 & 13 & 0.2570 & 0.60 & 3.3 \\
\hline 93SBB21(180-192) & 2.80 & $\mathrm{NA}$ & $\mathrm{NA}$ & NA & 0.63 & 221 & 55 & 23 & NA & 112 & $\mathrm{NA}$ & 2 & $\mathrm{NA}$ & 50 & 13 & 0.2350 & 0.57 & 3.2 \\
\hline
\end{tabular}


Appendix E. Chemical composition of samples analyzed, using 4 acid dissolution, by ICP-AES and ICP-MS at Eastern Washington University (EWU), Cheney, WA (Appendix_E.xls, Appendix_E.dbf).

\begin{tabular}{|l|r|r|r|r|r|}
\hline \multicolumn{1}{|c|}{ Sample No. } & $\begin{array}{c}\mathrm{V} \\
(\mathrm{ppm})\end{array}$ & $\begin{array}{c}\text { W } \\
(\mathrm{ppm})\end{array}$ & $\begin{array}{c}\mathrm{Y} \\
(\mathrm{ppm})\end{array}$ & $\begin{array}{c}\text { Zn } \\
(\mathrm{ppm})\end{array}$ & $\begin{array}{c}\mathrm{Zr} \\
(\mathrm{ppm})\end{array}$ \\
\hline \multicolumn{1}{|c|}{ SAMPLE_NO. } & V_PPM & w_PPM & Y_PPM & ZN_PPM & ZR_PPM \\
\hline 93CSC03(192-233) & 20 & 1 & 8 & 21399 & 22 \\
\hline 93CSC03(233-256) & 25 & 2 & 10 & 23420 & 27 \\
\hline 94JE06(0-80) & 24 & 1 & 9 & 8322 & 24 \\
\hline 94JE06(80-120) & 19 & 1 & 7 & 18569 & 20 \\
\hline 94JE06(120-150) & 34 & $<1$ & 11 & 7978 & 39 \\
\hline 94JE06(150-175) & 51 & 1 & 14 & 1724 & 56 \\
\hline 94JE06(175-200) & 53 & 1 & 18 & 1507 & 64 \\
\hline 94JE06B(0-5) & 39 & 2 & 10 & 3620 & 50 \\
\hline 93SBL34b & 38 & 3 & 14 & 4210 & 49 \\
\hline 93SBL34C & 41 & 3 & 17 & 14560 & 51 \\
\hline 93SBL34d & 49 & 2 & 23 & 4680 & 47 \\
\hline 93SBL34e & 43 & 3 & 17 & 15810 & 56 \\
\hline 93SBL34f & 44 & 2 & 22 & 4815 & 50 \\
\hline 93SBL34g & 40 & 2 & 16 & 5205 & 43 \\
\hline 93SBL34h & 33 & 2 & 13 & 12470 & 32 \\
\hline 93SBL34i & 32 & 2 & 11 & 6340 & 34 \\
\hline 93SBC15(0-15) & 42 & NA & NA & 540 & NA \\
\hline 93SBC15(15-35) & 41 & NA & NA & 785 & NA \\
\hline 93SBC15(35-90) & 34 & NA & NA & 1465 & NA \\
\hline 93SBC15(90-165) & 37 & NA & NA & 2835 & NA \\
\hline 93SBC15(165-214) & 40 & NA & NA & 2750 & NA \\
\hline 93SBC15(214-242) & 37 & NA & NA & 3760 & NA \\
\hline 93SBC15(242-261) & 43 & NA & NA & 3565 & NA \\
\hline 93SBK17(8-25) & 42 & NA & NA & 13600 & NA \\
\hline 93SBB21(0-9) & 23 & NA & NA & 4460 & NA \\
\hline 93SBB21(41-72) & 23 & NA & NA & 3310 & NA \\
\hline 93SBB21(72-84) & 24 & NA & NA & 2350 & NA \\
\hline 93SBB21(84-99) & 27 & NA & NA & 1860 & NA \\
\hline 93SBB21(99-111) & 27 & NA & NA & 2850 & NA \\
\hline 93SBB21(111-120) & 38 & NA & NA & 1129 & NA \\
\hline 93SBB21(120-130) & 37 & NA & NA & 225 & NA \\
\hline 93SBB21(130-148) & 41 & NA & NA & 187 & NA \\
\hline 93SBB21(148-160) & 38 & NA & NA & 84 & NA \\
\hline 93SBB21(160-180) & 43 & NA & NA & 108 & NA \\
\hline 93SBB21(173-180) & 49 & NA & NA & 336 & NA \\
\hline 93SBB21(180-192) & 49 & NA & NA & 240 & NA \\
\hline
\end{tabular}


Appendix E. Chemical composition of samples analyzed, using 4 acid dissolution, by ICP-AES and ICP-MS at Eastern Washington University (EWU), Cheney, WA (Appendix_E.xls, Appendix_E.dbf).

\begin{tabular}{|c|c|c|c|c|c|c|c|c|c|c|c|c|c|c|c|c|}
\hline Sample No. & Site ID & $\begin{array}{c}\text { Depth } \\
\text { Interval, } \\
\text { top }(\mathrm{cm})\end{array}$ & $\begin{array}{c}\text { Depth } \\
\text { Interval, } \\
\text { bottom }(\mathrm{cm})\end{array}$ & $\begin{array}{c}{ }^{*} \text { Other } \\
\text { analyses } \\
\text { (Appendix \#) }\end{array}$ & $\begin{array}{c}\mathrm{Ag} \\
(\mathrm{ppm})\end{array}$ & $\begin{array}{c}\mathrm{Al} \\
(\%)\end{array}$ & $\begin{array}{c}\text { As } \\
(\mathrm{ppm})\end{array}$ & $\begin{array}{c}\mathrm{Ba} \\
\text { (ppm) }\end{array}$ & $\begin{array}{c}\mathrm{Be} \\
(\mathrm{ppm})\end{array}$ & $\begin{array}{l}\mathrm{Ca} \\
(\%)\end{array}$ & $\begin{array}{c}\text { Cd } \\
\text { (ppm) }\end{array}$ & $\begin{array}{c}\mathrm{Ce} \\
(\mathrm{ppm})\end{array}$ & $\begin{array}{c}\text { Co } \\
\text { (ppm) }\end{array}$ & $\begin{array}{c}\mathrm{Cr} \\
(\mathrm{ppm})\end{array}$ & $\begin{array}{c}\text { Cs } \\
(\mathrm{ppm})\end{array}$ & $\begin{array}{c}\mathrm{Cu} \\
(\mathrm{ppm})\end{array}$ \\
\hline SAMPLE_NO. & $\overline{\text { SITE_ID }}$ & INT_TOP_CM & INT_BTM_CM & OTHR_ANLYS & $\overline{A G_{-} P P M}$ & AL_\% & AS_PPM & BA_PPM & BE_PPM & CA_\% & CD_PPM & CE_PPM & CO_PPM & \begin{tabular}{|l|} 
CR_PPM \\
\end{tabular} & CS_PPM & CU_PPM \\
\hline 93SBB21(192-203) & 93SBB21 & 192 & 203 & $\bar{D}$ & 0.2 & 4.1 & 4 & 407 & 1.5 & 0.07 & 0.4 & $\mathrm{NA}$ & 4.1 & $\mathrm{NA}$ & $\mathrm{NA}$ & 128 \\
\hline 93SBL27(0-7) & 93SBL27 & 0 & 7 & $\bar{D}$ & 15.0 & 3.8 & 127 & 606 & 1.3 & 0.23 & 29 & $\mathrm{NA}$ & 9.9 & $\overline{\mathrm{NA}}$ & $\mathrm{NA}$ & 145 \\
\hline 93SBL27(7-19) & 93SBL27 & 7 & 19 & $\bar{D}$ & 16.0 & 3.5 & 168 & 572 & 1.1 & 0.16 & 28 & NA & 11 & NA & NA & 143 \\
\hline 93SBL27(19-23) & 93SBL27 & 19 & 23 & $\bar{D}$ & 15.0 & 3.8 & 289 & 571 & 1.3 & 0.17 & 34 & NA & 14 & NA & NA & 160 \\
\hline 93SBL27(23-39) & 93SBL27 & 23 & 39 & $\mathrm{D}$ & 14.0 & 3.4 & 270 & 576 & 1.1 & 0.11 & 31 & NA & 15 & NA & NA & 125 \\
\hline 93SBL27(39-46) & 93SBL27 & 39 & 46 & $\bar{D}$ & 13.0 & 3.4 & 346 & 485 & 1.2 & 0.10 & 23 & $\mathrm{NA}$ & 14 & $\mathrm{NA}$ & $\mathrm{NA}$ & 128 \\
\hline 93SBL27(46-50) & 93SBL27 & 46 & 50 & $\bar{D}$ & 16.0 & 3.8 & 327 & 646 & 1.2 & 0.13 & 23 & $\mathrm{NA}$ & 14 & $\mathrm{NA}$ & NA & 162 \\
\hline 93SBL27(50-60) & 93SBL27 & 50 & 60 & $\bar{D}$ & 15.0 & 3.8 & 265 & 606 & 1.3 & 0.13 & 47 & $\mathrm{NA}$ & 14 & $\mathrm{NA}$ & $\mathrm{NA}$ & 160 \\
\hline 93SBL27(60-76) & 93SBL27 & 60 & 76 & $\mathrm{D}$ & 0.2 & 6.0 & 5 & 534 & 1.6 & 0.22 & 9.1 & $\mathrm{NA}$ & 5.2 & $\mathrm{NA}$ & NA & 34 \\
\hline 93SBL27(76-90) & 93SBL27 & 76 & 90 & $\mathrm{D}$ & 0.2 & 6.5 & 5 & 540 & 1.7 & 0.21 & 6.6 & $\mathrm{NA}$ & 5.1 & $\mathrm{NA}$ & NA & 32 \\
\hline 93SBL27(90-99) & 93SBL27 & 90 & 99 & $\mathrm{D}$ & 0.3 & 6.5 & 6 & 541 & 1.8 & 0.22 & 3.6 & $\mathrm{NA}$ & 5.6 & $\mathrm{NA}$ & $\mathrm{NA}$ & 47 \\
\hline 93SBL30(0-4) & 93SBL30 & 0 & 4 & $\mathrm{D}$ & 13.0 & 4.7 & 160 & 613 & 1.4 & 0.41 & 27 & $\mathrm{NA}$ & 18 & $\mathrm{NA}$ & $\mathrm{NA}$ & 118 \\
\hline 93SBL30(4-9) & 93SBL30 & 4 & 9 & $\bar{D}$ & 15.0 & 4.8 & 297 & 640 & 1.6 & 0.23 & 28 & $\mathrm{NA}$ & 8.8 & $\overline{\mathrm{NA}}$ & $\mathrm{NA}$ & 168 \\
\hline 93SBL30(9-18) & 93SBL30 & 9 & 18 & $\bar{D}$ & 13.0 & 3.6 & 289 & 515 & 1.3 & 0.16 & 18 & $\mathrm{NA}$ & 7.7 & $\mathrm{NA}$ & $\mathrm{NA}$ & 140 \\
\hline 93SBL30(18-23) & 93SBL30 & 18 & 23 & $\bar{D}$ & 14.0 & 4.1 & 120 & 603 & 1.5 & 0.18 & 13 & $\mathrm{NA}$ & 7.3 & $\mathrm{NA}$ & $\mathrm{NA}$ & 180 \\
\hline 93SBL30(23-28.5) & 93SBL30 & 23 & 28.5 & $\bar{D}$ & 17.0 & 3.9 & 112 & 618 & 1.4 & 0.18 & 11 & $\mathrm{NA}$ & 6.7 & $\overline{\mathrm{NA}}$ & $\mathrm{NA}$ & 154 \\
\hline 93SBL30(28.5-31.5) & 93SBL30 & 28.5 & 31.5 & D & 15.0 & 3.5 & 132 & 717 & 1.3 & 0.17 & 8.9 & NA & 6.6 & NA & NA & 145 \\
\hline 93SBL30(31.5-37) & 93SBL30 & 31.5 & 37 & $\bar{D}$ & 17.0 & 3.6 & 98 & 595 & 1.8 & 0.15 & 6.8 & $\mathrm{NA}$ & 12 & $\mathrm{NA}$ & $\mathrm{NA}$ & 128 \\
\hline 93SBL30(37-48) & 93SBL30 & 37 & 48 & $\bar{D}$ & 1.4 & 3.4 & 6 & 439 & 1.9 & 0.17 & 5.0 & $\mathrm{NA}$ & 6.1 & $\mathrm{NA}$ & $\mathrm{NA}$ & 33 \\
\hline 93SBL30(48-62) & 93SBL30 & 48 & 62 & $\mathrm{D}$ & 0.1 & 5.6 & 5 & 435 & 1.7 & 0.17 & 2.6 & $\mathrm{NA}$ & 4.5 & $\mathrm{NA}$ & NA & 22 \\
\hline 93SBL30(62-77) & 93SBL30 & 62 & 77 & D & 0.1 & 5.9 & 5 & 466 & 1.7 & 0.16 & 1.6 & $\mathrm{NA}$ & 4 & $\mathrm{NA}$ & NA & 35 \\
\hline 93SBL30(77-100) & 93SBL30 & 77 & 100 & D & 0.1 & 5.8 & 4 & 470 & 1.7 & 0.17 & 0.8 & NA & 3.5 & NA & NA & 27 \\
\hline 93SBL30(127-137.5) & 93SBL30 & 127 & 137.5 & $\bar{D}$ & 0.1 & 5.0 & 4 & 440 & 1.9 & 0.27 & 0.2 & $\mathrm{NA}$ & 3.9 & $\overline{N A}$ & NA & 92 \\
\hline 93SBL30(137.5-144.5) & 93SBL30 & 137.5 & 144.5 & $\bar{D}$ & 0.1 & 6.1 & 4 & 509 & 2.0 & 0.25 & 0.6 & $\mathrm{NA}$ & 5.9 & $\mathrm{NA}$ & NA & 134 \\
\hline 93SBL30(144.5-158.5) & 93SBL30 & 144.5 & 158.5 & $\mathrm{D}$ & 0.1 & 6.1 & 3 & 520 & 2.0 & 0.31 & 0.2 & $\mathrm{NA}$ & 4.4 & NA & NA & 75 \\
\hline 93SBL30(158.5-172) & 93SBL30 & 158.5 & 172 & $\mathrm{D}$ & 0.1 & 5.7 & 4 & 502 & 1.7 & 0.31 & 0.1 & NA & 4.6 & NA & NA & 91 \\
\hline 93SBL30(172-180) & 93SBL30 & 172 & 180 & $\bar{D}$ & 0.1 & 5.2 & 4 & 453 & 1.8 & 0.31 & 0.1 & NA & 8.6 & NA & NA & 127 \\
\hline 93SBL30(180-187) & 93SBL30 & 180 & 187 & $\bar{D}$ & 0.2 & 6.4 & 6 & 533 & 2.0 & 0.36 & 0.5 & $\mathrm{NA}$ & 10 & $\mathrm{NA}$ & $\mathrm{NA}$ & 245 \\
\hline 93SBK38(SAND) & 93SBK38 & 0 & 2 & $\bar{D}$ & 9.3 & 4.5 & 60 & 535 & 1.5 & 0.36 & 9.6 & $\mathrm{NA}$ & 40 & $\mathrm{NA}$ & $\mathrm{NA}$ & 138 \\
\hline 93SBK38(0-11) & 93SBK38 & 0 & 11 & $\bar{D}$ & 9.2 & 5.4 & 79 & 538 & 1.5 & 0.29 & 16 & $\mathrm{NA}$ & 13 & $\overline{\mathrm{NA}}$ & $\mathrm{NA}$ & 100 \\
\hline 93SBK38(11-20) & 93SBK38 & 11 & 20 & $\mathrm{D}$ & 15.0 & 4.7 & 133 & 671 & 1.4 & 0.16 & 9.0 & $\mathrm{NA}$ & 11 & $\mathrm{NA}$ & $\mathrm{NA}$ & 135 \\
\hline 93SBK38(20-55) & 93SBK38 & 20 & 55 & $\mathrm{D}$ & 7.2 & 4.9 & 64 & 547 & 1.6 & 0.13 & 4.7 & $\mathrm{NA}$ & 9.4 & $\mathrm{NA}$ & $\mathrm{NA}$ & 72 \\
\hline 93SBK38(55-100) & 93SBK38 & 55 & 100 & $\mathrm{D}$ & 15.0 & 4.1 & 117 & 559 & 1.4 & 0.17 & 11 & $\mathrm{NA}$ & 9.9 & $\mathrm{NA}$ & NA & 108 \\
\hline 93SBC39(SAND) & 93SBC39 & 0 & 2 & $\mathrm{D}$ & 12.0 & 3.6 & 128 & 435 & 1.2 & 0.13 & 13 & $\mathrm{NA}$ & 9.3 & $\mathrm{NA}$ & NA & 105 \\
\hline 93SBC39(0-5) & 93SBC39 & 0 & 5 & $\bar{D}$ & 10.0 & 3.5 & 132 & 447 & 1.2 & 0.13 & 13 & $\mathrm{NA}$ & 9.7 & $\mathrm{NA}$ & $\mathrm{NA}$ & 103 \\
\hline 93SBC39(5-10) & 93SBC39 & 5 & 10 & $\mathrm{D}$ & 16.0 & 3.9 & 302 & 542 & 1.2 & 0.13 & 21 & $\mathrm{NA}$ & 13 & $\mathrm{NA}$ & $\mathrm{NA}$ & 120 \\
\hline
\end{tabular}


Appendix E. Chemical composition of samples analyzed, using 4 acid dissolution, by ICP-AES and ICP-MS at Eastern Washington University (EWU), Cheney, WA (Appendix_E.xls, Appendix_E.dbf).

\begin{tabular}{|c|c|c|c|c|c|c|c|c|c|c|c|c|c|c|c|c|c|c|}
\hline Sample No. & $\begin{array}{c}\mathrm{Fe} \\
(\%)\end{array}$ & $\begin{array}{c}\mathrm{Ga} \\
(\mathrm{ppm})\end{array}$ & $\begin{array}{c}\mathrm{K} \\
(\%)\end{array}$ & $\begin{array}{c}\text { La } \\
(\mathrm{ppm})\end{array}$ & $\begin{array}{l}\mathrm{Mg} \\
(\%)\end{array}$ & $\begin{array}{c}\mathrm{Mn} \\
(\mathrm{ppm})\end{array}$ & $\begin{array}{c}\text { Mo } \\
\text { (ppm) }\end{array}$ & $\begin{array}{c}\mathrm{Ni} \\
(\mathrm{ppm})\end{array}$ & $\begin{array}{c}\mathrm{P} \\
(\mathrm{ppm})\end{array}$ & $\begin{array}{c}\mathrm{Pb} \\
(\mathrm{ppm})\end{array}$ & $\begin{array}{c}\mathrm{Rb} \\
(\mathrm{ppm})\end{array}$ & $\begin{array}{c}\mathrm{Sb} \\
(\mathrm{ppm})\end{array}$ & $\begin{array}{c}\mathrm{Sn} \\
(\mathrm{ppm})\end{array}$ & $\begin{array}{c}\mathrm{Sr} \\
(\mathrm{ppm})\end{array}$ & $\begin{array}{c}\text { Th } \\
(p p m)\end{array}$ & $\begin{array}{c}\mathrm{Ti} \\
(\%)\end{array}$ & $\begin{array}{c}\mathrm{TI} \\
(\mathrm{ppm})\end{array}$ & $\begin{array}{c}\mathrm{U} \\
(\mathrm{ppm})\end{array}$ \\
\hline SAMPLE_NO. & FE_\% & GA_PPM & K_\% & LA_PPM & MG_\% & MN_PPM & MO_PPM & NI_PPM & P_PPM & PB_PPM & RB_PPM & SB_PPM & SN_PPM & SR_PPM & TH_PPM & TI_\% & TL_PPM & U_PPM \\
\hline 93SBB21(192-203) & 2.50 & $\mathrm{NA}$ & $\overline{N A}$ & NA & 0.45 & 121 & 33 & 17 & NA & 39 & $\overline{N A}$ & 2 & $\mathrm{NA}$ & 36 & 6.8 & 0.2160 & 0.52 & 2.3 \\
\hline 93SBL27(0-7) & 10.00 & NA & $\mathrm{NA}$ & NA & 0.59 & 10610 & 8.1 & 12 & NA & 4335 & NA & 50 & NA & 36 & 6.9 & 0.1180 & 0.62 & 1.9 \\
\hline 93SBL27(7-19) & 11.00 & $\mathrm{NA}$ & $\mathrm{NA}$ & NA & 0.51 & 12040 & 9.2 & 12 & NA & 4240 & NA & 54 & $\mathrm{NA}$ & 25 & 6.5 & 0.0850 & 0.52 & 1.8 \\
\hline 93SBL27(19-23) & 12.00 & $\mathrm{NA}$ & $\overline{\mathrm{NA}}$ & NA & 0.51 & 11240 & 11 & 13 & NA & 4300 & $\mathrm{NA}$ & 59 & $\mathrm{NA}$ & 29 & 7.7 & 0.1010 & 0.63 & 2.5 \\
\hline 93SBL27(23-39) & 13.00 & $\mathrm{NA}$ & $\overline{\mathrm{NA}}$ & NA & 0.33 & 13770 & 6.3 & 14 & NA & 4390 & $\mathrm{NA}$ & 58 & $\mathrm{NA}$ & 25 & 6.6 & 0.0860 & 0.49 & 1.8 \\
\hline 93SBL27(46-50) & 11.00 & $\mathrm{NA}$ & $\overline{\mathrm{NA}}$ & NA & 0.32 & 11860 & 6 & 15 & NA & 5270 & $\mathrm{NA}$ & 55 & $\mathrm{NA}$ & 29 & 8.1 & 0.0960 & 0.47 & 2.3 \\
\hline 93SBL27(50-60) & 12.00 & $\mathrm{NA}$ & $\overline{\mathrm{NA}}$ & NA & 0.32 & 11830 & 8.2 & 18 & NA & 5085 & $\mathrm{NA}$ & 50 & $\mathrm{NA}$ & 27 & 7.6 & 0.0970 & 0.47 & 2.1 \\
\hline 93SBL27(60-76) & 2.00 & $\mathrm{NA}$ & $\mathrm{NA}$ & NA & 0.66 & 273 & 5.9 & 12 & NA & 59 & NA & 2 & $\mathrm{NA}$ & 50 & 12 & 0.1910 & 0.56 & 2.7 \\
\hline 93SBL27(76-90) & 2.10 & NA & $\mathrm{NA}$ & NA & 0.68 & 212 & 9.8 & 12 & NA & 44 & NA & 2 & $\mathrm{NA}$ & 52 & 14 & 0.2090 & 0.59 & 2.9 \\
\hline 93SBL27(90-99) & 2.20 & NA & $\mathrm{NA}$ & NA & 0.69 & 227 & 8.5 & 12 & NA & 28 & NA & 2 & $\mathrm{NA}$ & 54 & 14 & 0.2130 & 0.61 & 3.5 \\
\hline 93SBL30(0-4) & 8.80 & $\mathrm{NA}$ & $\mathrm{NA}$ & NA & 0.59 & 9320 & 7.3 & 17 & NA & 5100 & NA & 47 & $\mathrm{NA}$ & 62 & 6.8 & 0.1330 & 0.62 & 2.6 \\
\hline 93SBL30(4-9) & 11.00 & NA & $\mathrm{NA}$ & NA & 0.65 & 8880 & 9.2 & 22 & NA & 4850 & NA & 69 & $\mathrm{NA}$ & 40 & 8.8 & 0.1270 & 0.67 & 3.4 \\
\hline 93SBL30(9-18) & 11.00 & $\mathrm{NA}$ & $\mathrm{NA}$ & NA & 0.48 & 9290 & 5.4 & 16 & NA & 4700 & NA & 58 & $\mathrm{NA}$ & 25 & 6.7 & 0.0930 & 0.48 & 2.4 \\
\hline 93SBL30(18-23) & 10.00 & $\mathrm{NA}$ & $\mathrm{NA}$ & NA & 0.54 & 9160 & 6.9 & 15 & NA & 5910 & NA & 54 & $\mathrm{NA}$ & 32 & 7.7 & 0.1060 & 0.49 & 2.4 \\
\hline 93SBL30(28.5-31.5) & 12.00 & $\mathrm{NA}$ & $\overline{\mathrm{NA}}$ & NA & 0.56 & 10930 & 12 & 13 & NA & 5800 & NA & 54 & $\mathrm{NA}$ & 27 & 7.1 & 0.0980 & 0.45 & 2.8 \\
\hline 93SBL30(31.5-37) & 11.00 & $\overline{\mathrm{NA}}$ & $\overline{\mathrm{NA}}$ & NA & 0.42 & 6400 & 6.7 & 12 & NA & 5510 & NA & 45 & $\mathrm{NA}$ & 29 & 5.6 & 0.0870 & 0.53 & 1.6 \\
\hline 93SBL30(37-48) & 1.90 & $\mathrm{NA}$ & $\overline{\mathrm{NA}}$ & NA & 0.33 & 820 & 3.6 & 14 & NA & 428 & NA & 4 & $\mathrm{NA}$ & 39 & 4.5 & 0.1470 & 0.47 & 3.0 \\
\hline 93SBL30(48-62) & 2.00 & $\mathrm{NA}$ & $\mathrm{NA}$ & NA & 0.54 & 364 & 6 & 13 & NA & 97 & NA & 1 & $\mathrm{NA}$ & 42 & 9.7 & 0.1630 & 0.49 & 3.5 \\
\hline 93SBL30(62-77) & 2.20 & $\mathrm{NA}$ & $\mathrm{NA}$ & NA & 0.58 & 272 & 5.4 & 12 & NA & 47 & NA & 1 & NA & 44 & 9.9 & 590 & 0.59 & 3.1 \\
\hline 93SBL30 & 2.10 & $\mathrm{NA}$ & $\mathrm{NA}$ & NA & 0.58 & 208 & 6.1 & 11 & NA & 39 & $\mathrm{NA}$ & 1 & $\mathrm{NA}$ & 47 & 11 & 0.1590 & 0.58 & 2.7 \\
\hline 93SBL30 & 2.00 & $\mathrm{NA}$ & $\mathrm{NA}$ & NA & 0.48 & 122 & 15 & 13 & NA & 57 & $\mathrm{NA}$ & 1 & $\mathrm{NA}$ & 54 & 8.2 & 0.2290 & 0.55 & 2.8 \\
\hline 93SBL30(137.5-144.5) & 2.20 & NA & $\mathrm{NA}$ & NA & 0.60 & 251 & 28 & 16 & NA & 47 & NA & 2 & $\mathrm{NA}$ & 62 & 13 & 0.2460 & 0.59 & 4.1 \\
\hline 93SBL30(144.5-158.5) & 1.90 & $\mathrm{NA}$ & $\overline{\mathrm{NA}}$ & NA & 0.61 & 142 & 13 & 13 & $\overline{\mathrm{NA}}$ & 44 & NA & 1 & $\mathrm{NA}$ & 67 & 13 & 0.2290 & 0.59 & 3.8 \\
\hline 93SBL30(158.5-172) & 1.90 & $\mathrm{NA}$ & $\mathrm{NA}$ & NA & 0.58 & 95 & 7.8 & 12 & NA & 45 & NA & 1 & $\mathrm{NA}$ & 62 & 11 & 0.2090 & 0.58 & 3.1 \\
\hline 93SBL30(172-180) & 2.00 & $\mathrm{NA}$ & $\mathrm{NA}$ & NA & 0.51 & 110 & 13 & 13 & NA & 39 & NA & 1 & $\mathrm{NA}$ & 60 & 8.3 & 0.2220 & 0.56 & 2.7 \\
\hline 93SBL30(180-187) & 2.50 & $\mathrm{NA}$ & $\mathrm{NA}$ & NA & 0.65 & 1858 & 16 & 15 & NA & 99 & NA & 2 & $\mathrm{NA}$ & 68 & 11 & 0.2290 & 0.68 & 3.0 \\
\hline 93SBK38(SAND) & 10.00 & NA & $\mathrm{NA}$ & NA & 0.55 & 7740 & 228 & 1384 & NA & 2975 & $\mathrm{NA}$ & 49 & $\mathrm{NA}$ & 79 & 7.9 & 0.0840 & 0.59 & 1.8 \\
\hline 93SBK38(0-11) & 5.20 & $\mathrm{NA}$ & $\mathrm{NA}$ & NA & 0.46 & 3320 & 2.4 & 16 & NA & 2700 & NA & 42 & $\mathrm{NA}$ & 57 & 9.6 & 0.1550 & 0.65 & 2.6 \\
\hline 93SBK38(11-20) & 9.40 & $\mathrm{NA}$ & $\mathrm{NA}$ & NA & 0.51 & 6650 & 20 & 112 & NA & 4890 & NA & 46 & $\mathrm{NA}$ & 50 & 9.1 & 0.1250 & 0.68 & 2.2 \\
\hline 93SBK38(20-55) & 6.50 & $\mathrm{NA}$ & $\mathrm{NA}$ & NA & 0.73 & 4750 & 3 & 29 & NA & 2675 & NA & 34 & $\mathrm{NA}$ & 57 & 8.8 & 0.1080 & 0.70 & 1.9 \\
\hline 93SBK38(55-100) & 12.00 & $\mathrm{NA}$ & $\mathrm{NA}$ & NA & 0.59 & 10710 & 2.5 & 12 & NA & 4750 & NA & 74 & $\mathrm{NA}$ & 43 & 7.7 & 0.0810 & 0.63 & 1.8 \\
\hline 93SBC39(SAND) & 11.00 & NA & $\mathrm{NA}$ & NA & 0.53 & 9170 & 12 & 13 & NA & 3260 & NA & 52 & $\mathrm{NA}$ & 31 & 6.4 & 0.0770 & 0.51 & 1.7 \\
\hline 93SBC39(0-5) & 11.00 & $\mathrm{NA}$ & $\mathrm{NA}$ & NA & 0.55 & 9420 & 12 & 13 & NA & 3315 & NA & 60 & $\mathrm{NA}$ & 32 & 6.6 & 0.0820 & 0.55 & 1.7 \\
\hline
\end{tabular}


Appendix E. Chemical composition of samples analyzed, using 4 acid dissolution, by ICP-AES and ICP-MS at Eastern Washington University (EWU), Cheney, WA (Appendix_E.xls, Appendix_E.dbf).

\begin{tabular}{|c|c|c|c|c|c|}
\hline Sample No. & $\begin{array}{c}\text { V } \\
(p p m)\end{array}$ & $\begin{array}{c}\text { W } \\
(\mathrm{ppm})\end{array}$ & $\begin{array}{c}\mathrm{Y} \\
(\mathrm{ppm})\end{array}$ & $\begin{array}{c}\mathrm{Zn} \\
(\mathrm{ppm})\end{array}$ & $\begin{array}{c}\mathrm{Zr} \\
(\mathrm{ppm})\end{array}$ \\
\hline SAMPLE_NO. & $\begin{array}{l}\text { VPPM } \\
\end{array}$ & $\overline{\text { W_PPM }}$ & Y_PPM & ZN_PPM & ZR_PPM \\
\hline 93SBB21(192-203) & 43 & NA & NA & 166 & NA \\
\hline 93SBL27(0-7) & 32 & NA & NA & 3970 & NA \\
\hline 93SBL27(7-19) & 27 & $\mathrm{NA}$ & $\mathrm{NA}$ & 4445 & $\mathrm{NA}$ \\
\hline 93SBL27(19-23) & 29 & NA & NA & 3170 & $\overline{\mathrm{NA}}$ \\
\hline 93SBL27(23-39) & 25 & NA & NA & 4300 & $\overline{\mathrm{NA}}$ \\
\hline 93SBL27(39-46) & $\overline{26}$ & $\overline{\mathrm{NA}}$ & $\overline{\mathrm{NA}}$ & 4280 & $\overline{\mathrm{NA}}$ \\
\hline 93SBL27(46-50) & 29 & NA & NA & 4210 & $\mathrm{NA}$ \\
\hline 93SBL27(50-60) & 29 & NA & NA & 5605 & $\overline{\mathrm{NA}}$ \\
\hline 93SBL27(60-76) & 43 & NA & NA & 938 & $\mathrm{NA}$ \\
\hline 93SBL27(76-90) & 44 & NA & NA & 537 & $\mathrm{NA}$ \\
\hline 93SBL27(90-99) & 46 & NA & NA & 285 & $\mathrm{NA}$ \\
\hline 93SBL30(0-4) & 36 & NA & NA & 4140 & NA \\
\hline 93SBL30(4-9) & 39 & NA & NA & 5225 & NA \\
\hline 93SBL30(9-18) & 29 & NA & NA & 3410 & NA \\
\hline 93SBL30(18-23) & 32 & NA & NA & 3150 & NA \\
\hline 93SBL30(23-28.5) & 31 & NA & NA & 2780 & $\mathrm{NA}$ \\
\hline 93SBL30(28.5-31.5) & 26 & NA & NA & 2710 & NA \\
\hline 93SBL30(31.5-37) & 27 & $\overline{\mathrm{NA}}$ & $\overline{\mathrm{NA}}$ & 3140 & $\overline{\mathrm{NA}}$ \\
\hline 93SBL30(37-48) & 42 & NA & NA & 920 & $\overline{\mathrm{NA}}$ \\
\hline 93SBL30(48-62) & 41 & $\mathrm{NA}$ & NA & 560 & $\mathrm{NA}$ \\
\hline 93SBL30(62-77) & 45 & NA & NA & 387 & $\overline{N A}$ \\
\hline 93SBL30(77-100) & 46 & NA & NA & 285 & $\overline{\mathrm{NA}}$ \\
\hline 93SBL30(127-137.5) & 45 & NA & NA & 144 & NA \\
\hline 93SBL30(1 & 49 & $\mathrm{NA}$ & $\mathrm{NA}$ & 395 & $\mathrm{NA}$ \\
\hline 93SBL30(144.5-158.5) & 46 & NA & NA & 199 & NA \\
\hline 93SBL30(158.5-172) & 47 & $\mathrm{NA}$ & NA & 131 & NA \\
\hline 93SBL30(172-180) & 46 & NA & NA & 143 & NA \\
\hline 93SBL30(180-187) & 55 & NA & NA & 248 & NA \\
\hline 93SBK38(SAND) & 41 & NA & NA & 2090 & NA \\
\hline 93SBK38(0-11) & 43 & NA & NA & 1540 & NA \\
\hline 93SBK38(11-20) & 38 & NA & NA & 1665 & NA \\
\hline 93SBK38(20-55) & 38 & NA & NA & 950 & NA \\
\hline 93SBK38(55-100) & 35 & NA & NA & 1830 & $\mathrm{NA}$ \\
\hline 93SBC39(SAND) & 27 & NA & NA & 2165 & $\mathrm{NA}$ \\
\hline 93SBC39(0-5) & 29 & NA & NA & 2250 & $\mathrm{NA}$ \\
\hline 93SBC39(5-10) & 31 & NA & NA & 2705 & NA \\
\hline
\end{tabular}


Appendix E. Chemical composition of samples analyzed, using 4 acid dissolution, by ICP-AES and ICP-MS at Eastern Washington University (EWU), Cheney, WA (Appendix_E.xls, Appendix_E.dbf).

\begin{tabular}{|c|c|c|c|c|c|c|c|c|c|c|c|c|c|c|c|c|}
\hline Sample No. & Site ID & $\begin{array}{c}\text { Depth } \\
\text { Interval, } \\
\text { top }(\mathrm{cm})\end{array}$ & $\begin{array}{c}\text { Depth } \\
\text { Interval, } \\
\text { bottom }(\mathrm{cm})\end{array}$ & $\begin{array}{c}{ }^{*} \text { Other } \\
\text { analyses } \\
\text { (Appendix \#) }\end{array}$ & $\begin{array}{c}\mathrm{Ag} \\
(\mathrm{ppm})\end{array}$ & $\begin{array}{c}\mathrm{Al} \\
(\%)\end{array}$ & $\begin{array}{c}\text { As } \\
\text { (ppm) }\end{array}$ & $\begin{array}{c}\mathrm{Ba} \\
(\mathrm{ppm})\end{array}$ & $\begin{array}{c}\mathrm{Be} \\
(\mathrm{ppm})\end{array}$ & $\begin{array}{l}\mathrm{Ca} \\
(\%)\end{array}$ & $\begin{array}{c}\mathrm{Cd} \\
(\mathrm{ppm})\end{array}$ & $\begin{array}{c}\mathrm{Ce} \\
(\mathrm{ppm})\end{array}$ & $\begin{array}{c}\text { Co } \\
(\mathrm{ppm})\end{array}$ & $\begin{array}{c}\mathrm{Cr} \\
\text { (ppm) }\end{array}$ & $\begin{array}{c}\text { Cs } \\
\text { (ppm) }\end{array}$ & $\begin{array}{c}\mathrm{Cu} \\
(\mathrm{ppm})\end{array}$ \\
\hline SAMPLE_NO. & $\begin{array}{l}\text { SITE_ID } \\
\end{array}$ & INT_TOP_CM & INT_BTM_CM & OTHR_ANLYS & AG_PPM & AL_\% & AS_PPM & BA_PPM & BE_PPM & CA_\% & CD_PPM & CE_PPM & CO_PPM & CR_PPM & CS_PPM & CU_PPM \\
\hline 93SBC39(10-20) & 93SBC39 & 10 & 20 & $\bar{D}$ & 17.0 & 3.3 & 384 & 506 & 1.1 & 0.14 & 17 & $\mathrm{NA}$ & 16 & $\overline{\mathrm{NA}}$ & $\mathrm{NA}$ & 132 \\
\hline 93SBC39(20-27) & 93SBC39 & 20 & 27 & $\mathrm{D}$ & 16.0 & 3.2 & 346 & 539 & 1.1 & 0.11 & 21 & NA & 18 & NA & NA & 140 \\
\hline 93SBC39(27-32) & 93SBC39 & 27 & 32 & $\mathrm{D}$ & 13.0 & 4.6 & 105 & 707 & 1.6 & 0.15 & 20 & NA & 12 & $\mathrm{NA}$ & $\mathrm{NA}$ & 127 \\
\hline 93SBC39(32-40) & 93SBC39 & 32 & 40 & $\mathrm{D}$ & 0.6 & 6.6 & 11 & 691 & 1.9 & 0.23 & 17 & NA & 8.4 & NA & $\mathrm{NA}$ & 24 \\
\hline 93SBC39(40-58) & 93SBC39 & 40 & 58 & $\bar{D}$ & 0.3 & 6.4 & 9 & 596 & 1.9 & 0.20 & 2.8 & $\mathrm{NA}$ & 7.2 & $\mathrm{NA}$ & NA & 18 \\
\hline 93SBC39(58-72) & 93SBC39 & 58 & 72 & $\bar{D}$ & 0.3 & 6.1 & 11 & 571 & 1.9 & 0.20 & 0.8 & $\mathrm{NA}$ & 7.3 & $\mathrm{NA}$ & NA & 19 \\
\hline 94SB40(SAND) & 94SB40 & 0 & 2 & & 0.1 & 5.7 & 4 & 490 & 1.7 & 0.62 & 0.1 & $\mathrm{NA}$ & 7.1 & $\mathrm{NA}$ & NA & 16 \\
\hline 94SB40(0-15) & 94SB40 & 0 & 15 & & 0.1 & 5.9 & 8 & 506 & 1.7 & 0.71 & 0.2 & $\mathrm{NA}$ & 8.1 & $\mathrm{NA}$ & $\mathrm{NA}$ & 43 \\
\hline 94SB40(16-50) & 94SB40 & 16 & 50 & & 0.1 & 5.6 & 20 & 491 & 1.8 & 0.55 & 0.2 & $\mathrm{NA}$ & 8.6 & $\mathrm{NA}$ & NA & 130 \\
\hline 94SB40(50-75) & 94SB40 & 50 & 75 & & 0.1 & 4.9 & 8 & 462 & 1.9 & 0.38 & 0.2 & $\mathrm{NA}$ & 9.4 & $\mathrm{NA}$ & $\mathrm{NA}$ & 33 \\
\hline 94SB40(75-100) & 94SB40 & 75 & 100 & & 0.1 & 6.6 & 6 & 527 & 1.8 & 0.50 & 0.1 & $\mathrm{NA}$ & 8.8 & $\mathrm{NA}$ & $\mathrm{NA}$ & 25 \\
\hline 94SB40(100-125) & 94SB40 & 100 & 125 & & 0.1 & 5.9 & 7 & 487 & 1.8 & 0.45 & 0.2 & $\mathrm{NA}$ & 7.9 & $\mathrm{NA}$ & $\mathrm{NA}$ & 24 \\
\hline
\end{tabular}


Appendix E. Chemical composition of samples analyzed, using 4 acid dissolution, by ICP-AES and ICP-MS at Eastern Washington University (EWU), Cheney, WA (Appendix_E.xls, Appendix_E.dbf).

\begin{tabular}{|c|c|c|c|c|c|c|c|c|c|c|c|c|c|c|c|c|c|c|}
\hline Sample No. & $\begin{array}{l}\mathrm{Fe} \\
(\%)\end{array}$ & $\begin{array}{c}\mathrm{Ga} \\
(\mathrm{ppm})\end{array}$ & $\begin{array}{c}\mathrm{K} \\
(\%)\end{array}$ & $\begin{array}{c}\text { La } \\
(\mathrm{ppm})\end{array}$ & $\begin{array}{l}\mathrm{Mg} \\
(\%)\end{array}$ & $\begin{array}{c}\mathrm{Mn} \\
(\mathrm{ppm})\end{array}$ & $\begin{array}{c}\text { Mo } \\
(\mathrm{ppm})\end{array}$ & $\begin{array}{c}\mathrm{Ni} \\
(\mathrm{ppm})\end{array}$ & $\begin{array}{c}P \\
(p p m)\end{array}$ & $\begin{array}{c}\mathrm{Pb} \\
(\mathrm{ppm})\end{array}$ & $\begin{array}{c}\mathrm{Rb} \\
(\mathrm{ppm})\end{array}$ & $\begin{array}{c}\mathrm{Sb} \\
(\mathrm{ppm})\end{array}$ & $\begin{array}{c}\mathrm{Sn} \\
(\mathrm{ppm})\end{array}$ & $\begin{array}{c}\mathrm{Sr} \\
(\mathrm{ppm})\end{array}$ & $\begin{array}{c}\text { Th } \\
(p p m)\end{array}$ & $\begin{array}{c}\mathrm{Ti} \\
(\%)\end{array}$ & $\begin{array}{c}\text { TI } \\
(\mathrm{ppm})\end{array}$ & $\begin{array}{c}U \\
(p p m)\end{array}$ \\
\hline SAMPLE_NO. & FE_\% & GA_PPM & K_\% & LA_PPM & MG_\% & MN_PPM & MO_PPM & NI_PPM & P_PPM & PB_PPM & RB_PPM & SB_PPM & SN_PPM & SR_PPM & TH_PPM & TI_\% & TL_PPM & U_PPM \\
\hline 93SBC39(10-20) & 14.00 & NA & NA & NA & 0.39 & 11410 & 2.4 & 16 & NA & 3800 & NA & 81 & NA & 27 & 5.9 & 0.0960 & 0.47 & 1.8 \\
\hline 93SBC39(20-27) & 14.00 & NA & NA & NA & 0.36 & 12070 & 3.3 & 24 & NA & 3875 & NA & 77 & NA & 24 & 6.8 & 0.1030 & 0.41 & 1.7 \\
\hline 93SBC39(27-32) & 8.20 & $\mathrm{NA}$ & $\mathrm{NA}$ & $\mathrm{NA}$ & 0.47 & 6590 & 2.3 & 17 & NA & 3690 & $\mathrm{NA}$ & 48 & NA & 38 & 8.5 & 0.1350 & 0.49 & 2.6 \\
\hline 93SBC39(32-40) & 3.00 & $\mathrm{NA}$ & $\mathrm{NA}$ & NA & 0.75 & 1290 & 1.7 & 18 & NA & 83 & $\mathrm{NA}$ & 2 & NA & 58 & 13 & 0.2070 & 0.59 & 4.6 \\
\hline 93SBC39(40-58) & 2.80 & NA & $\mathrm{NA}$ & NA & 0.74 & 650 & 1.6 & 16 & NA & 97 & NA & 2 & NA & 51 & 12 & 0.1970 & 0.58 & 4.0 \\
\hline 94SB40(SAND) & 2.60 & NA & $\mathrm{NA}$ & NA & 0.83 & 307 & 1.3 & 13 & NA & 21 & NA & 1 & NA & 74 & 8.7 & 0.2570 & 0.37 & 2.6 \\
\hline 94 SB40(0-15) & 2.90 & NA & $\mathrm{NA}$ & NA & 0.86 & 383 & 2.3 & 17 & NA & 21 & NA & 1 & NA & 79 & 8.9 & 0.2780 & 0.40 & 3.3 \\
\hline 94SB40 (16-50) & 2.70 & NA & NA & NA & 0.79 & 314 & 1.2 & 16 & NA & 23 & NA & 1 & NA & 65 & 8.2 & 0.2720 & 0.42 & 3.6 \\
\hline 94SB40(50-75) & 3.00 & $\mathrm{NA}$ & $\mathrm{NA}$ & NA & 0.55 & 372 & 2.3 & 19 & NA & 22 & $\mathrm{NA}$ & 1 & NA & 54 & 5.7 & 0.2890 & 0.50 & 3.9 \\
\hline 94SB40(75-100) & 3.10 & $\mathrm{NA}$ & $\mathrm{NA}$ & NA & 0.62 & 418 & 2.7 & 18 & NA & 21 & $\mathrm{NA}$ & 1 & NA & 68 & 9.6 & 0.2930 & 0.45 & 4.3 \\
\hline 94SB40(100-125) & 3.00 & $\mathrm{NA}$ & $\mathrm{NA}$ & $\mathrm{NA}$ & 0.82 & 351 & 2 & 16 & $\mathrm{NA}$ & 23 & $\mathrm{NA}$ & 1 & $\mathrm{NA}$ & 65 & 9.1 & 0.2830 & 0.45 & 4.9 \\
\hline
\end{tabular}


Appendix E. Chemical composition of samples analyzed, using 4 acid dissolution, by ICP-AES and ICP-MS at Eastern Washington University (EWU), Cheney, WA (Appendix_E.xls, Appendix_E.dbf).

\begin{tabular}{|c|r|r|r|r|r|}
\hline Sample No. & $\begin{array}{c}\text { V } \\
(p p m)\end{array}$ & $\begin{array}{c}\text { W } \\
(\mathrm{ppm})\end{array}$ & $\begin{array}{c}\mathrm{Y} \\
(\mathrm{ppm})\end{array}$ & $\begin{array}{c}\mathrm{Zn} \\
(\mathrm{ppm})\end{array}$ & $\begin{array}{c}\mathrm{Zr} \\
(\mathrm{ppm})\end{array}$ \\
\hline SAMPLE_NO. & ﹎PPM & w_PPM & Y_PPM & ZN_PPM & ZR_PPM \\
\hline 93SBC39(10-20) & 30 & NA & NA & 2500 & NA \\
\hline 93SBC39(20-27) & 29 & NA & NA & 2930 & NA \\
\hline 93SBC39(27-32) & 37 & NA & NA & 2170 & NA \\
\hline 93SBC39(32-40) & 48 & NA & NA & 1275 & NA \\
\hline 93SBC39(40-58) & 47 & NA & NA & 1075 & NA \\
\hline 93SBC39(58-72) & 47 & NA & NA & 1030 & NA \\
\hline 94SB40(SAND) & 47 & NA & NA & 62 & NA \\
\hline 94SB40(0-15) & 52 & NA & NA & 94 & NA \\
\hline 94SB40(16-50) & 52 & NA & NA & 84 & NA \\
\hline 94SB40(50-75) & 56 & NA & NA & 62 & NA \\
\hline 94SB40(75-100) & 53 & NA & NA & 66 & NA \\
\hline 94SB40(100-125) & 51 & NA & NA & 88 & NA \\
\hline
\end{tabular}


Appendix F. Chemical composition of samples analyzed by ICP-AES at CHEMEX Labs in Reno, NV using nitric acid-aqua regia dissolution (Appendix_F.xls, Appendix_F.dbf).

\begin{tabular}{|c|c|c|c|c|c|c|c|c|c|c|c|c|c|c|c|}
\hline Sample No. & Site ID & $\begin{array}{l}\text { Depth } \\
\text { interval, } \\
\text { top }(\mathrm{cm})\end{array}$ & $\begin{array}{c}\text { Depth } \\
\text { interval, } \\
\text { bottom }(\mathrm{cm})\end{array}$ & $\begin{array}{l}\text { Size } \\
\text { fraction } \\
(\mathrm{mm})\end{array}$ & $\begin{array}{c}\text { *wt \% of } \\
\text { size } \\
\text { fraction }\end{array}$ & $\begin{array}{c}{ }^{* *} \text { Other } \\
\text { analyses } \\
(\text { Appendix \#) }\end{array}$ & $\begin{array}{c}\mathrm{Ag} \\
(\mathrm{ppm})\end{array}$ & $\begin{array}{c}\mathrm{Al} \\
(\%)\end{array}$ & $\begin{array}{c}\text { As } \\
\text { (ppm) }\end{array}$ & $\begin{array}{c}\mathrm{Ba} \\
(\mathrm{ppm})\end{array}$ & $\begin{array}{c}\mathrm{Be} \\
(\mathrm{ppm})\end{array}$ & $\begin{array}{c}\mathrm{Bi} \\
(\mathrm{ppm})\end{array}$ & $\begin{array}{l}\mathrm{Ca} \\
(\%)\end{array}$ & $\begin{array}{c}\mathrm{Cd} \\
(\mathrm{ppm})\end{array}$ & $\begin{array}{c}\text { Co } \\
\text { (ppm) }\end{array}$ \\
\hline SAMPLE_NO. & SITE_ID & INT_TOP_CM & INT_BTM_CM & SIZFRAC_MM & WT\%SIZFRAC & OTHR_ANLYS & AG_PPM & AL_\% & AS_PPM & BA_PPM & BE_PPM & BI_PPM & CA_\% & CD_PPM & CO_PPM \\
\hline 93ABM02(0-10) & 93ABM02 & 0 & 10 & $<0.83$ & $100 \%$ & $\bar{D}$ & 12.8 & 0.63 & 140 & 270 & $<0.5$ & $<2$ & 0.15 & 20.5 & 12 \\
\hline 93ABM02(11-22) & $93 \mathrm{ABM} 02$ & 11 & 22 & $<0.83$ & $100 \%$ & $\mathrm{D}, \mathrm{G}$ & 12.8 & 0.67 & 152 & 260 & $<0.5$ & $<2$ & 0.13 & 19.5 & 12 \\
\hline 93ABM02(22-36) & 93ABM02 & 22 & 36 & $<0.83$ & $100 \%$ & $\mathrm{D}$ & 14.2 & 0.66 & 332 & 280 & $<0.5$ & $<2$ & 0.12 & 50.0 & 17 \\
\hline 93ABM02(36-44) & 93ABM02 & 36 & 44 & $<0.83$ & $100 \%$ & $\mathrm{D}$ & 10.8 & 0.44 & 194 & 180 & $<0.5$ & $<2$ & 0.07 & 17.5 & 20 \\
\hline 93ABM02(44-66) & 93ABM02 & 44 & 66 & $<0.83$ & $100 \%$ & $\mathrm{D}$ & 13.0 & 0.52 & 318 & 290 & $<0.5$ & $<2$ & 0.07 & 16.0 & 16 \\
\hline 93ABM02(66-85) & 93ABM02 & 66 & 85 & $<0.83$ & $100 \%$ & $\mathrm{D}$ & 12.2 & 0.46 & 52 & 200 & $<0.5$ & $<2$ & 0.09 & 9.0 & 9 \\
\hline 93ABM02(85-97) & 93ABM02 & 85 & 97 & $<0.83$ & $100 \%$ & $\bar{D}$ & 9.2 & 0.81 & 60 & 260 & $<0.5$ & $<2$ & 0.09 & 33.0 & 8 \\
\hline 93ABM02(97-110) & 93ABM02 & 97 & 110 & $<0.83$ & $100 \%$ & $\mathrm{D}$ & $<0.2$ & 1.83 & 4 & 130 & 0.5 & 2 & 0.10 & 6.5 & 5 \\
\hline 93ABM02(110-126) & $93 \mathrm{ABM} 02$ & 110 & 126 & $<0.83$ & $100 \%$ & $\mathrm{D}$ & 0.2 & 1.90 & 4 & 120 & 0.5 & $<2$ & 0.10 & 7.5 & 6 \\
\hline 93ABM02(0-97) & 93ABM02 & 0 & 97 & $<0.83$ & $100 \%$ & $\bar{D}$ & 11.8 & 0.67 & 166 & 240 & $<0.5$ & $<2$ & 0.09 & 24.0 & $\overline{13}$ \\
\hline 93ABM02(97-126) & 93ABM02 & 97 & 126 & $<0.83$ & $100 \%$ & $\mathrm{D}$ & $<0.2$ & 1.70 & 4 & 120 & 0.5 & $<2$ & 0.09 & 6.5 & 5 \\
\hline 93ABM02(0-97)L & 93ABM02 & 0 & 97 & $<0.83$ & $100 \%$ & $\mathrm{D}$ & 12.2 & 0.46 & 218 & 220 & $<0.5$ & $<2$ & 0.09 & 27.0 & 14 \\
\hline 93ABM02(97-129)L & 93ABM02 & 97 & 129 & $<0.83$ & $100 \%$ & $\mathrm{D}$ & 0.2 & 1.89 & 14 & 120 & 0.5 & 2 & 0.10 & 7.5 & 6 \\
\hline 93ABM04(0-12) & 93ABM04 & 0 & 12 & $<0.83$ & $100 \%$ & $\mathrm{D}$ & 12.8 & 0.81 & 120 & 310 & $<0.5$ & $<2$ & 0.15 & 24.5 & 12 \\
\hline 93ABM04(13-18) & 93ABM04 & 13 & 18 & $<0.83$ & $100 \%$ & $\mathrm{D}$ & 12.8 & 1.23 & 134 & 350 & $<0.5$ & $<2$ & 0.15 & 20.0 & 11 \\
\hline 93ABM04(22-27) & 93ABM04 & 22 & 27 & $<0.83$ & $100 \%$ & $\bar{D}$ & 11.8 & 0.75 & 188 & 270 & $<0.5$ & $<2$ & 0.09 & 44.5 & 19 \\
\hline 93ABM04(32-37) & 93ABM04 & 32 & 37 & $<0.83$ & $100 \%$ & $\mathrm{D}$ & 12.2 & 0.96 & 170 & 300 & $<0.5$ & $<2$ & 0.08 & 20.5 & 13 \\
\hline 93ABM04(42-48) & 93ABM04 & 42 & 48 & $<0.83$ & $100 \%$ & $\mathrm{D}$ & 11.8 & 0.57 & 252 & 300 & $<0.5$ & $<2$ & 0.14 & 22.5 & 21 \\
\hline 93ABM04(48-76) & 93ABM04 & 48 & 76 & $<0.83$ & $100 \%$ & $\bar{D}$ & 24.2 & 1.12 & 198 & 600 & $<0.5$ & $<2$ & 0.11 & 22.0 & 12 \\
\hline 93ABM04(76-142) & 93ABM04 & 76 & 142 & $<0.83$ & $100 \%$ & $\mathrm{D}, \mathrm{G}$ & 0.8 & 2.09 & 6 & 240 & 0.5 & $<2$ & 0.08 & 4.0 & 9 \\
\hline 93ABM06A & 93ABM06 & & & $<0.83$ & $100 \%$ & $\mathrm{D}$ & 6.0 & 1.84 & 92 & 250 & $<0.5$ & $<2$ & 0.17 & 9.0 & 22 \\
\hline 93ABM06B & 93ABM06 & & & $<0.83$ & $100 \%$ & $\mathrm{D}$ & 0.2 & 2.51 & 8 & 150 & 0.5 & $<2$ & 0.04 & 0.5 & 4 \\
\hline 93ABL08(0-5) & 93ABL08 & 0 & 5 & $<0.83$ & $100 \%$ & $\mathrm{D}$ & 13.2 & 0.77 & 260 & 230 & $<0.5$ & $<2$ & 0.14 & 20.5 & 19 \\
\hline 93ABL09(0-83) & 93ABL09 & 0 & 83 & $<0.83$ & $100 \%$ & $\mathrm{D}$ & 14.0 & 0.41 & 104 & 130 & $<0.5$ & $<2$ & 0.22 & 32.5 & 8 \\
\hline 93SBC10(0-3) & 93SBC10 & 0 & 3 & $<0.83$ & $100 \%$ & $\mathrm{D}$ & 10.8 & 1.40 & 106 & 250 & $<0.5$ & $<2$ & 0.17 & 15.5 & 12 \\
\hline 93SBC10(3-30) & $93 \mathrm{SBC} 10$ & 3 & 30 & $<0.83$ & $100 \%$ & $\mathrm{D}$ & 13.4 & 1.17 & 198 & 270 & $<0.5$ & $<2$ & 0.09 & 16.0 & 14 \\
\hline 93SBC10(30-70) & 93SBC10 & 30 & 70 & $<0.83$ & $100 \%$ & $\mathrm{D}$ & 25.6 & 0.99 & 72 & 510 & $<0.5$ & $<2$ & 0.10 & 20.0 & 12 \\
\hline 93SBC10(70-90) & 93SBC10 & 70 & 90 & $<0.83$ & $100 \%$ & $\mathrm{D}, \mathrm{G}$ & 29.2 & 1.05 & 92 & 320 & $<0.5$ & $<2$ & 0.16 & 14.5 & 11 \\
\hline 93SBC10(90-100) & 93SBC10 & 90 & 100 & $<0.83$ & $100 \%$ & $\mathrm{D}$ & 31.4 & 0.78 & 36 & 370 & $<0.5$ & $<2$ & 0.08 & 15.0 & 8 \\
\hline 93SBC10(100-130) & $93 \mathrm{SBC} 10$ & 100 & 130 & $<0.83$ & $100 \%$ & $\mathrm{D}$ & 1.0 & 1.82 & 16 & 240 & 0.5 & $<2$ & 0.14 & 24.5 & 9 \\
\hline 93SBC10(130-160) & $93 \mathrm{SBC} 10$ & 130 & 160 & $<0.83$ & $100 \%$ & $\mathrm{D}$ & $<0.2$ & 2.18 & 2 & 300 & 1.0 & $<2$ & 0.13 & 39.5 & 10 \\
\hline 93SBR13(0-15) & 93SBR13 & 0 & 15 & $<0.83$ & $100 \%$ & $\bar{D}$ & 15.0 & 1.32 & 112 & 370 & $<0.5$ & $<2$ & 0.15 & 33.5 & 14 \\
\hline 93SBR13(16-52) & 93SBR13 & 16 & 52 & $<0.83$ & $100 \%$ & $\mathrm{D}$ & 15.6 & 0.91 & 100 & 310 & $<0.5$ & $<2$ & 0.17 & 24.5 & 10 \\
\hline
\end{tabular}

* Wt \% of size fraction: as \% by weight of bulk sample ( $\mathrm{nm}=$ not measured)

** Other analyses (Appendix \#): D =USGS-EDXRF, E =EWU, G =XRAL, ---- = none. 
Appendix F. Chemical composition of samples analyzed by ICP-AES at CHEMEX Labs in Reno, NV using nitric acid-aqua regia dissolution (Appendix_F.xls, Appendix_F.dbf).

\begin{tabular}{|c|c|c|c|c|c|c|c|c|c|c|c|c|c|c|c|c|c|c|c|}
\hline Sample No. & $\begin{array}{c}\mathrm{Cr} \\
(\mathrm{ppm})\end{array}$ & $\begin{array}{c}\mathrm{Cu} \\
(\mathrm{ppm})\end{array}$ & $\begin{array}{l}\mathrm{Fe} \\
(\%)\end{array}$ & $\begin{array}{c}\mathrm{Ga} \\
(\mathrm{ppm})\end{array}$ & $\begin{array}{c}\mathrm{Hg} \\
(\mathrm{ppm})\end{array}$ & $\begin{array}{c}K \\
(\%)\end{array}$ & $\begin{array}{c}\mathrm{La} \\
(\mathrm{ppm})\end{array}$ & $\begin{array}{l}\mathrm{Mg} \\
(\%)\end{array}$ & $\begin{array}{c}\mathrm{Mn} \\
(\mathrm{ppm})\end{array}$ & $\begin{array}{c}\text { Mo } \\
(\mathrm{ppm})\end{array}$ & $\begin{array}{l}\mathrm{Na} \\
(\%)\end{array}$ & $\begin{array}{c}\mathrm{Ni} \\
(\mathrm{ppm})\end{array}$ & $\begin{array}{c}\mathrm{P} \\
(\mathrm{ppm})\end{array}$ & $\begin{array}{c}\mathrm{Pb} \\
(\mathrm{ppm})\end{array}$ & $\begin{array}{c}\mathrm{Sb} \\
(\mathrm{ppm})\end{array}$ & $\begin{array}{c}\mathrm{Sc} \\
(\mathrm{ppm})\end{array}$ & $\begin{array}{c}\mathrm{Sr} \\
(\mathrm{ppm})\end{array}$ & $\begin{array}{c}\mathrm{Ti} \\
(\%)\end{array}$ & $\begin{array}{c}\mathrm{TI} \\
(\mathrm{ppm})\end{array}$ \\
\hline SAMPLE_NO. & PPM & CU_PPM & FE_\% & GA_PPM & HG_PPM & K_\% & LA_PPM & MG_\% & MN_PPM & MO_PPM & NA_\% & NI_PPM & P_PPM & PB_PPM & SB_PPM & SC_PPM & RPPM & TI_\% & TL_PPM \\
\hline 93ABM02(0-10) & 87 & 147 & 9.85 & $<10$ & 3 & 0.18 & 10 & 0.44 & 8550 & 4 & 0.01 & 10 & 320 & 3752 & 32 & 2 & 6 & 0.01 & $<10$ \\
\hline 93ABM02(11-22) & 55 & 139 & 9.23 & $<10$ & 6 & 0.17 & 10 & 0.33 & 7920 & 3 & $<0.01$ & 11 & 340 & 3592 & 34 & 2 & 7 & 0.01 & $<10$ \\
\hline 93ABM02(22-36) & 65 & 165 & 11.54 & $<10$ & 4 & 0.18 & 10 & 0.26 & 9245 & 3 & $<0.01$ & 14 & 420 & 3862 & 46 & 3 & 6 & $<0.01$ & $<10$ \\
\hline 93ABM02(36-44) & 85 & 120 & 9.06 & $<10$ & 2 & 0.17 & 10 & 0.17 & 8480 & 6 & $<0.01$ & 10 & 180 & 3136 & 36 & 2 & 6 & $<0.01$ & $<10$ \\
\hline 93ABM02(44-66) & 37 & 143 & 11.38 & $<10$ & 3 & 0.16 & 10 & 0.17 & 8930 & 2 & $<0.01$ & 12 & 240 & 4450 & 42 & 3 & 6 & $<0.01$ & $<10$ \\
\hline 93ABM02(66-85) & 34 & 121 & 8.44 & $<10$ & 3 & 0.15 & 10 & 0.21 & 7705 & 2 & $<0.01$ & 9 & 220 & 5526 & 34 & 2 & 7 & $<0.01$ & $<10$ \\
\hline 93ABM02(85-97) & 37 & 98 & 7.41 & $<10$ & 2 & 0.14 & 20 & 0.26 & 5450 & 2 & $<0.01$ & 12 & 390 & 4168 & 24 & 2 & 6 & 0.01 & $<10$ \\
\hline 93ABM02(97-110) & 55 & 36 & 2.07 & $<10$ & $<1$ & 0.17 & 30 & 0.48 & 165 & 2 & 0.01 & 13 & 550 & 80 & 2 & 3 & 6 & 0.02 & $<10$ \\
\hline 93ABM02(110-126) & 44 & 36 & 2.23 & $<10$ & $<1$ & 0.17 & 30 & 0.47 & 240 & 2 & 0.01 & 13 & 590 & 64 & $<2$ & 3 & 6 & 0.02 & $<10$ \\
\hline 93ABM02(0-97) & 83 & 127 & 9.18 & $<10$ & 4 & 0.16 & 10 & 0.24 & 7680 & 5 & $<0.01$ & 13 & 320 & 4340 & 36 & 2 & 6 & $<0.01$ & $<10$ \\
\hline 93ABM02(97-126) & 41 & 23 & 2.00 & $<10$ & $<1$ & 0.15 & 20 & 0.45 & 150 & 1 & 0.01 & 12 & 520 & 68 & $<2.0$ & 2 & 5 & 0.01 & $<10$ \\
\hline 93ABM02(0-97)L & 18 & 122 & 9.80 & $<10$ & 3 & 0.10 & 10 & 0.21 & 8100 & 1 & $<0.01$ & 12 & 290 & 4370 & 34 & 2 & 6 & $<0.01$ & $<10$ \\
\hline 93ABM02(97-129)L & 33 & 20 & 2.17 & $<10$ & $<1$ & 0.13 & 30 & 0.48 & 175 & 1 & 0.01 & 13 & 580 & 54 & 2 & 3 & 6 & 0.02 & $<10$ \\
\hline 93ABM04(0-12) & 261 & 127 & 10.08 & $<10$ & 4 & 0.30 & 10 & 0.46 & 8845 & 15 & 0.01 & 13 & 320 & 3638 & 36 & 3 & 7 & $<0.01$ & $<10$ \\
\hline 93ABM04(13-18) & 280 & 153 & 9.84 & $<10$ & 6 & 0.47 & 20 & 0.46 & 8430 & 15 & 0.02 & 13 & 360 & 3574 & 36 & 3 & 9 & 0.01 & $<10$ \\
\hline 93ABM04(22-27) & 259 & 125 & 10.07 & $<10$ & 3 & 0.29 & 10 & 0.26 & 9005 & 17 & 0.01 & 14 & 230 & 3668 & 40 & 2 & 7 & $<0.01$ & $<10$ \\
\hline 93ABM04(32-37) & 252 & 125 & 10.96 & $<10$ & 3 & 0.42 & 10 & 0.22 & 9470 & 13 & 0.01 & 14 & 200 & 3200 & 42 & 3 & 9 & $<0.01$ & $<10$ \\
\hline 93ABM04(42-48) & 260 & 125 & 11.43 & $<10$ & 3 & 0.23 & 10 & 0.33 & 9775 & 17 & 0.01 & 13 & 180 & 3568 & 40 & 2 & 6 & $<0.01$ & $<10$ \\
\hline 93ABM04(48-76) & 109 & 188 & 12.31 & $<10$ & 6 & 0.36 & 20 & 0.24 & $>10000$ & 6 & 0.01 & 16 & 380 & 9284 & 50 & 4 & 10 & 0.01 & $<10$ \\
\hline 93ABM04(76-142) & 222 & 28 & 2.32 & 10 & $<1$ & 0.36 & 30 & 0.47 & 1090 & 12 & 0.03 & 14 & 510 & 374 & 6 & 4 & 9 & 0.02 & $<10$ \\
\hline 93ABM06A & 334 & 60 & 5.96 & $<10$ & $<1$ & 0.58 & 20 & 0.43 & 5160 & 17 & 0.05 & 15 & 450 & 2798 & 20 & 4 & 21 & 0.03 & $<10$ \\
\hline 93АВМ06B & 203 & 21 & 2.32 & 10 & $<1$ & 0.42 & 40 & 0.49 & 160 & 13 & 0.03 & 14 & 510 & 78 & 4 & 4 & 7 & 0.03 & 10 \\
\hline 93ABL08(0-5) & 15 & 160 & 8.96 & $<10$ & 3 & 0.12 & 20 & 0.27 & 6690 & 1 & $<0.01$ & 16 & 320 & 4486 & 38 & 3 & 10 & 0.01 & $<10$ \\
\hline 93ABL09(0-83) & 16 & 146 & 9.75 & $<10$ & 5 & 0.08 & 10 & 0.47 & 8700 & 1 & $<0.01$ & 9 & 240 & 3552 & 36 & 2 & 8 & $<0.01$ & $<10$ \\
\hline 93SBC10(0-3) & 213 & 132 & 5.58 & $<10$ & 4 & 0.34 & 30 & 0.36 & 4020 & 12 & 0.02 & 14 & 570 & 3282 & 36 & 3 & 12 & 0.02 & $<10$ \\
\hline 93SBC10(3-30) & 257 & 150 & 7.89 & $<10$ & 4 & 0.31 & 20 & 0.32 & 6200 & 16 & 0.01 & 13 & 380 & 3734 & 46 & 3 & 9 & 0.01 & $<10$ \\
\hline 93SBC10(30-70) & 179 & 267 & 9.83 & $<10$ & 10 & 0.27 & 20 & 0.36 & 8640 & 10 & 0.01 & 13 & 280 & $>10000$ & 56 & 3 & 9 & 0.01 & $<10$ \\
\hline 93SBC10(70-90) & 270 & 317 & 10.14 & $<10$ & 12 & 0.28 & 10 & 0.53 & 8815 & 16 & 0.01 & 13 & 290 & $>10000$ & 58 & 3 & 9 & 0.01 & $<10$ \\
\hline 93SBC10(90-100) & 81 & 262 & 10.40 & $<10$ & 13 & 0.17 & 20 & 0.32 & 9190 & 4 & $<0.01$ & 10 & 240 & $>10000$ & 62 & 2 & 5 & 0.01 & $<10$ \\
\hline 93SBC10(100-130) & 193 & 86 & 2.16 & $<10$ & $<1$ & 0.32 & 30 & 0.53 & 965 & 10 & 0.02 & 14 & 470 & 682 & 4 & 3 & 12 & 0.02 & $<10$ \\
\hline 93SBC10(130-160) & 37 & 39 & 2.40 & 10 & $<1$ & 0.22 & 30 & 0.56 & 800 & 1 & 0.01 & 14 & 560 & 70 & 4 & 4 & 17 & 0.03 & $<10$ \\
\hline 93SBR13(0-15) & 322 & 199 & 9.25 & $<10$ & 6 & 0.40 & 20 & 0.45 & 8005 & 18 & 0.02 & 14 & 470 & 5002 & 42 & 3 & 9 & 0.01 & $<10$ \\
\hline 93SBR13(16-52) & 208 & 172 & 10.12 & $<10$ & 7 & 0.33 & 10 & 0.51 & 9145 & 10 & 0.01 & 11 & 230 & 5436 & 44 & 3 & 8 & 0.01 & $<10$ \\
\hline
\end{tabular}

* Wt \% of size fraction: as \% by weight of bulk sample ( $\mathrm{nm}=$ not measured)

** Other analyses (Appendix \#): D =USGS-EDXRF, E =EWU, G =XRAL, ---- = none. 
Appendix F. Chemical composition of samples analyzed by ICP-AES at CHEMEX Labs in Reno, NV using nitric acid-aqua regia dissolution (Appendix_F.Xls, Appendix_F.dbf).

\begin{tabular}{|l|r|r|r|r|}
\hline \multicolumn{1}{|c|}{ Sample No. } & $\begin{array}{c}\mathrm{U} \\
(\mathrm{ppm})\end{array}$ & $\begin{array}{c}\mathrm{V} \\
(\mathrm{ppm})\end{array}$ & $\begin{array}{c}\mathrm{W} \\
(\mathrm{ppm})\end{array}$ & $\begin{array}{c}\text { Zn } \\
(\mathrm{ppm})\end{array}$ \\
\hline SAMPLE_No. & U_PPM & V_pPM & w_pPM & ZN_PPM \\
\hline 93ABM02(0-10) & 10 & 12 & 10 & 3240 \\
\hline 93ABM02(11-22) & 10 & 12 & 10 & 3310 \\
\hline 93ABM02(22-36) & 20 & 13 & 10 & 5578 \\
\hline 93ABM02(36-44) & 10 & 9 & $<10$ & 2376 \\
\hline 93ABM02(44-66) & 10 & 11 & $<10$ & 2748 \\
\hline 93ABM02(66-85) & 10 & 9 & $<10$ & 1676 \\
\hline 93ABM02(85-97) & $<10$ & 12 & 10 & 3026 \\
\hline 93ABM02(97-110) & $<10$ & 18 & $<10$ & 596 \\
\hline 93ABM02(110-126) & $<10$ & 19 & $<10$ & 490 \\
\hline 93ABM02(0-97) & 10 & 12 & $<10$ & 3040 \\
\hline 93ABM02(97-126) & $<10$ & 17 & $<10$ & 530 \\
\hline 93ABM02(0-97)L & 10 & 10 & $<10$ & 3300 \\
\hline 93ABM02(97-129)L & $<10$ & 19 & $<10$ & 558 \\
\hline 93ABM04(0-12) & 10 & 14 & $<10$ & 4064 \\
\hline 93ABM04(13-18) & 20 & 17 & $<10$ & 2732 \\
\hline 93ABM04(22-27) & 10 & 13 & 10 & 6016 \\
\hline 93ABM04(32-37) & 10 & 14 & $<10$ & 3604 \\
\hline 93ABM04(42-48) & 20 & 12 & $<10$ & 2974 \\
\hline 93ABM04(48-76) & 10 & 17 & $<10$ & 4282 \\
\hline 93ABM04(76-142) & $<10$ & 21 & $<10$ & 382 \\
\hline 93ABM06A & 10 & 29 & $<10$ & 1112 \\
\hline 93ABM06B & $<10$ & 28 & $<10$ & 136 \\
\hline 93ABL08(0-5) & $<10$ & 12 & $<10$ & 3398 \\
\hline 93ABL09(0-83) & 10 & 9 & 10 & 5070 \\
\hline 93SBC10(0-3) & $<10$ & 21 & $<10$ & 1870 \\
\hline 93SBC10(3-30) & 10 & 17 & $<10$ & 2266 \\
\hline 93SBC10(30-70) & 20 & 15 & $<10$ & 3398 \\
\hline 93SBC10(70-90) & 20 & 16 & $<10$ & 2634 \\
\hline 93SBC10(90-100) & 10 & 12 & 10 & 2502 \\
\hline 93SBC10(100-130) & $<10$ & 20 & $<10$ & 1080 \\
\hline 93SBC10(130-160) & $<10$ & 22 & $<10$ & 1446 \\
\hline 93SBR13(0-15) & 10 & 19 & 10 & 4506 \\
\hline 93SBR13(16-52) & 10 & 15 & 10 & 4098 \\
\hline
\end{tabular}

* Wt \% of size fraction: as \% by weight of bulk sample ( $\mathrm{nm}=$ not measured)

** Other analyses (Appendix \#): D =USGS-EDXRF, E =EWU, G =XRAL, ---- = none 
Appendix F. Chemical composition of samples analyzed by ICP-AES at CHEMEX Labs in Reno, NV using nitric acid-aqua regia dissolution (Appendix_F.xls, Appendix_F.dbf).

\begin{tabular}{|c|c|c|c|c|c|c|c|c|c|c|c|c|c|c|c|}
\hline Sample No. & Site ID & $\begin{array}{c}\text { Depth } \\
\text { interval, } \\
\text { top }(\mathrm{cm})\end{array}$ & $\begin{array}{c}\text { Depth } \\
\text { interval, } \\
\text { bottom }(\mathrm{cm})\end{array}$ & $\begin{array}{c}\text { Size } \\
\text { fraction } \\
(\mathrm{mm})\end{array}$ & $\begin{array}{c}{ }^{*} \text { wt } \% \text { of } \\
\text { size } \\
\text { fraction }\end{array}$ & $\begin{array}{c}{ }^{* *} \text { Other } \\
\text { analyses } \\
(\text { Appendix \#) }\end{array}$ & $\begin{array}{c}\mathrm{Ag} \\
(\mathrm{ppm})\end{array}$ & $\begin{array}{c}\mathrm{Al} \\
(\%)\end{array}$ & $\begin{array}{c}\text { As } \\
\text { (ppm) }\end{array}$ & $\begin{array}{c}\mathrm{Ba} \\
(\mathrm{ppm})\end{array}$ & $\begin{array}{c}\mathrm{Be} \\
(\mathrm{ppm})\end{array}$ & $\begin{array}{c}\mathrm{Bi} \\
(\mathrm{ppm})\end{array}$ & $\begin{array}{l}\mathrm{Ca} \\
(\%)\end{array}$ & $\begin{array}{c}\mathrm{Cd} \\
(\mathrm{ppm})\end{array}$ & $\begin{array}{c}\text { Co } \\
\text { (ppm) }\end{array}$ \\
\hline SAMPLE_NO. & SITE_ID & INT_TOP_CM & INT_BTM_CM & SIZFRAC_MM & WT\%SIZFRAC & OTHR_ANLYS & AG_PPM & AL_\% & AS_PPM & BA_PPM & BE_PPM & BI_PPM & CA_\% & CD_PPM & CO_PPM \\
\hline 93SBR13(57-65) & 93SBR13 & 57 & 65 & $<0.83$ & $100 \%$ & $\bar{D}$ & 14.8 & 1.51 & 112 & 460 & $<0.5$ & $<2$ & 0.04 & 19.0 & 17 \\
\hline 93SBR13(221-237) & 93SBR13 & 221 & 237 & $<0.83$ & $100 \%$ & $\mathrm{D}$ & 45.0 & 1.20 & 50 & 660 & $<0.5$ & $<2$ & 0.13 & 27.0 & 8 \\
\hline 93SBR13(237-251) & 93SBR13 & 237 & 251 & $<0.83$ & $100 \%$ & $\mathrm{D}$ & 26.8 & 1.26 & 46 & 660 & $<0.5$ & $<2$ & 0.11 & 23.0 & 9 \\
\hline 93SBR13(251-291) & 93SBR13 & 251 & 291 & $<0.83$ & $100 \%$ & $\bar{D}$ & 23.2 & 1.44 & 64 & 800 & $<0.5$ & $<2$ & 0.09 & 25.0 & 11 \\
\hline 93SBM14 & 93SBM14 & & & & $\mathrm{nm}$ & $\mathrm{D}$ & 0.2 & 2.67 & 2 & 160 & 0.5 & $<2$ & 0.13 & $<0.5$ & 6 \\
\hline 93SBC16(0-5) & 93SBC16 & 0 & 5 & $<0.83$ & $\mathrm{~nm}$ & $\mathrm{D}$ & 5.6 & 0.70 & 88 & 100 & $<0.5$ & $<2$ & 0.09 & 6.0 & 8 \\
\hline 93SBK17(25-68) & 93SBK17 & 25 & 68 & $<0.83$ & $\mathrm{~nm}$ & $\mathrm{D}, \mathrm{G}$ & 54.2 & 0.41 & 58 & 120 & $<0.5$ & $<2$ & 0.20 & 70.5 & $\overline{13}$ \\
\hline 93SBK17(68-106) & 93SBK17 & 68 & 106 & $<0.83$ & $\mathrm{~nm}$ & $\mathrm{D}$ & \begin{tabular}{|l|}
171.0 \\
\end{tabular} & 0.84 & 118 & 140 & $<0.5$ & $<2$ & 0.06 & 39.5 & 10 \\
\hline 93SBK17(106-152) & 93SBK17 & 106 & 152 & $<0.83$ & $\mathrm{~nm}$ & $\mathrm{D}, \mathrm{G}$ & 4.4 & 1.88 & 16 & 290 & $<0.5$ & $<2$ & 0.07 & 36.0 & 29 \\
\hline 93SBK17(152-230) & $93 \mathrm{SBK} 17$ & 152 & 230 & $<0.83$ & $\mathrm{~nm}$ & $\mathrm{D}$ & 2.8 & 1.19 & 24 & 160 & $<0.5$ & $<2$ & 0.08 & 19.5 & 9 \\
\hline 93SBK18(7-10) & 93SBK18 & 7 & 10 & $<0.83$ & $\mathrm{~nm}$ & $\mathrm{D}, \mathrm{G}$ & 55.6 & 1.18 & 80 & 410 & $<0.5$ & $<2$ & 0.14 & 47.0 & 14 \\
\hline 93SBK18(10-26) & 93SBK18 & 10 & 26 & $<0.83$ & $\mathrm{~nm}$ & D & 80.4 & 1.32 & 66 & 420 & $<0.5$ & $<2$ & 0.17 & 47.5 & 10 \\
\hline 93SBK18(82-92) & 93SBK18 & 82 & 92 & $<0.83$ & $\mathrm{~nm}$ & $\bar{D}$ & 12.0 & 0.62 & 216 & 280 & $<0.5$ & $<2$ & 0.14 & 21.5 & 14 \\
\hline 93SBK18(92-109) & 93SBK18 & 92 & 109 & $<0.83$ & $\mathrm{~nm}$ & $\bar{D}$ & $<0.2$ & 2.03 & 12 & 290 & 0.5 & 2 & 0.09 & 64.0 & 12 \\
\hline 93SBK18(109-160) & 93SBK18 & 109 & 160 & $<0.83$ & $\mathrm{~nm}$ & $\mathrm{D}$ & 0.4 & 1.57 & 12 & 170 & 0.5 & 2 & 0.08 & 23.0 & 9 \\
\hline 93SBB20(0-5) & 93SBB20 & 0 & 5 & $<0.83$ & $100 \%$ & $\mathrm{D}, \mathrm{G}$ & 11.8 & 0.82 & 304 & 260 & $<0.5$ & $<2$ & 0.10 & 22.0 & 13 \\
\hline 93SBB22(0-4) & 93SBB22 & 0 & 4 & $<0.83$ & $100 \%$ & D & 4.6 & 1.20 & 18 & 160 & $<0.5$ & $<2$ & 0.34 & 4.5 & 6 \\
\hline 93SBB22(4.5-7.5) & 93SBB22 & 5 & 8 & $<0.83$ & $100 \%$ & $\mathrm{D}$ & 11.2 & 1.32 & 42 & 320 & $<0.5$ & $<2$ & 0.16 & 6.0 & 6 \\
\hline 93SBB22(7.5-12) & 93SBB22 & 8 & 12 & $<0.83$ & $100 \%$ & $\bar{D}$ & 13.8 & 0.88 & 248 & 280 & $<0.5$ & $<2$ & 0.12 & 13.5 & 7 \\
\hline 93SBB22(12-15) & 93SBB22 & 12 & 15 & $<0.83$ & $100 \%$ & $\mathrm{D}$ & 13.2 & 0.95 & 460 & 240 & $<0.5$ & $<2$ & 0.10 & 29.0 & 20 \\
\hline 93SBB22(15-19) & 93SBB22 & 15 & 19 & $<0.83$ & $100 \%$ & $\mathrm{D}$ & 12.8 & 0.81 & 246 & 280 & $<0.5$ & $<2$ & 0.10 & 10.5 & 13 \\
\hline 93SBB22(19-26) & 93SBB22 & 19 & 26 & $<0.83$ & $100 \%$ & $\overline{D, G}$ & 20.0 & 0.91 & 156 & 480 & $<0.5$ & $<2$ & 0.10 & 8.0 & 9 \\
\hline 93SBB22(26-29) & 93SBB22 & 26 & 29 & $<0.83$ & $100 \%$ & D & 41.6 & 1.49 & 78 & 750 & $<0.5$ & $<2$ & 0.12 & 4.0 & 6 \\
\hline 93SBB22(29-36) & 93SBB22 & 29 & 36 & $<0.83$ & $100 \%$ & $\bar{D}$ & 0.4 & 3.04 & 4 & 200 & 1.0 & $<2$ & 0.15 & 18.0 & 9 \\
\hline 93SBB22(36-48) & $93 \mathrm{SBB} 22$ & 36 & 48 & $<0.83$ & $100 \%$ & $\mathrm{D}$ & 0.2 & 3.65 & 8 & 270 & 1.0 & $<2$ & 0.11 & 5.0 & 6 \\
\hline 93SBB22(48-60) & 93SBB22 & 48 & 60 & $<0.83$ & $100 \%$ & $\bar{D}$ & 0.2 & 3.64 & 6 & 280 & 0.5 & $<2$ & 0.09 & 3.0 & 6 \\
\hline 93SBB22(60-82) & 93SBB22 & 60 & 82 & $<0.83$ & $100 \%$ & $\mathrm{D}$ & $<0.2$ & 2.53 & 4 & 190 & 0.5 & 2 & 0.07 & $<0.5$ & 5 \\
\hline 93SBB23(0-5) & 93SBB23 & 0 & 5 & $<0.83$ & $100 \%$ & $\bar{D}$ & 12.0 & 0.68 & 200 & 260 & $<0.5$ & $<2$ & 0.10 & 15.5 & 13 \\
\hline 93SBB23(5-12) & 93SBB23 & 5 & 12 & $<0.83$ & $100 \%$ & $\bar{D}$ & 11.0 & 0.49 & 122 & 250 & $<0.5$ & $<2$ & 0.23 & 17.5 & 9 \\
\hline 93SBB23(12-17) & 93SBB23 & 12 & 17 & $<0.83$ & $100 \%$ & $\mathrm{D}$ & 11.0 & 0.67 & 256 & 250 & $<0.5$ & $<2$ & 0.04 & 28.5 & 15 \\
\hline 93SBB23(17-22) & 93SBB23 & 17 & 22 & $<0.83$ & $100 \%$ & $\bar{D}$ & 12.4 & 0.63 & 270 & 260 & $<0.5$ & $<2$ & 0.03 & 16.5 & 15 \\
\hline 93SBB23(22-30) & 93SBB23 & 22 & 30 & $<0.83$ & $100 \%$ & $\mathrm{D}$ & 12.4 & 0.59 & 136 & 300 & $<0.5$ & $<2$ & 0.03 & 17.5 & 15 \\
\hline 93SBB23(30-46) & 93SBB23 & 30 & 46 & $<0.83$ & $100 \%$ & $\mathrm{D}$ & 13.4 & 0.59 & 106 & 260 & $<0.5$ & $<2$ & 0.08 & 17.0 & 11 \\
\hline
\end{tabular}

* Wt \% of size fraction: as \% by weight of bulk sample ( $\mathrm{nm}=$ not measured)

** Other analyses (Appendix \#): D =USGS-EDXRF, E =EWU, G =XRAL, ---- = none. 
Appendix F. Chemical composition of samples analyzed by ICP-AES at CHEMEX Labs in Reno, NV using nitric acid-aqua regia dissolution (Appendix_F.xls, Appendix_F.dbf).

\begin{tabular}{|c|c|c|c|c|c|c|c|c|c|c|c|c|c|c|c|c|c|c|c|}
\hline Sample No. & $\begin{array}{c}\mathrm{Cr} \\
(\mathrm{ppm})\end{array}$ & $\begin{array}{c}\mathrm{Cu} \\
(\mathrm{ppm})\end{array}$ & $\begin{array}{l}\mathrm{Fe} \\
(\%)\end{array}$ & $\begin{array}{c}\mathrm{Ga} \\
(\mathrm{ppm})\end{array}$ & $\begin{array}{c}\mathrm{Hg} \\
(\mathrm{ppm})\end{array}$ & $\begin{array}{c}K \\
(\%)\end{array}$ & $\begin{array}{c}\mathrm{La} \\
(\mathrm{ppm})\end{array}$ & $\begin{array}{l}\mathrm{Mg} \\
(\%)\end{array}$ & $\begin{array}{c}\mathrm{Mn} \\
(\mathrm{ppm})\end{array}$ & $\begin{array}{c}\text { Mo } \\
(\mathrm{ppm})\end{array}$ & $\begin{array}{l}\mathrm{Na} \\
(\%)\end{array}$ & $\begin{array}{c}\mathrm{Ni} \\
(\mathrm{ppm})\end{array}$ & $\begin{array}{c}\mathrm{P} \\
(\mathrm{ppm})\end{array}$ & $\begin{array}{c}\mathrm{Pb} \\
(\mathrm{ppm})\end{array}$ & $\begin{array}{c}\mathrm{Sb} \\
(\mathrm{ppm})\end{array}$ & $\begin{array}{c}\mathrm{Sc} \\
(\mathrm{ppm})\end{array}$ & $\begin{array}{c}\mathrm{Sr} \\
(\mathrm{ppm})\end{array}$ & $\begin{array}{c}\mathrm{Ti} \\
(\%)\end{array}$ & $\begin{array}{c}\mathrm{TI} \\
(\mathrm{ppm})\end{array}$ \\
\hline SAMPLE_NO. & PPM & CU_PPM & FE_\% & GA_PPM & HG_PPM & K_\% & LA_PPM & MG_\% & MN_PPM & MO_PPM & NA_\% & NI_PPM & P_PPM & PB_PPM & SB_PPM & SC_PPM & RPPM & TI_\% & TL_PPM \\
\hline 93SBR13(57-65) & 314 & 153 & 10.60 & $<10$ & 4 & 0.54 & 20 & 0.20 & 9240 & 19 & 0.03 & 14 & 330 & 6342 & 44 & 4 & 8 & 0.01 & $<10$ \\
\hline 93SBR13(221-237) & 236 & 420 & 11.57 & $<10$ & 15 & 0.41 & 10 & 0.38 & 9230 & 12 & 0.01 & 12 & 250 & $>10000$ & 86 & 3 & 9 & 0.01 & $<10$ \\
\hline 93SBR13(237-251) & 268 & 271 & 14.03 & $<10$ & 11 & 0.45 & 10 & 0.27 & $>10000$ & 14 & 0.02 & 19 & 230 & $>10000$ & 52 & 4 & 9 & 0.01 & $<10$ \\
\hline 93SBR13(251-291) & 363 & 225 & 12.40 & $<10$ & 12 & 0.56 & 20 & 0.23 & $>10000$ & 18 & 0.02 & 17 & 250 & $>10000$ & 50 & 4 & 10 & 0.01 & $<10$ \\
\hline 93SBM14 & 41 & 29 & 1.42 & 10 & $<1$ & 0.52 & 40 & 0.39 & 110 & 1 & 0.01 & 14 & 80 & 40 & 2 & 4 & 10 & $<0.01$ & $<10$ \\
\hline 93SBC16(0-5) & 196 & 60 & 5.14 & $<10$ & $<1$ & 0.23 & 20 & 0.31 & 3450 & 11 & 0.01 & 9 & 320 & 1988 & 26 & 1 & 7 & $<0.01$ & $<10$ \\
\hline 93SBK17(25-68) & 164 & 492 & 14.36 & $<10$ & 20 & 0.20 & $<10$ & 0.51 & $>10000$ & 11 & $<0.01$ & 11 & 170 & $>10000$ & 102 & 3 & 9 & $<0.01$ & $<10$ \\
\hline 93SBK17(68-106) & 7 & 498 & 11.49 & $<10$ & 20 & 0.10 & 10 & 0.18 & 6045 & 1 & $<0.01$ & 4 & 460 & $>10000$ & 228 & 3 & 35 & 0.01 & $<10$ \\
\hline 93SBK17(106-152) & 140 & 53 & 2.74 & $<10$ & 1 & 0.34 & 30 & 0.32 & 5665 & 7 & 0.01 & 12 & 600 & 3620 & 16 & 4 & 9 & 0.02 & $<10$ \\
\hline 93SBK17(152-230) & 186 & 39 & 2.96 & $<10$ & $<1$ & 0.35 & 20 & 0.27 & 2825 & 11 & 0.01 & 11 & 340 & 1182 & 10 & 2 & 10 & $<0.01$ & $<10$ \\
\hline 93SBK18(7-10) & 319 & 480 & 12.67 & $<10$ & 15 & 0.33 & 20 & 0.30 & $>10000$ & 20 & 0.04 & 15 & 360 & $>10000$ & 112 & 4 & 14 & 0.02 & $<10$ \\
\hline 93SBK18(10-26) & 98 & 599 & 11.56 & $<10$ & 20 & 0.31 & 20 & 0.44 & 9085 & 7 & 0.01 & 15 & 460 & $>10000$ & 146 & 4 & 23 & 0.02 & $<10$ \\
\hline 93SBK18(82-92) & 157 & 119 & 10.98 & $<10$ & 4 & 0.23 & 10 & 0.37 & 9260 & 9 & 0.01 & 13 & 330 & 3020 & 40 & 2 & 7 & $<0.01$ & $<10$ \\
\hline 93SBK18(92-109) & 182 & 21 & 1.79 & $<10$ & $<1$ & 0.34 & 30 & 0.37 & 885 & 12 & 0.01 & 14 & 350 & 258 & 6 & 4 & 12 & 0.02 & $<10$ \\
\hline 93SBK18(109-160) & 322 & 20 & 2.11 & $<10$ & $<1$ & 0.57 & 30 & 0.25 & 890 & 18 & 0.02 & 11 & 310 & 216 & 6 & 3 & 11 & 0.01 & $<10$ \\
\hline 93SBB20(0-5) & 101 & 122 & 11.02 & $<10$ & 3 & 0.20 & 20 & 0.23 & 8110 & 6 & 0.01 & 12 & 540 & 3214 & 42 & 3 & 7 & 0.01 & $<10$ \\
\hline 93SBB22(0-4) & 454 & 61 & 2.85 & $<10$ & 1 & 0.23 & 10 & 0.30 & 1740 & 25 & 0.25 & 14 & 600 & 1288 & 14 & 2 & 30 & 0.06 & $<10$ \\
\hline 93SBB22(4.5-7.5) & 457 & 197 & 7.19 & $<10$ & 2 & 0.37 & 20 & 0.39 & 5355 & 25 & 0.06 & 14 & 450 & 2662 & 34 & 3 & 12 & 0.01 & $<10$ \\
\hline 93SBB22(7.5-12) & 136 & 144 & 10.25 & $<10$ & 1 & 0.23 & 10 & 0.38 & 6490 & 8 & 0.01 & 8 & 470 & 3644 & 44 & 3 & 6 & $<0.01$ & $<10$ \\
\hline 93SBB22(12-15) & 132 & 170 & 12.25 & 10 & $<1$ & 0.28 & 10 & 0.27 & 4595 & 8 & 0.01 & 14 & 450 & \begin{tabular}{l|l|}
3538 \\
\end{tabular} & 52 & 3 & 6 & $<0.01$ & $<10$ \\
\hline 93SBB22(15-19) & 169 & 136 & 11.09 & $<10$ & $<1$ & 0.25 & 10 & 0.28 & 4925 & 11 & 0.01 & 14 & 320 & 4448 & 34 & 3 & 6 & $<0.01$ & $<10$ \\
\hline 93SBB22(19-26) & 67 & 161 & 11.31 & $<10$ & 1 & 0.24 & 10 & 0.29 & 3755 & 4 & 0.01 & 12 & 380 & 7908 & 42 & 3 & 8 & 0.01 & $<10$ \\
\hline 93SBB22(26-29) & 167 & 221 & 7.91 & $<10$ & 6 & 0.38 & 20 & 0.36 & 3460 & 9 & 0.02 & 11 & 530 & $>10000$ & 70 & 3 & 13 & 0.01 & $<10$ \\
\hline 93SBB22(29-36) & 91 & 64 & 1.82 & 10 & $<1$ & 0.26 & 40 & 0.38 & 1285 & 4 & 0.02 & 20 & 410 & 642 & 4 & 4 & 16 & 0.03 & $<10$ \\
\hline 93SBB22(36-48) & 577 & 188 & 2.15 & 10 & $<1$ & 0.88 & 30 & 0.46 & 505 & 35 & 0.08 & 17 & 170 & 68 & 6 & 7 & 17 & 0.06 & $<10$ \\
\hline 93SBB22(48-60) & 523 & 145 & 2.41 & 10 & $<1$ & 1.08 & 40 & 0.51 & 290 & 31 & 0.09 & 17 & 210 & 32 & 2 & 7 & 16 & 0.06 & $<10$ \\
\hline 93SBB22(60-82) & 219 & 32 & 2.20 & 10 & $<1$ & 0.63 & 40 & 0.46 & 160 & 12 & 0.05 & 13 & 280 & 34 & 4 & 4 & 11 & 0.03 & $<10$ \\
\hline 93SBB23(0-5) & 156 & 126 & 9.62 & $<10$ & $<1$ & 0.24 & 10 & 0.24 & 7875 & 11 & 0.01 & 10 & 330 & 3390 & 34 & 2 & 6 & $<0.01$ & $<10$ \\
\hline 93SBB23(5-12) & 144 & 106 & 9.62 & $<10$ & $<1$ & 0.19 & 10 & 0.42 & 8255 & 8 & $<0.01$ & 8 & 230 & 2162 & 30 & 2 & 8 & $<0.01$ & $<10$ \\
\hline 93SBB23(12-17) & 175 & 147 & 9.94 & $<10$ & $<1$ & 0.26 & 10 & 0.14 & 8325 & 12 & 0.01 & 13 & 210 & 3414 & 34 & 2 & 4 & $<0.01$ & $<10$ \\
\hline 93SBB23(17-22) & 135 & 139 & 10.12 & $<10$ & 1 & 0.23 & 10 & 0.14 & 8230 & 9 & 0.01 & 12 & 210 & 4032 & 36 & 2 & 4 & $<0.01$ & $<10$ \\
\hline 93SBB23(22-30) & 113 & 150 & 10.85 & $<10$ & $<1$ & 0.22 & 10 & 0.15 & 9040 & 8 & $<0.01$ & 12 & 210 & 4518 & 34 & 2 & 4 & $<0.01$ & $<10$ \\
\hline 93SBB23(30-46) & 86 & 146 & 10.11 & $<10$ & 1 & 0.21 & 10 & 0.20 & 8545 & 5 & $<0.01$ & 13 & 200 & 5486 & 36 & 2 & 8 & $<0.01$ & $<10$ \\
\hline
\end{tabular}

* Wt \% of size fraction: as \% by weight of bulk sample ( $\mathrm{nm}=$ not measured)

** Other analyses (Appendix \#): D =USGS-EDXRF, E =EWU, G =XRAL, ---- = none. 
Appendix F. Chemical composition of samples analyzed by ICP-AES at CHEMEX Labs in Reno, NV using nitric acid-aqua regia dissolution (Appendix_F.Xls, Appendix_F.dbf).

\begin{tabular}{|c|c|c|c|c|}
\hline No. & $\begin{array}{c}U \\
(p p m)\end{array}$ & $\begin{array}{c}\mathrm{V} \\
(\mathrm{ppm})\end{array}$ & $\begin{array}{c}\text { W } \\
(\mathrm{ppm})\end{array}$ & $\begin{array}{c}\mathrm{Zn} \\
(\mathrm{ppm})\end{array}$ \\
\hline SAMPLE_NO. & PPM & V_PPM & W_PPM & ZN_PPM \\
\hline 93SBR13(57-65) & 10 & 21 & $<10$ & 4224 \\
\hline 93SBR13(221-237) & 10 & 16 & 10 & 5702 \\
\hline 93SBR13(237-251) & 10 & 20 & $<10$ & 5448 \\
\hline 93SBR13(251-291) & 20 & 20 & $<10$ & 5334 \\
\hline 93SBM14 & $<10$ & 17 & $<10$ & 104 \\
\hline 93SBC16(0-5) & $<10$ & 11 & $<10$ & 1324 \\
\hline 93SBK17(25-68) & 20 & 11 & 10 & 9882 \\
\hline 93SBK17(68-106) & $<10$ & 12 & 10 & 7282 \\
\hline 93SBK17(106-152) & 10 & 22 & $<10$ & 1934 \\
\hline 93SBK17(152-230) & $<10$ & 15 & $<10$ & 1354 \\
\hline 93SBK18(7-10) & 20 & 21 & $<10$ & 6244 \\
\hline 93SBK18(10-26) & 10 & 20 & 10 & 6536 \\
\hline 93SBK18(82-92) & 20 & 12 & $<10$ & 2866 \\
\hline 93SBK18(92-109) & $<10$ & 22 & 10 & 5434 \\
\hline 93SBK18(109-160) & $<10$ & 18 & $<10$ & 1654 \\
\hline 93SBB20(0-5) & 10 & 16 & $<10$ & 3138 \\
\hline 93SBB22(0-4) & $<10$ & 31 & $<10$ & 504 \\
\hline 93SBB22(4.5-7.5) & $<10$ & 21 & $<10$ & 1012 \\
\hline 93SBB22(7.5-12) & $<10$ & 13 & $<10$ & 1882 \\
\hline 93SBB22(12-15) & $<10$ & 13 & $<10$ & 3798 \\
\hline 93SBB22(15-19) & $<10$ & 12 & $<10$ & 3128 \\
\hline 93SBB22(19-26) & $<10$ & 13 & $<10$ & 3210 \\
\hline 93SBB22(26-29) & $<10$ & 19 & $<10$ & 1694 \\
\hline 93SBB22(29-36) & $<10$ & 27 & $<10$ & 1106 \\
\hline 93SBB22(36-48) & $<10$ & 43 & $<10$ & 446 \\
\hline 93SBB22(48-60) & $<10$ & 45 & $<10$ & 278 \\
\hline 93SBB22(60-82) & $<10$ & 30 & $<10$ & 118 \\
\hline 93SBB23(0-5) & 10 & 11 & $<10$ & 2356 \\
\hline 93SBB23(5-12) & 10 & 10 & $<10$ & 3126 \\
\hline 93SBB23(12-17) & 10 & 11 & $<10$ & 4290 \\
\hline 93SBB23(17-22) & 10 & 10 & $<10$ & 3614 \\
\hline 93SBB2 & 10 & 11 & $<10$ & 2700 \\
\hline 93SBB23(30-46) & 10 & 11 & $<10$ & 2426 \\
\hline
\end{tabular}

* Wt \% of size fraction: as \% by weight of bulk sample ( $\mathrm{nm}=$ not measured)

** Other analyses (Appendix \#): D =USGS-EDXRF, E =EWU, G =XRAL, ---- = none 
Appendix F. Chemical composition of samples analyzed by ICP-AES at CHEMEX Labs in Reno, NV using nitric acid-aqua regia dissolution (Appendix_F.xls, Appendix_F.dbf).

\begin{tabular}{|c|c|c|c|c|c|c|c|c|c|c|c|c|c|c|c|}
\hline Sample No. & Site ID & $\begin{array}{c}\text { Depth } \\
\text { interval, } \\
\text { top }(\mathrm{cm})\end{array}$ & $\begin{array}{c}\text { Depth } \\
\text { interval, } \\
\text { bottom }(\mathrm{cm})\end{array}$ & $\begin{array}{c}\text { Size } \\
\text { fraction } \\
(\mathrm{mm})\end{array}$ & $\begin{array}{l}\text { *wt \% of } \\
\text { size } \\
\text { fraction }\end{array}$ & $\begin{array}{c}{ }^{* *} \text { Other } \\
\text { analyses } \\
(\text { Appendix \#) }\end{array}$ & $\begin{array}{c}\mathrm{Ag} \\
(\mathrm{ppm})\end{array}$ & $\begin{array}{c}\mathrm{Al} \\
(\%)\end{array}$ & $\begin{array}{c}\text { As } \\
\text { (ppm) }\end{array}$ & $\begin{array}{c}\mathrm{Ba} \\
(\mathrm{ppm})\end{array}$ & $\begin{array}{c}\mathrm{Be} \\
(\mathrm{ppm})\end{array}$ & $\begin{array}{c}\mathrm{Bi} \\
(\mathrm{ppm})\end{array}$ & $\begin{array}{l}\mathrm{Ca} \\
(\%)\end{array}$ & $\begin{array}{c}\mathrm{Cd} \\
(\mathrm{ppm})\end{array}$ & $\begin{array}{c}\text { Co } \\
\text { (ppm) }\end{array}$ \\
\hline SAMPLE_NO. & SITE_ID & INT_TOP_CM & INT_BTM_CM & SIZFRAC_MM & WT\%SIZFRAC & OTHR_ANLYS & AG_PPM & AL_\% & AS_PPM & BA_PPM & BE_PPM & BI_PPM & CA_\% & CD_PPM & CO_PPM \\
\hline 93SBB23(46-53) & 93SBB23 & 46 & 53 & $<0.83$ & $100 \%$ & $\mathrm{D}, \mathrm{G}$ & 16.0 & 0.83 & 166 & 440 & $<0.5$ & $<2$ & 0.12 & 34.5 & 15 \\
\hline 93SBB23(53-86) & 93SBB23 & 53 & 86 & $<0.83$ & $100 \%$ & D & 0.2 & 2.07 & 2 & 170 & 0.5 & $<2$ & 0.11 & 4.0 & 8 \\
\hline 93SBB23(86-117) & 93SBB23 & 86 & 117 & $<0.83$ & $100 \%$ & $\mathrm{D}$ & $<0.2$ & 2.13 & 6 & 140 & 0.5 & $<2$ & 0.10 & 0.5 & 8 \\
\hline 93SBL26(0-6) & 93SBL26 & 0 & 6 & $<0.83$ & $100 \%$ & $\mathrm{D}, \mathrm{G}$ & 10.4 & 2.53 & 66 & 310 & $<0.5$ & $<2$ & 0.26 & 11.0 & 8 \\
\hline 93SBL26(6-12) & 93SBL26 & 6 & 12 & $<0.83$ & $100 \%$ & $D$ & 13.2 & 1.64 & 158 & 230 & $<0.5$ & $<2$ & 0.20 & 54.5 & 21 \\
\hline 93SBL26(12-20) & 93SBL26 & 12 & 20 & $<0.83$ & $100 \%$ & $\bar{D}$ & 14.4 & 1.51 & 138 & 270 & $<0.5$ & $<2$ & 0.24 & 27.5 & 18 \\
\hline 93SBL26(20-28) & 93SBL26 & 20 & 28 & $<0.83$ & $100 \%$ & $\mathrm{D}$ & 21.2 & 2.29 & 26 & 360 & $<0.5$ & $<2$ & 0.19 & 14.5 & 7 \\
\hline 93SBL26(28-34) & 93SBL26 & 28 & 34 & $<0.83$ & $100 \%$ & $\mathrm{D}$ & 2.8 & 2.07 & 52 & 230 & $<0.5$ & $<2$ & 0.33 & 18.5 & 7 \\
\hline 93SBL26(34-47) & 93SBL26 & 34 & 47 & $<0.83$ & $100 \%$ & $\bar{D}$ & 0.6 & 1.99 & 14 & 180 & $<0.5$ & $<2$ & 0.50 & 1.5 & 3 \\
\hline 93SBL26(47-58) & 93SBL26 & 47 & 58 & $<0.83$ & $100 \%$ & $\mathrm{D}$ & 0.8 & 2.63 & 22 & 200 & $<0.5$ & $<2$ & 0.51 & 3.0 & 4 \\
\hline 93SBL27B(0-4) & 93SBL27B & 0 & 4 & $<0.83$ & $100 \%$ & $\mathrm{D}$ & 12.8 & 1.42 & 116 & 360 & $<0.5$ & $<2$ & 0.19 & 24.5 & 11 \\
\hline 93SBL27B(4-16) & 93SBL27B & 4 & 16 & $<0.83$ & $100 \%$ & $\mathrm{D}$ & 14.2 & 0.70 & 138 & 310 & $<0.5$ & $<2$ & 0.12 & 25.0 & 13 \\
\hline 93SBL27B(16-23) & 93SBL27B & 16 & 23 & $<0.83$ & $100 \%$ & $\bar{D}$ & 13.8 & 1.05 & 320 & 340 & $<0.5$ & $<2$ & 0.11 & 37.5 & 15 \\
\hline 93SBL27B(23-34) & 93SBL27B & 23 & 34 & $<0.83$ & $100 \%$ & $\bar{D}$ & 13.2 & 0.81 & 250 & 300 & $<0.5$ & $<2$ & 0.09 & 19.5 & 15 \\
\hline 93SBL27B(34-43) & 93SBL27B & 34 & 43 & $<0.83$ & $100 \%$ & $\mathrm{D}$ & 13.4 & 0.93 & 120 & 370 & $<0.5$ & $<2$ & 0.14 & 19.5 & 13 \\
\hline 93SBL27B(43-53) & 93SBL27B & 43 & 53 & $<0.83$ & $100 \%$ & $\mathrm{D}$ & 13.8 & 0.77 & 100 & 300 & $<0.5$ & $<2$ & 0.16 & 16.0 & 10 \\
\hline 93SBL27B(53-69) & 93SBL27B & 53 & 69 & $<0.83$ & $100 \%$ & $\bar{D}$ & 13.6 & 0.78 & 130 & 440 & $<0.5$ & $<2$ & 0.15 & 10.0 & 8 \\
\hline 93SBL27B(69-85) & 93SBL27B & 69 & 85 & $<0.83$ & $100 \%$ & $\mathrm{D}$ & 10.0 & 1.54 & 20 & 280 & $<0.5$ & $<2$ & 0.11 & 14.5 & 7 \\
\hline 93SBL28(0-11) & 93SBL28 & 0 & 11 & $<0.83$ & $100 \%$ & $\bar{D}$ & 13.8 & 2.20 & 154 & 310 & $<0.5$ & $<2$ & 0.17 & 56.0 & 9 \\
\hline 93SBL28(11-16) & 93SBL28 & 11 & 16 & $<0.83$ & $100 \%$ & $\bar{D}$ & 9.6 & 1.95 & 106 & 260 & $<0.5$ & $<2$ & 0.17 & 5.5 & 6 \\
\hline 93SBL28(16-25) & 93SBL28 & 16 & 25 & $<0.83$ & $100 \%$ & $\mathrm{D}$ & 17.4 & 1.39 & 318 & 260 & $<0.5$ & $<2$ & 0.17 & 48.0 & 25 \\
\hline 93SBL28(25-34) & 93SBL28 & 25 & $\overline{34}$ & $<0.83$ & $100 \%$ & $\bar{D}$ & 12.2 & 1.03 & 234 & 200 & $<0.5$ & $<2$ & 0.33 & 18.5 & 18 \\
\hline 93SBL28(34-37) & 93SBL28 & 34 & 37 & $<0.83$ & $100 \%$ & $\mathrm{D}$ & 12.4 & 1.58 & 120 & 250 & $<0.5$ & $<2$ & 0.37 & 20.0 & 16 \\
\hline 93SBL28(37-41) & 93SBL28 & 37 & 41 & $<0.83$ & $100 \%$ & $\bar{D}$ & 17.8 & 2.31 & 38 & 370 & $<0.5$ & $<2$ & 0.19 & 23.5 & 10 \\
\hline 93SBL28(41-46) & 93SBL28 & 41 & 46 & $<0.83$ & $100 \%$ & $\mathrm{D}$ & 21.2 & 2.50 & 16 & 400 & $<0.5$ & $<2$ & 0.13 & 10.0 & 6 \\
\hline 93SBL28C(0-25) & 93SBL28C & 0 & 25 & $<0.83$ & $100 \%$ & $\bar{D}$ & 4.8 & 1.19 & 292 & 230 & $<0.5$ & $<2$ & 0.36 & 12.5 & 11 \\
\hline 93SBL28C(25-28) & 93SBL28C & 25 & 28 & $<0.83$ & $100 \%$ & $\mathrm{D}$ & 15.8 & 2.29 & 210 & 310 & $<0.5$ & $<2$ & 0.16 & 19.0 & 8 \\
\hline 93SBL28C(28-37) & 93SBL28C & 28 & 37 & $<0.83$ & $100 \%$ & $\bar{D}$ & 14.8 & 1.35 & 308 & 240 & $<0.5$ & $<2$ & 0.17 & 37.0 & 21 \\
\hline 93SBL28C(37-48) & 93SBL28C & 37 & 48 & $<0.83$ & $100 \%$ & $\bar{D}$ & 12.2 & 1.03 & 198 & 260 & $<0.5$ & $<2$ & 0.37 & 18.5 & 18 \\
\hline 93SBL28C(48-54) & 93SBL28C & 48 & 54 & $<0.83$ & $100 \%$ & $\mathrm{D}$ & 14.8 & 1.33 & 86 & 290 & $<0.5$ & $<2$ & 0.31 & 22.5 & 13 \\
\hline 93SBL28C(54-64) & 93SBL28C & 54 & 64 & $<0.83$ & $100 \%$ & $\mathrm{D}$ & 17.4 & 2.34 & 26 & 320 & $<0.5$ & $<2$ & 0.14 & 12.5 & 7 \\
\hline 93SBL28C(64-72) & 93SBL28C & 64 & 72 & $<0.83$ & $100 \%$ & $\mathrm{D}$ & 1.0 & 1.80 & 82 & 180 & $<0.5$ & $<2$ & 0.17 & 20.0 & 8 \\
\hline 93SBL28C(72-83) & 93SBL28C & 72 & 83 & $<0.83$ & $100 \%$ & $\mathrm{D}$ & 0.2 & 1.45 & 34 & 140 & $<0.5$ & 2 & 0.21 & 1.0 & 4 \\
\hline
\end{tabular}

* Wt \% of size fraction: as \% by weight of bulk sample ( $\mathrm{nm}=$ not measured)

** Other analyses (Appendix \#): D =USGS-EDXRF, E =EWU, G =XRAL, ---- = none. 
Appendix F. Chemical composition of samples analyzed by ICP-AES at CHEMEX Labs in Reno, NV using nitric acid-aqua regia dissolution (Appendix_F.xls, Appendix_F.dbf).

\begin{tabular}{|c|c|c|c|c|c|c|c|c|c|c|c|c|c|c|c|c|c|c|c|}
\hline Sample No. & $\begin{array}{c}\mathrm{Cr} \\
(\mathrm{ppm})\end{array}$ & $\begin{array}{c}\mathrm{Cu} \\
(\mathrm{ppm})\end{array}$ & $\begin{array}{l}\mathrm{Fe} \\
(\%)\end{array}$ & $\begin{array}{c}\mathrm{Ga} \\
(\mathrm{ppm})\end{array}$ & $\begin{array}{c}\mathrm{Hg} \\
(\mathrm{ppm})\end{array}$ & $\begin{array}{c}K \\
(\%)\end{array}$ & $\begin{array}{c}\mathrm{La} \\
(\mathrm{ppm})\end{array}$ & $\begin{array}{l}\mathrm{Mg} \\
(\%)\end{array}$ & $\begin{array}{c}\mathrm{Mn} \\
(\mathrm{ppm})\end{array}$ & $\begin{array}{c}\text { Mo } \\
(\mathrm{ppm})\end{array}$ & $\begin{array}{l}\mathrm{Na} \\
(\%)\end{array}$ & $\begin{array}{c}\mathrm{Ni} \\
(\mathrm{ppm})\end{array}$ & $\begin{array}{c}\mathrm{P} \\
(\mathrm{ppm})\end{array}$ & $\begin{array}{c}\mathrm{Pb} \\
(\mathrm{ppm})\end{array}$ & $\begin{array}{c}\mathrm{Sb} \\
(\mathrm{ppm})\end{array}$ & $\begin{array}{c}\mathrm{Sc} \\
(\mathrm{ppm})\end{array}$ & $\begin{array}{c}\mathrm{Sr} \\
(\mathrm{ppm})\end{array}$ & $\begin{array}{c}\mathrm{Ti} \\
(\%)\end{array}$ & $\begin{array}{c}\mathrm{TI} \\
(\mathrm{ppm})\end{array}$ \\
\hline SAMPLE_NO. & CR_PPM & CU_PPM & FE_\% & GA_PPM & HG_PPM & K_\% & LA_PPM & MG_\% & MN_PPM & MO_PPM & NA_\% & NI_PPM & P_PPM & PB_PPM & SB_PPM & SC_PPM & SR_PPM & TI_\% & TL_PPM \\
\hline 93SBB23(46-53) & 90 & 190 & 11.29 & $<10$ & $<1$ & 0.29 & 10 & 0.22 & 9800 & 6 & 0.01 & 14 & 290 & 5918 & 40 & 3 & 13 & $<0.01$ & $<10$ \\
\hline 93SBB23(53-86) & 193 & 19 & 2.22 & 10 & $<1$ & 0.35 & 40 & 0.51 & 290 & 11 & 0.02 & 16 & 360 & 62 & $<2$ & 4 & 10 & 0.02 & $<10$ \\
\hline 93SBB23(86-117) & 141 & 21 & 2.27 & 10 & $<1$ & 0.29 & 40 & 0.50 & 260 & 8 & 0.01 & 15 & 290 & 44 & 4 & 4 & 10 & 0.02 & $<10$ \\
\hline 93SBL26(0-6) & 492 & 216 & 3.47 & 10 & $<1$ & 0.74 & 20 & 0.41 & 1440 & 26 & 0.11 & 17 & 600 & 2728 & 28 & 4 & 23 & 0.02 & $<10$ \\
\hline 93SBL26(6-12) & 285 & 368 & 6.55 & $<10$ & $<1$ & 0.43 & 20 & 0.40 & 4730 & 16 & 0.02 & 27 & 370 & 4556 & 46 & 3 & 13 & 0.01 & $<10$ \\
\hline 93SBL26(12-20) & 217 & 297 & 7.44 & $<10$ & $<1$ & 0.51 & 10 & 0.43 & 5950 & 11 & 0.02 & 18 & 300 & 4722 & 40 & 3 & 12 & $<0.01$ & $<10$ \\
\hline 93SBL26(20-28) & 255 & 344 & 4.52 & $<10$ & $<1$ & 0.49 & 20 & 0.41 & 3020 & 15 & 0.02 & 16 & 490 & 7116 & 32 & 4 & 15 & 0.02 & $<10$ \\
\hline 93SBL26(28-34) & 216 & 104 & 2.49 & $<10$ & $<1$ & 0.31 & 10 & 0.23 & 915 & 12 & 0.02 & 16 & 780 & 1360 & 12 & 3 & 26 & 0.02 & $<10$ \\
\hline 93SBL26(34-47) & 98 & 58 & 1.41 & $<10$ & $<1$ & 0.14 & 10 & 0.16 & 375 & 7 & 0.02 & 15 & 850 & 218 & 2 & 2 & 41 & 0.02 & $<10$ \\
\hline 93SBL26(47-58) & 134 & 177 & 1.42 & 10 & $<1$ & 0.24 & 10 & 0.26 & 445 & 7 & 0.03 & 19 & 710 & 242 & 2 & 3 & 41 & 0.03 & $<10$ \\
\hline 93SBL27B(0-4) & 401 & 222 & 8.74 & $<10$ & 1 & 0.43 & 20 & 0.44 & 7010 & 25 & 0.07 & 14 & 480 & 4250 & 28 & 3 & 14 & 0.02 & $<10$ \\
\hline 93SBL27B(4-16) & 186 & 170 & 10.36 & $<10$ & 2 & 0.27 & 10 & 0.34 & 9155 & 11 & 0.01 & 10 & 210 & 4464 & 36 & 2 & 7 & $<0.01$ & $<10$ \\
\hline 93SBL27B(16-23) & 179 & 173 & 11.04 & $<10$ & 2 & 0.30 & 20 & 0.33 & 8540 & 11 & 0.01 & 14 & 520 & 4144 & 40 & 3 & 8 & 0.01 & $<10$ \\
\hline 93SBL27B(23-34) & 113 & 167 & 10.28 & $<10$ & $<1$ & 0.25 & 10 & 0.23 & 8430 & 6 & 0.01 & 14 & 260 & 4610 & 38 & 3 & 9 & $<0.01$ & $<10$ \\
\hline 93SBL27B(34-43) & 239 & 179 & 10.22 & $<10$ & 1 & 0.34 & 10 & 0.39 & 8615 & 14 & 0.01 & 12 & 230 & 5144 & 34 & 3 & 21 & 0.01 & $<10$ \\
\hline 93SBL27B(43-53) & 138 & 165 & 10.11 & $<10$ & 2 & 0.25 & 10 & 0.43 & 8215 & 8 & 0.01 & 12 & 270 & 5736 & 34 & 2 & 8 & 0.01 & $<10$ \\
\hline 93SBL27B(53-69) & 169 & 142 & 10.94 & $<10$ & $<1$ & 0.26 & 10 & 0.46 & 8415 & 11 & 0.01 & 9 & 250 & 4860 & 34 & 3 & 8 & $<0.01$ & $<10$ \\
\hline 93SBL27B(69-85) & 117 & 94 & 2.89 & $<10$ & $<1$ & 0.28 & 30 & 0.41 & 1350 & 7 & 0.01 & 12 & 600 & 3718 & 18 & 2 & 9 & 0.02 & $<10$ \\
\hline 93SBL28(0-11) & 317 & 170 & 6.91 & 10 & 2 & 0.72 & 20 & 0.39 & 3435 & 20 & 0.04 & 15 & 620 & 2936 & 54 & 4 & 14 & 0.01 & $<10$ \\
\hline 93SBL28(11-16) & 511 & 168 & 4.48 & $<10$ & $<1$ & 0.57 & 20 & 0.37 & 1770 & 33 & 0.07 & 17 & 640 & 2608 & 26 & 3 & 15 & 0.01 & $<10$ \\
\hline 93SBL28(16-25) & 197 & 228 & 10.32 & $<10$ & 3 & 0.47 & 10 & 0.41 & 7160 & 12 & 0.02 & 19 & 430 & 3580 & 74 & 3 & 9 & $<0.01$ & $<10$ \\
\hline 93SBL28(25-34) & 173 & 151 & 9.87 & $<10$ & $<1$ & 0.34 & 10 & 0.47 & 7965 & 11 & 0.01 & 16 & 290 & 3870 & 36 & 3 & 12 & $<0.01$ & $<10$ \\
\hline 93SBL28(34-37) & 248 & 159 & 8.31 & $<10$ & $<1$ & 0.60 & 10 & 0.47 & 6950 & 13 & 0.03 & 17 & 300 & 4666 & 32 & 3 & 15 & 0.01 & $<10$ \\
\hline 93SBL28(37-41) & 399 & 206 & 6.68 & 10 & 1 & 0.89 & 20 & 0.43 & 5340 & 23 & 0.03 & 17 & 340 & 6272 & 44 & 4 & 13 & 0.01 & $<10$ \\
\hline 93SBL28(41-46) & 149 & 159 & 4.94 & 10 & $<1$ & 0.77 & 20 & 0.44 & 3480 & 8 & 0.03 & 13 & 420 & 5566 & 32 & 5 & 12 & 0.01 & $<10$ \\
\hline 93SBL28C(0-25) & 101 & 218 & 8.72 & $<10$ & $<1$ & 0.49 & 10 & 0.32 & 2255 & 7 & 0.09 & 12 & 1630 & 1944 & 22 & 1 & 28 & 0.02 & $<10$ \\
\hline 93SBL28C(25-28) & 756 & 296 & 5.47 & 10 & 4 & 0.72 & 20 & 0.39 & 2165 & 40 & 0.05 & 20 & 670 & 3422 & 48 & 4 & 14 & 0.01 & $<10$ \\
\hline 93SBL28C(28-37) & 134 & 229 & 10.91 & $<10$ & 1 & 0.45 & 10 & 0.40 & 7275 & 8 & 0.02 & 17 & 370 & 3148 & 70 & 3 & 9 & 0.01 & $<10$ \\
\hline 93SBL28C(37-48) & 143 & 177 & 9.84 & $<10$ & $<1$ & 0.32 & 10 & 0.48 & 8085 & 9 & 0.01 & 16 & 280 & 4200 & 34 & 3 & 13 & 0.01 & $<10$ \\
\hline 93SBL28C(48-54) & 164 & 226 & 8.32 & $<10$ & $<1$ & 0.46 & 20 & 0.46 & 6910 & 9 & 0.02 & 14 & 330 & 5482 & 38 & 3 & 13 & 0.01 & $<10$ \\
\hline 93SBL28C(54-64) & 72 & 162 & 3.67 & 10 & 1 & 0.42 & 20 & 0.39 & 1920 & 3 & 0.02 & 17 & 520 & 6566 & 28 & 4 & 14 & 0.04 & $<10$ \\
\hline 93SBL28C(64-72) & 97 & 112 & 1.79 & $<10$ & $<1$ & 0.20 & 10 & 0.17 & 300 & 6 & 0.01 & 18 & 760 & 290 & 4 & 2 & 17 & 0.02 & $<10$ \\
\hline 93SBL28C(72-83) & 41 & 116 & 1.42 & $<10$ & $<1$ & 0.10 & 10 & 0.13 & 190 & 3 & 0.01 & 12 & 780 & 54 & 2 & 1 & 21 & 0.02 & $<10$ \\
\hline
\end{tabular}

* Wt \% of size fraction: as \% by weight of bulk sample ( $\mathrm{nm}=$ not measured)

** Other analyses (Appendix \#): D =USGS-EDXRF, E =EWU, G =XRAL, ---- = none. 
Appendix F. Chemical composition of samples analyzed by ICP-AES at CHEMEX Labs in Reno, NV using nitric acid-aqua regia dissolution (Appendix_F.Xls, Appendix_F.dbf).

\begin{tabular}{|l|r|r|r|r|}
\hline Sample No. & $\begin{array}{c}\text { U } \\
\text { (ppm) }\end{array}$ & $\begin{array}{c}\text { V } \\
(\mathrm{ppm})\end{array}$ & $\begin{array}{c}\mathrm{W} \\
(\mathrm{ppm})\end{array}$ & $\begin{array}{c}\text { Zn } \\
\text { (ppm) }\end{array}$ \\
\hline SAMPLE_No. & U_PPM & V_PPM & W_PPM & ZN_PPM \\
\hline 93SBB23(46-53) & 10 & 14 & $<10$ & 5404 \\
\hline 93SBB23(53-86) & $<10$ & 22 & $<10$ & 656 \\
\hline 93SBB23(86-117) & $<10$ & 23 & $<10$ & 308 \\
\hline 93SBL26(0-6) & $<10$ & 30 & $<10$ & 1406 \\
\hline 93SBL26(6-12) & $<10$ & 21 & $<10$ & 6266 \\
\hline 93SBL26(12-20) & $<10$ & 17 & $<10$ & 3772 \\
\hline 93SBL26(20-28) & $<10$ & 24 & $<10$ & 2060 \\
\hline 93SBL26(28-34) & $<10$ & 21 & $<10$ & 1838 \\
\hline 93SBL26(34-47) & $<10$ & 20 & $<10$ & 182 \\
\hline 93SBL26(47-58) & $<10$ & 23 & $<10$ & 306 \\
\hline 93SBL27B(0-4) & $<10$ & 22 & $<10$ & 3428 \\
\hline 93SBL27B(4-16) & 10 & 12 & $<10$ & 4318 \\
\hline 93SBL27B(16-23) & 10 & 15 & $<10$ & 4790 \\
\hline 93SBL27B(23-34) & 10 & 12 & $<10$ & 3390 \\
\hline 93SBL27B(34-43) & 10 & 14 & $<10$ & 2502 \\
\hline 93SBL27B(43-53) & 10 & 13 & $<10$ & 2206 \\
\hline 93SBL27B(53-69) & 10 & 13 & $<10$ & 1812 \\
\hline 93SBL27B(69-85) & $<10$ & 17 & $<10$ & 1580 \\
\hline 93SBL28(0-11) & $<10$ & 24 & $<10$ & 2598 \\
\hline 93SBL28(11-16) & $<10$ & 23 & $<10$ & 858 \\
\hline 93SBL28(16-25) & $<10$ & 18 & $<10$ & 3884 \\
\hline 93SBL28(25-34) & 10 & 14 & $<10$ & 3126 \\
\hline 93SBL28(34-37) & $<10$ & 18 & $<10$ & 3272 \\
\hline 93SBL28(37-41) & $<10$ & 23 & $<10$ & 3342 \\
\hline 93SBL28(41-46) & $<10$ & 24 & $<10$ & 1562 \\
\hline 93SBL28C(0-25) & $<10$ & 17 & $<10$ & 1434 \\
\hline 93SBL28C(25-28) & $<10$ & 27 & $<10$ & 1478 \\
\hline 93SBL28C(28-37) & $<10$ & 17 & $<10$ & 3774 \\
\hline 93SBL28C(37-48) & 10 & 14 & $<10$ & 3164 \\
\hline 93SBL28C(48-54) & $<10$ & 17 & $<10$ & 3484 \\
\hline 93SBL28C(54-64) & $<10$ & 26 & $<10$ & 1788 \\
\hline 93SBL28C(64-72) & $<10$ & 19 & $<10$ & 1778 \\
\hline 93SBL28C(72-83) & $<10$ & 18 & $<10$ & 736 \\
\hline
\end{tabular}

* Wt \% of size fraction: as \% by weight of bulk sample ( $\mathrm{nm}=$ not measured)

** Other analyses (Appendix \#): D =USGS-EDXRF, E =EWU, G =XRAL, ---- = none 
Appendix F. Chemical composition of samples analyzed by ICP-AES at CHEMEX Labs in Reno, NV using nitric acid-aqua regia dissolution (Appendix_F.xls, Appendix_F.dbf).

\begin{tabular}{|c|c|c|c|c|c|c|c|c|c|c|c|c|c|c|c|}
\hline Sample No. & Site ID & $\begin{array}{c}\text { Depth } \\
\text { interval, } \\
\text { top }(\mathrm{cm})\end{array}$ & $\begin{array}{c}\text { Depth } \\
\text { interval, } \\
\text { bottom }(\mathrm{cm})\end{array}$ & $\begin{array}{c}\text { Size } \\
\text { fraction } \\
(\mathrm{mm})\end{array}$ & $\begin{array}{c}{ }^{*} \text { wt } \% \text { of } \\
\text { size } \\
\text { fraction }\end{array}$ & $\begin{array}{c}{ }^{* *} \text { Other } \\
\text { analyses } \\
(\text { Appendix \#) }\end{array}$ & $\begin{array}{c}\mathrm{Ag} \\
(\mathrm{ppm})\end{array}$ & $\begin{array}{c}\mathrm{Al} \\
(\%)\end{array}$ & $\begin{array}{c}\text { As } \\
\text { (ppm) }\end{array}$ & $\begin{array}{c}\mathrm{Ba} \\
(\mathrm{ppm})\end{array}$ & $\begin{array}{c}\mathrm{Be} \\
(\mathrm{ppm})\end{array}$ & $\begin{array}{c}\mathrm{Bi} \\
(p p m)\end{array}$ & $\begin{array}{l}\mathrm{Ca} \\
(\%)\end{array}$ & $\begin{array}{c}\text { Cd } \\
(\mathrm{ppm})\end{array}$ & $\begin{array}{c}\text { Co } \\
(\mathrm{ppm})\end{array}$ \\
\hline SAMPLE_NO. & SITE_ID & INT_TOP_CM & INT_BTM_CM & SIZFRAC_MM & WT\%SIZFRAC & OTHR_ANLYS & AG_PPM & AL_\% & AS_PPM & BA_PPM & BE_PPM & BI_PPM & CA_\% & CD_PPM & CO_PPM \\
\hline 93SBL28C(83-93) & 93SBL28C & 83 & $\overline{93}$ & $<0.83$ & $100 \%$ & $\bar{D}$ & 0.2 & 1.45 & 28 & 130 & $<0.5$ & $<2$ & 0.29 & 0.5 & 4 \\
\hline 93SBL28C(93-97) & 93SBL28C & 93 & 97 & $<0.83$ & $100 \%$ & $\mathrm{D}$ & 0.6 & 1.81 & 18 & 140 & $<0.5$ & $<2$ & 0.28 & 1.0 & 3 \\
\hline 93SBL31(0-6) & 93SBL31 & 0 & 6 & $<0.83$ & $100 \%$ & $\bar{D}$ & 12.6 & 1.58 & 132 & 370 & $<0.5$ & $<2$ & 0.18 & 16.0 & 11 \\
\hline 93SBL31(6.5-18) & 93SBL31 & 7 & 18 & $<0.83$ & $100 \%$ & $\bar{D}$ & 13.8 & 0.88 & 324 & 310 & $<0.5$ & $<2$ & 0.11 & 33.0 & 18 \\
\hline 93SBL31(18-23) & 93SBL31 & 18 & 23 & $<0.83$ & $100 \%$ & $\bar{D}$ & 12.6 & 0.61 & 326 & 300 & $<0.5$ & $<2$ & 0.08 & 20.5 & 16 \\
\hline 93SBL31(23-30) & 93SBL31 & 23 & 30 & $<0.83$ & $100 \%$ & $\bar{D}$ & 12.6 & 0.52 & 274 & 230 & $<0.5$ & $<2$ & 0.10 & 14.0 & 15 \\
\hline 93SBL31(30-35) & 93SBL31 & 30 & $\overline{35}$ & $<0.83$ & $100 \%$ & $\bar{D}$ & 12.6 & 0.55 & 314 & 210 & $<0.5$ & $<2$ & 0.12 & 12.5 & 12 \\
\hline 93SBL31(35-42) & 93SBL31 & 35 & 42 & $<0.83$ & $100 \%$ & $\bar{D}$ & 11.2 & 0.38 & 248 & 150 & $<0.5$ & $<2$ & 0.11 & 9.0 & 11 \\
\hline 93SBL31(42-48) & 93SBL31 & 42 & 48 & $<0.83$ & $100 \%$ & $\bar{D}$ & 12.2 & 0.37 & 274 & 120 & $<0.5$ & $<2$ & 0.13 & 9.5 & 7 \\
\hline 93SBL31(73-87) & 93SBL31 & 73 & 87 & $<0.83$ & $100 \%$ & $\bar{D}$ & 12.6 & 0.32 & 334 & 140 & $<0.5$ & $<2$ & 0.13 & 10.0 & 7 \\
\hline 93SBL31(130-137) & 93SBL31 & 130 & 137 & $<0.83$ & $100 \%$ & $\mathrm{D}$ & 12.2 & 0.62 & 104 & 210 & $<0.5$ & $<2$ & 0.35 & 59.5 & 11 \\
\hline 93SBL31(137-146) & 93SBL31 & 137 & 146 & $<0.83$ & $100 \%$ & $\mathrm{D}, \mathrm{G}$ & 11.2 & 0.83 & 90 & 220 & $<0.5$ & $<2$ & 0.42 & 74.5 & 14 \\
\hline 93SBL31(146-160) & 93SBL31 & 146 & 160 & $<0.83$ & $100 \%$ & $\mathrm{D}$ & 11.6 & 0.61 & 80 & 190 & $<0.5$ & $<2$ & 0.33 & 23.5 & 12 \\
\hline 93SBL31(185-200) & 93SBL31 & 185 & 200 & $<0.83$ & $100 \%$ & $\mathrm{D}, \mathrm{G}$ & 12.2 & 1.52 & 74 & 190 & $<0.5$ & $<2$ & 0.39 & 25.0 & 13 \\
\hline 93SBL31(200-211) & 93SBL31 & 200 & 211 & $<0.83$ & $100 \%$ & $\mathrm{D}$ & 11.2 & 1.04 & 52 & 290 & $<0.5$ & $<2$ & 0.34 & 25.5 & 12 \\
\hline 93SBL32(0-3) & 93SBL32 & 0 & 3 & $<0.83$ & $100 \%$ & $\bar{D}$ & 6.8 & 1.50 & 54 & 250 & $<0.5$ & $<2$ & 0.27 & 11.5 & 9 \\
\hline 93SBL32(3-10.5) & 93SBL32 & 3 & 11 & $<0.83$ & $100 \%$ & $\bar{D}$ & 12.8 & 0.93 & 214 & 260 & $<0.5$ & $<2$ & 0.10 & 21.0 & 11 \\
\hline 93SBL32(10.5-16) & 93SBL32 & 11 & 16 & $<0.83$ & $100 \%$ & $\mathrm{D}, \mathrm{G}$ & 4.8 & 1.91 & 6 & 310 & $<0.5$ & $<2$ & 0.08 & 6.0 & 9 \\
\hline 93SBL32(16-24) & 93SBL32 & 16 & 24 & $<0.83$ & $100 \%$ & $\mathrm{D}$ & 62.4 & 1.33 & 58 & 660 & $<0.5$ & $<2$ & 0.07 & 2.5 & 6 \\
\hline 93SBL32(24-31) & 93SBL32 & 24 & 31 & $<0.83$ & $100 \%$ & $\mathrm{D}$ & 1.2 & 1.99 & 12 & 250 & $<0.5$ & $<2$ & 0.07 & 3.0 & 8 \\
\hline 93SBL32(31-48) & 93SBL32 & 31 & 48 & $<0.83$ & $100 \%$ & $\bar{D}$ & $<0.2$ & 2.01 & 6 & 190 & $<0.5$ & $<2$ & 0.07 & 2.0 & 8 \\
\hline 93SBL34a & 93SBL34 & & & $<0.83$ & $100 \%$ & $\mathrm{D}$ & 18.2 & 0.68 & 300 & 160 & $<0.5$ & $<2$ & 0.31 & 36.0 & 17 \\
\hline 93SBL34b & 93SBL34 & & & $<0.83$ & $100 \%$ & $\mathrm{D}, \mathrm{E}$ & 25.2 & 0.83 & 34 & 280 & $<0.5$ & $<2$ & 0.12 & 17.0 & 5 \\
\hline 93SBL34C & 93SBL34 & & & $<0.83$ & $100 \%$ & $\mathrm{D}, \mathrm{E}$ & 45.2 & 1.58 & 30 & 80 & $<0.5$ & $<2$ & 0.32 & $>100$ & 11 \\
\hline 93SBL34d & 93SBL34 & & & $<0.83$ & $100 \%$ & $\overline{D, E}$ & 49.6 & 1.19 & 58 & 550 & $<0.5$ & $<2$ & 0.11 & 15.0 & 6 \\
\hline 93SBL34e & 93SBL34 & & & $<0.83$ & $100 \%$ & $\mathrm{D}, \mathrm{E}$ & 51.0 & 1.41 & 32 & 130 & $<0.5$ & $<2$ & 0.35 & $>100$ & 11 \\
\hline 93SBL34f & 93SBL34 & & & $<0.83$ & $100 \%$ & $\mathrm{D}, \mathrm{E}$ & 40.2 & 1.04 & 66 & 550 & $<0.5$ & $<2$ & 0.12 & 18.0 & 7 \\
\hline 93SBL34g & 93SBL34 & & & $<0.83$ & $100 \%$ & $\mathrm{D}, \mathrm{E}$ & 35.8 & 0.87 & 64 & 460 & $<0.5$ & $<2$ & 0.16 & 37.0 & 6 \\
\hline 93SBL34h & 93SBL34 & & & $<0.83$ & $100 \%$ & $\mathrm{D}, \mathrm{E}$ & 36.4 & 1.18 & 48 & 110 & $<0.5$ & $<2$ & 0.45 & $>100$ & 9 \\
\hline 93SBL34i & 93SBL34 & & & $<0.83$ & $100 \%$ & $\mathrm{D}, \mathrm{E}$ & 35.8 & 0.68 & 48 & 210 & $<0.5$ & $<2$ & 0.35 & 28.0 & 7 \\
\hline 94JE01(0-5) & 94JE01 & 0 & 5 & $0.25-2.0$ & $58 \%$ & ---- & $<0.2$ & \begin{tabular}{l|l|}
0.78 \\
\end{tabular} & 6 & 180 & $<0.5$ & $<2$ & 0.39 & $<0.5$ & 2 \\
\hline 94JE01(0-5) & 94JE01 & 0 & 5 & $0.063-0.25$ & $35 \%$ & $-\overline{---}$ & 0.2 & 0.72 & 4 & 130 & $<0.5$ & $<2$ & 0.19 & $<0.5$ & 2 \\
\hline 94JE01(0-5) & 94JE01 & 0 & 5 & $<0.063$ & $8 \%$ & $-\overline{---}$ & 0.6 & 1.37 & 8 & 260 & 0.5 & $<2$ & 0.50 & 0.5 & 4 \\
\hline
\end{tabular}

* Wt \% of size fraction: as \% by weight of bulk sample ( $\mathrm{nm}=$ not measured)

** Other analyses (Appendix \#): D =USGS-EDXRF, E =EWU, G =XRAL, ---- = none. 
Appendix F. Chemical composition of samples analyzed by ICP-AES at CHEMEX Labs in Reno, NV using nitric acid-aqua regia dissolution (Appendix_F.xls, Appendix_F.dbf).

\begin{tabular}{|c|c|c|c|c|c|c|c|c|c|c|c|c|c|c|c|c|c|c|c|}
\hline Sample No. & $\begin{array}{c}\mathrm{Cr} \\
(\mathrm{ppm})\end{array}$ & $\begin{array}{c}\mathrm{Cu} \\
(\mathrm{ppm})\end{array}$ & $\begin{array}{l}\mathrm{Fe} \\
(\%)\end{array}$ & $\begin{array}{c}\mathrm{Ga} \\
(\mathrm{ppm})\end{array}$ & $\begin{array}{c}\mathrm{Hg} \\
(\mathrm{ppm})\end{array}$ & $\begin{array}{c}\mathrm{K} \\
(\%)\end{array}$ & $\begin{array}{c}\mathrm{La} \\
(\mathrm{ppm})\end{array}$ & $\begin{array}{l}\mathrm{Mg} \\
(\%)\end{array}$ & $\begin{array}{c}\mathrm{Mn} \\
(\mathrm{ppm})\end{array}$ & $\begin{array}{c}\text { Mo } \\
(\mathrm{ppm})\end{array}$ & $\begin{array}{l}\mathrm{Na} \\
(\%)\end{array}$ & $\begin{array}{c}\mathrm{Ni} \\
(\mathrm{ppm})\end{array}$ & $\begin{array}{c}\mathrm{P} \\
(\mathrm{ppm})\end{array}$ & $\begin{array}{c}\mathrm{Pb} \\
(\mathrm{ppm})\end{array}$ & $\begin{array}{c}\mathrm{Sb} \\
(\mathrm{ppm})\end{array}$ & $\begin{array}{c}\mathrm{Sc} \\
(\mathrm{ppm})\end{array}$ & $\begin{array}{c}\mathrm{Sr} \\
(\mathrm{ppm})\end{array}$ & $\begin{array}{c}\mathrm{Ti} \\
(\%)\end{array}$ & $\begin{array}{c}\mathrm{TI} \\
(\mathrm{ppm})\end{array}$ \\
\hline SAMPLE_NO. & CR_PPM & CU_PPM & FE_\% & GA_PPM & HG_PPM & K_\% & LA_PPM & MG_\% & MN_PPM & MO_PPM & NA_\% & NI_PPM & P_PPM & PB_PPM & SB_PPM & SC_PPM & SR_PPM & TI_\% & TL_PPM \\
\hline 93SBL28C(83-93) & 114 & 129 & 1.30 & $<10$ & $<1$ & 0.08 & 10 & 0.14 & 135 & 9 & 0.01 & 13 & 760 & 46 & $<2$ & 2 & 30 & 0.01 & $<10$ \\
\hline 93SBL28C(93-97) & 216 & 336 & 1.44 & $<10$ & $<1$ & 0.14 & 10 & 0.22 & 240 & 13 & 0.02 & 16 & 760 & 220 & 2 & 3 & 30 & 0.02 & $<10$ \\
\hline 93SBL31(0-6) & 203 & 155 & 7.83 & $<10$ & 2 & 0.38 & 20 & 0.45 & 5620 & 12 & 0.04 & 12 & 670 & 4554 & 30 & 3 & 13 & 0.02 & $<10$ \\
\hline 93SBL31(6.5-18) & 117 & 166 & 11.23 & $<10$ & 1 & 0.24 & 10 & 0.26 & 9120 & 8 & 0.01 & 15 & 440 & 3998 & 46 & 3 & 7 & $<0.01$ & $<10$ \\
\hline 93SBL31(18-23) & 152 & 152 & 12.92 & $<10$ & $<1$ & 0.21 & 10 & 0.19 & $>10000$ & 10 & 0.01 & 13 & 250 & 3176 & 44 & 3 & 6 & $<0.01$ & $<10$ \\
\hline 93SBL31(23-30) & 120 & 140 & 10.95 & $<10$ & $<1$ & 0.18 & 10 & 0.21 & 9060 & 7 & $<0.01$ & 12 & 230 & 3594 & 40 & 2 & 6 & $<0.01$ & $<10$ \\
\hline 93SBL31(30-35) & 154 & 138 & 10.65 & $<10$ & 1 & 0.19 & 10 & 0.28 & 8665 & 10 & 0.01 & 11 & 210 & \begin{tabular}{l|l}
3252 \\
\end{tabular} & 40 & 2 & 5 & $<0.01$ & $<10$ \\
\hline 93SBL31(35-42) & 64 & 121 & 9.60 & $<10$ & 1 & 0.12 & $<10$ & 0.31 & 7785 & 4 & $<0.01$ & 8 & 160 & 2964 & 34 & 2 & 4 & $<0.01$ & $<10$ \\
\hline 93SBL31(42-48) & 8 & 111 & 10.03 & $<10$ & $<1$ & 0.07 & $<10$ & 0.37 & 7700 & 1 & $<0.01$ & 8 & 180 & \begin{tabular}{l|l}
3108 \\
\end{tabular} & 38 & 2 & 4 & $<0.01$ & $<10$ \\
\hline 93SBL31(73-87) & 6 & 120 & 11.60 & $<10$ & $<1$ & 0.07 & $<10$ & 0.42 & 8325 & 1 & $<0.01$ & 8 & 160 & 3966 & 32 & 2 & 4 & $<0.01$ & $<10$ \\
\hline 93SBL31(130-137) & 140 & 157 & 10.72 & $<10$ & 1 & 0.26 & 10 & 0.46 & 9010 & 7 & 0.01 & 10 & 180 & 4682 & 32 & 2 & 11 & $<0.01$ & $<10$ \\
\hline 93SBL31(137-146) & 173 & 158 & 8.76 & 10 & 1 & 0.27 & 10 & 0.45 & 7565 & 10 & 0.01 & 17 & 220 & \begin{tabular}{l|}
4482 \\
\end{tabular} & 30 & 2 & 15 & 0.01 & $<10$ \\
\hline 93SBL31(146-160) & 109 & 143 & 8.94 & 10 & 1 & 0.21 & 10 & 0.42 & 7810 & 6 & 0.01 & 13 & 210 & 4782 & 32 & 2 & 13 & $<0.01$ & $<10$ \\
\hline 93SBL31(185-200) & 491 & 249 & 9.54 & 10 & 1 & 0.61 & 10 & 0.47 & 7980 & 28 & 0.03 & 18 & 230 & 5220 & 26 & 3 & 17 & 0.01 & $<10$ \\
\hline 93SBL31(200-211) & 446 & 189 & 9.10 & 10 & 2 & 0.42 & 10 & 0.43 & 8045 & 23 & 0.02 & 14 & 170 & 4482 & 24 & 3 & 14 & 0.01 & $<10$ \\
\hline 93SBL32(0-3) & 382 & 153 & 4.76 & 10 & 2 & 0.41 & 10 & 0.47 & 3865 & 21 & 0.06 & 14 & 1460 & 2474 & 12 & 2 & 18 & 0.02 & $<10$ \\
\hline 93SBL32(3-10.5) & 16 & 137 & 8.93 & 10 & $<1$ & 0.12 & 20 & 0.30 & 5600 & 1 & $<0.01$ & 12 & 730 & 4790 & 32 & 3 & 7 & 0.01 & $<10$ \\
\hline 93SBL32(10.5-16) & 16 & 59 & 2.58 & 10 & 3 & 0.14 & 30 & 0.40 & 1255 & $<1$ & $<0.01$ & 11 & 1170 & 2260 & 8 & 2 & 9 & 0.03 & $<10$ \\
\hline 93SBL32(16-24) & 14 & 345 & 8.07 & 10 & 12 & 0.12 & 20 & 0.30 & 3270 & 1 & 0.01 & 8 & 770 & $>10000$ & 82 & 3 & 9 & 0.02 & $<10$ \\
\hline 93SBL32(24-31) & 15 & 35 & 2.33 & 10 & $<1$ & 0.16 & 30 & 0.40 & 695 & $<1$ & 0.01 & 11 & 1330 & 890 & 2 & 2 & 12 & 0.02 & $<10$ \\
\hline 93SBL32(31-48) & 15 & 22 & 2.16 & 10 & $<1$ & 0.15 & 30 & 0.40 & 465 & $<1$ & $<0.01$ & 11 & 1050 & 78 & $<2.0$ & 3 & 12 & 0.02 & $<10$ \\
\hline 93SBL34a & 134 & 145 & 12.00 & 10 & 2 & 0.24 & 10 & 0.55 & $>10000$ & 8 & 0.01 & 15 & 290 & 5156 & 42 & 3 & 12 & $<0.01$ & $<10$ \\
\hline 93SBL34b & 11 & 214 & 8.37 & $<10$ & 4 & 0.08 & 10 & 0.41 & 4880 & 1 & $<0.01$ & 9 & 310 & $>10000$ & 38 & 2 & 8 & 0.01 & $<10$ \\
\hline 93SBL34c & 194 & 391 & 8.47 & 10 & 10 & 0.37 & 10 & 0.58 & 7115 & 11 & 0.02 & 18 & 360 & $>10000$ & 62 & 4 & 15 & 0.02 & $<10$ \\
\hline 93SBL34d & 15 & 271 & 10.05 & 10 & 8 & 0.12 & 20 & 0.34 & 2630 & 1 & $<0.01$ & 11 & 480 & $>10000$ & 66 & 4 & 10 & 0.02 & $<10$ \\
\hline 93SBL34e & 127 & 398 & 8.53 & 10 & 12 & 0.29 & 10 & 0.58 & 7335 & 8 & 0.02 & 18 & 390 & $>10000$ & 62 & 4 & 19 & 0.02 & $<10$ \\
\hline 93SBL34f & 14 & 245 & 10.15 & 10 & 6 & 0.10 & 20 & 0.34 & 4150 & $<1$ & $<0.01$ & 10 & 440 & $>10000$ & 58 & 3 & 10 & 0.02 & $<10$ \\
\hline 93SBL34g & 12 & 267 & 11.15 & $<10$ & 6 & 0.10 & 10 & 0.41 & 6650 & 1 & $<0.01$ & 11 & 350 & $>10000$ & 52 & 3 & 9 & 0.02 & $<10$ \\
\hline 93SBL34h & 145 & 317 & 12.73 & 10 & 8 & 0.31 & 10 & 0.67 & $>10000$ & 8 & 0.02 & 12 & 280 & $>10000$ & 46 & 3 & 17 & 0.01 & $<10$ \\
\hline 93SBL34i & 10 & 296 & 13.65 & 10 & 8 & 0.10 & 10 & 0.46 & 8970 & $<1$ & $<0.01$ & 10 & 270 & $>10000$ & 52 & 3 & 14 & 0.01 & $<10$ \\
\hline 94JE01(0-5) & 6 & 15 & 0.82 & $<10$ & $<1$ & 0.11 & 20 & 0.24 & 75 & $<1$ & $<0.01$ & 6 & 410 & 78 & 2 & 1 & 21 & 0.01 & $<10$ \\
\hline 94JE01(0-5) & 6 & 13 & 0.92 & $<10$ & $<1$ & 0.09 & 20 & 0.19 & 150 & $<1$ & $<0.01$ & 6 & 290 & 64 & $<2$ & $<1$ & 13 & 0.01 & $<10$ \\
\hline 94JE01(0-5) & 34 & 30 & 1.31 & $<10$ & $<1$ & 0.11 & 20 & 0.31 & 175 & $<1$ & 0.01 & 18 & 650 & 132 & 2 & 2 & 27 & 0.02 & $<10$ \\
\hline
\end{tabular}

* Wt \% of size fraction: as \% by weight of bulk sample ( $\mathrm{nm}=$ not measured)

** Other analyses (Appendix \#): D =USGS-EDXRF, E =EWU, G =XRAL, ---- = none. 
Appendix F. Chemical composition of samples analyzed by ICP-AES at CHEMEX Labs in Reno, NV using nitric acid-aqua regia dissolution (Appendix_F.Xls, Appendix_F.dbf).

\begin{tabular}{|c|c|c|c|c|}
\hline Sample No. & $\begin{array}{c}U \\
(\mathrm{ppm})\end{array}$ & $\begin{array}{c}\mathrm{V} \\
(\mathrm{ppm})\end{array}$ & $\begin{array}{c}\text { W } \\
(\mathrm{ppm})\end{array}$ & $\begin{array}{c}\mathrm{Zn} \\
(\mathrm{ppm})\end{array}$ \\
\hline SAMPLE_NO. & PPM & \begin{tabular}{ll|} 
VPPM & \\
\end{tabular} & W_PPM & ZN_PPM \\
\hline 93SBL28C(83-93) & $<10$ & 19 & $<10$ & 406 \\
\hline 93SBL28C(93-97) & $<10$ & 22 & $<10$ & 378 \\
\hline 93SBL31(0-6) & $<10$ & 22 & $<10$ & 1978 \\
\hline 93SBL31(6.5-18) & 10 & 14 & $<10$ & 4122 \\
\hline 93SBL31(18-23) & 20 & 11 & $<10$ & 3296 \\
\hline 93SBL31(23-30) & 10 & 10 & $<10$ & 2460 \\
\hline 93SBL31(30-35) & 10 & 10 & $<10$ & 1844 \\
\hline 93SBL31(35-42) & 10 & 8 & $<10$ & 1416 \\
\hline 93SBL31(42-48) & 10 & 8 & $<10$ & 1516 \\
\hline 93SBL31(73-87) & 10 & 8 & $<10$ & 1998 \\
\hline 93SBL31(130-137) & 10 & 12 & $<10$ & 5280 \\
\hline 93SBL31(137-146) & 10 & 14 & 30 & 4506 \\
\hline 93SBL31(146-160) & 10 & 12 & 40 & 6792 \\
\hline 93SBL31(185-200) & 10 & 21 & 30 & 4438 \\
\hline 93SBL31(200-211) & 10 & 15 & 30 & 4394 \\
\hline 93SBL32(0-3) & $<10$ & 28 & 10 & 1502 \\
\hline 93SBL32(3-10.5) & $<10$ & 14 & 10 & 2220 \\
\hline 93SBL32(10.5-16) & $<10$ & 20 & 10 & 562 \\
\hline 93SBL32(16-24) & $<10$ & 17 & 20 & 1102 \\
\hline 93SBL32(24-31) & $<10$ & 20 & $<10$ & 308 \\
\hline 93SBL32(31-48) & $<10$ & 20 & $<10$ & 272 \\
\hline 93SBL34a & 20 & 12 & 40 & 5910 \\
\hline 93SBL34b & $<10$ & 13 & 20 & 3790 \\
\hline 93SBL34c & 10 & 21 & 60 & $>10000$ \\
\hline 93SBL34d & $<10$ & 16 & 20 & 4160 \\
\hline 93SBL34e & 10 & 19 & 60 & $>10000$ \\
\hline 93SBL34f & $<10$ & 14 & 20 & 4230 \\
\hline 93SBL34g & $<10$ & 14 & 30 & 4640 \\
\hline 93SBL34h & 20 & 19 & 70 & $>10000$ \\
\hline 93SBL34i & 10 & 15 & 50 & 5868 \\
\hline 94JE01(0-5) & $<10$ & 7 & $<10$ & 86 \\
\hline 94JE01(0-5) & $<10$ & 7 & $<10$ & 104 \\
\hline 94JE01(0-5) & $<10$ & 15 & $<10$ & 146 \\
\hline
\end{tabular}

* Wt \% of size fraction: as \% by weight of bulk sample ( $\mathrm{nm}=$ not measured)

** Other analyses (Appendix \#): D =USGS-EDXRF, E =EWU, G =XRAL, ---- = none 
Appendix F. Chemical composition of samples analyzed by ICP-AES at CHEMEX Labs in Reno, NV using nitric acid-aqua regia dissolution (Appendix_F.xls, Appendix_F.dbf).

\begin{tabular}{|c|c|c|c|c|c|c|c|c|c|c|c|c|c|c|c|}
\hline Sample No. & Site ID & $\begin{array}{l}\text { Depth } \\
\text { interval, } \\
\text { top }(\mathrm{cm})\end{array}$ & $\begin{array}{c}\text { Depth } \\
\text { interval, } \\
\text { bottom }(\mathrm{cm})\end{array}$ & $\begin{array}{c}\text { Size } \\
\text { fraction } \\
(\mathrm{mm})\end{array}$ & $\begin{array}{c}\text { *wt \% of } \\
\text { size } \\
\text { fraction }\end{array}$ & $\begin{array}{c}{ }^{* *} \text { Other } \\
\text { analyses } \\
\text { (Appendix \#) }\end{array}$ & $\begin{array}{c}\mathrm{Ag} \\
(\mathrm{ppm})\end{array}$ & $\begin{array}{c}\mathrm{Al} \\
(\%)\end{array}$ & $\begin{array}{c}\text { As } \\
\text { (ppm) }\end{array}$ & $\begin{array}{c}\mathrm{Ba} \\
(\mathrm{ppm})\end{array}$ & $\begin{array}{c}\mathrm{Be} \\
(\mathrm{ppm})\end{array}$ & $\begin{array}{c}\mathrm{Bi} \\
(\mathrm{ppm})\end{array}$ & $\begin{array}{l}\mathrm{Ca} \\
(\%)\end{array}$ & $\begin{array}{c}\mathrm{Cd} \\
(\mathrm{ppm})\end{array}$ & $\begin{array}{c}\text { Co } \\
\text { (ppm) }\end{array}$ \\
\hline SAMPLE_NO. & SITE_ID & INT_TOP_CM & INT_BTM_CM & SIZFRAC_MM & WT\%SIZFRAC & OTHR_ANLYS & AG_PPM & AL_\% $\%$ & AS_PPM & BA_PPM & BE_PPM & BI_PPM & CA_\% & CD_PPM & CO_PPM \\
\hline 94JE02(0-5) & 94JE02 & 0 & 5 & $0.25-2.0$ & $88 \%$ & ---- & 3.4 & 0.85 & 20 & 560 & $<0.5$ & 2 & 1.44 & 3.5 & 45 \\
\hline 94JE02(0-5) & 94JE02 & 0 & 5 & $0.063-0.25$ & $11 \%$ & ---- & 8.2 & 0.90 & 40 & 750 & $<0.5$ & $<2$ & 0.96 & 6.5 & 29 \\
\hline 94JE03(0-5) & 94JE03 & 0 & 5 & $0.25-2.0$ & $65 \%$ & ---- & 4.2 & 0.79 & 24 & 550 & $<0.5$ & $<2$ & 1.37 & 4.5 & 49 \\
\hline 94JE03(0-5) & 94JE03 & 0 & 5 & $0.063-0.25$ & $30 \%$ & ---- & 7.0 & 0.59 & 46 & 610 & $<0.5$ & $<2$ & 0.65 & 7.0 & 26 \\
\hline 94JE03(0-5) & 94JE03 & 0 & 5 & $<0.063$ & $5 \%$ & --- & 12.0 & 1.15 & 64 & 780 & $<0.5$ & $<2$ & 0.45 & 20.0 & 25 \\
\hline 94JE04(0-5) & 94JE04 & 0 & 5 & $0.25-2.0$ & $68 \%$ & $\overline{----}$ & 1.2 & 0.57 & 32 & 170 & $<0.5$ & $<2$ & 0.38 & 3.5 & 12 \\
\hline 94JE04(0-5) & 94JE04 & 0 & 5 & $0.063-0.25$ & $29 \%$ & ---- & 8.0 & 0.46 & 32 & 340 & $<0.5$ & $<2$ & 0.45 & 6.0 & 17 \\
\hline 94JE04(0-5) & 94JE04 & 0 & 5 & $<0.063$ & $3 \%$ & ---- & 13.4 & 0.79 & 86 & 520 & $<0.5$ & $<2$ & 0.42 & 13.5 & 19 \\
\hline 94JE05(0-5) & 94JE05 & 0 & 5 & $0.25-2.0$ & $69 \%$ & $\begin{array}{ll}--- \\
\end{array}$ & 11.8 & 0.61 & 20 & 170 & $<0.5$ & $<2$ & 0.37 & 17.0 & 13 \\
\hline 94JE06A(0-5) & 94JE06A & 0 & 5 & $0.25-2.0$ & $68 \%$ & ---- & 5.8 & 0.85 & 22 & 150 & $<0.5$ & $<2$ & 0.15 & 12.0 & 11 \\
\hline 94JE07(0-5) & 94JE07 & 0 & 5 & $0.25-2.0$ & $93 \%$ & ---- & $<0.2$ & 0.59 & 2 & 70 & $<0.5$ & $<2$ & 0.03 & $<0.5$ & 3 \\
\hline 94JE08(0-5) & 94JE08 & 0 & 5 & $0.25-2.0$ & $83 \%$ & ---- & 6.0 & 0.80 & 90 & 320 & $<0.5$ & $<2$ & 1.14 & 8.0 & 36 \\
\hline 94JE09(0-5) & 94JE09 & 0 & 5 & $0.25-2.0$ & $74 \%$ & ---- & 7.0 & 0.73 & 30 & 300 & $<0.5$ & $<2$ & 1.11 & 6.5 & 36 \\
\hline 94JE10(0-5) & 94JE10 & 0 & 5 & $0.25-2.0$ & $82 \%$ & ---- & 13.4 & 0.64 & 28 & 250 & $<0.5$ & $<2$ & 0.99 & 13.5 & 30 \\
\hline 94JE11(0-5) & 94JE11 & 0 & 5 & $0.25-2.0$ & $84 \%$ & ---- & 14.8 & 0.57 & 28 & 130 & $<0.5$ & $<2$ & 0.44 & 14.0 & 17 \\
\hline 94JE12(0-5) & 94JE12 & 0 & 5 & $0.25-2.0$ & $75 \%$ & ---- & 8.6 & 1.11 & 16 & 440 & 0.5 & $<2$ & 2.18 & 13.0 & 49 \\
\hline 94JE13(0-5) & 94JE13 & 0 & 5 & $0.25-2.0$ & $74 \%$ & ---- & 4.6 & 1.03 & 24 & 490 & $<0.5$ & $<2$ & 2.74 & 6.5 & 52 \\
\hline 94JE13(0-5) & 94JE13 & 0 & 5 & $0.063-0.25$ & $23 \%$ & ---- & 18.2 & 0.74 & 52 & 270 & $<0.5$ & $<2$ & 1.28 & 14.0 & 31 \\
\hline 94JE13(0-5) & 94JE13 & 0 & 5 & $<0.063$ & $3 \%$ & ---- & 18.4 & 0.97 & 68 & 230 & $<0.5$ & $<2$ & 0.52 & 15.5 & 15 \\
\hline 94JE14(0-5) & 94JE14 & 0 & 5 & $0.25-2.0$ & $64 \%$ & ---- & 2.8 & 0.61 & 48 & 740 & 0.5 & $<2$ & 0.79 & 0.5 & 9 \\
\hline 94JE15(0-5) & 94JE15 & 0 & 5 & $0.25-2.0$ & $92 \%$ & ---- & 8.2 & 2.20 & 52 & 400 & 0.5 & $<2$ & 7.37 & 2.0 & 197 \\
\hline 94JE16(0-5) & 94JE16 & 0 & 5 & $0.25-2.0$ & $87 \%$ & ---- & 14.2 & 0.73 & 32 & 250 & $<0.5$ & $<2$ & 0.95 & 11.0 & 27 \\
\hline 94JE17(0-5) & 94JE17 & 0 & 5 & $0.25-2.0$ & $78 \%$ & $\begin{array}{l}--- \\
\end{array}$ & 18.4 & 0.90 & 32 & 490 & $<0.5$ & $<2$ & 2.22 & 11.5 & 59 \\
\hline 94JE18(0-5) & 94JE18 & 0 & 5 & $0.25-2.0$ & $92 \%$ & ---- & 10.6 & 0.79 & 20 & 420 & $<0.5$ & 2 & 1.62 & 7.5 & 43 \\
\hline 94JE18(0-5) & 94JE18 & 0 & 5 & $0.063-0.25$ & $7 \%$ & $\overline{----}$ & 21.4 & 0.59 & 60 & 220 & $<0.5$ & $<2$ & 0.82 & 18.5 & 24 \\
\hline 94JE19(0-5) & 94JE19 & 0 & 5 & $0.25-2.0$ & $89 \%$ & ---- & 22.6 & 1.47 & 126 & 660 & 0.5 & $<2$ & 5.17 & 1.0 & 125 \\
\hline 94JE20(0-5) & 94JE20 & 0 & 5 & $0.25-2.0$ & $79 \%$ & ---- & 2.4 & 0.77 & 34 & 240 & 0.5 & $<2$ & 0.24 & 0.5 & 9 \\
\hline 94JE21(0-5) & 94JE21 & 0 & 5 & $0.25-2.0$ & $89 \%$ & ---- & 1.6 & 2.02 & 32 & 1020 & 0.5 & $<2$ & 5.98 & 1.0 & 157 \\
\hline 94JE22(0-5) & 94JE22 & 0 & 5 & $0.25-2.0$ & $85 \%$ & ---- & 6.2 & 0.80 & 24 & 300 & $<0.5$ & $<2$ & 1.14 & 8.0 & 32 \\
\hline 94JE23(0-5) & 94JE23 & 0 & 5 & $0.25-2.0$ & $87 \%$ & ---- & 0.4 & 0.91 & 14 & 120 & 0.5 & $<2$ & 0.16 & $<0.5$ & 8 \\
\hline 94JE24(0-5) & 94JE24 & 0 & 5 & $0.25-2.0$ & $89 \%$ & ---- & 15.4 & 0.54 & 28 & 170 & $<0.5$ & $<2$ & 0.52 & 7.5 & 17 \\
\hline 94JE25(0-5) & 94JE25 & 0 & 5 & $0.25-2.0$ & $87 \%$ & ---- & 10.2 & 0.40 & 38 & 720 & $<0.5$ & $<2$ & 0.84 & 1.5 & 23 \\
\hline 94JE26(0-5) & 94JE26 & 0 & 5 & $0.25-2.0$ & $87 \%$ & ---- & 0.6 & 1.50 & 28 & 910 & 0.5 & $<2$ & 4.60 & 1.0 & 122 \\
\hline
\end{tabular}

* Wt \% of size fraction: as \% by weight of bulk sample ( $\mathrm{nm}=$ not measured)

** Other analyses (Appendix \#): D =USGS-EDXRF, E =EWU, G =XRAL, ---- = none. 
Appendix F. Chemical composition of samples analyzed by ICP-AES at CHEMEX Labs in Reno, NV using nitric acid-aqua regia dissolution (Appendix_F.xls, Appendix_F.dbf).

\begin{tabular}{|c|c|c|c|c|c|c|c|c|c|c|c|c|c|c|c|c|c|c|c|}
\hline Sample No. & $\left|\begin{array}{c}\mathrm{Cr} \\
(\mathrm{ppm})\end{array}\right|$ & $\begin{array}{c}\mathrm{Cu} \\
(\mathrm{ppm})\end{array}$ & $\begin{array}{l}\mathrm{Fe} \\
(\%)\end{array}$ & $\begin{array}{c}\mathrm{Ga} \\
(\mathrm{ppm})\end{array}$ & $\begin{array}{c}\mathrm{Hg} \\
(\mathrm{ppm})\end{array}$ & $\begin{array}{c}K \\
(\%)\end{array}$ & $\begin{array}{c}\mathrm{La} \\
(\mathrm{ppm})\end{array}$ & $\begin{array}{l}\mathrm{Mg} \\
(\%)\end{array}$ & $\begin{array}{c}\mathrm{Mn} \\
(\mathrm{ppm})\end{array}$ & $\begin{array}{c}\text { Mo } \\
(\mathrm{ppm})\end{array}$ & $\begin{array}{l}\mathrm{Na} \\
(\%)\end{array}$ & $\begin{array}{c}\mathrm{Ni} \\
(\mathrm{ppm})\end{array}$ & $\begin{array}{c}\mathrm{P} \\
(\mathrm{ppm})\end{array}$ & $\begin{array}{c}\mathrm{Pb} \\
(\mathrm{ppm})\end{array}$ & $\begin{array}{c}\mathrm{Sb} \\
(\mathrm{ppm})\end{array}$ & $\begin{array}{c}\mathrm{Sc} \\
(\mathrm{ppm})\end{array}$ & $\begin{array}{c}\mathrm{Sr} \\
(\mathrm{ppm})\end{array}$ & $\begin{array}{c}\mathrm{Ti} \\
(\%)\end{array}$ & $\begin{array}{c}\mathrm{TI} \\
(\mathrm{ppm})\end{array}$ \\
\hline SAMPLE_NO. & CR_PPM & CU_PPM & $\mathrm{FE}_{2} \%$ & GA_PPM & HG_PPM & K_\% & LA_PPM & MG_\% & MN_PPM & MO_PPM & NA_\% & NI_PPM & P_PPM & PB_PPM & SB_PPM & SC_PPM & SR_PPM & TI_\% & TL_PPM \\
\hline 94JE02(0-5) & 32 & 214 & 5.02 & $<10$ & 3 & 0.20 & 10 & 0.39 & 3800 & 10 & 0.04 & 11 & 400 & 1065 & 6 & 1 & 236 & 0.02 & $<10$ \\
\hline 94JE02(0-5) & 33 & 226 & 5.13 & $<10$ & 1 & 0.12 & 10 & 0.30 & 4780 & 7 & 0.03 & 11 & 540 & 1485 & 18 & 1 & 142 & 0.02 & $<10$ \\
\hline 94JE03(0-5) & 30 & 203 & 5.99 & $<10$ & 2 & 0.17 & $<10$ & 0.36 & 4660 & 9 & 0.04 & 12 & 390 & 1655 & 8 & 1 & 269 & 0.02 & $<10$ \\
\hline 94JE03(0-5) & 25 & 146 & 4.86 & $<10$ & $<1$ & 0.09 & $<10$ & 0.25 & 4280 & 3 & 0.02 & 11 & 390 & 1670 & 16 & 1 & 105 & 0.02 & $<10$ \\
\hline 94JE03(0-5) & 38 & 224 & 4.15 & $<10$ & 2 & 0.12 & 20 & 0.30 & 7530 & 2 & 0.02 & 18 & 950 & 3560 & 16 & 2 & 30 & 0.04 & $<10$ \\
\hline 94JE04(0-5) & 8 & 58 & 2.72 & $<10$ & $<1$ & 0.13 & 10 & 0.28 & 1810 & 1 & 0.01 & 9 & 440 & 982 & 4 & 2 & 36 & $<0.01$ & $<10$ \\
\hline 94JE04(0-5) & 13 & 97 & 4.59 & $<10$ & 2 & 0.07 & $<10$ & 0.25 & 4310 & 2 & 0.01 & 10 & 320 & 2340 & 20 & 1 & 61 & 0.01 & $<10$ \\
\hline 94JE04(0-5) & 205 & 239 & 4.52 & $<10$ & 1 & 0.08 & 10 & 0.27 & 5220 & 4 & 0.01 & 89 & 750 & 4080 & 42 & 2 & 25 & 0.03 & $<10$ \\
\hline 94JE05(0-5) & 9 & 135 & 3.49 & $<10$ & 6 & 0.16 & 10 & 0.15 & 2430 & 2 & 0.02 & 9 & 320 & 6450 & 34 & 1 & 62 & 0.01 & $<10$ \\
\hline 94JE06A(0-5) & 8 & 78 & 3.48 & $<10$ & 3 & 0.13 & 10 & 0.27 & 3550 & 1 & 0.01 & 10 & 360 & 3140 & 14 & 1 & 17 & 0.01 & $<10$ \\
\hline 94JE07(0-5) & 5 & 12 & 1.27 & $<10$ & 2 & 0.13 & 30 & 0.16 & 245 & $<1$ & $<0.01$ & 6 & 170 & 32 & $<2$ & $<1$ & 4 & $<0.01$ & $<10$ \\
\hline 94JE08(0-5) & 23 & 221 & 5.28 & $<10$ & 2 & 0.24 & 10 & 0.21 & 2440 & 8 & 0.03 & 11 & 400 & 2530 & 28 & 1 & 198 & 0.01 & $<10$ \\
\hline 94JE09(0-5) & 22 & 204 & 4.71 & $<10$ & 2 & 0.19 & 10 & 0.20 & 2600 & 8 & 0.03 & 11 & 360 & 3930 & 30 & 1 & 196 & 0.02 & $<10$ \\
\hline 94JE10(0-5) & 19 & 178 & 4.93 & $<10$ & 4 & 0.17 & $<10$ & 0.21 & 2840 & 7 & 0.03 & 11 & 380 & 6450 & 40 & 1 & 163 & 0.02 & $<10$ \\
\hline 94JE11(0-5) & 9 & 144 & 3.79 & $<10$ & 7 & 0.14 & 10 & 0.15 & 2510 & 2 & 0.01 & 8 & 290 & 6330 & 56 & 1 & 73 & 0.01 & $<10$ \\
\hline 94JE12(0-5) & 35 & 271 & 6.30 & $<10$ & 4 & 0.27 & 10 & 0.28 & 3960 & 16 & 0.06 & 10 & 400 & 4630 & 14 & 2 & 299 & 0.04 & $<10$ \\
\hline 94JE13(0-5) & 40 & 266 & 6.79 & 10 & 2 & 0.26 & 10 & 0.46 & 4130 & 19 & 0.07 & 12 & 410 & 2150 & 12 & 2 & 331 & 0.03 & $<10$ \\
\hline 94JE13(0-5) & 25 & 253 & 7.57 & $<10$ & 3 & 0.13 & $<10$ & 0.36 & 4830 & 9 & 0.03 & 16 & 350 & 5260 & 34 & 1 & 159 & 0.02 & $<10$ \\
\hline 94JE13(0-5) & 35 & 189 & 3.86 & $<10$ & 6 & 0.11 & 10 & 0.34 & 2810 & 2 & 0.01 & 23 & 610 & 5730 & 28 & 2 & 40 & 0.03 & $<10$ \\
\hline 94JE14(0-5) & 14 & 71 & 2.35 & $<10$ & $<1$ & 0.19 & 10 & 0.24 & 1260 & 1 & 0.01 & 8 & 420 & 72 & 6 & 1 & 27 & $<0.01$ & $<10$ \\
\hline 94JE15(0-5) & 130 & 836 & $>15.00$ & 30 & 4 & 0.51 & 10 & 0.72 & 9270 & 56 & 0.22 & 26 & 670 & 250 & 24 & 4 & 1235 & 0.08 & $<10$ \\
\hline 94JE16(0-5) & 17 & 184 & 5.89 & $<10$ & 4 & 0.21 & 10 & 0.34 & 4100 & 6 & 0.03 & 11 & 320 & 6270 & 24 & 1 & 132 & 0.01 & $<10$ \\
\hline 94JE17(0-5) & 38 & 296 & 9.63 & $<10$ & 10 & 0.21 & $<10$ & 0.44 & 6390 & 15 & 0.06 & 14 & 380 & 6200 & 32 & 1 & 353 & 0.03 & $<10$ \\
\hline 94JE18(0-5) & 27 & 204 & 7.20 & $<10$ & 3 & 0.21 & $<10$ & 0.39 & 5060 & 11 & 0.05 & 11 & 300 & 4130 & 22 & 1 & 256 & 0.02 & $<10$ \\
\hline 94JE18(0-5) & 18 & 282 & 8.63 & $<10$ & 4 & 0.12 & $<10$ & 0.32 & 5620 & 4 & 0.03 & 15 & 330 & 6980 & 40 & 1 & 111 & 0.02 & $<10$ \\
\hline 94JE19(0-5) & 85 & 723 & 13.80 & 20 & $<1$ & 0.35 & $<10$ & 0.57 & 8290 & 40 & 0.15 & 20 & 500 & 574 & 44 & 3 & 784 & 0.05 & $<10$ \\
\hline 94JE20(0-5) & 7 & 63 & 3.04 & $<10$ & 2 & 0.18 & 10 & 0.21 & 4100 & 1 & $<0.01$ & 18 & 440 & 76 & 14 & 2 & 11 & $<0.01$ & $<10$ \\
\hline 94JE21(0-5) & 105 & 622 & 12.95 & 30 & 6 & 0.41 & 10 & 0.59 & 6980 & 47 & 0.19 & 22 & 680 & 166 & 8 & 3 & 1005 & 0.07 & $<10$ \\
\hline 94JE22(0-5) & 21 & 152 & 5.20 & $<10$ & 1 & 0.19 & 10 & 0.34 & 3690 & 7 & 0.03 & 10 & 320 & 3060 & 18 & 1 & 178 & 0.02 & $<10$ \\
\hline 94JE23(0-5) & 10 & 28 & 3.51 & $<10$ & $<1$ & 0.17 & 30 & 0.12 & 645 & 1 & 0.01 & 15 & 440 & 58 & 2 & 1 & 25 & $<0.01$ & $<10$ \\
\hline 94JE24(0-5) & 10 & 145 & 6.55 & $<10$ & $<1$ & 0.13 & 10 & 0.35 & 4980 & 3 & 0.02 & 9 & 260 & 7020 & 24 & 1 & 77 & 0.01 & $<10$ \\
\hline 94JE25(0-5) & 12 & 396 & 12.25 & $<10$ & $<1$ & 0.12 & $<10$ & 0.34 & 8600 & 6 & 0.02 & 9 & 280 & 302 & 94 & 1 & 130 & $<0.01$ & $<10$ \\
\hline 94JE26(0-5) & 78 & 423 & 10.30 & 20 & 1 & 0.32 & $<10$ & 0.46 & 5540 & 36 & 0.13 & 18 & 500 & 174 & 14 & 2 & 766 & 0.04 & $<10$ \\
\hline
\end{tabular}

* Wt \% of size fraction: as \% by weight of bulk sample ( $\mathrm{nm}=$ not measured)

** Other analyses (Appendix \#): D =USGS-EDXRF, E =EWU, G =XRAL, ---- = none. 
Appendix F. Chemical composition of samples analyzed by ICP-AES at CHEMEX Labs in Reno, NV using nitric acid-aqua regia dissolution (Appendix_F.Xls, Appendix_F.dbf).

\begin{tabular}{|l|r|r|r|r|}
\hline Sample No. & $\begin{array}{c}\text { U } \\
(\mathrm{ppm})\end{array}$ & $\begin{array}{c}\text { V } \\
(\mathrm{ppm})\end{array}$ & $\begin{array}{c}\text { W } \\
(\mathrm{ppm})\end{array}$ & $\begin{array}{c}\text { Zn } \\
(\mathrm{ppm})\end{array}$ \\
\hline SAMPLE_No. & U_PPM & V_PPM & W_PPM & ZN_PPM \\
\hline 94JE02(0-5) & $<10$ & 12 & $<10$ & 3650 \\
\hline 94JE02(0-5) & $<10$ & 13 & $<10$ & 2810 \\
\hline 94JE03(0-5) & $<10$ & 12 & $<10$ & 3560 \\
\hline 94JE03(0-5) & $<10$ & 12 & $<10$ & 2470 \\
\hline 94JE03(0-5) & $<10$ & 18 & $<10$ & 3910 \\
\hline 94JE04(0-5) & $<10$ & 6 & $<10$ & 1200 \\
\hline 94JE04(0-5) & $<10$ & 7 & $<10$ & 2230 \\
\hline 94JE04(0-5) & $<10$ & 14 & $<10$ & 3020 \\
\hline 94JE05(0-5) & $<10$ & 9 & $<10$ & 5380 \\
\hline 94JE06A(0-5) & $<10$ & 10 & 10 & 2720 \\
\hline 94JE07(0-5) & $<10$ & 4 & $<10$ & 48 \\
\hline 94JE08(0-5) & $<10$ & 10 & $<10$ & 3990 \\
\hline 94JE09(0-5) & $<10$ & 11 & $<10$ & 3690 \\
\hline 94JE10(0-5) & $<10$ & 12 & $<10$ & 5490 \\
\hline 94JE11(0-5) & $<10$ & 9 & $<10$ & 5090 \\
\hline 94JE12(0-5) & $<10$ & 24 & $<10$ & 8250 \\
\hline 94JE13(0-5) & $<10$ & 17 & $<10$ & 6810 \\
\hline 94JE13(0-5) & $<10$ & 18 & $<10$ & 6160 \\
\hline 94JE13(0-5) & $<10$ & 17 & $<10$ & 4560 \\
\hline 94JE14(0-5) & $<10$ & 7 & $<10$ & 642 \\
\hline 94JE15(0-5) & $<10$ & 32 & $<10$ & $>10000$ \\
\hline 94JE16(0-5) & $<10$ & 11 & $<10$ & 4980 \\
\hline 94JE17(0-5) & $<10$ & 17 & $<10$ & 7170 \\
\hline 94JE18(0-5) & $<10$ & 13 & $<10$ & 5210 \\
\hline 94JE18(0-5) & $<10$ & 18 & $<10$ & 6450 \\
\hline 94JE19(0-5) & $<10$ & 22 & $<10$ & $>10000$ \\
\hline 94JE20(0-5) & $<10$ & 9 & $<10$ & 320 \\
\hline 94JE21(0-5) & $<10$ & 28 & $<10$ & $>10000$ \\
\hline 94JE22(0-5) & $<10$ & 12 & $<10$ & 4300 \\
\hline 94JE23(0-5) & $<10$ & 11 & $<10$ & 156 \\
\hline 94JE24(0-5) & $<10$ & 8 & $<10$ & 2980 \\
\hline 94JE25(0-5) & $<10$ & 4 & $<10$ & 1820 \\
\hline 94JE26(0-5) & $<10$ & 22 & $<10$ & 9440 \\
\hline
\end{tabular}

* Wt \% of size fraction: as \% by weight of bulk sample ( $\mathrm{nm}=$ not measured)

** Other analyses (Appendix \#): D =USGS-EDXRF, E =EWU, G =XRAL, ---- = none 
Appendix F. Chemical composition of samples analyzed by ICP-AES at CHEMEX Labs in Reno, NV using nitric acid-aqua regia dissolution (Appendix_F.xls, Appendix_F.dbf).

\begin{tabular}{|c|c|c|c|c|c|c|c|c|c|c|c|c|c|c|c|}
\hline Sample No. & Site ID & $\begin{array}{l}\text { Depth } \\
\text { interval, } \\
\text { top }(\mathrm{cm})\end{array}$ & $\begin{array}{c}\text { Depth } \\
\text { interval, } \\
\text { bottom }(\mathrm{cm})\end{array}$ & $\begin{array}{c}\text { Size } \\
\text { fraction } \\
(\mathrm{mm})\end{array}$ & $\begin{array}{l}\text { *wt \% of } \\
\text { size } \\
\text { fraction }\end{array}$ & $\begin{array}{c}{ }^{* *} \text { Other } \\
\text { analyses } \\
(\text { Appendix \#) }\end{array}$ & $\begin{array}{c}\mathrm{Ag} \\
(\mathrm{ppm})\end{array}$ & $\begin{array}{c}\mathrm{Al} \\
(\%)\end{array}$ & $\begin{array}{c}\text { As } \\
\text { (ppm) }\end{array}$ & $\begin{array}{c}\mathrm{Ba} \\
(\mathrm{ppm})\end{array}$ & $\begin{array}{c}\mathrm{Be} \\
(\mathrm{ppm})\end{array}$ & $\begin{array}{c}\mathrm{Bi} \\
(\mathrm{ppm})\end{array}$ & $\begin{array}{l}\mathrm{Ca} \\
(\%)\end{array}$ & $\begin{array}{c}\text { Cd } \\
(\mathrm{ppm})\end{array}$ & $\begin{array}{c}\text { Co } \\
\text { (ppm) }\end{array}$ \\
\hline SAMPLE_NO. & SITE_ID & INT_TOP_CM & INT_BTM_CM & SIZFRAC_MM & WT\%SIZFRAC & OTHR_ANLYS & AG_PPM & AL_\% & AS_PPM & BA_PPM & BE_PPM & BI_PPM & CA_\% & CD_PPM & CO_PPM \\
\hline 94JE27(0-5) & 94JE27 & 0 & 5 & $0.25-2.0$ & $71 \%$ & ---- & 0.2 & 0.44 & 34 & 70 & $<0.5$ & $<2$ & 0.17 & 0.5 & 5 \\
\hline 94JE28(0-5) & 94JE28 & 0 & 5 & $0.25-2.0$ & $91 \%$ & ---- & 3.8 & 0.56 & 22 & 120 & $<0.5$ & $<2$ & 0.29 & 4.0 & 12 \\
\hline 94JE28(0-5) & 94JE28 & 0 & 5 & $0.063-0.25$ & $8 \%$ & ---- & 17.0 & 0.48 & 76 & 230 & $<0.5$ & $<2$ & 0.53 & 10.0 & 20 \\
\hline 94JE29(0-5) & 94JE29 & 0 & 5 & $0.25-2.0$ & $85 \%$ & ---- & 63.2 & 0.75 & 134 & 430 & $<0.5$ & $<2$ & 1.46 & 0.5 & 51 \\
\hline 94JE30(0-5) & 94JE30 & 0 & 5 & $0.25-2.0$ & $93 \%$ & ---- & 7.8 & 0.62 & 32 & 150 & $<0.5$ & $<2$ & 0.29 & 5.0 & 17 \\
\hline 94JE31(0-5) & 94JE31 & 0 & 5 & $0.25-2.0$ & $97 \%$ & ---- & 4.0 & 0.68 & 32 & 170 & $<0.5$ & $<2$ & 0.49 & 5.0 & 19 \\
\hline 94JE32(0-5) & 94JE32 & 0 & 5 & $0.25-2.0$ & $74 \%$ & ---- & 0.4 & 0.89 & 50 & 100 & $<0.5$ & $<2$ & 0.19 & 1.5 & 18 \\
\hline 94JE33(0-5) & 94JE33 & 0 & 5 & $0.25-2.0$ & $89 \%$ & ---- & 2.0 & 0.62 & 26 & 80 & $<0.5$ & $<2$ & 0.12 & 3.0 & 8 \\
\hline 94JE34(0-5) & 94JE34 & 0 & 5 & $0.25-2.0$ & $60 \%$ & ---- & 10.0 & 0.59 & 40 & 80 & $<0.5$ & $<2$ & 0.16 & 8.0 & 9 \\
\hline 94JE34(0-5) & 94JE34 & 0 & 5 & $0.063-0.25$ & $35 \%$ & ---- & 11.8 & 0.53 & 60 & 170 & $<0.5$ & $<2$ & 0.21 & 6.0 & 11 \\
\hline 94JE34(0-5) & 94JE34 & 0 & 5 & $<0.063$ & $5 \%$ & ---- & 31.4 & 0.90 & 134 & 260 & $<0.5$ & $<2$ & 0.26 & 11.0 & 14 \\
\hline 94JE35(0-5) & 94JE35 & 0 & 5 & $0.25-2.0$ & $93 \%$ & ---- & 8.4 & 0.59 & 42 & 90 & $<0.5$ & $<2$ & 0.11 & 2.0 & 8 \\
\hline 94JE36(0-5) & 94JE36 & 0 & 5 & $0.25-2.0$ & $43 \%$ & ---- & 19.0 & 0.47 & 82 & 100 & $<0.5$ & $<2$ & 0.13 & 8.0 & 8 \\
\hline 94JE37(0-5) & 94JE37 & 0 & 5 & $0.25-2.0$ & $73 \%$ & ---- & 19.4 & 0.40 & 50 & 100 & $<0.5$ & $<2$ & 0.13 & 17.5 & 10 \\
\hline 94JE37(0-5) & 94JE37 & 0 & 5 & $0.063-0.25$ & $22 \%$ & ---- & 21.0 & 0.58 & 94 & 200 & $<0.5$ & $<2$ & 0.27 & 17.5 & 16 \\
\hline 94JE37(0-5) & 94JE37 & 0 & 5 & $<0.063$ & $5 \%$ & ---- & 43.6 & 1.33 & 134 & 330 & 0.5 & $<2$ & 0.27 & 36.5 & 30 \\
\hline 94JE38(0-5) & 94JE38 & 0 & 5 & $0.25-2.0$ & $76 \%$ & ---- & 10.0 & 0.93 & 70 & 490 & $<0.5$ & $<2$ & 1.81 & 8.0 & 54 \\
\hline 94JE39A(0-5) & 94JE39A & 0 & 5 & $0.25-2.0$ & $94 \%$ & ---- & 3.0 & 0.62 & 48 & 90 & $<0.5$ & $<2$ & 0.18 & 5.5 & 13 \\
\hline 94JE40(0-5) & 94JE40 & 0 & 5 & $0.25-2.0$ & $87 \%$ & ---- & 5.2 & 0.53 & 46 & 80 & $<0.5$ & $<2$ & 0.19 & 5.5 & 11 \\
\hline 94JE41(0-5) & 94JE41 & 0 & 5 & $0.25-2.0$ & $9 \%$ & ---- & 16.8 & 0.30 & 114 & 50 & $<0.5$ & $<2$ & 0.20 & 14.5 & 4 \\
\hline 94JE41(0-5) & 94JE41 & 0 & 5 & $0.063-0.25$ & $75 \%$ & ---- & 18.4 & 0.30 & 274 & 90 & $<0.5$ & $<2$ & 0.13 & 11.0 & 8 \\
\hline 94JE41(0-5) & 94JE41 & 0 & 5 & $<0.063$ & $16 \%$ & ---- & 27.0 & 0.37 & 1095 & 80 & $<0.5$ & 2 & 0.17 & 23.5 & 17 \\
\hline 94JE42(0-5) & 94JE42 & 0 & 5 & $0.25-2.0$ & $98 \%$ & ---- & $<0.2$ & 0.58 & 14 & 40 & $<0.5$ & $<2$ & 0.03 & 0.5 & 5 \\
\hline 94JE43(0-5) & 94JE43 & 0 & 5 & $0.25-2.0$ & $70 \%$ & ---- & 7.6 & 0.47 & 62 & 70 & $<0.5$ & $<2$ & 0.15 & 6.0 & 7 \\
\hline 94JE44(0-5) & 94JE44 & 0 & 5 & $0.25-2.0$ & $74 \%$ & ---- & 0.8 & 0.64 & 44 & 50 & $<0.5$ & $<2$ & 0.06 & 1.0 & 7 \\
\hline 94JE45(0-5) & 94JE45 & 0 & 5 & $0.25-2.0$ & $44 \%$ & ---- & 6.6 & 0.58 & 66 & 70 & $<0.5$ & $<2$ & 0.13 & 5.5 & 8 \\
\hline 94JE46(0-5) & 94JE46 & 0 & 5 & $0.25-2.0$ & $54 \%$ & ---- & 6.2 & 0.54 & 60 & 100 & $<0.5$ & $<2$ & 0.24 & 4.5 & 13 \\
\hline 94JE47(0-5) & 94JE47 & 0 & 5 & $0.25-2.0$ & $51 \%$ & ---- & 6.8 & 0.54 & 82 & 100 & $<0.5$ & $<2$ & 0.16 & 6.5 & 8 \\
\hline 94JE48(0-5) & 94JE48 & 0 & 5 & $0.25-2.0$ & $91 \%$ & ---- & 6.8 & 0.58 & 54 & 110 & $<0.5$ & $<2$ & 0.28 & 4.0 & 11 \\
\hline 94JE49(0-5) & 94JE49 & 0 & 5 & $0.25-2.0$ & $30 \%$ & ---- & 9.4 & 0.54 & 110 & 120 & $<0.5$ & $<2$ & 0.14 & 9.0 & 11 \\
\hline 94JE49(0-5) & 94JE49 & 0 & 5 & $0.063-0.25$ & $65 \%$ & ---- & 15.2 & 0.42 & 156 & 160 & $<0.5$ & $<2$ & 0.14 & 9.5 & 6 \\
\hline 94JE49(0-5) & 94JE49 & 0 & 5 & $<0.063$ & $5 \%$ & $-\cdots$ & 23.0 & 0.77 & 330 & 160 & $<0.5$ & $<2$ & 0.16 & 25.0 & 16 \\
\hline $94 \mathrm{VC}-2(0-10)$ & $94 \mathrm{VC}-2$ & 0 & 10 & $<0.83$ & $100 \%$ & ---- & 12.0 & 0.67 & 78 & 190 & 0.5 & $<2$ & 0.26 & 28.5 & 10 \\
\hline
\end{tabular}

* Wt \% of size fraction: as \% by weight of bulk sample ( $\mathrm{nm}=$ not measured)

** Other analyses (Appendix \#): D =USGS-EDXRF, E =EWU, G =XRAL, ---- = none. 
Appendix F. Chemical composition of samples analyzed by ICP-AES at CHEMEX Labs in Reno, NV using nitric acid-aqua regia dissolution (Appendix_F.xls, Appendix_F.dbf).

\begin{tabular}{|c|c|c|c|c|c|c|c|c|c|c|c|c|c|c|c|c|c|c|c|}
\hline Sample No. & $\begin{array}{c}\mathrm{Cr} \\
(\mathrm{ppm})\end{array}$ & $\begin{array}{c}\mathrm{Cu} \\
(\mathrm{ppm})\end{array}$ & $\begin{array}{l}\mathrm{Fe} \\
(\%)\end{array}$ & $\begin{array}{c}\mathrm{Ga} \\
(\mathrm{ppm})\end{array}$ & $\begin{array}{c}\mathrm{Hg} \\
(\mathrm{ppm})\end{array}$ & $\begin{array}{c}\mathrm{K} \\
(\%)\end{array}$ & $\begin{array}{c}\mathrm{La} \\
(\mathrm{ppm})\end{array}$ & $\begin{array}{l}\mathrm{Mg} \\
(\%)\end{array}$ & $\begin{array}{c}\mathrm{Mn} \\
(\mathrm{ppm})\end{array}$ & $\begin{array}{c}\text { Mo } \\
\text { (ppm) }\end{array}$ & $\begin{array}{l}\mathrm{Na} \\
(\%)\end{array}$ & $\begin{array}{c}\mathrm{Ni} \\
(\mathrm{ppm})\end{array}$ & $\begin{array}{c}\mathrm{P} \\
(\mathrm{ppm})\end{array}$ & $\begin{array}{c}\mathrm{Pb} \\
(\mathrm{ppm})\end{array}$ & $\begin{array}{c}\mathrm{Sb} \\
(\mathrm{ppm})\end{array}$ & $\begin{array}{c}\mathrm{Sc} \\
(\mathrm{ppm})\end{array}$ & $\begin{array}{c}\mathrm{Sr} \\
(\mathrm{ppm})\end{array}$ & $\begin{array}{c}\mathrm{Ti} \\
(\%)\end{array}$ & $\begin{array}{c}\mathrm{TI} \\
(\mathrm{ppm})\end{array}$ \\
\hline SAMPLE_NO. & CR_PPM & CU_PPM & FE_\% & GA_PPM & HG_PPM & K_\% & LA_PPM & MG_\% & MN_PPM & MO_PPM & NA_\% & NI_PPM & P_PPM & PB_PPM & SB_PPM & SC_PPM & SR_PPM & TI_\% & TL_PPM \\
\hline 94JE27(0-5) & 6 & 15 & 1.87 & $<10$ & $<1$ & 0.08 & 10 & 0.07 & 705 & $<1$ & $<0.01$ & 11 & 340 & 42 & 2 & 2 & 7 & $<0.01$ & $<10$ \\
\hline 94JE28(0-5) & 8 & 77 & 4.15 & $<10$ & $<1$ & 0.14 & 10 & 0.29 & 3050 & $<1$ & 0.01 & 9 & 290 & 2360 & 14 & 1 & 39 & $<0.01$ & $<10$ \\
\hline 94JE28(0-5) & 11 & 250 & 12.35 & $<10$ & $<1$ & 0.10 & $<10$ & 0.38 & 9350 & 3 & 0.02 & 13 & 250 & 5130 & 30 & 1 & 72 & 0.01 & $<10$ \\
\hline 94JE29(0-5) & 30 & 389 & 7.53 & $<10$ & 4 & 0.16 & $<10$ & 0.36 & 4750 & 11 & 0.04 & 12 & 350 & 950 & 112 & 2 & 259 & 0.01 & $<10$ \\
\hline 94JE30(0-5) & 8 & 120 & 4.92 & $<10$ & 2 & 0.17 & 10 & 0.30 & 3420 & $<1$ & 0.01 & 10 & 350 & 2780 & 18 & 1 & 36 & 0.01 & $<10$ \\
\hline 94JE31(0-5) & 16 & 82 & 3.31 & $<10$ & 1 & 0.14 & 10 & 0.28 & 2290 & 2 & 0.02 & 11 & 350 & 1685 & 12 & 1 & 75 & 0.01 & $<10$ \\
\hline 94JE32(0-5) & 12 & 80 & 3.49 & $<10$ & $<1$ & 0.14 & 30 & 0.20 & 870 & 2 & 0.01 & 19 & 350 & 328 & 4 & 1 & 35 & $<0.01$ & $<10$ \\
\hline 94JE33(0-5) & 7 & 47 & 2.62 & $<10$ & $<1$ & 0.14 & 20 & 0.25 & 1390 & 1 & 0.01 & 9 & 290 & 1580 & 10 & 1 & 15 & $<0.01$ & $<10$ \\
\hline 94JE34(0-5) & 7 & 95 & 6.59 & $<10$ & 5 & 0.14 & 10 & 0.34 & 5110 & 1 & 0.01 & 11 & 290 & 3970 & 18 & 1 & 18 & $<0.01$ & $<10$ \\
\hline 94JE34(0-5) & 9 & 142 & 6.72 & $<10$ & 2 & 0.09 & 10 & 0.27 & 5030 & 1 & 0.01 & 9 & 300 & 3770 & 28 & 1 & 27 & 0.01 & $<10$ \\
\hline 94JE34(0-5) & 42 & 272 & 5.38 & $<10$ & 5 & 0.11 & 10 & 0.28 & 3490 & 2 & 0.01 & 26 & 630 & 6500 & 40 & 2 & 21 & 0.02 & $<10$ \\
\hline 94JE35(0-5) & 7 & 82 & 4.97 & $<10$ & 2 & 0.14 & 20 & 0.25 & 3180 & $<1$ & 0.01 & 9 & 320 & 2450 & 20 & 1 & 12 & $<0.01$ & $<10$ \\
\hline 94JE36(0-5) & 4 & 139 & 9.36 & $<10$ & $<1$ & 0.10 & 10 & 0.30 & 6090 & $<1$ & 0.01 & 10 & 500 & 9140 & 32 & 1 & 12 & $<0.01$ & $<10$ \\
\hline 94JE37(0-5) & 3 & 130 & 9.86 & $<10$ & 6 & 0.10 & $<10$ & 0.25 & 8280 & $<1$ & 0.01 & 8 & 250 & 9770 & 38 & 1 & 12 & $<0.01$ & $<10$ \\
\hline 94JE37(0-5) & 9 & 214 & 9.72 & $<10$ & 6 & 0.09 & $<10$ & 0.27 & 8260 & 2 & 0.01 & 11 & 520 & 8050 & 48 & 1 & 34 & 0.01 & $<10$ \\
\hline 94JE37(0-5) & 29 & 296 & 7.49 & $<10$ & 8 & 0.13 & 10 & 0.26 & 9570 & $\overline{1}$ & 0.02 & 22 & 1710 & $>10000$ & 70 & 2 & 18 & 0.02 & $<10$ \\
\hline 94JE38(0-5) & 31 & 259 & 10.40 & $<10$ & 1 & 0.19 & 10 & 0.39 & 7350 & 14 & 0.06 & 11 & 540 & 2950 & 20 & 1 & 267 & 0.02 & $<10$ \\
\hline 94JE39A(0-5) & 8 & 68 & 3.06 & $<10$ & 2 & 0.13 & 20 & 0.22 & 2160 & 1 & 0.01 & 8 & 400 & 2060 & 16 & 1 & 16 & $<0.01$ & $<10$ \\
\hline 94JE40(0-5) & 8 & 75 & 3.89 & $<10$ & 2 & 0.10 & 10 & 0.22 & 2660 & 2 & 0.01 & 8 & 350 & 2400 & 14 & 1 & 23 & $<0.01$ & $<10$ \\
\hline 94JE41(0-5) & 2 & 113 & 12.95 & $<10$ & $<1$ & 0.09 & $<10$ & 0.52 & $>10000$ & 1 & 0.01 & 8 & 280 & 8570 & 34 & 1 & 14 & $<0.01$ & $<10$ \\
\hline 94JE41(0-5) & 3 & 133 & 11.65 & $<10$ & 1 & 0.07 & $<10$ & 0.37 & 8720 & $<1$ & 0.01 & 9 & 260 & 3160 & 46 & 1 & 4 & $<0.01$ & $<10$ \\
\hline 94JE41(0-5) & 7 & 209 & $>15.00$ & $<10$ & 4 & 0.06 & $<10$ & 0.48 & $>10000$ & 1 & 0.01 & 18 & 530 & 6050 & 56 & 1 & 5 & 0.01 & $<10$ \\
\hline 94JE42(0-5) & 6 & 17 & 1.69 & $<10$ & $<1$ & 0.11 & 20 & 0.16 & 365 & $<1$ & $<0.01$ & 6 & 190 & 238 & 2 & $<1$ & 4 & $<0.01$ & $<10$ \\
\hline 94JE43(0-5) & 6 & 79 & 6.82 & $<10$ & 1 & 0.11 & 10 & 0.30 & 4780 & $<1$ & 0.01 & 8 & 260 & 3220 & 26 & 1 & 14 & $<0.01$ & $<10$ \\
\hline 94JE44(0-5) & 9 & 29 & 2.33 & $<10$ & 1 & 0.10 & 20 & 0.20 & 670 & $<1$ & $<0.01$ & 8 & 240 & 896 & 52 & $<1$ & 7 & $<0.01$ & $<10$ \\
\hline 94JE45(0-5) & 8 & 71 & 4.22 & $<10$ & 2 & 0.11 & 10 & 0.24 & 2670 & $<1$ & 0.01 & 7 & 320 & 2760 & 36 & 1 & 15 & $<0.01$ & $<10$ \\
\hline 94JE46(0-5) & 9 & 92 & 6.51 & $<10$ & $<1$ & 0.11 & 10 & 0.30 & 4730 & 2 & 0.01 & 7 & 470 & 2700 & 22 & 1 & 28 & $<0.01$ & $<10$ \\
\hline 94JE47(0-5) & 7 & 88 & 7.21 & $<10$ & 1 & 0.09 & 10 & 0.29 & 4610 & 2 & 0.01 & 7 & 650 & 2900 & 24 & 1 & 18 & $<0.01$ & $<10$ \\
\hline 94JE48(0-5) & 8 & 81 & 7.73 & $<10$ & $<1$ & 0.11 & 10 & 0.32 & 5560 & 1 & 0.01 & 8 & 330 & 2860 & 26 & 1 & 40 & $<0.01$ & $<10$ \\
\hline 94JE49(0-5) & 8 & 86 & 7.09 & $<10$ & 2 & 0.10 & 10 & 0.27 & 5210 & $<1$ & 0.01 & 8 & 560 & 4330 & 30 & 1 & 15 & $<0.01$ & $<10$ \\
\hline 94JE49(0-5) & 4 & 132 & 11.10 & $<10$ & 2 & 0.07 & 10 & 0.32 & 7830 & 2 & 0.01 & 10 & 480 & 3580 & 42 & 1 & 8 & $<0.01$ & $<10$ \\
\hline 94JE49(0-5) & 17 & 222 & 9.27 & $<10$ & 8 & 0.07 & 10 & 0.27 & 5760 & 1 & 0.01 & 16 & 1290 & 6810 & 50 & 1 & 10 & 0.02 & $<10$ \\
\hline 94VC-2(0-10) & 101 & 109 & 11.00 & $<10$ & 3 & 0.19 & 10 & 0.54 & 9790 & 3 & 0.01 & 9 & 260 & 4380 & 12 & 2 & 10 & 0.01 & $<10$ \\
\hline
\end{tabular}

* Wt \% of size fraction: as \% by weight of bulk sample ( $\mathrm{nm}=$ not measured)

** Other analyses (Appendix \#): D =USGS-EDXRF, E =EWU, G =XRAL, ---- = none. 
Appendix F. Chemical composition of samples analyzed by ICP-AES at CHEMEX Labs in Reno, NV using nitric acid-aqua regia dissolution (Appendix_F.Xls, Appendix_F.dbf).

\begin{tabular}{|l|r|r|r|r|}
\hline Sample No. & $\begin{array}{c}\text { U } \\
(\mathrm{ppm})\end{array}$ & $\begin{array}{c}\mathrm{V} \\
(\mathrm{ppm})\end{array}$ & $\begin{array}{c}\mathrm{W} \\
(\mathrm{ppm})\end{array}$ & $\begin{array}{c}\text { Zn } \\
(\mathrm{ppm})\end{array}$ \\
\hline SAMPLE_No. & U_PPM & V_PPM $_{-}$ & W_PPM & ZN_PPM \\
\hline 94JE27(0-5) & $<10$ & 7 & $<10$ & 92 \\
\hline 94JE28(0-5) & $<10$ & 7 & $<10$ & 1675 \\
\hline 94JE28(0-5) & $<10$ & 14 & $<10$ & 3690 \\
\hline 94JE29(0-5) & $<10$ & 10 & $<10$ & 2980 \\
\hline 94JE30(0-5) & $<10$ & 8 & $<10$ & 1740 \\
\hline 94JE31(0-5) & $<10$ & 10 & $<10$ & 2080 \\
\hline 94JE32(0-5) & $<10$ & 9 & $<10$ & 912 \\
\hline 94JE33(0-5) & $<10$ & 7 & $<10$ & 1160 \\
\hline 94JE34(0-5) & $<10$ & 7 & $<10$ & 2090 \\
\hline 94JE34(0-5) & $<10$ & 10 & $<10$ & 1910 \\
\hline 94JE34(0-5) & $<10$ & 16 & $<10$ & 2290 \\
\hline 94JE35(0-5) & $<10$ & 8 & $<10$ & 1135 \\
\hline 94JE36(0-5) & $<10$ & 5 & $<10$ & 2910 \\
\hline 94JE37(0-5) & $<10$ & 3 & $<10$ & 6230 \\
\hline 94JE37(0-5) & $<10$ & 9 & $<10$ & 5240 \\
\hline 94JE37(0-5) & $<10$ & 17 & $<10$ & 8470 \\
\hline 94JE38(0-5) & $<10$ & 14 & $<10$ & 5190 \\
\hline 94JE39A(0-5) & $<10$ & 8 & $<10$ & 1555 \\
\hline 94JE40(0-5) & $<10$ & 7 & $<10$ & 1780 \\
\hline 94JE41(0-5) & $<10$ & 2 & $<10$ & 3620 \\
\hline 94JE41(0-5) & $<10$ & 4 & $<10$ & 2890 \\
\hline 94JE41(0-5) & $<10$ & 7 & $<10$ & 4000 \\
\hline 94JE42(0-5) & $<10$ & 6 & $<10$ & 288 \\
\hline 94JE43(0-5) & $<10$ & 6 & 10 & 2520 \\
\hline 94JE44(0-5) & $<10$ & 9 & $<10$ & 474 \\
\hline 94JE45(0-5) & $<10$ & 7 & $<10$ & 1670 \\
\hline 94JE46(0-5) & $<10$ & 8 & $<10$ & 1390 \\
\hline 94JE47(0-5) & $<10$ & 8 & $<10$ & 1675 \\
\hline 94JE48(0-5) & $<10$ & 8 & $<10$ & 1780 \\
\hline 94JE49(0-5) & $<10$ & 7 & $<10$ & 2580 \\
\hline 94JE49(0-5) & $<10$ & 7 & $<10$ & 2940 \\
\hline 94JE49(0-5) & $<10$ & 14 & $<10$ & 4460 \\
\hline 94VC-2(0-10) & $<10$ & 3 & $<10$ & 5280 \\
\hline
\end{tabular}

* Wt \% of size fraction: as \% by weight of bulk sample ( $\mathrm{nm}=$ not measured)

** Other analyses (Appendix \#): D =USGS-EDXRF, E =EWU, G =XRAL, ---- = none 
Appendix F. Chemical composition of samples analyzed by ICP-AES at CHEMEX Labs in Reno, NV using nitric acid-aqua regia dissolution (Appendix_F.xls, Appendix_F.dbf).

\begin{tabular}{|c|c|c|c|c|c|c|c|c|c|c|c|c|c|c|c|}
\hline Sample No. & Site ID & $\begin{array}{c}\text { Depth } \\
\text { interval, } \\
\text { top }(\mathrm{cm})\end{array}$ & $\begin{array}{c}\text { Depth } \\
\text { interval, } \\
\text { bottom }(\mathrm{cm})\end{array}$ & $\begin{array}{l}\text { Size } \\
\text { fraction } \\
(\mathrm{mm})\end{array}$ & $\begin{array}{c}\text { *wt \% of } \\
\text { size } \\
\text { fraction }\end{array}$ & $\begin{array}{c}{ }^{* *} \text { Other } \\
\text { analyses } \\
(\text { Appendix \#) }\end{array}$ & $\begin{array}{c}\mathrm{Ag} \\
(\mathrm{ppm})\end{array}$ & $\begin{array}{c}\mathrm{Al} \\
(\%)\end{array}$ & $\begin{array}{c}\text { As } \\
\text { (ppm) }\end{array}$ & $\begin{array}{c}\mathrm{Ba} \\
(\mathrm{ppm})\end{array}$ & $\begin{array}{c}\mathrm{Be} \\
(\mathrm{ppm})\end{array}$ & $\begin{array}{c}\mathrm{Bi} \\
(\mathrm{ppm})\end{array}$ & $\begin{array}{l}\mathrm{Ca} \\
(\%)\end{array}$ & $\begin{array}{c}\text { Cd } \\
(p p m)\end{array}$ & $\begin{array}{c}\text { Co } \\
\text { (ppm) }\end{array}$ \\
\hline SAMPLE_NO. & SITE_ID & INT_TOP_CM & INT_BTM_CM & SIZFRAC_MM & WT\%SIZFRAC & OTHR_ANLYS & AG_PPM & AL_\% & AS_PPM & BA_PPM & BE_PPM & BI_PPM & CA_\% & CD_PPM & CO_PPM \\
\hline 94VC-2(10-20) & 94VC-2 & 10 & 20 & $<0.83$ & $100 \%$ & ---- & 16.8 & 0.67 & 80 & 130 & $<0.5$ & $<2$ & 0.37 & 50.5 & 12 \\
\hline 94VC-2(20-30) & 94VC-2 & 20 & 30 & $<0.83$ & $100 \%$ & ---- & 16.2 & 0.52 & 90 & 90 & $<0.5$ & $<2$ & 0.37 & 48.0 & 12 \\
\hline 94VC-2(30-40) & 94VC-2 & 30 & 40 & $<0.83$ & $100 \%$ & ---- & 22.2 & 0.48 & 98 & 50 & $<0.5$ & $<2$ & 0.52 & 66.5 & 14 \\
\hline 94VC-2(40-50) & 94VC-2 & 40 & 50 & $<0.83$ & $100 \%$ & ---- & 21.8 & 0.43 & 102 & 40 & $<0.5$ & $<2$ & 0.53 & 79.0 & $\overline{14}$ \\
\hline 94VC-2(50-60) & 94VC-2 & 50 & 60 & $<0.83$ & $100 \%$ & ---- & 23.6 & 0.53 & 126 & 60 & 0.5 & $<2$ & 0.56 & 66.5 & 15 \\
\hline 94VC-2(60-70) & 94VC-2 & 60 & 70 & $<0.83$ & $100 \%$ & ---- & 24.0 & 0.56 & 122 & 50 & $<0.5$ & $<2$ & 0.56 & 71.5 & 15 \\
\hline 94VC-2(70-80) & 94VC-2 & 70 & 80 & $<0.83$ & $100 \%$ & ---- & 26.6 & 0.71 & 94 & 50 & 0.5 & $<2$ & 0.51 & 80.0 & 15 \\
\hline 94VC-2(80-90) & 94VC-2 & 80 & 90 & $<0.83$ & $100 \%$ & ---- & 30.4 & 0.83 & 60 & 40 & 0.5 & $<2$ & 0.52 & $>100.0$ & 14 \\
\hline 94VC-2(90-100) & 94VC-2 & 90 & 100 & $<0.83$ & $100 \%$ & ---- & 29.0 & 0.63 & 62 & 40 & $<0.5$ & $<2$ & 0.53 & $>100.0$ & 13 \\
\hline 94VC-2(100-110) & 94VC-2 & 100 & 110 & $<0.83$ & $100 \%$ & ---- & 30.4 & 0.71 & 52 & 50 & $<0.5$ & $<2$ & 0.47 & 95.0 & 13 \\
\hline 94VC-2(110-120) & 94VC-2 & 110 & 120 & $<0.83$ & $100 \%$ & ---- & 28.2 & 0.62 & 56 & 40 & 0.5 & $<2$ & 0.49 & $>100.0$ & 12 \\
\hline 94VC-2(120-130) & 94VC-2 & 120 & 130 & $<0.83$ & $100 \%$ & ---- & 40.0 & 0.82 & 70 & 50 & 0.5 & $<2$ & 0.50 & $>100.0$ & 15 \\
\hline 94VC-2(130-140) & 94VC-2 & 130 & 140 & $<0.83$ & $100 \%$ & ---- & 45.0 & 0.99 & 76 & 70 & 0.5 & $<2$ & 0.48 & 92.0 & 15 \\
\hline 94VC-2(140-150) & 94VC-2 & 140 & 150 & $<0.83$ & $100 \%$ & ---- & 29.8 & 1.09 & 44 & 140 & 0.5 & $<2$ & 0.29 & 67.5 & 11 \\
\hline 94VC-2(150-160) & 94VC-2 & 150 & 160 & $<0.83$ & $100 \%$ & $\begin{array}{ll}--- \\
\end{array}$ & 11.0 & 0.89 & 16 & 210 & 0.5 & $<2$ & 0.17 & 43.0 & 7 \\
\hline 94VC-2(160-164) & 94VC-2 & 160 & 164 & $<0.83$ & $100 \%$ & $\overline{----}$ & 19.8 & 0.92 & 30 & 100 & 0.5 & $<2$ & 0.30 & 89.0 & 9 \\
\hline 94VC-3(0-11) & 94VC-3 & 0 & 11 & $<0.83$ & $100 \%$ & $E$ & 12.4 & 0.99 & 90 & 320 & $<0.5$ & $<2$ & 0.21 & 35.0 & 8 \\
\hline 94VC-3(11-22) & 94VC-3 & 11 & 22 & $<0.83$ & $100 \%$ & $E$ & 13.0 & 0.97 & 74 & 260 & $<0.5$ & $<2$ & 0.24 & 30.5 & 7 \\
\hline 94VC-3(22-33) & 94VC-3 & 22 & 33 & $<0.83$ & $100 \%$ & $\bar{E}$ & 11.6 & 0.92 & 104 & 260 & $<0.5$ & $<2$ & 0.23 & 33.5 & 8 \\
\hline 94VC-3(33-44) & 94VC-3 & 33 & 44 & $<0.83$ & $100 \%$ & $E$ & 11.0 & 0.89 & 80 & 240 & $<0.5$ & $<2$ & 0.24 & 28.5 & 8 \\
\hline 94VC-3(44-55) & 94VC-3 & 44 & 55 & $<0.83$ & $100 \%$ & $E$ & 11.6 & 0.84 & 72 & 260 & $<0.5$ & $<2$ & 0.29 & 31.5 & 8 \\
\hline 94VC-3(55-66) & 94VC-3 & 55 & 66 & $<0.83$ & $100 \%$ & $\bar{E}$ & 12.0 & 0.90 & 76 & 290 & $<0.5$ & $<2$ & 0.32 & 31.5 & 9 \\
\hline 94VC-3(66-77) & 94VC-3 & 66 & 77 & $<0.83$ & $100 \%$ & $E$ & 14.0 & 0.86 & 72 & 300 & $<0.5$ & $<2$ & 0.34 & 32.0 & 9 \\
\hline 94VC-3(77-88) & 94VC-3 & 77 & 88 & $<0.83$ & $100 \%$ & $E$ & 13.6 & 0.80 & 76 & 280 & $<0.5$ & $<2$ & 0.32 & 30.5 & 8 \\
\hline 94VC-3(88-99) & 94VC-3 & 88 & 99 & $<0.83$ & $100 \%$ & $E$ & 12.6 & 0.81 & 70 & 260 & $<0.5$ & $<2$ & 0.31 & 28.5 & 7 \\
\hline 94VC-3(99-110) & 94VC-3 & 99 & 110 & $<0.83$ & $100 \%$ & $E$ & 14.0 & 0.76 & 96 & 270 & $<0.5$ & $<2$ & 0.32 & 33.5 & 9 \\
\hline 94VC-3(110-120) & 94VC-3 & 110 & 120 & $<0.83$ & $100 \%$ & $E$ & 15.2 & 0.62 & 122 & 200 & $<0.5$ & $<2$ & 0.34 & 33.5 & 9 \\
\hline 94VC-3(120-130) & 94VC-3 & 120 & 130 & $<0.83$ & $100 \%$ & $\bar{E}$ & 16.0 & 0.59 & 176 & 250 & $<0.5$ & $<2$ & 0.37 & 31.5 & 8 \\
\hline 94VC-3(130-140) & 94VC-3 & 130 & 140 & $<0.83$ & $100 \%$ & $E$ & 17.8 & 0.57 & 250 & 240 & $<0.5$ & $<2$ & 0.35 & 32.0 & 12 \\
\hline 94VC-3(140-150) & 94VC-3 & 140 & 150 & $<0.83$ & $100 \%$ & $E$ & 13.0 & 0.87 & 190 & 340 & $<0.5$ & $<2$ & 0.32 & 27.0 & 10 \\
\hline 94VC-3(150-160) & 94VC-3 & 150 & 160 & $<0.83$ & $100 \%$ & $\bar{E}$ & 15.0 & 0.73 & 238 & 270 & $<0.5$ & $<2$ & 0.33 & 30.0 & 11 \\
\hline 94VC-3(160-170) & 94VC-3 & 160 & 170 & $<0.83$ & $100 \%$ & $E$ & 17.8 & 0.72 & 454 & 300 & $<0.5$ & $<2$ & 0.41 & 24.0 & 17 \\
\hline 94VC-3(170-180) & 94VC-3 & 170 & 180 & $<0.83$ & $100 \%$ & $E$ & 19.8 & 0.78 & 354 & 290 & $<0.5$ & $<2$ & 0.38 & 26.5 & 19 \\
\hline
\end{tabular}

* Wt \% of size fraction: as \% by weight of bulk sample ( $\mathrm{nm}=$ not measured)

** Other analyses (Appendix \#): D =USGS-EDXRF, E =EWU, G =XRAL, ---- = none. 
Appendix F. Chemical composition of samples analyzed by ICP-AES at CHEMEX Labs in Reno, NV using nitric acid-aqua regia dissolution (Appendix_F.xls, Appendix_F.dbf).

\begin{tabular}{|c|c|c|c|c|c|c|c|c|c|c|c|c|c|c|c|c|c|c|c|}
\hline Sample No. & $\begin{array}{c}\mathrm{Cr} \\
(\mathrm{ppm})\end{array}$ & $\begin{array}{c}\mathrm{Cu} \\
(\mathrm{ppm})\end{array}$ & $\begin{array}{l}\mathrm{Fe} \\
(\%)\end{array}$ & $\begin{array}{c}\mathrm{Ga} \\
(\mathrm{ppm})\end{array}$ & $\begin{array}{c}\mathrm{Hg} \\
(\mathrm{ppm})\end{array}$ & $\begin{array}{c}\mathrm{K} \\
(\%)\end{array}$ & $\begin{array}{c}\mathrm{La} \\
(\mathrm{ppm})\end{array}$ & $\begin{array}{l}\mathrm{Mg} \\
(\%)\end{array}$ & $\begin{array}{c}\mathrm{Mn} \\
(\mathrm{ppm})\end{array}$ & $\begin{array}{c}\text { Mo } \\
(\mathrm{ppm})\end{array}$ & $\begin{array}{l}\mathrm{Na} \\
(\%)\end{array}$ & $\begin{array}{c}\mathrm{Ni} \\
(\mathrm{ppm})\end{array}$ & $\begin{array}{c}\mathrm{P} \\
(\mathrm{ppm})\end{array}$ & $\begin{array}{c}\mathrm{Pb} \\
(\mathrm{ppm})\end{array}$ & $\begin{array}{c}\mathrm{Sb} \\
(\mathrm{ppm})\end{array}$ & $\begin{array}{c}\mathrm{Sc} \\
(\mathrm{ppm})\end{array}$ & $\begin{array}{c}\mathrm{Sr} \\
(\mathrm{ppm})\end{array}$ & $\begin{array}{c}\mathrm{Ti} \\
(\%)\end{array}$ & $\begin{array}{c}\mathrm{TI} \\
(\mathrm{ppm})\end{array}$ \\
\hline SAMPLE_NO. & CR_PPM & CU_PPM & $\mathrm{FE}_{2} \%$ & GA_PPM & HG_PPM & K_\% & LA_PPM & MG_\% & MN_PPM & MO_PPM & NA_\% & NI_PPM & P_PPM & PB_PPM & SB_PPM & SC_PPM & SR_PPM & TI_\% & TL_PPM \\
\hline 94VC-2(10-20) & 102 & 160 & 13.85 & $<10$ & 4 & 0.21 & 10 & 0.62 & $>10000$ & 3 & 0.01 & 11 & 210 & 6210 & 14 & 2 & 13 & $<0.01$ & $<10$ \\
\hline 94VC-2(20-30) & 64 & 155 & 14.30 & $<10$ & 4 & 0.14 & 10 & 0.62 & $>10000$ & 4 & 0.01 & 7 & 180 & 6080 & 14 & 2 & 12 & $<0.01$ & $<10$ \\
\hline 94VC-2(30-40) & 40 & 197 & 14.90 & $<10$ & 6 & 0.13 & 10 & 0.63 & $>10000$ & 4 & $<0.01$ & 12 & 160 & 9230 & 16 & 2 & 16 & $<0.01$ & $<10$ \\
\hline 94VC-2(40-50) & 64 & 208 & $>15.00$ & $<10$ & 7 & 0.11 & 10 & 0.64 & $>10000$ & 4 & $<0.01$ & 11 & 130 & 9240 & 18 & 2 & 16 & $<0.01$ & $<10$ \\
\hline 94VC-2(50-60) & 32 & 222 & 14.50 & $<10$ & 6 & 0.14 & 10 & 0.63 & $>10000$ & 3 & $<0.01$ & 13 & 200 & $>10000$ & 24 & 3 & 18 & $<0.01$ & $<10$ \\
\hline 94VC-2(60-70) & 32 & 202 & 14.75 & $<10$ & 6 & 0.12 & 10 & 0.64 & $>10000$ & 4 & $<0.01$ & 13 & 200 & $>10000$ & 22 & 3 & 18 & $<0.01$ & $<10$ \\
\hline 94VC-2(70-80) & 56 & 220 & 13.70 & $<10$ & 7 & 0.19 & 10 & 0.60 & $>10000$ & 3 & 0.01 & 12 & 200 & $>10000$ & 20 & 3 & 17 & $<0.01$ & $<10$ \\
\hline 94VC-2(80-90) & 69 & 259 & 12.60 & $<10$ & 9 & 0.20 & 10 & 0.60 & $>10000$ & 3 & 0.01 & 13 & 200 & $>10000$ & 22 & 2 & 18 & 0.01 & $<10$ \\
\hline 94VC-2(90-100) & 107 & 264 & 13.65 & $<10$ & 10 & 0.13 & 10 & 0.60 & $>10000$ & 3 & $<0.01$ & 11 & 160 & $>10000$ & 22 & 2 & 18 & 0.01 & $<10$ \\
\hline 94VC-2(100-110) & 71 & 245 & 11.45 & $<10$ & 11 & 0.17 & 10 & 0.55 & $>10000$ & 3 & $<0.01$ & 12 & 210 & $>10000$ & 22 & 2 & 17 & 0.01 & $<10$ \\
\hline 94VC-2(110-120) & 68 & 257 & 12.05 & $<10$ & 12 & 0.17 & 10 & 0.55 & $>10000$ & 3 & 0.01 & 12 & 160 & $>10000$ & 22 & 2 & 17 & $<0.01$ & $<10$ \\
\hline 94VC-2(120-130) & 56 & 327 & 13.15 & $<10$ & 11 & 0.20 & 10 & 0.57 & $>10000$ & 4 & $<0.01$ & 13 & 230 & $>10000$ & 28 & 3 & 19 & 0.01 & $<10$ \\
\hline 94VC-2(130-140) & 95 & 333 & 11.65 & $<10$ & 8 & 0.19 & 10 & 0.55 & $>10000$ & 3 & $<0.01$ & 15 & 330 & $>10000$ & 34 & 3 & 19 & 0.01 & $<10$ \\
\hline 94VC-2(140-150) & 99 & 261 & 6.99 & $<10$ & 6 & 0.27 & 20 & 0.45 & 5990 & 2 & 0.01 & 12 & 300 & $>10000$ & 22 & 2 & 15 & 0.01 & $<10$ \\
\hline 94VC-2(150-160) & 128 & 127 & 4.53 & $<10$ & 5 & 0.26 & 20 & 0.40 & 3800 & 1 & 0.01 & 10 & 200 & 5420 & 12 & 1 & 9 & 0.01 & $<10$ \\
\hline 94VC-2(160-164) & 237 & 249 & 7.15 & $<10$ & 8 & 0.28 & 20 & 0.44 & 6460 & 2 & 0.01 & 12 & 250 & $>10000$ & 22 & 2 & 13 & 0.01 & $<10$ \\
\hline 94VC-3(0-11) & 91 & 108 & 9.36 & $<10$ & $<1$ & 0.29 & 10 & 0.44 & 7570 & $<1$ & 0.01 & 11 & 260 & 3080 & 18 & 1 & 9 & $<0.01$ & $<10$ \\
\hline 94VC-3(11-22) & 85 & 104 & 8.67 & $<10$ & $<1$ & 0.34 & 10 & 0.46 & 7290 & $<1$ & 0.01 & 10 & 250 & 3040 & 26 & 1 & 10 & $<0.01$ & $<10$ \\
\hline 94VC-3(22-33) & 100 & 100 & 7.94 & $<10$ & $<1$ & 0.28 & 10 & 0.42 & 6500 & 1 & 0.01 & 11 & 390 & 3360 & 24 & 1 & 10 & $<0.01$ & $<10$ \\
\hline 94VC-3(33-44) & 103 & 92 & 7.78 & $<10$ & $<1$ & 0.29 & 10 & 0.42 & 6580 & $<1$ & 0.01 & 11 & 230 & 3240 & 28 & 1 & 10 & $<0.01$ & $<10$ \\
\hline 94VC-3(44-55) & 69 & 95 & 8.67 & $<10$ & $<1$ & 0.29 & 10 & 0.42 & 7480 & 1 & 0.01 & 9 & 180 & 3770 & 24 & 1 & 11 & $<0.01$ & $<10$ \\
\hline 94VC-3(55-66) & 98 & 95 & 9.12 & $<10$ & $<1$ & 0.35 & 10 & 0.43 & 7920 & 2 & 0.01 & 11 & 150 & 4200 & 24 & 1 & 12 & $<0.01$ & $<10$ \\
\hline 94VC-3(66-77) & 83 & 105 & 9.26 & $<10$ & $<1$ & 0.36 & 10 & 0.45 & 8030 & $<1$ & 0.01 & 10 & 170 & 4480 & 28 & 1 & 13 & $<0.01$ & $<10$ \\
\hline 94VC-3(77-88) & 88 & 103 & 8.45 & $<10$ & $<1$ & 0.33 & 10 & 0.45 & 7480 & 1 & 0.01 & 10 & 170 & 4350 & 28 & 1 & 12 & $<0.01$ & $<10$ \\
\hline 94VC-3(88-99) & 85 & 101 & 7.66 & $<10$ & $<1$ & 0.35 & 10 & 0.44 & 6900 & 1 & 0.01 & 9 & 160 & 4080 & 24 & 1 & 12 & $<0.01$ & $<10$ \\
\hline 94VC-3(99-110) & 61 & 100 & 9.34 & $<10$ & $<1$ & 0.28 & 10 & 0.45 & 8300 & $<1$ & 0.01 & 11 & 170 & 4300 & 24 & 1 & 12 & $<0.01$ & $<10$ \\
\hline 94VC-3(110-120) & 66 & 100 & 9.56 & $<10$ & $<1$ & 0.24 & $<10$ & 0.46 & 8540 & 1 & 0.01 & 11 & 170 & 4480 & 30 & 1 & 12 & $<0.01$ & $<10$ \\
\hline 94VC-3(120-130) & 64 & 112 & 9.78 & $<10$ & $<1$ & 0.26 & $<10$ & 0.49 & 8630 & 1 & 0.01 & 10 & 170 & 4680 & 26 & 1 & 14 & $<0.01$ & $<10$ \\
\hline 94VC-3(130-140) & 79 & 121 & 9.85 & $<10$ & $<1$ & 0.21 & $<10$ & 0.48 & 8830 & 2 & $<0.01$ & 12 & 200 & 5260 & 28 & 1 & 13 & $<0.01$ & $<10$ \\
\hline 94VC-3(140-150) & 80 & 88 & 9.50 & $<10$ & $<1$ & 0.30 & 10 & 0.45 & 8200 & 2 & 0.01 & 11 & 200 & 3890 & 26 & 1 & 13 & $<0.01$ & $<10$ \\
\hline 94VC-3(150-160) & 96 & 101 & 9.63 & $<10$ & $<1$ & 0.25 & 10 & 0.46 & 8270 & $<1$ & 0.01 & 12 & 210 & 4350 & 28 & 1 & 13 & $<0.01$ & $<10$ \\
\hline 94VC-3(160-170) & 55 & 95 & 12.20 & $<10$ & $<1$ & 0.25 & 10 & 0.51 & $>10000$ & 2 & 0.01 & 16 & 250 & 4420 & 34 & 2 & 16 & $<0.01$ & $<10$ \\
\hline 94VC-3(170-180) & 50 & 118 & 11.80 & $<10$ & $<1$ & 0.28 & 10 & 0.50 & 9510 & 1 & 0.01 & 15 & 260 & 4520 & 34 & 2 & 15 & $<0.01$ & $<10$ \\
\hline
\end{tabular}

* Wt \% of size fraction: as \% by weight of bulk sample ( $\mathrm{nm}=$ not measured)

** Other analyses (Appendix \#): D =USGS-EDXRF, E =EWU, G =XRAL, ---- = none. 
Appendix F. Chemical composition of samples analyzed by ICP-AES at CHEMEX Labs in Reno, NV using nitric acid-aqua regia dissolution (Appendix_F.Xls, Appendix_F.dbf).

\begin{tabular}{|l|r|r|r|r|}
\hline Sample No. & $\begin{array}{c}\text { U } \\
(\mathrm{ppm})\end{array}$ & $\begin{array}{c}\mathrm{V} \\
(\mathrm{ppm})\end{array}$ & $\begin{array}{c}\mathrm{W} \\
(\mathrm{ppm})\end{array}$ & $\begin{array}{c}\text { Zn } \\
(\mathrm{ppm})\end{array}$ \\
\hline SAMPLE_No. & U_PPM & V_PPM & W_PPM & ZN_PPM \\
\hline 94VC-2(10-20) & $<10$ & 2 & $<10$ & 8370 \\
\hline 94VC-2(20-30) & $<10$ & $<1$ & $<10$ & 8100 \\
\hline 94VC-2(30-40) & $<10$ & $<1$ & $<10$ & $>10000$ \\
\hline 94VC-2(40-50) & $<10$ & $<1$ & $<10$ & $>10000$ \\
\hline 94VC-2(50-60) & $<10$ & $<1$ & $<10$ & $>10000$ \\
\hline 94VC-2(60-70) & $<10$ & $<1$ & $<10$ & $>10000$ \\
\hline 94VC-2(70-80) & $<10$ & 1 & $<10$ & $>10000$ \\
\hline 94VC-2(80-90) & $<10$ & 4 & $<10$ & $>10000$ \\
\hline 94VC-2(90-100) & $<10$ & 3 & $<10$ & $>10000$ \\
\hline 94VC-2(100-110) & $<10$ & 3 & $<10$ & $>10000$ \\
\hline 94VC-2(110-120) & $<10$ & 1 & $<10$ & $>10000$ \\
\hline 94VC-2(120-130) & $<10$ & 4 & $<10$ & $>10000$ \\
\hline 94VC-2(130-140) & $<10$ & 6 & $<10$ & $>10000$ \\
\hline 94VC-2(140-150) & $<10$ & 8 & $<10$ & $>10000$ \\
\hline 94VC-2(150-160) & $<10$ & 6 & $<10$ & 6600 \\
\hline 94VC-2(160-164) & $<10$ & 7 & $<10$ & $>10000$ \\
\hline 94VC-3(0-11) & $<10$ & 14 & $<10$ & 4950 \\
\hline 94VC-3(11-22) & $<10$ & 13 & $<10$ & 4920 \\
\hline 94VC-3(22-33) & $<10$ & 13 & $<10$ & 4510 \\
\hline 94VC-3(33-44) & $<10$ & 12 & $<10$ & 4390 \\
\hline 94VC-3(44-55) & $<10$ & 12 & $<10$ & 4660 \\
\hline 94VC-3(55-66) & $<10$ & 12 & $<10$ & 4820 \\
\hline 94VC-3(66-77) & $<10$ & 12 & $<10$ & 5170 \\
\hline 94VC-3(77-88) & $<10$ & 11 & $<10$ & 4960 \\
\hline 94VC-3(88-99) & $<10$ & 11 & $<10$ & 4730 \\
\hline 94VC-3(99-110) & $<10$ & 11 & $<10$ & 5080 \\
\hline 94VC-3(110-120) & $<10$ & 10 & $<10$ & 5060 \\
\hline 94VC-3(120-130) & $<10$ & 10 & $<10$ & 5030 \\
\hline 94VC-3(130-140) & $<10$ & 10 & $<10$ & 4440 \\
\hline 94VC-3(140-150) & $<10$ & 12 & $<10$ & 4050 \\
\hline 94VC-3(150-160) & $<10$ & 11 & $<10$ & 4310 \\
\hline 94VC-3(160-170) & $<10$ & 11 & $<10$ & 3400 \\
\hline 94VC-3(170-180) & $<10$ & 13 & $<10$ & 4000 \\
\hline
\end{tabular}

* Wt \% of size fraction: as \% by weight of bulk sample ( $\mathrm{nm}=$ not measured)

** Other analyses (Appendix \#): D =USGS-EDXRF, E =EWU, G =XRAL, ---- = none 
Appendix F. Chemical composition of samples analyzed by ICP-AES at CHEMEX Labs in Reno, NV using nitric acid-aqua regia dissolution (Appendix_F.Xls, Appendix_F.dbf).

\begin{tabular}{|c|c|c|c|c|c|c|c|c|c|c|c|c|c|c|c|}
\hline Sample No. & Site ID & $\begin{array}{c}\text { Depth } \\
\text { interval, } \\
\text { top }(\mathrm{cm})\end{array}$ & $\begin{array}{c}\text { Depth } \\
\text { interval, } \\
\text { bottom }(\mathrm{cm})\end{array}$ & $\begin{array}{c}\text { Size } \\
\text { fraction } \\
(\mathrm{mm})\end{array}$ & $\begin{array}{c}\text { *wt } \% \text { of } \\
\text { size } \\
\text { fraction }\end{array}$ & $\begin{array}{c}{ }^{* *} \text { Other } \\
\text { analyses } \\
(\text { Appendix \#) }\end{array}$ & $\begin{array}{c}\mathrm{Ag} \\
(\mathrm{ppm})\end{array}$ & $\begin{array}{c}\mathrm{Al} \\
(\%)\end{array}$ & $\begin{array}{c}\text { As } \\
\text { (ppm) }\end{array}$ & $\begin{array}{c}\mathrm{Ba} \\
(\mathrm{ppm})\end{array}$ & $\begin{array}{c}\mathrm{Be} \\
(\mathrm{ppm})\end{array}$ & $\begin{array}{c}\mathrm{Bi} \\
(\mathrm{ppm})\end{array}$ & $\begin{array}{l}\mathrm{Ca} \\
(\%)\end{array}$ & $\begin{array}{c}\mathrm{Cd} \\
(\mathrm{ppm})\end{array}$ & $\begin{array}{c}\text { Co } \\
\text { (ppm) }\end{array}$ \\
\hline SAMPLE_NO. & SITE_ID & INT_TOP_CM & INT_BTM_CM & SIZFRAC_MM & WT\%SIZFRAC & OTHR_ANLYS & AG_PPM & AL_\% & AS_PPM & BA_PPM & BE_PPM & BI_PPM & CA_\% & CD_PPM & CO_PPM \\
\hline 94VC-3(180-190) & 94VC-3 & 180 & 190 & $<0.83$ & $100 \%$ & $\bar{E}$ & 19.0 & 0.83 & 112 & 330 & $<0.5$ & $<2$ & 0.40 & 38.0 & 11 \\
\hline 94VC-3(190-200) & 94VC-3 & 190 & 200 & $<0.83$ & $100 \%$ & $E$ & 16.4 & 0.77 & 90 & 340 & $<0.5$ & $<2$ & 0.38 & 38.0 & 9 \\
\hline 94VC-3(200-210) & 94VC-3 & 200 & 210 & $<0.83$ & $100 \%$ & $\mathrm{E}$ & 18.4 & 0.60 & 100 & 210 & $<0.5$ & $<2$ & 0.39 & 46.0 & 10 \\
\hline 94VC-3(210-220) & 94VC-3 & 210 & 220 & $<0.83$ & $100 \%$ & $\mathrm{E}$ & 14.6 & 0.67 & 84 & 240 & $<0.5$ & $<2$ & 0.34 & 37.5 & 9 \\
\hline 94VC-3(220-230) & 94VC-3 & 220 & 230 & $<0.83$ & $100 \%$ & $E$ & 14.4 & 0.71 & 88 & 230 & $<0.5$ & $<2$ & 0.33 & 36.0 & 8 \\
\hline
\end{tabular}


Appendix F. Chemical composition of samples analyzed by ICP-AES at CHEMEX Labs in Reno, NV using nitric acid-aqua regia dissolution (Appendix F.xls, Appendix F.dbf).

\begin{tabular}{|c|c|c|c|c|c|c|c|c|c|c|c|c|c|c|c|c|c|c|c|}
\hline Sample No. & $\begin{array}{c}\mathrm{Cr} \\
(\mathrm{ppm})\end{array}$ & $\begin{array}{c}\mathrm{Cu} \\
(\mathrm{ppm})\end{array}$ & $\begin{array}{l}\mathrm{Fe} \\
(\%)\end{array}$ & $\begin{array}{c}\mathrm{Ga} \\
(\mathrm{ppm})\end{array}$ & $\begin{array}{c}\mathrm{Hg} \\
(\mathrm{ppm})\end{array}$ & $\begin{array}{c}\mathrm{K} \\
(\%)\end{array}$ & $\begin{array}{c}\mathrm{La} \\
(\mathrm{ppm})\end{array}$ & $\begin{array}{l}\mathrm{Mg} \\
(\%)\end{array}$ & $\begin{array}{c}\mathrm{Mn} \\
(\mathrm{ppm})\end{array}$ & $\begin{array}{c}\text { Mo } \\
\text { (ppm) }\end{array}$ & $\begin{array}{l}\mathrm{Na} \\
(\%)\end{array}$ & $\begin{array}{c}\mathrm{Ni} \\
(\mathrm{ppm})\end{array}$ & $\begin{array}{c}P \\
(p p m)\end{array}$ & $\begin{array}{c}\mathrm{Pb} \\
(\mathrm{ppm})\end{array}$ & $\begin{array}{c}\mathrm{Sb} \\
\text { (ppm) }\end{array}$ & $\begin{array}{c}\mathrm{Sc} \\
(\mathrm{ppm})\end{array}$ & $\begin{array}{c}\mathrm{Sr} \\
(\mathrm{ppm})\end{array}$ & $\begin{array}{c}\mathrm{Ti} \\
(\%)\end{array}$ & $\begin{array}{c}\mathrm{TI} \\
(\mathrm{ppm})\end{array}$ \\
\hline SAMPLE_NO. & CR_PPM & CU_PPM & FE_\% & GA_PPM & HG_PPM & K_\% & LA_PPM & MG_\% & MN_PPM & MO_PPM & NA_\% & NI_PPM & P_PPM & PB_PPM & SB_PPM & SC_PPM & SR_PPM & TI_\% & TL_PPM \\
\hline 94VC-3(180-190) & 64 & 126 & 10.95 & $<10$ & $<1$ & 0.29 & 10 & 0.50 & 9300 & 1 & 0.01 & 11 & 200 & 5490 & 28 & $\overline{1}$ & 13 & $<0.01$ & $<10$ \\
\hline 94VC-3(190-200) & 64 & 121 & 9.69 & $<10$ & $<1$ & 0.32 & 10 & 0.50 & 8530 & 1 & 0.01 & 11 & 170 & 5230 & 28 & 1 & 14 & $<0.01$ & $<10$ \\
\hline 94VC-3(200-210) & 73 & 140 & 10.35 & $<10$ & $<1$ & 0.25 & $<10$ & 0.52 & 9330 & $<1$ & 0.01 & 10 & 170 & 5750 & 36 & 1 & 13 & $<0.01$ & $<10$ \\
\hline 94VC-3(210-220) & 99 & 122 & 8.38 & $<10$ & $<1$ & 0.28 & 10 & 0.47 & 7350 & 1 & 0.01 & 11 & 180 & 5450 & 28 & 1 & 13 & $<0.01$ & $<10$ \\
\hline 94VC-3(220-230) & 94 & 114 & 8.69 & $<10$ & $<1$ & 0.28 & 10 & 0.47 & 7660 & 1 & 0.01 & 10 & 180 & 4890 & 30 & 1 & 12 & $<0.01$ & $<10$ \\
\hline
\end{tabular}


Appendix F. Chemical composition of samples analyzed by ICP-AES at CHEMEX Labs in Reno, NV using nitric acid-aqua regia dissolution (Appendix_F.xls, Appendix_F.dbf).

\begin{tabular}{|c|r|r|r|c|}
\hline Sample No. & $\begin{array}{c}\text { U } \\
(\mathrm{ppm})\end{array}$ & $\begin{array}{c}\text { V } \\
(\mathrm{ppm})\end{array}$ & $\begin{array}{c}\mathrm{W} \\
(\mathrm{ppm})\end{array}$ & $\begin{array}{c}\text { Zn } \\
(\mathrm{ppm})\end{array}$ \\
\hline SAMPLE_No. & U_PPM & V_PPM & w_PPM & ZN_PPM \\
\hline 94VC-3(180-190) & $<10$ & 13 & $<10$ & 6010 \\
\hline 94VC-3(190-200) & $<10$ & 12 & $<10$ & 5790 \\
\hline 94VC-3(200-210) & $<10$ & 11 & $<10$ & 6890 \\
\hline 94VC-3(210-220) & $<10$ & 11 & $<10$ & 5630 \\
\hline 94VC-3(220-230) & $<10$ & 11 & $<10$ & 5430 \\
\hline
\end{tabular}


Appendix G. Chemical composition of samples analyzed by ICP-AES at XRAL Labs in Golden, CO using 4 acid dissolution (Appendix_G.xls, Appendix_G.dbf).

\begin{tabular}{|c|c|c|c|c|c|c|c|c|c|c|c|c|c|c|c|c|c|}
\hline Sample No. & Site ID & $\begin{array}{c}\text { Depth } \\
\text { interval, } \\
\text { top }(\mathrm{cm})\end{array}$ & $\begin{array}{c}\text { Depth } \\
\text { interval, } \\
\text { bottom }(\mathrm{cm})\end{array}$ & $\begin{array}{c}{ }^{*} \text { Other } \\
\text { Analyses } \\
\text { (appendix \#) }\end{array}$ & $\begin{array}{c}\mathrm{Ag} \\
(\mathrm{ppm})\end{array}$ & $\begin{array}{c}\mathrm{Al} \\
(\%)\end{array}$ & $\begin{array}{c}\text { As } \\
\text { (ppm) }\end{array}$ & $\mid \begin{array}{c}\mathrm{Au} \\
(\mathrm{ppm})\end{array}$ & $\begin{array}{c}\mathrm{Ba} \\
(\mathrm{ppm})\end{array}$ & $\begin{array}{c}\mathrm{Be} \\
(\mathrm{ppm})\end{array}$ & $\begin{array}{c}\mathrm{Bi} \\
(\mathrm{ppm})\end{array}$ & $\begin{array}{l}\mathrm{Ca} \\
(\%)\end{array}$ & $\begin{array}{c}\mathrm{Cd} \\
(\mathrm{ppm})\end{array}$ & $\begin{array}{c}\mathrm{Ce} \\
(\mathrm{ppm})\end{array}$ & $\begin{array}{c}\text { Co } \\
(\mathrm{ppm})\end{array}$ & $\begin{array}{c}\mathrm{Cr} \\
(\mathrm{ppm})\end{array}$ & $\begin{array}{c}\mathrm{Cu} \\
(\mathrm{ppm})\end{array}$ \\
\hline SAMPLE_NO. & SITE_ID & INT_TOP_CM & INT_BTM_CM & OTHR_ANLYS & AG_PPM & AL_(\%) & AS_PPM & AU_PPM & BA_PPM & BE_PPM & BI_PPM & CA_(\%) & CD_PPM & CE_PPM & CO_PPM & CR_PPM & CU_PPM \\
\hline T98C-01(0-4) & T98C-01 & 0 & 4 & $\begin{array}{ll}--- \\
--\end{array}$ & $<2$ & 4.7 & 18 & $<8$ & 408 & 1 & $<50$ & 0.818 & 36 & 51 & 9 & 80 & 46 \\
\hline T98C-01(18-22) & T98C-01 & 18 & 22 & ---- & $<2$ & 6.2 & 11 & $<8$ & 576 & 2 & $<50$ & 0.235 & 6 & 84 & 9 & 102 & 22 \\
\hline T98C-01(38-42) & T98C-01 & 38 & 42 & ---- & $<2$ & 6.1 & 22 & $<8$ & 549 & 2 & $<50$ & 0.219 & 7 & 89 & 8 & 87 & 27 \\
\hline T98C-01(58-62) & T98C-01 & 58 & 62 & --- & $<2$ & 7.4 & 14 & $<8$ & 621 & 2 & $<50$ & 0.481 & $<2$ & 108 & 10 & 64 & 32 \\
\hline T98C-05(0-4) & T98C-05 & 0 & 4 & ---- & $<2$ & 6.1 & 55 & $<8$ & 579 & 2 & $<50$ & 0.593 & 4 & 112 & 16 & 94 & 223 \\
\hline T98C-05(18-22) & T98C-05 & 18 & 22 & ---- & $<2$ & 6.3 & 33 & $<8$ & 569 & 2 & $<50$ & 0.454 & $<2$ & 122 & 8 & 88 & 210 \\
\hline T98C-05(38-42) & T98C-05 & 38 & 42 & ---- & $<2$ & 5.5 & 24 & $<8$ & 484 & 2 & $<50$ & 0.516 & $<2$ & 122 & 5 & 71 & 184 \\
\hline T98C-05(58-62) & T98C-05 & 58 & 62 & ---- & $<2$ & 6.1 & 71 & $<8$ & 549 & 2 & $<50$ & 0.563 & $<2$ & 142 & 13 & 75 & 514 \\
\hline T98C-05(118-122) & T98C-05 & 118 & 122 & ---- & $<2$ & 8.1 & 16 & $<8$ & 593 & 2 & $<50$ & 0.390 & $<2$ & \begin{tabular}{|c|}
104 \\
\end{tabular} & 8 & 92 & 42 \\
\hline T98C-06(0-4) & T98C-06 & 0 & 4 & ---- & 4 & 5.2 & 33 & $<8$ & 478 & 1 & $<50$ & 1.299 & 10 & 54 & 9 & 81 & 51 \\
\hline T98C-06(0-4) & T98C-06 & 0 & 4 & ---- & 5 & 5.1 & 34 & $<8$ & 530 & 1 & $<50$ & 1.048 & 12 & 69 & 9 & 81 & 65 \\
\hline T98C-06(18-22) & T98C-06 & 18 & 22 & $\begin{array}{ll}--- \\
--\end{array}$ & $<2$ & 5.9 & 13 & $<8$ & 602 & 2 & $<50$ & 0.246 & 21 & 87 & 8 & 85 & 24 \\
\hline T98C-06(38-42) & T98C-06 & 38 & 42 & $\begin{array}{ll}--- \\
--\end{array}$ & $<2$ & 6.2 & 11 & $<8$ & 627 & 2 & $<50$ & 0.251 & $<2$ & 84 & 8 & 89 & 29 \\
\hline T98C-06(58-62) & T98C-06 & 58 & 62 & ---- & $<2$ & 6.3 & 11 & $<8$ & 656 & 2 & $<50$ & 0.262 & $<2$ & 94 & 8 & 99 & 15 \\
\hline T98C-06(78-82) & T98C-06 & $\overline{78}$ & 82 & ---- & $<2$ & 5.9 & 13 & $<8$ & 483 & 2 & $<50$ & 0.246 & $<2$ & 86 & 7 & 84 & 13 \\
\hline T98C-06(98-102) & T98C-06 & 98 & 102 & ---- & $<2$ & 5.5 & 11 & $<8$ & 511 & 2 & $<50$ & 0.192 & $<2$ & 103 & 6 & 40 & 15 \\
\hline T98C-06(133-137) & T98C-06 & 133 & 137 & ---- & $<2$ & 5.4 & $<10$ & $<8$ & 484 & 2 & $<50$ & 0.181 & $<2$ & 78 & 5 & 81 & 10 \\
\hline T98C-06(153-157) & T98C-06 & 153 & 157 & ---- & $<2$ & 5.4 & $<10$ & $<8$ & 479 & 2 & $<50$ & 0.203 & $<2$ & 89 & 6 & 42 & 12 \\
\hline T98C-06(173-177) & T98C-06 & 173 & 177 & ---- & $<2$ & & 13 & $<8$ & 458 & 2 & $<50$ & 0.192 & $<2$ & 80 & 6 & 38 & 13 \\
\hline T98C-06(198-202) & T98C-06 & 198 & 202 & ---- & $<2$ & 5.6 & 13 & $<8$ & 480 & 2 & $<50$ & 0.197 & $<2$ & 86 & 6 & 90 & 13 \\
\hline T98C-07(0-4) & T98C-07 & 0 & 4 & ---- & 14 & 5.3 & 60 & $<8$ & 520 & 2 & $<50$ & 1.000 & 24 & 50 & 9 & 75 & 85 \\
\hline T98C-08(0-4) & T98C-08 & 0 & 4 & ---- & 17 & 5.5 & 118 & $<8$ & 698 & 2 & $<50$ & 0.540 & 9 & 64 & 14 & 84 & 147 \\
\hline T98C-10(21-25) & T98C-10 & 21 & 25 & ---- & 8 & 6.6 & 65 & $<8$ & 608 & 2 & $<50$ & 0.952 & 16 & 72 & 14 & 81 & 118 \\
\hline T98C-11B(0-14) & T98C-11B & 0 & 14 & ---- & \begin{tabular}{c|c|}
13 \\
\end{tabular} & 5.0 & 225 & $<8$ & 539 & 2 & $<50$ & 0.336 & 42 & 65 & 21 & 74 & 115 \\
\hline T98C-11B(14-34) & T98C-11B & 14 & 34 & ---- & \begin{tabular}{l|l}
16 \\
\end{tabular} & 3.8 & 435 & $<8$ & 536 & 1 & $<50$ & 0.347 & 36 & 62 & 29 & \begin{tabular}{l|l}
66 \\
\end{tabular} & 123 \\
\hline T98C-11B(34-55) & T98C-11B & 34 & 55 & ---- & 16 & 3.9 & 232 & $<8$ & 586 & 1 & $<50$ & 0.508 & 26 & 71 & 21 & 73 & 130 \\
\hline T98C-11B(55-71) & T98C-11B & 55 & 71 & ---- & 14 & 4.0 & 111 & $<8$ & 706 & 1 & $<50$ & 0.497 & 22 & 70 & 16 & 56 & 127 \\
\hline T98C-11B(71-92) & T98C-11B & 71 & 92 & $-\cdots$ & 17 & 3.3 & 78 & $<8$ & 634 & 1 & $<50$ & 0.347 & 34 & 51 & 14 & 73 & 126 \\
\hline T98C-11B(92-111) & T98C-11B & 92 & 111 & $\begin{array}{ll}--- \\
\end{array}$ & 13 & 3.5 & 89 & $<8$ & 598 & 1 & $<50$ & 0.363 & 29 & 53 & 15 & 73 & 129 \\
\hline T98C-12(6-30) & T98C-12 & 6 & 30 & ---- & 13 & 4.9 & 308 & $<8$ & 622 & 2 & $<50$ & 0.208 & 22 & 85 & 22 & 85 & 136 \\
\hline T98R-13(0-4) & T98R-13 & 0 & 4 & ---- & 10 & 4.2 & 66 & $<8$ & 560 & 1 & $<50$ & 1.016 & 87 & 37 & 290 & 73 & 85 \\
\hline T98R-13(18-22) & T98R-13 & 18 & 22 & ---- & 15 & 3.9 & 161 & $<8$ & 617 & 1 & $<50$ & 0.181 & 12 & 57 & 11 & 84 & 158 \\
\hline T98R-13(38-42) & T98R-13 & 38 & 42 & ---- & $<2$ & 7.4 & 10 & $<8$ & 714 & 2 & $<50$ & 0.443 & 18 & 76 & 20 & 80 & 10 \\
\hline T98R-14(0-4) & T98R-14 & 0 & 4 & ---- & 8 & 5.4 & 170 & $<8$ & 354 & 1 & $<50$ & 1.497 & 32 & 47 & 56 & 67 & 77 \\
\hline T98R-14(18-22) & T98R-14 & 18 & 22 & $-\cdots$ & 13 & 2.9 & 274 & $<8$ & 365 & $<1$ & $<50$ & 0.133 & 4 & 55 & 8 & 45 & 79 \\
\hline
\end{tabular}

*Other analyses (Appendix \#): D =USGS-EDXRF, E =EWU,

$\mathrm{F}=\mathrm{CHEMEX}, \mathrm{H}=\mathrm{ACZ},-\cdots=$ none. 
Appendix G. Chemical composition of samples analyzed by ICP-AES at XRAL Labs in Golden, CO using 4 acid dissolution (Appendix_G.xls, Appendix_G.dbf).

\begin{tabular}{|c|c|c|c|c|c|c|c|c|c|c|c|c|c|c|c|c|c|c|c|c|}
\hline Sample No. & $\begin{array}{c}\text { Eu } \\
(p p m)\end{array}$ & $\begin{array}{l}\mathrm{Fe} \\
(\%)\end{array}$ & $\begin{array}{c}\mathrm{Ga} \\
(\mathrm{ppm})\end{array}$ & $\begin{array}{c}\text { Ho } \\
(\mathrm{ppm})\end{array}$ & $\begin{array}{c}K \\
(\%)\end{array}$ & $\begin{array}{c}\mathrm{La} \\
(\mathrm{ppm})\end{array}$ & $\begin{array}{c}\mathrm{Li} \\
(\mathrm{ppm})\end{array}$ & $\begin{array}{l}\mathrm{Mg} \\
(\%)\end{array}$ & $\begin{array}{c}\mathrm{Mn} \\
(\mathrm{ppm})\end{array}$ & $\begin{array}{c}\text { Mo } \\
\text { (ppm) }\end{array}$ & $\begin{array}{l}\mathrm{Na} \\
(\%)\end{array}$ & $\begin{array}{c}\mathrm{Nb} \\
(\mathrm{ppm})\end{array}$ & $\begin{array}{c}\mathrm{Nd} \\
(\mathrm{ppm})\end{array}$ & $\begin{array}{c}\mathrm{Ni} \\
(\mathrm{ppm})\end{array}$ & $\begin{array}{c}P \\
(p p m)\end{array}$ & $\begin{array}{c}\mathrm{Pb} \\
(\mathrm{ppm})\end{array}$ & $\begin{array}{c}\text { Sc } \\
(\mathrm{ppm})\end{array}$ & $\begin{array}{c}\text { Sn } \\
\text { (ppm) }\end{array}$ & $\begin{array}{c}\mathrm{Sr} \\
(\mathrm{ppm})\end{array}$ & $\begin{array}{c}\mathrm{Ta} \\
\text { (ppm) }\end{array}$ \\
\hline SAMPLE_NO. & PPM & FE__\%) & GA_PPM & HO_PPM & K_(\%) & LA_PPM & LI_PPM & MG_(\%) & MN_PPM & MO_PPM & NA_(\%) & NB_PPM & ND_PPM & NI_PPM & P_PPM & PB_PPM & SC_PPM & SN_PPM & SR_PPM & TA_PPM \\
\hline T98C-01(0-4) & $<2$ & 2.53 & 20 & $<4$ & 1.35 & 27 & 25 & 0.525 & 1000 & 4 & 0.860 & 7 & 27 & 15 & 0.125 & 712 & 7 & $<50$ & 108 & $<40$ \\
\hline T98C-01(18-22) & $<2$ & 2.73 & 18 & $<4$ & 2.17 & 43 & 42 & 0.655 & 768 & 4 & 0.615 & 9 & 42 & 18 & 0.055 & 385 & 9 & $<50$ & 56 & $<40$ \\
\hline T98C-01(38-42) & $<2$ & 3.09 & 19 & $<4$ & 2.14 & 43 & 37 & 0.600 & 1120 & 4 & 0.590 & 12 & 41 & 18 & 0.050 & 815 & $\overline{9}$ & $<50$ & 54 & $<40$ \\
\hline T98C-01(58-62) & 2 & 2.68 & 24 & $<4$ & 1.80 & 66 & 42 & 0.545 & 299 & 5 & 0.760 & 12 & 63 & 25 & 0.040 & 259 & 13 & $<50$ & 115 & $<40$ \\
\hline T98C-05(0-4) & $<2$ & 3.20 & 18 & $<4$ & 1.88 & 52 & 35 & 0.485 & 2100 & 4 & 0.670 & \begin{tabular}{c|c|}
13 \\
\end{tabular} & 52 & 21 & 0.065 & 2710 & 12 & $<50$ & 107 & $<40$ \\
\hline T98C-05(18-22) & 3 & 2.40 & 17 & $<4$ & 1.90 & 61 & 36 & 0.470 & 287 & 3 & 0.675 & 11 & 60 & 17 & 0.045 & 2780 & 12 & $<50$ & 99 & $<40$ \\
\hline T98C-05(38-42) & 4 & 2.26 & 16 & $<4$ & 1.69 & 51 & 24 & 0.411 & 281 & $<2$ & 0.716 & $<4$ & 65 & 10 & 0.042 & 2680 & 9 & $<50$ & 111 & $<40$ \\
\hline T98C-05(58-62) & 3 & 2.40 & 19 & 5 & 1.76 & 57 & 30 & 0.451 & 896 & 3 & 0.811 & 8 & 85 & 12 & 0.042 & 4420 & 12 & $<50$ & 120 & $<40$ \\
\hline T98C-05(118-122) & 3 & 2.45 & 24 & $<4$ & 1.94 & 58 & 52 & 0.595 & 334 & 5 & 0.545 & 5 & 54 & 29 & 0.050 & 35 & 14 & $<50$ & 69 & $<40$ \\
\hline T98C-06(0-4) & $<2$ & 3.81 & 15 & $<4$ & 1.46 & 25 & 24 & 0.560 & 2250 & 5 & 1.155 & 10 & 30 & 13 & 0.080 & 1890 & 7 & $<50$ & 146 & $<40$ \\
\hline T98C-06(0-4) & $<2$ & 4.43 & 11 & $<4$ & 1.56 & 37 & 26 & 0.550 & 2910 & 5 & 0.970 & 13 & 32 & 15 & 0.085 & 2500 & 7 & $<50$ & 123 & $<40$ \\
\hline T98C-06(18-22) & $<2$ & 2.72 & 19 & $<4$ & 2.20 & 46 & 39 & 0.630 & 867 & 4 & 0.575 & 7 & 40 & 16 & 0.055 & 552 & 9 & $<50$ & 54 & $<40$ \\
\hline T98C-06(38-42) & $<2$ & 2.51 & 21 & $<4$ & 2.21 & 43 & 40 & 0.685 & 578 & 3 & 0.595 & 6 & 39 & 18 & 0.050 & 87 & 10 & $<50$ & 57 & $<40$ \\
\hline T98C-06(58-62) & $<2$ & 2.53 & 19 & $<4$ & 2.28 & 48 & 44 & 0.710 & 470 & 3 & 0.615 & 7 & 44 & 18 & 0.035 & 23 & 11 & $<50$ & 58 & $<40$ \\
\hline T98C-06(78-82) & $<2$ & 2.33 & 14 & $<4$ & 2.26 & 46 & 37 & 0.585 & 296 & 3 & 0.570 & 6 & 41 & 15 & 0.030 & 60 & 8 & $<50$ & 51 & $<40$ \\
\hline T98C-06(98-102) & $<2$ & 2.13 & 23 & $<4$ & 2.20 & 50 & 39 & 0.630 & 260 & 4 & 0.490 & 5 & 48 & 16 & 0.030 & 27 & 9 & $<50$ & 43 & $<40$ \\
\hline T98C-06(133-137) & $<2$ & 2.05 & 16 & $<4$ & 2.23 & 43 & 36 & 0.605 & 211 & 3 & 0.485 & 5 & 39 & 14 & 0.025 & 26 & 8 & $<50$ & 44 & $<40$ \\
\hline T98C-06(153-157) & $<2$ & 2.05 & 17 & $<4$ & 2.13 & 44 & 35 & 0.610 & 210 & 3 & 0.510 & 8 & 37 & 15 & 0.025 & 10 & 8 & $<50$ & 47 & $<40$ \\
\hline T98C-06(173-177) & $<2$ & 2.10 & 19 & $<4$ & 2.16 & 41 & 35 & 0.585 & 227 & 3 & 0.505 & 17 & 38 & 15 & 0.025 & 19 & 8 & $<50$ & 46 & $<40$ \\
\hline T98C-06(198-202) & $<2$ & 2.16 & 18 & $<4$ & 2.22 & 44 & 37 & 0.620 & 192 & 4 & 0.520 & 8 & 39 & 18 & 0.030 & 11 & $\overline{9}$ & $<50$ & 46 & $<40$ \\
\hline T98C-071 & $<2$ & 4.00 & $<4$ & $<4$ & 1.55 & 24 & 28 & 0.555 & 1650 & 4 & 1.030 & 11 & 21 & 18 & 0.125 & 4300 & 8 & $<50$ & 130 & $<40$ \\
\hline T98C-08 & 3 & 8.99 & 11 & $<4$ & 1.83 & 33 & 30 & 0.510 & 7250 & 7 & 0.770 & 8 & 33 & 15 & 0.050 & 6160 & 8 & $<50$ & 89 & $<40$ \\
\hline T98C-10 & $<2$ & 4.49 & 14 & $<4$ & 1.89 & 36 & 34 & 0.605 & 2140 & 5 & 1.300 & 13 & 33 & 18 & 0.105 & 2900 & 8 & $<50$ & 143 & $<40$ \\
\hline T98C-11 & $<2$ & 8.25 & 16 & $<4$ & 1.79 & 34 & 29 & 0.565 & 6030 & 9 & 0.455 & 5 & 31 & 23 & 0.065 & 3510 & 8 & $<50$ & 47 & $<40$ \\
\hline T98C-11 & 3 & 10.30 & $<4$ & $<4$ & 1.42 & 25 & 22 & 0.580 & 9160 & 8 & 0.290 & 4 & 23 & 23 & 0.035 & 3890 & 6 & $<50$ & 34 & $<40$ \\
\hline T98C-11 & 3 & 9.44 & $<4$ & $<4$ & 1.49 & 30 & 22 & 0.585 & 8760 & 7 & 0.280 & 6 & 27 & 18 & 0.030 & 5350 & 6 & $<50$ & 38 & $<40$ \\
\hline T98C-11 & 3 & 9.30 & $<4$ & $<4$ & 1.55 & 32 & 23 & 0.595 & 8420 & 9 & 0.315 & 8 & 31 & 18 & 0.035 & 6140 & 6 & $<50$ & 41 & $<40$ \\
\hline T98C-11E & 3 & 9.14 & 8 & $<4$ & 1.41 & 26 & 21 & 0.535 & 8630 & 9 & 0.205 & 5 & 26 & 14 & 0.025 & 7290 & 5 & $<50$ & 32 & $<40$ \\
\hline T98C-11B(92-111) & 3 & 9.21 & 7 & $<4$ & 1.47 & 27 & 21 & 0.515 & 8350 & 7 & 0.230 & 5 & 24 & 15 & 0.025 & 6580 & 5 & $<50$ & 34 & $<40$ \\
\hline T98C-12(6-30) & 3 & 9.33 & $<4$ & $<4$ & 1.77 & 39 & 29 & 0.435 & 7820 & 8 & 0.415 & 10 & 37 & 20 & 0.050 & 4720 & 8 & $<50$ & 43 & $<40$ \\
\hline T98R-13(0-4) & $<2$ & 6.46 & 10 & $<4$ & 1.39 & 16 & 24 & 0.515 & 22450 & 7 & 0.540 & 8 & 16 & 38 & 0.145 & 1500 & 6 & $<50$ & 80 & $<40$ \\
\hline T98R-13 & 3 & 9.83 & $<4$ & $<4$ & 1.60 & 27 & 20 & 0.485 & 7970 & 5 & 0.160 & 5 & 27 & 15 & 0.025 & 6050 & 5 & $<50$ & 21 & $<40$ \\
\hline T98R-13(38-42) & $<2$ & 2.35 & 26 & $<4$ & 2.46 & 39 & 48 & 0.760 & 1100 & 3 & 0.725 & 5 & 33 & 16 & 0.015 & 80 & 11 & $<50$ & 81 & $<40$ \\
\hline T98R-14(0-4) & 2 & 6.65 & 10 & $<4$ & 1.26 & 18 & 23 & 0.635 & 7510 & 6 & 1.560 & 8 & 16 & 26 & 0.080 & 1610 & 6 & $<50$ & 179 & $<40$ \\
\hline T98R-14(18-22) & 3 & 9.64 & 5 & $<4$ & 1.27 & 27 & 15 & 0.405 & 7580 & 5 & 0.175 & 8 & 23 & & 0.020 & 2360 & 4 & $<50$ & 20 & $<40$ \\
\hline
\end{tabular}

*Other analyses (Appendix \#): D =USGS-EDXRF, E =EWU,

$\mathrm{F}=$ CHEMEX, $\mathrm{H}=\mathrm{ACZ},---=$ none. 
Appendix G. Chemical composition of samples analyzed by ICP-AES at XRAL Labs in Golden, CO using 4 acid dissolution (Appendix_G.xls, Appendix_G.dbf).

\begin{tabular}{|c|c|c|c|c|c|c|c|}
\hline No. & $\begin{array}{c}\text { Th } \\
(\mathrm{ppm})\end{array}$ & $\begin{array}{c}\mathrm{Ti} \\
(\%)\end{array}$ & $\begin{array}{c}\mathrm{U} \\
(\mathrm{ppm})\end{array}$ & $\begin{array}{c}\mathrm{V} \\
(\mathrm{ppm})\end{array}$ & $\begin{array}{c}Y \\
(p p m)\end{array}$ & $\begin{array}{c}\mathrm{Yb} \\
(\mathrm{ppm})\end{array}$ & $\begin{array}{c}\mathrm{Zn} \\
(\mathrm{ppm})\end{array}$ \\
\hline SAMPLE_NO. & PPM & TI_\% & \begin{tabular}{l|l} 
U_PPM \\
\end{tabular} & V_PPM & YPPPM & YB_PPM & ZN_PPM \\
\hline $98 \mathrm{C}-01(0-4)$ & 9 & 0.219 & $<100$ & 43 & 15 & 2 & 1150 \\
\hline T98C-01(18-22) & 16 & 0.263 & $<100$ & 52 & 19 & 2 & 548 \\
\hline T98C-01(38-42) & 11 & 0.275 & $<100$ & 49 & 17 & 2 & 719 \\
\hline T98C-01(58-62) & 16 & 0.413 & $<100$ & 73 & 31 & 3 & 378 \\
\hline T98C-05(0-4) & 14 & 0.288 & $<100$ & 62 & $\overline{23}$ & 2 & 465 \\
\hline T98C-05(18-22) & 16 & 0.363 & $<100$ & 66 & 27 & 3 & 160 \\
\hline T98C-05(38-42) & 8 & 0.351 & $<100$ & 51 & 19 & 2 & 167 \\
\hline T98C-05(58-62) & $<6$ & 0.385 & $<100$ & 57 & 31 & 4 & 207 \\
\hline T98C-05(118-122) & 15 & 0.306 & $<100$ & 59 & 36 & 3 & 86 \\
\hline T98C-06(0-4) & 8 & 0.244 & $<100$ & 46 & 11 & 1 & 1130 \\
\hline T98C-06(0-4) & 10 & 0.238 & $<100$ & 48 & 12 & 2 & 1440 \\
\hline T98C-06(18-22) & 14 & 0.288 & $<100$ & 52 & 17 & 2 & 1760 \\
\hline T98C-06(38-42) & 16 & 0.281 & $<100$ & 55 & 18 & 2 & 1100 \\
\hline T98C-06( & 17 & 0.294 & $<100$ & 58 & 20 & 2 & 140 \\
\hline T98C-0 & 15 & 0.275 & $<100$ & 48 & $\overline{16}$ & 2 & 76 \\
\hline T98C-0 & 15 & 0.231 & $<100$ & 52 & 15 & 2 & 56 \\
\hline T98C-0 & 12 & 0.238 & $<100$ & 48 & 13 & 1 & 53 \\
\hline T98C-0 & 15 & 0.269 & $<100$ & 48 & 15 & 2 & 45 \\
\hline T98C-0 & 13 & 0.238 & $<100$ & 47 & 14 & 2 & 51 \\
\hline T98C-0 & 13 & 0.263 & $<100$ & 49 & 15 & 2 & 48 \\
\hline T98C-0 & $<6$ & 0.231 & $<100$ & 48 & 13 & 2 & 2550 \\
\hline T98C-0 & 10 & 0.213 & $<100$ & 50 & 14 & 1 & 2190 \\
\hline T98C-1 & 14 & 0.269 & $<100$ & 54 & 15 & 2 & 1350 \\
\hline T98C-11E & 13 & 0.163 & $<100$ & 44 & 15 & 1 & 4420 \\
\hline$\overline{T 98 C-111}$ & 12 & 0.119 & $<100$ & 35 & 12 & 1 & 4080 \\
\hline T98C-11B & 13 & 0.125 & $<100$ & 35 & 12 & $<1$ & 3500 \\
\hline T98C-11B(55-71) & 13 & 0.131 & $<100$ & 37 & 11 & $<1$ & 3200 \\
\hline T98C-11B(71-92) & 12 & 0.106 & $<100$ & 32 & 9 & $<1$ & 4690 \\
\hline T98C-11B(92-111) & 11 & 0.119 & $<100$ & 31 & 9 & $<1$ & 4130 \\
\hline T98C-12( & 15 & 0.163 & $<100$ & 43 & 15 & 1 & 2290 \\
\hline T98R-13( & 9 & 0.15 & $<100$ & 40 & 9 & 2 & 6960 \\
\hline T98R-13( & 11 & 0.1 & $<100$ & 33 & 9 & $<1$ & 2000 \\
\hline T98R-13 & 12 & 0.3 & $<100$ & 66 & 12 & 1 & 875 \\
\hline T98R-14(C & $<6$ & 0.25 & $<100$ & 42 & $\overline{11}$ & 2 & 7310 \\
\hline T98R-14(18-22) & 9 & 0.081 & $<100$ & 24 & 8 & $<1$ & 831 \\
\hline
\end{tabular}

*Other analyses (Appendix \#): D =USGS-EDXRF, E =EWU,

$\mathrm{F}=$ CHEMEX, $\mathrm{H}=\mathrm{ACZ},----=$ none. 
Appendix G. Chemical composition of samples analyzed by ICP-AES at XRAL Labs in Golden, CO using 4 acid dissolution (Appendix_G.xls, Appendix_G.dbf).

\begin{tabular}{|c|c|c|c|c|c|c|c|c|c|c|c|c|c|c|c|c|c|}
\hline Sample No. & Site ID & $\begin{array}{c}\text { Depth } \\
\text { interval, } \\
\text { top }(\mathrm{cm})\end{array}$ & $\begin{array}{c}\text { Depth } \\
\text { interval, } \\
\text { bottom }(\mathrm{cm})\end{array}$ & $\begin{array}{c}{ }^{*} \text { Other } \\
\text { Analyses } \\
\text { (appendix \#) }\end{array}$ & $\begin{array}{c}\mathrm{Ag} \\
(\mathrm{ppm})\end{array}$ & $\begin{array}{c}\mathrm{Al} \\
(\%)\end{array}$ & $\begin{array}{c}\text { As } \\
\text { (ppm) }\end{array}$ & $\mid \begin{array}{c}\mathrm{Au} \\
(\mathrm{ppm})\end{array}$ & $\begin{array}{c}\mathrm{Ba} \\
(\mathrm{ppm})\end{array}$ & $\begin{array}{c}\mathrm{Be} \\
(\mathrm{ppm})\end{array}$ & $\begin{array}{c}\mathrm{Bi} \\
(\mathrm{ppm})\end{array}$ & $\begin{array}{l}\mathrm{Ca} \\
(\%)\end{array}$ & $\begin{array}{c}\mathrm{Cd} \\
(\mathrm{ppm})\end{array}$ & $\begin{array}{c}\mathrm{Ce} \\
(\mathrm{ppm})\end{array}$ & $\begin{array}{c}\text { Co } \\
(\mathrm{ppm})\end{array}$ & $\begin{array}{c}\mathrm{Cr} \\
(\mathrm{ppm})\end{array}$ & $\begin{array}{c}\mathrm{Cu} \\
\text { (ppm) }\end{array}$ \\
\hline SAMPLE_NO. & SITE_ID & INT_TOP_CM & INT_BTM_CM & OTHR_ANLYS & AG_PPM & AL_(\%) & AS_PPM & AU_PPM & BA_PPM & BE_PPM & BI_PPM & CA_(\%) & CD_PPM & CE_PPM & CO_PPM & CR_PPM & CU_PPM \\
\hline T98R-14(38-42) & T98R-14 & 38 & 42 & $\begin{array}{ll}--- \\
--\end{array}$ & 16 & 2.9 & 261 & $<8$ & 396 & 1 & $<50$ & 0.144 & 6 & 58 & 9 & 62 & 99 \\
\hline T98R-14(58-62) & T98R-14 & 58 & 62 & ---- & 10 & 2.8 & 232 & $<8$ & 363 & 1 & $<50$ & 0.278 & 12 & 49 & 12 & 35 & 84 \\
\hline T98R-14(78-82) & T98R-14 & $\overline{78}$ & 82 & ---- & 10 & 3.1 & 148 & $<8$ & 404 & 1 & $<50$ & 0.363 & 15 & 49 & 16 & 47 & 85 \\
\hline T98R-14(98-102) & T98R-14 & 98 & 102 & --- & 14 & 3.0 & 110 & $<8$ & 528 & 1 & $<50$ & 0.310 & 25 & 53 & 15 & 43 & 99 \\
\hline T98R-14(118-122) & T98R-14 & 118 & 122 & ---- & 12 & 3.2 & 97 & $<8$ & 525 & 1 & $<50$ & 0.272 & 24 & 51 & 12 & 42 & 107 \\
\hline T98C-15(0-4) & T98C-15 & 0 & 4 & ---- & 21 & 5.1 & 102 & $<8$ & 760 & 2 & $<50$ & 0.304 & 18 & 73 & 14 & 76 & 171 \\
\hline T98C-15(18-22) & T98C-15 & 18 & 22 & ---- & 35 & 5.3 & 96 & $<8$ & 1060 & 2 & $<50$ & 0.240 & 27 & 84 & 17 & 64 & 238 \\
\hline T98C-15(38-42) & T98C-15 & 38 & 42 & ---- & 40 & 5.2 & 57 & $<8$ & 1050 & 2 & $<50$ & 0.208 & 10 & 80 & 12 & 74 & 278 \\
\hline T98C-15(58-62) & T98C-15 & 58 & 62 & ---- & $<2$ & 7.0 & 17 & $<8$ & 650 & 2 & $<50$ & 0.240 & 4 & 87 & 9 & 38 & 18 \\
\hline T98C-16(0-4) & T98C-16 & 0 & 4 & ---- & 5 & 6.8 & 56 & $<8$ & 638 & 2 & $<50$ & 0.347 & 13 & 89 & 14 & 77 & 69 \\
\hline T98C-16(18-22) & T98C-16 & 18 & 22 & ---- & 15 & 5.2 & 174 & $<8$ & 642 & 2 & $<50$ & 0.262 & 16 & 82 & 12 & 77 & 120 \\
\hline T98C-16(38-42) & T98C-16 & 38 & 42 & $\begin{array}{ll}--- \\
--\end{array}$ & 17 & 2.8 & 413 & $<8$ & 499 & 1 & $<50$ & 0.106 & 21 & 60 & 26 & 56 & 110 \\
\hline T98C-16(58-62) & T98C-16 & 58 & 62 & $\begin{array}{ll}--- \\
--\end{array}$ & 21 & 2.9 & 101 & $<8$ & 610 & 1 & $<50$ & 0.106 & 10 & 53 & 13 & 52 & 116 \\
\hline T98C-16(78-82) & T98C-16 & 78 & 82 & ---- & 18 & 3.5 & 102 & $<8$ & 699 & 1 & $<50$ & 0.187 & 15 & 62 & 10 & 78 & 132 \\
\hline T98C-16(98-102) & T98C-16 & 98 & 102 & ---- & 19 & 3.1 & 118 & $<8$ & 635 & 1 & $<50$ & 0.187 & 17 & 60 & 10 & 48 & 126 \\
\hline T98C-16(118-122) & T98C-16 & 118 & 122 & ---- & 22 & 2.9 & 128 & $<8$ & 678 & 1 & $<50$ & 0.213 & 19 & 54 & 11 & 51 & 132 \\
\hline T98C-16(143-147) & T98C-16 & 143 & 147 & ---- & 18 & 3.2 & 104 & $<8$ & 635 & 1 & $<50$ & 0.358 & 57 & 54 & 13 & 55 & 121 \\
\hline T98C-16(168-172) & T98C-16 & 168 & 172 & ---- & 14 & 2.8 & 113 & $<8$ & 654 & 1 & $<50$ & 0.353 & 34 & 55 & 15 & 51 & 108 \\
\hline T98C-16(178-182) & T98C-16 & 178 & 182 & ---- & 21 & 3.1 & 136 & $<8$ & 667 & 1 & $<50$ & 0.283 & 27 & 56 & 13 & 44 & 111 \\
\hline T98C-16(208-212) & T98C-16 & 208 & 212 & ---- & 19 & 2.9 & 138 & $<8$ & 624 & $<1$ & $<50$ & 0.310 & 29 & 58 & 14 & 46 & 110 \\
\hline T98C-17(0-4) & T98C-17 & 0 & 4 & ---- & 5 & 7.1 & 46 & $<8$ & 668 & 2 & $<50$ & 0.379 & 10 & 89 & 14 & 84 & 63 \\
\hline T98C-17(18-22) & T98C-17 & 18 & 22 & ---- & 18 & 5.0 & 92 & $<8$ & 698 & 2 & $<50$ & 0.171 & 14 & 84 & 14 & 70 & 148 \\
\hline T98C-17(38-42) & T98C-17 & 38 & 42 & ---- & 15 & 4.3 & 318 & $<8$ & 587 & 1 & $<50$ & 0.171 & 18 & 67 & 19 & 67 & 127 \\
\hline T98C-17(58-62) & T98C-17 & 58 & 62 & ---- & 23 & 5.5 & 115 & $<8$ & 902 & 2 & $<50$ & 0.235 & 31 & 88 & 14 & 87 & 163 \\
\hline T98C-17(78-82) & T98C-17 & 78 & 82 & ---- & 31 & 5.1 & 101 & $<8$ & 1010 & 2 & $<50$ & 0.187 & 18 & 77 & 14 & 79 & 205 \\
\hline T98C-17(108-112) & T98C-17 & 108 & 112 & ---- & $<2$ & 6.2 & 13 & $<8$ & 572 & 2 & $<50$ & 0.219 & 3 & 96 & 8 & 48 & 18 \\
\hline T98C-17(128-132) & T98C-17 & 128 & 132 & --- & $<2$ & 6.3 & 17 & $<8$ & 573 & 2 & $<50$ & 0.208 & $<2$ & 97 & 8 & 41 & 18 \\
\hline T98C-17(153-157) & T98C-17 & 153 & 157 & $-\cdots$ & $<2$ & 5.6 & 11 & $<8$ & 507 & 2 & $<50$ & 0.176 & $<2$ & 84 & 7 & 62 & 14 \\
\hline T98C-17(181-185) & T98C-17 & 181 & 185 & $\begin{array}{ll}--- \\
\end{array}$ & $<2$ & 6.1 & 12 & $<8$ & 538 & 2 & $<50$ & 0.192 & $<2$ & 90 & 5 & 48 & 16 \\
\hline T98C-17(198-202) & T98C-17 & 198 & 202 & ---- & $<2$ & 5.9 & 16 & $<8$ & 564 & 2 & $<50$ & 0.197 & $<2$ & 99 & 5 & 42 & 16 \\
\hline T98C-18(0-4) & T98C-18 & 0 & 4 & ---- & 4 & 6.6 & 56 & $<8$ & 569 & 2 & $<50$ & 0.941 & 16 & 71 & 12 & 77 & 50 \\
\hline T98C-18(18-22) & T98C-18 & 18 & 22 & ---- & 30 & 4.9 & 102 & $<8$ & 961 & 2 & $<50$ & 0.197 & 16 & 80 & 12 & 86 & 202 \\
\hline T98C-18(38-42) & T98C-18 & 38 & 42 & ---- & $<2$ & 6.0 & $<10$ & $<8$ & 572 & 2 & $<50$ & 0.208 & 8 & 86 & 8 & 72 & 16 \\
\hline T98C-18(58-62) & T98C-18 & 58 & 62 & ---- & $<2$ & 6.3 & 72 & $<8$ & 601 & 2 & $<50$ & 0.197 & 6 & 97 & 9 & 49 & 17 \\
\hline T98C-18(83-87) & T98C-18 & 83 & 87 & $-\cdots$ & $<2$ & 6.4 & 12 & $<8$ & 570 & 2 & $<50$ & 0.181 & 3 & 87 & 9 & 94 & 19 \\
\hline
\end{tabular}

*Other analyses (Appendix \#): D =USGS-EDXRF, E =EWU,

$\mathrm{F}=\mathrm{CHEMEX}, \mathrm{H}=\mathrm{ACZ},----=$ none. 
Appendix G. Chemical composition of samples analyzed by ICP-AES at XRAL Labs in Golden, CO using 4 acid dissolution (Appendix_G.xls, Appendix_G.dbf).

\begin{tabular}{|c|c|c|c|c|c|c|c|c|c|c|c|c|c|c|c|c|c|c|c|c|}
\hline Sample No. & $\begin{array}{c}\mathrm{Eu} \\
(\mathrm{ppm})\end{array}$ & $\begin{array}{l}\mathrm{Fe} \\
(\%)\end{array}$ & $\begin{array}{c}\mathrm{Ga} \\
(\mathrm{ppm})\end{array}$ & $\begin{array}{c}\text { Ho } \\
(\mathrm{ppm})\end{array}$ & $\begin{array}{c}\mathrm{K} \\
(\%)\end{array}$ & $\begin{array}{c}\mathrm{La} \\
(\mathrm{ppm})\end{array}$ & $\begin{array}{c}\mathrm{Li} \\
(\mathrm{ppm})\end{array}$ & $\begin{array}{l}\mathrm{Mg} \\
(\%)\end{array}$ & $\begin{array}{c}\mathrm{Mn} \\
(\mathrm{ppm})\end{array}$ & $\begin{array}{c}\text { Mo } \\
(\mathrm{ppm})\end{array}$ & $\begin{array}{l}\mathrm{Na} \\
(\%)\end{array}$ & $\begin{array}{c}\mathrm{Nb} \\
(\mathrm{ppm})\end{array}$ & $\begin{array}{c}\mathrm{Nd} \\
(\mathrm{ppm})\end{array}$ & $\begin{array}{c}\mathrm{Ni} \\
(p p m)\end{array}$ & $\begin{array}{c}\mathrm{P} \\
(\mathrm{ppm})\end{array}$ & $\begin{array}{c}\mathrm{Pb} \\
(\mathrm{ppm})\end{array}$ & $\begin{array}{c}\mathrm{Sc} \\
(\mathrm{ppm})\end{array}$ & $\begin{array}{c}\mathrm{Sn} \\
(\mathrm{ppm})\end{array}$ & $\begin{array}{c}\mathrm{Sr} \\
(\mathrm{ppm})\end{array}$ & $\begin{array}{c}\mathrm{Ta} \\
(\mathrm{ppm})\end{array}$ \\
\hline SAMPLE_NO. & PPM & (\%) & PPM & HO_PPM & K_(\%) & LA_PPM & LI_PPM & G_(\%) & MN_PPM & O_PPM & IA_(\%) & NB_PPM & DPPM & NI_PPM & P_PPM & PB_PPM & SCPPM & SN_PPM & SR_PPM & TA_PPM \\
\hline T98R-14(38-42) & 3 & 10.30 & 9 & $<4$ & 1.31 & 25 & 18 & 0.465 & 9080 & 5 & 0.145 & $<4$ & 21 & 9 & 0.020 & 2410 & 4 & $<50$ & 18 & $<40$ \\
\hline T98R-14(58-62) & 3 & 8.48 & $<4$ & $<4$ & 1.24 & 21 & 15 & 0.415 & 7770 & 5 & 0.110 & 6 & 18 & 9 & 0.015 & 2460 & 4 & $<50$ & 18 & $<40$ \\
\hline T98R-14(78-82) & 2 & 7.51 & $<4$ & $<4$ & 1.38 & 24 & 17 & 0.420 & 6990 & 3 & 0.145 & 5 & 25 & 13 & 0.015 & 3040 & 4 & $<50$ & 23 & $<40$ \\
\hline T98R-14(98-102) & 3 & 10.30 & $<4$ & $<4$ & 1.33 & 23 & 16 & 0.490 & 9460 & 5 & 0.150 & 5 & 24 & 13 & 0.015 & 4560 & 4 & $<50$ & 24 & $<40$ \\
\hline T98R-14(118-122) & 3 & 9.38 & $<4$ & $<4$ & 1.42 & 25 & 19 & 0.475 & 8710 & 5 & 0.160 & 5 & 25 & 11 & 0.015 & 4930 & 4 & $<50$ & 25 & $<40$ \\
\hline T98C-15(0-4) & 3 & 7.45 & 10 & $<4$ & 1.74 & 35 & 29 & 0.405 & 6420 & 4 & 0.480 & 9 & 31 & 15 & 0.050 & 7460 & 8 & $<50$ & 62 & $<40$ \\
\hline T98C-15(18-22) & 3 & 8.85 & 5 & $<4$ & 1.86 & 40 & 35 & 0.445 & 7560 & 9 & 0.470 & 10 & 40 & 19 & 0.055 & 13970 & 8 & $<50$ & 57 & $<40$ \\
\hline T98C-15(38-42) & 3 & 7.28 & 9 & $<4$ & 1.84 & 37 & 32 & 0.445 & 5770 & 4 & 0.450 & 8 & 35 & 14 & 0.050 & 15410 & 8 & $<50$ & 50 & $<40$ \\
\hline T98C-15(58-62) & $<2$ & 2.61 & 25 & $<4$ & 2.26 & 47 & 45 & 0.720 & 399 & 4 & 0.680 & $<4$ & 43 & 19 & 0.045 & 42 & 11 & $<50$ & 64 & $<40$ \\
\hline T98C-16(0-4) & $<2$ & 3.83 & 19 & $<4$ & 2.43 & 43 & 43 & 0.635 & 1950 & 5 & 0.685 & 8 & 40 & 24 & 0.075 & 1960 & 11 & $<50$ & 63 & $<40$ \\
\hline T98C-16(18-22) & $<2$ & 6.18 & 6 & $<4$ & 1.87 & 43 & 34 & 0.500 & 3710 & 4 & 0.530 & 11 & 38 & 16 & 0.055 & 4150 & 8 & $<50$ & 59 & $<40$ \\
\hline T98C-16(38-42) & 4 & 12.50 & $<4$ & $<4$ & 1.16 & 25 & 16 & 0.240 & 11970 & 7 & 0.180 & 7 & 21 & 15 & 0.025 & 3720 & 4 & $<50$ & 26 & $<40$ \\
\hline T98C-16(58-62) & 3 & 12.40 & $<4$ & $<4$ & 1.27 & 25 & 15 & 0.295 & 12190 & 6 & 0.130 & $<4$ & 23 & 12 & 0.020 & 7230 & 4 & $<50$ & 19 & $<40$ \\
\hline T98C-16(78-82) & $2 \mid$ & 10.30 & $<4$ & $<4$ & 1.46 & 30 & 22 & 0.495 & 9530 & 4 & 0.200 & 6 & 28 & 13 & 0.025 & 7820 & 5 & $<50$ & 26 & $<40$ \\
\hline T98C-16(98-102) & $4 \sqrt{1}$ & 11.00 & $<4$ & $<4$ & 1.32 & 26 & 18 & 0.520 & 10360 & 5 & 0.155 & 6 & 25 & 13 & 0.020 & 6910 & 4 & $<50$ & 23 & $<40$ \\
\hline T98C-16(118-122) & 3 - & 11.40 & $<4$ & $<4$ & 1.22 & 25 & 17 & 0.545 & 10810 & 6 & 0.145 & 5 & 22 & 11 & 0.020 & 6730 & 4 & $<50$ & 22 & $<40$ \\
\hline T98C-16(143-147) & 2 & 9.84 & $<4$ & $<4$ & 1.35 & 27 & 19 & 0.535 & 9540 & 7 & 0.175 & 7 & 25 & 16 & 0.025 & 6990 & 5 & $<50$ & 28 & $<40$ \\
\hline T98C-16(168-172) & 3 & 10.60 & $<4$ & $<4$ & 1.24 & 24 & 16 & 0.530 & 10450 & 6 & 0.125 & 7 & 23 & 14 & 0.020 & 5630 & 4 & $<50$ & 24 & $<40$ \\
\hline T98C-16(178-182) & 4 & 11.20 & $<4$ & 5 & 1.40 & 24 & 18 & 0.565 & 11230 & 7 & 0.140 & 7 & 20 & 12 & 0.015 & 5620 & 4 & $<50$ & 24 & $<40$ \\
\hline T98C-16(208-212) & 31 & 11.30 & $<4$ & $<4$ & 1.30 & 23 & 18 & 0.550 & 11440 & 6 & 0.120 & 4 & 22 & 12 & 0.015 & 5420 & 4 & $<50$ & 24 & $<40$ \\
\hline T98C-17(0-4) & 2 & 3.61 & 13 & $<4$ & 2.59 & 45 & 45 & 0.680 & 1800 & 5 & 0.705 & 10 & 40 & 21 & 0.075 & 1630 & 11 & $<50$ & 66 & $<40$ \\
\hline T98C-17 & 3 & 7.99 & 9 & $<4$ & 1.84 & 39 & 28 & 0.390 & 6490 & 6 & 0.430 & 10 & 37 & 16 & 0.045 & 7550 & 8 & $<50$ & 44 & $<40$ \\
\hline T98C-1 & 3 & 10.20 & $<4$ & $<4$ & 1.58 & 32 & 26 & 0.345 & 8320 & 8 & 0.345 & 12 & 33 & 21 & 0.045 & 4650 & 6 & $<50$ & 40 & $<40$ \\
\hline T98C-1 & 3 & 8.09 & 11 & $<4$ & 1.95 & 43 & 35 & 0.490 & 6670 & 7 & 0.460 & 12 & 41 & 22 & 0.065 & 9440 & 9 & $<50$ & 51 & $<40$ \\
\hline T98C-1 & 31 & 9.68 & 4 & $<4$ & 1.85 & 37 & 32 & 0.460 & 9200 & 12 & 0.390 & 9 & 38 & 16 & 0.060 & 14490 & 8 & $<50$ & 43 & $<40$ \\
\hline 112) & $<2$ & 2.48 & 16 & $<4$ & 2.19 & 48 & 41 & 0.700 & 382 & 3 & 0.640 & 4 & 45 & 17 & 0.050 & 237 & 10 & $<50$ & 55 & $<40$ \\
\hline T98C-1 & \begin{tabular}{l|}
$<2$ \\
\end{tabular} & 2.51 & 21 & $<4$ & 2.23 & 48 & 43 & 0.700 & 362 & 3 & 0.635 & $<4$ & 46 & 17 & 0.050 & 91 & 10 & $<50$ & 55 & $<40$ \\
\hline T98C-1 & \begin{tabular}{l|}
$<2$ \\
\end{tabular} & 2.16 & 17 & $<4$ & 2.05 & 45 & 36 & 0.610 & 215 & 3 & 0.555 & 7 & 42 & 15 & 0.045 & 38 & 9 & $<50$ & 48 & $<40$ \\
\hline T98C-1 & \begin{tabular}{l|}
$<2$ \\
\end{tabular} & 2.28 & 18 & $<4$ & 2.11 & 48 & 38 & 0.630 & 138 & 4 & 0.625 & 9 & 43 & 17 & 0.045 & 33 & 10 & $<50$ & 53 & $<40$ \\
\hline T98C-17 & $<2$ & 2.10 & 18 & $<4$ & 2.07 & 51 & 42 & 0.675 & 122 & 4 & 0.620 & 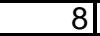 & 52 & 18 & 0.045 & 26 & 10 & $<50$ & 53 & $<40$ \\
\hline T98C-1 & $<2$ & 3.67 & 25 & $<4$ & 1.88 & 32 & 38 & 0.645 & 1660 & 5 & 1.175 & 11 & 28 & 22 & 0.090 & 1380 & 9 & $<50$ & 134 & $<40$ \\
\hline T98C-1 & 3 & 8.36 & $<4$ & $<4$ & 1.75 & 37 & 30 & 0.420 & 6850 & 7 & 0.435 & 8 & 34 & 16 & 0.050 & 11330 & 7 & $<50$ & 49 & $<40$ \\
\hline 42) & $<2$ & 2.36 & 15 & $<4$ & 2.12 & 45 & 39 & 0.635 & 426 & 4 & 0.575 & 7 & 40 & 17 & 0.065 & 137 & 9 & $<50$ & 53 & $<40$ \\
\hline 62) & $<2$ & 2.52 & 23 & $<4$ & 2.19 & 48 & 41 & 0.685 & 347 & 3 & 0.600 & 4 & 43 & 18 & 0.065 & 44 & 10 & $<50$ & 54 & $<40$ \\
\hline T98C-18(z & 2 & 2.62 & 17 & $<4$ & 2.20 & 47 & 41 & 0.655 & 345 & 4 & 0.585 & 10 & 48 & 20 & 0.070 & 26 & 10 & $<50$ & 52 & $<40$ \\
\hline
\end{tabular}

*Other analyses (Appendix \#): D =USGS-EDXRF, E =EWU,

$\mathrm{F}=$ CHEMEX, $\mathrm{H}=\mathrm{ACZ},---=$ none. 
Appendix G. Chemical composition of samples analyzed by ICP-AES at XRAL Labs in Golden, CO using 4 acid dissolution (Appendix_G.xls, Appendix_G.dbf).

\begin{tabular}{|c|c|c|c|c|c|c|c|}
\hline No. & $\begin{array}{c}\text { Th } \\
(\mathrm{ppm})\end{array}$ & $\begin{array}{c}\mathrm{Ti} \\
(\%)\end{array}$ & $\begin{array}{c}\mathrm{U} \\
(\mathrm{ppm})\end{array}$ & $\begin{array}{c}\mathrm{V} \\
(\mathrm{ppm})\end{array}$ & $\begin{array}{c}Y \\
(p p m)\end{array}$ & $\begin{array}{c}\mathrm{Yb} \\
(\mathrm{ppm})\end{array}$ & $\begin{array}{c}\mathrm{Zn} \\
(\mathrm{ppm})\end{array}$ \\
\hline SAMPLE & PPM & TI_\% & \begin{tabular}{l|l} 
U_PPM \\
\end{tabular} & V_PPM & YPPPM & YB_PPM & ZN_PPM \\
\hline $98 \mathrm{R}-14(38-42)$ & 11 & 0.088 & $<100$ & 25 & 7 & $<1$ & 801 \\
\hline $98 \mathrm{R}-14(58-62)$ & 11 & 0.075 & $<100$ & 22 & 6 & $<1$ & 2100 \\
\hline T98R-14(78-82) & 9 & 0.081 & $<100$ & 25 & $\frac{6}{6}$ & $<1$ & 2500 \\
\hline T98R-14(98-102) & 9 & 0.094 & $<100$ & 26 & 7 & $<1$ & 3780 \\
\hline T98R-14(118-122) & 8 & 0.1 & $<100$ & 28 & 7 & $<1$ & 3720 \\
\hline T98C-15(0-4) & 12 & 0.206 & $<100$ & 46 & 15 & 1 & 2030 \\
\hline T98C-15(18-22) & 13 & 0.231 & $<100$ & 48 & 17 & 1 & 3380 \\
\hline T98C-15(38-42) & 12 & 0.188 & $<100$ & 45 & 16 & 1 & 1770 \\
\hline T98C-15(58-62) & 14 & 0.294 & $<100$ & 62 & 17 & 2 & 335 \\
\hline T98C-16(0-4) & 14 & 0.219 & $<100$ & 58 & 18 & 2 & 1210 \\
\hline T98C-16(18-22) & 14 & 0.194 & $<100$ & 49 & 16 & 2 & 1580 \\
\hline T98C-16 & 10 & 0.094 & $<100$ & 26 & 10 & $<1$ & 2260 \\
\hline T98C-16 & 8 & 0.1 & $<100$ & 26 & 8 & $<1$ & 1510 \\
\hline T98C-16 & 12 & 0.106 & $<100$ & 32 & 9 & $<1$ & 1790 \\
\hline$\overline{\text { T98C-1 }}$ & 10 & 0.119 & $<100$ & 28 & $\overline{8}$ & $<1$ & 1570 \\
\hline T98C-1 & 11 & 0.1 & $<100$ & 27 & 8 & $<1$ & 1910 \\
\hline T98C-1 & 11 & 0.094 & $<100$ & 29 & 9 & $<1$ & 5180 \\
\hline T98C- & 10 & 0.094 & $<100$ & 26 & $\overline{7}$ & $<1$ & 5520 \\
\hline T98C & 7 & 0.11 & $<100$ & 27 & $\overline{7}$ & $<1$ & 4450 \\
\hline T98C-1 & 9 & 0.075 & $<100$ & 25 & $\overline{7}$ & $<1$ & 4740 \\
\hline T98C-17 & 14 & 0.256 & $<100$ & 62 & 19 & 2 & 1110 \\
\hline T98C-1 & 14 & 0.163 & $<100$ & 45 & 13 & 1 & 2470 \\
\hline T98C-1 & 12 & 0.156 & $<100$ & 39 & 13 & 1 & 2360 \\
\hline T98C-17 & 14 & 0.188 & $<100$ & 51 & 16 & 1 & 2970 \\
\hline T98C-1 & 15 & 0.163 & $<100$ & 48 & 15 & 1 & 3360 \\
\hline T98C-17 & 16 & 0.275 & $<100$ & 55 & 18 & 2 & 305 \\
\hline T98C-17( & 16 & 0.281 & $<100$ & 56 & 21 & 2 & 195 \\
\hline T98C-17(1) & 15 & 0.256 & $<100$ & 49 & 17 & 2 & 139 \\
\hline T98C-17(1 & 15 & 0.269 & $<100$ & 51 & 19 & 2 & 139 \\
\hline T98C-17( & 18 & 0.294 & $<100$ & 54 & 20 & 2 & 138 \\
\hline T98C-18 & 10 & 0.275 & $<100$ & 58 & 17 & 2 & 1440 \\
\hline T98C-18 & 13 & 0.175 & $<100$ & 45 & 15 & 1 & 2200 \\
\hline T98C-18 & 10 & 0.263 & $<100$ & 51 & 18 & 2 & 442 \\
\hline T98C-18 & 16 & 0.269 & $<100$ & 56 & 20 & 2 & 303 \\
\hline T98C-18(83-87) & 15 & 0.244 & $<100$ & 56 & 19 & 2 & 257 \\
\hline
\end{tabular}

*Other analyses (Appendix \#): D =USGS-EDXRF, E =EWU,

$\mathrm{F}=$ CHEMEX, $\mathrm{H}=\mathrm{ACZ},---=$ none. 
Appendix G. Chemical composition of samples analyzed by ICP-AES at XRAL Labs in Golden, CO using 4 acid dissolution (Appendix_G.xls, Appendix_G.dbf).

\begin{tabular}{|c|c|c|c|c|c|c|c|c|c|c|c|c|c|c|c|c|c|}
\hline Sample No. & Site ID & $\begin{array}{c}\text { Depth } \\
\text { interval, } \\
\text { top }(\mathrm{cm})\end{array}$ & $\begin{array}{c}\text { Depth } \\
\text { interval, } \\
\text { bottom }(\mathrm{cm})\end{array}$ & $\begin{array}{c}{ }^{*} \text { Other } \\
\text { Analyses } \\
\text { (appendix \#) }\end{array}$ & $\begin{array}{c}\mathrm{Ag} \\
(\mathrm{ppm})\end{array}$ & $\begin{array}{c}\mathrm{Al} \\
(\%)\end{array}$ & $\begin{array}{c}\text { As } \\
\text { (ppm) }\end{array}$ & $\mid \begin{array}{c}\mathrm{Au} \\
(\mathrm{ppm})\end{array}$ & $\begin{array}{c}\mathrm{Ba} \\
(\mathrm{ppm})\end{array}$ & $\begin{array}{c}\mathrm{Be} \\
(\mathrm{ppm})\end{array}$ & $\begin{array}{c}\mathrm{Bi} \\
(\mathrm{ppm})\end{array}$ & $\begin{array}{l}\mathrm{Ca} \\
(\%)\end{array}$ & $\begin{array}{c}\mathrm{Cd} \\
(\mathrm{ppm})\end{array}$ & $\begin{array}{c}\mathrm{Ce} \\
(\mathrm{ppm})\end{array}$ & $\begin{array}{c}\text { Co } \\
(\mathrm{ppm})\end{array}$ & $\begin{array}{c}\mathrm{Cr} \\
(\mathrm{ppm})\end{array}$ & $\begin{array}{c}\mathrm{Cu} \\
\text { (ppm) }\end{array}$ \\
\hline SAMPLE_NO. & SITE_ID & INT_TOP_CM & INT_BTM_CM & OTHR_ANLYS & AG_PPM & AL_(\%) & AS_PPM & AU_PPM & BA_PPM & BE_PPM & BI_PPM & CA_(\%) & CD_PPM & CE_PPM & CO_PPM & CR_PPM & CU_PPM \\
\hline T98C-18(103-107) & T98C-18 & 103 & 107 & $\begin{array}{ll}--- \\
--\end{array}$ & $<2$ & 6.3 & 15 & $<8$ & 536 & 2 & $<50$ & 0.165 & $<2$ & 85 & 8 & 92 & 17 \\
\hline T98C-18(128-132) & T98C-18 & 128 & 132 & ---- & $<2$ & 6.4 & 16 & $<8$ & 532 & 2 & $<50$ & 0.165 & $<2$ & 86 & 9 & 54 & 19 \\
\hline T98C-18(158-162) & T98C-18 & 158 & 162 & ---- & $<2$ & 6.6 & 13 & $<8$ & 561 & 2 & $<50$ & 0.181 & $<2$ & 85 & 8 & 92 & 17 \\
\hline T98C-18(173-177) & T98C-18 & 173 & 177 & ---- & $<2$ & 6.3 & 10 & $<8$ & 583 & 2 & $<50$ & 0.181 & $<2$ & 96 & 7 & 67 & 26 \\
\hline T98C-20(0-4) & T98C-20 & 0 & 4 & ---- & 4 & 6.7 & 23 & $<8$ & 527 & 2 & $<50$ & 0.866 & 6 & 61 & 8 & 78 & 62 \\
\hline T98C-20(18-22) & T98C-20 & 18 & 22 & ---- & 32 & 6.2 & 80 & $<8$ & 946 & 2 & $<50$ & 0.278 & 26 & 91 & 15 & 78 & 191 \\
\hline T98C-20(38-42) & T98C-20 & 38 & 42 & ---- & 28 & 6.6 & 41 & $<8$ & 829 & 2 & $<50$ & 0.304 & 12 & 76 & 11 & 86 & 165 \\
\hline T98C-20(43-47) & T98C-20 & 43 & 47 & ---- & $<2$ & 7.3 & 13 & $<8$ & 630 & 2 & $<50$ & 0.256 & 8 & 93 & 10 & 80 & 22 \\
\hline T98C-21(0-4) & T98C-21 & 0 & 4 & ---- & 5 & 6.5 & 52 & $<8$ & 647 & 2 & $<50$ & 0.401 & 15 & 83 & 14 & 94 & 64 \\
\hline T98C-21(18-22) & T98C-21 & 18 & 22 & ---- & 11 & 6.5 & 136 & $<8$ & 677 & 2 & $<50$ & 0.379 & 19 & 89 & 16 & 91 & 118 \\
\hline T98C-21(38-42) & T98C-21 & 38 & 42 & --- & 16 & 3.6 & 521 & $<8$ & 480 & $\overline{1}$ & $<50$ & 0.251 & 26 & 66 & 13 & 63 & 117 \\
\hline T98C-21(58-62) & T98C-21 & 58 & 62 & $\begin{array}{ll}--- \\
--\end{array}$ & 14 & 3.2 & 262 & $<8$ & 432 & 1 & $<50$ & 0.267 & 41 & 52 & 17 & 36 & 109 \\
\hline T98C-21(78-82) & T98C-21 & 78 & 82 & ---- & 15 & 3.0 & 213 & $<8$ & 482 & 1 & $<50$ & 0.229 & 16 & 51 & 16 & 59 & 110 \\
\hline T98C-21(108-112) & T98C-21 & 108 & 112 & ---- & 16 & 3.3 & 469 & $<8$ & 570 & 1 & $<50$ & 0.315 & 22 & 54 & 24 & 55 & 130 \\
\hline T98C-21(128-132) & T98C-21 & 128 & 132 & ---- & 14 & 3.1 & 235 & $<8$ & 499 & 1 & $<50$ & 0.304 & 19 & 58 & 17 & 58 & 110 \\
\hline T98C-21(153-157) & T98C-21 & 153 & 157 & ---- & 18 & 3.0 & 316 & $<8$ & 489 & $<1$ & $<50$ & 0.310 & 16 & 52 & 19 & 41 & 113 \\
\hline T98C-21(183-187) & T98C-21 & 183 & 187 & ---- & 15 & 2.8 & 276 & $<8$ & 439 & $<1$ & $<50$ & 0.347 & 17 & 41 & 16 & 77 & 108 \\
\hline T98C-21(198-202) & T98C-21 & 198 & 202 & ---- & 20 & 2.4 & 377 & $<8$ & 361 & $<1$ & $<50$ & 0.336 & 14 & 46 & 19 & 54 & 122 \\
\hline T98C-22(0-4) & T98C-22 & 0 & 4 & ---- & 7 & 5.0 & 32 & $<8$ & 425 & 1 & $<50$ & 0.770 & 7 & 47 & 8 & 69 & 87 \\
\hline T98C-22(18-22) & T98C-22 & 18 & 22 & ---- & 8 & 6.9 & 32 & $<8$ & 688 & 2 & $<50$ & 0.288 & 5 & 87 & 9 & 64 & 69 \\
\hline T98C-22(38-42) & T98C-22 & 38 & 42 & ---- & \begin{tabular}{l|l|}
$<2$ \\
\end{tabular} & 6.1 & $<10$ & $<8$ & 495 & 2 & $<50$ & 0.262 & $<2$ & 78 & 7 & 87 & 20 \\
\hline T98C-22(48-52) & T98C-22 & 48 & 52 & ---- & $<2$ & 6.3 & 12 & $<8$ & 514 & 2 & $<50$ & 0.299 & $<2$ & 80 & 8 & 38 & 20 \\
\hline T98C-22(58-62) & T98C-22 & 58 & 62 & ---- & $<2$ & 6.7 & 16 & $<8$ & 597 & 2 & $<50$ & 0.358 & $<2$ & 99 & 8 & 103 & 19 \\
\hline T98C-22(78-82) & T98C-22 & 78 & 82 & ---- & $<2$ & 6.4 & 11 & $<8$ & 580 & 2 & $<50$ & 0.379 & $<2$ & 92 & 8 & 73 & 15 \\
\hline T98R-23(0-4) & T98R-23 & 0 & 4 & ---- & 6 & 5.6 & 138 & $<8$ & 551 & 2 & $<50$ & 0.807 & 15 & 49 & 15 & 78 & 173 \\
\hline T98R-23(18-22) & T98R-23 & 18 & 22 & ---- & 21 & 8.0 & 112 & $<8$ & 1020 & 3 & $<50$ & 0.235 & 13 & 56 & 12 & 69 & 189 \\
\hline T98R-23(28-32) & T98R-23 & 28 & 32 & ---- & $<2$ & 6.8 & 11 & $<8$ & 641 & 2 & $<50$ & 0.427 & 10 & 66 & 13 & 85 & 24 \\
\hline T98R-23(38-42) & T98R-23 & 38 & 42 & $-\cdots$ & $<2$ & 6.8 & 14 & $<8$ & 702 & 2 & $<50$ & 0.401 & $<2$ & 78 & 11 & 46 & 19 \\
\hline T98R-24(0-4) & T98R-24 & 0 & 4 & $\begin{array}{ll}--- \\
\end{array}$ & 5 & 5.5 & 28 & $<8$ & 333 & 1 & $<50$ & 1.925 & 262 & 32 & 105 & 60 & 79 \\
\hline T98R-24(18-22) & T98R-24 & 18 & 22 & ---- & 11 & 2.5 & 378 & $<8$ & 349 & $<1$ & $<50$ & 0.160 & 6 & 43 & 14 & 38 & 75 \\
\hline T98R-24(38-42) & T98R-24 & 38 & 42 & ---- & 14 & 2.6 & 357 & $<8$ & 377 & $<1$ & $<50$ & 0.497 & 9 & 49 & 14 & 50 & 91 \\
\hline T98R-24(58-62) & T98R-24 & 58 & 62 & ---- & 18 & 3.3 & 434 & $<8$ & 467 & 1 & $<50$ & 0.636 & 27 & 40 & 25 & 56 & 134 \\
\hline T98R-24(78-82) & T98R-24 & 78 & 82 & ---- & 31 & 5.0 & 485 & $<8$ & 655 & 2 & $<50$ & 0.433 & 54 & 55 & 41 & 62 & 258 \\
\hline T98C-25(0-4) & T98C-25 & 0 & 4 & ---- & 3 & 6.4 & 35 & $<8$ & 575 & 2 & $<50$ & 0.989 & 6 & 66 & 10 & 85 & $\overline{43}$ \\
\hline T98C-25(18-22) & T98C-25 & 18 & 22 & ---- & $<2$ & 6.3 & 13 & $<8$ & 629 & 2 & $<50$ & 0.262 & 9 & 83 & 9 & 93 & 20 \\
\hline
\end{tabular}

*Other analyses (Appendix \#): D =USGS-EDXRF, E =EWU,

$\mathrm{F}=$ CHEMEX, $\mathrm{H}=\mathrm{ACZ},---=$ none. 
Appendix G. Chemical composition of samples analyzed by ICP-AES at XRAL Labs in Golden, CO using 4 acid dissolution (Appendix_G.xls, Appendix_G.dbf).

\begin{tabular}{|c|c|c|c|c|c|c|c|c|c|c|c|c|c|c|c|c|c|c|c|c|}
\hline Sample No. & $\begin{array}{c}\text { Eu } \\
\text { (ppm) }\end{array}$ & $\begin{array}{l}\mathrm{Fe} \\
(\%)\end{array}$ & $\begin{array}{c}\mathrm{Ga} \\
(\mathrm{ppm})\end{array}$ & $\begin{array}{c}\mathrm{Ho} \\
(\mathrm{ppm})\end{array}$ & $\begin{array}{c}\mathrm{K} \\
(\%)\end{array}$ & $\begin{array}{c}\mathrm{La} \\
(\mathrm{ppm})\end{array}$ & $\begin{array}{c}\mathrm{Li} \\
(\mathrm{ppm})\end{array}$ & $\begin{array}{l}\mathrm{Mg} \\
(\%)\end{array}$ & $\begin{array}{c}\mathrm{Mn} \\
\text { (ppm) }\end{array}$ & $\begin{array}{c}\text { Mo } \\
\text { (ppm) }\end{array}$ & $\begin{array}{l}\mathrm{Na} \\
(\%)\end{array}$ & $\begin{array}{c}\mathrm{Nb} \\
(\mathrm{ppm})\end{array}$ & $\begin{array}{c}\mathrm{Nd} \\
(\mathrm{ppm})\end{array}$ & $\begin{array}{c}\mathrm{Ni} \\
(\mathrm{ppm})\end{array}$ & $\begin{array}{c}P \\
(p p m)\end{array}$ & $\begin{array}{c}\mathrm{Pb} \\
(\mathrm{ppm})\end{array}$ & $\begin{array}{c}\text { Sc } \\
(\mathrm{ppm})\end{array}$ & $\begin{array}{c}\text { Sn } \\
(p p m)\end{array}$ & $\begin{array}{c}\mathrm{Sr} \\
(\mathrm{ppm})\end{array}$ & $\begin{array}{c}\text { Ta } \\
\text { (ppm) }\end{array}$ \\
\hline SAMPLE_NO. & PPM & FE_(\%) & GA_PPM & HO_PPM & K_(\%) & LA_PPM & LI_PPM & MG_(\%) & MN_PPM & MO_PPM & NA_(\%) & NB_PPM & ND_PPM & NI_PPM & P_PPM & B_PPM & SC_PPM & SN_PPM & SR_PPM & TA_PPM \\
\hline T98C-18(103-107) & $<2$ & 2.59 & 21 & $<4$ & 2.12 & 45 & 39 & 0.640 & 324 & 5 & 0.570 & 6 & 40 & 18 & 0.065 & 19 & 11 & $<50$ & 51 & $<40$ \\
\hline T98C-18(128-132) & $<2$ & 2.74 & 23 & $<4$ & 2.12 & 46 & 39 & 0.645 & 317 & 4 & 0.580 & $<4$ & 40 & 19 & 0.060 & 19 & 10 & $<50$ & 52 & $<40$ \\
\hline T98C-18(158-162) & 2 & 2.68 & 22 & $<4$ & 2.27 & 46 & 42 & 0.670 & 285 & $\overline{4}$ & 0.635 & $<4$ & 41 & 18 & 0.050 & 21 & 10 & $<50$ & 55 & $<40$ \\
\hline T98C-18(173-177) & $<2$ & 2.47 & 23 & $<4$ & 2.19 & 49 & 41 & 0.695 & 254 & 4 & 0.615 & $<4$ & 44 & 22 & 0.045 & 19 & 11 & $<50$ & 55 & $<40$ \\
\hline T98C-20(0-4) & $<2$ & 2.48 & 22 & $<4$ & 1.95 & 28 & 33 & 0.620 & 769 & 5 & 1.055 & 9 & 24 & 20 & 0.105 & 1150 & 10 & $<50$ & 118 & $<40$ \\
\hline T98C-20(18-22) & 3 & 6.95 & 16 & $<4$ & 2.08 & 44 & 39 & 0.545 & 4580 & 5 & 0.565 & 15 & 41 & 20 & 0.065 & |10970 & 10 & $<50$ & 61 & $<40$ \\
\hline T98C-20(38-42) & 3 & 5.55 & 11 & $<4$ & 2.25 & 38 & 41 & 0.555 & 3460 & 5 & 0.590 & 10 & 35 & 19 & 0.080 & |10250 & 10 & $<50$ & 62 & $<40$ \\
\hline T98C-20(43-47) & $<2$ & 2.73 & 25 & $<4$ & 2.25 & 47 & 50 & 0.780 & 455 & 5 & 0.695 & 5 & 44 & 21 & 0.055 & 82 & 12 & $<50$ & 61 & $<40$ \\
\hline T98C-21(0-4) & $<2$ & 3.79 & 21 & $<4$ & 2.38 & 39 & 41 & 0.700 & 2240 & 4 & 0.570 & 17 & 37 & 23 & 0.095 & 1870 & 11 & $<50$ & 57 & $<40$ \\
\hline T98C-21(18-22) & 2 & 4.86 & 10 & $<4$ & 2.15 & 42 & 41 & 0.660 & 2750 & 5 & 0.560 & 13 & 41 & 23 & 0.080 & 3290 & 11 & $<50$ & 63 & $<40$ \\
\hline T98C-21(38-42) & 4 & 11.20 & $<4$ & $<4$ & 1.36 & 30 & 20 & 0.335 & 8880 & 8 & 0.255 & 7 & 27 & 15 & 0.060 & 3580 & 5 & $<50$ & 41 & $<40$ \\
\hline T98C-21(58-62) & 2 & 7.43 & $<4$ & 5 & 1.18 & 25 & 17 & 0.455 & 6190 & 4 & 0.260 & 8 & 22 & 17 & 0.030 & 3740 & 5 & $<50$ & 31 & $<40$ \\
\hline T98C-21(78-82) & 3 & 9.90 & $<4$ & $<4$ & 1.31 & 25 & 15 & 0.505 & 8970 & 6 & 0.165 & 6 & 19 & 14 & 0.020 & 3700 & 4 & $<50$ & 23 & $<40$ \\
\hline T98C-21(108-112) & 3 & 9.78 & $<4$ & $<4$ & 1.32 & 25 & 16 & 0.515 & 8650 & 8 & 0.215 & 11 & 23 & 18 & 0.030 & 4080 & 5 & $<50$ & 29 & $<40$ \\
\hline T98C-21(128-132) & 3 & 10.40 & $<4$ & $<4$ & 1.32 & 24 & 17 & 0.510 & 9720 & 6 & 0.175 & 8 & 23 & 14 & 0.020 & 4320 & 4 & $<50$ & 24 & $<40$ \\
\hline T98C-21(153-157) & 3 & 10.70 & $<4$ & $<4$ & 1.21 & 25 & 14 & 0.500 & 9240 & 7 & 0.175 & 11 & 16 & 14 & 0.020 & 3440 & 4 & $<50$ & 25 & $<40$ \\
\hline T98C-21(183-187) & 2 & 10.80 & $<4$ & 4 & 1.16 & 20 & 16 & 0.515 & 9810 & 6 & 0.170 & 5 & 18 & 15 & 0.020 & 3700 & 4 & $<50$ & 23 & $<40$ \\
\hline T98C-21(198-202) & 3 & 12.70 & $<4$ & $<4$ & 1.09 & 19 & 14 & 0.540 & 11820 & 6 & 0.115 & 8 & 15 & 13 & 0.015 & 2630 & 3 & $<50$ & 18 & $<40$ \\
\hline T98C-22(0-4) & $<2$ & 1.90 & 19 & $<4$ & 1.35 & 22 & 20 & 0.425 & 595 & 4 & 0.830 & 8 & 18 & 13 & 0.120 & 1550 & 8 & $<50$ & 112 & $<40$ \\
\hline T98C-22(18-22) & 2 & 3.56 & 12 & $<4$ & 1.84 & 45 & 40 & 0.610 & 542 & 5 & 0.560 & 11 & 36 & 22 & 0.100 & 2660 & 12 & $<50$ & 63 & $<40$ \\
\hline T98C-22 & $<2$ & 2.18 & 17 & $<4$ & 1.77 & 39 & 35 & 0.540 & 198 & 4 & 0.525 & 5 & 34 & 19 & 0.070 & 35 & 10 & $<50$ & 59 & $<40$ \\
\hline T98C-22( & $<2$ & 2.46 & 17 & $<4$ & 1.91 & 42 & 36 & 0.560 & 147 & 3 & 0.570 & 5 & 34 & 17 & 0.065 & 26 & 10 & $<50$ & 68 & $<40$ \\
\hline T98C-22 & $<2$ & 2.53 & 23 & $<4$ & 2.07 & 47 & 44 & 0.695 & 104 & 3 & 0.625 & 8 & 40 & 21 & 0.040 & 33 & 12 & $<50$ & 73 & $<40$ \\
\hline T98C-22 & $<2$ & 2.37 & 20 & $<4$ & 2.05 & 45 & 39 & 0.670 & 93 & 3 & 0.690 & 5 & 38 & 17 & 0.025 & 22 & 10 & $<50$ & 81 & $<40$ \\
\hline T98R-23(0-4) & $<2$ & 5.46 & 16 & $<4$ & 1.57 & 23 & 25 & 0.515 & 400 & 6 & 0.825 & 11 & 24 & 20 & 0.175 & 3400 & 8 & $<50$ & 108 & $<40$ \\
\hline T98R-23 & $<2$ & 6.05 & 14 & $<4$ & 3.21 & 29 & 30 & 0.450 & 695 & 6 & 0.305 & 11 & 23 & 19 & 0.055 & 5800 & 11 & $<50$ & 42 & $<40$ \\
\hline T98R-23(28-32) & $<2$ & 2.72 & 29 & $<4$ & 1.84 & 34 & 40 & 0.660 & 447 & 5 & 0.540 & 10 & 30 & 23 & 0.065 & 43 & 12 & $<50$ & 71 & $<40$ \\
\hline T98R-23(38-42) & $<2$ & 2.35 & 29 & $<4$ & 2.24 & 43 & 39 & 0.720 & 537 & 4 & 0.600 & 4 & 31 & 23 & 0.010 & 28 & 12 & $<50$ & 80 & $<40$ \\
\hline T98R-24(0-4) & $<2$ & 2.25 & 14 & $<4$ & 1.03 & 14 & 17 & 0.645 & 12730 & 5 & 1.845 & $\overline{6}$ & 16 & 43 & 0.100 & 242 & $\overline{6}$ & $<50$ & 238 & $<40$ \\
\hline T98R-24(18-22) & 3 & 10.60 & $<4$ & $<4$ & 1.06 & 24 & 11 & 0.435 & 8660 & 5 & 0.125 & 7 & 16 & 10 & 0.015 & 1890 & 3 & $<50$ & 16 & $<40$ \\
\hline T98R-24(38-42) & 2 & 10.30 & 5 & $<4$ & 1.12 & 24 & 12 & 0.495 & 8800 & 6 & 0.155 & $<4$ & 18 & 11 & 0.020 & 2330 & 4 & $<50$ & 23 & $<40$ \\
\hline T98R-24(58-62) & 2 & 10.40 & $<4$ & $<4$ & 1.37 & 23 & 14 & 0.515 & 9450 & $\overline{7}$ & 0.160 & 5 & 18 & 19 & 0.020 & 3260 & $\overline{4}$ & $<50$ & 26 & $<40$ \\
\hline T98R-24(78-82) & 2 & 10.70 & 13 & $<4$ & 2.07 & 30 & 20 & 0.610 & 10040 & 9 & 0.175 & 6 & 26 & 29 & 0.030 & 5840 & 7 & $<50$ & 30 & $<40$ \\
\hline T98C-25(0-4) & $<2$ & 2.91 & 20 & $<4$ & 1.93 & 30 & 34 & 0.630 & 1030 & 4 & 1.055 & 6 & 27 & 16 & 0.095 & 839 & 10 & $<50$ & 129 & $<40$ \\
\hline T98C-25(18-22) & $<2$ & 2.64 & 18 & $<4$ & 2.12 & 44 & 42 & 0.645 & 669 & 5 & 0.625 & 7 & 39 & 19 & 0.075 & 325 & 10 & $<50$ & 57 & $<40$ \\
\hline
\end{tabular}

*Other analyses (Appendix \#): D =USGS-EDXRF, E =EWU,

$\mathrm{F}=$ CHEMEX, $\mathrm{H}=\mathrm{ACZ},---=$ none. 
Appendix G. Chemical composition of samples analyzed by ICP-AES at XRAL Labs in Golden, CO using 4 acid dissolution (Appendix_G.xls, Appendix_G.dbf).

\begin{tabular}{|c|c|c|c|c|c|c|c|}
\hline Sample No. & $\begin{array}{c}\text { Th } \\
\text { (ppm) }\end{array}$ & $\begin{array}{l}\mathrm{Ti} \\
(\%)\end{array}$ & $\begin{array}{c}U \\
(p p m)\end{array}$ & $\begin{array}{c}\mathrm{V} \\
(\mathrm{ppm})\end{array}$ & $\begin{array}{c}\text { Y } \\
\text { (ppm) }\end{array}$ & $\begin{array}{c}\mathrm{Yb} \\
(\mathrm{ppm})\end{array}$ & $\begin{array}{c}\mathrm{Zn} \\
\text { (ppm) }\end{array}$ \\
\hline SAMPL & PPM & TI_\% & U_PPM & V_PPM & Y_PPM & YB_PPM & ZN_PPM \\
\hline T98C-18(1 & 16 & 0.244 & $<100$ & 55 & 19 & 2 & 188 \\
\hline T98C-18(128-1 & 16 & 0.294 & $<100$ & 56 & 21 & 2 & 176 \\
\hline T98C-18(158-162) & 15 & 0.281 & $<100$ & 58 & 20 & 2 & 183 \\
\hline T98C-18(173-177) & 15 & 0.281 & $<100$ & 58 & 20 & 2 & 179 \\
\hline T98C-20(0-4) & $\overline{9}$ & 0.256 & $<100$ & 55 & 14 & 2 & 857 \\
\hline T98C-20(18-22) & 14 & 0.219 & $<100$ & 56 & 20 & 3 & 3140 \\
\hline T98C-20(38-42) & 14 & 0.25 & $<100$ & 55 & 17 & 2 & 1850 \\
\hline T98C-20(43-47) & 15 & 0.294 & $<100$ & 65 & 20 & 2 & 509 \\
\hline T98C-21(0-4) & 13 & 0.219 & $<100$ & 59 & 17 & 2 & 1370 \\
\hline T98C-21(18-22) & 13 & 0.231 & $<100$ & 59 & 20 & 2 & 1610 \\
\hline T98C-21(38-42) & 11 & 0.106 & $<100$ & 32 & 12 & 1 & 2010 \\
\hline T98C-21(58-62) & 12 & 0.15 & $<100$ & 30 & 15 & $<1$ & 3270 \\
\hline T98C-21(78-82) & 11 & 0.106 & $<100$ & 28 & 8 & $<1$ & 2440 \\
\hline T98C-21(108-112) & 11 & 0.125 & $<100$ & 29 & 11 & $<1$ & 2660 \\
\hline T98C-21(128-132) & 11 & 0.106 & $<100$ & 26 & 9 & $<1$ & 2690 \\
\hline T98C-21(153-157) & 10 & 0.131 & $<100$ & 26 & 10 & $<1$ & 2300 \\
\hline T98C-21(183-187) & 9 & 0.081 & $<100$ & 25 & 8 & $<1$ & 2320 \\
\hline T98C-21( & 11 & 0.063 & $<100$ & 22 & 7 & $<1$ & 2360 \\
\hline T98C-22(C & 6 & 0.225 & $<100$ & 45 & 14 & 1 & 437 \\
\hline T98C-22(18-22) & 15 & 0.294 & $<100$ & 62 & 24 & 3 & 659 \\
\hline T98C-22( & 13 & 0.275 & $<100$ & 56 & 20 & 2 & 228 \\
\hline T98C-22 & 12 & 0.263 & $<100$ & 60 & 22 & 2 & 151 \\
\hline T98C-22 & 14 & 0.281 & $<100$ & 69 & 21 & 2 & 83 \\
\hline T98C-22 & 14 & 0.294 & $<100$ & 59 & 18 & 2 & 61 \\
\hline T98R-2 & 9 & 0.206 & $<100$ & 50 & 17 & 2 & 2020 \\
\hline T98R-2 & 12 & 0.181 & $<100$ & 61 & 13 & 2 & 2420 \\
\hline T98R-2 & 11 & 0.263 & $<100$ & 59 & 19 & 2 & 1240 \\
\hline T98R-2i & 13 & 0.281 & $<100$ & 70 & 21 & 2 & 214 \\
\hline T98R-24(C & $<6$ & 0.275 & $<100$ & 42 & 12 & 1 & 5430 \\
\hline T98R-24 & 9 & 0.075 & $<100$ & 21 & 7 & $<1$ & 1150 \\
\hline T98R-24( & 11 & 0.081 & $<100$ & 24 & 8 & $<1$ & 1480 \\
\hline T98R-24( & 9 & 0.081 & $<100$ & 27 & 9 & $<1$ & 2860 \\
\hline T98R-24 & 10 & 0.106 & $<100$ & 39 & 11 & 1 & 5090 \\
\hline T98C-25 & 8 & 0.263 & $<100$ & 57 & 15 & 2 & 770 \\
\hline T98C-25(18-22) & 13 & 0.256 & $<100$ & 54] & 19 & 2 & 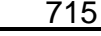 \\
\hline
\end{tabular}

*Other analyses (Appendix \#): D =USGS-EDXRF, E =EWU,

$\mathrm{F}=$ CHEMEX, $\mathrm{H}=\mathrm{ACZ},----=$ none. 
Appendix G. Chemical composition of samples analyzed by ICP-AES at XRAL Labs in Golden, CO using 4 acid dissolution (Appendix_G.xls, Appendix_G.dbf).

\begin{tabular}{|c|c|c|c|c|c|c|c|c|c|c|c|c|c|c|c|c|c|}
\hline Sample No. & Site ID & $\begin{array}{c}\text { Depth } \\
\text { interval, } \\
\text { top }(\mathrm{cm})\end{array}$ & $\begin{array}{c}\text { Depth } \\
\text { interval, } \\
\text { bottom }(\mathrm{cm})\end{array}$ & $\begin{array}{c}{ }^{*} \text { Other } \\
\text { Analyses } \\
\text { (appendix \#) }\end{array}$ & $\begin{array}{c}\mathrm{Ag} \\
(\mathrm{ppm})\end{array}$ & $\begin{array}{c}\mathrm{Al} \\
(\%)\end{array}$ & $\begin{array}{c}\text { As } \\
\text { (ppm) }\end{array}$ & $\mid \begin{array}{c}\mathrm{Au} \\
(\mathrm{ppm})\end{array}$ & $\begin{array}{c}\mathrm{Ba} \\
(\mathrm{ppm})\end{array}$ & $\left|\begin{array}{c}\mathrm{Be} \\
(\mathrm{ppm})\end{array}\right|$ & $\begin{array}{c}\mathrm{Bi} \\
(\mathrm{ppm})\end{array}$ & $\begin{array}{l}\mathrm{Ca} \\
(\%)\end{array}$ & $\begin{array}{c}\text { Cd } \\
(p p m)\end{array}$ & $\begin{array}{c}\mathrm{Ce} \\
(\mathrm{ppm})\end{array}$ & $\begin{array}{c}\text { Co } \\
\text { (ppm) }\end{array}$ & $\begin{array}{c}\mathrm{Cr} \\
(\mathrm{ppm})\end{array}$ & $\begin{array}{c}\mathrm{Cu} \\
(\mathrm{ppm})\end{array}$ \\
\hline SAMPLE_NO. & SITE_ID & IT_TOP_CM & INT_BTM_CM & OTHR_ANLYS & \begin{tabular}{|l|} 
AG_PPM \\
\end{tabular} & AL_(\%) & AS_PPM & AU_PPM & BA_PPM & BE_PPM & BI_PPM & CA_(\%) & CD_PPM & CE_PPM & CO_PPM & CR_PPM & CU_PPM \\
\hline T98C-25(38-42) & $98 C-25$ & 38 & 42 & ---- & $<2$ & 6.8 & 13 & $<8$ & 637 & 2 & $<50$ & 0.267 & $<2$ & 84 & 10 & 92 & 19 \\
\hline T98C-25(58-62) & $98 \mathrm{C}-25$ & 58 & 62 & ---- & $<2$ & 7.4 & 14 & $<8$ & 689 & 2 & $<50$ & 0.229 & $<2$ & 99 & 11 & 100 & 20 \\
\hline T98C-25(78-82) & T98C-25 & 78 & 82 & ---- & $<2$ & 6.5 & 13 & $<8$ & 584 & 2 & $<50$ & 0.208 & $<2$ & 90 & 10 & 48 & 18 \\
\hline T98C-25(98-102) & $98 C-25$ & 98 & 102 & ---- & $<2$ & 6.0 & 16 & $<8$ & 556 & 2 & $<50$ & 0.219 & $<2$ & 92 & 8 & 80 & 14 \\
\hline T98C-25(118-122) & T98C-25 & 118 & 122 & $-\overline{---}$ & $<2$ & 5.7 & 10 & $<8$ & 513 & 2 & $<50$ & 0.213 & $<2$ & 86 & 8 & 66 & 14 \\
\hline T98C-25(138-142) & T98C-25 & 138 & 142 & ---- & $<2$ & 6.0 & 13 & $<8$ & 529 & 2 & $<50$ & 0.213 & $<2$ & 90 & 8 & 88 & 15 \\
\hline T98C-25(153-157) & T98C-25 & 153 & 157 & ---- & $<2$ & 6.6 & 12 & $<8$ & 554 & 2 & $<50$ & 0.224 & $<2$ & 87 & 8 & 107 & 17 \\
\hline T98C-25(173-177) & T98C-25 & 173 & 177 & ---- & $<2$ & 7.3 & 12 & $<8$ & 586 & 2 & $<50$ & 0.219 & $<2$ & 96 & 6 & 96 & 17 \\
\hline T98C-26(0-4) & T98C-26 & 0 & 4 & ---- & $<2$ & 6.5 & 20 & $<8$ & 601 & 2 & $<50$ & 0.467 & 6 & 100 & 10 & 64 & 35 \\
\hline T98C-26(18-22) & T98C-26 & 18 & 22 & ---- & 4 & 7.8 & 23 & $<8$ & 772 & 3 & $<50$ & 0.415 & 5 & 119 & 8 & 46 & 52 \\
\hline T98C-26(38-42) & T98C-26 & 38 & 42 & ---- & $<2$ & 7.7 & $<10$ & $<8$ & 677 & 2 & $<50$ & 0.368 & $<2$ & 109 & 7 & 86 & 24 \\
\hline T98R-27(0-4) & T98R-27 & 0 & 4 & ---- & 8 & 5.9 & 58 & $<8$ & 673 & 2 & $<50$ & 0.798 & 16 & 64 & 10 & 71 & 65 \\
\hline T98R-27(8-12) & T98R-27 & 8 & 12 & --- & 29 & 5.2 & 42 & $<8$ & 866 & 2 & $<50$ & 0.305 & 9 & 78 & 6 & 53 & 184 \\
\hline T98R-27(18-22) & T98R-27 & 18 & 22 & ---- & $<2$ & 6.2 & $<10$ & $<8$ & 653 & 2 & $<50$ & 0.326 & 4 & 73 & 4 & 36 & 16 \\
\hline T98R-27(38-42) & T98R-27 & 38 & 42 & $\overline{----}$ & $<2$ & 5.8 & $<10$ & $<8$ & 567 & 2 & $<50$ & 0.273 & $<2$ & 82 & 4 & 45 & 11 \\
\hline T98R-27(58-62) & T98R-27 & 58 & 62 & ---- & $<2$ & 6.0 & $<10$ & $<8$ & 504 & 2 & $<50$ & 0.278 & $<2$ & 90 & 7 & 52 & 11 \\
\hline T98R-27(78-82) & T98R-27 & 78 & 82 & ---- & $<2$ & 6.3 & 10 & $<8$ & 575 & 2 & $<50$ & 0.406 & $<2$ & 87 & 7 & 40 & 16 \\
\hline T98R-27(96-100) & T98R-27 & 96 & 100 & ---- & $<2$ & 7.0 & 13 & $<8$ & 624 & 2 & $<50$ & 0.588 & $<2$ & 90 & 9 & 39 & 24 \\
\hline T98R-27(118-122) & T98R-27 & 118 & 122 & --- & $<2$ & 7.4 & 16 & $<8$ & 685 & 2 & $<50$ & 0.802 & $<2$ & 88 & 11 & 34 & 28 \\
\hline T98R-27(138-142) & T98R-27 & 138 & 142 & ---- & $<2$ & 5.1 & $<10$ & $<8$ & 473 & 2 & $<50$ & 0.181 & $<2$ & 92 & 10 & 88 & 13 \\
\hline T98R-27(163-167) & T98R-27 & 163 & 167 & ---- & $<2$ & 5.1 & 55 & $<8$ & 455 & 2 & $<50$ & 0.192 & $<2$ & 87 & 11 & 39 & 12 \\
\hline T98R-27(178-182) & T98R-27 & 178 & 182 & ---- & $<2$ & 4.5 & 48 & $<8$ & 468 & 2 & $<50$ & 0.165 & $<2$ & 87 & 18 & 50 & 9 \\
\hline T98R-28(0-4) & T98R-28 & 0 & 4 & $-\cdots$ & 18 & 4.7 & 82 & $<8$ & 697 & 2 & $<50$ & 0.525 & 14 & 56 & 6 & 59 & 99 \\
\hline T98R-28(8-12) & T98R-28 & 8 & 12 & ---- & 26 & 4.9 & 107 & $<8$ & 925 & 2 & $<50$ & 0.215 & 9 & 77 & 8 & 56 & 154 \\
\hline T98R-28(18-22) & T98R-28 & 18 & 22 & ---- & 17 & 5.2 & 36 & $<8$ & 710 & 2 & $<50$ & 0.315 & 14 & 67 & 9 & 71 & 108 \\
\hline T98R-28(38-42) & T98R-28 & 38 & 42 & ---- & $<2$ & 6.6 & $<10$ & $<8$ & 498 & 2 & $<50$ & 0.173 & 13 & 75 & 3 & 80 & 29 \\
\hline T98R-28(58-62) & T98R-28 & 58 & 62 & ---- & $<2$ & 6.8 & $<10$ & $<8$ & 589 & 2 & $<50$ & 0.168 & 4 & 85 & 3 & 43 & 20 \\
\hline T98R-28(78-82) & T98R-28 & 78 & 82 & $-\cdots$ & $<2$ & 6.0 & 10 & $<8$ & 503 & 2 & $<50$ & 0.165 & $<2$ & 74 & 5 & 68 & 9 \\
\hline T98R-28(88-92) & T98R-28 & 88 & 92 & ---- & \begin{tabular}{l|l}
$<2$ \\
\end{tabular} & 7.1 & $<10$ & $<8$ & 606 & 2 & $<50$ & 0.187 & $<2$ & 98 & 4 & 98 & 11 \\
\hline T98R-28 & T98R-28 & 108 & 112 & ---- & $<2$ & 6.7 & $<10$ & $<8$ & 567 & 2 & $<50$ & 0.197 & $<2$ & 96 & 5 & 54 & 12 \\
\hline T98R-28(133-137) & T98R-28 & 133 & 137 & --- & $<2$ & 6.8 & $<10$ & $<8$ & 573 & 2 & $<50$ & 0.256 & $<2$ & 94 & 6 & 87 & 18 \\
\hline T98R-29(0-4) & T98R-29 & 0 & 4 & --- & 16 & 5.6 & 43 & $<8$ & 602 & 2 & $<50$ & 0.683 & 15 & 57 & 5 & 72 & 90 \\
\hline T98R-29| & T98R-29 & 18 & 22 & --- & 84 & 5.1 & 80 & $<8$ & 1720 & 2 & $<50$ & 0.158 & 6 & 72 & 7 & 75 & 495 \\
\hline$\overline{T 98 R-29}$ & T98R-29 & 38 & 42 & $\overline{----}$ & $<2$ & 6.9 & $<10$ & $<8$ & 652 & 2 & $<50$ & 0.226 & 3 & 91 & 5 & 87 & $\overline{18}$ \\
\hline T98R-29(68-72) & T98R-29 & 68 & 72 & ---- & $<2$ & 6.6 & $<10$ & $<8$ & 592 & 2 & $<50$ & 0.289 & 3 & 85 & 5 & 22 & 16 \\
\hline
\end{tabular}

*Other analyses (Appendix \#): D =USGS-EDXRF, E =EWU,

$\mathrm{F}=\mathrm{CHEMEX}, \mathrm{H}=\mathrm{ACZ},-\cdots=$ none. 
Appendix G. Chemical composition of samples analyzed by ICP-AES at XRAL Labs in Golden, CO using 4 acid dissolution (Appendix_G.xls, Appendix_G.dbf).

\begin{tabular}{|c|c|c|c|c|c|c|c|c|c|c|c|c|c|c|c|c|c|c|c|c|}
\hline Sample No. & $\begin{array}{c}\mathrm{Eu} \\
(\mathrm{ppm})\end{array}$ & $\begin{array}{l}\mathrm{Fe} \\
(\%)\end{array}$ & $\begin{array}{c}\mathrm{Ga} \\
(\mathrm{ppm})\end{array}$ & $\begin{array}{c}\mathrm{Ho} \\
(\mathrm{ppm})\end{array}$ & $\begin{array}{c}\mathrm{K} \\
(\%)\end{array}$ & $\begin{array}{c}\mathrm{La} \\
(\mathrm{ppm})\end{array}$ & $\begin{array}{c}\mathrm{Li} \\
(\mathrm{ppm})\end{array}$ & $\begin{array}{l}\mathrm{Mg} \\
(\%)\end{array}$ & $\begin{array}{c}\mathrm{Mn} \\
(\mathrm{ppm})\end{array}$ & $\begin{array}{c}\text { Mo } \\
(\mathrm{ppm})\end{array}$ & $\begin{array}{l}\mathrm{Na} \\
(\%)\end{array}$ & $\begin{array}{c}\mathrm{Nb} \\
(\mathrm{ppm})\end{array}$ & $\begin{array}{c}\mathrm{Nd} \\
(\mathrm{ppm})\end{array}$ & $\begin{array}{c}\mathrm{Ni} \\
(p p m)\end{array}$ & $\begin{array}{c}\mathrm{P} \\
(\mathrm{ppm})\end{array}$ & $\begin{array}{c}\mathrm{Pb} \\
(\mathrm{ppm})\end{array}$ & $\begin{array}{c}\mathrm{Sc} \\
(\mathrm{ppm})\end{array}$ & $\begin{array}{c}\mathrm{Sn} \\
(\mathrm{ppm})\end{array}$ & $\begin{array}{c}\mathrm{Sr} \\
(\mathrm{ppm})\end{array}$ & $\begin{array}{c}\mathrm{Ta} \\
(\mathrm{ppm})\end{array}$ \\
\hline SAMPLE_NO. & PPM & (\%) & GA_PPM & HO_PPM & K_(\%) & LA_PPM & LI_PPM & MG_(\%) & MN_PPM & MO_PPM & NA_(\%) & NB_PPM & ND_PPM & NI_PPM & P_PPM & PB_PPM & SC_PPM & SN_PPM & SR_PPM & TA_PPM \\
\hline T98C-25(38-42) & $<2$ & 2.81 & 20 & $<4$ & 2.17 & 46 & 44 & .715 & 628 & 4 & 0.580 & 10 & 43 & 22 & 0.075 & 39 & 12 & $<50$ & 54 & $<40$ \\
\hline T98C-25(58-62) & 2 & 3.04 & 19 & $<4$ & 2.34 & 51 & 49 & 0.820 & 629 & 4 & 0.630 & 5 & 47 & 22 & 0.060 & 26 & 13 & $<50$ & 54 & $<40$ \\
\hline T98C-25(78-82) & $<2$ & 2.62 & 23 & $<4$ & 2.15 & 46 & 42 & 0.685 & 453 & 3 & 0.615 & 5 & 40 & 18 & 0.050 & 31 & 11 & $<50$ & 53 & $<40$ \\
\hline T98C-25(98-102) & $<2$ & 2.33 & 17 & $<4$ & 2.13 & 48 & 39 & 0.625 & 360 & 3 & 0.635 & $<4$ & 43 & 15 & 0.040 & 34 & 10 & $<50$ & 56 & $<40$ \\
\hline T98C-25(118-122) & $<2$ & 2.17 & 23 & $<4$ & 2.07 & 45 & 36 & 0.600 & 308 & 3 & 0.615 & 5 & 38 & 15 & 0.035 & 16 & $\overline{9}$ & $<50$ & 55 & $<40$ \\
\hline T98C-25(138-142) & $<2$ & 2.40 & 24 & $<4$ & 2.14 & 46 & 38 & 0.625 & 397 & 3 & 0.625 & $<4$ & 41 & 16 & 0.040 & 16 & 10 & $<50$ & 57 & $<40$ \\
\hline T98C-25(153-157) & $<2$ & 2.58 & 22 & $<4$ & 2.24 & 45 & 42 & 0.685 & 263 & 4 & 0.630 & 5 & 39 & 17 & 0.055 & 17 & 11 & $<50$ & 55 & $<40$ \\
\hline T98C-25(173-177) & $<2$ & 2.26 & 26 & $<4$ & 2.35 & 50 & 45 & 0.745 & 102 & 5 & 0.640 & $<4$ & 45 & 20 & 0.050 & 18 & 12 & $<50$ & 56 & $<40$ \\
\hline T98C-26(0-4) & 2 & 3.26 & 14 & $<4$ & 2.01 & 46 & 26 & 0.535 & 847 & 6 & 0.705 & 5 & 45 & 18 & 0.095 & 491 & 12 & $<50$ & 86 & $<40$ \\
\hline T98C-26(18-22) & 3 & 3.04 & 28 & $<4$ & 2.03 & 56 & 31 & 0.595 & 420 & 5 & 0.695 & 5 & 55 & 22 & 0.080 & 1950 & 15 & $<50$ & 90 & $<40$ \\
\hline T98C-26(38-42) & $<2$ & 2.78 & 26 & $<4$ & 1.72 & 54 & 28 & 0.535 & 288 & 5 & 0.605 & 9 & 52 & 23 & 0.085 & 49 & 14 & $<50$ & 81 & $<40$ \\
\hline T98R-27(0-4) & 2 & 5.23 & 20 & $<4$ & 1.91 & 31 & 28 & 0.645 & 4240 & 5 & 0.910 & 9 & 27 & 10 & 0.085 & 2540 & 10 & $<50$ & 107 & $<40$ \\
\hline T98R-27(8-12) & 2 & 6.03 & 7 & $<4$ & 1.95 & 37 & 27 & 0.515 & 4200 & 5 & 0.560 & 11 & 37 & 8 & 0.055 & 10930 & 9 & $<50$ & 62 & $<40$ \\
\hline T98R-27(18-22) & $<2$ & 1.92 & 24 & $<4$ & 2.28 & 42 & 34 & 0.645 & 241 & 4 & 0.715 & 4 & 35 & 11 & 0.045 & 191 & 10 & $<50$ & 72 & $<40$ \\
\hline T98R-27(38-42) & $<2$ & 2.04 & 21 & $<4$ & 2.28 & 44 & 33 & 0.650 & 118 & 3 & 0.630 & $<4$ & 39 & 11 & 0.020 & 24 & 10 & $<50$ & 66 & $<40$ \\
\hline T98R-27(58-62) & $<2$ & 2.29 & 21 & $<4$ & 2.16 & 44 & 35 & 0.610 & 142 & 2 & 0.640 & 6 & 36 & 14 & 0.010 & 17 & 9 & $<50$ & 68 & $<40$ \\
\hline T98R-27(78-82) & $<2$ & 2.20 & 27 & $<4$ & 2.12 & 45 & 35 & 0.625 & 187 & 3 & 0.795 & 4 & 39 & 15 & 0.010 & 15 & 10 & $<50$ & 91 & $<40$ \\
\hline T98R-27(96-100) & 2 & 2.47 & 23 & $<4$ & 2.16 & 47 & 37 & 0.620 & 385 & 3 & 1.090 & $<4$ & 40 & 17 & 0.015 & 14 & 10 & $<50$ & 128 & $<40$ \\
\hline T98R-27(118-122) & $<2$ & 2.71 & 26 & $<4$ & 1.88 & 39 & 36 & 0.560 & 717 & 4 & 1.450 & 6 & 34 & 21 & 0.020 & 11 & 10 & $<50$ & 172 & $<40$ \\
\hline T98R-27(1 & $<2$ & 2.00 & 15 & $<4$ & 2.10 & 47 & 31 & 0.545 & 252 & 2 & 0.500 & 6 & 40 & 12 & 0.015 & 10 & 8 & $<50$ & 50 & $<40$ \\
\hline T98R-27( & $<2$ & 3.98 & 13 & $<4$ & 1.99 & 46 & 29 & 0.525 & 307 & 3 & 0.470 & 5 & 41 & 16 & 0.025 & 8 & 8 & $<50$ & 50 & $<40$ \\
\hline T98R-27( & $<2$ & 6.89 & 8 & $<4$ & 1.78 & 43 & 26 & 0.485 & 1590 & 4 & 0.405 & 11 & 37 & 12 & 0.035 & 8 & 7 & $<50$ & 44 & $<40$ \\
\hline T98R-28( & 2 & 6.99 & $<4$ & $<4$ & 1.56 & 27 & 23 & 0.535 & 3710 & 4 & 0.630 & 9 & 27 & 8 & 0.095 & 6130 & 8 & $<50$ & 76 & $<40$ \\
\hline T98R-28( & 3 & 9.94 & 5 & $<4$ & 1.92 & 36 & 27 & 0.575 & 6930 & 8 & 0.350 & 11 & 33 & 8 & 0.050 & 9810 & 9 & $<50$ & 40 & $<40$ \\
\hline T98R-28( & $<2$ & 4.79 & 9 & $<4$ & 1.63 & 30 & 23 & 0.420 & 2740 & 4 & 0.530 & 10 & 30 & 13 & 0.070 & 6490 & 10 & $<50$ & 56 & $<40$ \\
\hline T98R-28 & $<2$ & 1.32 & 27 & $<4$ & 1.68 & 33 & 29 & 0.385 & 181 & 4 & 0.445 & 4 & 31 & 21 & 0.115 & 163 & 13 & $<50$ & 45 & $<40$ \\
\hline T98R-28( & $<2$ & 1.48 & 27 & $<4$ & 2.06 & 40 & 36 & 0.540 & 151 & 4 & 0.555 & 1 & 37 & 15 & 0.050 & 87 & 13 & $<50$ & 52 & $<40$ \\
\hline T98R-28(7) & $<2$ & 1.38 & 26 & $<4$ & 1.86 & 37 & 35 & 0.485 & 136 & 5 & 0.545 & 8 & 34 & 17 & 0.015 & 33 & 10 & $<50$ & 53 & $<40$ \\
\hline T98R-28(88-92) & $<2$ & 1.76 & 29 & $<4$ & 2.23 & 50 & 42 & 0.610 & 110 & 4 & 0.635 & 8 & 45 & 16 & 0.015 & 19 & 12 & $<50$ & 60 & $<40$ \\
\hline T98R-28(108-112) & $<2$ & 1.71 & 26 & $<4$ & 2.13 & 44 & 40 & 0.560 & 75 & 3 & 0.640 & 5 & 39 & 14 & 0.010 & 18 & 11 & $<50$ & 61 & $<40$ \\
\hline T98R-28(1 & $<2$ & 1.87 & 28 & $<4$ & 2.15 & 46 & 39 & 0.595 & 86 & 3 & 0.700 & 5 & 40 & 17 & 0.015 & 27 & 11 & $<50$ & 71 & $<40$ \\
\hline T98R-29(0-4) & $<2$ & 3.79 & 14 & $<4$ & 1.70 & 26 & 24 & 0.480 & 2520 & 5 & 0.800 & 11 & 26 & 13 & 0.095 & 4950 & 9 & $<50$ & 98 & $<40$ \\
\hline T98R-29(18-22) & 4 & 10.70 & 11 & $<4$ & 1.97 & 34 & 26 & 0.435 & 6030 & 10 & 0.325 & 8 & 35 & 3 & 0.050 & 30680 & 9 & $<50$ & 36 & $<40$ \\
\hline T98R-29( & $<2$ & 2.40 & 26 & $<4$ & 2.35 & 46 & 40 & 0.760 & 312 & 4 & 0.650 & 6 & 42 & 15 & 0.060 & 343 & 13 & $<50$ & 58 & $<40$ \\
\hline T98R-29(68-72) & $<2$ & 2.27 & 18 & $<4$ & 2.29 & 44 & 38 & 0.705 & 327 & 4 & 0.745 & 51 & 39 & 13 & 0.035 & 231 & 12 & $<50$ & 67 & $<40$ \\
\hline
\end{tabular}

*Other analyses (Appendix \#): D =USGS-EDXRF, E =EWU,

$\mathrm{F}=\mathrm{CHEMEX}, \mathrm{H}=\mathrm{ACZ},---=$ none. 
Appendix G. Chemical composition of samples analyzed by ICP-AES at XRAL Labs in Golden, CO using 4 acid dissolution (Appendix_G.xls, Appendix_G.dbf).

\begin{tabular}{|c|c|c|c|c|c|c|c|}
\hline e No. & $\begin{array}{c}\text { Th } \\
(\mathrm{ppm})\end{array}$ & $\begin{array}{c}\mathrm{Ti} \\
(\%)\end{array}$ & $\begin{array}{c}\mathrm{U} \\
(\mathrm{ppm})\end{array}$ & $\begin{array}{c}\mathrm{V} \\
(\mathrm{ppm})\end{array}$ & $\begin{array}{c}\mathrm{Y} \\
(\mathrm{ppm})\end{array}$ & $\begin{array}{c}\mathrm{Yb} \\
(\mathrm{ppm})\end{array}$ & $\begin{array}{c}\mathrm{Zn} \\
(\mathrm{ppm})\end{array}$ \\
\hline SAMPLE_NO. & PPM & TI_\% & \begin{tabular}{l|l} 
U_PPM \\
\end{tabular} & V_PPM & $\overline{Y P P P M}$ & YB_PPM & ZN_PPM \\
\hline $98 C-25(38-42)$ & 15 & 0.263 & $<100$ & 60 & 22 & 2 & 545 \\
\hline T98C-25(58-62) & 17 & 0.319 & $<100$ & 69 & 26 & 3 & 131 \\
\hline T98C-25(78-82) & 13 & 0.256 & $<100$ & 58 & 20 & 2 & 72 \\
\hline T98C-25(98-102) & 13 & 0.281 & $<100$ & 53 & 20 & 2 & 68 \\
\hline T98C-25(118-122) & 15 & 0.275 & $<100$ & 50 & 18 & 2 & 53 \\
\hline T98C-25(138-142) & 15 & 0.275 & $<100$ & 53 & 19 & 2 & 54 \\
\hline T98C-25(153-157) & 15 & 0.288 & $<100$ & 59 & 20 & 2 & 58 \\
\hline T98C-25(173-177) & 15 & 0.306 & $<100$ & 60 & 20 & 2 & 60 \\
\hline T98C-26(0-4) & 15 & 0.288 & $<100$ & 9 & 21 & 3 & 435 \\
\hline T98C-26(18-22) & 19 & 0.364 & $<100$ & 75 & 27 & 3 & 604 \\
\hline T98C-26(38-42) & 17 & 0.37 & $<100$ & 69 & 26 & 3 & 258 \\
\hline T98R-27(0-4) & 8 & 0.253 & $<100$ & 56 & 16 & 2 & 1230 \\
\hline T98R-27(8-12) & 12 & 0.241 & $<100$ & 48 & 13 & 2 & 1130 \\
\hline T98R-27 & 10 & 0.306 & $<100$ & 53 & 12 & 2 & 293 \\
\hline$\overline{T 98 R-27}$ & 14 & 0.282 & $<100$ & 52 & 14 & 2 & 160 \\
\hline T98R-27 & 13 & 0.269 & $<100$ & 55 & 14 & 2 & 118 \\
\hline T98R-27 & 13 & 0.294 & $<100$ & 55 & 17 & 2 & 133 \\
\hline T98R-2 & 13 & 0.313 & $<100$ & 55 & 20 & 2 & 135 \\
\hline T98R-2 & 11 & 0.344 & $<100$ & 51 & 25 & 2 & 185 \\
\hline T98R-27 & 16 & 0.256 & $<100$ & 49 & 17 & 2 & 91 \\
\hline T98R-27 & 13 & 0.275 & $<100$ & 47 & 17 & 2 & 97 \\
\hline T98R-27 & 14 & 0.219 & $<100$ & 43 & 14 & 2 & 56 \\
\hline T98R-28 & 3 & 0.188 & $<100$ & 44 & 13 & 1 & 2210 \\
\hline T98R-28 & 12 & 0.17 & $<100$ & 45 & 15 & 2 & 2340 \\
\hline T98R-28 & 6 & 0.206 & $<100$ & 44 & 16 & 2 & 984 \\
\hline T98R-28 & 13 & 0.264 & $<100$ & 52 & 21 & 2 & 285 \\
\hline T98R-28 & 14 & 0.294 & $<100$ & 59 & 16 & 2 & 185 \\
\hline T98R-28(7 & 13 & 0.25 & $<100$ & 55 & 13 & 1 & 134 \\
\hline T98R-28(8 & 16 & 0.313 & $<100$ & 65 & 18 & 2 & 157 \\
\hline T98R-28( & 14 & 0.275 & $<100$ & 57 & 18 & 2 & 121 \\
\hline T98R-28( & 13 & 0.275 & $<100$ & 57 & 22 & 2 & 120 \\
\hline T98R-29( & 10 & 0.223 & $<100$ & 49 & 13 & 2 & 1350 \\
\hline T98R-29 & 14 & 0.153 & $<100$ & 44 & 15 & 2 & 2160 \\
\hline T98R-29 & 17 & 0.282 & $<100$ & 60 & 17 & 2 & 319 \\
\hline T98R-29(68-72) & 9 & 0.276 & $<100$ & 56 & 17 & 2 & 236 \\
\hline
\end{tabular}

*Other analyses (Appendix \#): D =USGS-EDXRF, E =EWU,

$\mathrm{F}=$ CHEMEX, $\mathrm{H}=\mathrm{ACZ},---=$ none. 
Appendix G. Chemical composition of samples analyzed by ICP-AES at XRAL Labs in Golden, CO using 4 acid dissolution (Appendix_G.xls, Appendix_G.dbf).

\begin{tabular}{|c|c|c|c|c|c|c|c|c|c|c|c|c|c|c|c|c|c|}
\hline Sample No. & Site ID & $\begin{array}{c}\text { Depth } \\
\text { interval, } \\
\text { top }(\mathrm{cm})\end{array}$ & $\begin{array}{c}\text { Depth } \\
\text { interval, } \\
\text { bottom }(\mathrm{cm})\end{array}$ & $\begin{array}{c}{ }^{*} \text { Other } \\
\text { Analyses } \\
\text { (appendix \#) }\end{array}$ & $\begin{array}{c}\mathrm{Ag} \\
(\mathrm{ppm})\end{array}$ & $\begin{array}{c}\mathrm{Al} \\
(\%)\end{array}$ & $\begin{array}{c}\text { As } \\
\text { (ppm) }\end{array}$ & $\begin{array}{c}\mathrm{Au} \\
(\mathrm{ppm})\end{array}$ & $\begin{array}{c}\mathrm{Ba} \\
(\mathrm{ppm})\end{array}$ & $\left|\begin{array}{c}\mathrm{Be} \\
(\mathrm{ppm})\end{array}\right|$ & $\begin{array}{c}\mathrm{Bi} \\
(\mathrm{ppm})\end{array}$ & $\begin{array}{l}\mathrm{Ca} \\
(\%)\end{array}$ & $\begin{array}{c}\text { Cd } \\
\text { (ppm) }\end{array}$ & $\begin{array}{c}\mathrm{Ce} \\
(\mathrm{ppm})\end{array}$ & $\begin{array}{c}\text { Co } \\
\text { (ppm) }\end{array}$ & $\begin{array}{c}\mathrm{Cr} \\
(\mathrm{ppm})\end{array}$ & $\begin{array}{c}\mathrm{Cu} \\
(\mathrm{ppm})\end{array}$ \\
\hline SAMPLE_NO. & SITE_ID & UT_TOP_CM & INT_BTM_CM & OTHR_ANLYS & AG_PPM & AL_(\%) & AS_PPM & AU_PPM & BA_PPM & BE_PPM & BI_PPM & CA_(\%) & CD_PPM & CE_PPM & CO_PPM & CR_PPM & CU_PPM \\
\hline T98R-29(88-92) & 98R-29 & 88 & 92 & ---- & $<2$ & 6.4 & $<10$ & $<8$ & 536 & 2 & $<50$ & 0.315 & $<2$ & 86 & 5 & 91 & 10 \\
\hline T98R-29(98-102) & T98R-29 & 98 & 102 & ---- & $<2$ & 6.1 & $<10$ & $<8$ & 518 & 2 & $<50$ & 0.262 & $<2$ & 92 & 4 & 72 & 11 \\
\hline T98R-29(118-122) & T98R-29 & 118 & 122 & ---- & $<2$ & 5.9 & 12 & $<8$ & 528 & 2 & $<50$ & 0.267 & $<2$ & 89 & 4 & 57 & 8 \\
\hline T98R-30(0-4) & T98R-30 & 0 & 4 & ---- & 5 & 6.1 & 33 & $<8$ & 617 & 2 & $<50$ & 1.024 & 10 & 60 & 9 & 64 & 48 \\
\hline T98R-30(6-10) & T98R-30 & 6 & 10 & ---- & 12 & 5.2 & 78 & $<8$ & 691 & 2 & $<50$ & 0.462 & 12 & 71 & 8 & 76 & 68 \\
\hline T98R-30(18-22) & T98R-30 & 18 & 22 & ---- & $<2$ & 6.1 & 12 & $<8$ & 720 & 2 & $<50$ & 0.404 & $<2$ & 95 & 6 & 82 & 19 \\
\hline T98R-30(38-42) & T98R-30 & 38 & 42 & ---- & $<2$ & 6.3 & $<10$ & $<8$ & 643 & 2 & $<50$ & 0.352 & $<2$ & 95 & 6 & 69 & 18 \\
\hline T98R-30(58-62) & T98R-30 & 58 & 62 & ---- & $<2$ & 6.5 & 14 & $<8$ & 568 & 2 & $<50$ & 0.326 & $<2$ & 85 & 8 & 92 & 17 \\
\hline T98R-30(78-82) & T98R-30 & 78 & 82 & $-\cdots$ & $<2$ & 6.1 & 15 & $<8$ & 564 & 2 & $<50$ & 0.294 & $<2$ & 95 & 8 & 53 & 18 \\
\hline T98R-31(0-4) & T98R-31 & 0 & 4 & ---- & 6 & 6.6 & 49 & $<8$ & 653 & 2 & $<50$ & 0.368 & 11 & 83 & 9 & 81 & 58 \\
\hline T98R-31(18-22) & 98R-31 & 18 & 22 & ---- & 19 & 3.9 & 333 & $<8$ & 636 & 1 & $<50$ & 0.168 & 23 & 77 & $\overline{14}$ & 53 & 113 \\
\hline T98R-31(38-42) & T98R-31 & 38 & 42 & ---- & 34 & 3.7 & 270 & $<8$ & 947 & 1 & $<50$ & 0.126 & 5 & 72 & 9 & 54 & 184 \\
\hline T98R-31(58-62) & T98R-31 & 58 & 62 & ---- & 34 & 3.6 & 98 & $<8$ & 848 & 1 & $<50$ & 0.105 & 12 & 67 & 11 & 51 & 197 \\
\hline T98R-31(73-77) & T98R-31 & 73 & 77 & ---- & 28 & 3.2 & 140 & $<8$ & 786 & 1 & $<50$ & 0.110 & 24 & 57 & 11 & 53 & 132 \\
\hline T98R-31(108-112) & T98R-31 & 108 & 112 & $\begin{array}{ll}--- \\
-\end{array}$ & 34 & 2.9 & 61 & $<8$ & 1020 & 1 & $<50$ & 0.194 & 15 & 56 & 9 & 49 & 217 \\
\hline T98R-31(133-137) & T98R-31 & 133 & 137 & ---- & 35 & 3.1 & 71 & $<8$ & 1100 & 1 & $<50$ & 0.247 & 21 & 56 & 9 & 53 & 195 \\
\hline T98R-31(158-162) & T98R-31 & 158 & 162 & ---- & 43 & 3.7 & 90 & $<8$ & 1270 & 1 & $<50$ & 0.284 & 31 & 59 & 7 & 55 & 241 \\
\hline T98R-31(173-177) & T98R-31 & 173 & 177 & ---- & 33 & 3.1 & 96 & $<8$ & 1340 & 1 & $<50$ & 0.268 & 21 & 58 & 7 & 55 & 215 \\
\hline T98R-31(188-192) & T98R-31 & 188 & 192 & --- & 38 & 3.4 & 57 & $<8$ & 1020 & 1 & $<50$ & 0.268 & 41 & 54 & 8 & 55 & 277 \\
\hline T98R-32(0-4) & T98R-32 & 0 & 4 & ---- & 8 & 5.5 & 90 & $<8$ & 653 & 2 & $<50$ & 0.315 & 12 & 91 & 11 & 84 & 70 \\
\hline T98R-32(18-22) & T98R-32 & 18 & 22 & ---- & 19 & 3.7 & 444 & $<8$ & 572 & 1 & $<50$ & 0.189 & 29 & 68 & 24 & 60 & 119 \\
\hline T98R-32(38-42) & T98R-32 & 38 & 42 & ---- & 17 & 2.9 & 438 & $<8$ & 421 & $<1$ & $<50$ & 0.168 & 14 & 60 & 12 & 56 & 109 \\
\hline T98R-32(58-62) & T98R-32 & 58 & 62 & ---- & 17 & 3.3 & 733 & $<8$ & 518 & 1 & $<50$ & 0.168 & 10 & 65 & 13 & 55 & 118 \\
\hline T98R-32(78-82) & T98R-32 & 78 & 82 & ---- & 15 & 3.3 & 145 & $<8$ & 574 & 1 & $<50$ & 0.137 & 8 & 63 & 10 & 58 & 102 \\
\hline T98R-32(98-102) & T98R-32 & 98 & 102 & ---- & 20 & 4.1 & 102 & $<8$ & 650 & 1 & $<50$ & 0.168 & 13 & 78 & 9 & 54 & 126 \\
\hline T98R-32(118-122) & T98R-32 & 118 & 122 & ---- & 25 & 3.7 & 84 & $<8$ & 641 & 1 & $<50$ & 0.184 & 34 & 64 & 10 & 56 & 134 \\
\hline T98R-32 & T98R-32 & 138 & 142 & $\begin{array}{ll}--- \\
\end{array}$ & 13 & 3.0 & 85 & $<8$ & 542 & 1 & $<50$ & 0.289 & 28 & 53 & 13 & 62 & 102 \\
\hline T98R-32 & T98R-32 & 158 & 162 & $-\cdots$ & 23 & 3.4 & 220 & $<8$ & 818 & 1 & $<50$ & 0.389 & 34 & 63 & 16 & 58 & 102 \\
\hline T98R-32(178-182) & T98R-32 & 178 & 182 & $\begin{array}{c}--- \\
\end{array}$ & 24 & 3.3 & 179 & $<8$ & 704 & 1 & $<50$ & 0.467 & 34 & 43 & 13 & 54 & 107 \\
\hline T98R-3 & T98R-33 & 0 & 4 & ---- & 14 & 4.5 & 70 & $<8$ & 645 & 1 & $<50$ & 0.305 & 14 & 73 & 8 & 51 & 85 \\
\hline T98R-3 & T98R-33 & 18 & 22 & --- & 20 & 3.5 & 54 & $<8$ & 661 & 1 & $<50$ & 0.084 & 23 & 60 & 8 & 54 & 133 \\
\hline T98R-3 & T98R-33 & 38 & 42 & --- & $<2$ & 5.4 & 13 & $<8$ & 608 & 2 & $<50$ & 0.294 & 27 & 81 & 5 & 75 & 23 \\
\hline T98R-3 & T98R-33 & 58 & 62 & --- & $<2$ & 5.8 & $<10$ & $<8$ & 539 & 2 & $<50$ & 0.215 & $<2$ & 85 & 6 & 29 & 13 \\
\hline T98R-3 & T98R-33 & 78 & 82 & $\overline{----}$ & $<2$ & 5.6 & 71 & $<8$ & 501 & 2 & $<50$ & 0.187 & $<2$ & 86 & 5 & 41 & $\overline{9}$ \\
\hline T98R-33(108-112) & T98R-33 & 108 & 112 & ---- & $<2$ & 5.4 & 11 & $<8$ & 471 & 2 & $<50$ & 0.197 & $<2$ & 85 & 7 & 34 & 13 \\
\hline
\end{tabular}

*Other analyses (Appendix \#): D =USGS-EDXRF, E =EWU,

$\mathrm{F}=\mathrm{CHEMEX}, \mathrm{H}=\mathrm{ACZ},-\cdots=$ none. 
Appendix G. Chemical composition of samples analyzed by ICP-AES at XRAL Labs in Golden, CO using 4 acid dissolution (Appendix_G.xls, Appendix_G.dbf).

\begin{tabular}{|c|c|c|c|c|c|c|c|c|c|c|c|c|c|c|c|c|c|c|c|c|}
\hline Sample No. & $\begin{array}{c}\text { Eu } \\
\text { (ppm) }\end{array}$ & $\begin{array}{l}\mathrm{Fe} \\
(\%)\end{array}$ & $\begin{array}{c}\mathrm{Ga} \\
(\mathrm{ppm})\end{array} \mid$ & $\begin{array}{c}\mathrm{Ho} \\
(\mathrm{ppm})\end{array}$ & $\begin{array}{c}\mathrm{K} \\
(\%)\end{array}$ & $\begin{array}{c}\mathrm{La} \\
(\mathrm{ppm})\end{array}$ & $\begin{array}{c}\mathrm{Li} \\
(\mathrm{ppm})\end{array}$ & $\begin{array}{l}\mathrm{Mg} \\
(\%)\end{array}$ & $\begin{array}{c}\mathrm{Mn} \\
(\mathrm{ppm})\end{array}$ & $\begin{array}{c}\text { Mo } \\
\text { (ppm) }\end{array}$ & $\begin{array}{l}\mathrm{Na} \\
(\%)\end{array}$ & $\begin{array}{c}\mathrm{Nb} \\
(\mathrm{ppm})\end{array}$ & $\begin{array}{c}\mathrm{Nd} \\
(\mathrm{ppm})\end{array}$ & $\begin{array}{c}\mathrm{Ni} \\
(\mathrm{ppm})\end{array}$ & $\begin{array}{c}\mathrm{P} \\
(\mathrm{ppm})\end{array}$ & $\begin{array}{c}\mathrm{Pb} \\
(\mathrm{ppm})\end{array}$ & $\begin{array}{c}\text { Sc } \\
(\mathrm{ppm})\end{array}$ & $\begin{array}{c}\text { Sn } \\
(p p m)\end{array}$ & $\begin{array}{c}\mathrm{Sr} \\
(\mathrm{ppm})\end{array}$ & $\begin{array}{c}\text { Ta } \\
\text { (ppm) }\end{array}$ \\
\hline SAMPLE_NO. & PPM & FE_(\%) & GA_PPM & HO_PPM & K_(\%) & LA_PPM & LI_PPM & MG_(\%) & \begin{tabular}{|l|} 
MN_PPM \\
\end{tabular} & MO_PPM & NA_(\%) & NB_PPM & ND_PPM & NI_PPM & P_PPM & PB_PPM & SC_PPM & SN_PPM & SR_PPM & TA_PPM \\
\hline T98R-29(88-92) & $<2$ & 1.83 & 31 & $<4$ & 2.16 & 43 & 40 & 0.625 & 143 & 3 & 0.810 & 10 & 37 & 14 & 0.025 & 18 & 10 & $<50$ & 78 & $<40$ \\
\hline T98R-29(98-102) & $<2$ & 1.76 & 22 & $<4$ & 2.12 & 45 & 37 & 0.605 & 95 & 3 & 0.730 & 11 & 39 & 14 & 0.020 & 17 & 9 & $<50$ & 69 & $<40$ \\
\hline T98R-29(118-122) & $<2$ & 1.79 & 24 & $<4$ & 2.21 & 46 & 38 & 0.610 & 105 & 3 & 0.710 & 7 & 40 & 13 & 0.015 & 10 & 9 & $<50$ & 70 & $<40$ \\
\hline T98R-30(0-4) & $<2$ & 3.70 & 17 & $<4$ & 1.86 & 29 & 26 & 0.650 & 2320 & 4 & 1.145 & 10 & 26 & 12 & 0.120 & 1820 & 10 & $<50$ & 136 & $<40$ \\
\hline T98R-30(6-10) & 3 & 6.56 & 9 & $<4$ & 1.82 & $\overline{34}$ & 26 & 0.580 & 4560 & 4 & 0.540 & 7 & 31 & 9 & 0.165 & 5440 & $\overline{10}$ & $<50$ & 70 & $<40$ \\
\hline T98R-30(18-22) & $<2$ & 2.38 & 24 & $<4$ & 2.19 & 50 & 33 & 0.660 & 519 & 4 & 0.670 & 7 & 44 & 15 & 0.120 & 52 & 12 & $<50$ & 77 & $<40$ \\
\hline T98R-30(38-42) & 2 & 2.49 & 21 & $<4$ & 2.27 & 50 & 33 & 0.675 & 421 & 5 & 0.665 & $<4$ & 44 & 14 & 0.080 & 25 & 12 & $<50$ & 72 & $<40$ \\
\hline T98R-30(58-62) & $<2$ & 2.54 & 28 & $<4$ & 2.23 & 49 & 38 & 0.630 & 306 & $\overline{4}$ & 0.675 & $\overline{7}$ & 45 & 18 & 0.065 & 19 & 10 & $<50$ & 72 & $<40$ \\
\hline T98R-30(78-82) & $<2$ & 2.41 & 21 & $<4$ & 2.10 & 50 & 36 & 0.625 & 275 & 3 & 0.650 & 6 & 47 & 17 & 0.050 & 16 & 10 & $<50$ & 69 & $<40$ \\
\hline T98R-31(0-4) & $<2$ & 3.52 & 22 & $<4$ & 2.35 & 44 & 33 & 0.625 & 1490 & 5 & 0.700 & 8 & 41 & 17 & 0.070 & 1520 & 12 & $<50$ & 67 & $<40$ \\
\hline T98R-31(18-22) & 3 & 9.98 & $<4$ & $<4$ & 1.53 & 36 & 19 & 0.350 & 8340 & 7 & 0.365 & 4 & 27 & 9 & 0.040 & 3980 & 7 & $<50$ & 39 & $<40$ \\
\hline T98R-31(38-42) & 4 & 12.30 & $<4$ & $<4$ & 1.48 & 31 & 18 & 0.285 & 8080 & 8 & 0.285 & 8 & 30 & $<3$ & 0.035 & 10590 & 7 & $<50$ & 37 & $<40$ \\
\hline T98R-31(58-62) & 3 & 9.94 & $<4$ & $<4$ & 1.45 & 30 & 18 & 0.280 & 11490 & 6 & 0.235 & 7 & 29 & 6 & 0.035 & 12950 & 7 & $<50$ & 30 & $<40$ \\
\hline T98R-31(73-77) & 4 & 12.40 & $<4$ & $<4$ & 1.35 & 27 & 16 & 0.280 & 13000 & 5 & 0.185 & 10 & 29 & 6 & 0.025 & 8650 & 6 & $<50$ & 25 & $<40$ \\
\hline T98R-31(108-112) & 4 & 12.40 & $<4$ & $<4$ & 1.31 & 22 & 15 & 0.545 & 13940 & 5 & 0.135 & 8 & 22 & $<3$ & 0.020 & 10470 & $\overline{6}$ & $<50$ & 23 & $<40$ \\
\hline T98R-31(133-137) & 4 & 11.40 & $<4$ & $<4$ & 1.28 & 24 & 17 & 0.610 & 12170 & 6 & 0.180 & 5 & 24 & $<3$ & 0.025 & 11240 & 6 & $<50$ & 27 & $<40$ \\
\hline T98R-31(158-162) & 4 & 11.40 & $<4$ & $<4$ & 1.44 & 28 & 20 & 0.645 & 10730 & 7 & 0.265 & 5 & 28 & 4 & 0.035 & 14520 & 7 & $<50$ & 35 & $<40$ \\
\hline T98R-31(173-177) & $<2$ & 13.00 & $<4$ & $<4$ & 1.31 & 26 & 19 & 0.690 & 13550 & 6 & 0.170 & 5 & 25 & $<3$ & 0.025 & 12740 & 6 & $<50$ & 28 & $<40$ \\
\hline T98R-31(188-192) & 4 & 12.70 & 10 & $<4$ & 1.39 & 24 & 20 & 0.695 & 12100 & 8 & 0.230 & $<4$ & 25 & $<3$ & 0.030 & 13440 & $\overline{6}$ & $<50$ & 30 & $<40$ \\
\hline T98R-32(0-4) & $<2$ & 5.14 & 24 & $<4$ & 1.98 & 42 & 32 & 0.605 & 3280 & 5 & 0.525 & 10 & 43 & 12 & 0.075 & 2240 & 11 & $<50$ & 56 & $<40$ \\
\hline T98R-32 & 4 & 12.00 & $<4$ & $<4$ & 1.44 & 30 & 18 & 0.310 & 11830 & 8 & 0.260 & 7 & 27 & 10 & 0.050 & 3390 & 7 & $<50$ & 35 & $<40$ \\
\hline T98R-32 & $<2$ & 13.30 & $<4$ & $<4$ & 1.16 & 25 & 15 & 0.290 & 11220 & 7 & 0.170 & $<4$ & 23 & 7 & 0.030 & 2920 & $\overline{6}$ & $<50$ & 22 & $<40$ \\
\hline T98R-32 & 4 & 11.80 & $<4$ & $<4$ & 1.34 & 29 & 17 & 0.300 & 8910 & 7 & 0.200 & $<4$ & 28 & 7 & 0.030 & 4020 & 7 & $<50$ & 25 & $<40$ \\
\hline T98R-32 & 4 & 9.50 & $<4$ & $<4$ & 1.36 & 30 & 15 & 0.275 & 7870 & $\overline{9}$ & 0.190 & 6 & 27 & 9 & 0.025 & 4640 & 6 & $<50$ & 23 & $<40$ \\
\hline T98R-32 & 3 & 9.62 & 4 & $<4$ & 1.67 & 34 & 21 & 0.345 & 7080 & 6 & 0.285 & 7 & 34 & 8 & 0.035 & 7080 & 8 & $<50$ & 32 & $<40$ \\
\hline T98R-32 & 3 & 11.00 & 5 & $<4$ & 1.51 & 29 & 18 & 0.370 & 10620 & 4 & 0.225 & 7 & 28 & 7 & 0.030 & 8990 & 7 & $<50$ & 28 & $<40$ \\
\hline$\overline{\text { T98R-32 }}$ & 3 & 11.00 & $<4$ & $<4$ & 1.33 & 25 & 15 & 0.520 & 10470 & 8 & 0.120 & 7 & 22 & 6 & 0.025 & 4790 & 6 & $<50$ & 22 & $<40$ \\
\hline T98R-32(158-162) & 3 & 10.90 & $<4$ & $<4$ & 1.38 & 28 & 18 & 0.630 & 11070 & 8 & 0.205 & 4 & 27 & 10 & 0.035 & 6680 & 7 & $<50$ & 34 & $<40$ \\
\hline T98R-32(178-182) & 4 & 11.00 & $<4$ & $<4$ & 1.34 & 24 & 17 & 0.645 & 11180 & 7 & 0.215 & 6 & 25 & 9 & 0.030 & 7180 & 6 & $<50$ & 35 & $<40$ \\
\hline T98R-33(0-4) & $<2$ & 6.47 & 8 & $<4$ & 1.66 & 34 & 23 & 0.540 & 5340 & 4 & 0.480 & 7 & 32 & 7 & 0.045 & 3870 & 8 & $<50$ & 51 & $<40$ \\
\hline T98R-33(18-22) & 3 & 9.38 & $<4$ & $<4$ & 1.57 & 27 & 20 & 0.330 & 9230 & 7 & 0.190 & 7 & 26 & 4 & 0.025 & 5920 & $\overline{6}$ & $<50$ & 25 & $<40$ \\
\hline T98R-33(38-42) & $<2$ & 2.16 & 15 & $<4$ & 2.03 & 45 & 29 & 0.595 & 836 & $\overline{3}$ & 0.605 & 5 & 42 & 11 & 0.050 & 949 & $\overline{9}$ & $<50$ & 64 & $<40$ \\
\hline T98R-33(58-62) & $<2$ & 2.11 & 19 & $<4$ & 2.26 & 45 & 31 & 0.615 & 247 & 3 & 0.585 & $<4$ & 39 & 12 & 0.020 & 19 & 9 & $<50$ & 59 & $<40$ \\
\hline T98R-33 & $<2$ & 2.00 & 21 & $<4$ & 2.28 & 48 & 34 & 0.620 & 119 & 3 & 0.545 & 6 & 41 & 13 & 0.020 & 10 & $\overline{9}$ & $<50$ & 54 & $<40$ \\
\hline T98R-33(108-112) & $<2$ & 2.25 & 20 & $<4$ & 2.16 & 46 & 32 & 0.580 & 186 & 4 & 0.520 & $<4$ & 45 & 13 & 0.030 & 9 & 9 & $<50$ & 55 & $<40$ \\
\hline
\end{tabular}

*Other analyses (Appendix \#): D =USGS-EDXRF, E =EWU,

$\mathrm{F}=$ CHEMEX, $\mathrm{H}=\mathrm{ACZ},---=$ none. 
Appendix G. Chemical composition of samples analyzed by ICP-AES at XRAL Labs in Golden, CO using 4 acid dissolution (Appendix_G.xls, Appendix_G.dbf).

\begin{tabular}{|c|c|c|c|c|c|c|c|}
\hline Sample No. & $\begin{array}{c}\text { Th } \\
\text { (ppm) }\end{array}$ & $\begin{array}{l}\mathrm{Ti} \\
(\%)\end{array}$ & $\begin{array}{c}U \\
(\mathrm{ppm})\end{array}$ & $\begin{array}{c}\mathrm{V} \\
(\mathrm{ppm})\end{array}$ & $\begin{array}{c}\mathrm{Y} \\
(\mathrm{ppm})\end{array}$ & $\begin{array}{c}\mathrm{Yb} \\
(\mathrm{ppm})\end{array}$ & $\begin{array}{c}\mathrm{Zn} \\
(\mathrm{ppm})\end{array}$ \\
\hline SAMPLE_NO. & PPM & TI_\% & PPM & V_PPM & Y_PPM & YB_PPM & ZN_PPM \\
\hline T98R-29(88-92) & 15 & 0.269 & $<100$ & 52 & 15 & 2 & 172 \\
\hline T98R-29(98-102) & 14 & 0.269 & $<100$ & 52 & 14 & 2 & 162 \\
\hline T98R-29(118-122) & $\overline{14}$ & 0.294 & $<100$ & 51 & 14 & 2 & 147 \\
\hline T98R-30(0-4) & 8 & 0.259 & $<100$ & 53 & 14 & 2 & 1060 \\
\hline T98R-30(6-10) & 10 & 0.217 & $<100$ & 50 & 14 & 2 & 998 \\
\hline T98R-30(18-22) & 16 & 0.288 & $<100$ & 56 & 18 & 2 & 190 \\
\hline T98R-30(38-42) & 16 & 0.3 & $<100$ & 59 & 21 & 3 & 70 \\
\hline T98R-30(58-62) & 12 & 0.269 & $<100$ & 56 & 18 & 2 & 65 \\
\hline T98R-30(78-82) & 16 & 0.294 & $<100$ & 57 & 18 & 2 & 53 \\
\hline T98R-31(0-4) & 12 & 0.288 & $<100$ & 57 & 19 & 3 & 925 \\
\hline T98R-31(18-22) & 9 & 0.153 & $<100$ & 36 & 12 & 1 & 2610 \\
\hline T98R-31(38-42) & 11 & 0.165 & $<100$ & 34 & 10 & 1 & 2630 \\
\hline T98R-31(58-62) & 9 & 0.129 & $<100$ & 32 & 14 & 2 & 2770 \\
\hline T98R-31(73-77) & 9 & 0.112 & $<100$ & 29 & 11 & 1 & 2120 \\
\hline T98R-31(108-112) & 8 & 0.106 & $<100$ & 26 & 9 & 1 & 1110 \\
\hline T98R-31( & 7 & 0.123 & $<100$ & 27 & 11 & 1 & 1480 \\
\hline T98R-31(158-162) & 9 & 0.135 & $<100$ & 33 & 12 & 1 & 2560 \\
\hline T98R-31(173-177) & 12 & 0.106 & $<100$ & 29 & 11 & 1 & 1990 \\
\hline T98R-31(188-192) & 9 & 0.129 & $<100$ & 31 & 14 & 1 & 4050 \\
\hline T98R-32 & 16 & 0.223 & $<100$ & 54 & 17 & 3 & 1160 \\
\hline T98R-3 & 10 & 0.118 & $<100$ & 33 & 12 & 1 & 2070 \\
\hline T98R-3 & 9 & 0.088 & $<100$ & 26 & 10 & 1 & 1850 \\
\hline T98R-3 & 13 & 0.112 & $<100$ & 30 & 11 & 1 & 2010 \\
\hline T98R-3 & 9 & 0.1 & 00 & 28 & 9 & 1 & 1520 \\
\hline T98R-3 & 10 & 0.141 & 00 & 38 & 13 & 1 & 2250 \\
\hline T98R-3 & 13 & 0.118 & $<100$ & 34 & 10 & 1 & 2930 \\
\hline T98R-3 & 11 & 0.094 & $<100$ & 30 & 8 & $<1$ & 2730 \\
\hline T98R-32( & 13 & 0.112 & $<100$ & 31 & 11 & 1 & 4010 \\
\hline T98R-32(178-182) & 7 & 0.1 & $<100$ & 29 & 10 & 1 & 4530 \\
\hline T98R-33( & 10 & 0.188 & $<100$ & 40 & 12 & 2 & 1800 \\
\hline T98R-3 & 7 & 0.118 & $<100$ & 31 & 9 & 1 & 3430 \\
\hline T98R-3 & 9 & 0.282 & $<100$ & 49 & 13 & 2 & 923 \\
\hline T98R-3 & 13 & 0.3 & $<100$ & 51 & 12 & 2 & 153 \\
\hline T98R-3 & 14 & 0.306 & $<100$ & 53 & 13 & 1 & 164 \\
\hline-112$)$ & 14 & 0.294 & $<100$ & 52 & 20 & 2 & 217 \\
\hline
\end{tabular}

*Other analyses (Appendix \#): D =USGS-EDXRF, E =EWU,

$\mathrm{F}=$ CHEMEX, $\mathrm{H}=\mathrm{ACZ},---=$ none. 
Appendix G. Chemical composition of samples analyzed by ICP-AES at XRAL Labs in Golden, CO using 4 acid dissolution (Appendix_G.xls, Appendix_G.dbf).

\begin{tabular}{|c|c|c|c|c|c|c|c|c|c|c|c|c|c|c|c|c|c|}
\hline Sample No. & Site ID & $\begin{array}{c}\text { Depth } \\
\text { interval, } \\
\text { top }(\mathrm{cm})\end{array}$ & $\begin{array}{c}\text { Depth } \\
\text { interval, } \\
\text { bottom }(\mathrm{cm})\end{array}$ & $\begin{array}{c}{ }^{*} \text { Other } \\
\text { Analyses } \\
\text { (appendix \#) }\end{array}$ & $\begin{array}{c}\mathrm{Ag} \\
(\mathrm{ppm})\end{array}$ & $\begin{array}{c}\mathrm{Al} \\
(\%)\end{array}$ & $\begin{array}{c}\text { As } \\
\text { (ppm) }\end{array}$ & $\mid \begin{array}{c}\mathrm{Au} \\
(\mathrm{ppm})\end{array}$ & $\begin{array}{c}\mathrm{Ba} \\
(\mathrm{ppm})\end{array}$ & $\begin{array}{c}\mathrm{Be} \\
(\mathrm{ppm})\end{array}$ & $\begin{array}{c}\mathrm{Bi} \\
(\mathrm{ppm})\end{array}$ & $\begin{array}{l}\mathrm{Ca} \\
(\%)\end{array}$ & $\begin{array}{c}\mathrm{Cd} \\
(\mathrm{ppm})\end{array}$ & $\begin{array}{c}\mathrm{Ce} \\
(\mathrm{ppm})\end{array}$ & $\begin{array}{c}\text { Co } \\
(\mathrm{ppm})\end{array}$ & $\begin{array}{c}\mathrm{Cr} \\
(\mathrm{ppm})\end{array}$ & $\begin{array}{c}\mathrm{Cu} \\
\text { (ppm) }\end{array}$ \\
\hline SAMPLE_NO. & SITE_ID & INT_TOP_CM & INT_BTM_CM & OTHR_ANLYS & AG_PPM & AL_(\%) & AS_PPM & AU_PPM & BA_PPM & BE_PPM & BI_PPM & CA_(\%) & CD_PPM & CE_PPM & CO_PPM & CR_PPM & CU_PPM \\
\hline T98R-33(123-127) & T98R-33 & 123 & 127 & $\begin{array}{ll}--- \\
--\end{array}$ & $<2$ & 5.6 & 12 & $<8$ & 521 & 2 & $<50$ & 0.213 & $<2$ & 94 & 8 & 29 & 14 \\
\hline T98R-33(143-147) & T98R-33 & 143 & 147 & ---- & $<2$ & 5.9 & 15 & $<8$ & 536 & 2 & $<50$ & 0.240 & 2 & 91 & 9 & 57 & 14 \\
\hline T98R-33(163-167) & T98R-33 & 163 & 167 & ---- & $<2$ & 5.6 & $<10$ & $<8$ & 499 & 2 & $<50$ & 0.219 & $<2$ & 84 & 8 & 52 & 11 \\
\hline T98R-33(183-187) & T98R-33 & 183 & 187 & --- & $<2$ & 5.6 & $<10$ & $<8$ & 510 & 2 & $<50$ & 0.181 & $<2$ & 85 & 7 & 47 & 11 \\
\hline T98R-34(8-12) & T98R-34 & 8 & 12 & ---- & 18 & 6.4 & 21 & $<8$ & 741 & 2 & $<50$ & 0.315 & 11 & 69 & 5 & 79 & 98 \\
\hline T98R-34(28-32) & T98R-34 & 28 & 32 & ---- & $<2$ & 5.9 & 14 & $<8$ & 549 & 2 & $<50$ & 0.294 & 4 & 68 & 5 & 79 & 27 \\
\hline T98R-34(48-52) & T98R-34 & 48 & 52 & ---- & $<2$ & 6.0 & $<10$ & $<8$ & 564 & 2 & $<50$ & 0.242 & $<2$ & 77 & 4 & 87 & 25 \\
\hline T98R-34(68-72) & T98R-34 & 68 & 72 & ---- & $<2$ & 4.6 & 11 & $<8$ & 377 & 2 & $<50$ & 0.358 & $<2$ & 64 & 8 & 87 & 28 \\
\hline T98R-34(88-92) & T98R-34 & 88 & 92 & ---- & $<2$ & 5.1 & $<10$ & $<8$ & 414 & 2 & $<50$ & 0.331 & $<2$ & 68 & 7 & 87 & 27 \\
\hline T98L-35(0-4) & T98L-35 & 0 & 4 & ---- & 6 & 5.4 & 80 & $<8$ & 642 & 2 & $<50$ & 0.257 & 11 & 51 & 13 & 80 & 53 \\
\hline T98L-35(18-22) & T98L-35 & 18 & 22 & ---- & 19 & 6.3 & 123 & $<8$ & 743 & 2 & $<50$ & 0.194 & 19 & 63 & 8 & 95 & 136 \\
\hline T98L-35(38-42) & T98L-35 & 38 & 42 & $\begin{array}{ll}--- \\
--\end{array}$ & 3 & 7.0 & 33 & $<8$ & 634 & 2 & $<50$ & 0.263 & 4 & 87 & $\frac{1}{6}$ & 82 & 39 \\
\hline T98L-35(58-62) & T98L-35 & 58 & 62 & $\begin{array}{ll}--- \\
--\end{array}$ & $<2$ & 6.0 & 11 & $<8$ & 523 & 2 & $<50$ & 0.189 & $<2$ & 91 & 6 & 80 & 16 \\
\hline T98L-35(78-82) & T98L-35 & 78 & 82 & ---- & $<2$ & 5.8 & 11 & $<8$ & 479 & 2 & $<50$ & 0.160 & $<2$ & 84 & 6 & 67 & 11 \\
\hline T98L-35(98-102) & T98L-35 & 98 & 102 & ---- & $<2$ & 5.3 & 13 & $<8$ & 451 & 2 & $<50$ & 0.160 & $<2$ & 91 & 5 & 87 & 14 \\
\hline T98L-35(118-122) & T98L-35 & 118 & 122 & ---- & $<2$ & 5.5 & 12 & $<8$ & 498 & 2 & $<50$ & 0.155 & $<2$ & 100 & 5 & 61 & 8 \\
\hline T98L-35(143-147) & T98L-35 & 143 & 147 & ---- & $<2$ & 6.3 & 10 & $<8$ & 515 & 2 & $<50$ & 0.219 & $<2$ & 89 & 5 & 43 & 16 \\
\hline T98L-35(168-172) & T98L-35 & 168 & 172 & ---- & $<2$ & 6.3 & $<10$ & $<8$ & 496 & 2 & $<50$ & 0.192 & $<2$ & 79 & 4 & 88 & 13 \\
\hline T98L-35(183-187) & T98L-35 & 183 & 187 & ---- & $<2$ & 6.8 & $<10$ & $<8$ & 561 & 2 & $<50$ & 0.208 & $<2$ & 91 & 5 & 35 & 17 \\
\hline T98L-36(0-4) & T98L-36 & 0 & 4 & ---- & 17 & 4.4 & 88 & $<8$ & 696 & 1 & $<50$ & 0.389 & 35 & 67 & 10 & 55 & 96 \\
\hline T98L-36(18-22) & T98L-36 & 18 & 22 & --- & 21 & 3.1 & 115 & $<8$ & 649 & 1 & $<50$ & 0.131 & 23 & 55 & 10 & 50 & 141 \\
\hline T98L-36(23-27) & T98L-36 & 23 & 27 & ---- & 19 & 3.9 & 338 & $<8$ & 589 & 1 & $<50$ & 0.221 & 36 & 65 & 10 & 56 & 127 \\
\hline T98L-36(38-42) & T98L-36 & 38 & 42 & ---- & 20 & 3.0 & 216 & $<8$ & 574 & 1 & $<50$ & 0.137 & 17 & 56 & 14 & 48 & 114 \\
\hline T98L-36(63-67) & T98L-36 & 63 & 67 & ---- & 17 & 2.9 & 347 & $<8$ & 531 & $<1$ & $<50$ & 0.168 & 8 & 46 & 8 & 49 & 87 \\
\hline T98L-36(83-87) & T98L-36 & 83 & 87 & ---- & 14 & 3.1 & 131 & $<8$ & 496 & 1 & $<50$ & 0.163 & 9 & 54 & 8 & 47 & 88 \\
\hline T98L-36(98-102) & T98L-36 & 98 & 102 & ---- & 15 & 3.1 & 124 & $<8$ & 583 & 1 & $<50$ & 0.189 & 15 & 54 & 8 & 46 & 116 \\
\hline T98L-36(118-122) & T98L-36 & 118 & 122 & ---- & 17 & 3.1 & 107 & $<8$ & 563 & 1 & $<50$ & 0.200 & 33 & 55 & 8 & 48 & 90 \\
\hline T98L-36(138-142) & T98L-36 & 138 & 142 & $-\cdots$ & 15 & 3.2 & 78 & $<8$ & 550 & 1 & $<50$ & 0.173 & 25 & 55 & 8 & 46 & 121 \\
\hline T98L-36(158-162) & T98L-36 & 158 & 162 & $\begin{array}{ll}--- \\
\end{array}$ & 19 & 3.3 & 95 & $<8$ & 598 & 1 & $<50$ & 0.252 & 46 & 56 & 10 & 54 & 116 \\
\hline T98L-36(178-182) & T98L-36 & 178 & 182 & ---- & 21 & 3.2 & 91 & $<8$ & 580 & 1 & $<50$ & 0.404 & 46 & 48 & 11 & 51 & 101 \\
\hline T98L-36(193-197) & T98L-36 & 193 & 197 & ---- & 23 & 3.2 & 99 & $<8$ & 733 & 1 & $<50$ & 0.263 & 34 & 50 & 10 & 52 & 116 \\
\hline T98L-37(0-4) & T98L-37 & 0 & 4 & ---- & 10 & 5.9 & 69 & $<8$ & 725 & 2 & $<50$ & 0.352 & 22 & 61 & 19 & 79 & 78 \\
\hline T98L-37(18-22) & T98L-37 & 18 & 22 & ---- & 22 & 6.0 & 101 & $<8$ & 730 & 2 & $<50$ & 0.152 & 12 & 73 & 6 & 64 & 113 \\
\hline T98L-37(38-42) & T98L-37 & 38 & 42 & ---- & 5 & 6.0 & 37 & $<8$ & 647 & 2 & $<50$ & 0.299 & 19 & 70 & 6 & 81 & 51 \\
\hline T98L-37(58-62) & T98L-37 & 58 & 62 & $-\cdots$ & $<2$ & 6.2 & 13 & $<8$ & 573 & 2 & $<50$ & 0.236 & 5 & 89 & 5 & 84 & 29 \\
\hline
\end{tabular}

*Other analyses (Appendix \#): D =USGS-EDXRF, E =EWU,

$\mathrm{F}=\mathrm{CHEMEX}, \mathrm{H}=\mathrm{ACZ},-\cdots=$ none. 
Appendix G. Chemical composition of samples analyzed by ICP-AES at XRAL Labs in Golden, CO using 4 acid dissolution (Appendix_G.xls, Appendix_G.dbf).

\begin{tabular}{|c|c|c|c|c|c|c|c|c|c|c|c|c|c|c|c|c|c|c|c|c|}
\hline Sample No. & $\begin{array}{c}\text { Eu } \\
(\mathrm{ppm})\end{array}$ & $\begin{array}{l}\mathrm{Fe} \\
(\%)\end{array}$ & $\begin{array}{c}\mathrm{Ga} \\
(\mathrm{ppm})\end{array}$ & $\begin{array}{c}\mathrm{Ho} \\
(\mathrm{ppm})\end{array}$ & $\begin{array}{c}K \\
(\%)\end{array}$ & $\begin{array}{c}\mathrm{La} \\
(\mathrm{ppm})\end{array}$ & $\begin{array}{c}\mathrm{Li} \\
(\mathrm{ppm})\end{array}$ & $\begin{array}{l}\mathrm{Mg} \\
(\%)\end{array}$ & $\begin{array}{c}\mathrm{Mn} \\
(\mathrm{ppm})\end{array}$ & $\begin{array}{c}\text { Mo } \\
\text { (ppm) }\end{array}$ & $\begin{array}{l}\mathrm{Na} \\
(\%)\end{array}$ & $\begin{array}{c}\mathrm{Nb} \\
(\mathrm{ppm})\end{array}$ & $\begin{array}{c}\mathrm{Nd} \\
(\mathrm{ppm})\end{array}$ & $\begin{array}{c}\mathrm{Ni} \\
(\mathrm{ppm})\end{array}$ & $\begin{array}{c}\mathrm{P} \\
(\mathrm{ppm})\end{array}$ & $\begin{array}{c}\mathrm{Pb} \\
(\mathrm{ppm})\end{array}$ & $\begin{array}{c}\text { Sc } \\
(\mathrm{ppm})\end{array}$ & $\begin{array}{c}\text { Sn } \\
\text { (ppm) }\end{array}$ & $\begin{array}{c}\mathrm{Sr} \\
(\mathrm{ppm})\end{array}$ & $\begin{array}{c}\text { Ta } \\
\text { (ppm) }\end{array}$ \\
\hline SAMPLE_NO. & PPM & FE__\%) & GA_PPM & HO_PPM & K_(\%) & LA_PPM & LI_PPM & MG_(\%) & MN_PPM & MO_PPM & NA_(\%) & NB_PPM & ND_PPM & NI_PPM & P_PPM & PB_PPM & SC_PPM & SN_PPM & SR_PPM & TA_PPM \\
\hline T98R-33(123-127) & $<2$ & 2.23 & 23 & $<4$ & 2.21 & 49 & 34 & 0.630 & 207 & 4 & 0.565 & $<4$ & 43 & 14 & 0.025 & 9 & 9 & $<50$ & 59 & $<40$ \\
\hline T98R-33(143-147) & $<2$ & 2.24 & 23 & $<4$ & 2.30 & 48 & 34 & 0.635 & 228 & 3 & 0.605 & 5 & 42 & 15 & 0.025 & 12 & 9 & $<50$ & 66 & $<40$ \\
\hline T98R-33(163-167) & $<2$ & 2.19 & 20 & $<4$ & 2.18 & 43 & 33 & 0.585 & 173 & 4 & 0.570 & 10 & 40 & 15 & 0.025 & 9 & $\overline{8}$ & $<50$ & 63 & $<40$ \\
\hline T98R-33(183-187) & $<2$ & 2.02 & 17 & $<4$ & 2.30 & 44 & 35 & 0.600 & 162 & 3 & 0.540 & 7 & 36 & 14 & 0.025 & 8 & 8 & $<50$ & 57 & $<40$ \\
\hline T98R-34(8-12) & $<2$ & 3.56 & 21 & $<4$ & 1.90 & 33 & 29 & 0.500 & 1880 & $\overline{4}$ & 0.520 & 12 & 30 & 16 & 0.100 & 4630 & 12 & $<50$ & 61 & $<40$ \\
\hline T98R-34(28-32) & $<2$ & 1.58 & 18 & $<4$ & 1.50 & 31 & 27 & 0.395 & 245 & 4 & 0.505 & 9 & 27 & 20 & 0.105 & 363 & 11 & $<50$ & 59 & $<40$ \\
\hline T98R-34(48-52) & $<2$ & 1.54 & 19 & $<4$ & 1.82 & 36 & 29 & 0.525 & 204 & 3 & 0.455 & 12 & 34 & 16 & 0.065 & 20 & 12 & $<50$ & 53 & $<40$ \\
\hline T98R-34(68-72) & $<2$ & 1.34 & 15 & $<4$ & 1.22 & 29 & 20 & 0.370 & 205 & 4 & 0.295 & 7 & 28 & 19 & 0.070 & 16 & $\overline{9}$ & $<50$ & 53 & $<40$ \\
\hline T98R-34(88-92) & $<2$ & 1.32 & 26 & $<4$ & 1.57 & 34 & 27 & 0.475 & 97 & 5 & 0.490 & 9 & 29 & 16 & 0.045 & 17 & 8 & $<50$ & 65 & $<40$ \\
\hline T98L-35(0-4) & $<2$ & 4.51 & 12 & $<4$ & 1.97 & 27 & 26 & 0.510 & 2990 & 5 & 0.395 & 9 & 24 & 17 & 0.135 & 2120 & 10 & $<50$ & 42 & $<40$ \\
\hline T98L-35(18-22) & $<2$ & 5.43 & 14 & $<4$ & 2.35 & 32 & 25 & 0.450 & 2600 & 5 & 0.315 & 7 & 30 & 14 & 0.075 & 3500 & 11 & $<50$ & 36 & $<40$ \\
\hline T98L-35(38-42) & $<2$ & 2.93 & 26 & $<4$ & 1.94 & 45 & 33 & 0.610 & 403 & 4 & 0.585 & 4 & 40 & 19 & 0.075 & 880 & 13 & $<50$ & 57 & $<40$ \\
\hline T98L-35(58-62) & 2 & 2.08 & 25 & $<4$ & 2.01 & 45 & 31 & 0.580 & 149 & 4 & 0.555 & 7 & 38 & 14 & 0.030 & 134 & 11 & $<50$ & 51 & $<40$ \\
\hline T98L-35(78-82) & $<2$ & 1.74 & 22 & $<4$ & 2.02 & 45 & 36 & 0.565 & 106 & 3 & 0.530 & 5 & 39 & 19 & 0.020 & 55 & 9 & $<50$ & 48 & $<40$ \\
\hline T98L-35(98-102) & $<2$ & 1.63 & 18 & $<4$ & 1.87 & 47 & 35 & 0.495 & 110 & 3 & 0.475 & 12 & 39 & 14 & 0.030 & 32 & $\overline{9}$ & $<50$ & 46 & $<40$ \\
\hline T98L-35(118-122) & $<2$ & 1.61 & 20 & $<4$ & 2.14 & 50 & 36 & 0.595 & 84 & 3 & 0.530 & 7 & 44 & 14 & 0.015 & 17 & 9 & $<50$ & 49 & $<40$ \\
\hline T98L-35(143-147) & $<2$ & 1.56 & 18 & $<4$ & 2.02 & 49 & 37 & 0.510 & 61 & 3 & 0.650 & 6 & 43 & 16 & 0.020 & 20 & 10 & $<50$ & 63 & $<40$ \\
\hline T98L-35(168-172) & $<2$ & 1.48 & 18 & $<4$ & 2.00 & 41 & 36 & 0.485 & 61 & 3 & 0.605 & 10 & 38 & 15 & 0.025 & 22 & 10 & $<50$ & 58 & $<40$ \\
\hline T98L-35(183-187) & $<2$ & 1.56 & 18 & $<4$ & 2.13 & 46 & 40 & 0.555 & 62 & 3 & 0.665 & 8 & 40 & 18 & 0.035 & 21 & 11 & $<50$ & 63 & $<40$ \\
\hline T98L-36(0-4) & 2 & 7.80 & $<4$ & $<4$ & 1.69 & 31 & 23 & 0.605 & 7010 & $\overline{7}$ & 0.390 & $4 \mid$ & 31 & 10 & 0.045 & 5100 & 8 & $<50$ & 47 & $<40$ \\
\hline T98L-36( & 3 & 11.20 & $<4$ & $<4$ & 1.40 & 27 & 16 & 0.435 & 11150 & 6 & 0.180 & $4 \mid$ & 23 & $<3$ & 0.020 & 4670 & 6 & $<50$ & 22 & $<40$ \\
\hline T98L-36( & 3 & 9.91 & $<4$ & $<4$ & 1.52 & 32 & 19 & 0.415 & 7760 & 8 & 0.285 & 6 & 34 & 4 & 0.075 & 4320 & 7 & $<50$ & 32 & $<40$ \\
\hline T98L-36 & 3 & 11.00 & $<4$ & $<4$ & 1.33 & 23 & 13 & 0.345 & 10820 & 7 & 0.145 & $<4$ & 18 & $<3$ & 0.020 & 4110 & 5 & $<50$ & 18 & $<40$ \\
\hline T98L-36 & 3 & 10.30 & $<4$ & $<4$ & 1.24 & 22 & 12 & 0.440 & 8870 & 6 & 0.160 & 5 & 18 & $<3$ & 0.020 & 3640 & 5 & $<50$ & 19 & $<40$ \\
\hline T98L-36 & 2 & 8.86 & $<4$ & $<4$ & 1.34 & 25 & 13 & 0.450 & 8000 & 5 & 0.170 & 4 & 22 & $<3$ & 0.015 & 3710 & 5 & $<50$ & 21 & $<40$ \\
\hline T98L-36( & 3 & 10.20 & $<4$ & $<4$ & 1.34 & 27 & 14 & 0.530 & 9450 & 7 & 0.160 & $<4$ & 27 & $<3$ & 0.020 & 4790 & 6 & $<50$ & 22 & $<40$ \\
\hline T98L-36( & 3 & 10.20 & $<4$ & $<4$ & 1.35 & 27 & 14 & 0.530 & 9670 & 4 & 0.165 & 5 & 23 & 3 & 0.020 & 4110 & 5 & $<50$ & 22 & $<40$ \\
\hline T98L-36(138-142) & 3 & 8.99 & 5 & $<4$ & 1.45 & 26 & 14 & 0.510 & 8590 & 4 & 0.165 & 6 & 25 & 5 & 0.020 & 4580 & 6 & $<50$ & 24 & $<40$ \\
\hline T98L-36(158-162) & 3 & 10.00 & $<4$ & $<4$ & 1.42 & 25 & 16 & 0.565 & 9480 & 6 & 0.200 & $<4$ & 22 & 6 & 0.025 & 4920 & 6 & $<50$ & 27 & $<40$ \\
\hline T98L-36(178-182) & 3 & 9.77 & $<4$ & $<4$ & 1.35 & 23 & 14 & 0.535 & 9480 & 6 & 0.200 & 5 & 21 & 8 & 0.025 & 5680 & 6 & $<50$ & 30 & $<40$ \\
\hline T98L-36(193-197) & 3 & 9.87 & $<4$ & $<4$ & 1.35 & 23 & 15 & 0.600 & 10210 & 9 & 0.175 & 4 & 22 & 7 & 0.020 & 6530 & 6 & $<50$ & 26 & $<40$ \\
\hline T98L-37(0-4) & $<2$ & 5.27 & 18 & $<4$ & 2.07 & 31 & 27 & 0.595 & 5540 & 4 & 0.545 & 9 & 30 & 14 & 0.105 & 3250 & 11 & $<50$ & 57 & $<40$ \\
\hline T98L-37(18-22) & 2 & 5.10 & 15 & $<4$ & 2.38 & 35 & 23 & 0.415 & 2140 & 4 & 0.290 & 8 & 32 & 9 & 0.040 & 5540 & 11 & $<50$ & 32 & $<40$ \\
\hline T98L-37(3 & $<2$ & 2.45 & 11 & $<4$ & 1.75 & 35 & 29 & 0.510 & 808 & 3 & 0.590 & 7 & 31 & 18 & 0.070 & 2010 & 11 & $<50$ & 58 & $<40$ \\
\hline T98L-37(58-62) & $<2$ & 1.85 & 20 & $<4$ & 1.80 & 41 & 30 & 0.475 & 620 & 3 & 0.555 & 7 & 40 & 16 & 0.075 & 333 & 12 & $<50$ & 52 & $<40$ \\
\hline
\end{tabular}

*Other analyses (Appendix \#): D =USGS-EDXRF, E =EWU,

$\mathrm{F}=$ CHEMEX, $\mathrm{H}=\mathrm{ACZ},---=$ none. 
Appendix G. Chemical composition of samples analyzed by ICP-AES at XRAL Labs in Golden, CO using 4 acid dissolution (Appendix_G.xls, Appendix_G.dbf).

\begin{tabular}{|c|c|c|c|c|c|c|c|}
\hline No. & $\begin{array}{c}\text { Th } \\
(\mathrm{ppm})\end{array}$ & $\begin{array}{c}\mathrm{Ti} \\
(\%)\end{array}$ & $\begin{array}{c}\mathrm{U} \\
(\mathrm{ppm})\end{array}$ & $\begin{array}{c}\mathrm{V} \\
(\mathrm{ppm})\end{array}$ & $\begin{array}{c}Y \\
(p p m)\end{array}$ & $\begin{array}{c}\mathrm{Yb} \\
(\mathrm{ppm})\end{array}$ & $\begin{array}{c}\mathrm{Zn} \\
(\mathrm{ppm})\end{array}$ \\
\hline SAMPLE_NO. & PPM & TI_\% & \begin{tabular}{l|l} 
U_PPM \\
\end{tabular} & V_PPM & YPPPM & YB_PPM & ZN_PPM \\
\hline T98R-33(123-127) & 15 & 0.3 & $<100$ & 56 & 17 & 2 & 251 \\
\hline T98R-33(143-147) & 16 & 0.294 & $<100$ & 57 & 15 & 2 & 303 \\
\hline T98R-33(163-167) & 14 & 0.269 & $<100$ & 52 & 13 & 2 & 272 \\
\hline T98R-33(183-187) & 12 & 0.231 & $<100$ & 50 & 12 & 1 & 232 \\
\hline T98R-34(8-12) & $\overline{9}$ & 0.247 & $<100$ & 58 & $\overline{16}$ & 2 & 1380 \\
\hline T98R-34(28-32) & 8 & 0.253 & $<100$ & 54 & 16 & 2 & 537 \\
\hline T98R-34(48-52) & 15 & 0.241 & $<100$ & 60 & 16 & 2 & 286 \\
\hline T98R-34(68-72) & 11 & 0.194 & $<100$ & 62 & 20 & 2 & 503 \\
\hline T98R-34(88-92) & 12 & 0.263 & $<100$ & 51 & 16 & 2 & 64 \\
\hline T98L-35(0-4) & 7 & 0.165 & $<100$ & 48 & 13 & 2 & 1020 \\
\hline T98L-35(18-22) & 10 & 0.17 & $<100$ & 52 & 13 & 2 & 1500 \\
\hline T98L-35(38-42) & 13 & 0.276 & $<100$ & 58 & 23 & 3 & 322 \\
\hline T98L-35( & 13 & 0.241 & $<100$ & 53 & 18 & 2 & 128 \\
\hline T98L-35( & 12 & 0.3 & $<100$ & 51 & 15 & 2 & 75 \\
\hline T98L-35 & 14 & 0.263 & $<100$ & 50 & 17 & 2 & 54 \\
\hline T98L-35 & 13 & 0.319 & $<100$ & 53 & 14 & 2 & 44 \\
\hline$\overline{T 98 L-35}$ & 12 & 0.281 & $<100$ & 55 & 18 & 2 & 35 \\
\hline T98L-35 & 11 & 0.269 & $<100$ & 55 & 16 & 2 & 34 \\
\hline T98L-3 & 13 & 0.288 & $<100$ & 58 & 19 & 2 & 35 \\
\hline T98L-36 & 10 & 0.165 & $<100$ & 40 & 12 & 1 & 4230 \\
\hline T98L-36 & 7 & 0.112 & $<100$ & 29 & 9 & 1 & 3640 \\
\hline T98L-36( & 10 & 0.135 & $<100$ & 36 & 13 & 1 & 3030 \\
\hline T98L-36 & 10 & 0.088 & $<100$ & 27 & 7 & $<1$ & 2870 \\
\hline T98L-36( & 10 & 0.1 & $<100$ & 24 & 8 & $<1$ & 1100 \\
\hline T98L-36 & 10 & 0.112 & $<100$ & 26 & 8 & $<1$ & 1080 \\
\hline T98L-36 & $<6$ & 0.106 & $<100$ & 28 & 8 & $<1$ & 1440 \\
\hline T98L-36( & 7 & 0.118 & $<100$ & 27 & 8 & $<1$ & 2340 \\
\hline T98L-36(1 & 11 & 0.135 & $<100$ & 28 & 9 & $<1$ & 2610 \\
\hline T98L-36( & 8 & 0.123 & $<100$ & 31 & 9 & $<1$ & 3750 \\
\hline T98L-36( & 7 & 0.118 & $<100$ & 29 & 10 & 1 & 4250 \\
\hline T98L-36( & 6 & 0.118 & $<100$ & 29 & 10 & 1 & 5370 \\
\hline T98L-37(C & 9 & 0.2 & $<100$ & 51 & 15 & 2 & 1690 \\
\hline T98L-37(1 & 10 & 0.159 & $<100$ & 49 & $\overline{11}$ & 2 & 1640 \\
\hline T98L-37(38-42) & 11 & 0.217 & $<100$ & 53 & 17 & 2 & 942 \\
\hline T98L-37(58-62) & 14 & 0.259 & $<100$ & 56 & 19 & 2 & 603 \\
\hline
\end{tabular}

*Other analyses (Appendix \#): D =USGS-EDXRF, E =EWU,

$\mathrm{F}=$ CHEMEX, $\mathrm{H}=\mathrm{ACZ},---=$ none. 
Appendix G. Chemical composition of samples analyzed by ICP-AES at XRAL Labs in Golden, CO using 4 acid dissolution (Appendix_G.xls, Appendix_G.dbf).

\begin{tabular}{|c|c|c|c|c|c|c|c|c|c|c|c|c|c|c|c|c|c|}
\hline Sample No. & Site ID & $\begin{array}{c}\text { Depth } \\
\text { interval, } \\
\text { top }(\mathrm{cm})\end{array}$ & $\begin{array}{c}\text { Depth } \\
\text { interval, } \\
\text { bottom }(\mathrm{cm})\end{array}$ & $\begin{array}{c}{ }^{*} \text { Other } \\
\text { Analyses } \\
\text { (appendix \#) }\end{array}$ & $\begin{array}{c}\mathrm{Ag} \\
(\mathrm{ppm})\end{array}$ & $\begin{array}{c}\mathrm{Al} \\
(\%)\end{array}$ & $\begin{array}{c}\text { As } \\
\text { (ppm) }\end{array}$ & $\mid \begin{array}{c}\mathrm{Au} \\
(\mathrm{ppm})\end{array}$ & $\begin{array}{c}\mathrm{Ba} \\
(\mathrm{ppm})\end{array}$ & $\begin{array}{c}\mathrm{Be} \\
(\mathrm{ppm})\end{array}$ & $\begin{array}{c}\mathrm{Bi} \\
(\mathrm{ppm})\end{array}$ & $\begin{array}{l}\mathrm{Ca} \\
(\%)\end{array}$ & $\begin{array}{c}\mathrm{Cd} \\
(\mathrm{ppm})\end{array}$ & $\begin{array}{c}\mathrm{Ce} \\
(\mathrm{ppm})\end{array}$ & $\begin{array}{c}\text { Co } \\
(\mathrm{ppm})\end{array}$ & $\begin{array}{c}\mathrm{Cr} \\
(\mathrm{ppm})\end{array}$ & $\begin{array}{c}\mathrm{Cu} \\
\text { (ppm) }\end{array}$ \\
\hline SAMPLE_NO. & SITE_ID & INT_TOP_CM & INT_BTM_CM & OTHR_ANLYS & AG_PPM & AL_(\%) & AS_PPM & AU_PPM & BA_PPM & BE_PPM & BI_PPM & CA_(\%) & CD_PPM & CE_PPM & CO_PPM & CR_PPM & CU_PPM \\
\hline T98L-37(98-102) & T98L-37 & 98 & 102 & $\begin{array}{ll}--- \\
--\end{array}$ & $<2$ & 6.4 & $<10$ & $<8$ & 464 & 2 & $<50$ & 0.229 & $<2$ & 82 & 5 & 96 & 19 \\
\hline T98L-37(118-122) & T98L-37 & 118 & 122 & ---- & $<2$ & 6.3 & $<10$ & $<8$ & 485 & 2 & $<50$ & 0.213 & $<2$ & 77 & 5 & 94 & 17 \\
\hline T98L-37(138-142) & T98L-37 & 138 & 142 & ---- & $<2$ & 7.0 & $<10$ & $<8$ & 591 & 2 & $<50$ & 0.281 & 2 & 93 & 8 & 78 & 23 \\
\hline T98L-37(168-172) & T98L-37 & 168 & 172 & --- & $<2$ & 9.4 & $<10$ & $<8$ & 794 & 2 & $<50$ & 0.428 & $<2$ & 83 & 5 & 44 & 16 \\
\hline T98L-38(0-4) & T98L-38 & 0 & 4 & ---- & 11 & 6.1 & 36 & $<8$ & 665 & 2 & $<50$ & 0.273 & 7 & 75 & 5 & 86 & 77 \\
\hline T98L-38(18-22) & T98L-38 & 18 & 22 & ---- & 16 & 6.2 & 137 & $<8$ & 685 & 2 & $<50$ & 0.357 & 38 & 71 & 8 & 89 & 116 \\
\hline T98L-38(43-47) & T98L-38 & 43 & 47 & ---- & 18 & 5.4 & 90 & $<8$ & 679 & 2 & $<50$ & 0.373 & 33 & 61 & 9 & 78 & 112 \\
\hline T98L-38(63-67) & T98L-38 & 63 & 67 & ---- & 2 & 4.6 & 21 & $<8$ & 397 & 1 & $<50$ & 0.200 & 8 & 58 & 4 & 73 & 26 \\
\hline T98L-38(88-92) & T98L-38 & 88 & 92 & ---- & $<2$ & 6.3 & $\overline{14}$ & $<8$ & 473 & 2 & $<50$ & 0.247 & 6 & 95 & 5 & 93 & 27 \\
\hline T98L-38(113-117) & T98L-38 & 113 & 117 & ---- & $<2$ & 7.1 & $\overline{14}$ & $<8$ & 501 & 2 & $<50$ & 0.225 & 4 & 92 & 6 & 79 & 23 \\
\hline T98L-38(138-142) & T98L-38 & 138 & 142 & ---- & $<2$ & 6.5 & $<10$ & $<8$ & 450 & 2 & $<50$ & 0.218 & 3 & 106 & 5 & 72 & 25 \\
\hline T98L-38(163-167) & T98L-38 & 163 & 167 & $\begin{array}{ll}--- \\
--\end{array}$ & $<2$ & 5.2 & 13 & $<8$ & 402 & 2 & $<50$ & 0.243 & 6 & 78 & 5 & 68 & 29 \\
\hline T98L-38(188-192) & T98L-38 & 188 & 192 & $\begin{array}{ll}--- \\
--\end{array}$ & $<2$ & 6.9 & $<10$ & $<8$ & 580 & 2 & $<50$ & 0.256 & $<2$ & 79 & 5 & 82 & 16 \\
\hline T98M-39(0-4) & T98M-39 & 0 & 4 & ---- & $<2$ & 5.8 & $<10$ & $<8$ & 417 & 2 & $<50$ & 0.304 & $<2$ & 83 & 6 & 71 & 22 \\
\hline T98M-39(18-22) & T98M-39 & 18 & 22 & ---- & $<2$ & 6.1 & $<10$ & $<8$ & 418 & 2 & $<50$ & 0.298 & 3 & 90 & 4 & 81 & 19 \\
\hline T98M-39(38-42) & T98M-39 & 38 & 42 & ---- & $<2$ & 7.4 & $<10$ & $<8$ & 499 & 2 & $<50$ & 0.148 & $<2$ & 111 & 5 & 79 & 8 \\
\hline T98M-39(58-62) & T98M-39 & 58 & 62 & ---- & $<2$ & 8.4 & $<10$ & $<8$ & 578 & 2 & $<50$ & 0.163 & $<2$ & 89 & 4 & 32 & 13 \\
\hline T98M-39(78-82) & T98M-39 & 78 & 82 & ---- & $<2$ & 8.2 & $<10$ & $<8$ & 655 & 2 & $<50$ & 0.177 & $<2$ & 105 & 7 & 88 & 12 \\
\hline T98M-39(93-97) & T98M-39 & 93 & 97 & ---- & $<2$ & 8.2 & $<10$ & $<8$ & 669 & 2 & $<50$ & 0.179 & $<2$ & 98 & 4 & 77 & 14 \\
\hline T98M-39(110-114) & T98M-39 & 110 & 114 & ---- & $<2$ & 9.1 & $<10$ & $<8$ & 762 & 2 & $<50$ & 0.207 & $<2$ & 115 & 5 & 78 & 11 \\
\hline T98M-39(133-137) & T98M-39 & 133 & 137 & ---- & $<2$ & 8.7 & $<10$ & $<8$ & 813 & 2 & $<50$ & 0.957 & $<2$ & 87 & 5 & 53 & 25 \\
\hline T98M-39(143-147) & T98M-39 & 143 & 147 & ---- & $<2$ & 7.9 & $<10$ & $<8$ & 830 & 2 & $<50$ & 1.177 & $<2$ & 50 & 5 & 61 & 21 \\
\hline T98M-39(161-165) & T98M-39 & 161 & 165 & ---- & $<2$ & 7.9 & $<10$ & $<8$ & 709 & 2 & $<50$ & 0.182 & $<2$ & 85 & 7 & 85 & $\overline{14}$ \\
\hline T98M-39(170-174) & T98M-39 & 170 & 174 & ---- & $<2$ & 7.7 & $<10$ & $<8$ & 699 & 3 & $<50$ & 0.162 & \begin{tabular}{l|l}
$<2$ \\
\end{tabular} & 101 & 6 & 41 & 12 \\
\hline T98M-39(198-202) & T98M-39 & 198 & 202 & ---- & $<2$ & 8.3 & $<10$ & $<8$ & 736 & 2 & $<50$ & 0.150 & $<2$ & 103 & 11 & 89 & $\overline{16}$ \\
\hline T98M-40(0-4) & T98M-40 & 0 & 4 & ---- & 8 & 6.1 & 27 & $<8$ & 567 & 2 & $<50$ & 0.488 & 8 & 75 & 6 & 76 & 55 \\
\hline T98M-40(18-22) & T98M-40 & 18 & 22 & ---- & $<2$ & 5.8 & $<10$ & $<8$ & 470 & 2 & $<50$ & 0.226 & 4 & 91 & 4 & 82 & 26 \\
\hline T98M-40(38-42) & T98M-40 & 38 & 42 & $-\cdots$ & $<2$ & 4.9 & $<10$ & $<8$ & 320 & 2 & $<50$ & 0.163 & 2 & 101 & 3 & 79 & 24 \\
\hline T98M-40(58-62) & T98M-40 & 58 & 62 & $\begin{array}{ll}--- \\
\end{array}$ & $<2$ & 5.6 & $<10$ & $<8$ & 358 & 2 & $<50$ & 0.126 & \begin{tabular}{l|l}
$<2$ \\
\end{tabular} & 86 & 5 & 71 & 18 \\
\hline T98M-40(88-92) & T98M-40 & 88 & 92 & ---- & $<2$ & 6.3 & $<10$ & $<8$ & 403 & 2 & $<50$ & 0.137 & $<2$ & 86 & 3 & 71 & 20 \\
\hline T98M-40(98-102) & T98M-40 & 98 & 102 & ---- & $<2$ & 6.7 & $<10$ & $<8$ & 435 & 2 & $<50$ & 0.131 & $<2$ & 104 & 5 & 79 & 9 \\
\hline T98M-40(123-127) & T98M-40 & 123 & 127 & ---- & $<2$ & 6.8 & $<10$ & $<8$ & 526 & 2 & $<50$ & 0.133 & $<2$ & 109 & 3 & 72 & 11 \\
\hline T98M-40(143-147) & T98M-40 & 143 & 147 & ---- & 2 & 7.7 & 14 & $<8$ & 638 & 3 & $<50$ & 0.278 & $<2$ & 95 & 8 & 112 & 103 \\
\hline T98M-40(173-177) & T98M-40 & 173 & 177 & ---- & $<2$ & 8.9 & 16 & $<8$ & 775 & 2 & $<50$ & 0.704 & $<2$ & 79 & 7 & 49 & $\overline{54}$ \\
\hline T98M-40(188-192) & T98M-40 & 188 & 192 & $-\cdots$ & $<2$ & 8.1 & $<10$ & $<8$ & 824 & 2 & $<50$ & 1.167 & $<2$ & 67 & 5 & 54 & 25 \\
\hline
\end{tabular}

*Other analyses (Appendix \#): D =USGS-EDXRF, E =EWU,

$\mathrm{F}=$ CHEMEX, $\mathrm{H}=\mathrm{ACZ},---=$ none. 
Appendix G. Chemical composition of samples analyzed by ICP-AES at XRAL Labs in Golden, CO using 4 acid dissolution (Appendix_G.xls, Appendix_G.dbf).

\begin{tabular}{|c|c|c|c|c|c|c|c|c|c|c|c|c|c|c|c|c|c|c|c|c|}
\hline Sample No. & $\begin{array}{c}\mathrm{Eu} \\
(\mathrm{ppm})\end{array}$ & $\begin{array}{l}\mathrm{Fe} \\
(\%)\end{array}$ & $\begin{array}{c}\mathrm{Ga} \\
(\mathrm{ppm})\end{array}$ & $\begin{array}{c}\mathrm{Ho} \\
(\mathrm{ppm})\end{array}$ & $\begin{array}{c}\mathrm{K} \\
(\%)\end{array}$ & $\begin{array}{c}\mathrm{La} \\
\text { (ppm) }\end{array}$ & $\begin{array}{c}\mathrm{Li} \\
(\mathrm{ppm})\end{array}$ & $\begin{array}{l}\mathrm{Mg} \\
(\%)\end{array}$ & $\begin{array}{c}\mathrm{Mn} \\
\text { (ppm) }\end{array}$ & $\begin{array}{c}\text { Mo } \\
\text { (ppm) }\end{array}$ & $\begin{array}{l}\mathrm{Na} \\
(\%)\end{array}$ & $\begin{array}{c}\mathrm{Nb} \\
(\mathrm{ppm})\end{array}$ & $\begin{array}{c}\mathrm{Nd} \\
(\mathrm{ppm})\end{array}$ & $\begin{array}{c}\mathrm{Ni} \\
(\mathrm{ppm})\end{array}$ & $\begin{array}{c}\mathrm{P} \\
(\mathrm{ppm})\end{array}$ & $\begin{array}{c}\mathrm{Pb} \\
(\mathrm{ppm})\end{array}$ & $\begin{array}{c}\text { Sc } \\
(\mathrm{ppm})\end{array}$ & $\begin{array}{c}\text { Sn } \\
(p p m)\end{array}$ & $\begin{array}{c}\mathrm{Sr} \\
(\mathrm{ppm})\end{array}$ & $\begin{array}{c}\text { Ta } \\
\text { (ppm) }\end{array}$ \\
\hline SAMPLE_NO. & PPM & FE_(\%) & GA_PPM & HO_PPM & K_(\%) & LA_PPM & LI_PPM & MG_(\%) & MN_PPM & MO_PPM & NA_(\%) & NB_PPM & ND_PPM & NI_PPM & P_PPM & B_PPM & $\overline{\text { SC_PPM }}$ & SN_PPM & SR_PPM & TA_PPM \\
\hline T98L-37(98-102) & $<2$ & 1.41 & 18 & $<4$ & 1.56 & 35 & 38 & 0.415 & 638 & 4 & 0.450 & 12 & 35 & 17 & 0.045 & 51 & 11 & $<50$ & 47 & $<40$ \\
\hline T98L-37(118-122) & $<2$ & 1.43 & 24 & $<4$ & 1.75 & 35 & 36 & 0.485 & 455 & 3 & 0.490 & 11 & 34 & 16 & 0.040 & 93 & 10 & $<50$ & 48 & $<40$ \\
\hline T98L-37(138-142) & $<2$ & 1.58 & $<4$ & $<4$ & 1.94 & 39 & 35 & 0.562 & 487 & $<2$ & 0.598 & $<4$ & 42 & 17 & 0.064 & 90 & 12 & $<50$ & 63 & $<40$ \\
\hline T98L-37(168-172) & $<2$ & 1.91 & 29 & $<4$ & 2.93 & 35 & 43 & 0.722 & 153 & $<2$ & 1.193 & 6 & 34 & 17 & 0.016 & 17 & 11 & $<50$ & 106 & $<40$ \\
\hline T98L-38(0-4) & 2 & 4.25 & 21 & $<4$ & 2.15 & 36 & 30 & 0.615 & 1670 & 4 & 0.505 & $\overline{11}$ & 34 & 16 & 0.110 & 3570 & $\overline{12}$ & $<50$ & 51 & $<40$ \\
\hline T98L-38(18-22) & 2 & 4.42 & 15 & $<4$ & 2.13 & 33 & 27 & 0.550 & 2230 & 5 & 0.545 & 10 & 32 & 21 & 0.080 & 3990 & 11 & $<50$ & 61 & $<40$ \\
\hline T98L-38(43-47) & $<2$ & 5.90 & 6 & $<4$ & 1.85 & 29 & 24 & 0.530 & 3950 & 5 & 0.545 & 8 & 25 & 12 & 0.050 & 5020 & 10 & $<50$ & 61 & $<40$ \\
\hline T98L-38(63-67) & $<2$ & 2.08 & 8 & $<4$ & 1.02 & 27 & 21 & 0.300 & 545 & $\overline{3}$ & 0.260 & $<4$ & 29 & 15 & 0.080 & 744 & $\overline{9}$ & $<50$ & 36 & $<40$ \\
\hline T98L-38(88-92) & $<2$ & 2.02 & 21 & $<4$ & 1.24 & 36 & 30 & 0.355 & 471 & 4 & 0.295 & 7 & 37 & 20 & 0.135 & 352 & 13 & $<50$ & 40 & $<40$ \\
\hline T98L-38(113-117) & $<2$ & 1.83 & 25 & $<4$ & 1.58 & 36 & 37 & 0.475 & 342 & $<2$ & 0.396 & 17 & 32 & 17 & 0.060 & 412 & 11 & $<50$ & 43 & $<40$ \\
\hline T98L-38(138-142) & $<2$ & 1.71 & 17 & $<4$ & 1.34 & 33 & 31 & 0.384 & 256 & $<2$ & 0.305 & $<4$ & 40 & 19 & 0.060 & 532 & 10 & $<50$ & 41 & $<40$ \\
\hline T98L-38(163-167) & $<2$ & 1.59 & 11 & $<4$ & 1.30 & 26 & 26 & 0.356 & 266 & $<2$ & 0.336 & 5 & 25 & 14 & 0.062 & 644 & 9 & $<50$ & 45 & $<40$ \\
\hline T98L-38(188-192) & $<2$ & 1.70 & 27 & $<4$ & 2.02 & 31 & 39 & 0.573 & 98 & $<2$ & 0.466 & 11 & 25 & 15 & 0.048 & 246 & 10 & $<50$ & 54 & $<40$ \\
\hline T98M-39(0-4) & $<2$ & 1.69 & 18 & $<4$ & 1.51 & 34 & 32 & 0.462 & 316 & $<2$ & 0.470 & $<4$ & 42 & 18 & 0.164 & 479 & 9 & $<50$ & 49 & $<40$ \\
\hline T98M-39(18-22) & $<2$ & 1.56 & 17 & $<4$ & 1.37 & 33 & 28 & 0.363 & 97 & $<2$ & 0.503 & 13 & 37 & 15 & 0.131 & 377 & 10 & $<50$ & 54 & $<40$ \\
\hline T98M-39(38-42) & $<2$ & 1.45 & 28 & $<4$ & 1.82 & 41 & 43 & 0.447 & 54 & $<2$ & 0.444 & 14 & 38 & 15 & 0.055 & 17 & 11 & $<50$ & 40 & $<40$ \\
\hline T98M-39(58-62) & $<2$ & 1.56 & 20 & $<4$ & 2.10 & 43 & 46 & 0.479 & 52 & 3 & 0.473 & 5 & 43 & 15 & 0.031 & 20 & 13 & $<50$ & 44 & $<40$ \\
\hline T98M-39(78-82) & $<2$ & 1.54 & 17 & $<4$ & 2.34 & 39 & 48 & 0.564 & 46 & $<2$ & 0.529 & 8 & 42 & 14 & 0.022 & 8 & 13 & $<50$ & 47 & $<40$ \\
\hline T98M-39(93-97) & $<2$ & 1.68 & 28 & $<4$ & 2.51 & 41 & 49 & 0.649 & 54 & $<2$ & 0.583 & 4 & 45 & 15 & 0.023 & 20 & 13 & $<50$ & 53 & $<40$ \\
\hline T98M-39(110-114) & $<2$ & 2.03 & 32 & $<4$ & 2.87 & 44 & 48 & 0.678 & 63 & $<2$ & 0.694 & $\overline{6}$ & 38 & 15 & 0.010 & 17 & 13 & $<50$ & 63 & $<40$ \\
\hline T98M-39 & $<2$ & 2.00 & 16 & $<4$ & 1.72 & 22 & 31 & 0.385 & 208 & 2 & 1.911 & 4 & 24 & 13 & 0.024 & 4 & 8 & $<50$ & 214 & $<40$ \\
\hline T98M-39 & 2 & 1.62 & 27 & $<4$ & 2.01 & 23 & 33 & 0.337 & 298 & 2 & 2.831 & 5 & 30 & 7 & 0.026 & 8 & 7 & $<50$ & 257 & $<40$ \\
\hline T98M-39 & $<2$ & 1.97 & 35 & $<4$ & 2.94 & 49 & 45 & 0.794 & 72 & 3 & 0.646 & 11 & 51 & 13 & 0.008 & 18 & 13 & $<50$ & 56 & $<40$ \\
\hline T98M-39 & $<2$ & 1.92 & 25 & $<4$ & 2.91 & 51 & 44 & 0.796 & 74 & $<2$ & 0.670 & $<4$ & 48 & 14 & 0.008 & 21 & 12 & $<50$ & 54 & $<40$ \\
\hline T98M-39 & 3 & 2.18 & 30 & $<4$ & 3.13 & 50 & 48 & 0.847 & 71 & $<2$ & 0.669 & 5 & 48 & 18 & 0.008 & 20 & 13 & $<50$ & 53 & $<40$ \\
\hline T98M-40(0-4) & $<2$ & 2.53 & 11 & $<4$ & 1.69 & 33 & 27 & 0.480 & 693 & 4 & 0.715 & 6 & 32 & 15 & 0.095 & 2350 & 11 & $<50$ & 85 & $<40$ \\
\hline T98M-40(18-22) & $<2$ & 1.64 & 17 & $<4$ & 1.52 & 35 & 28 & 0.410 & 204 & 4 & 0.455 & 10 & 30 & 15 & 0.095 & 652 & 11 & $<50$ & 47 & $<40$ \\
\hline T98M-40(38-42) & $<2$ & 1.13 & 24 & $<4$ & 1.01 & 33 & 19 & 0.250 & 141 & 2 & 0.270 & 7 & 36 & 17 & 0.105 & 26 & 10 & $<50$ & 34 & $<40$ \\
\hline T98M-40(58-62) & $<2$ & 1.12 & 19 & $<4$ & 1.17 & 34 & 26 & 0.294 & 115 & $<2$ & 0.278 & $<4$ & 33 & 16 & 0.091 & 34 & 9 & $<50$ & 31 & $<40$ \\
\hline T98M-40(88-92) & $<2$ & 1.22 & 28 & $<4$ & 1.40 & 34 & 33 & 0.344 & 100 & 2 & 0.364 & 16 & 42 & 15 & 0.070 & 77 & 10 & $<50$ & 38 & $<40$ \\
\hline T98M-40(98-102) & $<2$ & 1.42 & 20 & $<4$ & 1.43 & 33 & 34 & 0.403 & 61 & $<2$ & 0.335 & 15 & 28 & 15 & 0.040 & 16 & 10 & $<50$ & 35 & $<40$ \\
\hline T98M-40(123-127) & $<2$ & 1.41 & 11 & $<4$ & 1.80 & 34 & 37 & 0.470 & 48 & $<2$ & 0.440 & 15 & 41 & 13 & 0.031 & 23 & 11 & $<50$ & 43 & $<40$ \\
\hline T98M-40(143-147) & $<2$ & 1.93 & 45 & $<4$ & 2.31 & 46 & 42 & 0.658 & 276 & $<2$ & 0.602 & $<4$ & 58 & 22 & 0.039 & 736 & 13 & $<50$ & 68 & $<40$ \\
\hline T98M-40(173-177) & $<2$ & 1.98 & 25 & $<4$ & 2.41 & 34 & 39 & 0.567 & 339 & 3 & 1.609 & 6 & 43 & 12 & 0.024 & 400 & 10 & $<50$ & 156 & $<40$ \\
\hline T98M-40(188-192) & 2 & 1.62 & 18 & $<4$ & 2.16 & 27 & 30 & 0.424 & 291 & 3 & 2.593 & $<4$ & 27 & 7 & 0.034 & 9 & & $<50$ & 255 & $<40$ \\
\hline
\end{tabular}

*Other analyses (Appendix \#): D =USGS-EDXRF, E =EWU,

$\mathrm{F}=$ CHEMEX, $\mathrm{H}=\mathrm{ACZ},---=$ none. 
Appendix G. Chemical composition of samples analyzed by ICP-AES at XRAL Labs in Golden, CO using 4 acid dissolution (Appendix_G.xls, Appendix_G.dbf).

\begin{tabular}{|c|c|c|c|c|c|c|c|}
\hline No. & $\begin{array}{c}\text { Th } \\
(\mathrm{ppm})\end{array}$ & $\begin{array}{c}\mathrm{Ti} \\
(\%)\end{array}$ & $\begin{array}{c}\mathrm{U} \\
(\mathrm{ppm})\end{array}$ & $\begin{array}{c}\mathrm{V} \\
(\mathrm{ppm})\end{array}$ & $\begin{array}{c}Y \\
(p p m)\end{array}$ & $\begin{array}{c}\mathrm{Yb} \\
(\mathrm{ppm})\end{array}$ & $\begin{array}{c}\mathrm{Zn} \\
(\mathrm{ppm})\end{array}$ \\
\hline SAMPLE_NO. & PPM & TI_\% & \begin{tabular}{l|l} 
U_PPM \\
\end{tabular} & V_PPM & YPPPM & YB_PPM & ZN_PPM \\
\hline $98 \mathrm{~L}-37(98-102)$ & 14 & 0.25 & $<100$ & 57 & 19 & 2 & 695 \\
\hline T98L-37(118-122) & 13 & 0.25 & $<100$ & 57 & 15 & 2 & 471 \\
\hline T98L-37(138-142) & 13 & 0.31 & $<100$ & 67 & 20 & 3 & 676 \\
\hline T98L-37(168-172) & $<6$ & 0.363 & $<100$ & 65 & 14 & 2 & 94 \\
\hline T98L-38(0-4) & 11 & 0.217 & $<100$ & 59 & 19 & 3 & 655 \\
\hline T98L-38(18-22) & 12 & 0.217 & $<100$ & 56 & 17 & 3 & 2000 \\
\hline T98L-38(43-47) & 10 & 0.182 & $<100$ & 48 & 14 & 2 & 2420 \\
\hline T98L-38(63-67) & 11 & 0.159 & $<100$ & 40 & 18 & 2 & 608 \\
\hline T98L-38(88-92) & 16 & 0.217 & $<100$ & 58 & 25 & 3 & 675 \\
\hline T98L-38(113-117) & 9 & 0.262 & $<100$ & 57 & 18 & 2 & 473 \\
\hline T98L-38(1 & 14 & 0.246 & $<100$ & 59 & 19 & 2 & 390 \\
\hline T98L-38(1 & $<6$ & 0.206 & $<100$ & 50 & 16 & 2 & 402 \\
\hline T98L-38( & $<6$ & 0.257 & $<100$ & 60 & 13 & 2 & 102 \\
\hline T98M-39 & $<6$ & 0.226 & $<100$ & 48 & 17 & 2 & 217 \\
\hline T98M-33 & 7 & 0.253 & $<100$ & 49 & 18 & 2 & 128 \\
\hline T98M-3 & 16 & 0.289 & $<100$ & 56 & 17 & 2 & 70 \\
\hline T98M-39 & 14 & 0.339 & $<100$ & 69 & 21 & 2 & 59 \\
\hline T98M-33 & 14 & 0.309 & $<100$ & 68 & 16 & 2 & 37 \\
\hline T98M-3 & $<6$ & 0.325 & $<100$ & 69 & 16 & 2 & 46 \\
\hline T98M-3 & 8 & 0.352 & $<100$ & 76 & 15 & 2 & 191 \\
\hline T98M-39 & $<6$ & 0.383 & $<100$ & 40 & 15 & 2 & 82 \\
\hline T98M-39 & $<6$ & 0.349 & $<100$ & 28 & 19 & 3 & 57 \\
\hline T98M-3s & 21 & 0.31 & $<100$ & 73 & 18 & 2 & 72 \\
\hline T98M-39 & $<6$ & 0.297 & $<100$ & 72 & 17 & 2 & 71 \\
\hline T98M-39 & 16 & 0.273 & $<100$ & 75 & 15 & 2 & 78 \\
\hline T98M-40 & 6 & 0.247 & $<100$ & 54 & 20 & 2 & 587 \\
\hline T98M-40 & 10 & 0.212 & $<100$ & 52 & 21 & 2 & 329 \\
\hline T98M-40 & 11 & 0.188 & $<100$ & 47 & 24 & 3 & 308 \\
\hline T98M-40 & 8 & 0.2 & $<100$ & 46 & 16 & 2 & 259 \\
\hline T98M-40 & 22 & 0.241 & $<100$ & 51 & 16 & 2 & 184 \\
\hline T98M-40 & $<6$ & 0.249 & $<100$ & 54 & 16 & 2 & 83 \\
\hline T98M-40( & 17 & 0.281 & $<100$ & 57 & 15 & 2 & 38 \\
\hline T98M-40( & 20 & 0.335 & $<100$ & 80 & 23 & 3 & 112 \\
\hline T98M-40(1 & 13 & 0.39 & $<100$ & 53 & $\overline{16}$ & 2 & 114 \\
\hline T98M-40(188-192) & 10 & 0.385 & $<100$ & 36 & 19 & 2 & 64 \\
\hline
\end{tabular}

*Other analyses (Appendix \#): D =USGS-EDXRF, E =EWU,

$\mathrm{F}=$ CHEMEX, $\mathrm{H}=\mathrm{ACZ},---=$ none. 
Appendix G. Chemical composition of samples analyzed by ICP-AES at XRAL Labs in Golden, CO using 4 acid dissolution (Appendix_G.xls, Appendix_G.dbf).

\begin{tabular}{|c|c|c|c|c|c|c|c|c|c|c|c|c|c|c|c|c|c|}
\hline Sample No. & Site ID & $\begin{array}{c}\text { Depth } \\
\text { interval, } \\
\text { top }(\mathrm{cm})\end{array}$ & $\begin{array}{c}\text { Depth } \\
\text { interval, } \\
\text { bottom }(\mathrm{cm})\end{array}$ & $\begin{array}{c}{ }^{*} \text { Other } \\
\text { Analyses } \\
\text { (appendix \#) }\end{array}$ & $\begin{array}{c}\mathrm{Ag} \\
(\mathrm{ppm})\end{array}$ & $\begin{array}{c}\mathrm{Al} \\
(\%)\end{array}$ & $\begin{array}{c}\text { As } \\
\text { (ppm) }\end{array}$ & $\begin{array}{c}\mathrm{Au} \\
(\mathrm{ppm})\end{array}$ & $\begin{array}{c}\mathrm{Ba} \\
(\mathrm{ppm})\end{array}$ & $\left|\begin{array}{c}\mathrm{Be} \\
(\mathrm{ppm})\end{array}\right|$ & $\begin{array}{c}\mathrm{Bi} \\
(\mathrm{ppm})\end{array}$ & $\begin{array}{l}\mathrm{Ca} \\
(\%)\end{array}$ & $\begin{array}{c}\text { Cd } \\
\text { (ppm) }\end{array}$ & $\begin{array}{c}\mathrm{Ce} \\
(\mathrm{ppm})\end{array}$ & $\begin{array}{c}\text { Co } \\
\text { (ppm) }\end{array}$ & $\begin{array}{c}\mathrm{Cr} \\
(\mathrm{ppm})\end{array}$ & $\begin{array}{c}\mathrm{Cu} \\
(\mathrm{ppm})\end{array}$ \\
\hline SAMPLE_NO. & SITE_ID & IT_TOP_CM & INT_BTM_CM & OTHR_ANLYS & AG_PPM & AL_(\%) & AS_PPM & AU_PPM & BA_PPM & BE_PPM & BI_PPM & CA_(\%) & CD_PPM & CE_PPM & CO_PPM & \begin{tabular}{|l|} 
CR_PPM \\
\end{tabular} & CU_PPM \\
\hline T98L-41(0-4) & $98 \mathrm{~L}-41$ & 0 & 4 & ---- & 9 & 5.7 & 58 & $<8$ & 728 & 2 & $<50$ & 0.457 & 18 & 70 & 19 & 74 & 55 \\
\hline T98L-41(18-22) & $98 \mathrm{~L}-41$ & 18 & 22 & ---- & 20 & 6.7 & 166 & $<8$ & 768 & 2 & $<50$ & 0.147 & 12 & 73 & 8 & 86 & 122 \\
\hline T98L-41(38-42) & T98L-41 & 38 & 42 & ---- & 24 & 6.1 & 72 & $<8$ & 795 & 2 & $<50$ & 0.184 & 24 & 63 & 9 & 60 & 145 \\
\hline T98L-41(63-67) & T98L-41 & 63 & 67 & ---- & $<2$ & 3.7 & 11 & $<8$ & 279 & 1 & $<50$ & 0.236 & 19 & 58 & 5 & 68 & 28 \\
\hline T98L-41(88-92) & T98L-41 & 88 & 92 & ---- & $<2$ & 5.8 & $<10$ & $<8$ & 497 & 1 & $<50$ & 0.215 & $<2$ & 88 & 4 & 49 & $\overline{9}$ \\
\hline T98L-41(103-107) & T98L-41 & 103 & 107 & $\begin{array}{ll}--- \\
\end{array}$ & $<2$ & 6.4 & $<10$ & $<8$ & 545 & 2 & $<50$ & 0.243 & $<2$ & 105 & 4 & 48 & 8 \\
\hline T98L-42(0-4) & T98L-42 & 0 & 4 & $\begin{array}{ll}--- \\
\end{array}$ & 9 & 5.2 & 129 & $<8$ & 644 & 2 & $<50$ & 0.252 & 22 & 67 & 21 & 74 & 61 \\
\hline T98L-42(8-12) & T98L-42 & 8 & 12 & ---- & 20 & 6.2 & 273 & $<8$ & 769 & 2 & $<50$ & 0.158 & 11 & 75 & 8 & 90 & 136 \\
\hline T98L-42(18-22) & T98L-42 & 18 & 22 & $-\cdots$ & 14 & 6.6 & 42 & $<8$ & 736 & 2 & $<50$ & 0.294 & 12 & 84 & 7 & 85 & 89 \\
\hline T98L-42(43-47) & T98L-42 & 43 & 47 & ---- & $<2$ & 5.9 & 17 & $<8$ & 538 & 2 & $<50$ & 0.289 & 5 & 79 & 6 & 84 & 18 \\
\hline T98L-42(63-67) & T98L-42 & 63 & 67 & ---- & $<2$ & 6.5 & $\overline{14}$ & $<8$ & 478 & 2 & $<50$ & 0.200 & $<2$ & 88 & 5 & 82 & 17 \\
\hline T98L-42(88-94) & T98L-42 & 88 & 94 & ---- & $<2$ & 6.8 & $<10$ & $<8$ & 542 & 2 & $<50$ & 0.168 & $<2$ & 92 & 6 & 80 & 14 \\
\hline T98L-42(108-112) & T98L-42 & 108 & 112 & --- & 14 & 3.4 & 136 & $<8$ & 756 & 1 & $<50$ & 0.219 & 19 & 54 & 12 & 47 & 115 \\
\hline T98M-43(0-4) & T98M-43 & 0 & 4 & ---- & 5 & 6.7 & 37 & $<8$ & 586 & 2 & $<50$ & 1.139 & 12 & 55 & 11 & 72 & 47 \\
\hline T98M-43(8-12) & T98M-43 & 8 & 12 & $\begin{array}{ll}--- \\
-\end{array}$ & 17 & 6.4 & 124 & $<8$ & 785 & 2 & $<50$ & 0.179 & 13 & 73 & 12 & 85 & 120 \\
\hline T98M-43(18-22) & T98M-43 & 18 & 22 & ---- & 6 & 6.8 & 23 & $<8$ & 855 & 2 & $<50$ & 0.347 & 7 & 87 & 9 & 88 & 47 \\
\hline T98M-43(38-42) & T98M-43 & 38 & 42 & ---- & $<2$ & 7.3 & 13 & $<8$ & 717 & 2 & $<50$ & 0.252 & $<2$ & 95 & 9 & 94 & 19 \\
\hline T98M-43(58-62) & T98M-43 & 58 & 62 & ---- & $<2$ & 6.9 & 16 & $<8$ & 660 & 2 & $<50$ & 0.210 & $<2$ & 93 & 8 & 90 & 20 \\
\hline T98M-43(83-87) & T98M-43 & 83 & 87 & --- & $<2$ & 7.1 & 20 & $<8$ & 654 & 2 & $<50$ & 0.221 & $<2$ & 129 & 11 & 79 & 21 \\
\hline T98M-43(98-102) & T98M-43 & 98 & 102 & --- & $<2$ & 7.2 & $<10$ & $<8$ & 686 & 2 & $<50$ & 0.280 & $<2$ & 89 & 12 & 29 & 23 \\
\hline T98M-43(118-122) & T98M-43 & 118 & 122 & --- & $<2$ & 7.1 & $<10$ & $<8$ & 669 & 2 & $<50$ & 0.284 & $<2$ & 99 & 12 & 77 & 17 \\
\hline T98M-43(138-142) & T98M-43 & 138 & 142 & ---- & $<2$ & 7.0 & 15 & $<8$ & 692 & 2 & $<50$ & 0.328 & $<2$ & 89 & 11 & 63 & 17 \\
\hline T98M-43(161-165) & T98M-43 & 161 & 165 & ---- & $<2$ & 6.9 & $<10$ & $<8$ & 654 & 2 & $<50$ & 0.330 & $<2$ & 100 & 11 & 79 & 15 \\
\hline T98M-43(178-182) & T98M-43 & 178 & 182 & ---- & $<2$ & 6.7 & $<10$ & $<8$ & 648 & 2 & $<50$ & 0.370 & $<2$ & 92 & 11 & 71 & 17 \\
\hline T98M-43(188-192) & T98M-43 & 188 & 192 & $\overline{----}$ & $<2$ & 6.9 & $<10$ & $<8$ & 674 & 2 & $<50$ & 0.382 & $<2$ & 78 & 10 & 62 & 15 \\
\hline 93ABM02(11-22) & 93ABM02 & 11 & 22 & $\mathrm{D}, \mathrm{F}$ & 18 & 3.8 & 164 & $<8$ & 623 & 1 & $<50$ & 0.184 & 21 & 72 & 13 & 78 & 116 \\
\hline 93ABM04(76-142) & 93ABM04 & 76 & 142 & $\mathrm{D}, \mathrm{F}$ & $<2$ & 5.7 & 10 & $<8$ & 551 & 2 & $<50$ & 0.231 & 3 & 91 & 8 & 142 & 22 \\
\hline 93SBC10(70-90) & $93 \mathrm{SBC} 10$ & 70 & 90 & $\mathrm{D}, \mathrm{F}$ & 38 & 3.9 & 94 & $<8$ & 903 & 1 & $<50$ & 0.215 & 16 & 72 & 10 & 245 & 284 \\
\hline 93SBK1 & $93 \mathrm{SBK} 17$ & 152 & 230 & $\mathrm{D}, \mathrm{F}$ & 4 & 5.4 & 20 & $<8$ & 617 & 2 & $<50$ & 0.105 & 19 & 73 & 7 & 145 & 31 \\
\hline 93SBK & 93SBK17 & 25 & 68 & $\mathrm{D}, \mathrm{F}$ & 65 & 2.8 & 71 & $<8$ & 1140 & 1 & $<50$ & 0.200 & 78 & 47 & 13 & 209 & 421 \\
\hline $93 \mathrm{SBK} 1$ & 93SBK18 & 7 & 10 & $\mathrm{D}, \mathrm{F}$ & 69 & 4.0 & 74 & $<8$ & 1520 & 1 & $<50$ & 0.373 & 46 & 66 & 12 & 230 & 382 \\
\hline 93SBB2 & 93SBB20 & 0 & 5 & $\mathrm{D}, \mathrm{F}$ & 15 & 3.7 & 268 & $<8$ & 515 & 1 & $<50$ & 0.200 & 21 & 57 & 12 & 106 & 89 \\
\hline 93SBB2 & 93SBB22 & 19 & 26 & $D, F$ & 28 & 4.2 & 169 & $<8$ & 886 & 2 & $<50$ & 0.194 & 10 & 71 & 11 & 132 & 148 \\
\hline $93 \mathrm{SBB}^{2}$ & 93SBB23 & 46 & 53 & $\mathrm{D}, \mathrm{F}$ & 22 & 3.7 & 176 & $<8$ & 757 & 1 & $<50$ & 0.168 & 39 & 70 & 16 & 111 & 171 \\
\hline 93SBK18(26-82) & 93SBK18 & 26 & 82 & $\mathrm{D}, \mathrm{F}$ & 103 & 3.1 & 67 & $<8$ & 1550 & & $<50$ & 0.257 & 62 & 57 & 8 & 69 & 695 \\
\hline
\end{tabular}

*Other analyses (Appendix \#): D =USGS-EDXRF, E =EWU,

$\mathrm{F}=$ CHEMEX, $\mathrm{H}=\mathrm{ACZ},----=$ none. 
Appendix G. Chemical composition of samples analyzed by ICP-AES at XRAL Labs in Golden, CO using 4 acid dissolution (Appendix_G.xls, Appendix_G.dbf).

\begin{tabular}{|c|c|c|c|c|c|c|c|c|c|c|c|c|c|c|c|c|c|c|c|c|}
\hline Sample No. & $\begin{array}{c}\text { Eu } \\
(p p m)\end{array}$ & $\begin{array}{l}\mathrm{Fe} \\
(\%)\end{array}$ & $\begin{array}{c}\mathrm{Ga} \\
(\mathrm{ppm})\end{array}$ & $\begin{array}{c}\text { Ho } \\
(\mathrm{ppm})\end{array}$ & $\begin{array}{c}\mathrm{K} \\
(\%)\end{array}$ & $\begin{array}{c}\mathrm{La} \\
(\mathrm{ppm})\end{array}$ & $\begin{array}{c}\mathrm{Li} \\
(\mathrm{ppm})\end{array}$ & $\begin{array}{l}\mathrm{Mg} \\
(\%)\end{array}$ & $\begin{array}{c}\mathrm{Mn} \\
(\mathrm{ppm})\end{array}$ & $\begin{array}{c}\text { Mo } \\
(\mathrm{ppm})\end{array}$ & $\begin{array}{l}\mathrm{Na} \\
(\%)\end{array}$ & $\begin{array}{c}\mathrm{Nb} \\
(\mathrm{ppm})\end{array}$ & $\begin{array}{c}\mathrm{Nd} \\
(\mathrm{ppm})\end{array}$ & $\begin{array}{c}\mathrm{Ni} \\
(\mathrm{ppm})\end{array}$ & $\begin{array}{c}\mathrm{P} \\
(\mathrm{ppm})\end{array}$ & $\begin{array}{c}\mathrm{Pb} \\
(\mathrm{ppm})\end{array}$ & $\begin{array}{c}\mathrm{Sc} \\
(\mathrm{ppm})\end{array}$ & $\begin{array}{c}\mathrm{Sn} \\
(\mathrm{ppm})\end{array}$ & $\begin{array}{c}\mathrm{Sr} \\
(\mathrm{ppm})\end{array}$ & $\begin{array}{c}\text { Ta } \\
\text { (ppm) }\end{array}$ \\
\hline SAMPLE_NO. & PPM & $=(\%)$ & GA_PPM & HO_PPM & $\mathrm{K}_{-}(\%)$ & LA_PPM & LI_PPM & MG_(\%) & MN_PPM & MO_PPM & NA_(\%) & NB_PPM & ND_PPM & NI_PPM & P_PPM & PB_PPM & SC_PPM & SN_PPM & SR_PPM & TA_PPM \\
\hline T98L-41(0-4) & $<2$ & 4.63 & 16 & $<4$ & 2.13 & 31 & 27 & 0.620 & 8380 & 5 & 0.450 & 5 & 28 & 17 & 0.125 & 2120 & 10 & $<50$ & 51 & $<40$ \\
\hline T98L-41(18-22) & 3 & 5.78 & 11 & $<4$ & 2.56 & 37 & 27 & 0.465 & 1120 & 6 & 0.355 & 9 & 32 & 11 & 0.070 & 3390 & 12 & $<50$ & 35 & $<40$ \\
\hline T98L-41(38-42) & 4 & 8.07 & 14 & $<4$ & 2.45 & 33 & 24 & 0.485 & 6110 & 7 & 0.280 & 8 & 30 & 8 & 0.040 & 6610 & 10 & $<50$ & 29 & $<40$ \\
\hline T98L-41(63-67) & $<2$ & 1.85 & 11 & $<4$ & 0.75 & 20 & 13 & 0.240 & 1090 & 3 & 0.230 & $<4$ & 19 & 15 & 0.130 & 290 & 8 & $<50$ & 29 & $<40$ \\
\hline T98L-41(88-92) & $<2$ & 1.41 & 18 & $<4$ & 1.78 & 44 & 30 & 0.450 & 206 & 4 & 0.670 & 6 & 36 & 19 & 0.015 & 30 & 10 & $<50$ & 61 & $<40$ \\
\hline T98L-41(103-107) & $<2$ & 1.83 & 26 & $<4$ & 2.35 & 56 & 37 & 0.602 & 123 & $<2$ & 0.807 & $<4$ & 42 & 12 & 0.011 & 24 & 9 & $<50$ & 69 & $<40$ \\
\hline T98L-42(0-4) & $<2$ & 6.44 & 15 & $<4$ & 1.94 & 29 & 26 & 0.535 & 5260 & 5 & 0.405 & 9 & 26 & 16 & 0.145 & 2200 & 10 & $<50$ & 40 & $<40$ \\
\hline T98L-42(8-12) & 3 & 8.34 & 11 & $<4$ & 2.43 & 37 & 26 & 0.460 & 1300 & 8 & 0.310 & 11 & 33 & 10 & 0.110 & 4830 & 11 & $<50$ & 31 & $<40$ \\
\hline T98L-42(18-22) & 2 & 3.60 & 20 & $<4$ & 2.23 & 39 & 31 & 0.560 & 1250 & $4 \mid$ & 0.595 & 10 & 33 & 18 & 0.050 & 4810 & 12 & $<50$ & 61 & $<40$ \\
\hline T98L-42(43-47) & $<2$ & 1.92 & 19 & $<4$ & 1.82 & 41 & 31 & 0.540 & 249 & 4 & 0.670 & 6 & 40 & 15 & 0.070 & 522 & 11 & $<50$ & 61 & $<40$ \\
\hline T98L-42(63-67) & $<2$ & 2.17 & 22 & $<4$ & 1.75 & 43 & 31 & 0.535 & 178 & 4 & 0.530 & 6 & 37 & 18 & 0.050 & 63 & 13 & $<50$ & 51 & $<40$ \\
\hline T98L-42(88-94) & 2 & 2.12 & 16 & $<4$ & 2.13 & 45 & 38 & 0.608 & 138 & $<2$ & 0.599 & 7 & 41 & 16 & 0.027 & 40 & 11 & $<50$ & 50 & $<40$ \\
\hline T98L-42(108-112) & 4 & 11.30 & $<4$ & $<4$ & 1.42 & 24 & 17 & 0.562 & 10580 & 5 & 0.189 & $<4$ & 27 & $<3$ & 0.022 & 6030 & 4 & $<50$ & 26 & $<40$ \\
\hline T98M-43(0-4) & $<2$ & 3.59 & 24 & $<4$ & 1.94 & 25 & 26 & 0.660 & 2700 & 5 & 1.315 & 11 & 23 & 12 & 0.095 & 1620 & 11 & $<50$ & 148 & $<40$ \\
\hline T98M-43(8-12) & 3 & 6.87 & 15 & $<4$ & 2.58 & 35 & 27 & 0.500 & 5280 & 6 & 0.320 & 8 & 33 & 11 & 0.095 & 5110 & 12 & $<50$ & 34 & $<40$ \\
\hline T98M-43(18-22) & $<2$ & 3.10 & 26 & $<4$ & 2.26 & 46] & 35 & 0.715 & 1240 & 5 & 0.635 & 9 & 44 & 19 & 0.160 & 2060 & 13 & $<50$ & 67 & $<40$ \\
\hline T98M-43(38-42) & $<2$ & 2.99 & 23 & $<4$ & 2.42 & 51 & 38 & 0.755 & 501 & 5 & 0.660 & $<4$ & 42 & 18 & 0.090 & 46 & 13 & $<50$ & 61 & $<40$ \\
\hline T98M-43(58-62) & 3 & 2.82 & 26 & $<4$ & 2.30 & 50 & 37 & 0.735 & 355 & 4 & 0.630 & 6 & 48 & 18 & 0.070 & 28 & 14 & $<50$ & 55 & $<40$ \\
\hline T98M-43(83-87) & $<2$ & 2.89 & $<4$ & 7 & 2.41 & 47 & 40 & 0.703 & 499 & $<2$ & 0.665 & $<4$ & 43 & 16 & 0.059 & 158 & 11 & $<50$ & 57 & $<40$ \\
\hline$\overline{\mathrm{T} 98 \mathrm{M}-43}$ & $<2$ & 2.97 & 18 & $<4$ & 2.26 & 50 & 39 & 0.722 & 344 & $4 \mid$ & 0.709 & $<4$ & 45 & 17 & 0.052 & 21 & 11 & $<50$ & 66 & $<40$ \\
\hline T98M-43 & $<2$ & 2.69 & 21 & $<4$ & 2.42 & 52 & 39 & 0.677 & 384 & 3 & 0.736 & 9 & 51 & 13 & 0.042 & 20 & 10 & $<50$ & 69 & $<40$ \\
\hline T98M-4 & 2 & 2.66 & 18 & $<4$ & 2.38 & 49 & 37 & 0.679 & 528 & $<2$ & 0.746 & 4 & 53 & 16 & 0.037 & 22 & 10 & $<50$ & 75 & $<40$ \\
\hline T98M-43 & $<2$ & 2.55 & 22 & $<4$ & 2.34 & 47 & 36 & 0.672 & 423 & 2 & 0.769 & $<4$ & 50 & 14 & 0.030 & 22 & 10 & $<50$ & 77 & $<40$ \\
\hline T98M-4 & $<2$ & 2.47 & 28 & $<4$ & 2.30 & 48 & 36 & 0.665 & 311 & $<2$ & 0.780 & $<4$ & 42 & 14 & 0.027 & 40 & 10 & $<50$ & 82 & $<40$ \\
\hline T98M-4 & $<2$ & 2.48 & 20 & $<4$ & 2.43 & 45 & 33 & 0.641 & 359 & $<2$ & 0.879 & 9 & 54 & 13 & 0.029 & 21 & 9 & $<50$ & 87 & $<40$ \\
\hline 93ABMC & 3 & 9.17 & 4 & $<4$ & 1.57 & 33 & 20 & 0.465 & 8350 & 10 & 0.260 & 8 & 33 & 8 & 0.040 & 4030 & 7 & $<50$ & 30 & $<40$ \\
\hline 93ABM04( & $<2$ & 2.41 & 27 & $<4$ & 2.00 & 44 & 29 & 0.600 & 920 & 14 & 0.610 & $<4$ & 38 & 15 & 0.055 & 296 & 10 & $<50$ & 57 & $<40$ \\
\hline 93SBC10( & 4 & 9.59 & $<4$ & $<4$ & 1.60 & 31 & 22 & 0.645 & 9310 & 24 & 0.265 & 6 & 29 & 12 & 0.035 & 11180 & 7 & $<50$ & 33 & $<40$ \\
\hline 93SBK17(152-230) & $<2$ & 3.10 & 15 & $<4$ & 2.29 & 37 & 29 & 0.440 & 2550 & 13 & 0.495 & 8 & 37 & 13 & 0.035 & 1130 & 9 & $<50$ & 52 & $<40$ \\
\hline 93SBK17( & 2 & 13.50 & $<4$ & $<4$ & 1.32 & 20 & 14 & 0.530 & 13850 & 24 & 0.105 & 4 & 22 & 5 & 0.020 & 17330 & 5 & $<50$ & 20 & $<40$ \\
\hline 93SBK18(7-10) & 4 & 10.80 & 5 & $<4$ & 1.42 & 28 & 20 & 0.390 & 11490 & 23 & 0.490 & 5 & 26 & 8 & 0.045 & 21510 & 7 & $<50$ & 63 & $<40$ \\
\hline 93SBB20 & 3 & 9.61 & $<4$ & $<4$ & 1.44 & 30 & 15 & 0.325 & 7910 & 12 & 0.250 & 7 & 24 & 8 & 0.055 & 3020 & 7 & $<50$ & 32 & $<40$ \\
\hline 93SBB22(19-26) & 4 & 11.70 & $<4$ & $<4$ & 1.62 & 33 & 20 & 0.425 & 4350 & 12 & 0.265 & 10 & 33 & 8 & 0.050 & 9340 & 8 & $<50$ & 34 & $<40$ \\
\hline 93SBB23(4 & 4 & 11.70 & $<4$ & $<4$ & 1.56 & 33 & 16 & 0.305 & 11540 & 13 & 0.190 & 7 & 33 & 11 & 0.040 & 6740 & 7 & $<50$ & 32 & $<40$ \\
\hline 93SBK18(26-82) & 4 & 11.20 & 5 & $<4$ & 1.30 & 24 & 17 & 0.580 & 11890 & 8 & 0.165 & $<4$ & 24 & & 0.030 & 32730 & 6 & $<50$ & 40 & $<40$ \\
\hline
\end{tabular}

*Other analyses (Appendix \#): D =USGS-EDXRF, E =EWU,

$\mathrm{F}=$ CHEMEX, $\mathrm{H}=\mathrm{ACZ},---=$ none. 
Appendix G. Chemical composition of samples analyzed by ICP-AES at XRAL Labs in Golden, CO using 4 acid dissolution (Appendix_G.xls, Appendix_G.dbf).

\begin{tabular}{|c|c|c|c|c|c|c|c|}
\hline Sample No. & $\begin{array}{c}\text { Th } \\
(\mathrm{ppm})\end{array}$ & $\begin{array}{l}\mathrm{Ti} \\
(\%)\end{array}$ & $\begin{array}{c}U \\
(p p m)\end{array}$ & $\begin{array}{c}\mathrm{V} \\
(\mathrm{ppm})\end{array}$ & $\begin{array}{c}\text { Y } \\
\text { (ppm) }\end{array}$ & $\begin{array}{c}\mathrm{Yb} \\
(\mathrm{ppm})\end{array}$ & $\begin{array}{c}\mathrm{Zn} \\
\text { (ppm) }\end{array}$ \\
\hline SAMPLE_NO. & PPM & TI_\% & IPPM & V_PPM & Y_PPM & YB_PPM & ZN_PPM \\
\hline T98L-41(0-4) & 11 & 0.188 & $<100$ & 49 & 13 & 2 & 1520 \\
\hline T98L-41(18-22) & 12 & 0.182 & $<100$ & 57 & 15 & 3 & 1280 \\
\hline T98L-41(38-42) & 10 & 0.153 & $<100$ & 50 & 14 & 2 & 2510 \\
\hline T98L-41(63-67) & 8 & 0.129 & $<100$ & 35 & 15 & 2 & 1820 \\
\hline T98L-41(88-92) & 10 & 0.306 & $<100$ & 50 & 15 & 2 & 166 \\
\hline T98L-41(103-107) & $<6$ & 0.326 & $<100$ & 55 & 17 & 2 & 70 \\
\hline T98L-42(0-4) & 8 & 0.17 & $<100$ & 45 & 15 & 2 & 2240 \\
\hline T98L-42(8-12) & 10 & 0.176 & $<100$ & 56 & 15 & 2 & 1880 \\
\hline T98L-42(18-22) & 12 & 0.247 & $<100$ & 56 & 17 & 2 & 1700 \\
\hline T98L-42(43-47) & 9 & 0.264 & $<100$ & 48 & 18 & 2 & 355 \\
\hline T98L-42(63-67) & 12 & 0.282 & $<100$ & 53 & 20 & 2 & 217 \\
\hline T98L-42(88-94) & 11 & 0.273 & $<100$ & 59 & 17 & 2 & 201 \\
\hline T98L-42(108-112) & $<6$ & 0.122 & 155 & 32 & 8 & $<1$ & 2030 \\
\hline T98M-43(0-4) & 7 & 0.259 & $<100$ & 55 & 14 & 2 & 1000 \\
\hline T98M-43(8-12) & 13 & 0.188 & $<100$ & 56 & 14 & 3 & 1360 \\
\hline T98M-43(18-22) & 15 & 0.27 & $<100$ & 63 & 19 & 2 & 812 \\
\hline T98M-43(38-42) & 16 & 0.294 & $<100$ & 67 & 21 & 3 & 402 \\
\hline T98M-4 & 20 & 0.282 & $<100$ & 65 & 23 & 3 & 155 \\
\hline T98M-43 & 15 & 0.277 & $<100$ & 62 & 21 & 3 & 209 \\
\hline T98M-43(98-102) & 18 & 0.32 & $<100$ & 63 & 20 & 3 & 77 \\
\hline T98M-43 & 13 & 0.286 & 179 & 58 & 17 & 2 & 67 \\
\hline T98M-4 & 7 & 0.317 & $<100$ & 60 & 20 & 3 & 69 \\
\hline T98M-4 & 15 & 0.297 & $<100$ & 57 & 17 & 2 & 58 \\
\hline T98M-4 & 8 & 0.32 & $<100$ & 58 & 18 & 2 & 58 \\
\hline T98M-4 & 11 & 0.275 & $<100$ & 54 & 16 & 2 & 99 \\
\hline 2) & 9 & 0.147 & $<100$ & 35 & 12 & 1 & 2790 \\
\hline 3ABM0 & 10 & 0.294 & $<100$ & 48 & 20 & 2 & 247 \\
\hline SBC1 & 9 & 0.182 & $<100$ & 37 & 14 & 1 & 2120 \\
\hline 93SBK17 & 9 & 0.206 & $<100$ & 45 & 15 & 2 & 985 \\
\hline 93SBK1 & $<6$ & 0.088 & $<100$ & 25 & 12 & 1 & 8990 \\
\hline 93SBK1 & 0 & 0.165 & $<100$ & 35 & 14 & 1 & 4760 \\
\hline 93SBB2 & 10 & 0.17 & $<100$ & 36 & 12 & 1 & 2270 \\
\hline & 13 & 0.147 & $<100$ & 39 & 16 & 2 & 2890 \\
\hline 3) & 11 & 0.135 & $<100$ & 34 & 11 & 1 & 4720 \\
\hline 93SBK18( & - & 0.1 & $<100$ & 27 & 10 & & 4580 \\
\hline
\end{tabular}

*Other analyses (Appendix \#): D =USGS-EDXRF, E =EWU,

$\mathrm{F}=$ CHEMEX, $\mathrm{H}=\mathrm{ACZ},---=$ none. 
Appendix G. Chemical composition of samples analyzed by ICP-AES at XRAL Labs in Golden, CO using 4 acid dissolution (Appendix_G.xls, Appendix_G.dbf).

\begin{tabular}{|c|c|c|c|c|c|c|c|c|c|c|c|c|c|c|c|c|c|}
\hline Sample No. & Site ID & $\begin{array}{c}\text { Depth } \\
\text { interval, } \\
\text { top }(\mathrm{cm})\end{array}$ & $\begin{array}{c}\text { Depth } \\
\text { interval, } \\
\text { bottom }(\mathrm{cm})\end{array}$ & $\begin{array}{c}{ }^{*} \text { Other } \\
\text { Analyses } \\
\text { (appendix \#) }\end{array}$ & $\begin{array}{c}\mathrm{Ag} \\
(\mathrm{ppm})\end{array}$ & $\begin{array}{c}\mathrm{Al} \\
(\%)\end{array}$ & $\begin{array}{c}\text { As } \\
\text { (ppm) }\end{array}$ & $\begin{array}{c}\mathrm{Au} \\
(\mathrm{ppm})\end{array}$ & $\begin{array}{c}\mathrm{Ba} \\
(\mathrm{ppm})\end{array}$ & $\begin{array}{c}\mathrm{Be} \\
(\mathrm{ppm})\end{array}$ & $\begin{array}{c}\mathrm{Bi} \\
(\mathrm{ppm})\end{array}$ & $\begin{array}{c}\mathrm{Ca} \\
(\%)\end{array}$ & $\begin{array}{c}\text { Cd } \\
\text { (ppm) }\end{array}$ & $\begin{array}{c}\mathrm{Ce} \\
(\mathrm{ppm})\end{array}$ & $\begin{array}{c}\text { Co } \\
\text { (ppm) }\end{array}$ & $\begin{array}{c}\mathrm{Cr} \\
(\mathrm{ppm})\end{array}$ & $\begin{array}{c}\mathrm{Cu} \\
\text { (ppm) }\end{array}$ \\
\hline SAMPLE_NO. & SITE_ID & INT_TOP_CM & INT_BTM_CM & OTHR_ANLYS & AG_PPM & AL_(\%) & AS_PPM & AU_PPM & BA_PPM & BE_PPM & BI_PPM & CA_(\%) & CD_PPM & CE_PPM & CO_PPM & CR_PPM & CU_PPM \\
\hline 93SBL27B(23-34) & 3SBL27B & 23 & 34 & $\mathrm{D}, \mathrm{F}$ & 20 & 3.5 & 252 & $<8$ & 619 & 1 & $<50$ & 0.152 & 21 & 77 & 16 & 126 & 154 \\
\hline 93SBL26(6-12) & 93SBL26 & 6 & 12 & $\mathrm{D}, \mathrm{F}$ & 17 & 5.6 & 194 & $<8$ & 603 & 2 & $<50$ & 0.310 & 53 & 69 & 18 & 303 & 296 \\
\hline 93SBL31(146-160) & 93SBL31 & 146 & 160 & $\mathrm{D}, \mathrm{F}$ & 16 & 3.2 & 98 & $<8$ & 542 & 1 & $<50$ & 0.394 & 26 & 56 & 14 & 119 & 128 \\
\hline 93SBL32(10.5-16) & 93SBL32 & 11 & 16 & $\mathrm{D}, \mathrm{F}$ & 6 & 6.3 & 19 & $<8$ & 738 & 2 & $<50$ & 0.294 & 5 & 88 & 9 & 84 & 51 \\
\hline 93SBL31(185-200) & 93SBL31 & 185 & 200 & $\mathrm{D}, \mathrm{F}$ & 18 & 3.3 & 84 & $<8$ & 530 & 1 & $<50$ & 0.457 & 28 & 62 & 13 & 374 & 227 \\
\hline 94Gid2(0-30) & 94Gid2 & 0 & 30 & $\mathrm{E}, \mathrm{H}$ & 15 & 4.1 & 122 & $<8$ & 649 & 1 & $<50$ & 0.336 & 22 & 69 & 8 & 233 & 107 \\
\hline 94Gid2(0-30) & 94Gid2 & 0 & 30 & $E, H$ & 13 & 4.0 & 109 & $<8$ & 570 & 1 & $<50$ & 0.331 & 19 & 64 & 9 & 191 & 102 \\
\hline 94Gid2(0-30) & 94Gid2 & 0 & 30 & $\mathrm{E}, \mathrm{H}$ & 16 & 4.0 & 112 & $<8$ & 645 & 1 & $<50$ & 0.326 & 21 & 68 & 8 & 213 & 103 \\
\hline 94Gid2(250-280) & 94Gid2 & 250 & 280 & $E, H$ & 44 & 3.8 & 67 & $<8$ & 1210 & 1 & $<50$ & 0.630 & 99 & 69 & 13 & 113 & 241 \\
\hline 94Gid2(250-280) & 94Gid2 & 250 & 280 & $\bar{E}, \mathrm{H}$ & 36 & 3.8 & 69 & $<8$ & 1060 & 1 & $<50$ & 0.641 & 98 & 69 & 15 & 105 & 258 \\
\hline 94Gid2(30-60) & 94Gid2 & 30 & 60 & $E, H$ & 19 & 3.8 & 132 & $<8$ & 655 & 1 & $<50$ & 0.268 & 37 & 70 & 9 & 149 & 107 \\
\hline 94Gid2(30-60) & 94Gid2 & 30 & 60 & $\mathrm{E}, \mathrm{H}$ & 16 & 3.9 & 133 & $<8$ & 638 & 1 & $<50$ & 0.272 & 38 & 73 & 11 & 148 & 114 \\
\hline 94Gid2(60-90) & 94Gid2 & 60 & 90 & $\mathrm{E}, \mathrm{H}$ & 20 & 2.7 & 405 & $<8$ & 516 & $<1$ & $<50$ & 0.320 & 30 & 51 & 17 & 134 & 104 \\
\hline 94Gid2(60-90) & 94Gid2 & 60 & 90 & $\mathrm{E}, \mathrm{H}$ & 15 & 2.8 & 401 & $<8$ & 479 & $<1$ & $<50$ & 0.331 & 31 & 58 & 20 & 134 & 124 \\
\hline 94Gid3(244-284) & 94Gid3 & 244 & 284 & $\overline{E, H}$ & $<2$ & 5.1 & 18 & $<8$ & 500 & 1 & $<50$ & 0.168 & 2 & 84 & 11 & 38 & 17 \\
\hline 94Gid3(244-284) & 94Gid3 & 244 & 284 & $\mathrm{E}, \mathrm{H}$ & $<2$ & 5.3 & 16 & $<8$ & 497 & 2 & $<50$ & 0.176 & $<2$ & 82 & 14 & 64 & 17 \\
\hline 94Gid3(3-12) & 94Gid3 & 3 & 12 & $\mathrm{E}, \mathrm{H}$ & 19 & 3.5 & 110 & $<8$ & 638 & 1 & $<50$ & 0.179 & 25 & 62 & 11 & 185 & 115 \\
\hline 94Gid3(3-12) & 94Gid3 & 3 & 12 & $\mathrm{E}, \mathrm{H}$ & 17 & 3.6 & 116 & $<8$ & 627 & 1 & $<50$ & 0.181 & 25 & 64 & 13 & 153 & 127 \\
\hline 94Gid4(31-63) & 94Gid4 & 31 & 63 & $\mathrm{E}, \mathrm{H}$ & 38 & 4.0 & 106 & $<8$ & 1020 & 1 & $<50$ & 0.142 & 18 & 72 & 8 & 117 & 217 \\
\hline 94Gid4(31-63) & 94Gid4 & 31 & 63 & $\mathrm{E}, \mathrm{H}$ & 34 & 4.2 & 100 & $<8$ & 983 & 2 & $<50$ & 0.144 & 18 & 77 & 10 & 115 & 246 \\
\hline 94Gid6(0-20) & 94Gid6 & 0 & 20 & $\mathrm{E}, \mathrm{H}$ & 26 & 4.7 & 103 & $<8$ & 805 & 1 & $<50$ & 0.273 & 16 & 69 & 11 & 163 & 131 \\
\hline 94Gid6(0-20) & 94Gid6 & 0 & 20 & $\bar{E}, \mathrm{H}$ & 18 & 5.0 & 111 & $<8$ & 804 & 2 & $<50$ & 0.288 & 16 & 80 & 13 & 153 & 147 \\
\hline 94VC1(10-20) & 94VC1 & 10 & 20 & $\mathrm{H}$ & 10 & 3.9 & 50 & $<8$ & 437 & 1 & $<50$ & 0.189 & 19 & 55 & 6 & 69 & 72 \\
\hline 94VC11 & $94 \mathrm{VC1}$ & 10 & 20 & $\mathrm{H}$ & 8 & 3.9 & 51 & $<8$ & 436 & 1 & $<50$ & 0.187 & 19 & 55 & 8 & 97 & 79 \\
\hline 94VC11 & $94 \mathrm{VC1}$ & 40 & 50 & $\mathrm{E}, \mathrm{H}$ & 39 & 2.4 & 58 & $<8$ & 969 & $<1$ & $<50$ & 0.814 & 135 & 45 & 14 & 77 & 240 \\
\hline 94VC1(140-150) & $94 \mathrm{VC1}$ & 40 & 50 & $\mathrm{E}, \mathrm{H}$ & 28 & 2.3 & 58 & $<8$ & 456 & 1 & $<50$ & 0.823 & 131 & 48 & 17 & 76 & 276 \\
\hline 94VC1(150-160) & 94VC1 & 50 & 60 & $\mathrm{E}, \mathrm{H}$ & 51 & 3.2 & 76 & $<8$ & 1280 & 1 & $<50$ & 0.830 & 132 & 50 & 15 & 80 & 238 \\
\hline 94VC1(180-190) & 94VC1 & 180 & 190 & $\mathrm{E}, \mathrm{H}$ & 46 & 2.9 & 67 & $<8$ & 1110 & 1 & $<50$ & 0.767 & 109 & 52 & 15 & 76 & 218 \\
\hline 94VC1(180-190) & $94 \mathrm{VC1}$ & 180 & 190 & $\mathrm{E}, \mathrm{H}$ & 37 & 3.2 & 63 & $<8$ & 289 & 1 & $<50$ & 0.829 & 114 & 54 & 18 & 77 & 262 \\
\hline 94VC1(190-200) & 94VC1 & 190 & 200 & $E, H$ & 56 & 2.3 & 55 & $<8$ & 832 & $<1$ & $<50$ & 0.646 & 173 & 42 & 14 & 83 & 408 \\
\hline $94 \mathrm{VC} 1(2$ & 94VC1 & 200 & 207 & $E, H$ & 44 & 2.1 & 56 & $<8$ & 736 & $<1$ & $<50$ & 0.719 & 167 & 42 & 13 & 96 & 397 \\
\hline 94VC1(200-207) & 94VC1 & 200 & 207 & $\mathrm{E}, \mathrm{H}$ & 50 & 2.0 & 47 & $<8$ & 691 & $<1$ & $<50$ & 0.688 & 166 & 43 & 13 & 87 & 388 \\
\hline 94VCD3(67-100) & 94VCD3 & 67 & 100 & $E, H$ & $<2$ & 5.6 & 11 & $<8$ & 540 & 2 & $<50$ & 0.215 & $<2$ & 85 & 11 & 43 & 14 \\
\hline 94VCD3(67-100) & 94VCD3 & 67 & 100 & $E, H$ & $<2$ & 5.5 & 11 & $<8$ & 493 & 2 & $<50$ & 0.208 & $<2$ & 87 & 12 & 28 & 13 \\
\hline 94VCK1(0-33) & 94VCK1 & 0 & 33 & $\mathrm{E}, \mathrm{H}$ & $17 \mid$ & 3.5 & 82 & $<8$ & 540 & 1 & $<50$ & 0.263 & 34 & 59 & 9 & 84 & 100 \\
\hline
\end{tabular}

*Other analyses (Appendix \#): D =USGS-EDXRF, E =EWU,

$\mathrm{F}=$ CHEMEX, $\mathrm{H}=\mathrm{ACZ},----=$ none. 
Appendix G. Chemical composition of samples analyzed by ICP-AES at XRAL Labs in Golden, CO using 4 acid dissolution (Appendix_G.xls, Appendix_G.dbf).

\begin{tabular}{|c|c|c|c|c|c|c|c|c|c|c|c|c|c|c|c|c|c|c|c|c|}
\hline Sample No. & $\begin{array}{c}\text { Eu } \\
(p p m)\end{array}$ & $\begin{array}{l}\mathrm{Fe} \\
(\%)\end{array}$ & $\begin{array}{c}\mathrm{Ga} \\
(\mathrm{ppm})\end{array}$ & $\begin{array}{c}\text { Ho } \\
(\mathrm{ppm})\end{array}$ & $\begin{array}{c}\mathrm{K} \\
(\%)\end{array}$ & $\begin{array}{c}\mathrm{La} \\
(\mathrm{ppm})\end{array}$ & $\begin{array}{c}\mathrm{Li} \\
(\mathrm{ppm})\end{array}$ & $\begin{array}{l}\mathrm{Mg} \\
(\%)\end{array}$ & $\begin{array}{c}\mathrm{Mn} \\
(\mathrm{ppm})\end{array}$ & $\begin{array}{c}\text { Mo } \\
\text { (ppm) }\end{array}$ & $\begin{array}{l}\mathrm{Na} \\
(\%)\end{array}$ & $\begin{array}{c}\mathrm{Nb} \\
(\mathrm{ppm})\end{array}$ & $\begin{array}{c}\mathrm{Nd} \\
(\mathrm{ppm})\end{array}$ & $\begin{array}{c}\mathrm{Ni} \\
(\mathrm{ppm})\end{array}$ & $\begin{array}{c}P \\
(p p m)\end{array}$ & $\begin{array}{c}\mathrm{Pb} \\
(\mathrm{ppm})\end{array}$ & $\begin{array}{c}\text { Sc } \\
(\mathrm{ppm})\end{array}$ & $\begin{array}{c}\text { Sn } \\
(p p m)\end{array}$ & $\begin{array}{c}\mathrm{Sr} \\
(\mathrm{ppm})\end{array}$ & $\begin{array}{c}\text { Ta } \\
\text { (ppm) }\end{array}$ \\
\hline SAMPLE_NO. & PPM & FE_(\%) & GA_PPM & HO_PPM & K_(\%) & LA_PPM & LI_PPM & MG_(\%) & MN_PPM & MO_PPM & NA_(\%) & NB_PPM & ND_PPM & NI_PPM & P_PPM & PB_PPM & SC_PPM & SN_PPM & SR_PPM & TA_PPM \\
\hline 93SBL27B(23-34) & 4 & 9.94 & $<4$ & $<4$ & 1.43 & 32 & 17 & 0.355 & 9400 & 12 & 0.220 & $<4$ & 29 & 11 & 0.030 & 5360 & 7 & $<50$ & 29 & $<40$ \\
\hline 93SBL26(6-12) & 3 & 6.42 & 5 & $<4$ & 2.04 & 34 & 22 & 0.520 & 4870 & 19 & 0.380 & 7 & 30 & 28 & 0.045 & 4410 & 10 & $<50$ & 48 & $<40$ \\
\hline 93SBL31(146-160) & 3 & 9.10 & 8 & $<4$ & 1.40 & 26 & $\overline{15}$ & 0.520 & 8980 & 7 & 0.205 & 7 & 24 & 12 & 0.025 & 5630 & 6 & $<50$ & 31 & $<40$ \\
\hline 93SBL32(10.5-16) & $<2$ & 2.89 & 24 & $<4$ & 2.06 & 44 & 31 & 0.650 & 1030 & 5 & 0.645 & 6 & 38 & 15 & 0.145 & 2120 & 13 & $<50$ & 65 & $<40$ \\
\hline 93SBL31(185-200) & 3 & 9.59 & $<4$ & $<4$ & 1.38 & 29 & 15 & 0.530 & 9250 & 31 & 0.220 & 4 & 25 & 14 & 0.030 & 6100 & $\overline{6}$ & $<50$ & 33 & $<40$ \\
\hline 94Gid2(0-30) & 3 & 8.64 & 7 & $<4$ & 1.53 & 33 & 20 & 0.555 & 6770 & 20 & 0.435 & 5 & 30 & 7 & 0.060 & 4300 & 7 & $<50$ & 50 & $<40$ \\
\hline 94Gid2(0-30) & 3 & 8.46 & 6 & $<4$ & 1.49 & 30 & 21 & 0.510 & 6560 & 18 & 0.435 & 7 & 28 & 14 & 0.050 & 4210 & 5 & $<50$ & 51 & $<40$ \\
\hline 94Gid2(0-30) & 3 & 8.31 & $<4$ & $<4$ & 1.50 & 31 & 19 & 0.550 & 6610 & 19 & 0.425 & 5 & 33 & 8 & 0.060 & 4280 & $\overline{7}$ & $<50$ & 49 & $<40$ \\
\hline 94Gid2(250-280) & 5 & 12.00 & $<4$ & $<4$ & 1.49 & 31 & 20 & 0.760 & 12710 & 12 & 0.245 & 5 & 31 & 7 & 0.035 & 17200 & 7 & $<50$ & 44 & $<40$ \\
\hline 94Gid2(250-280) & 5 & 12.40 & $<4$ & $<4$ & 1.48 & 31 & 25 & 0.735 & 12690 & 14 & 0.255 & 6 & 31 & 21 & 0.030 & 17740 & 5 & $<50$ & 45 & $<40$ \\
\hline 94Gid2(30-60) & 3 & 8.31 & 12 & $<4$ & 1.51 & 32 & 19 & 0.550 & 6790 & 14 & 0.325 & 6 & 30 & 8 & 0.055 & 4550 & 7 & $<50$ & 38 & $<40$ \\
\hline 94Gid2(30-60) & 3 & 8.76 & 6 & $<4$ & 1.51 & 33 & 23 & 0.550 & 6910 & 16 & 0.335 & 6 & 31 & 15 & 0.050 & 4870 & 6 & $<50$ & 40 & $<40$ \\
\hline 94Gid2(60-90) & 4 & 12.40 & $<4$ & $<4$ & 1.12 & 22 & 12 & 0.550 & 11770 & 13 & 0.160 & $<4$ & 23 & 7 & 0.035 & 3200 & 5 & $<50$ & 23 & $<40$ \\
\hline 94Gid2(60-90) & $<2$ & 13.00 & $<4$ & $<4$ & 1.14 & 25 & 15 & 0.570 & 12120 & 13 & 0.170 & $<4$ & 25 & 17 & 0.035 & 3610 & 4 & $<50$ & 26 & $<40$ \\
\hline 94Gid3(244-284) & $<2$ & 2.52 & 20 & $<4$ & 2.02 & 41 & 26 & 0.575 & 892 & 3 & 0.475 & $<4$ & 36 & 12 & 0.035 & 397 & $\overline{9}$ & $<50$ & 45 & $<40$ \\
\hline 94Gid3(244-284) & $<2$ & 2.66 & 13 & $<4$ & 2.07 & 42 & 34 & 0.595 & 920 & $\overline{3}$ & 0.500 & $<4$ & 40 & 14 & 0.035 & 430 & 8 & $<50$ & 48 & $<40$ \\
\hline 94Gid3(3-12) & 3 & 10.60 & $<4$ & $<4$ & 1.50 & 29 & 16 & 0.420 & 10610 & 13 & 0.240 & $<4$ & 26 & 5 & 0.025 & 3950 & 6 & $<50$ & 29 & $<40$ \\
\hline 94Gid3(3-12) & 4 & 11.20 & $<4$ & $<4$ & 1.54 & 30 & 20 & 0.430 & 10630 & 17 & 0.250 & 9 & 32 & 13 & 0.025 & 4340 & 5 & $<50$ & 31 & $<40$ \\
\hline 94Gid4(31-63) & 4 & 10.70 & $<4$ & $<4$ & 1.63 & 34 & 21 & 0.325 & 6470 & 10 & 0.265 & 8 & 33 & 6 & 0.035 & 12960 & 7 & $<50$ & 32 & $<40$ \\
\hline 94Gid4(3 & 4 & 11.00 & $<4$ & $<4$ & 1.66 & 35 & 25 & 0.325 & 6560 & 11 & 0.280 & 10 & 33 & 14 & 0.035 & 13960 & $\overline{6}$ & $<50$ & 34 & $<40$ \\
\hline 94Gid6(0 & 3 & 7.67 & 7 & $<4$ & 1.73 & 36 & 22 & 0.485 & 6200 & 12 & 0.465 & 11 & 33 & 12 & 0.085 & 7300 & $\overline{9}$ & $<50$ & 52 & $<40$ \\
\hline 94Gid6(0 & 3 & 8.16 & 5 & $<4$ & 1.80 & 38 & 29 & 0.510 & 6450 & 16 & 0.505 & 11 & 35 & 17 & 0.090 & 8100 & 7 & $<50$ & 56 & $<40$ \\
\hline 94VC1(1 & 2 & 6.36 & 16 & $<4$ & 1.79 & 27 & 19 & 0.510 & 5610 & 6 & 0.270 & 7 & 27 & 6 & 0.020 & 2490 & 6 & $<50$ & 31 & $<40$ \\
\hline 94VC1(1 & 2 & 6.51 & 9 & $<4$ & 1.78 & 30 & 24 & 0.530 & 5580 & 5 & 0.280 & 6 & 29 & 12 & 0.020 & 2780 & 5 & $<50$ & 31 & $<40$ \\
\hline 94VC1(1 & 2 & 16.10 & $<4$ & $<4$ & 1.03 & 18 & 13 & 0.800 & 17120 & 11 & 0.120 & 10 & 18 & $<3$ & 0.015 & 13430 & $\overline{4}$ & $<50$ & 37 & $<40$ \\
\hline $94 \mathrm{VC1}(12$ & 3 & 16.00 & $<4$ & $<4$ & 1.01 & 18 & 17 & 0.780 & 16780 & 13 & 0.120 & 13 & 23 & 15 & 0.015 & 13950 & 3 & $<50$ & 37 & $<40$ \\
\hline 94VC1(150-160) & 3 & 15.30 & $<4$ & $<4$ & 1.35 & 20 & 17 & 0.875 & 17820 & 13 & 0.130 & 8 & 23 & 8 & 0.025 & 19220 & 5 & $<50$ & 42 & $<40$ \\
\hline 94VC1(180-190) & 3 & 14.80 & $<4$ & $<4$ & 1.22 & 20 & 15 & 0.775 & 15820 & 16 & 0.135 & 7 & 17 & 5 & 0.025 & 17280 & 5 & $<50$ & 37 & $<40$ \\
\hline 94VC1(180-190) & 3 & 16.00 & $<4$ & $<4$ & 1.29 & 23 & 20 & 0.825 & 16710 & 15 & 0.145 & 10 & 24 & 17 & 0.025 & 19270 & 4 & $<50$ & 41 & $<40$ \\
\hline 94VC1 $1(190-200)$ & 3 & 17.00 & $<4$ & $<4$ & 0.99 & 19 & 12 & 0.865 & 20740 & 11 & 0.100 & 10 & 21 & $<3$ & 0.020 & 15310 & 5 & $<50$ & 29 & $<40$ \\
\hline 94VC1(200-207) & 3 & 17.30 & $<4$ & $<4$ & 0.95 & 21 & 12 & 0.920 & 22120 & 21 & 0.095 & 13 & 26 & $<3$ & 0.015 & 13260 & 4 & $<50$ & 30 & $<40$ \\
\hline 94VC1(200-207) & 3 & 17.70 & $<4$ & $<4$ & 0.90 & 19 & 12 & 0.885 & 21110 & 16 & 0.090 & 10 & 21 & $<3$ & 0.015 & 12640 & 5 & $<50$ & 29 & $<40$ \\
\hline 94VCD3(67-100) & $<2$ & 1.89 & 20 & $<4$ & 2.07 & 44 & 30 & 0.600 & 110 & 4 & 0.570 & 5 & 38 & 14 & 0.030 & 22 & 10 & $<50$ & 53 & $<40$ \\
\hline 94VCD3(67-100) & $<2$ & 1.88 & 14 & $<4$ & 2.02 & 43 & 34 & 0.575 & 112 & 3 & 0.570 & 6 & 38 & 16 & 0.030 & 23 & 9 & $<50$ & 53 & $<40$ \\
\hline 94VCK1(0-33) & 3 & 9.32 & 6 & $<4$ & 1.57 & 27 & 17 & 0.600 & 9120 & 12 & 0.220 & 5 & 23 & 6 & 0.025 & 2780 & 6 & $<50$ & 28 & $<40$ \\
\hline
\end{tabular}

*Other analyses (Appendix \#): D =USGS-EDXRF, E =EWU,

$\mathrm{F}=$ CHEMEX, $\mathrm{H}=\mathrm{ACZ},---=$ none. 
Appendix G. Chemical composition of samples analyzed by ICP-AES at XRAL Labs in Golden, CO using 4 acid dissolution (Appendix_G.xls, Appendix_G.dbf).

\begin{tabular}{|c|c|c|c|c|c|c|c|}
\hline San & $\begin{array}{c}\text { Th } \\
(\mathrm{ppm})\end{array}$ & $\begin{array}{c}\mathrm{Ti} \\
(\%)\end{array}$ & $\begin{array}{c}\mathrm{U} \\
(\mathrm{ppm})\end{array}$ & $\begin{array}{c}\mathrm{V} \\
(\mathrm{ppm})\end{array}$ & $\begin{array}{c}Y \\
(p p m)\end{array}$ & $\begin{array}{c}\mathrm{Yb} \\
(\mathrm{ppm})\end{array}$ & $\begin{array}{c}\mathrm{Zn} \\
(\mathrm{ppm})\end{array}$ \\
\hline SAMPLE_NO. & PPM & TI_\% & \begin{tabular}{l|l} 
U_PPM \\
\end{tabular} & V_PPM & YPPPM & YB_PPM & ZN_PPM \\
\hline 93SBL27B(23-34) & 12 & 0.135 & $<100$ & 34 & 12 & 1 & 2970 \\
\hline 93SBL26(6-12) & $<6$ & 0.2 & $<100$ & 48 & 15 & 2 & 4720 \\
\hline 93SBL31(146-160) & 9 & 0.129 & $<100$ & 31 & 10 & 1 & 6030 \\
\hline 93SBL32(10.5-16) & 12 & 0.3 & $<100$ & 57 & 20 & 3 & 418 \\
\hline 93SBL31(185-200) & $<6$ & 0.159 & $<100$ & 32 & 16 & 2 & 3840 \\
\hline 94Gid2(0-30) & 6 & 0.188 & $<100$ & 38 & 14 & 2 & 1940 \\
\hline 94Gid2(0-30) & 11 & 0.194 & $<100$ & 36 & 13 & 1 & 1940 \\
\hline 94Gid2(0-30) & 10 & 0.188 & $<100$ & 38 & 14 & 1 & 1900 \\
\hline 94Gid2(250-280) & 10 & 0.141 & $<100$ & 36 & 15 & 2 & 12720 \\
\hline 94Gid2(250-280) & 10 & 0.163 & $<100$ & 36 & 18 & 2 & 13910 \\
\hline 94Gid2(30-60) & 12 & 0.165 & $<100$ & 35 & 13 & 1 & 3710 \\
\hline 94Gid2(30-60) & 10 & 0.15 & $<100$ & 37 & 13 & $<1$ & 4160 \\
\hline $4 \mathrm{Gid2} 6$ & 8 & 0.094 & $<100$ & 24 & 11 & 1 & 4020 \\
\hline 94Gid2(6 & 12 & 0.1 & $<100$ & 25 & 10 & $<1$ & 4620 \\
\hline 94Gid3( & 11 & 0.282 & $<100$ & 44 & 15 & 2 & 310 \\
\hline 4Gid3 & 12 & 0.275 & $<100$ & 47 & 14 & $\overline{2}$ & 368 \\
\hline 94Gid31 & 11 & 0.159 & $<100$ & 31 & 13 & 1 & 3520 \\
\hline Gid3 & 13 & 0.163 & $<100$ & 33 & 12 & $<1$ & 4020 \\
\hline Gid & 10 & 0.153 & $<100$ & 37 & 14 & 2 & 3580 \\
\hline Gid4 & 14 & 0.15 & $<100$ & 39 & 14 & 1 & 3950 \\
\hline 4Gid6( & 10 & 0.229 & $<100$ & 43 & 16 & 2 & 1430 \\
\hline 94Gid6( & 12 & 0.219 & $<100$ & 46 & 16 & 1 & 1650 \\
\hline tVC1(1 & 6 & 0.135 & $<100$ & 31 & 9 & 2 & 2660 \\
\hline $4 \mathrm{VC} 1(1$ & 10 & 0.131 & $<100$ & 32 & 9 & 2 & 3050 \\
\hline $\operatorname{tVC} 1(1$ & 8 & 0.088 & $<100$ & 26 & 11 & 1 & 16320 \\
\hline 94VC1(1 & 10 & 0.075 & $<100$ & 27 & 11 & $<1$ & 17500 \\
\hline 94VC1(1 & $<6$ & 0.094 & $<100$ & 31 & 14 & 1 & 15800 \\
\hline 94VC1(1 & 9 & 0.094 & $<100$ & 28 & 12 & 1 & 13540 \\
\hline 94VC1(180-190) & 12 & 0.1 & 104 & 30 & 13 & 1 & 15850 \\
\hline 94VC1(1 & 7 & 0.112 & $<100$ & 24 & 11 & 1 & 20890 \\
\hline $94 \mathrm{VC} 1(2$ & 11 & 0.106 & $<100$ & 23 & 12 & 1 & 20230 \\
\hline 94VC112 & 8 & 0.118 & $<100$ & 22 & 12 & 1 & 19810 \\
\hline 94VCD3( & 13 & 0.294 & $<100$ & 49 & 18 & 2 & 64 \\
\hline 94 VCD3 & 12 & 0.313 & $<100$ & 48 & $\overline{18}$ & 2 & 66 \\
\hline 94VCK1(0-33) & 6 & 0.147 & $<100$ & 31 & 10 & 1 & 4680 \\
\hline
\end{tabular}

*Other analyses (Appendix \#): D =USGS-EDXRF, E =EWU,

$\mathrm{F}=$ CHEMEX, $\mathrm{H}=\mathrm{ACZ},---=$ none. 
Appendix G. Chemical composition of samples analyzed by ICP-AES at XRAL Labs in Golden, CO using 4 acid dissolution (Appendix_G.xls, Appendix_G.dbf).

\begin{tabular}{|c|c|c|c|c|c|c|c|c|c|c|c|c|c|c|c|c|c|}
\hline Sample No. & Site ID & $\begin{array}{c}\text { Depth } \\
\text { interval, } \\
\text { top }(\mathrm{cm})\end{array}$ & $\begin{array}{c}\text { Depth } \\
\text { interval, } \\
\text { bottom }(\mathrm{cm})\end{array}$ & $\begin{array}{c}{ }^{*} \text { Other } \\
\text { Analyses } \\
\text { (appendix \#) }\end{array}$ & $\begin{array}{c}\mathrm{Ag} \\
(\mathrm{ppm})\end{array}$ & $\begin{array}{c}\text { Al } \\
(\%)\end{array}$ & $\begin{array}{c}\text { As } \\
(\mathrm{ppm})\end{array}$ & $\begin{array}{c}\mathrm{Au} \\
(\mathrm{ppm})\end{array}$ & $\begin{array}{c}\mathrm{Ba} \\
(\mathrm{ppm})\end{array}$ & $\begin{array}{c}\mathrm{Be} \\
(\mathrm{ppm})\end{array}$ & $\begin{array}{c}\mathrm{Bi} \\
(\mathrm{ppm})\end{array}$ & $\begin{array}{c}\mathrm{Ca} \\
(\%)\end{array}$ & $\begin{array}{c}\text { Cd } \\
\text { (ppm) }\end{array}$ & $\begin{array}{c}\mathrm{Ce} \\
(\mathrm{ppm})\end{array}$ & $\begin{array}{c}\text { Co } \\
(\mathrm{ppm})\end{array}$ & $\begin{array}{c}\mathrm{Cr} \\
(\mathrm{ppm})\end{array}$ & $\begin{array}{c}\mathrm{Cu} \\
(\mathrm{ppm})\end{array}$ \\
\hline SAMPLE_NO. & SITE_ID & -TOP_CM & T_BTM_CM & LYS & SPM & AL_(\%) & 5.PPM & U_PPM & BA_PPM & E_PPM & I_PPM & $\mathrm{CA}_{-}(\%)$ & DPPM & CE_PPM & CO_PPM & R_PPM & CU_PPM \\
\hline 94VCK1(0-33) & $4 \mathrm{VCK} 1$ & 0 & 8 & & 13 & 3.6 & 90 & $<8$ & 530 & 1 & $<50$ & 0.278 & 37 & 56 & 12 & 123 & 111 \\
\hline 94VCK & 94VCK1 & 233 & 2 & $\mathrm{E}, \mathrm{H}$ & 23 & 2.9 & 78 & $<8$ & 639 & $<1$ & $<50$ & 0.415 & 47 & 52 & 11 & 127 & 119 \\
\hline 94VCK1(233-267) & 94VCK1 & 233 & 267 & $\mathrm{E}, \mathrm{H}$ & 17 & 3.1 & 78 & $<8$ & 622 & 1 & $<50$ & 0.449 & 47 & 55 & 13 & 127 & 132 \\
\hline 94VCK2(300-333) & 94VCK2 & 300 & 333 & $\mathrm{E}, \mathrm{H}$ & 21 & 3.0 & 121 & $<8$ & 680 & 1 & $<50$ & 0.431 & 41 & 50 & 14 & 100 & 117 \\
\hline 95PCK1(260-262) & 95PCK1 & 260 & 262 & $\mathrm{E}, \mathrm{H}$ & 39 & 4.0 & 146 & $<8$ & 1220 & 2 & $<50$ & 0.583 & 72 & 68 & 15 & 100 & 189 \\
\hline 95PCK1(297-326) & 95PCK1 & 297 & 326 & $\mathrm{E}, \mathrm{H}$ & 94 & 4.8 & 57 & $<8$ & 1200 & 2 & $<50$ & 0.457 & 98 & 80 & 11 & 97 & 325 \\
\hline 95PCK1(415-418) & 95PCK1 & 415 & 418 & $\mathrm{E}, \mathrm{H}$ & 3 & 5.1 & 24 & $<8$ & 575 & 2 & $<50$ & 0.236 & 5 & 83 & 7 & 176 & 24 \\
\hline 95PCK1(415-418) & 95PCK1 & 415 & 418 & $\mathrm{E}, \mathrm{H}$ & $<2$ & 5.1 & 29 & $<8$ & 565 & 2 & $<50$ & 0.236 & 5 & 90 & 6 & 99 & 24 \\
\hline 95PCUD2(0-25) & 95PCUD2 & 0 & 25 & $\mathrm{E}, \mathrm{H}$ & 96 & 5.0 & 78 & $<8$ & 1240 & 2 & $<50$ & 0.462 & 99 & 68 & 13 & 101 & 332 \\
\hline 95VCD3(402-423) & 95VCD3 & 402 & 423 & $\mathrm{E}, \mathrm{H}$ & 74 & 5.0 & 54 & $<8$ & 822 & 2 & $<50$ & 0.362 & 69 & 68 & 9 & 138 & 238 \\
\hline 95VCD3(195.5-214) & 95VCD3 & 195.5 & 214 & $\mathrm{E}, \mathrm{H}$ & 34 & 2.8 & 62 & $<8$ & 736 & $<1$ & $<50$ & 0.504 & 82 & 48 & 11 & 83 & 208 \\
\hline 95VCD3(195.5-214) & 95VCD3 & 195.5 & 214 & $\mathrm{E}, \mathrm{H}$ & 27 & 2.8 & 73 & $<8$ & 774 & 1 & $<50$ & 0.515 & 88 & 46 & 11 & 83 & 205 \\
\hline 95VCUD1(266-286) & 95VCUD1 & 266 & 286 & $\mathrm{E}, \mathrm{H}$ & 32 & 3.5 & 55 & $<8$ & 783 & 1 & $<50$ & 0.520 & 87 & 54 & 11 & 79 & 215 \\
\hline 95VCUD1(25-50) & 95VCUD1 & 25 & 50 & $\mathrm{E}, \mathrm{H}$ & 9 & 3.8 & 45 & $<8$ & 450 & 1 & $<50$ & 0.158 & 11 & 51 & 6 & 86 & 57 \\
\hline $96 \mathrm{~K} 114 \mathrm{E}(140-160)$ & $96 \mathrm{~K} 114 \mathrm{E}$ & 140 & 160 & $\mathrm{E}, \mathrm{H}$ & 23 & 3.7 & 123 & $<8$ & 803 & 1 & $<50$ & 0.294 & 27 & 65 & 11 & 58 & 131 \\
\hline $96 \mathrm{~K} 114 \mathrm{E}(140-160)$ & 96K114E & 140 & 160 & $\mathrm{E}, \mathrm{H}$ & 19 & 3.6 & 108 & $<8$ & 699 & 1 & $<50$ & 0.288 & 26 & 56 & 12 & 58 & 133 \\
\hline
\end{tabular}


Appendix G. Chemical composition of samples analyzed by ICP-AES at XRAL Labs in Golden, CO using 4 acid dissolution (Appendix_G.xls, Appendix_G.dbf).

\begin{tabular}{|c|c|c|c|c|c|c|c|c|c|c|c|c|c|c|c|c|c|c|c|c|}
\hline Sample No. & $\begin{array}{c}\text { Eu } \\
\text { (ppm) }\end{array}$ & $\begin{array}{l}\mathrm{Fe} \\
(\%)\end{array}$ & $\begin{array}{c}\mathrm{Ga} \\
(\mathrm{ppm})\end{array} \mid$ & $\begin{array}{c}\mathrm{Ho} \\
\text { (ppm) }\end{array}$ & $\begin{array}{c}\mathrm{K} \\
(\%)\end{array}$ & $\begin{array}{c}\mathrm{La} \\
(\mathrm{ppm})\end{array}$ & $\begin{array}{c}\mathrm{Li} \\
(\mathrm{ppm})\end{array}$ & $\begin{array}{l}\mathrm{Mg} \\
(\%)\end{array}$ & $\begin{array}{c}\mathrm{Mn} \\
\text { (ppm) }\end{array}$ & $\begin{array}{c}\text { Mo } \\
\text { (ppm) }\end{array}$ & $\begin{array}{l}\mathrm{Na} \\
(\%)\end{array}$ & $\begin{array}{c}\mathrm{Nb} \\
(\mathrm{ppm})\end{array}$ & $\begin{array}{c}\mathrm{Nd} \\
(\mathrm{ppm})\end{array}$ & $\begin{array}{c}\mathrm{Ni} \\
\text { (ppm) }\end{array}$ & $\begin{array}{c}\mathrm{P} \\
(\mathrm{ppm})\end{array}$ & $\begin{array}{c}\mathrm{Pb} \\
(\mathrm{ppm})\end{array}$ & $\begin{array}{c}\text { Sc } \\
(\mathrm{ppm})\end{array}$ & $\begin{array}{c}\text { Sn } \\
(\mathrm{ppm})\end{array}$ & $\begin{array}{c}\mathrm{Sr} \\
(\mathrm{ppm})\end{array}$ & $\begin{array}{c}\text { Ta } \\
\text { (ppm) }\end{array}$ \\
\hline SAMPLE_NO. & PPM & $\mathrm{FE}_{-}(\%)$ & APPM & HO_PPM & K_(\%) & LA_PPM & LI_PPM & MG_(\%) & MN_PPM & MO_PPM & NA_(\%) & B_PPM & ND_PPM & NI_PPM & P_PPM & PB_PPM & SC_PPM & SN_PPM & SR_PPM & TA_PPM \\
\hline 94VCK1(0-33) & 3 & 10.20 & 5 & $<4$ & 1.65 & 30 & 22 & 0.615 & 9770 & 15 & 0.240 & 5 & 27 & $\overline{14}$ & 0.025 & 3080 & 5 & $<50$ & 30 & $<40$ \\
\hline 94VCK1(233-267) & 3 & 9.98 & $<4$ & $<4$ & 1.31 & 23 & 14 & 0.580 & 10680 & 15 & 0.135 & $<4$ & 19 & 6 & 0.020 & 5590 & 5 & $<50$ & 27 & $<40$ \\
\hline 94VCK1(233-267) & 4 & 10.80 & $<4$ & $<4$ & 1.38 & 23 & 18 & 0.585 & 11320 & 15 & 0.145 & $\overline{6}$ & 23 & 13 & 0.015 & 6050 & $\overline{4}$ & $<50$ & 29 & $<40$ \\
\hline 94VCK2(300-333) & 3 & 10.90 & $<4$ & $<4$ & 1.37 & 24 & 13 & 0.585 & 11370 & 15 & 0.130 & $<4$ & 22 & 7 & 0.020 & 5250 & 5 & $<50$ & 26 & $<40$ \\
\hline 95PCK1(260-262) & 4 & 12.50 & 6 & $<4$ & 1.71 & 29 & 19 & 0.730 & 13590 & 11 & 0.155 & 9 & 27 & 9 & 0.030 & 12260 & 7 & $<50$ & 37 & $<40$ \\
\hline 95PCK1(297-326) & 4 & 8.02 & 12 & $<4$ & 1.85 & 38 & 23 & 0.645 & 7350 & 10 & 0.375 & 7 & 38 & 18 & 0.050 & 31970 & 9 & $<50$ & 50 & $<40$ \\
\hline 95PCK1 & $<2$ & 2.97 & 21 & $<4$ & 2.06 & 43 & 30 & 0.625 & 1390 & 5 & 0.490 & $<4$ & 38 & 14 & 0.035 & 780 & 9 & $<50$ & 46 & $<40$ \\
\hline 95PCK1( & $<2$ & 2.92 & 21 & $<4$ & 2.04 & 43 & 28 & 0.610 & 1350 & 5 & 0.485 & $<4$ & 38 & 15 & 0.035 & 767 & 9 & $<50$ & 46 & $<40$ \\
\hline 95PCUD & 4 & 9.78 & 17 & $<4$ & 1.94 & 33 & 25 & 0.700 & 9220 & 13 & 0.295 & 5 & 35 & 12 & 0.045 & 34950 & 9 & $<50$ & 43 & $<40$ \\
\hline 95VCD3 & 3 & 6.52 & 7 & $<4$ & 1.94 & 33 & 24 & 0.635 & 5430 & 8 & 0.375 & 8 & 28 & 13 & 0.035 & 22150 & 10 & $<50$ & 45 & $<40$ \\
\hline 95VCD3 & 4 & 12.30 & 6 & $<4$ & 1.24 & 18 & 13 & 0.660 & 13710 & 10 & 0.135 & 10 & 18 & 6 & 0.020 & 8280 & 5 & $<50$ & 26 & $<40$ \\
\hline 95VCD3(195.5-214) & 4 & 12.60 & $<4$ & $<4$ & 1.29 & 20 & 15 & 0.680 & 14170 & 6 & 0.140 & 9 & 24 & 5 & 0.020 & 8620 & 5 & $<50$ & 28 & $<40$ \\
\hline 95VCUD1(266-286) & 3 & 10.50 & 12 & $<4$ & 1.53 & 24 & 18 & 0.680 & 11180 & 11 & 0.185 & 4 & 23 & 5 & 0.020 & 10370 & 6 & $<50$ & 34 & $<40$ \\
\hline 95VCUD1(25-50) & 2 & 5.24 & 10 & $<4$ & 1.78 & 28 & 19 & 0.490 & 4460 & 4 & 0.290 & 5 & 23 & 8 & 0.020 & 2530 & $\overline{6}$ & $<50$ & 35 & $<40$ \\
\hline $96 \mathrm{~K} 114 \mathrm{E}(140-160)$ & 2 & 9.85 & 6 & $<4$ & 1.50 & 31 & 19 & 0.625 & 9460 & $\overline{7}$ & 0.265 & $\overline{7}$ & 29 & $\overline{12}$ & 0.035 & 7650 & $\overline{7}$ & $<50$ & 35 & $<40$ \\
\hline $96 \mathrm{~K} 114 \mathrm{E}(140-160)$ & 3 & 9.88 & $<4$ & $<4$ & 1.45 & 28 & 21 & 0.575 & 9100 & 7 & 0.265 & 9 & 26 & 17 & 0.030 & 7620 & 5 & $<50$ & 35 & $<40$ \\
\hline
\end{tabular}


Appendix G. Chemical composition of samples analyzed by ICP-AES at XRAL Labs in Golden, CO using 4 acid dissolution (Appendix_G.xls, Appendix_G.dbf).

\begin{tabular}{|c|r|c|c|r|r|r|r|}
\hline Sample No. & $\begin{array}{c}\text { Th } \\
(\mathrm{ppm})\end{array}$ & $\begin{array}{c}\mathrm{Ti} \\
(\%)\end{array}$ & $\begin{array}{c}\mathrm{U} \\
(\mathrm{ppm})\end{array}$ & $\begin{array}{c}\mathrm{V} \\
(\mathrm{ppm})\end{array}$ & $\begin{array}{c}\mathrm{Y} \\
(\mathrm{ppm})\end{array}$ & $\begin{array}{c}\mathrm{Yb} \\
(\mathrm{ppm})\end{array}$ & $\begin{array}{c}\mathrm{Zn} \\
(\mathrm{ppm})\end{array}$ \\
\hline SAMPLE_NO. & TH_PPM & $\mathrm{TI} \%$ & U_PPM & V_PPM & Y_PPM & YB_PPM & ZN_PPM \\
\hline 94VCK1(0-33) & 12 & 0.144 & $<100$ & 33 & 10 & $<1$ & 5440 \\
\hline 94VCK1(233-267) & 7 & 0.106 & $<100$ & 25 & 9 & $<1$ & 6180 \\
\hline 94VCK1(233-267) & 10 & 0.113 & $<100$ & 27 & 9 & $<1$ & 6920 \\
\hline 94VCK2(300-333) & 7 & 0.106 & $<100$ & 27 & 10 & 1 & 5450 \\
\hline 95PCK1(260-262) & 10 & 0.112 & $<100$ & 35 & 12 & 1 & 9260 \\
\hline 95PCK1(297-326) & 13 & 0.182 & $<100$ & 42 & 15 & 2 & 11690 \\
\hline 95PCK1(415-418) & 14 & 0.288 & $<100$ & 47 & 16 & 2 & 750 \\
\hline 95PCK1(415-418) & 13 & 0.276 & $<100$ & 45 & 16 & 2 & 690 \\
\hline 95PCUD2(0-25) & 11 & 0.165 & $<100$ & 44 & 15 & 2 & 12140 \\
\hline 95VCD3(402-423) & 11 & 0.2 & $<100$ & 43 & 14 & 2 & 8430 \\
\hline 95VCD3(195.5-214) & 8 & 0.106 & $<100$ & 24 & 9 & $<1$ & 10470 \\
\hline 95VCD3(195.5-214) & 7 & 0.1 & $<100$ & 25 & 9 & $<1$ & 11130 \\
\hline 95VCUD1(266-286) & 10 & 0.123 & $<100$ & 31 & 11 & 1 & 10490 \\
\hline 95VCUD1(25-50) & 7 & 0.135 & $<100$ & 31 & 9 & 2 & 1860 \\
\hline 96K114E(140-160) & 9 & 0.141 & $<100$ & 35 & 11 & 1 & 3260 \\
\hline 96K114E(140-160) & 13 & 0.138 & $<100$ & 34 & 11 & 1 & 3400 \\
\hline
\end{tabular}


Appendix H. Chemical composition of samples analyzed by ICP-AES at ACZ Labs in Steamboat Springs, CO using nitric acid dissolution (Appendix_H.xls, Appendix_H.dbf).

\begin{tabular}{|c|c|c|c|c|c|c|c|c|c|c|}
\hline Sample No. & Site ID & $\begin{array}{l}\text { Depth interval, } \\
\text { top }(\mathrm{cm})\end{array}$ & $\begin{array}{c}\text { Depth interval, } \\
\text { bottom }(\mathrm{cm})\end{array}$ & $\begin{array}{c}{ }^{\star} \text { Other } \\
\text { Analyses } \\
(\text { Appendix \#) }\end{array}$ & $\begin{array}{c}\text { As } \\
(p p m)\end{array}$ & $\begin{array}{c}\mathrm{Cd} \\
(\mathrm{ppm})\end{array}$ & $\begin{array}{l}\mathrm{Fe} \\
(\%)\end{array}$ & $\begin{array}{c}\mathrm{Pb} \\
(\mathrm{ppm})\end{array}$ & $\begin{array}{c}\mathrm{Mn} \\
(\mathrm{ppm})\end{array}$ & $\begin{array}{c}\mathrm{Zn} \\
(\mathrm{ppm})\end{array}$ \\
\hline SAMPLE_NO. & SITE_ID & INT_TOP_CM & INT_BTM_CM & OTHR_ANLYS & AS_PPM & CD_PPM & $\mathrm{FE}_{-} \%$ & PB_PPM & MN_PPM & ZN_PPM \\
\hline 94Gid2(0-30) & 94Gid2 & 0 & 30 & $\mathrm{E}, \mathrm{G}$ & 116 & 16.8 & 8.89 & 4380 & 7150 & 2390 \\
\hline 94Gid2(30-60) & 94Gid2 & 30 & 60 & $\mathrm{E}, \mathrm{G}$ & 130 & 34.2 & 9.17 & 4750 & 7580 & 4790 \\
\hline 94Gid2(60-90) & 94Gid2 & 60 & 90 & $\mathrm{E}, \mathrm{G}$ & 433 & 28.2 & 13.2 & 3470 & 12500 & 5300 \\
\hline 94Gid2(250-280) & 94Gid2 & 250 & 280 & $\mathrm{E}, \mathrm{G}$ & 63.1 & 83.7 & 12.9 & 17600 & 13700 & 16700 \\
\hline 94Gid3(3-12) & 94Gid3 & 3 & 12 & $\mathrm{E}, \mathrm{G}$ & 108 & 22.4 & 11.8 & 4320 & 11800 & 4730 \\
\hline 94Gid3(244-284) & 94Gid3 & 244 & 284 & $\mathrm{E}, \mathrm{G}$ & 12.5 & 1.5 & 1.9 & 380 & 872 & 362 \\
\hline 94Gid4(31-63) & 94Gid4 & 31 & 63 & $\mathrm{E}, \mathrm{G}$ & 107 & 16.1 & 11 & 13100 & 6810 & 4390 \\
\hline 94Gid6(0-20) & 94Gid6 & 0 & 20 & $\mathrm{E}, \mathrm{G}$ & 101 & 12.8 & 7.85 & 7500 & 6660 & 1790 \\
\hline 94VC1(10-20) & 94VC1 & 10 & 20 & $\mathrm{E}, \mathrm{G}$ & 52.5 & 14.7 & 6.4 & 2640 & 6050 & 3470 \\
\hline 94VC1(140-150) & 94VC1 & 140 & 150 & $\mathrm{E}, \mathrm{G}$ & 76.4 & 119 & 17.7 & 14300 & 18700 & 21500 \\
\hline 94VC1(150-160) & 94VC1 & 150 & 160 & $\mathrm{E}, \mathrm{G}$ & & 138 & 15.5 & 20200 & 19200 & 20700 \\
\hline 94VC1(180-190) & 94VC1 & 180 & 190 & $\mathrm{E}, \mathrm{G}$ & 69 & 102 & 16.9 & 19200 & 17800 & 18500 \\
\hline 94VC1(190-200) & 94VC1 & 190 & 200 & $\mathrm{E}, \mathrm{G}$ & 80.2 & 165 & 19.3 & 15500 & 21900 & 27100 \\
\hline 94VC1(200-207) & 94VC1 & 200 & 207 & $\mathrm{E}, \mathrm{G}$ & & 174 & 19.3 & 13600 & 24300 & 26900 \\
\hline 94VCD3(67-100) & 94VCD3 & 67 & 100 & $\mathrm{E}, \mathrm{G}$ & & & 1.13 & 26.3 & 73.8 & 64.8 \\
\hline 94VCK1(0-33) & 94VCK1 & 0 & 33 & $\mathrm{E}, \mathrm{G}$ & 46.4 & 30.1 & 9.79 & 2820 & 9800 & 5920 \\
\hline 94VCK1(233-267) & 94VCK1 & 233 & 267 & $\mathrm{E}, \mathrm{G}$ & 74 & 43.1 & 11 & 5910 & 11700 & 8120 \\
\hline 94VCK2(300-333) & 94VCK2 & 300 & 333 & $\mathrm{E}, \mathrm{G}$ & 116 & 38 & 11.6 & 5450 & 12300 & 7030 \\
\hline 95PCK1(260-262) & 95PCK1 & 260 & 262 & $\mathrm{E}, \mathrm{G}$ & 141 & 65 & 13.8 & 12600 & 14800 & 11800 \\
\hline 95PCK1(297-326) & 95PCK1 & 297 & 326 & $\mathrm{E}, \mathrm{G}$ & 16.1 & 92.8 & 8.01 & 32900 & 8130 & 15100 \\
\hline 95PCK1(415-418) & 95PCK1 & 415 & 418 & $\mathrm{E}, \mathrm{G}$ & 19.3 & 4 & 2.3 & 697 & 1290 & 752 \\
\hline 95PCUD2(0-25) & 95PCUD2 & 0 & 25 & $\mathrm{E}, \mathrm{G}$ & 51.2 & 90.1 & 9.33 & 35800 & 9760 & 15400 \\
\hline 95VCD3(195.5-214 & 95VCD3 & 195.5 & 214 & $\mathrm{E}, \mathrm{G}$ & 70.2 & 76.8 & 13.4 & 9300 & 15400 & 14400 \\
\hline 95VCD3(402-423) & 95VCD3 & 402 & 423 & $\mathrm{E}, \mathrm{G}$ & 13.9 & 64.8 & 6.12 & 23200 & 5870 & 10700 \\
\hline 95VCUD1(25-50) & 95VCUD1 & 25 & 50 & $\mathrm{E}, \mathrm{G}$ & 37 & 9.4 & 4.61 & 2200 & 4260 & 1950 \\
\hline 95VCUD1(266-286) & 95VCUD1 & 266 & 286 & $\mathrm{E}, \mathrm{G}$ & & 74.3 & 11.2 & 10500 & 12100 & 13500 \\
\hline 96K114E(140-160) & $96 \mathrm{~K} 114 \mathrm{E}$ & 140 & 160 & $\mathrm{E}, \mathrm{G}$ & 103 & 25 & 10 & 7400 & 9860 & 3620 \\
\hline
\end{tabular}

*Other analyses (Appendix \#): E =EWU, G =XRAL. 


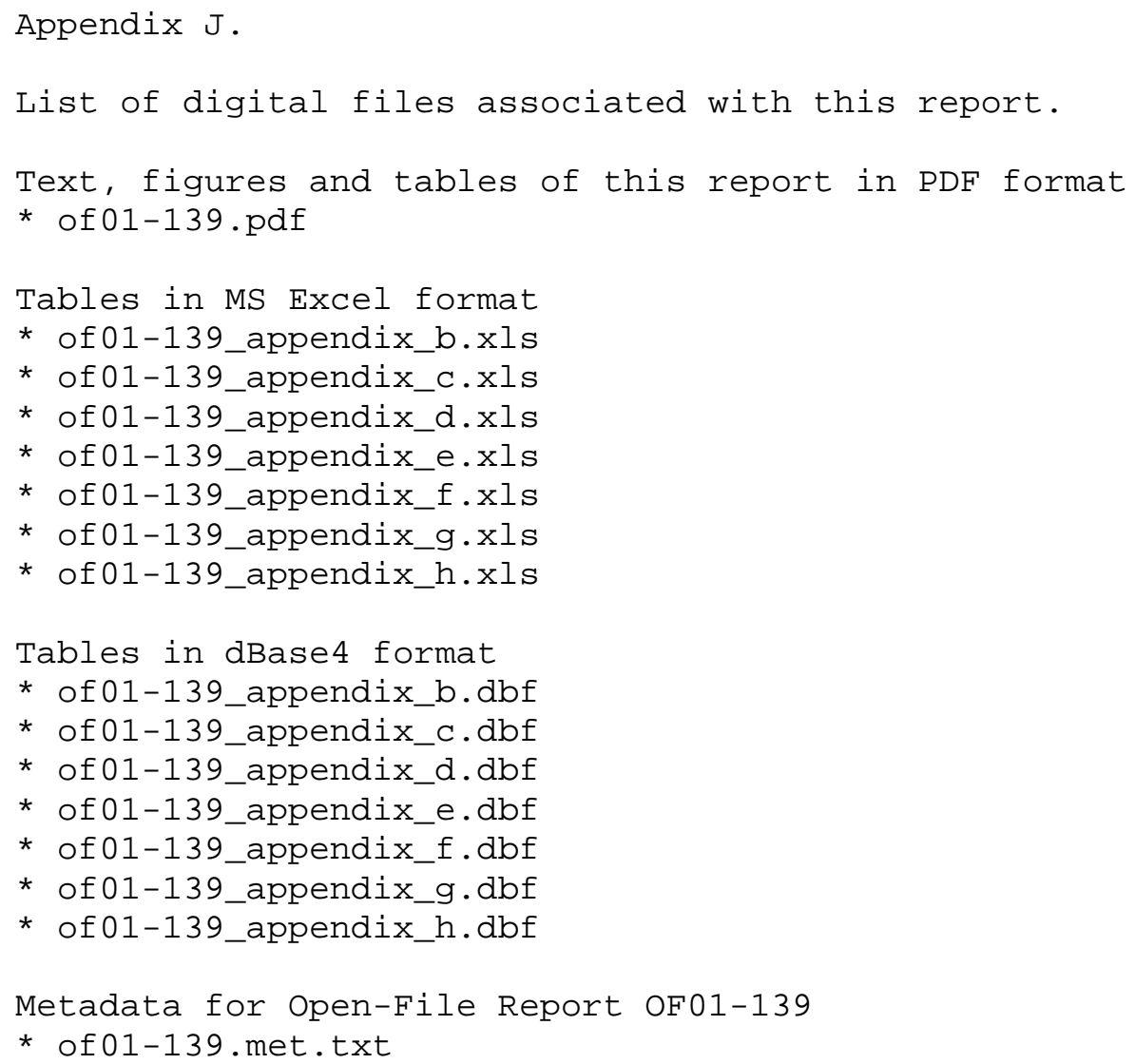

